SELECTED WATER-QUALITY DATA

FOR A COASTAL DUNES AQUIFER

NEAR COOS BAY, OREGON - 1971 TO 1983

By R. A. Dobberpuhl, J. E. Luzier, and C. A. Collins

U. S. Geological SuRvey

OPEN-FILE REPORT 84-858

PREPARED IN COOPERATION WITH THE

COOS BAY - NORTH BEND WATER BOARD 
U.S. DEPARTMENT OF THE INTERIOR

WILLIAM P. CLARK, Secretary

GEOLOGICAL SURVEY

Dallas Peck, Director

For additional information write to:

Oregon Office Chief U.S. Geological Survey 847 N.E. 19th Ave., Suite 300 Portland, Oregon 97232
Copies of this report can

be purchased from:

Open-File Services Section Western Distribution Branch Box 25425, Federal Center Denver, Colorado 80225 (Telephone: (303) 776-7476 
CONTENTS

Page

Abstract--

Introduction-- 1

Acknowledgements-a 3

Description of wells-- 3

Methods of sampling and analysis- 3

Selected references-_- 6

Data tables-_- 7

\section{ILLUSTRATIONS}

[Plate is in pocket]

Plate 1. Location of wells sampled for water-chemistry analyses, and cross sections west of Saunders Lake and Horsfall Beach study areas, Coos Bay, Oregon

Figure 1. Location of the coastal dunes study area------

\section{TABLES}

Table 1. Water-chemistry data from jetted wells in the deflation plain, July and August 1977-

2. Field water-chemistry data from jetted wells in the deflation plain, July and August 1977-..-

3. Water-chemistry data collected during a pumping test of well B4 near Horsfall Beach, October 1979 through December 1979---

4. Water-chemistry data collected from west of Saunders Lake, 1978-79-_ 28

5. Water-chemistry data collected from wells near Horsfall Beach, and from wells B5 and 44, 1978-82--- 29

6. Water-chemistry analyses from wells in areas west of Saunders Lake and near Horsfall Beach 1978-82--_--- 40

7. Water-chemistry data from profile well B5, 1981-83---- 43

8. Water-chemistry data from production wells, 1971-83--- 90

9. Miscellaneous water-quality data from wells in the study area--_- 189

10. Selected data for production wells of the Coos Bay-North Bend Water Board, 1983-_-_-_- 191

11. Construction data for profile and test wells in the coastal dunes aquifer-_-_- 


\title{
SELECTED WATER-QUALITY DATA FOR A COASTAL DUNES AQUIFER NEAR COOS BAY, OREGON - 1971 TO 1983
}

By R. A. Dobberpuhl, J.E. Luzier, and C. A. Collins

\begin{abstract}
An extensive amount of water-chemistry data were collected from the coastal dunes aquifer north of Coos Bay, Oregon from 1971 to 1983. The data were collected to support an iron geochemistry study of the dune area. The main study area was near Horsfall Beach. Additional areas of study were west of Saunders Lake and along the deflation plain between the Horsfall Beach area and Saunders Lake area. The Coos Bay-North Bend Water Board has monitored the water chemistry of a series of production wells in the dunes.
\end{abstract}

\section{INTRODUCTION}

The U.S. Geological Survey and the Coos Bay-North Bend Water Board have collected water-chemistry data from the coastal dunes aquifer north of Coos Bay, Oregon since 1971 ( $f$ ig. 1). The data were collected in response to long-term monitoring needs and in support of special studies of the behavior of iron-rich ground water. Most of the data were collected during the period 1978-83 at a cross section near Horsfall Beach ( $p \mid$. 1). The section consists of a number of pipe piezometers, two test wells, and 11 tube-screen profile wells (packages of vertically spaced sampling tubes) used to monitor water-chemistry changes through the full thickness of the aquifer under pumping and natural flow conditions.

A second section was also established near the beach, west of Saunders Lake, about 6 miles north of Horsfall Beach. The section consists of a shallow production well, two profile wells, and several piezometers ( $p$ l. 1).

Fifty-two test holes were jetted to depths in coastal deflation plain of approximately 20 feet and water samples collected after pumping the test holes at rates of abcut 40 gallons per minute (gal $/ \mathrm{min}$ ) for 30 minutes. The deflation plain as defined here is the areas east of the foredune where sand has been removed by wind action approximately to the elevation of the water table. The screens and casing were removed and the holes filled after sampling. The water-chemistry data are presented in tables 1 and 2 . 


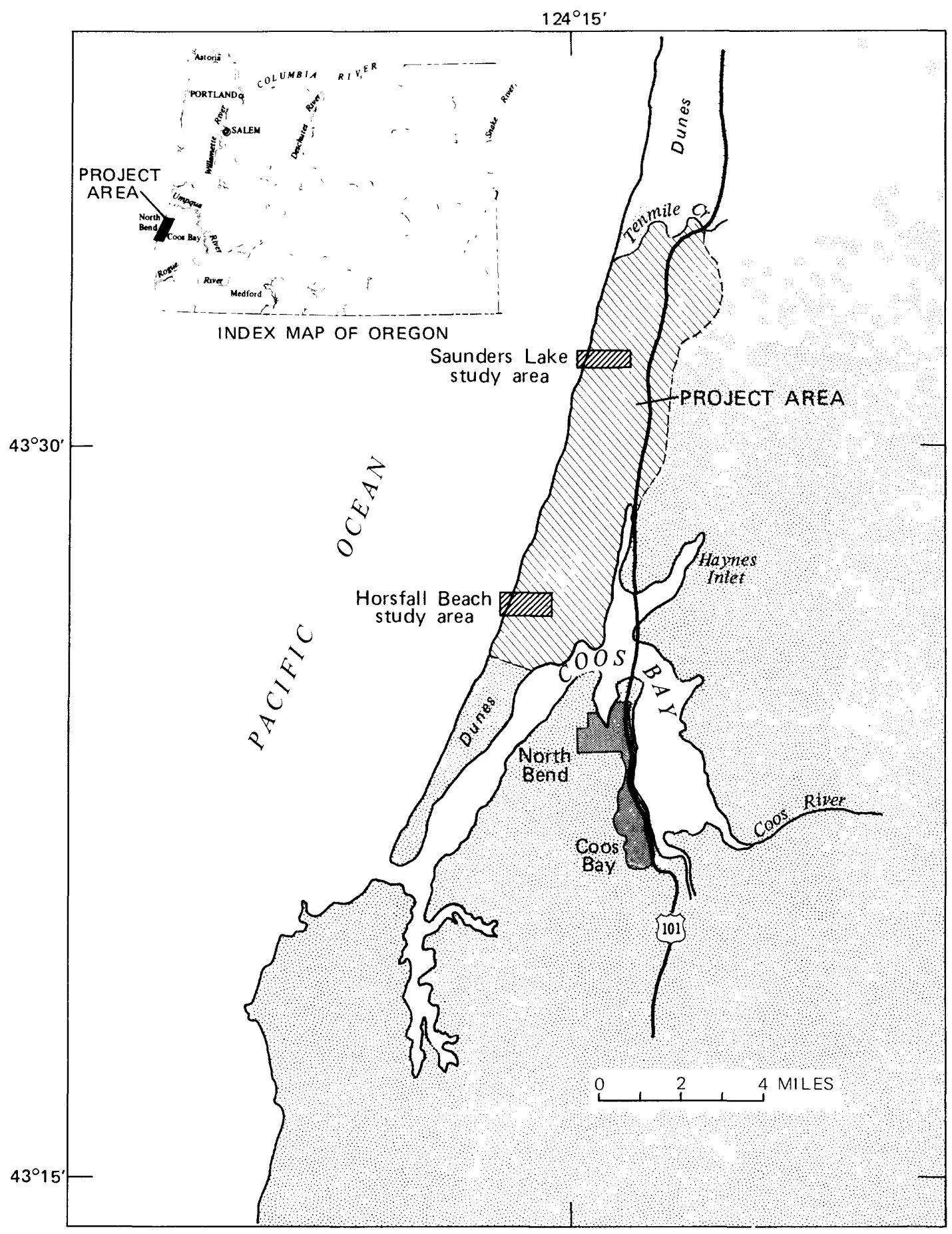

FIGURE 1. - Study area. 


\section{ACKNOWLEDGEMENTS}

The authors would like to give special thanks to the Coos Bay-North Bend Water Board personnel for their outstanding cooperation and technical assistance.

\section{DESCRIPTION OF WELLS}

Wells B1, B2, B3, B5, and P1 through P11 are tube-screen profile wells. Each of the $B$-wells has about 30 tube screens embedded vertically every 4 to 6 feet through the thickness of the aquifer. The P-wells penetrate only the upper one-third of the aquifer and contain about 10 tube screens each. The tube screens were constructed by perforating a one-foot section at the bottom of a length of .25-inch I.D., flexible polyvinyl cloride (PVC) tubing. The openings were then triple wrapped in 54 mesh stainless steel cloth, and the wrap retained with nylon ties and heat-shrunk tubing. Each of the deep profile wells (B1, B2, B3, B5) also contain a 1.25-inch PVC pipe perforated near the bottom. Special purpose piezometer wells, such as 100E, P44, 205, (mostly installed for pumping tests prior to 1978) consist of standard 1.25 to 2.0-inch galvanized pipe with a 3-foot stainless steel screen and drive point. Wells 41 through 60 are production wells operated by Coos Bay-North Bend Water Board ( $\mathrm{l}$. . 1). Screened aquifer-test wells drilled as part of the study include HB (Horsfall Beach), NTW (North Test Well), and B4. Tables 10 and 11 provide summarized information concerning the construction of all wells and piezometers.

\section{METHODS OF SAMPLING AND ANALYSIS}

Methods of data collection and analysis for tables 1-9 are described in the following sections. Bicarbonate was derived from field measurements of alkalinity in tables $1,2,3,5$, and 6 .

Table 1 and 2: Temperature, specific conductance, dissolved iron, $\mathrm{pH}$, and dissolved oxygen were measured by Coos Bay-North Bend Water Board personnel using (1) a yellow Springs Model 57 DO meter, (2) an Orion Model 399A pH meter, (3) the colorimetric test of the Hach kit DRI Region 854 for dissolved iron, and (4) a Lab-line Meter and cell for specific conductance.1/ In table 2, where two analyses are shown at the same depth for a given date, the first analysis listed was determined at the sampling site, and the second analysis listed was determined at the Coos Bay-North Bend water laboratory. Titrations for alkalinity and chloride were conducted at the laboratory of the Coos Bay-North Bend Water Board. All other determinations were made by the U.S. Geological Survey Central Laboratory, Arvada, Colorado, using methods described by Skougstad and others, (1979).

1/ Use of brand names in this report are for identification purposes only and does not constitute endorsement by the U.S. Geological Survey. 
Table 3: Data measurements at Horsfall Beach from October, 1979 through December, 1979 were performed by U.S. Geological Survey personnel using (1) a Lab-line meter and cell for specific conductance, (2) an Orion Meter and probe Model 399A, or Corning Meter and probe Model 3D for $\mathrm{pH}$, and (3) hand-held thermometer for temperature. Dissolved iron determinations were performed by U.S. Geological Survey personnel at the Coos Bay-North Bend Water Board Laboratory using the colorimetric, bipyridine method with a Bausch and Lomb spectrometer Model 20. Alkalinity was determined by electrometric titration (Skougstad and others, 1979).

Table 4: Measurements west of Saunders Lake during the period 1978-79 were performed by U.S. Geologial Survey personnel using the same methods and instrumentation described for table 3 , except dissolved iron, and some chloride determinations which were conducted by the U.S. Geological Survey Central Laboratory, Arvada, Colorado.

Table 5: For seasonal measurements prior to October, 1981, the same procedures were used by U.S. Geological Survey personnel as for table 3 except that iron and chloride values were determined by the U.S. Geological Survey Central Laboratory. In 1981 a vertical flow-through water chamber was developed by the authors to accommodate probes for the simultaneous determination of $\mathrm{pH}$, temperature, specific conductance, Eh, and DO. The chamber was flooded from below using a peristaltic pump, and readings were recorded at 5- to 10-minute intervals for 20 to 30 minutes. Parameter equilibration was usually reached within 5 to 10 minutes after initial flooding of the chamber. The flow was stopped for final readings, and water was then diverted ahead of the chamber by use of a valve for sample collection and immediate field titrations. An Orion Model 399A Meter and Model 91-04 probe were used for $\mathrm{pH}$ measurements. A Yellow Springs Instruments (YSI) thermistor (thermometer Model 3220), a YSI conductance cell, and a YSI Meter Model 32 were used for temperature and conductance. A YSI probe Model 5739 and meter Model 57 were used for measuring DO. Eh was determined using an Orion meter Model 399A, with a Model 967800 combination platinum redox probe, using methods outlined by Wood (1976), and Nordstrom (1977). Alkalinity and sulfide titrations were performed in the field after August, 1981, using standard methods described by Skougstad and others, (1979). Dissolved iron was determined at the District Laboratory, U.S. Geological Survey, Portland, Oregon using the colorimetric, bipyridine method with a Bausch and Lomb spectrometer. Checks on quality control were performed by sending blind samples to the U.S. Geological Survey Central Laboratory, Arvada, Colorado. 
Table 6: Determinations for hardness, potassium, calcium, magnesium, sulfate, silica, sodium, and chloride were performed by U.S. Geological Survey Central Laboratory. All other measurements were conducted by U.S. Geological Survey personnel using methods described previously.

Table 7 and 8: Temperature and specific conductance were read with a YSI digital conductance meter. Dissolved iron concentrations were determined using a Hach kit DRI Region 854 colorimetric test. An Orion Meter Model 399A was used to measure $\mathrm{pH}$. Total alkalinity, total hardness, and chloride were determined using titration methods. Carbon dioxide was determined from a Languir chart, and fluoride with a specific-ion electrode and Orion Model 407 meter.

Table 9: Determinations for arsenic, boron, manganese, aluminum, bromide, strontium, and organic carbon were performed by U.S. Geological Survey Central Laboratory. 


\section{SELECTED REFERENCES}

Nordstrom, D. K., 1977, Thermochemical redox equilibria of Zobell's solution: Geochimica et Cosmochimica Acta, v. 44, p. 1835-1841.

Robison, J. H., 1973, Hydrology of the dunes area north of Coos Bay, Oregon: U.S. Geological Survey Open-File report, 62 p.

Skougstad, M. W., Fishman, M. J., Friedman, L. C., Erdmann, D. E., and Duncan, S. S., [eds.], 1979, Methods for analysis of inorganic substances in water and fluvial sediments: U.S. Geological Survey Techniques of Water-Resources Investigations, Book 5, Chap. A1, $626 \mathrm{p}$.

Wood, W. W., 1976, Guidelines for collection and field analysis of ground-water samples for unstable constituents: U.S. Geological Survey Techniques of Water-Resources Investigations, Book 1, Chap. D2, $24 p$. 


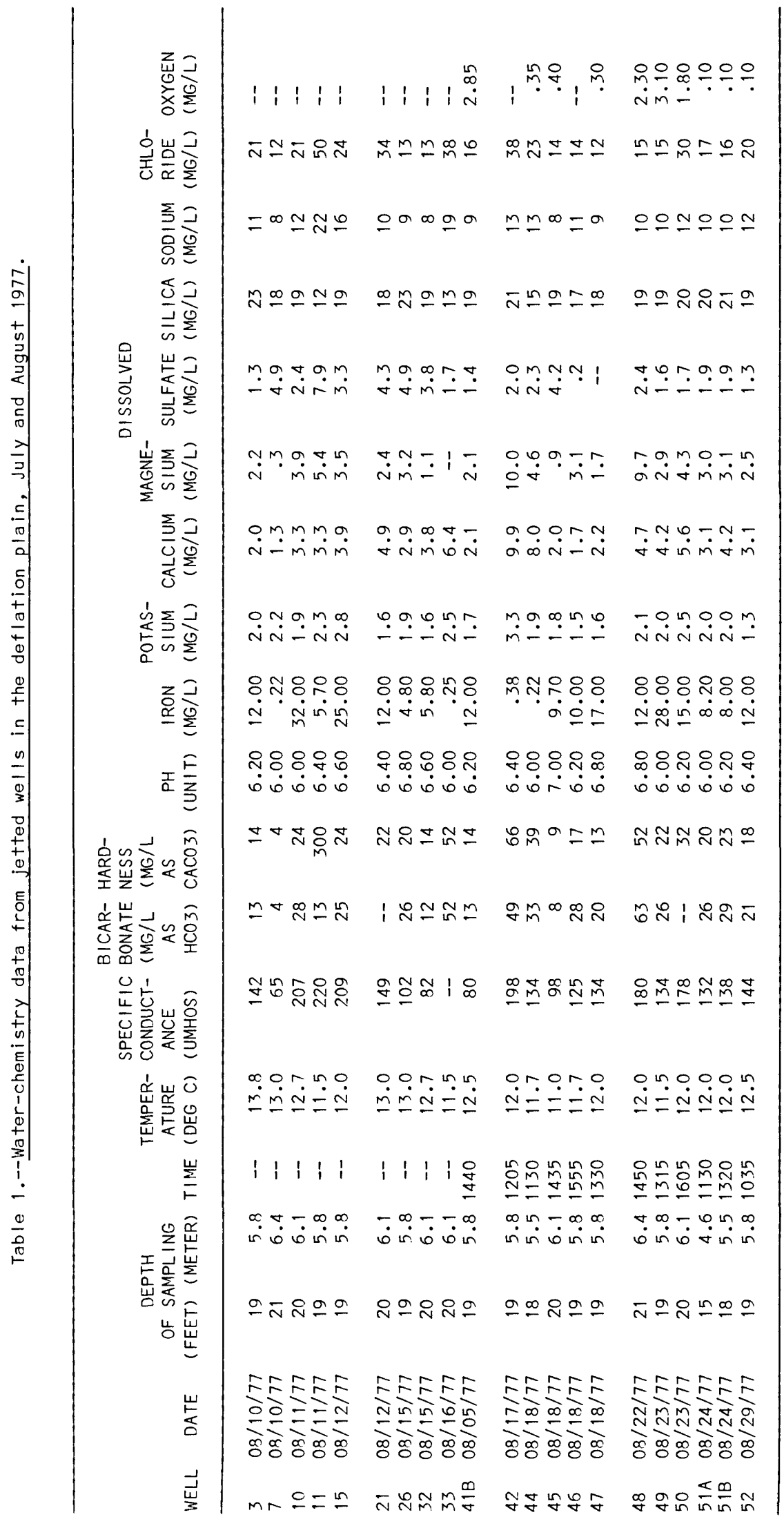


Table 2.--Field water-chemistry data from jetted wells in the deflation plain, July and August 1977

\begin{tabular}{|c|c|c|c|c|c|c|c|c|c|}
\hline WELL & DATE & $\begin{array}{r}\text { OF } \\
\text { (FEFT) }\end{array}$ & $\begin{array}{l}\text { DEPTH } \\
\text { SAMPL ING } \\
\text { (METER) }\end{array}$ & $\begin{array}{l}\text { TEMPER- } \\
\text { ATURE } \\
(\text { DEG C) }\end{array}$ & $\begin{array}{l}\text { SPECIFIC } \\
\text { CONDUCT- } \\
\text { ANCE } \\
\text { (UMHOS) }\end{array}$ & $\begin{array}{c}\text { BICAR- } \\
\text { BONATE } \\
\text { (MG/L } \\
\text { AS } \\
\text { HCO3) }\end{array}$ & $\begin{array}{l}\text { IRON } \\
\text { DIS- } \\
\text { SOLVED } \\
(M G / L)\end{array}$ & $\begin{array}{c}\text { PH } \\
(\text { UNIT) }\end{array}$ & $\begin{array}{l}\text { CHLO- } \\
\text { RIDE } \\
\text { DIS- } \\
\text { SOLVED } \\
(M G / L)\end{array}$ \\
\hline 1 & $07 / 13 / 77$ & 17 & 5.2 & 15.0 & -- & -- & 9.60 & -- & - \\
\hline 2 & $07 / 13 / 77$ & 21 & 6.4 & -- & - & 85 & 2.50 & 6.20 & 24 \\
\hline 2 & $07 / 13 / 77$ & 21 & 5.4 & 12.7 & -- & -- & 3.80 & -- & - \\
\hline 3 & $07 / 14 / 77$ & 19 & 5.8 & 13.3 & -- & -- & 12.00 & 6.00 & -- \\
\hline 3 & $07 / 14 / 77$ & 19 & 5.8 & -- & -- & 61 & 15.00 & 5.20 & 20 \\
\hline 4 & $07 / 15 / 77$ & 20 & 6.1 & 12.2 & -- & -- & 2.20 & 6.00 & -- \\
\hline 4 & $07 / 15 / 77$ & 20 & 6.1 & -- & -- & 6 & .30 & 6.10 & 30 \\
\hline 5 & $07 / 15 / 77$ & - & -- & 13.3 & - & -- & 19.00 & 6.00 & - \\
\hline 5 & $07 / 15 / 77$ & -- & -- & -- & -- & 85 & 27.50 & 6.00 & 48 \\
\hline 5 & $07 i 15 / 77$ & 19 & 5.8 & 13.3 & -- & -- & 5.40 & 6.20 & -- \\
\hline 6 & $07 / 15 / 77$ & 19 & 5.8 & -- & - & 12 & 6.50 & 6.00 & 23 \\
\hline 7 & $07 / 18 / 77$ & 21 & 6.4 & 13.6 & -- & 10 & 6.00 & 6.10 & 15 \\
\hline 8 & $07 / 18 / 77$ & 21 & 6.4 & 11.9 & -- & -- & .80 & 6.20 & -- \\
\hline 8 & $07 / 18 / 77$ & 21 & 6.4 & -- & -- & -- & .10 & 6.00 & 18 \\
\hline 9 & $07 / 18 / 77$ & 19 & 5.8 & 12.7 & -- & -- & 15.40 & -- & -- \\
\hline 9 & $07 / 18 / 77$ & 19 & 5.8 & -- & -- & 44 & 20.00 & & 18 \\
\hline 10 & $07 / 19 / 77$ & 20 & 5.1 & 13.3 & -- & -- & 25.60 & 6.20 & -- \\
\hline 10 & $07 / 19 / 77$ & 20 & 6.1 & -- & -- & 73 & 30.00 & 5.90 & 22 \\
\hline 11 & $07 / 19 / 77$ & 19 & 5.8 & 12.7 & -- & -- & 5.80 & 6.10 & -- \\
\hline 11 & $07 / 19 / 77$ & 19 & 5.8 & -- & -- & 24 & 10.40 & 5.90 & 20 \\
\hline 12 & $07 / 19 / 77$ & 19 & 5.8 & 13.3 & -- & -- & 5.60 & 6.40 & -- \\
\hline 12 & $07 / 19 / 77$ & 19 & 5.8 & -- & -- & 20 & 10.00 & 5.90 & 25 \\
\hline 13 & $07 / 19 / 77$ & 20 & 6.1 & 13.3 & -- & -- & 5.00 & 6.60 & -- \\
\hline 13 & $07 / 19 / 77$ & 20 & 6.1 & - & -- & 22 & 9.50 & 5.90 & 12 \\
\hline
\end{tabular}


Table 2.--Field water-chemistry data from jetted wells in the deflation plain, July and August 1977--Continued

\begin{tabular}{|c|c|c|c|c|c|c|c|c|c|}
\hline WELL & DATE & $\begin{array}{c}\text { OF } S A \\
(F E E T)\end{array}$ & $\begin{array}{l}\text { EPTH } \\
\text { AMPL ING } \\
\text { (METER) }\end{array}$ & $\begin{array}{l}\text { TEMPER- } \\
\text { ATURE } \\
(\text { DEG C) }\end{array}$ & $\begin{array}{l}\text { SPECIFIC } \\
\text { CONDUCT- } \\
\text { ANCE } \\
\text { (UMHOS) }\end{array}$ & $\begin{array}{l}\text { BICAR- } \\
\text { BONATE } \\
\text { (MG/L } \\
\text { AS } \\
\mathrm{HCO} 3 \text { ) }\end{array}$ & $\begin{array}{l}\text { IRON } \\
\text { DIS- } \\
\text { SOLVED } \\
(M G / L)\end{array}$ & $\begin{array}{c}\mathrm{PH} \\
(\text { UNIT) }\end{array}$ & $\begin{array}{l}\text { CHLO- } \\
\text { RIDE } \\
\text { חIS- } \\
\text { SOLVED } \\
(M G / L)\end{array}$ \\
\hline 14 & $07 / 21 / 77$ & 16 & 4.9 & 12.7 & -- & -- & .80 & 6.00 & -- \\
\hline 14 & $07 / 21 / 77$ & 16 & 4.9 & -- & -- & 34 & .20 & 5.90 & 48 \\
\hline 15 & $07 / 21 / 77$ & 19 & 5.8 & 12.2 & -- & -- & 3.00 & 6.40 & -- \\
\hline 15 & $07 / 21 / 77$ & 19 & 5.8 & -- & -- & 73 & 25.00 & 5.90 & -- \\
\hline 16 & $07 / 21 / 77$ & 21 & 6.4 & 12.2 & -- & -- & 15.20 & 6.40 & -- \\
\hline 16 & $07 / 21 / 77$ & 21 & 5.4 & -- & -- & -- & 20.00 & 6.30 & 23 \\
\hline 17 & $07 / 22 / 77$ & 22 & 6.7 & 12.7 & -- & 10 & 7.00 & 6.20 & -- \\
\hline 17 & $07 / 22 / 77$ & 22 & 6.7 & -- & -- & 37 & 10.50 & 5.80 & 15 \\
\hline 18 & $07 / 72 / 77$ & 20 & 6.1 & 13.3 & -- & -- & 7.50 & 6.20 & -- \\
\hline 18 & $07 / 22 / 77$ & 20 & 6.1 & -- & -- & 27 & 3.00 & 5.90 & 13 \\
\hline 19 & $07 / 26 / 77$ & 20 & 6.1 & 12.2 & -- & -- & 1.00 & 6.00 & -- \\
\hline 19 & $07 / 26 / 77$ & 20 & 6.1 & -- & -- & 37 & .35 & 5.80 & 66 \\
\hline 20 & $07 / 26 / 77$ & 20 & 6.1 & 12.7 & -- & -- & .80 & 6.00 & -- \\
\hline 20 & $07 / 26 / 77$ & 20 & 6.1 & -- & -- & 102 & .25 & 6.10 & 24 \\
\hline 21 & $07 / 26 / 77$ & 20 & 6.1 & 13.3 & -- & -- & 14.80 & 6.00 & -- \\
\hline 21 & $07 / 26 / 77$ & 20 & 6.1 & -- & -- & 51 & 15.00 & 6.00 & 24 \\
\hline 22 & $07 / 27 / 77$ & 21 & 5.4 & 13.8 & -- & -- & 11.00 & 5.00 & -- \\
\hline 22 & $07 / 27 / 77$ & 21 & 6.4 & -- & -- & 37 & 15.00 & 5.80 & 10 \\
\hline 23 & $07 / 27 / 77$ & 16 & 4.9 & 11.6 & -- & -- & 15.00 & 6.00 & -- \\
\hline 23 & $07 / 27 / 77$ & 16 & 4.9 & -- & -- & 85 & 17.50 & 6.00 & 30 \\
\hline 24 & $07 / 28 / 77$ & 20 & 6.1 & 12.7 & -- & -- & 14.00 & 6.60 & -- \\
\hline 24 & $07 / 28 / 77$ & 20 & 6.1 & -- & -- & 85 & 20.00 & 6.20 & 18 \\
\hline 25 & $07 / 28 / 77$ & 20 & 6.1 & 13.3 & -- & -- & 8.00 & 6.60 & -- \\
\hline 25 & $07 / 28 / 77$ & 20 & 6.1 & -- & -- & 37 & 10.00 & 6.10 & 18 \\
\hline 26 & $07 / 28 / 77$ & 19 & 5.8 & -- & -- & -- & 5.50 & 5.40 & -- \\
\hline 26 & $07 / 28 / 77$ & 19 & 5.8 & -- & -- & 37 & 7.50 & 6.10 & 12 \\
\hline
\end{tabular}


Table 2.--Field water-chemistry data from jetted wells in the deflation plain, July and August 1977--Continued

\begin{tabular}{|c|c|c|c|c|c|c|c|c|c|}
\hline WELL & DATE & $\begin{array}{r}\text { OF } \\
(F E E T)\end{array}$ & $\begin{array}{l}\text { EPTH } \\
\text { AMPL ING } \\
\text { (METER) }\end{array}$ & $\begin{array}{l}\text { TEMPER- } \\
\text { ATURE } \\
(\text { DEG C) }\end{array}$ & $\begin{array}{l}\text { SPECIFIC } \\
\text { CONDUCT- } \\
\text { ANCE } \\
\text { (UMHOS) }\end{array}$ & $\begin{array}{c}\text { BICAR- } \\
\text { BONATE } \\
\text { (MG/L } \\
\text { AS } \\
\text { HCO3) }\end{array}$ & $\begin{array}{l}\text { IRON } \\
\text { DIS- } \\
\text { SOLVED } \\
(M G / L)\end{array}$ & $\begin{array}{c}\text { PH } \\
(\text { UNIT) }\end{array}$ & $\begin{array}{l}\text { CHLO- } \\
\text { RIDE } \\
\text { DIS- } \\
\text { SOLVED } \\
(M G / L)\end{array}$ \\
\hline 27 & $07 / 28 / 77$ & 20 & 6.1 & 13.3 & -- & -- & 4.50 & 6.60 & -- \\
\hline 27 & $07 / 28 / 77$ & 20 & 5.1 & -- & -- & 37 & 7.50 & 6.20 & 12 \\
\hline 28 & $07 / 29 / 77$ & 19 & 5.8 & 12.2 & -- & -- & 3.00 & 6.40 & - \\
\hline 28 & $07 / 29 / 77$ & 19 & 5.8 & -- & -- & 37 & 1.70 & 6.30 & 13 \\
\hline 29 & $07 / 29 / 77$ & 19 & 5.8 & -- & -- & -- & 5.50 & 6.80 & - \\
\hline 29 & $07 / 29 / 77$ & 19 & 5.8 & -- & -- & 27 & 8.80 & 6.30 & 13 \\
\hline 30 & $07 / 29 / 77$ & 20 & 6.1 & -- & -- & - & 9.50 & 6.80 & - \\
\hline 30 & $07 / 29 / 77$ & 20 & 6.1 & -- & -- & 39 & 15.00 & 6.30 & 14 \\
\hline 31 & $08 / 01 / 77$ & 20 & 6.1 & -- & -- & -- & 15.50 & 6.40 & -- \\
\hline 31 & $08 / 01 / 77$ & 20 & 6.1 & -- & -- & 37 & 20.00 & 5.80 & 15 \\
\hline 32. & $08 / 01 / 77$ & 20 & 5.1 & -- & -- & -- & 5.20 & 6.60 & -- \\
\hline 32 & $08 / 01 / 77$ & 20 & 6.1 & -- & -- & 20 & 8.75 & 5.90 & 17 \\
\hline 33 & $0.9 / 01 / 77$ & 20 & 6.1 & -- & -- & -- & 1.20 & 6.00 & - \\
\hline 33 & $08 / 01 / 77$ & 20 & 6.1 & -- & -- & 49 & 2.50 & 5.90 & 30 \\
\hline 34 & $08 / 02 / 77$ & 20 & 6.1 & 12.7 & -- & -- & 6.20 & 5.00 & -- \\
\hline 34 & $08 / 02 / 77$ & 20 & 6.1 & -- & -- & 49 & 13.00 & 5.90 & 20 \\
\hline 35 & $08 / 02 / 77$ & 20 & 6.1 & 12.7 & -- & -- & 3.50 & 6.40 & -- \\
\hline 35 & $08 / 02 / 77$ & 20 & 6.1 & - & -- & 37 & 5.00 & 6.00 & 20 \\
\hline 36 & $08 / 02 / 77$ & 20 & 6.1 & 12.7 & -- & -- & 7.20 & 6.60 & -- \\
\hline 36 & $08 / 02 / 77$ & 20 & 6.1 & -- & -- & 37 & 12.00 & 5.90 & 15 \\
\hline 37 & $08 / 03 / 77$ & 20 & 6.1 & 12.2 & 180 & -- & 18.00 & 6.60 & - \\
\hline $38 \mathrm{~A}$ & $08 / 03 / 77$ & - & -- & 13.0 & 126 & -- & 8.00 & 6.60 & -- \\
\hline $38 B$ & $08 / 03 / 77$ & 20 & 6.1 & 12.5 & 180 & -- & 30.40 & 6.60 & - \\
\hline $39 A$ & $08 / 04 / 77$ & 14 & 4.3 & 13.0 & 142 & -- & 18.00 & 6.40 & - \\
\hline 398 & $08 / 04 / 77$ & 20 & 6.1 & 12.8 & 126 & -- & 15.00 & 6.40 & -- \\
\hline $40 \mathrm{~A}$ & $08 / 05 / 77$ & 14 & 4.3 & 13.0 & 142 & - & 22.00 & 6.00 & -- \\
\hline $40 B$ & $08 / 05 / 77$ & $? 1$ & 6.4 & 12.5 & 131 & - & 20.80 & 6.40 & -- \\
\hline
\end{tabular}


Table 2.--Field water-chemistry data from jetted wells in the deflation plain, July and August 1977--Continued

\begin{tabular}{|c|c|c|c|c|c|c|c|c|c|}
\hline WELL & DATE & $\begin{array}{c}\text { DE } S F \\
\text { (FEET) }\end{array}$ & $\begin{array}{l}\text { EPTH } \\
\text { AMPL ING } \\
\text { (METER) }\end{array}$ & $\begin{array}{c}\text { TEMPER- } \\
\text { ATURE } \\
(D E G \quad C)\end{array}$ & $\begin{array}{l}\text { SPECIFIC } \\
\text { CONDUCT- } \\
\text { ANCE } \\
\text { (UMHOS) }\end{array}$ & $\begin{array}{c}\text { BICAR- } \\
\text { BONATE } \\
\text { (MG/L } \\
\text { AS } \\
\text { HCO3) }\end{array}$ & $\begin{array}{l}\text { IRON } \\
\text { DIS- } \\
\text { SOLVED } \\
(M G / L)\end{array}$ & $\begin{array}{c}\mathrm{PH} \\
(\mathrm{UN} I T)\end{array}$ & $\begin{array}{l}\text { CHLO- } \\
\text { RIDE } \\
\text { DIS- } \\
\text { SOLVED } \\
(M G / L)\end{array}$ \\
\hline $41 B$ & $08 / 05 / 77$ & 19 & 5.8 & 12.5 & 111 & -- & 13.00 & 6.20 & - \\
\hline 42 & $08 / 17 / 77$ & 19 & 5.8 & 13.0 & 198 & -- & 1.60 & 6.20 & - \\
\hline $43 A$ & $03 / 05 / 77$ & 15 & 4.6 & 11.0 & 290 & -- & 20.00 & 6.20 & - \\
\hline $43 B$ & $08 / 08 / 77$ & 21 & 6.4 & 10.5 & 160 & -- & 12.40 & 6.00 & - \\
\hline 44 & $08 / 18 / 77$ & 18 & 5.5 & 11.8 & 134 & -- & 1.40 & 6.00 & - \\
\hline 45 & $08 / 18 / 77$ & 20 & 6.1 & 13.6 & 98 & -- & 7.00 & -- & - \\
\hline 46 & $08 / 18 / 77$ & 19 & 5.8 & 14.0 & 125 & -- & 11.75 & 6.20 & - \\
\hline 47 & $08 / 18 / 77$ & 19 & 5.8 & 12.0 & 1.34 & -- & 16.80 & 6.80 & - \\
\hline 48 & $08 / 22 / 77$ & 21 & 6.4 & 14.0 & 190 & -- & 14.00 & 6.80 & -- \\
\hline 49 & $08 / 23 / 77$ & 19 & 5.8 & 11.5 & 134 & - & 14.00 & 6.00 & -- \\
\hline 50 & $08 / 23 / 77$ & 20 & 6.1 & 12.0 & 178 & -- & 14.00 & 6.20 & -- \\
\hline $51 \mathrm{~A}$ & $08 / 24 / 77$ & 15 & 4.6 & 12.0 & 132 & -- & 12.00 & 6.00 & -- \\
\hline $51 \mathrm{~B}$ & $08 / 24 / 77$ & 18 & 5.5 & 12.0 & 138 & -- & 12.00 & -- & -- \\
\hline
\end{tabular}


Table 3.--Water-chemistry data collected during a pumping test of well B4, near Horsfall Beach, October 1979 through December 1979

\begin{tabular}{|c|c|c|c|c|c|c|c|c|c|}
\hline WELL & DATE & $\begin{array}{l}\text { ELEVA } \\
\text { (FEET) }\end{array}$ & $\begin{array}{l}\text { AT ION } \\
\text { (METER) }\end{array}$ & TIME & $\begin{array}{l}\text { TEMPER- } \\
\text { ATURE } \\
(\text { DEG C) }\end{array}$ & $\begin{array}{l}\text { SPECIFIC } \\
\text { CONDUCT- } \\
\text { ANCE } \\
\text { (UMHOS) }\end{array}$ & $\begin{array}{c}\text { BICAR- } \\
\text { BONATE } \\
\text { (MG/L } \\
\text { AS } \\
\text { HCO3) }\end{array}$ & $\begin{array}{l}\text { IRON } \\
\text { DIS- } \\
\text { SOLVED } \\
(M G / L)\end{array}$ & $\begin{array}{c}\mathrm{PH} \\
(\text { UNIT) }\end{array}$ \\
\hline B1 & $10 / 18 / 79$ & -2 & -0.6 & 1030 & 11.8 & 154 & -- & 2.40 & 6.69 \\
\hline B1 & $10 / 18 / 79$ & -9 & -2.7 & 1040 & 12.8 & 155 & -- & 1.30 & 6.76 \\
\hline B1 & $10 / 18 / 79$ & -15 & -4.6 & 1050 & 12.8 & 157 & -- & .77 & 6.41 \\
\hline B1 & $10 / 18 / 79$ & -21 & -6.4 & 1100 & 12.6 & 178 & -- & .96 & 6.30 \\
\hline B1 & $10 / 18 / 79$ & -27 & -8.2 & 1110 & 12.3 & 178 & -- & 1.10 & 6.38 \\
\hline B1 & $10 / 18 / 79$ & -33 & -10.1 & 1120 & 12.2 & 179 & -- & 1.10 & 6.38 \\
\hline B1 & $10 / 18 / 79$ & -39 & -11.9 & 1130 & 12.2 & 200 & -- & 1.40 & 6.30 \\
\hline B1 & $10 / 18 / 79$ & -45 & -13.7 & 1140 & 12.2 & 207 & -- & .64 & 6.42 \\
\hline B1 & $10 / 18 / 79$ & -51 & -15.5 & 1145 & 12.3 & 209 & -- & .50 & 6.44 \\
\hline B1 & $10 / 18 / 79$ & -57 & -17.4 & 1150 & 12.1 & 218 & -- & .36 & 6.34 \\
\hline B1 & $10 / 18 / 79$ & -63 & -19.2 & 1200 & 12.3 & 239 & -- & .44 & 6.70 \\
\hline B1 & $10 / 18 / 79$ & -69 & -21.0 & 1210 & 12.2 & 289 & -- & .46 & 6.80 \\
\hline B1 & $10 / 18 / 79$ & -75 & -22.9 & 1220 & 12.3 & 348 & -- & 2.10 & 6.70 \\
\hline B1 & $10 / 18 / 79$ & -81 & -24.7 & 1230 & 12.4 & 352 & -- & 1.90 & 6.86 \\
\hline B1 & $10 / 18 / 79$ & -87 & -26.5 & 1240 & 12.4 & 455 & -- & 1.40 & 7.18 \\
\hline B1 & $10 / 18 / 79$ & -93 & -28.3 & 1250 & 12.4 & 450 & -- & .29 & 7.60 \\
\hline B1 & $10 / 18 / 79$ & -99 & -30.2 & 1300 & 12.6 & 422 & -- & .19 & 7.80 \\
\hline B1 & $10 / 18 / 79$ & -105 & -32.0 & 1310 & 12.6 & 392 & -- & .04 & 7.92 \\
\hline $\mathrm{B1}$ & $10 / 18 / 79$ & -110 & -33.5 & 1320 & 12.6 & 408 & -- & .04 & 7.90 \\
\hline $\mathrm{B1}$ & $10 / 18 / 79$ & -122 & -37.2 & 1330 & 12.6 & 418 & -- & .01 & 7.92 \\
\hline B1 & $10 / 18 / 79$ & -129 & -39.3 & 1340 & 12.5 & 390 & -- & .06 & 7.96 \\
\hline B1 & $10 / 18 / 79$ & -135 & -41.1 & 1350 & 12.6 & 402 & -- & .13 & 7.85 \\
\hline$B 1$ & $10 / 18 / 79$ & -141 & -43.0 & 1400 & 12.6 & 435 & -- & .23 & 7.70 \\
\hline B1 & $10 / 18 / 79$ & -147 & -44.8 & 1410 & 13.0 & 2100 & -- & .34 & 7.55 \\
\hline B1 & $10 / 18 / 79$ & -153 & -46.6 & 1420 & 12.4 & 3640 & -- & .26 & 7.88 \\
\hline B1 & $10 / 18 / 79$ & -159 & -48.5 & 1430 & 12.4 & 4220 & -- & .41 & 7.70 \\
\hline B1 & $10 / 18 / 79$ & -165 & -50.3 & 1440 & 12.4 & 8160 & -- & 5.29 & 6.88 \\
\hline $\mathrm{B1}$ & $10 / 18 / 79$ & -173 & -52.7 & 1500 & 12.4 & 9500 & -- & 9.20 & 7.12 \\
\hline B1 & $10 / 18 / 79$ & -194 & -59.1 & 1510 & 12.4 & 14800 & -- & 46.00 & 7.42 \\
\hline B1 & $10 / 18 / 79$ & -200 & -61.0 & 1520 & 12.8 & 13200 & -- & 22.80 & 7.06 \\
\hline B1 & $11 / 02 / 79$ & -122 & -37.2 & 1340 & 13.2 & 400 & -- & .04 & 7.18 \\
\hline B1 & $11 / 02 / 79$ & -147 & -44.8 & 1330 & 14.8 & 750 & -- & .47 & 6.90 \\
\hline $\mathrm{B1}$ & $11 / 02 / 79$ & -153 & -46.6 & 1320 & 13.6 & 2850 & -- & .27 & 7.74 \\
\hline $\mathrm{B1}$ & $11 / 02 / 79$ & -159 & -48.5 & 1310 & 13.3 & 4480 & -- & .43 & 7.74 \\
\hline B1 & $11 / 02 / 79$ & -165 & -50.3 & 1300 & 13.3 & 8700 & -- & 1.90 & 7.41 \\
\hline B1 & $11 / 02 / 79$ & -173 & -52.7 & 1250 & 13.7 & 9330 & -- & 7.60 & 6.86 \\
\hline B1 & $11 / 02 / 79$ & -194 & -59.1 & 1240 & 13.4 & 17800 & -- & 25.70 & 6.74 \\
\hline B1 & $11 / 02 / 79$ & -200 & -61.0 & 1230 & 13.7 & 13600 & -- & 12.00 & 6.76 \\
\hline
\end{tabular}


Table 3.--Water-chemistry data collected during a pumping test of well B4, near Horsfall Beach, October 1979 through December 1979--Continued

\begin{tabular}{|c|c|c|c|c|c|c|c|c|c|}
\hline WELL & DATE & $\begin{array}{l}\text { ELEVA } \\
\text { (FEET) }\end{array}$ & $\begin{array}{l}\text { ATION } \\
\text { (METER) }\end{array}$ & TIME & $\begin{array}{c}\text { TEMPER- } \\
\text { ATURE } \\
(\text { DEG C) }\end{array}$ & $\begin{array}{l}\text { SPECIFIC } \\
\text { CONDUCT- } \\
\text { ANCE } \\
\text { (UMHOS) }\end{array}$ & $\begin{array}{l}\text { BICAR- } \\
\text { BONATE } \\
\text { (MG/L } \\
\text { AS } \\
\text { HCO3) }\end{array}$ & $\begin{array}{l}\text { IRON } \\
\text { DIS- } \\
\text { SOLVED } \\
(M G / L)\end{array}$ & $\begin{array}{c}P H \\
\text { (UNIT) }\end{array}$ \\
\hline B1 & $11 / 09 / 79$ & $1 /$ & $1 /$ & 1100 & 13.2 & 508 & -- & -- & -- \\
\hline B1 & $11 / 09 / 79$ & $1 /$ & $1 /$ & 1115 & 12.8 & 525 & -- & -- & -- \\
\hline B1 & $11 / 09 / 79$ & $\overline{1} /$ & $\overline{1} /$ & 1125 & 12.8 & 525 & -- & -- & -- \\
\hline B1 & $11 / 09 / 79$ & $\overline{1} /$ & $\overline{1} /$ & 1130 & 12.5 & 510 & -- & -- & -- \\
\hline B1 & $11 / 09 / 79$ & $\overline{1}$ & $\overline{1}$ & 1135 & 13.0 & 508 & -- & -- & -- \\
\hline B1 & $11 / 09 / 79$ & -141 & -43.0 & 1057 & 13.1 & 430 & -- & -- & 8.10 \\
\hline B1 & $11 / 09 / 79$ & -147 & -44.8 & 1055 & 14.0 & 636 & -- & -- & 7.20 \\
\hline B1 & $11 / 09 / 79$ & -153 & -46.6 & 1050 & 13.0 & 760 & -- & -- & 7.70 \\
\hline B1 & $11 / 09 / 79$ & -159 & -48.5 & 1045 & 12.9 & 758 & -- & -- & 7.40 \\
\hline$B 1$ & $11 / 09 / 79$ & -165 & -50.3 & 1040 & 12.6 & 2540 & -- & -- & 7.30 \\
\hline B1 & $11 / 09 / 79$ & -173 & -52.7 & 1035 & 12.9 & 2980 & -- & -- & 6.90 \\
\hline B1 & $11 / 09 / 79$ & -194 & -59.1 & 1030 & 13.0 & 2420 . & -- & -- & 6.40 \\
\hline B1 & $11 / 09 / 79$ & -200 & -61.0 & 1022 & 13.6 & 2090 & -- & -- & 6.70 \\
\hline B1 & $11 / 15 / 79$ & -153 & -46.6 & 1.730 & 12.1 & 3380 & -- & -- & -- \\
\hline $\mathrm{B1}$ & $11 / 15 / 79$ & -153 & -46.6 & 2350 & 12.2 & 3500 & -- & -- & -- \\
\hline B1 & $11 / 15 / 79$ & -159 & -48.5 & 1630 & 12.1 & 3850 & -- & -- & -- \\
\hline B1 & $11 / 15 / 79$ & -159 & -48.5 & 2340 & 12.2 & 3700 & -- & -- & -- \\
\hline B1 & $11 / 16 / 79$ & -141 & -43.0 & 1145 & 13.7 & 470 & -- & -- & 7.50 \\
\hline B1 & $11 / 16 / 79$ & -147 & -44.8 & 1135 & 13.8 & 789 & -- & -- & 6.82 \\
\hline$B 1$ & $11 / 16 / 79$ & -153 & -46.5 & 1000 & 13.1 & 3800 & -- & -- & 7.60 \\
\hline B1 & $11 / 16 / 79$ & -159 & -48.5 & 0900 & 13.6 & 4030 & -- & -- & 7.31 \\
\hline B1 & $11 / 16 / 79$ & -165 & -50.3 & 1140 & 13.7 & 4500 & -- & -- & - \\
\hline B1 & $11 / 16 / 79$ & -173 & -52.7 & 1130 & 13.8 & 9850 & -- & -- & 6.97 \\
\hline B1 & $11 / 17 / 79$ & -153 & -46.6 & 1035 & 13.7 & 3890 & -- & -- & - \\
\hline B1 & $11 / 17 / 79$ & -159 & -48.5 & 1030 & 16.2 & 3940 & -- & -- & -- \\
\hline B1 & $11 / 20 / 79$ & -141 & -43.0 & 1625 & 12.5 & 340 & -- & -- & 3.08 \\
\hline B1 & $11 / 20 / 79$ & -153 & -46.6 & 1706 & 11.8 & 4070 & -- & -- & 7.80 \\
\hline B1 & $11 / 20 / 79$ & -165 & -50.3 & 1624 & 12.4 & 4250 & -- & -- & 7.56 \\
\hline B1 & $11 / 20 / 79$ & -194 & -59.1 & 1702 & 11.6 & 18000 & -- & -- & 6.80 \\
\hline B1 & $11 / 28 / 79$ & -122 & -37.2 & 1300 & 12.9 & 420 & -- & -- & 7.70 \\
\hline$B 1$ & $11 / 28 / 79$ & -129 & -39.3 & 1235 & 12.5 & 410 & -- & -- & 7.80 \\
\hline B1 & $11 / 28 / 79$ & -135 & -41.1 & 1250 & 12.5 & 430 & -- & -- & 7.80 \\
\hline B1 & $11 / 28 / 79$ & -141 & -43.0 & 1245 & 12.6 & 440 & -- & -- & 7.70 \\
\hline
\end{tabular}


Table 3.--Water-chem istry data collected during a pumping test of well B4, near Horsfall Beach, October 1979 through December 1979--Continued

\begin{tabular}{|c|c|c|c|c|c|c|c|c|c|}
\hline WELL & DATE & $\begin{array}{c}\text { ELEV } \\
\text { (FEET) }\end{array}$ & $\begin{array}{l}\text { ATION } \\
\text { (METER) }\end{array}$ & TIME & $\begin{array}{l}\text { TEMPER- } \\
\text { ATURE } \\
\text { (DEG C) }\end{array}$ & $\begin{array}{l}\text { SPECIFIC } \\
\text { CONDUCT- } \\
\text { ANCE } \\
\text { (UMHOS) }\end{array}$ & $\begin{array}{c}\text { BICAR- } \\
\text { BONATE } \\
\text { (MG/L } \\
\text { AS } \\
\text { HCO3) }\end{array}$ & $\begin{array}{l}\text { IRON } \\
\text { DIS- } \\
\text { SOLVED } \\
(M G / L)\end{array}$ & $\begin{array}{c}\mathrm{PH} \\
(U N \mid T)\end{array}$ \\
\hline B1 & $11 / 28 / 79$ & -153 & -46.5 & 1240 & 13.4 & 5500 & -- & -- & 7.35 \\
\hline B1 & $11 / 28 / 79$ & -159 & -48.5 & -- & 12.8 & 6500 & -- & -- & 7.20 \\
\hline B1 & $11 / 28 / 79$ & -165 & -50.3 & 1230 & 12.5 & 4600 & -- & -- & 7.30 \\
\hline B1 & $11 / 28 / 79$ & -173 & -52.7 & 1225 & 12.4 & 9300 & -- & -- & 7.00 \\
\hline B1 & $11 / 28 / 79$ & -194 & -59.1 & 1220 & 12.9 & 23000 & -- & -- & 6.60 \\
\hline B1 & $11 / 28 / 79$ & -200 & -61.0 & 1215 & 14.6 & 15500 & -- & -- & 6.90 \\
\hline B1 & $12 / 04 / 79$ & -3 & -0.9 & 1150 & 12.8 & 219 & -- & -- & 6.10 \\
\hline B1 & $12 / 04 / 79$ & -15 & -4.6 & 1140 & 12.6 & 1160 & -- & -- & 5.70 \\
\hline B1 & $12 / 04 / 79$ & -39 & -11.9 & 1130 & 12.3 & 3250 & -- & -- & 5.70 \\
\hline B1 & $12 / 04 / 79$ & -51 & -15.5 & 1120 & 12.3 & 98 & -- & -- & 6.20 \\
\hline B1 & $12 / 04 / 79$ & -63 & -19.2 & 1110 & 12.2 & 103 & -- & -- & 6.70 \\
\hline B1 & $12 / 04 / 79$ & -75 & -22.9 & 1100 & 12.2 & 127 & -- & -- & 6.70 \\
\hline B1 & $12 / 04 / 79$ & -87 & -26.5 & 1050 & $12 . ?$ & 210 & -- & -- & 7.10 \\
\hline B1 & $12 / 04 / 79$ & -99 & -30.2 & 1040 & 12.3 & 236 & -- & -- & 7.70 \\
\hline B1 & $12 / 04 / 79$ & -110 & -33.5 & 1030 & 12.2 & 220 & -- & -- & 7.70 \\
\hline B1 & $12 / 04 / 79$ & -122 & -37.2 & 1020 & 12.2 & 223 & -- & -- & 7.80 \\
\hline B1 & $12 / 04 / 79$ & -135 & -41.1 & 1010 & 12.1 & 4800 & -- & -- & 7.60 \\
\hline B1 & $12 / 04 / 79$ & -153 & -46.6 & 1000 & 12.7 & 7500 & -- & -- & 7.40 \\
\hline B1 & $12 / 04 / 79$ & -159 & -48.5 & 0950 & 12.1 & 6200 & -- & -- & 7.00 \\
\hline B1 & $12 / 04 / 79$ & -173 & -52.7 & 0940 & 12.1 & 8000 & -- & -- & 6.80 \\
\hline B1 & $12 / 04 / 79$ & -200 & -61.0 & 0930 & 12.1 & 9200 & -- & -- & 6.70 \\
\hline B2 & $10 / 02 / 79$ & 0 & 0.0 & 1020 & 14.2 & 45540 & -- & -- & -- \\
\hline B2 & $10 / 02 / 79$ & -5 & -1.5 & 1010 & 14.2 & 36050 & -- & -- & -- \\
\hline B2 & $10 / 02 / 79$ & -133 & -40.5 & 1000 & 15.9 & 12580 & -- & -- & -- \\
\hline B2 & $10 / 02 / 79$ & -140 & -42.7 & 0950 & 16.8 & 19790 & -- & -- & -- \\
\hline B2 & $10 / 02 / 79$ & -146 & -44.5 & 0940 & 17.4 & 23780 & -- & -- & -- \\
\hline B2 & $10 / 02 / 79$ & -158 & -48.2 & 0930 & 15.8 & 18830 & -- & -- & -- \\
\hline B2 & $10 / 04 / 79$ & 0 & 0.0 & 1245 & -- & 47400 & -- & -- & 7.47 \\
\hline B2 & $10 / 04 / 79$ & -5 & -1.5 & 1240 & -- & 31200 & -- & -- & 7.78 \\
\hline B2 & $10 / 04 / 79$ & -12 & -3.7 & 1235 & -- & 9720 & -- & -- & 8.31 \\
\hline B2 & $10 / 04 / 79$ & -18 & -5.5 & 1230 & -- & 7149 & -- & -- & 8.54 \\
\hline B2 & $10 / 04 / 79$ & -30 & -9.1 & 1225 & -- & 560 & -- & -- & 8.99 \\
\hline B2 & $10 / 04 / 79$ & -36 & -11.0 & 1220 & -- & 1675 & -- & -- & 8.51 \\
\hline B2 & $10 / 04 / 79$ & -42 & -12.8 & 1215 & -- & 590 & -- & -- & 8.70 \\
\hline B2 & $10 / 04 / 79$ & -49 & -14.9 & 1212 & -- & 910 & -- & -- & 7.82 \\
\hline B2 & $10 / 04 / 79$ & -55 & -16.8 & 1210 & -- & 705 & -- & -- & 8.17 \\
\hline
\end{tabular}


Table 3.- Water-chem istry data collected during a pumping test of well B4, near Horsfall Beach, October 1979 through December 1979--Continued

\begin{tabular}{|c|c|c|c|c|c|c|c|c|}
\hline WELL & DATE & $\begin{array}{c}\text { ELEVATION } \\
\text { (FEET) (METER) }\end{array}$ & TIME & $\begin{array}{c}\text { TEMPER- } \\
\text { ATURE } \\
\text { (DEG C) }\end{array}$ & $\begin{array}{l}\text { SPECIFIC } \\
\text { CONDUCT- } \\
\text { ANCE } \\
\text { (UMHOS) }\end{array}$ & $\begin{array}{c}\text { BICAR- } \\
\text { BONATE } \\
\text { (MG/L } \\
\text { AS } \\
\text { HCO3) }\end{array}$ & $\begin{array}{l}\text { IRON } \\
\text { DIS- } \\
\text { SOLVED } \\
(M G / L)\end{array}$ & $\begin{array}{c}\mathrm{PH} \\
(\mathrm{UNIT})\end{array}$ \\
\hline
\end{tabular}

\begin{tabular}{|c|c|c|c|c|c|c|c|c|c|}
\hline B2 & $10 / 04 / 79$ & -61 & -18.6 & 1205 & -- & 310 & - & - & 7.48 \\
\hline B2 & $10 / 04 / 79$ & -67 & -20.4 & 1200 & -- & 370 & -- & -- & 7.18 \\
\hline B2 & $10 / 04 / 79$ & -73 & -22.3 & 1150 & -- & 420 & - & -- & 7.12 \\
\hline B2 & $10 / 04 / 79$ & -79 & -24.1 & 1145 & -- & 445 & -- & -- & 7.14 \\
\hline B2 & $10 / 04 / 79$ & -85 & -25.9 & 1140 & -- & 510 & -- & - & 7.19 \\
\hline B2 & $10 / 04 / 79$ & -90 & -27.4 & 1135 & - & 930 & -- & -- & 7.37 \\
\hline B2 & $10 / 04 / 79$ & -97 & -29.6 & 1130 & -- & 665 & -- & - & 7.51 \\
\hline B2 & $10 / 04 / 79$ & -103 & -31.4 & 1125 & -- & 765 & -- & -- & 8.01 \\
\hline B2 & $10 / 04 / 79$ & -109 & -33.2 & 1120 & - & 620 & - & - & 8.42 \\
\hline B2 & $10 / 04 / 79$ & -115 & -35.1 & 1115 & - & 670 & -- & -- & 8.05 \\
\hline B2 & $10 / 04 / 79$ & -127 & -38.7 & 1110 & -- & 580 & -- & -- & 8.05 \\
\hline B2 & $10 / 04 / 79$ & -133 & -40.5 & 1105 & - & 15549 & - & -- & 7.36 \\
\hline B2 & $10 / 04 / 79$ & -140 & -42.7 & 1100 & -- & 24400 & - & - & 7.62 \\
\hline B2 & $10 / 04 / 79$ & -146 & -44.5 & 1055 & - & 26540 & -- & -- & 7.52 \\
\hline B2 & $10 / 04 / 79$ & -158 & -48.2 & 1050 & -- & 30000 & -- & -- & 7.52 \\
\hline B2 & $10 / 04 / 79$ & -164 & -50.0 & 1045 & - & 9840 & -- & -- & 7.28 \\
\hline B2 & $10 / 04 / 79$ & -170 & -51.8 & 1040 & -- & 3750 & -- & -- & 7.10 \\
\hline $\mathrm{B} 2$ & $10 / 04 / 79$ & -179 & -54.6 & 1030 & - & 10800 & - & -- & 6.78 \\
\hline B2 & $11 / 01 / 79$ & 0 & 0.0 & 1750 & 13.5 & 41600 & -- & 1.20 & 7.40 \\
\hline B2 & $11 / 01 / 79$ & -5 & -1.5 & 1740 & 13.6 & 43500 & - & .01 & 7.63 \\
\hline B2 & $11 / 01 / 79$ & -12 & -3.7 & 1730 & 13.5 & 43500 & -- & .01 & 7.67 \\
\hline B2 & $11 / 01 / 79$ & -18 & -5.5 & 1720 & 13.4 & 38400 & -- & .01 & 7.61 \\
\hline B2 & $11 / 01 / 79$ & -24 & $-7 \cdot 3$ & 1710 & 13.2 & 40000 & -- & -- & 7.37 \\
\hline B2 & $11 / 01 / 79$ & -30 & -9.1 & 1700 & 13.2 & 16300 & -- & - & 7.26 \\
\hline B2 & $11 / 01 / 79$ & -36 & -11.0 & 1650 & 13.2 & 23000 & - & .07 & 7.48 \\
\hline B2 & $11 / 01 / 79$ & -42 & -12.8 & 1640 & 13.7 & 19100 & -- & .41 & 7.42 \\
\hline B2 & $11 / 01 / 79$ & -49 & -14.9 & 1630 & 13.3 & 14000 & - & .63 & 6.40 \\
\hline B2 & $11 / 01 / 79$ & -55 & -16.8 & 1628 & 13.7 & 445 & -- & .11 & 7.31 \\
\hline B2 & $11 / 01 / 79$ & -61 & -18.6 & 1625 & 12.9 & 320 & -- & .11 & 7.00 \\
\hline B2 & $11 / 01 / 79$ & -67 & -20.4 & 1622 & 13.2 & 212 & -- & .13 & 7.14 \\
\hline B2 & $11 / 01 / 79$ & -73 & -22.3 & 1620 & 13.4 & 940 & - & .06 & 7.15 \\
\hline B2 & $11 / 01 / 79$ & -79 & -24.1 & 1615 & 13.4 & 265 & -- & .06 & 7.22 \\
\hline B2 & $11 / 01 / 79$ & -85 & -25.9 & 1612 & 12.8 & 345 & -- & .06 & 7.38 \\
\hline B2 & $11 / 01 / 79$ & -90 & -27.4 & 1610 & 14.0 & 350 & -- & .06 & 7.48 \\
\hline $\mathrm{B} 2$ & $11 / 01 / 79$ & -97 & -29.6 & 1600 & 14.0 & 410 & - & .07 & 7.66 \\
\hline B2 & $11 / 01 / 79$ & -103 & -31.4 & 1552 & 13.4 & 615 & - & .06 & 7.96 \\
\hline B2 & $11 / 01 / 79$ & -109 & -33.2 & 1550 & 14.0 & 520 & - & .07 & 8.05 \\
\hline B2 & $11 / 01 / 79$ & -115 & -35.1 & 1540 & 13.3 & 450 & -- & .01 & 7.68 \\
\hline B2 & $11 / 01 / 79$ & -121 & -36.9 & 1530 & 13.4 & 415 & -- & .03 & 7.71 \\
\hline B2 & $11 / 01 / 79$ & -127 & -38.7 & 1520 & 13.4 & 450 & -- & .06 & 7.78 \\
\hline
\end{tabular}


Table 3.--Water-chem istry data collected during a pumping test of well B4, near Horsfall Beach, October 1979 through December 1979--Continued

\begin{tabular}{|c|c|c|c|c|c|c|c|c|c|}
\hline WELL & DATE & $\begin{array}{l}\text { ELEVA } \\
\text { (FEET) }\end{array}$ & $\begin{array}{l}\text { ATION } \\
\text { (METER) }\end{array}$ & TIME & $\begin{array}{l}\text { TEMPER- } \\
\text { ATURE } \\
(\text { DEG C) }\end{array}$ & $\begin{array}{l}\text { SPECIFIC } \\
\text { CONDUCT- } \\
\text { ANCE } \\
\text { (UMHOS) }\end{array}$ & $\begin{array}{c}\text { BICAR- } \\
\text { BONATE } \\
\text { (MG/L } \\
\text { AS } \\
\text { HCO3) }\end{array}$ & $\begin{array}{l}\text { IRON } \\
\text { DIS- } \\
\text { SOLVED } \\
\text { (MG/L) }\end{array}$ & $\begin{array}{c}P H \\
(U N \mid T)\end{array}$ \\
\hline B2 & $11 / 01 / 79$ & -133 & -40.5 & 1510 & 14.4 & 600 & -- & .02 & 8.09 \\
\hline$B 2$ & $11 / 01 / 79$ & -140 & -42.7 & 1500 & 14.6 & 23600 & -- & 1.20 & 6.99 \\
\hline B2 & $11 / 01 / 79$ & -146 & -44.5 & 1450 & 13.7 & 26200 & -- & 2.60 & 7.05 \\
\hline$B 2$ & $11 / 01 / 79$ & -152 & -46.3 & 1440 & 14.0 & 36600 & -- & 1.40 & 6.88 \\
\hline B2 & $11 / 01 / 79$ & -158 & -48.2 & 1430 & 14.1 & 31099 & -- & 2.40 & 7.20 \\
\hline B2 & $11 / 01 / 79$ & -164 & -50.0 & 1420 & 13.7 & 9860 & -- & 1.30 & 7.87 \\
\hline B2 & $11 / 01 / 79$ & -170 & -51.8 & 1410 & 14.0 & 4760 & -- & 6.40 & 7.47 \\
\hline B2 & $11 / 01 / 79$ & -179 & -54.6 & 1400 & 13.0 & 10000 & -- & 9.60 & 7.43 \\
\hline$B 2$ & $11 / 06 / 79$ & -12 & -3.7 & 1435 & 14.5 & 49000 & 134 & -- & 6.90 \\
\hline B2 & $11 / 06 / 79$ & -24 & -7.3 & 1430 & 14.6 & 32000 & 112 & -- & 6.50 \\
\hline$B 2$ & $11 / 06 / 79$ & -36 & -11.0 & 1420 & 14.4 & 14000 & 70 & -- & 6.50 \\
\hline $\mathrm{B2}$ & $11 / 06 / 79$ & -49 & -14.9 & 1410 & 14.3 & 590 & 84 & -- & 6.30 \\
\hline B2 & $11 / 06 / 79$ & -115 & -35.1 & 1400 & 14.6 & 480 & -- & -- & 7.20 \\
\hline 82 & $11 / 06 / 79$ & -127 & -38.7 & 1350 & 14.6 & 440 & -- & -- & 7.70 \\
\hline B2 & $11 / 06 / 79$ & -133 & -40.5 & 1340 & 14.8 & 630 & 256 & -- & 7.90 \\
\hline$B 2$ & $11 / 06 / 79$ & -140 & -42.7 & 1330 & 16.0 & 13000 & -- & -- & 7.30 \\
\hline $\mathrm{B2}$ & $11 / 06 / 79$ & -152 & -46.3 & 1310 & 13.2 & 16500 & 134 & 1.00 & 7.50 \\
\hline B2 & $11 / 06 / 79$ & -158 & -48.2 & 1320 & 14.6 & 18700 & -- & -- & 7.40 \\
\hline B2 & $11 / 06 / 79$ & -164 & -50.0 & 1300 & 14.8 & 5700 & 390 & .84 & 7.00 \\
\hline B2 & $11 / 06 / 79$ & -170 & -51.8 & 1245 & 14.8 & 6800 & 329 & 5.80 & 6.60 \\
\hline B2 & $11 / 06 / 79$ & -179 & -54.6 & 1610 & 13.4 & 8200 & 488 & 19.00 & 6.90 \\
\hline B2 & $11 / 07 / 79$ & -61 & -18.6 & 1410 & 14.3 & 520 & 56 & .13 & 5.80 \\
\hline$B 2$ & $11 / 07 / 79$ & -73 & -22.3 & 1400 & 14.4 & 160 & 96 & .01 & 6.20 \\
\hline B2 & $11 / 07 / 79$ & -97 & -29.6 & 1350 & 14.6 & 310 & 220 & .03 & 7.30 \\
\hline B2 & $11 / 07 / 79$ & -109 & -33.2 & 1340 & 14.4 & 520 & 268 & .04 & 7.80 \\
\hline B2 & $11 / 07 / 79$ & -121 & -36.9 & 1330 & 14.4 & 490 & 244 & -- & 7.90 \\
\hline$B 2$ & $11 / 07 / 79$ & -146 & -44.5 & 1320 & 14.7 & 15000 & 134 & 2.10 & 7.10 \\
\hline B2 & $11 / 09 / 79$ & -127 & -38.7 & 1520 & 14.0 & 408 & -- & -- & 7.90 \\
\hline$B 2$ & $11 / 09 / 79$ & -133 & -40.5 & 1515 & 13.7 & 525 & -- & -- & 8.39 \\
\hline B2 & $11 / 09 / 79$ & -140 & -42.7 & 1510 & 14.6 & 9700 & -- & -- & 7.50 \\
\hline B2 & $11 / 09 / 79$ & -146 & -44.5 & 1505 & 13.6 & 9900 & -- & -- & 7.60 \\
\hline B2 & $11 / 09 / 79$ & -152 & -46.3 & 1500 & 13.9 & 9800 & -- & -- & 7.30 \\
\hline B2 & $11 / 09 / 79$ & -158 & -48.2 & 1455 & 14.0 & 10300 & -- & -- & 7.60 \\
\hline B2 & $11 / 09 / 79$ & -164 & -50.0 & 1450 & 13.9 & 13200 & -- & -- & 7.10 \\
\hline $\mathrm{B} 2$ & $11 / 09 / 79$ & -170 & -51.8 & 1445 & 14.1 & 9300 & -- & -- & 6.90 \\
\hline B2 & $11 / 09 / 79$ & -179 & -54.6 & 1440 & 14.2 & 13000 & -- & -- & 6.80 \\
\hline
\end{tabular}


Table 3.--Water-chem istry data collected during a pumping test of well B4, near Horsfall Beach, October 1979 through December 1979--Continued

\begin{tabular}{|c|c|c|c|c|c|c|c|c|}
\hline WELL & DATE & $\begin{array}{c}\text { ELEVATION } \\
\text { (FEET) (METER) }\end{array}$ & TIME & $\begin{array}{c}\text { TEMPER- } \\
\text { ATURE } \\
\text { (DEG C) }\end{array}$ & $\begin{array}{l}\text { SPECIFIC } \\
\text { CONDUCT- } \\
\text { ANCE } \\
\text { (UMHOS) }\end{array}$ & $\begin{array}{c}\text { B ICAR- } \\
\text { BONATE } \\
\text { (MG/L } \\
\text { AS } \\
\text { HCO3) }\end{array}$ & $\begin{array}{l}\text { IRON } \\
\text { DIS- } \\
\text { SOLVED } \\
(M G / L)\end{array}$ & $\begin{array}{c}\text { PH } \\
(\text { UNIT) }\end{array}$ \\
\hline
\end{tabular}

\begin{tabular}{|c|c|c|c|c|c|c|c|c|c|}
\hline B2 & $11 / 15 / 79$ & -140 & -42.7 & 1130 & 12.6 & 8000 & -- & -- & -- \\
\hline B2 & $11 / 15 / 79$ & -140 & -42.7 & 1520 & 11.6 & 6600 & -- & -- & - \\
\hline B2 & $11 / 15 / 79$ & -146 & -44.5 & 1120 & 12.6 & 20500 & -- & -- & -1 \\
\hline B2 & $11 / 15 / 79$ & -146 & -44.5 & 1510 & 12.5 & 26400 & -- & -- & -- \\
\hline B2 & $11 / 15 / 79$ & -158 & -48.2 & 1110 & 12.2 & 33800 & -- & -- & - \\
\hline B2 & $11 / 15 / 79$ & -158 & -48.2 & 1505 & 11.6 & 16000 & -- & -- & -- \\
\hline B2 & $11 / 16 / 79$ & -127 & -38.7 & 1000 & 13.7 & 429 & -- & -- & - \\
\hline B2 & $11 / 16 / 79$ & -133 & -40.5 & 0950 & 14.0 & 1385 & -- & -- & -- \\
\hline B2 & $11 / 16 / 79$ & -140 & -42.7 & 0940 & 13.6 & 7700 & -- & -- & 7.86 \\
\hline B2 & $11 / 16 / 79$ & -146 & -44.5 & 0930 & 13.6 & 27800 & -- & -- & 6.93 \\
\hline B2 & $11 / 16 / 79$ & -152 & $-46 \cdot 3$ & 0920 & 14.0 & 34500 & -- & -- & 7.25 \\
\hline B2 & $11 / 16 / 79$ & -158 & -48.2 & 0910 & 13.6 & 30000 & -- & -- & 6.84 \\
\hline B2 & $11 / 16 / 79$ & -164 & -50.0 & 0905 & 13.8 & 19000 & -- & -- & -- \\
\hline B2 & $11 / 16 / 79$ & -170 & -51.8 & 0900 & 13.8 & 9400 & -- & -- & - \\
\hline B2 & $11 / 17 / 79$ & -152 & -46.3 & 1040 & 14.0 & 34500 & -- & -- & -- \\
\hline B2 & $11 / 17 / 79$ & -158 & -48.2 & 1030 & 13.6 & 30000 & -- & -- & - \\
\hline B2 & $11 / 17 / 79$ & -164 & -50.0 & 1020 & 13.8 & 19000 & -- & -- & - \\
\hline B2 & $11 / 17 / 79$ & -170 & -51.8 & 1010 & 13.8 & 9400 & -- & -- & -- \\
\hline B2 & $11 / 18 / 79$ & -18 & -5.5 & 1415 & 11.8 & 46600 & -- & -- & 7.56 \\
\hline B2 & $11 / 18 / 79$ & -30 & -9.1 & 1410 & 11.3 & 11000 & -- & -- & 7.19 \\
\hline B2 & $11 / 18 / 79$ & -42 & -12.8 & 1405 & 11.3 & 1600 & -- & -- & 7.63 \\
\hline B2 & $11 / 18 / 79$ & -55 & -16.8 & 1400 & 10.2 & 24500 & -- & -- & 7.07 \\
\hline B2 & $11 / 18 / 79$ & -67 & -20.4 & 1355 & 10.4 & 1760 & -- & -- & 7.29 \\
\hline B2 & $11 / 18 / 79$ & -79 & -24.1 & 1350 & 12.1 & 310 & -- & -- & 7.20 \\
\hline B2 & $11 / 18 / 79$ & -90 & -27.4 & 1345 & 12.2 & 380 & -- & -- & 7.68 \\
\hline B2 & $11 / 18 / 79$ & -103 & -31.4 & 1340 & 12.1 & 397 & -- & -- & 8.19 \\
\hline B2 & $11 / 18 / 79$ & -115 & -35.1 & 1335 & 12.2 & 487 & -- & -- & 8.00 \\
\hline B2 & $11 / 18 / 79$ & -127 & -38.7 & 1330 & 12.5 & 417 & -- & -- & 7.75 \\
\hline B2 & $11 / 18 / 79$ & -140 & -42.7 & 1325 & 11.8 & 3800 & -- & -- & 8.10 \\
\hline B2 & $11 / 18 / 79$ & -152 & -46.3 & 1315 & 11.2 & 35250 & -- & -- & 7.20 \\
\hline B2 & $11 / 18 / 79$ & -158 & -48.2 & 1310 & 11.0 & 35500 & -- & -- & 7.20 \\
\hline B2 & $11 / 18 / 79$ & -164 & -50.0 & 1305 & 12.1 & 18000 & -- & -- & 7.30 \\
\hline B2 & $11 / 18 / 79$ & -170 & -51.8 & 1300 & 11.6 & 24000 & -- & -- & 7.00 \\
\hline B2 & $11 / 28 / 79$ & -85 & -25.9 & 1455 & 13.0 & 16000 & -- & -- & 6.20 \\
\hline B2 & $11 / 28 / 79$ & -97 & -29.6 & 1450 & 13.0 & 390 & -- & -- & 7.70 \\
\hline B2 & $11 / 28 / 79$ & -109 & -33.2 & 1445 & 12.9 & -- & -- & -- & 8.20 \\
\hline B2 & $11 / 28 / 79$ & -121 & -36.9 & 1440 & 12.5 & 420 & -- & -- & 7.50 \\
\hline
\end{tabular}


Table 3.--Water-chemistry data collected during a pumping test of well B4, near Horsfall Beach, October 1979 through December 1979--Continued

\begin{tabular}{|c|c|c|c|c|c|c|c|c|c|}
\hline WELL & DATE & $\begin{array}{l}\text { ELEVA } \\
\text { (FEET) }\end{array}$ & $\begin{array}{l}\text { ATION } \\
\text { (METER) }\end{array}$ & TIME & $\begin{array}{l}\text { TEMPER- } \\
\text { ATURE } \\
\left(\begin{array}{ll}D E G & C\end{array}\right)\end{array}$ & $\begin{array}{l}\text { SPECIFIC } \\
\text { CONDUCT- } \\
\text { ANCE } \\
\text { (UMHOS) }\end{array}$ & $\begin{array}{c}\text { BICAR- } \\
\text { BONATE } \\
\text { (MG/L } \\
\text { AS } \\
\text { HCO3) }\end{array}$ & $\begin{array}{l}\text { IRON } \\
\text { DIS- } \\
\text { SOLVED } \\
\text { (MG/L) }\end{array}$ & $\begin{array}{c}P H \\
(U N \mid T)\end{array}$ \\
\hline B2 & $11 / 28 / 79$ & -133 & -40.5 & 1435 & 12.8 & 550 & -- & -- & 8.39 \\
\hline B2 & $11 / 28 / 79$ & -146 & -44.5 & 1430 & 12.8 & 34500 & -- & -- & 7.20 \\
\hline B2 & $11 / 28 / 79$ & -158 & $-48 \cdot 2$ & 1425 & 13.1 & 47000 & -- & -- & 6.70 \\
\hline B2 & $11 / 28 / 79$ & -170 & -51.8 & 1420 & 13.0 & 24000 & -- & -- & 6.60 \\
\hline B2 & $12 / 04 / 79$ & 0 & 0.0 & 1005 & 11.6 & 45000 & -- & -- & 5.80 \\
\hline B2 & $12 / 04 / 79$ & -12 & -3.7 & 1000 & 11.6 & 50000 & -- & -- & 6.70 \\
\hline 32 & $12 / 04 / 79$ & -24 & -7.3 & 0955 & 11.8 & 42000 & -- & -- & 6.20 \\
\hline $\mathrm{B} 2$ & $12 / 04 / 79$ & -36 & -11.0 & 0950 & 12.5 & 16500 & -- & -- & 5.30 \\
\hline B2 & $12 / 04 / 79$ & -49 & -14.9 & 0945 & 11.8 & 12000 & -- & -- & 5.80 \\
\hline B2 & $12 / 04 / 79$ & -61 & -18.6 & 0940 & 12.0 & 418 & -- & -- & 6.60 \\
\hline B2 & $12 / 04 / 79$ & -85 & -25.9 & 0935 & 12.0 & 4000 & -- & -- & 6.60 \\
\hline B2 & $12 / 04 / 79$ & -97 & -29.6 & 0930 & 11.8 & 4350 & -- & -- & 7.00 \\
\hline B2 & $12 / 04 / 79$ & -109 & -33.2 & 0925 & 12.0 & 402 & -- & -- & 7.50 \\
\hline B2 & $12 / 04 / 79$ & -121 & -36.9 & 0920 & 12.0 & 398 & -- & -- & 7.50 \\
\hline B2 & $12 / 04 / 79$ & -133 & -40.5 & 0915 & 12.0 & 552 & -- & -- & 7.50 \\
\hline B2 & $12 / 04 / 79$ & -146 & -44.5 & 0910 & 12.1 & 29000 & -- & -- & 6.90 \\
\hline B2 & $12 / 04 / 79$ & -158 & $-48 \cdot 2$ & 0905 & 12.0 & 38500 & -- & -- & 6.70 \\
\hline B2 & $12 / 04 / 79$ & -170 & -51.8 & 0900 & 12.2 & 30000 & -- & -- & 6.60 \\
\hline B3 & $10 / 25 / 79$ & 4 & 1.2 & 1410 & 13.8 & 260 & -- & -- & 6.70 \\
\hline B3 & $10 / 25 / 79$ & -1 & -0.3 & 1400 & 13.6 & 315 & -- & -- & 6.50 \\
\hline B3 & $10 / 25 / 79$ & -5 & -1.5 & 1350 & 13.2 & 250 & -- & -- & 6.60 \\
\hline B3 & $10 / 25 / 79$ & -11 & -3.4 & 1340 & 13.0 & 275 & -- & -- & 6.65 \\
\hline B3 & $10 / 25 / 79$ & -16 & -4.9 & 1330 & 13.0 & 240 & -- & -- & 6.80 \\
\hline B3 & $10 / 25 / 79$ & -21 & -6.4 & 1320 & 13.0 & 235 & -- & -- & 6.70 \\
\hline B3 & $10 / 25 / 79$ & -26 & -7.9 & 1310 & 12.8 & 225 & -- & -- & 6.70 \\
\hline B3 & $10 / 25 / 79$ & -31 & -9.4 & 1300 & 13.3 & 262 & -- & -- & 6.10 \\
\hline B3 & $10 / 25 / 79$ & -36 & -11.0 & 1250 & 13.1 & 266 & -- & -- & 6.60 \\
\hline$B 3$ & $10 / 25 / 79$ & -41 & -12.5 & 1240 & 14.1 & 260 & -- & -- & 6.40 \\
\hline B3 & $10 / 25 / 79$ & -46 & -14.0 & 1230 & 13.3 & 255 & -- & -- & 6.80 \\
\hline B3 & $10 / 25 / 79$ & -51 & -15.5 & 1220 & 13.2 & 265 & -- & -- & 6.80 \\
\hline B3 & $10 / 25 / 79$ & -57 & -17.4 & 1210 & 13.2 & 260 & -- & -- & 7.05 \\
\hline B3 & $10 / 25 / 79$ & -63 & -19.2 & 1200 & 13.0 & 240 & -- & -- & 6.90 \\
\hline B3 & $10 / 25 / 79$ & -69 & -21.0 & 1150 & 13.2 & 290 & -- & -- & 6.85 \\
\hline B3 & $10 / 25 / 79$ & -75 & -22.9 & 1140 & 13.0 & 325 & -- & -- & 7.00 \\
\hline B3 & $10 / 25 / 79$ & -81 & -24.7 & 1130 & 13.0 & 340 & -- & -- & 6.80 \\
\hline B3 & $10 / 25 / 79$ & -87 & -26.5 & 1120 & 13.2 & 385 & -- & -- & 7.10 \\
\hline
\end{tabular}


Table 3.--Water-chemistry data collected during a pumping test of well B4, near Horsfall Beach, October 1979 through December 1979--Continued

\begin{tabular}{|c|c|c|c|c|c|c|c|c|c|}
\hline WELL & DATE & $\begin{array}{l}\text { ELEVA } \\
\text { (FEET) }\end{array}$ & $\begin{array}{l}\text { ATION } \\
\text { (METER) }\end{array}$ & TIME & $\begin{array}{l}\text { TEMPER- } \\
\text { ATURE } \\
(\text { DEG C) }\end{array}$ & $\begin{array}{l}\text { SPECIFIC } \\
\text { CONDUCT- } \\
\text { ANCE } \\
\text { (UMHOS) }\end{array}$ & $\begin{array}{c}\text { BICAR- } \\
\text { BONATE } \\
\text { (MG/L } \\
\text { AS } \\
\text { HCO3) }\end{array}$ & $\begin{array}{l}\text { IRON } \\
\text { DIS- } \\
\text { SOLVED } \\
(M G / L)\end{array}$ & $\begin{array}{c}\mathrm{PH} \\
(\text { UNIT) }\end{array}$ \\
\hline B3 & $10 / 25 / 79$ & -93 & -28.3 & 1110 & 13.0 & 450 & -- & -- & 7.30 \\
\hline B3 & $10 / 25 / 79$ & -99 & -30.2 & 1100 & 13.0 & 382 & -- & -- & 7.90 \\
\hline B3 & $10 / 25 / 79$ & -105 & -32.0 & 1050 & 12.8 & 378 & -- & -- & 7.90 \\
\hline B3 & $10 / 25 / 79$ & -110 & -33.5 & 1040 & 13.2 & 370 & -- & -- & 8.00 \\
\hline B3 & $10 / 25 / 79$ & -117 & -35.7 & 1030 & 13.1 & 405 & -- & -- & 8.00 \\
\hline B3 & $10 / 25 / 79$ & -122 & -37.2 & 1020 & 13.4 & 390 & -- & -- & 8.00 \\
\hline B3 & $10 / 25 / 79$ & -129 & -39.3 & 1010 & 12.8 & 410 & -- & -- & 8.00 \\
\hline B3 & $10 / 25 / 79$ & -135 & -41.1 & 1000 & 12.7 & 395 & -- & -- & 8.05 \\
\hline B3 & $10 / 25 / 79$ & -141 & -43.0 & 0950 & 12.8 & 430 & -- & -- & 7.85 \\
\hline B3 & $10 / 25 / 79$ & -147 & -44.8 & 0940 & 12.7 & 682 & -- & -- & 8.05 \\
\hline B3 & $10 / 25 / 79$ & -153 & -46.6 & 0930 & 13.1 & 635 & -- & -- & 7.70 \\
\hline B3 & $10 / 25 / 79$ & -159 & $-48 \cdot 5$ & 0920 & 13.0 & 820 & -- & -- & 7.75 \\
\hline B3 & $10 / 25 / 79$ & -165 & -50.3 & 0910 & 12.8 & 1200 & -- & -- & 7.70 \\
\hline B3 & $10 / 25 / 79$ & -171 & -52.1 & 0900 & 12.7 & 1400 & -- & -- & 8.60 \\
\hline B3 & $10 / 31 / 79$ & 4 & 1.2 & 1410 & 13.5 & 255 & -- & 5.60 & 6.95 \\
\hline B3 & $10 / 31 / 79$ & -1 & -0.3 & 1400 & 14.3 & 340 & -- & 10.70 & 6.87 \\
\hline B3 & $10 / 31 / 79$ & -5 & -1.5 & 1350 & 13.6 & 248 & -- & 5.60 & 6.86 \\
\hline B3 & $10 / 31 / 79$ & -11 & -3.4 & 1340 & 13.4 & 262 & -- & 7.90 & 6.68 \\
\hline B3 & $10 / 31 / 79$ & -16 & -4.9 & 1330 & 13.0 & 245 & -- & 5.40 & 6.87 \\
\hline B3 & $10 / 31 / 79$ & -21 & -6.4 & 1320 & 13.8 & 245 & -- & 4.40 & 7.00 \\
\hline B3 & $10 / 31 / 79$ & -26 & -7.9 & 1310 & 13.5 & 220 & -- & 3.50 & 6.60 \\
\hline B3 & $10 / 31 / 79$ & -31 & -9.4 & 1300 & 12.8 & 230 & -- & 8.90 & 6.67 \\
\hline B3 & $10 / 31 / 79$ & -36 & -11.0 & 1250 & 13.2 & 295 & -- & 4.70 & 7.00 \\
\hline B3 & $10 / 31 / 79$ & -41 & -12.5 & 1240 & 13.4 & 247 & -- & 10.40 & 6.89 \\
\hline B3 & $10 / 31 / 79$ & -46 & -14.0 & 1230 & 13.6 & 235 & -- & 11.80 & 6.84 \\
\hline B3 & $10 / 31 / 79$ & -51 & -15.5 & 1228 & 13.2 & 255 & -- & 18.60 & 6.84 \\
\hline B3 & $10 / 31 / 79$ & -57 & -17.4 & 1210 & 12.9 & 220 & -- & 7.60 & 7.25 \\
\hline B3 & $10 / 31 / 79$ & -63 & -19.2 & 1200 & 12.9 & 215 & -- & 11.10 & 7.16 \\
\hline B3 & $10 / 31 / 79$ & -69 & -21.0 & 1150 & 12.8 & 315 & -- & 21.60 & 6.98 \\
\hline B3 & $10 / 31 / 79$ & -75 & -22.9 & 1140 & 13.4 & 360 & -- & 15.89 & 6.84 \\
\hline B3 & $10 / 31 / 79$ & -81 & -24.7 & 1130 & 13.4 & 375 & -- & 10.80 & 6.80 \\
\hline B3 & $10 / 31 / 79$ & -87 & -26.5 & 1120 & 13.6 & 395 & -- & 9.70 & 7.04 \\
\hline B3 & $10 / 31 / 79$ & -93 & -28.3 & 1110 & 13.2 & 385 & -- & .90 & 7.63 \\
\hline B3 & $10 / 31 / 79$ & -99 & -30.2 & 1100 & 13.2 & 370 & -- & .06 & 7.53 \\
\hline B3 & $10 / 31 / 79$ & -105 & -32.0 & 1050 & 13.2 & 360 & -- & .07 & 7.30 \\
\hline B3 & $10 / 31 / 79$ & -110 & -33.5 & 1040 & 13.2 & 388 & -- & -- & 7.15 \\
\hline B3 & $10 / 31 / 79$ & -117 & -35.7 & 1030 & 13.3 & 380 & -- & -- & 7.13 \\
\hline
\end{tabular}


Table 3.--Water-chemistry data collected during a pumping test of well B4, near Horsfall Beach, October 1979 through December 1979--Continued

\begin{tabular}{|c|c|c|c|c|c|c|c|c|c|}
\hline WELL & DATE & $\begin{array}{l}\text { ELEVA } \\
\text { (FEET) }\end{array}$ & $\begin{array}{l}\text { ATION } \\
\text { (METER) }\end{array}$ & TIME & $\begin{array}{l}\text { TEMPER- } \\
\text { ATURE } \\
\left(\begin{array}{ll}\text { DEG } & \text { C) }\end{array}\right.\end{array}$ & $\begin{array}{l}\text { SPECIFIC } \\
\text { CONDUCT- } \\
\text { ANCE } \\
\text { (UMHOS) }\end{array}$ & $\begin{array}{c}\text { BICAR- } \\
\text { BONATE } \\
\text { (MG/L } \\
\text { AS } \\
\text { HCO3) }\end{array}$ & $\begin{array}{l}\text { IRON } \\
\text { DIS- } \\
\text { SOLVED } \\
\text { (MG/L) }\end{array}$ & $\begin{array}{c}\mathrm{PH} \\
(\mathrm{UN} I T)\end{array}$ \\
\hline B3 & $10 / 31 / 79$ & -122 & -37.2 & 1020 & 13.2 & 420 & -- & -- & 7.96 \\
\hline B3 & $10 / 31 / 79$ & -129 & -39.3 & 1010 & 13.2 & 420 & -- & .01 & 7.96 \\
\hline B3 & $10 / 31 / 79$ & -135 & -41.1 & 1000 & 13.2 & 390 & -- & .06 & 7.64 \\
\hline B3 & $10 / 31 / 79$ & -141 & -43.0 & 0950 & 13.2 & 410 & - & .06 & 7.12 \\
\hline B3 & $10 / 31 / 79$ & -147 & -44.8 & 0940 & 13.6 & 645 & -- & .18 & 7.70 \\
\hline B3 & $10 / 31 / 79$ & -153 & -46.6 & 0930 & 13.6 & 620 & -- & .29 & 7.31 \\
\hline B3 & $10 / 31 / 79$ & -159 & -48.5 & 0920 & 13.2 & 800 & -- & 1.10 & 7.47 \\
\hline B3 & $10 / 31 / 79$ & -165 & -50.3 & 0910 & 13.1 & 1225 & -- & .81 & 7.40 \\
\hline B3 & $10 / 31 / 79$ & -171 & -52.1 & 0900 & 13.4 & 1280 & -- & .89 & 7.63 \\
\hline B3 & $11 / 07 / 79$ & -11 & -3.4 & 1630 & 12.6 & 254 & -- & 8.90 & 5.99 \\
\hline B3 & $11 / 07 / 79$ & -21 & -6.4 & 1620 & 12.4 & 226 & - & 4.50 & 6.09 \\
\hline B3 & $11 / 07 / 79$ & -31 & -9.4 & 1610 & 12.6 & 234 & -- & 18.00 & 6.06 \\
\hline B3 & $11 / 07 / 79$ & -36 & -11.0 & 1600 & 12.6 & 290 & -- & 5.10 & 6.19 \\
\hline B3 & $11 / 07 / 79$ & -41 & -12.5 & 1550 & 12.8 & 254 & -- & 12.00 & 6.03 \\
\hline B3 & $11 / 07 / 79$ & -46 & -14.0 & 1540 & 12.8 & 227 & -- & 14.00 & 6.01 \\
\hline B3 & $11 / 07 / 79$ & -51 & -15.5 & 1530 & 12.8 & 245 & -- & 21.00 & 6.05 \\
\hline B3 & $11 / 07 / 79$ & -57 & -17.4 & 1510 & 13.2 & 220 & -- & 7.10 & 6.15 \\
\hline B3 & $11 / 07 / 79$ & -63 & -19.2 & 1400 & 13.4 & 252 & -- & 13.00 & 6.63 \\
\hline B3 & $11 / 07 / 79$ & -69 & -21.0 & 1350 & 13.8 & 330 & -- & 22.00 & 6.48 \\
\hline B3 & $11 / 07 / 79$ & -75 & -22.9 & 1330 & 13.4 & 360 & -- & 15.00 & 6.55 \\
\hline B3 & $11 / 07 / 79$ & -81 & -24.7 & 1315 & 13.8 & 374 & -- & 11.00 & 6.45 \\
\hline B3 & $11 / 07 / 79$ & -87 & -26.5 & 1240 & 13.2 & 410 & -- & 5.80 & 6.72 \\
\hline B3 & $11 / 07 / 79$ & -93 & -28.3 & 1215 & 14.1 & 396 & -- & .60 & 6.95 \\
\hline B3 & $11 / 07 / 79$ & -159 & -48.5 & 1640 & 12.8 & -- & -- & 1.70 & 7.20 \\
\hline B3 & $11 / 08 / 79$ & -93 & -28.3 & 1500 & 12.8 & 388 & -- & .43 & 7.80 \\
\hline B3 & $11 / 08 / 79$ & -99 & -30.2 & 1445 & 12.8 & 380 & -- & .03 & 8.01 \\
\hline B3 & $11 / 08 / 79$ & -117 & -35.7 & 1440 & 12.8 & 386 & - & -- & 8.03 \\
\hline B3 & $11 / 16 / 79$ & -46 & -14.0 & 0950 & 13.0 & 185 & -- & 18.00 & 6.61 \\
\hline B3 & $11 / 16 / 79$ & -75 & -22.9 & 0940 & 12.8 & 340 & - & 16.00 & 6.79 \\
\hline 83 & $11 / 16 / 79$ & -99 & -30.2 & 0930 & 12.7 & 365 & -- & .07 & 7.64 \\
\hline 83 & $11 / 16 / 79$ & -117 & -35.7 & 0920 & 12.6 & 410 & -- & .03 & 7.80 \\
\hline B3 & $11 / 16 / 79$ & -147 & -44.8 & 0910 & 12.8 & 558 & -- & 1.50 & 7.03 \\
\hline B3 & $11 / 16 / 79$ & -159 & -48.5 & 0900 & 13.0 & 925 & -- & 2.20 & 7.57 \\
\hline
\end{tabular}


Table 3.--Water-chemistry data collected during a pumping test of well B4, near Horsfall Beach, October 1979 through December 1979--Continued

\begin{tabular}{|c|c|c|c|c|c|c|c|c|c|}
\hline WELL & DATE & $\begin{array}{c}\text { ELEVA } \\
\text { (FEET) }\end{array}$ & $\begin{array}{l}\text { AT I ON } \\
\text { ( METER) }\end{array}$ & TIME & $\begin{array}{l}\text { TEMPER- } \\
\text { ATURE } \\
\text { (DEG C) }\end{array}$ & $\begin{array}{l}\text { SPECIFIC } \\
\text { CONDUCT- } \\
\text { ANCE } \\
\text { (UMHOS) }\end{array}$ & $\begin{array}{c}\text { BICAR- } \\
\text { BONATE } \\
\text { (MG/L } \\
\text { AS } \\
\text { HCO3) }\end{array}$ & $\begin{array}{l}\text { IRON } \\
\text { DIS- } \\
\text { SOLVED } \\
(M G / L)\end{array}$ & $\begin{array}{c}P H \\
(U N \mid T)\end{array}$ \\
\hline B3 & $11 / 18 / 79$ & -46 & -14.0 & 0950 & 11.2 & 240 & -- & 22.00 & 6.80 \\
\hline B3 & $11 / 18 / 79$ & -75 & -22.9 & 0940 & 10.7 & 330 & -- & 14.00 & 6.92 \\
\hline B3 & $11 / 18 / 79$ & -99 & -30.2 & 0930 & 11.3 & 390 & -- & .07 & 8.08 \\
\hline B3 & $11 / 18 / 79$ & -117 & -35.7 & 0920 & 11.4 & 430 & -- & .07 & 8.01 \\
\hline B3 & $11 / 18 / 79$ & -147 & -44.8 & 0910 & 11.4 & 550 & -- & .41 & 7.73 \\
\hline B3 & $11 / 18 / 79$ & -159 & -48.5 & 0900 & 11.2 & 1080 & -- & 1.40 & 7.66 \\
\hline B3 & $11 / 19 / 79$ & -46 & -14.0 & 0950 & 12.4 & 190 & -- & 21.00 & 7.10 \\
\hline B3 & $11 / 19 / 79$ & -75 & -22.9 & 0940 & 12.5 & 260 & -- & 13.00 & 7.00 \\
\hline B3 & $11 / 19 / 79$ & -99 & -30.2 & 0930 & 12.6 & 310 & -- & .05 & 7.90 \\
\hline B3 & $11 / 19 / 79$ & -117 & -35.7 & 0920 & 12.6 & 350 & -- & .04 & 7.90 \\
\hline B3 & $11 / 19 / 79$ & -147 & -44.8 & 0910 & 12.5 & 450 & -- & .32 & 7.50 \\
\hline B3 & $11 / 19 / 79$ & -159 & -48.5 & 0900 & 12.5 & 925 & -- & 1.30 & 7.20 \\
\hline B3 & $11 / 20 / 79$ & -1 & -0.3 & 1455 & 13.5 & 150 & -- & 15.00 & 6.60 \\
\hline B3 & $11 / 20 / 79$ & -11 & -3.4 & 1445 & 13.1 & 150 & -- & 12.00 & 6.60 \\
\hline B3 & $11 / 20 / 79$ & -21 & -6.4 & 1435 & 12.7 & 140 & -- & 11.00 & 7.00 \\
\hline B3 & $11 / 20 / 79$ & -31 & -9.4 & 1425 & 12.6 & 160 & -- & 18.00 & 6.80 \\
\hline B3 & $11 / 20 / 79$ & -41 & -12.5 & 1415 & 12.8 & 150 & -- & 19.00 & 6.60 \\
\hline B3 & $11 / 20 / 79$ & -57 & -17.4 & 1405 & 12.8 & 110 & -- & 17.00 & 6.70 \\
\hline B3 & $11 / 20 / 79$ & -69 & -21.0 & 1355 & 12.6 & 150 & -- & 18.00 & 6.90 \\
\hline B3 & $11 / 20 / 79$ & -81 & -24.7 & 1345 & 12.8 & 180 & -- & 6.90 & 6.70 \\
\hline B3 & $11 / 20 / 79$ & -93 & -28.3 & 1335 & 12.7 & 225 & -- & .04 & 7.90 \\
\hline B3 & $11 / 20 / 79$ & -110 & -33.5 & 1325 & 12.6 & 230 & -- & .40 & 8.00 \\
\hline B3 & $11 / 20 / 79$ & -122 & -37.2 & 1315 & 12.8 & 250 & -- & .01 & 7.90 \\
\hline B3 & $11 / 20 / 79$ & -129 & -39.3 & 1305 & 12.8 & 225 & -- & .89 & 7.30 \\
\hline B3 & $11 / 20 / 79$ & -135 & -41.1 & 1255 & 12.6 & 230 & -- & .70 & 7.40 \\
\hline B3 & $11 / 20 / 79$ & -141 & -43.0 & 1245 & 12.8 & 250 & -- & .56 & 7.70 \\
\hline B3 & $11 / 20 / 79$ & -153 & -46.6 & 1235 & 12.8 & 500 & -- & .22 & 7.70 \\
\hline B3 & $11 / 20 / 79$ & -165 & -50.3 & 1225 & 12.8 & 4100 & -- & 7.79 & 7.60 \\
\hline B3 & $11 / 27 / 79$ & -21 & -6.4 & 1210 & -- & -- & -- & 8.00 & -- \\
\hline B3 & $11 / 27 / 79$ & -31 & -9.4 & 1200 & 12.0 & -- & -- & 21.00 & 5.89 \\
\hline B3 & $11 / 27 / 79$ & -41 & -12.5 & 1150 & 11.7 & -- & -- & 20.00 & 5.80 \\
\hline B3 & $11 / 27 / 79$ & -57 & -17.4 & 1140 & -- & -- & -- & 18.00 & -- \\
\hline B3 & $11 / 27 / 79$ & -59 & -21.0 & 1130 & 11.9 & -- & -- & 14.00 & 6.30 \\
\hline B3 & $11 / 27 / 79$ & -81 & -24.7 & 1120 & 12.0 & -- & -- & 5.70 & 6.50 \\
\hline B3 & $11 / 27 / 79$ & -93 & -28.3 & 1110 & 12.0 & -- & -- & .20 & 7.50 \\
\hline B3 & $11 / 27 / 79$ & -110 & -33.5 & 1100 & -- & -- & -- & .02 & -- \\
\hline B3 & $11 / 27 / 79$ & -122 & -37.2 & 1050 & 12.0 & -- & -- & .08 & 7.70 \\
\hline B3 & $11 / 27 / 79$ & -129 & -39.3 & 1040 & 12.0 & -- & -- & .06 & 7.60 \\
\hline
\end{tabular}


Table 3.--Water-chemistry data collected during a pumping test of well B4, near Horsfall Beach, October 1979 through December 1979--Continued

\begin{tabular}{|c|c|c|c|c|c|c|c|c|c|}
\hline WELL & DATE & $\begin{array}{c}\text { ELEV } \\
\text { (FEET) }\end{array}$ & $\begin{array}{l}\text { ATION } \\
\text { (METER) }\end{array}$ & TIME & $\begin{array}{l}\text { TEMPER- } \\
\text { ATURE } \\
(\text { DEG C) }\end{array}$ & $\begin{array}{l}\text { SPECIFIC } \\
\text { CONDUCT- } \\
\text { ANCE } \\
\text { (UMHOS) }\end{array}$ & $\begin{array}{c}\text { BICAR- } \\
\text { BONATE } \\
\text { (MG/L } \\
\text { AS } \\
\text { HCO3) }\end{array}$ & $\begin{array}{l}\text { IRON } \\
\text { DIS- } \\
\text { SOLVED } \\
\text { (MG/L) }\end{array}$ & $\begin{array}{c}\text { PH } \\
\text { (UNIT) }\end{array}$ \\
\hline B3 & $11 / 27 / 79$ & -135 & -41.1 & 1030 & 12.1 & -- & -- & .09 & 7.60 \\
\hline B3 & $11 / 27 / 79$ & -141 & -43.0 & 1020 & 11.9 & -- & -- & .16 & 7.20 \\
\hline B3 & $11 / 27 / 79$ & -147 & -44.8 & 1010 & 11.9 & -- & -- & .51 & 7.40 \\
\hline B3 & $11 / 27 / 79$ & -165 & -50.3 & 1000 & -- & -- & -- & 9.00 & -- \\
\hline B3 & $12 / 04 / 79$ & -1 & -0.3 & 1310 & -- & -- & -- & 6.40 & -- \\
\hline B3 & $12 / 04 / 79$ & -1 & -0.3 & 1300 & 13.6 & 155 & -- & 6.40 & 7.00 \\
\hline B3 & $12 / 04 / 79$ & -11 & -3.4 & 1250 & 12.2 & 174 & -- & 5.89 & 6.30 \\
\hline B3 & $12 / 04 / 79$ & -21 & -6.4 & 1240 & 12.7 & 205 & -- & 3.50 & 6.81 \\
\hline B3 & $12 / 04 / 79$ & -31 & -9.4 & 1230 & 15.0 & 202 & -- & 15.00 & 6.71 \\
\hline B3 & $12 / 04 / 79$ & -41 & -12.5 & 1220 & 15.6 & 295 & -- & 29.00 & 6.64 \\
\hline B3 & $12 / 04 / 79$ & -46 & -14.0 & 1210 & 15.0 & 235 & -- & 23.00 & 6.71 \\
\hline B3 & $12 / 04 / 79$ & -57 & -17.4 & 1200 & 13.5 & 240 & -- & 16.00 & 6.90 \\
\hline B3 & $12 / 04 / 7$ & -69 & -21.0 & 1140 & 13.1 & 258 & -- & 15.00 & 6.78 \\
\hline B3 & $12: 04 / 79$ & -75 & -22.9 & 1130 & 14.6 & 271 & -- & 8.10 & 7.00 \\
\hline B3 & $12 / 04 / 79$ & -81 & -24.7 & 1120 & 14.4 & 295 & -- & 5.00 & 7.41 \\
\hline B3 & $12 / 04 / 79$ & -87 & -26.5 & 1110 & 12.8 & 375 & -- & .55 & 8.13 \\
\hline B3 & $12 / 04 / 79$ & -93 & -28.3 & 1100 & 13.5 & 412 & -- & .15 & 8.00 \\
\hline B3 & $12 / 04 / 79$ & -99 & -30.2 & 1050 & 13.4 & 405 & -- & .04 & 8.10 \\
\hline B3 & $12 / 04 / 79$ & -110 & -33.5 & 1040 & 14.2 & 359 & -- & -- & 8.26 \\
\hline B3 & $12 / 04 / 79$ & -117 & -35.7 & 1030 & 13.8 & 390 & -- & -- & 8.20 \\
\hline B3 & $12 / 04 / 79$ & -122 & -37.2 & 1020 & 13.2 & 411 & -- & .04 & 8.10 \\
\hline B3 & $12 / 04 / 79$ & -129 & -39.3 & 1010 & 14.7 & 418 & -- & .04 & 7.76 \\
\hline B3 & $12 / 04 / 79$ & -135 & $-41 \cdot 1$ & 1000 & 14.5 & 425 & -- & .08 & 8.00 \\
\hline B3 & $12 / 04 / 79$ & -141 & -43.0 & 0950 & 13.0 & 438 & -- & .14 & 7.57 \\
\hline B3 & $12 / 04 / 79$ & -147 & -44.8 & 0940 & 13.2 & 472 & -- & .47 & 7.76 \\
\hline B3 & $12 / 04 / 79$ & -159 & -48.5 & 0930 & $14 \cdot 3$ & 960 & -- & .98 & 7.81 \\
\hline B4 & $11 / 06 / 79$ & -157 & -47.9 & 1525 & 13.8 & 870 & -- & .04 & 8.00 \\
\hline B4 & $11 / 08 / 79$ & -157 & -47.9 & 1155 & 13.8 & 890 & -- & .70 & 7.00 \\
\hline B4 & $11 / 08 / 79$ & -157 & -47.9 & 1210 & 13.5 & 870 & -- & .70 & 7.20 \\
\hline B4 & $11 / 08 / 79$ & -157 & -47.9 & 1225 & 13.4 & 800 & -- & .66 & 7.20 \\
\hline B4 & $11 / 08 / 79$ & -157 & -47.9 & 1240 & 13.7 & 800 & -- & .63 & 7.30 \\
\hline B4 & $11 / 08 / 79$ & -157 & -47.9 & 1255 & 13.5 & 810 & -- & .71 & 7.40 \\
\hline B4 & $11 / 08 / 79$ & -157 & -47.9 & 1325 & 13.5 & 800 & -- & .59 & 7.40 \\
\hline B4 & $11 / 08 / 79$ & -157 & -47.9 & 1425 & 13.4 & 760 & -- & .46 & 7.50 \\
\hline B4 & $11 / 08 / 79$ & -157 & -47.9 & 1615 & 12.9 & -- & -- & .43 & 7.50 \\
\hline
\end{tabular}


Table 3.--Water-chemistry data collected during a pumping test of well B4, near Horsfall Beach, October 1979 through December 1979--Continued

\begin{tabular}{|c|c|c|c|c|c|c|c|c|c|}
\hline WELL & DATE & $\begin{array}{l}\text { ELEVA } \\
\text { (FEET) }\end{array}$ & $\begin{array}{l}\text { ATION } \\
\text { (METER) }\end{array}$ & TIME & $\begin{array}{l}\text { TEMPER- } \\
\text { ATURE } \\
\left(\begin{array}{ll}\text { DEG } & \text { C) }\end{array}\right.\end{array}$ & $\begin{array}{l}\text { SPECIFIC } \\
\text { CONDUCT- } \\
\text { ANCE } \\
\text { (UMHOS) }\end{array}$ & $\begin{array}{c}\text { BICAR- } \\
\text { BONATE } \\
\text { (MG/L } \\
\text { AS } \\
\text { HCO3) }\end{array}$ & $\begin{array}{l}\text { IRON } \\
\text { DIS- } \\
\text { SOLVED } \\
\text { (MG/L) }\end{array}$ & $\begin{array}{c}\text { PH } \\
(\text { UNIT) }\end{array}$ \\
\hline B4 & $11 / 17 / 79$ & -157 & -47.9 & 1125 & -- & -- & -- & .42 & -- \\
\hline B4 & $11 / 17 / 79$ & -157 & -47.9 & 1545 & -- & -- & -- & .38 & -- \\
\hline B4 & $11 / 17 / 79$ & -157 & -47.9 & 1705 & -- & -- & -- & .43 & -- \\
\hline B4 & $11 / 19 / 79$ & -157 & -47.9 & 1345 & -- & -- & -- & .31 & -- \\
\hline B4 & $11 / 20 / 79$ & -157 & -47.9 & 1015 & -- & -- & -- & .35 & -- \\
\hline B4 & $11 / 20 / 79$ & -157 & -47.9 & 1545 & -- & -- & -- & .28 & -- \\
\hline B4 & $11 / 27 / 79$ & -157 & -47.9 & 1530 & -- & -- & -- & .28 & -- \\
\hline B4 & $11 / 28 / 79$ & -157 & -47.9 & 1015 & -- & -- & -- & .21 & -- \\
\hline $\mathrm{B}_{4} 21$ & $12 / 05 / 79$ & 9 & 2.7 & 1100 & -- & - & - & .54 & -- \\
\hline B4 & $12 / 05 / 79$ & -157 & -47.9 & 1015 & -- & -- & -- & .22 & -- \\
\hline P1 & $10 / 16 / 79$ & 6 & 1.8 & 1400 & 15.5 & 260 & -- & 1.29 & 5.48 \\
\hline P1 & $10 / 16 / 79$ & 2 & 0.6 & 1410 & 15.0 & 266 & -- & 22.00 & 6.16 \\
\hline P1 & $10 / 16 / 79$ & -2 & -0.6 & 1420 & 14.2 & 240 & - & 21.60 & 6.40 \\
\hline P1 & $10 / 16 / 79$ & -6 & -1.8 & 1430 & 13.5 & 302 & -- & 30.00 & 6.34 \\
\hline P1 & $10 / 16 / 79$ & -10 & -3.0 & 1440 & 13.4 & 348 & -- & 36.40 & 6.53 \\
\hline P1 & $10 / 16 / 79$ & -14 & $-4 \cdot 3$ & 1450 & 13.2 & 232 & -- & 30.60 & 6.65 \\
\hline P1 & $10 / 16 / 79$ & -18 & -5.5 & 1500 & 13.0 & 210 & -- & 25.30 & 6.64 \\
\hline P1 & $10 / 16 / 79$ & -22 & -6.7 & 1510 & 13.0 & 221 & -- & 25.60 & 6.70 \\
\hline P1 & $10 / 16 / 79$ & -26 & -7.9 & 1520 & 13.2 & 215 & -- & 23.10 & 6.77 \\
\hline P2 & $10 / 16 / 79$ & 4 & 1.2 & 1000 & 14.9 & 167 & -- & 2.07 & 6.02 \\
\hline $\mathrm{P} 2$ & $10 / 16 / 79$ & -1 & -0.3 & 1015 & 14.4 & 152 & -- & .54 & 6.10 \\
\hline P2 & $10 / 16 / 79$ & -6 & -1.8 & 1030 & 13.9 & 154 & -- & .80 & 6.17 \\
\hline P2 & $10 / 16 / 79$ & -11 & -3.4 & 1045 & 12.8 & 156 & -- & 1.03 & 6.22 \\
\hline P2 & $10 / 16 / 79$ & -16 & -4.9 & 1100 & 12.5 & 159 & -- & 1.38 & 6.41 \\
\hline P2 & $10 / 16 / 79$ & -21 & -6.4 & 1115 & -- & -- & -- & .82 & -- \\
\hline P2 & $10 / 16 / 79$ & -26 & -7.9 & 1130 & 12.5 & 333 & -- & 3.86 & 6.98 \\
\hline P2 & $10 / 16 / 79$ & -31 & -9.4 & 1145 & 12.5 & 240 & -- & 12.70 & 6.75 \\
\hline P2 & $10 / 16 / 79$ & -36 & -11.0 & 1200 & 12.5 & 310 & -- & 17.10 & 6.76 \\
\hline P2 & $10 / 16 / 79$ & -41 & -12.5 & 1215 & 12.5 & 302 & -- & 17.70 & 6.79 \\
\hline P2 & $10 / 31 / 79$ & -21 & -6.4 & 1520 & 12.2 & 200 & -- & 1.40 & 6.40 \\
\hline
\end{tabular}


Table 3.--Water-chem istry data collected during a pumping test of well B4, near Horsfall Beach, October 1979 through December 1979--Continued

\begin{tabular}{|c|c|c|c|c|c|c|c|c|c|}
\hline WELL & DATE & $\begin{array}{l}\text { ELEVA } \\
\text { (FEET) }\end{array}$ & $\begin{array}{l}\text { ATION } \\
\text { (METER) }\end{array}$ & TIME & $\begin{array}{l}\text { TEMPER- } \\
\text { ATURE } \\
(\text { DEG C) }\end{array}$ & $\begin{array}{l}\text { SPECIFIC } \\
\text { CONDUCT- } \\
\text { ANCE } \\
\text { (UMHOS) }\end{array}$ & $\begin{array}{c}\text { BICAR- } \\
\text { BONATE } \\
\text { (MG/L } \\
\text { AS } \\
\text { HCO3) }\end{array}$ & $\begin{array}{l}\text { IRON } \\
\text { DIS- } \\
\text { SOLVED } \\
\text { (MG/L) }\end{array}$ & $\begin{array}{c}\mathrm{PH} \\
(\mathrm{UN} \mid \mathrm{T})\end{array}$ \\
\hline P2 & $10 / 31 / 79$ & -26 & -7.9 & 1510 & 11.9 & 255 & -- & 3.20 & 6.79 \\
\hline P2 & $10 / 31 / 79$ & -31 & -9.4 & 1530 & 12.0 & 232 & -- & 12.70 & 6.48 \\
\hline P2 & $10 / 31 / 79$ & -36 & -11.0 & 1540 & 12.0 & 280 & -- & 15.70 & 6.48 \\
\hline P2 & $10 / 31 / 79$ & -41 & -12.5 & 1550 & 13.4 & 318 & -- & 18.40 & 6.52 \\
\hline P2 & $11 / 06 / 79$ & -21 & -6.4 & 1200 & 12.6 & 120 & -- & 2.20 & 6.60 \\
\hline P2 & $11 / 06 / 79$ & -26 & -7.9 & 1215 & 12.8 & 230 & -- & 3.30 & 6.50 \\
\hline P2 & $11 / 06 / 79$ & -31 & -9.4 & 1230 & 12.5 & 160 & -- & 13.10 & 6.50 \\
\hline P2 & $11 / 06 / 79$ & -36 & -11.0 & 1245 & 13.0 & 170 & -- & 13.90 & 6.40 \\
\hline P2 & $11 / 06 / 79$ & -41 & -12.5 & 1300 & 12.6 & 260 & -- & 19.40 & 6.30 \\
\hline P2 & $11 / 16 / 79$ & -41 & -12.5 & 1010 & 12.4 & 245 & -- & -14.00 & 6.78 \\
\hline P2 & $11 / 18 / 7 i$ & -41 & -12.5 & 1010 & 11.8 & 280 & -- & 14.00 & 6.72 \\
\hline P2 & $11 / 19 / 79$ & -41 & -12.5 & 1010 & 12.0 & 210 & -- & 14.00 & 6.50 \\
\hline P2 & $11 / 20 / 79$ & -6 & -1.8 & 1545 & 12.7 & 70 & -- & .60 & 6.20 \\
\hline P2 & $11 / 20 / 79$ & -16 & -4.9 & 1555 & 12.1 & 80 & -- & 1.20 & 6.70 \\
\hline P2 & $11 / 20 / 79$ & -26 & -7.9 & 1605 & 11.9 & 150 & -- & 1.40 & 6.90 \\
\hline P2 & $11 / 20 / 79$ & -36 & -11.0 & 1615 & 11.8 & 110 & -- & 13.00 & 6.80 \\
\hline P2 & $11 / 27 / 79$ & -6 & -1.8 & 1320 & 12.4 & -- & -- & .53 & 5.30 \\
\hline P2 & $11 / 27 / 79$ & -16 & -4.9 & 1330 & -- & -- & -- & .78 & -- \\
\hline P2 & $11 / 27 / 79$ & -26 & -7.9 & 1300 & -- & -- & -- & 4.10 & -- \\
\hline P2 & $11 / 27 / 79$ & -36 & -11.0 & 1310 & -- & -- & -- & 12.00 & -- \\
\hline P2 & $12 / 05 / 79$ & -6 & -1.8 & 0900 & 11.8 & 124 & -- & .25 & 6.40 \\
\hline P2 & $12 / 05 / 79$ & -16 & -4.9 & 0910 & 12.6 & 151 & -- & .54 & 6.10 \\
\hline $\mathrm{P} 2$ & $12 / 05 / 79$ & -21 & -6.4 & 0920 & 12.1 & 175 & -- & .67 & 6.80 \\
\hline P2 & $12 / 05 / 79$ & -26 & -7.9 & 0930 & 11.9 & 185 & -- & 3.50 & 6.80 \\
\hline P2 & $12 / 05 / 79$ & -31 & -9.4 & 0940 & 11.6 & 262 & -- & 12.00 & 6.70 \\
\hline P2 & $12 / 05 / 79$ & -36 & -11.0 & 0950 & 11.6 & 262 & -- & 14.00 & 6.70 \\
\hline P2 & $12 / 05 / 79$ & -41 & -12.5 & 1000 & 11.6 & 274 & -- & 15.00 & 7.10 \\
\hline
\end{tabular}


Table 3.--Water-chemistry data collected during a pumping test of well B4, near Horsfall Beach, October 1979 through December 1979--Continued

\begin{tabular}{|c|c|c|c|c|c|c|c|c|c|}
\hline WELL & DATE & $\begin{array}{c}\text { ELEV } \\
\text { (FEET) }\end{array}$ & $\begin{array}{l}\text { ATION } \\
\text { (METER) }\end{array}$ & TIME & $\begin{array}{l}\text { TEMPER- } \\
\text { ATURE } \\
(\text { DEG C) }\end{array}$ & $\begin{array}{l}\text { SPECIFIC } \\
\text { CONDUCT- } \\
\text { ANCE } \\
\text { (UMHOS) }\end{array}$ & $\begin{array}{c}\text { BICAR- } \\
\text { BONATE } \\
\text { (MG/L } \\
\text { AS } \\
\text { HCO3) }\end{array}$ & $\begin{array}{l}\text { IRON } \\
\text { DIS- } \\
\text { SOLVED } \\
(M G / L)\end{array}$ & $\begin{array}{c}P H \\
(U N \mid T)\end{array}$ \\
\hline P3 & $10 / 17 / 79$ & 13 & 4.0 & 1300 & 16.4 & 200 & -- & .37 & 5.33 \\
\hline P3 & $10 / 17 / 79$ & 10 & 3.0 & 1310 & 15.8 & 210 & -- & 3.20 & 6.04 \\
\hline P3 & $10 / 17 / 79$ & 7 & 2.1 & 1320 & 15.0 & 179 & -- & 7.70 & 6.03 \\
\hline P3 & $10 / 17 / 79$ & 4 & 1.2 & 1330 & 14.8 & 198 & -- & 8.00 & 6.17 \\
\hline P3 & $10 / 17 / 79$ & 1 & 0.3 & 1340 & 14.6 & 174 & -- & 9.30 & 6.12 \\
\hline P3 & $10 / 17 / 79$ & -2 & -0.6 & 1350 & 13.8 & 271 & -- & 18.70 & 6.05 \\
\hline P3 & $10 / 17 / 79$ & -7 & -2.1 & 1400 & 13.4 & 235 & -- & 19.10 & 6.24 \\
\hline P3 & $10 / 17 / 79$ & -12 & -3.7 & 1410 & 13.4 & 340 & -- & 31.00 & 6.32 \\
\hline P3 & $10 / 17 / 79$ & -17 & -5.2 & 1420 & 13.4 & 207 & -- & 27.39 & 6.57 \\
\hline P3 & $10 / 17 / 79$ & -22 & -6.7 & 1430 & 13.4 & 164 & -- & 20.70 & 6.68 \\
\hline P3 & $10 / 17 / 79$ & -27 & -8.2 & 1440 & 13.6 & 200 & -- & 28.20 & 6.76 \\
\hline P5 & $10 / 16 / 79$ & 2 & 0.6 & 1200 & 15.4 & 152: & -- & .84 & 6.28 \\
\hline P5 & $10 / 16 / 79$ & -2 & -0.6 & 1215 & 14.6 & 142 & -- & 1.21 & 6.37 \\
\hline P5 & $10 / 16 / 79$ & -6 & -1.8 & 1230 & 14.5 & 165 & -- & 1.21 & 6.20 \\
\hline P5 & $10 / 16 / 79$ & -10 & -3.0 & 1245 & 13.8 & 144 & -- & 5.00 & 6.52 \\
\hline P5 & $10 / 16 / 79$ & -15 & -4.6 & 1300 & 12.8 & 250 & -- & 12.00 & 6.62 \\
\hline P5 & $10 / 16 / 79$ & -20 & -6.1 & 1315 & 12.8 & 268 & -- & 10.30 & 6.84 \\
\hline P5 & $10 / 16 / 79$ & -25 & -7.6 & 1330 & 12.8 & 390 & -- & 28.70 & 6.78 \\
\hline P5 & $10 / 16 / 79$ & -30 & -9.1 & 1345 & 13.0 & 329 & -- & 24.30 & 6.85 \\
\hline P5 & $10 / 16 / 79$ & -35 & -10.7 & 1400 & 13.8 & 345 & -- & 25.79 & 6.82 \\
\hline P5 & $11 / 01 / 79$ & -15 & -4.6 & 1420 & 12.2 & 230 & -- & 11.40 & 6.40 \\
\hline P5 & $11 / 01 / 79$ & -20 & -6.1 & 1430 & 12.0 & 235 & -- & 14.60 & 6.54 \\
\hline P5 & $11 / 01 / 79$ & -25 & -7.6 & 1440 & 12.2 & 352 & -- & 27.80 & 6.50 \\
\hline P5 & $11 / 01 / 79$ & -30 & -9.1 & 1450 & 12.2 & 288 & -- & 22.60 & 6.64 \\
\hline P5 & $11 / 01 / 79$ & -35 & -10.7 & 1500 & 12.4 & 325 & -- & 27.10 & 6.61 \\
\hline P5 & $11 / 06 / 79$ & -15 & -4.6 & 1315 & 12.6 & 160 & 49 & 12.10 & 6.40 \\
\hline P5 & $11 / 06 / 79$ & -20 & -6.1 & 1330 & 13.1 & 160 & - & 13.69 & 6.50 \\
\hline P5 & $11 / 06 / 79$ & -25 & -7.6 & 1345 & 13.1 & 280 & 110 & 27.60 & 6.50 \\
\hline P5 & $11 / 06 / 79$ & -30 & -9.1 & 1400 & 13.2 & 220 & -- & 22.80 & 6.50 \\
\hline P5 & $11 / 06 / 79$ & -35 & -10.7 & 1415 & 12.7 & 260 & 122 & 27.10 & 6.40 \\
\hline P5 & $11 / 09 / 79$ & -15 & -4.6 & 1100 & 13.6 & 150 & -- & 11.00 & 6.35 \\
\hline P5 & $11 / 09 / 79$ & -20 & -6.1 & 1110 & 13.0 & 150 & -- & 12.00 & 6.45 \\
\hline P5 & $11 / 09 / 79$ & -25 & -7.6 & 1120 & 13.1 & 300 & -- & 26.00 & 6.35 \\
\hline P5 & $11 / 09 / 79$ & -30 & -9.1 & 1130 & 13.2 & 230 & -- & 23.00 & 6.35 \\
\hline P5 & $11 / 09 / 79$ & -35 & -10.7 & 1140 & 13.2 & 220 & -- & 23.00 & 6.40 \\
\hline
\end{tabular}


Table 3.--Water-chemistry data collected during a pumping test of well B4, near Horsfall Beach, October 1979 through December 1979--Continued

\begin{tabular}{|c|c|c|c|c|c|c|c|c|c|}
\hline WELL & DATE & $\begin{array}{l}\text { ELEV } \\
\text { (FEET) }\end{array}$ & $\begin{array}{l}\text { ATI ON } \\
\text { (METER) }\end{array}$ & TIME & $\begin{array}{c}\text { TEMPER- } \\
\text { ATURE } \\
(\text { DEG C) }\end{array}$ & $\begin{array}{l}\text { SPECIFIC } \\
\text { CONDUCT- } \\
\text { ANCE } \\
\text { (UMHOS) }\end{array}$ & $\begin{array}{c}\text { BICAR- } \\
\text { BONATE } \\
\text { (MG/L } \\
\text { AS } \\
\text { HCO3) }\end{array}$ & $\begin{array}{l}\text { IRON } \\
\text { DIS- } \\
\text { SOLVED } \\
(M G / L)\end{array}$ & $\begin{array}{c}\text { PH } \\
(U N \mid T)\end{array}$ \\
\hline P5 & $11 / 16 / 79$ & -35 & -10.7 & 1000 & 12.6 & -- & - & 22.00 & 6.42 \\
\hline P5 & $11 / 18 / 79$ & -35 & -10.7 & 0900 & 11.4 & -- & -- & 20.00 & 6.78 \\
\hline P5 & $11 / 19 / 79$ & -35 & $-10 \cdot 7$ & 1000 & 12.4 & 210 & -- & 19.00 & 6.40 \\
\hline $\begin{array}{l}\text { P5 } \\
\text { P5 } \\
\text { P5 } \\
\text { P5 }\end{array}$ & $\begin{array}{l}11 / 20 / 79 \\
11 / 20 / 79 \\
11 / 20 / 79 \\
11 / 20 / 79\end{array}$ & $\begin{array}{r}-2 \\
-10 \\
-20 \\
-30\end{array}$ & $\begin{array}{l}-0.6 \\
-3.0 \\
-6.1 \\
-9.1\end{array}$ & $\begin{array}{l}1455 \\
1505 \\
1515 \\
1525\end{array}$ & $\begin{array}{l}14.0 \\
13.3 \\
12.8 \\
12.8\end{array}$ & $\begin{array}{r}50 \\
45 \\
75 \\
225\end{array}$ & $\begin{array}{l}-- \\
-- \\
--\end{array}$ & $\begin{array}{r}1.60 \\
3.80 \\
11.00 \\
27.00\end{array}$ & $\begin{array}{l}6.10 \\
6.30 \\
6.70 \\
6.70\end{array}$ \\
\hline $\begin{array}{l}\text { P5 } \\
\text { P5 } \\
\text { P5 } \\
\text { P5 }\end{array}$ & $\begin{array}{l}11 / 28 / 79 \\
11 / 28 / 79 \\
11 / 28 / 79 \\
11 / 28 / 79\end{array}$ & $\begin{array}{r}-2 \\
-10 \\
-20 \\
-30\end{array}$ & $\begin{array}{l}-0.6 \\
-3.0 \\
-6.1 \\
-9.1\end{array}$ & $\begin{array}{l}1000 \\
1010 \\
1020 \\
1030\end{array}$ & $\begin{array}{l}13.2 \\
12.9 \\
12.0 \\
11.7\end{array}$ & $\begin{array}{l}-- \\
-- \\
--\end{array}$ & $\begin{array}{l}-- \\
-- \\
-- \\
--\end{array}$ & $\begin{array}{r}1.40 \\
4.20 \\
11.00 \\
21.00\end{array}$ & $\begin{array}{l}5.30 \\
5.80 \\
6.00 \\
6.00\end{array}$ \\
\hline $\begin{array}{l}\text { P5 } \\
\text { P5 } \\
\text { P5 } \\
\text { P5 } \\
\text { P5 }\end{array}$ & $\begin{array}{l}12 / 05 / 79 \\
12 / 05 / 79 \\
12 / 05 / 79 \\
12 / 05 / 79 \\
12 / 05 / 79\end{array}$ & $\begin{array}{l}-2 \\
-10 \\
-20 \\
-30 \\
-35\end{array}$ & $\begin{array}{r}-0.6 \\
-3.0 \\
-6.1 \\
-9.1 \\
-10.7\end{array}$ & $\begin{array}{l}1120 \\
1130 \\
1140 \\
1150 \\
1200\end{array}$ & $\begin{array}{l}12.7 \\
12.8 \\
12.2 \\
12.0 \\
12.0\end{array}$ & $\begin{array}{l}175 \\
133 \\
162 \\
338 \\
324\end{array}$ & $\begin{array}{l}-- \\
-- \\
-- \\
-- \\
--\end{array}$ & $\begin{array}{r}.84 \\
4.60 \\
8.80 \\
26.00 \\
29.00\end{array}$ & $\begin{array}{l}5.50 \\
5.89 \\
6.00 \\
6.00 \\
5.89\end{array}$ \\
\hline $\begin{array}{l}\text { P6 } \\
\text { P6 } \\
\text { P6 } \\
\text { P6 } \\
\text { P6 } \\
\text { P6 } \\
\text { P6 } \\
\text { P6 } \\
\text { P6 }\end{array}$ & $\begin{array}{l}10 / 16 / 79 \\
10 / 16 / 79 \\
10 / 16 / 79 \\
10 / 16 / 79 \\
10 / 16 / 79 \\
10 / 16 / 79 \\
10 / 16 / 79 \\
10 / 16 / 79 \\
10 / 16 / 79\end{array}$ & $\begin{array}{r}5 \\
1 \\
-3 \\
-7 \\
-11 \\
-17 \\
-21 \\
-26 \\
-31\end{array}$ & $\begin{array}{r}1.5 \\
0.3 \\
-0.9 \\
-2.1 \\
-3.4 \\
-5.2 \\
-6.4 \\
-7.9 \\
-9.4\end{array}$ & $\begin{array}{l}1600 \\
1610 \\
1620 \\
1630 \\
1640 \\
1650 \\
1700 \\
1710 \\
1720\end{array}$ & $\begin{array}{l}16.4 \\
15.8 \\
15.2 \\
14.5 \\
14.0 \\
13.5 \\
13.2 \\
13.2 \\
13.4\end{array}$ & $\begin{array}{l}300 \\
410 \\
440 \\
369 \\
410 \\
246 \\
211 \\
213 \\
217\end{array}$ & $\begin{array}{l}-- \\
-- \\
-- \\
-- \\
-- \\
-- \\
--\end{array}$ & $\begin{array}{r}7.71 \\
15.60 \\
34.30 \\
33.40 \\
45.00 \\
29.70 \\
25.70 \\
23.00 \\
17.30\end{array}$ & $\begin{array}{l}6.10 \\
6.19 \\
6.24 \\
6.38 \\
6.57 \\
6.78 \\
6.90 \\
6.78 \\
6.95\end{array}$ \\
\hline
\end{tabular}


Table 3.--Water-chemistry data collected during a pumping test of well B4, near Horsfall Beach, October 1979 through December 1979--Continued

\begin{tabular}{|c|c|c|c|c|c|c|c|c|c|}
\hline WELL & DATE & $\begin{array}{l}\text { ELEV } \\
\text { (FEET) }\end{array}$ & $\begin{array}{l}\text { ATION } \\
\text { (METER) }\end{array}$ & TIME & $\begin{array}{c}\text { TEMPER- } \\
\text { ATURE } \\
(\text { DEG C) }\end{array}$ & $\begin{array}{l}\text { SPECIFIC } \\
\text { CONDUCT- } \\
\text { ANCE } \\
\text { (UMHOS) }\end{array}$ & $\begin{array}{c}\text { BICAR- } \\
\text { BONATE } \\
\text { (MG/L } \\
\text { AS } \\
\text { HCO3) }\end{array}$ & $\begin{array}{l}\text { IRON } \\
\text { DIS- } \\
\text { SOLVED } \\
(M G / L)\end{array}$ & $\begin{array}{c}P H \\
(U N I T)\end{array}$ \\
\hline P6 & $11 / 20 / 79$ & -3 & -0.9 & 1615 & 14.2 & 250 & -- & 33.00 & 6.10 \\
\hline P6 & $11 / 20 / 79$ & -11 & -3.4 & 1625 & 13.6 & 225 & -- & 38.00 & 6.40 \\
\hline P6 & $11 / 20 / 79$ & -21 & -6.4 & 1635 & 12.9 & 125 & -- & 24.00 & 6.80 \\
\hline P6 & $11 / 20 / 79$ & -31 & -9.4 & 1645 & 12.9 & 100 & - & 14.00 & 6.80 \\
\hline P7 & $10 / 17 / 79$ & 4 & 1.2 & 1140 & 15.0 & 150 & -- & 2.90 & 5.78 \\
\hline P7 & $10 / 17 / 79$ & 0 & 0.0 & 1150 & 14.4 & 131 & -- & 6.10 & 5.81 \\
\hline P7 & $10 / 17 / 79$ & -4 & -1.2 & 1200 & 13.8 & 280 & -- & 22.60 & 5.86 \\
\hline P7 & $10 / 17 / 79$ & -8 & -2.4 & 1210 & 13.4 & 324 & -- & 30.10 & 6.12 \\
\hline P7 & $10 / 17 / 79$ & -13 & -4.0 & 1220 & 13.2 & 490 & -- & 58.49 & 6.16 \\
\hline P7 & $10 / 17 / 79$ & -18 & -5.5 & 1230 & 13.0 & 590 & -- & 66.40 & 6.24 \\
\hline
\end{tabular}

1/ Sampled from 1-1/4 inch PVC pipe with screen at elevation ranging from -142 to -147 feet $(-43.3$ to $-44.8 \mathrm{~m})$.

2f Water sampled at water table next to well. 
Table 4.--Water-chemistry data collected from wells west of Saunders Lake, 1978-79

\begin{tabular}{|c|c|c|c|c|c|c|c|c|c|}
\hline WELL & DATE & $\begin{array}{l}\text { ELEVA } \\
\text { (FEET) }\end{array}$ & $\begin{array}{l}\text { ATION } \\
\text { (METER) }\end{array}$ & TIME & $\begin{array}{l}\text { TEMPER- } \\
\text { ATURE } \\
(\text { DEG C) }\end{array}$ & $\begin{array}{l}\text { SPECIFIC } \\
\text { CONDUCT- } \\
\text { ANCE } \\
\text { (UMHOS) }\end{array}$ & $\begin{array}{l}\text { IRON } \\
\text { DIS } \\
\text { SOLVED } \\
\text { (MG/L) }\end{array}$ & $\begin{array}{c}P H \\
(U N \mid T)\end{array}$ & $\begin{array}{l}\text { CHLO- } \\
\text { RIDE } \\
\text { DIS- } \\
\text { SOLVED } \\
(M G / L)\end{array}$ \\
\hline $100 E$ & $03 / 08 / 79$ & -13 & $-4 \cdot 0$ & 1710 & 9.7 & 150 & 2.60 & -- & 24 \\
\hline $100 \mathrm{~S}$ & $03 / 08 / 79$ & -24 & -7.3 & 1645 & 11.9 & 290 & .08 & 6.10 & 57 \\
\hline $250 \mathrm{~W}$ & $03 / 08 / 79$ & -9 & -2.7 & 1815 & 11.2 & 160 & .41 & 6.00 & 29 \\
\hline $400 \mathrm{~N}$ & $03 / 08 / 79$ & -3 & -.9 & 1740 & 10.3 & 130 & .22 & 7.10 & 17 \\
\hline P11 & $08 / 02 / 78$ & 8 & 2.4 & 1900 & 13.0 & 102 & 7.50 & 6.30 & 14 \\
\hline P11 & $08 / 02 / 78$ & 6 & 1.8 & 1730 & 13.2 & 109 & 7.50 & 6.80 & 15 \\
\hline P11 & $08 / 02 / 78$ & 1 & 0.3 & 1745 & 12.5 & 192 & 16.00 & 6.90 & 23 \\
\hline P11 & $08 / 02 / 79$ & -4 & -1.2 & 1800 & 12.2 & 126 & 9.50 & 6.50 & 16 \\
\hline P11 & $09 / 02 / 78$ & -5 & -1.8 & 1645 & -- & 117 & 10.00 & 7.10 & 20 \\
\hline P11 & $09 / 02 / 78$ & -9 & -2.7 & 1815 & 12.1 & 12.5 & 13.00 & 6.50 & 17 \\
\hline P11 & $08 / 02 / 78$ & -14 & $-4 \cdot 3$ & 1830 & 12.2 & 108 & 7.40 & 6.30 & 17 \\
\hline P11 & $08 / 02 / 78$ & -19 & -5.8 & 1845 & 12.4 & 104 & 6.70 & 6.20 & 13 \\
\hline P8 & $07 / 14 / 78$ & -14 & $-4 \cdot 3$ & 1620 & 16.0 & 131 & .28 & 5.70 & -- \\
\hline$P Q$ & $07 / 14 / 78$ & -19 & -5.8 & 1630 & 15.5 & 127 & .82 & 5.80 & -- \\
\hline P8 & $07 / 14 / 78$ & -24 & -7.3 & 1640 & 14.0 & 138 & .56 & 5.90 & -- \\
\hline PQ & $07 / 14 / 78$ & -29 & -8.8 & 1655 & 14.0 & 115 & 1.90 & 6.20 & -- \\
\hline P8 & $07 / 14 / 78$ & -34 & -10.4 & 1703 & 14.0 & 120 & 3.20 & 6.30 & -- \\
\hline P8 & $07 / 14 / 79$ & -39 & -11.9 & 1600 & 14.0 & 125 & 4.80 & 6.10 & -- \\
\hline P8 & $07 / 15 / 78$ & 1 & .3 & 1115 & 14.7 & 145 & .26 & 5.80 & -- \\
\hline P8 & $07 / 15 / 78$ & -4 & -1.2 & 0903 & 15.0 & 137 & .20 & 6.00 & -- \\
\hline PQ & $07 / 15 / 78$ & -9 & -2.7 & 0920 & 14.8 & 139 & .26 & 5.80 & -- \\
\hline P8 & $07 / 15 / 78$ & -14 & -4.3 & 0935 & 14.5 & 136 & .16 & 5.80 & -- \\
\hline P8 & $07 / 15 / 78$ & -19 & -5.8 & 0955 & 14.6 & 132 & .29 & 5.80 & -- \\
\hline P8 & $07 / 15 / 78$ & -2.4 & $-7 \cdot 3$ & 1015 & 12.4 & 124 & .58 & 6.20 & -- \\
\hline P\& & $07 / 15 / 78$ & -29 & -8.8 & 1025 & 12.4 & 108 & 2.10 & 6.20 & -- \\
\hline PQ & $07 / 15 / 79$ & -34 & -10.4 & 1040 & 12.4 & 120 & 3.10 & 6.40 & -- \\
\hline P8 & $07 / 15 / 78$ & -39 & -11.9 & 1058 & 12.7 & 118 & 5.50 & 6.40 & -- \\
\hline PR & $08 / 02 / 78$ & 1 & .3 & 1430 & 14.0 & 126 & .19 & 5.89 & 20 \\
\hline P8 & $08 / 02 / 78$ & -4 & -1.2 & 1445 & 13.4 & 126 & 4.50 & 6.00 & 19 \\
\hline$p q$ & $03 / 02 / 78$ & -9 & -2.7 & 1500 & 12.5 & 134 & .23 & 5.00 & 19 \\
\hline P8 & $08 / 02 / 78$ & -14 & -4.3 & 1515 & 12.4 & 115 & .35 & 6.00 & -- \\
\hline P8 & $08 / 02 / 78$ & -19 & -5.8 & 1530 & 12.0 & 107 & .26 & 6.20 & 12 \\
\hline P8 & $08 / 02 / 79$ & -24 & -7.3 & 1545 & 11.5 & 130 & .55 & 6.40 & 12 \\
\hline PQ & $08 / 02 / 78$ & -29 & -8.8 & 1600 & 11.9 & 134 & 2.60 & 6.50 & 10 \\
\hline PQ & $08 / 02 / 78$ & -34 & -10.4 & 1615 & 12.0 & 130 & 2.90 & 6.70 & 13 \\
\hline P8 & $08 / 02 / 78$ & -39 & -11.9 & 1630 & 12.2 & 125 & 4.20 & 6.70 & 13 \\
\hline$P R$ & $03 / 08 / 79$ & PIT & $1 /$ & 1457 & 12.4 & 106 & .34 & 5.89 & 19 \\
\hline $\mathrm{P} 8$ & $03 / 08 / 79$ & 6 & $\overline{1} .8$ & 1503 & 9.6 & 100 & .37 & 6.00 & 20 \\
\hline$P 8$ & $03 / 08 / 79$ & 1 & .3 & 1509 & 8.8 & 100 & .41 & 5.10 & 19 \\
\hline P8 & $03 / 08 / 79$ & -4 & -1.2 & 1515 & 8.5 & 100 & .40 & 6.20 & 18 \\
\hline$P Q$ & $03 / 08 / 79$ & -9 & -2.7 & 1519 & 8.7 & 106 & .38 & 6.20 & 20 \\
\hline P8 & $03 / 08 / 79$ & -14 & -4.3 & 1524 & 8.3 & 108 & .17 & 6.10 & 20 \\
\hline P8 & $03 / 08 / 79$ & -19 & -5.8 & 1529 & 8.8 & 114 & .21 & 6.10 & 21 \\
\hline P8 & $03 / 08 / 79$ & -24 & -7.3 & 1534 & 8.3 & 124 & .54 & 5.30 & 22 \\
\hline P8 & $03 / 08 / 79$ & -29 & -8.8 & 1539 & 9.2 & 126 & .98 & 6.40 & 24 \\
\hline P8 & $03 / 08 / 79$ & -34 & -10.4 & 1544 & 9.0 & 148 & 4.80 & 5.40 & 25 \\
\hline$P 8$ & $03 / 08 / 79$ & -39 & -11.9 & 1549 & 9.8 & 190 & 5.50 & 6.50 & 24 \\
\hline TIDE & $07 / 18 / 79$ & -5 & -1.8 & 1130 & 13.8 & 135 & 4.80 & 6.50 & 18 \\
\hline
\end{tabular}

1/ Samples collected from exposed water table near the well. 
Table 5.--Water-chemistry data collected from wells near Horsfall Beach, and from wells B5 and 44, 1978-82

\begin{tabular}{|c|c|c|c|c|c|c|c|c|c|c|}
\hline WELL & DATE & $\begin{array}{c}\text { ELEV } \\
\text { (FEET) }\end{array}$ & $\begin{array}{l}\text { ATION } \\
\text { (METER) }\end{array}$ & TIME & $\begin{array}{c}\text { TEMPER- } \\
\text { ATURE } \\
(\text { DEG C) }\end{array}$ & $\begin{array}{l}\text { SPECIFIC } \\
\text { CONDUCT- } \\
\text { ANCE } \\
\text { (UMHOS) }\end{array}$ & $\begin{array}{c}\text { BICAR- } \\
\text { BONATE } \\
\text { (MG/L } \\
\text { AS } \\
\text { HCO3) }\end{array}$ & $\begin{array}{l}\text { IRON } \\
\text { DIS- } \\
\text { SOLVED } \\
\text { (MG/L) }\end{array}$ & $\begin{array}{c}\text { PH } \\
(U N \mid T)\end{array}$ & $\begin{array}{l}\text { CHLO- } \\
\text { RIDE } \\
\text { DIS- } \\
\text { SOLVED } \\
\text { (MG/L) }\end{array}$ \\
\hline $100 \mathrm{~W}$ & $03 / 23 / 78$ & -12 & -3.7 & 1330 & 12.0 & 170 & -- & .35 & 6.70 & 2 \\
\hline $200 N$ & $03 / 23 / 78$ & -15 & $-4 \cdot 6$ & 1300 & 10.5 & 410 & -- & 27.00 & 6.40 & 5 \\
\hline $200 W$ & $03 / 23 / 78$ & -12 & -3.7 & 1400 & -- & 239 & -- & .29 & 6.70 & 12 \\
\hline $400 W$ & $03 / 23 / 78$ & -8 & -2.4 & 1430 & 10.5 & 120 & -- & .03 & 6.30 & 22 \\
\hline B1 & $10 / 22 / 81$ & -87 & -26.5 & 1829 & 12.8 & 318 & 117 & .71 & 7.89 & -- \\
\hline B1 & $10 / 22 / 81$ & -99 & -30.2 & 1728 & 13.4 & 505 & 254 & .23 & 8.32 & -- \\
\hline B1 & $10 / 22 / 81$ & -129 & -39.3 & 1535 & 14.9 & 487 & 239 & .13 & 8.15 & -- \\
\hline B1 & $10 / 22 / 81$ & -135 & -41.1 & 1452 & 14.6 & 464 & 222 & .15 & 8.25 & -- \\
\hline B1 & $10 / 21 / 81$ & -147 & -44.8 & 1842 & 14.3 & 1315 & 244 & .25 & 7.55 & -- \\
\hline B1 & $05 / 21 / 82$ & -15 & -4.6 & 1203 & 12.9 & 88 & 20 & .16 & 6.41 & -- \\
\hline B1 & $05 / 21 / 82$ & -33 & -10.1 & 1155 & 14.6 & 91 & 24 & .39 & 6.28 & -- \\
\hline B1 & $05 / 21 / 82$ & -51 & -15.5 & 1131 & 14.6 & 139 & 43 & 1.10 & 6.41 & -- \\
\hline B1 & $05 / 21 / 82$ & -63 & -19.2 & 1121 & 13.5 & 177 & 48 & .11 & 6.50 & -- \\
\hline B1 & $05 / 21 / 82$ & -69 & -21.0 & 1058 & 14.0 & 201 & 52 & .31 & 6.52 & -- \\
\hline B1 & $05 / 21 / 82$ & -75 & -22.9 & 1026 & 12.8 & 192 & 79 & .33 & 6.62 & -- \\
\hline B1 & $05 / 20 / 82$ & -87 & -26.5 & 1948 & 12.5 & 221 & 75 & .59 & 7.20 & -- \\
\hline$B 1$ & $05 / 20 / 82$ & -99 & -30.2 & 1930 & 13.7 & 311 & 145 & .05 & 8.01 & -- \\
\hline B1 & $05 / 20 / 82$ & -123 & -37.5 & 1915 & 13.8 & 410 & 292 & .03 & 8.01 & -- \\
\hline B1 & $05 / 20 / 82$ & -129 & -39.3 & 1840 & 14.0 & 410 & 301 & .05 & 7.99 & -- \\
\hline B1 & $05 / 20 / 82$ & -141 & -43.0 & 1811 & 14.0 & 449 & 287 & .12 & 8.10 & -- \\
\hline B1 & $05 / 20 / 82$ & -147 & -44.8 & 1753 & 14.7 & 562 & 324 & -- & 7.63 & -- \\
\hline B1 & $05 / 20 / 82$ & -153 & -46.6 & 1735 & 15.6 & 3037 & 636 & .33 & 7.60 & -- \\
\hline B1 & $05 / 20 / 82$ & -159 & -48.5 & 1718 & 15.1 & 3440 & 637 & .32 & 7.50 & -- \\
\hline$B 1$ & $05 / 20 / 82$ & -165 & -50.3 & 1701 & 15.0 & 3480 & 639 & .61 & 7.45 & -- \\
\hline $\mathrm{B} 1$ & $05 / 20 / 82$ & -173 & -52.7 & 1642 & 17.0 & 6835 & 593 & 4.10 & 7.50 & -- \\
\hline $\mathrm{B1}$ & $05 / 20 / 82$ & -194 & -59.1 & 1629 & 18.3 & 21142 & 419 & 27.00 & 6.79 & -- \\
\hline B1 & $05 / 20 / 82$ & -200 & -61.0 & 1523 & 17.2 & 19631 & 591 & 23.00 & 7.11 & -- \\
\hline B2 & $07 / 23 / 80$ & -12 & -3.7 & 1610 & 16.0 & 20000 & -- & -- & 7.60 & -- \\
\hline B2 & $07 / 23 / 80$ & -24 & -7.3 & 1600 & 16.0 & 17000 & -- & -- & 7.60 & -- \\
\hline B2 & $07 / 23 / 80$ & -36 & -11.0 & 1550 & 18.0 & 7000 & -- & -- & 6.80 & -- \\
\hline B2 & $07 / 23 / 80$ & -49 & -14.9 & 1540 & 18.0 & 300 & -- & -- & 6.80 & -- \\
\hline$B 2$ & $07 / 23 / 80$ & -61 & -18.6 & 1530 & 17.0 & 200 & -- & -- & 6.80 & -- \\
\hline$B 2$ & $07 / 23 / 80$ & -79 & -24.1 & 1520 & 18.0 & 750 & -- & -- & 6.70 & -- \\
\hline B2 & $07 / 23 / 80$ & -90 & -27.4 & 1510 & 16.0 & 400 & -- & -- & 7.20 & -- \\
\hline B2 & $07 / 23 / 80$ & -97 & -29.6 & 1500 & 17.0 & 475 & -- & -- & 7.40 & -- \\
\hline B2 & $07 / 23 / 80$ & -109 & -33.2 & 1430 & 17.0 & 475 & -- & -- & 7.40 & -- \\
\hline B2 & $07 / 23 / 80$ & -121 & -36.9 & 1420 & 18.0 & 475 & -- & -- & 7.40 & -- \\
\hline $\mathrm{B} 2$ & $07 / 23 / 80$ & -133 & -40.5 & 1410 & 19.0 & 475 & -- & -- & 7.50 & -- \\
\hline
\end{tabular}


Table 5.--Water-chemistry data collected from wells near Horsfall Beach, and from wells B5 and 44, 1978-82--Continued

\begin{tabular}{|c|c|c|c|c|c|c|c|c|c|c|}
\hline WELL & DATE & $\begin{array}{l}\text { ELEVA } \\
\text { (FEET) }\end{array}$ & $\begin{array}{l}\text { ATION } \\
\text { (METER) }\end{array}$ & TIME & $\begin{array}{l}\text { TEMPER- } \\
\text { ATURE } \\
(\text { DEG C) }\end{array}$ & $\begin{array}{l}\text { SPECIFIC } \\
\text { CONDUCT- } \\
\text { ANCE } \\
\text { (UMHOS) }\end{array}$ & $\begin{array}{c}\text { BICAR- } \\
\text { BONATE } \\
\text { (MG/L } \\
\text { AS } \\
\text { HCO3) }\end{array}$ & $\begin{array}{l}\text { IRON } \\
\text { DIS- } \\
\text { SOLVED } \\
\text { (MG/L) }\end{array}$ & $\begin{array}{c}P H \\
(U N \mid T)\end{array}$ & $\begin{array}{l}\text { CHLO- } \\
\text { RIDE } \\
\text { DIS- } \\
\text { SOLVED } \\
(M G / L)\end{array}$ \\
\hline B2 & $07 / 23 / 80$ & -140 & -42.7 & 1400 & 15.0 & 475 & -- & -- & 7.40 & -- \\
\hline B2 & $07 / 23 / 80$ & -146 & -44.5 & 1350 & 16.0 & 1600 & -- & -- & 7.50 & -- \\
\hline B2 & $07 / 23 / 80$ & -158 & -48.2 & 1335 & 19.0 & 10500 & -- & -- & 7.50 & -- \\
\hline B2 & $07 / 23 / 80$ & -170 & -51.8 & 1315 & 17.5 & 12000 & -- & -- & 6.80 & -- \\
\hline B2 & $05 / 21 / 82$ & -6 & -1.8 & 1608 & 14.6 & 7788 & 131 & .05 & 8.09 & -- \\
\hline B2 & $05 / 21 / 82$ & -24 & -7.3 & 1600 & 14.4 & 163 & 58 & .03 & 8.71 & -- \\
\hline B2 & $05 / 21 / 82$ & -30 & -9.1 & 1505 & 14.6 & 183 & 29 & .05 & 9.18 & -- \\
\hline B2 & $05 / 21 / 82$ & -42 & -12.8 & 1500 & 14.4 & 151 & 58 & .02 & 7.70 & -- \\
\hline B2 & $05 / 21 / 82$ & -79 & -24.1 & 1443 & 16.0 & 199 & 58 & .03 & 6.99 & -- \\
\hline B2 & $05 / 21 / 82$ & -97 & -29.6 & 1330 & 15.7 & 290 & 128 & .07 & 7.81 & -- \\
\hline B2 & $05 / 21 / 82$ & -103 & -31.4 & 1433 & 15.4 & 317 & 148 & .02 & 7.79 & -- \\
\hline B2 & $05 / 21 / 82$ & -115 & -35.1 & 1325 & 16.1 & 323 & 224 & .02 & 7.72 & -- \\
\hline B2 & $05 / 21 / 82$ & -121 & -36.9 & 1256 & 15.4 & 499 & 382 & .06 & 8.09 & -- \\
\hline B2 & $05 / 21 / 82$ & -152 & -46.3 & 1247 & 15.7 & 880 & 358 & .03 & 8.80 & -- \\
\hline B3 & $/ 22 / 81$ & 4 & 1.2 & & 12.2 & 342 & 88 & 36.00 & & -- \\
\hline B3 & $10 / 22 / 81$ & -1 & -0.3 & 1824 & 13.6 & 242 & 46 & 11.00 & 6.50 & -- \\
\hline B3 & $10 / 22 / 81$ & -6 & -1.8 & 1800 & 12.9 & 183 & 34 & 5.20 & 6.20 & -- \\
\hline B3 & $10 / 22 / 81$ & -16 & -4.9 & 1600 & 12.4 & 166 & 36 & 3.00 & 6.40 & -- \\
\hline B3 & $10 / 22 / 81$ & -21 & -6.4 & 1630 & 12.4 & 178 & 34 & 2.80 & 6.40 & -- \\
\hline B3 & $10 / 22 / 81$ & -31 & -9.4 & 1445 & 12.6 & 175 & 39 & 11.00 & 6.90 & -- \\
\hline B3 & $10 / 22 / 81$ & -36 & -11.0 & 1422 & 12.6 & 230 & 74 & 2.40 & 7.20 & -- \\
\hline B3 & $10 / 22 / 81$ & -41 & -12.5 & 1353 & 12.6 & 244 & 76 & 25.00 & 6.70 & -- \\
\hline B3 & $10 / 21 / 81$ & -57 & -17.4 & 1754 & 12.7 & 241 & 42 & 19.00 & 7.00 & -- \\
\hline B3 & $10 / 21 / 81$ & -63 & -19.2 & 1724 & 12.8 & 321 & 104 & 24.00 & 6.90 & -- \\
\hline B3 & $10 / 21 / 81$ & -75 & -22.9 & 1625 & 13.0 & 236 & 108 & 7.30 & & -- \\
\hline$B 3$ & $10 / 21 / 81$ & -99 & -30.2 & 1429 & 13.3 & 310 & 201 & .05 & & -- \\
\hline B3 & $10 / 21 / 81$ & -105 & -32.0 & 1359 & 13.4 & 362 & 209 & .03 & 8.10 & -- \\
\hline B3 & $10 / 21 / 81$ & -117 & -35.7 & 1323 & 13.5 & 338 & 196 & .03 & 8.30 & -- \\
\hline B3 & $10 / 21 / 81$ & -123 & -37.5 & 1305 & 13.4 & 355 & 200 & .08 & 8.10 & -- \\
\hline B3 & $10 / 21 / 81$ & -135 & -41.1 & 1229 & 13.6 & 414 & 236 & .21 & 8.20 & -- \\
\hline B3 & $05 / 19 / 82$ & 4 & & 151 & & 211 & 90 & 29.00 & & - \\
\hline B3 & $05 / 19 / 82$ & -1 & -0.3 & 1438 & 14.2 & 118 & 53 & 6.40 & & -- \\
\hline B3 & $05 / 19 / 32$ & -6 & -1.8 & 1348 & 13.4 & 106 & 34 & 3.10 & & -- \\
\hline B3 & $05 / 19 / 82$ & -41 & -12.5 & 1116 & 13.1 & 208 & 70 & 17.00 & 6.86 & \\
\hline B3 & $05 / 18 / 82$ & -75 & -22.9 & 2016 & 11.8 & 270 & 114 & 10.00 & 6.90 & -- \\
\hline B3 & $05 / 18 / 82$ & -99 & -30.2 & 1842 & 13.2 & 268 & 159 & .01 & 8.30 & -- \\
\hline B3 & $05 / 18 / 82$ & -117 & -35.7 & 1646 & 13.8 & 378 & 273 & .01 & 8.22 & -- \\
\hline B3 & $05 / 19 / 82$ & -165 & -50.3 & 1600 & 14.2 & 2322 & -- & -- & -- & -- \\
\hline
\end{tabular}


Table 5.--Water-chemistry data collected from wells near Horsfall Beach, and from wells 35 and $44,1978-82--C o n t i n u e d$

\begin{tabular}{|c|c|c|c|c|c|c|c|c|c|c|}
\hline WELL & DATE & $\begin{array}{l}\text { ELEVA } \\
\text { (FEET) }\end{array}$ & $\begin{array}{l}\text { 4TION } \\
\text { (METER) }\end{array}$ & TIME & 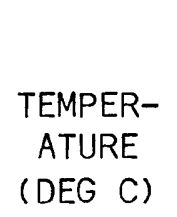 & $\begin{array}{l}\text { SPECIFIC } \\
\text { CONDUCT- } \\
\text { ANCE } \\
\text { (UMHOS) }\end{array}$ & $\begin{array}{c}\text { BICAR- } \\
\text { BONATE } \\
\text { (MG/L } \\
\text { AS } \\
\text { HCO3) }\end{array}$ & $\begin{array}{l}\text { IRON } \\
\text { DIS- } \\
\text { SOLVED } \\
\text { (MG/L) }\end{array}$ & $\begin{array}{c}P H \\
(U N \mid T)\end{array}$ & $\begin{array}{l}\text { CHLO- } \\
\text { RIDE } \\
\text { DIS- } \\
\text { SOLVED } \\
\text { (MG/L) }\end{array}$ \\
\hline B5 & $10 / 20 / 81$ & -45 & -13.7 & 1348 & 14.4 & 172 & 102 & 26.00 & 6.43 & -- \\
\hline B5 & $10 / 20 / 81$ & -70 & $-21 \cdot 3$ & 1413 & 14.5 & 305 & 154 & 12.00 & 7.08 & -- \\
\hline B5 & $10 / 20 / 81$ & -75 & -22.9 & 1435 & 14.0 & 284 & 156 & 11.00 & 7.11 & -- \\
\hline B5 & $10 / 20 / 81$ & -155 & -47.2 & 1610 & 14.2 & 246 & 124 & .05 & 8.60 & -- \\
\hline B5 & $10 / 23 / 81$ & -30 & -9.1 & 1245 & 14.3 & 145 & 88 & 20.00 & 6.20 & -- \\
\hline B5 & $10 / 23 / 81$ & -55 & -16.8 & 1320 & 14.3 & 151 & 87 & 26.00 & 6.40 & -- \\
\hline B5 & $10 / 23 / 81$ & -65 & -19.8 & 1350 & 14.3 & 277 & 146 & 12.00 & 6.90 & -- \\
\hline B5 & $10 / 23 / 81$ & -80 & -24.4 & 1425 & 14.6 & 258 & 144 & .80 & 7.60 & -- \\
\hline B5 & $10 / 23 / 81$ & -90 & -27.4 & 1450 & 14.3 & 332 & 180 & .71 & 7.80 & -- \\
\hline B5 & $10 / 23 / 81$ & -100 & -30.5 & 1505 & 14.2 & 231 & 116 & 14.00 & 7.10 & -- \\
\hline B5 & $10 / 23 / 81$ & -115 & -35.1 & 1540 & 14.6 & 164 & 68 & 13.00 & 6.90 & -- \\
\hline B5 & $10 / 23 / 81$ & -135 & -41.1 & 1410 & 14.8 & 175 & 66 & 6.40 & 7.10 & -- \\
\hline 85 & $10 / 23 / 81$ & -150 & -45.7 & 1637 & 14.3 & 254 & 128 & .10 & 8.10 & -- \\
\hline B5 & $10 / 23 / 81$ & -165 & -50.3 & 1655 & 14.3 & 277 & 152 & .12 & 8.30 & -- \\
\hline HB & $03 / 23 / 78$ & -14 & $-4 \cdot 3$ & 1000 & 10.0 & 145 & -- & 8.90 & 6.30 & 25 \\
\hline $\mathrm{HB}$ & $03 / 23 / 78$ & -18 & -5.5 & 1030 & 11.0 & 162 & -- & 10.00 & 6.20 & 26 \\
\hline HB & $03 / 23 / 78$ & -22 & -6.7 & 1100 & 10.5 & 162 & -- & 11.00 & 6.50 & 26 \\
\hline $\mathrm{HB}$ & $03 / 23 / 78$ & -26 & -7.9 & 1130 & 10.5 & 170 & -- & 11.00 & 6.60 & 27 \\
\hline $\mathrm{HB}$ & $03 / 23 / 78$ & -30 & -10.7 & 1630 & 11.0 & 182 & -- & 12.00 & 6.70 & 30 \\
\hline HB & $03 / 23 / 78$ & -35 & -9.1 & 1200 & 10.5 & 242 & -- & 18.00 & 6.70 & 42 \\
\hline HB & $07 / 18 / 78$ & -14 & $-4 \cdot 3$ & 1500 & 13.8 & 400 & -- & 33.00 & 5.90 & 49 \\
\hline $\mathrm{HB}$ & $07 / 18 / 78$ & -18 & -5.5 & 1510 & 14.2 & 355 & -- & 32.00 & 6.00 & 48 \\
\hline HB & $07 / 18 / 78$ & -22 & -6.7 & 1520 & 14.7 & 360 & -- & 34.00 & -- & 48 \\
\hline HB & $07 / 18 / 78$ & -26 & -7.9 & 1530 & 13.7 & 355 & -- & 30.00 & 5.80 & 48 \\
\hline$H B$ & $07 / 18 / 78$ & -30 & -10.7 & 1550 & 13.1 & 342 & -- & 34.00 & 5.80 & 48 \\
\hline HB & $07 / 18 / 78$ & -35 & -9.1 & 1540 & 13.2 & 350 & -- & 35.00 & 5.90 & 50 \\
\hline P1 & $06 / 21 / 78$ & 6 & 1.8 & 1745 & 16.0 & 258 & -- & 6.00 & 5.60 & -- \\
\hline P1 & $06 / 21 / 78$ & 2 & 0.6 & 1750 & 14.0 & 325 & -- & 25.00 & 6.30 & -- \\
\hline P1 & $06 / 21 / 78$ & -2 & -0.6 & 1755 & 14.0 & 322 & -- & 36.00 & 6.30 & -- \\
\hline P1 & $06 / 21 / 78$ & -6 & -1.8 & 1800 & 13.5 & 146 & -- & 20.00 & 6.40 & -- \\
\hline P1 & $06 / 21 / 78$ & -10 & -3.0 & 1805 & 14.0 & 195 & -- & 25.00 & 6.60 & -- \\
\hline P1 & $06 / 21 / 78$ & -14 & $-4 \cdot 3$ & 1810 & 14.0 & 191 & -- & 35.00 & 6.50 & -- \\
\hline$P 1$ & $06 / 21 / 78$ & -18 & -5.5 & 1815 & 14.0 & 165 & -- & 28.00 & 6.60 & -- \\
\hline P1 & $06 / 21 / 78$ & -22 & -6.7 & 1820 & 14.0 & 157 & -- & 25.00 & 6.60 & -- \\
\hline P1 & $06 / 21 / 78$ & -26 & -7.9 & 1825 & 14.5 & 160 & -- & 25.00 & 6.50 & -- \\
\hline
\end{tabular}


Table 5.--Water-chemistry data collected from wells near Horsfall Beach, and from wells B5 and 44, 1978-82--Continued

\begin{tabular}{|c|c|c|c|c|c|c|c|c|c|c|}
\hline WELL & DATE & $\begin{array}{l}\text { ELEVA } \\
\text { (FEET) }\end{array}$ & $\begin{array}{l}\text { ATION } \\
\text { (METER) }\end{array}$ & TIME & $\begin{array}{l}\text { TEMPER- } \\
\text { ATURE } \\
\text { (DEG C) }\end{array}$ & $\begin{array}{l}\text { SPECIFIC } \\
\text { CONDUCT- } \\
\text { ANCE } \\
\text { (UMHOS) }\end{array}$ & $\begin{array}{c}\text { BICAR- } \\
\text { BONATE } \\
\text { (MG/L } \\
\text { AS } \\
\text { HCO3) }\end{array}$ & $\begin{array}{l}\text { IRON } \\
\text { DIS- } \\
\text { SOLVED } \\
\text { (MG/L) }\end{array}$ & $\begin{array}{c}P H \\
(U N I T)\end{array}$ & $\begin{array}{l}\text { CHLO- } \\
\text { RIDE } \\
\text { DIS- } \\
\text { SOLVED } \\
\text { (MG/L) }\end{array}$ \\
\hline P1 & $07 / 17 / 78$ & 6 & 1.8 & 1610 & 17.2 & 225 & -- & 2.10 & 5.30 & 54 \\
\hline P1 & $07 / 17 / 78$ & 2 & 0.6 & 1600 & 16.2 & 650 & -- & 47.00 & 3.50 & 85 \\
\hline P1 & $07 / 17 / 78$ & -2 & -0.6 & 1550 & 15.4 & 242 & -- & 21.00 & 6.00 & 31 \\
\hline P1 & $07 / 17 / 78$ & -6 & -1.8 & 1540 & 15.2 & 205 & -- & 21.00 & 5.90 & 27 \\
\hline P1 & $07 / 17 / 78$ & -10 & -3.0 & 1530 & 15.4 & 310 & -- & 29.00 & 5.40 & 41 \\
\hline$P 1$ & $07 / 17 / 78$ & -14 & -4.3 & 1520 & 15.4 & 295 & -- & 39.00 & 5.90 & 23 \\
\hline P1 & $07 / 17 / 78$ & -18 & -5.5 & 1510 & 15.8 & 240 & -- & 24.00 & 5.70 & 29 \\
\hline P1 & $07 / 17 / 78$ & -22 & -6.7 & 1500 & 15.6 & 225 & -- & 26.00 & 3.50 & 24 \\
\hline P1 & $07 / 17 / 78$ & -26 & -7.9 & 1446 & 15.8 & 242 & -- & 24.00 & 6.00 & 26 \\
\hline P1 & $08 / 01 / 78$ & 6 & 1.8 & 1830 & 14.6 & 177 & -- & 2.30 & 5.40 & 40 \\
\hline P1 & $08 / 01 / 78$ & 2 & 0.6 & 1845 & 13.5 & 585 & -- & 42.00 & 6.00 & 88 \\
\hline P1 & $08 / 01 / 78$ & -2 & -0.6 & 1900 & 13.4 & 218 & -- & 18.00 & 6.30 & 23 \\
\hline P1 & $08 / 01 / 78$ & -6 & -1.8 & 1915 & 13.0 & 210 & -- & 21.00 & 6.30 & 19 \\
\hline P1 & $08 / 01 / 78$ & -10 & -3.0 & 1930 & 12.7 & 299 & -- & 28.00 & 6.50 & 33 \\
\hline P1 & $08 / 01 / 78$ & -14 & $-4 \cdot 3$ & 1945 & 12.6 & 259 & -- & 32.00 & 6.50 & 17 \\
\hline P1 & $08 / 01 / 78$ & -18 & -5.5 & 2000 & 12.6 & 232 & -- & 26.00 & 6.60 & 19 \\
\hline P1 & $08 / 01 / 78$ & -22 & -6.7 & 2015 & 12.6 & 211 & -- & 23.00 & 6.70 & 15 \\
\hline P1 & $08 / 01 / 78$ & -26 & -7.9 & 2030 & 12.6 & 224 & -- & 22.00 & 6.80 & 17 \\
\hline P1 & $08 / 18 / 78$ & 6 & 1.8 & $1240^{\circ}$ & 15.5 & 580 & -- & 18.00 & 5.50 & 62 \\
\hline P1 & $08 / 18 / 78$ & 2 & 0.6 & 1245 & 14.2 & 580 & -- & 44.00 & 6.10 & 100 \\
\hline P1 & $08 / 18 / 78$ & -2 & -0.6 & 1250 & 13.6 & 225 & -- & 20.00 & 6.40 & 33 \\
\hline P1 & $08 / 18 / 78$ & -6 & -1.8 & 1255 & 13.2 & 195 & -- & 18.00 & 6.50 & 27 \\
\hline P1 & $08 / 18 / 78$ & -10 & -3.0 & 1300 & 13.0 & 295 & -- & 27.00 & 6.65 & 43 \\
\hline P1 & $08 / 18 / 78$ & -14 & -4.3 & 1305 & 13.0 & 255 & -- & 34.00 & 6.65 & 26 \\
\hline P1 & $08 / 18 / 78$ & -18 & -5.5 & 1310 & 13.0 & 225 & -- & 25.00 & 6.60 & 26 \\
\hline P1 & $08 / 18 / 78$ & -22 & -6.7 & 1315 & 13.1 & 205 & -- & 23.00 & 6.70 & 23 \\
\hline$P 1$ & $08 / 18 / 78$ & -26 & -7.9 & 1320 & 13.1 & 225 & -- & 24.00 & 6.70 & 29 \\
\hline P1 & $09 / 14 / 78$ & -2 & -0.6 & 1630 & 14.4 & 255 & 20 & 27.00 & 6.40 & 25 \\
\hline P1 & $09 / 14 / 78$ & -6 & -1.8 & 1640 & 14.0 & 178 & 19 & 21.00 & 6.55 & 22 \\
\hline P1 & $09 / 14 / 78$ & -10 & -3.0 & 1650 & 13.7 & 300 & 20 & 34.00 & 6.70 & 42 \\
\hline P1 & $09 / 14 / 78$ & -18 & -5.5 & 1710 & 13.6 & 200 & 19 & 24.00 & 6.80 & 26 \\
\hline P1 & $09 / 14 / 78$ & -22 & -6.7 & 1720 & 13.8 & 202 & 31 & 32.00 & 6.90 & 18 \\
\hline
\end{tabular}


Table 5.- -Water-chemistry data collected from well s near Horstall Beach, and from wells B5 and 44, 1978-82--Continued

\begin{tabular}{|c|c|c|c|c|c|c|c|c|c|c|}
\hline WELL & DATE & $\begin{array}{l}\text { ELEV } \\
\text { (FEET) }\end{array}$ & $\begin{array}{l}\text { ATION } \\
\text { (METER) }\end{array}$ & TIME & $\begin{array}{l}\text { TEMPER- } \\
\text { ATURE } \\
\text { (DEG C) }\end{array}$ & $\begin{array}{l}\text { SPECIFIC } \\
\text { CONDUCT- } \\
\text { ANCE } \\
\text { (UMHOS) }\end{array}$ & $\begin{array}{c}\text { BICAR- } \\
\text { BONATE } \\
\text { (MG/L } \\
\text { AS } \\
\text { HCO3) }\end{array}$ & $\begin{array}{l}\text { IRON } \\
\text { DIS- } \\
\text { SOLVED } \\
\text { (MG/L) }\end{array}$ & $\begin{array}{c}\mathrm{PH} \\
(\mathrm{UNIT})\end{array}$ & $\begin{array}{l}\text { CHLO- } \\
\text { RIDE } \\
\text { DIS- } \\
\text { SOLVED } \\
\text { (MG/L) }\end{array}$ \\
\hline P1 & $10 / 18 / 78$ & 6 & 1.8 & 1450 & 15.4 & 320 & 4 & .54 & 4.90 & 75 \\
\hline P1 & $10 / 18 / 78$ & 2 & 0.6 & 1505 & 14.8 & 401 & 34 & 27.00 & 6.10 & 31 \\
\hline P1 & $10 / 18 / 78$ & -2 & -0.6 & 1515 & 14.3 & 162 & 57 & 14.00 & 6.20 & 16 \\
\hline P1 & $10 / 18 / 78$ & -6 & -1.8 & 1525 & 13.9 & 209 & 52 & 20.00 & 6.20 & 27 \\
\hline P1 & $10 / 18 / 78$ & -14 & -4.3 & 1545 & 13.4 & 300 & 91 & 39.00 & 6.45 & 37 \\
\hline P1 & $10 / 18 / 78$ & -18 & -5.5 & 1555 & 13.2 & 206 & 65 & 24.00 & 6.50 & 27 \\
\hline P1 & $10 / 18 / 78$ & -22 & -6.7 & 1605 & 13.0 & 192 & 87 & 24.00 & 6.55 & 18 \\
\hline $\mathrm{Pl}$ & $03 / 06 / 79$ & 6 & 1.8 & 1410 & 9.9 & 230 & -- & .63 & 4.90 & 31 \\
\hline P1 & $03 / 06 / 79$ & 2 & 0.6 & 1420 & 10.3 & 480 & -- & 44.00 & 6.00 & 66 \\
\hline$P i$ & $03 / 06 / 79$ & -2 & -0.6 & 1425 & 11.1 & 245 & -- & 24.00 & 6.20 & 31 \\
\hline P1 & $03 / 06 / 79$ & -6 & -1.8 & 1431 & 11.8 & 230 & - & 22.00 & 6.30 & 41 \\
\hline $\mathrm{P1}$ & $03 / 06 / 79$ & -10 & -3.0 & 1436 & 12.1 & 320 & -- & 36.00 & 6.50 & 34 \\
\hline P1 & $03 / 06 / 79$ & -14 & -4.3 & 1443 & 12.3 & 370 & -- & 50.00 & 6.50 & 74 \\
\hline $\mathrm{P1}$ & $03 / 06 / 79$ & -18 & -5.5 & 1447 & 12.3 & 390 & -- & 50.00 & 6.50 & 72 \\
\hline P1 & $03 / 06 / 79$ & -22 & -6.7 & 1454 & 12.3 & 310 & -- & 40.00 & 6.60 & 45 \\
\hline P2 & $08 / 03 / 78$ & 4 & 1.2 & 1800 & 13.9 & 128 & -- & .42 & 6.40 & 28 \\
\hline P2 & $08 / 03 / 78$ & -1 & -0.3 & 1815 & 14.0 & 171 & -- & .16 & 6.30 & 27 \\
\hline $\mathrm{P} 2$ & $08 / 03 / 78$ & -6 & -1.8 & 1830 & 13.6 & 128 & -- & .14 & 6.30 & 29 \\
\hline P2 & $08 / 03 / 78$ & -11 & -3.4 & 1845 & 13.0 & 118 & -- & .12 & 6.40 & 23 \\
\hline P2 & $08 / 03 / 78$ & -16 & -4.9 & 1900 & 13.6 & 140 & -- & .19 & 6.60 & 27 \\
\hline P2 & $08 / 03 / 78$ & -21 & -6.4 & 1915 & 13.6 & 141 & -- & .23 & 6.50 & 27 \\
\hline $\mathrm{P} 2$ & $08 / 03 / 78$ & -26 & -7.9 & 1930 & 13.6 & 150 & -- & .40 & 6.80 & 25 \\
\hline P2 & $08 / 03 / 78$ & -31 & -9.4 & 1945 & 13.6 & 142 & -- & 3.50 & 6.90 & 27 \\
\hline P2 & $08 / 03 / 78$ & -36 & -11.0 & 2000 & 13.4 & 142 & -- & 5.50 & 7.00 & 27 \\
\hline P2 & $08 / 03 / 78$ & -41 & -12.5 & 2015 & 13.1 & 142 & -- & 4.50 & 7.00 & 28 \\
\hline P2 & $08 / 18 / 78$ & 4 & 1.2 & 1050 & 15.8 & 165 & -- & 1.20 & 6.15 & 28 \\
\hline P2 & $08 / 18 / 78$ & -1 & -0.3 & 1057 & 14.8 & 175 & -- & .31 & 6.10 & 39 \\
\hline P2 & $08 / 18 / 78$ & -6 & -1.8 & 1105 & 14.0 & 138 & -- & .35 & 6.25 & 29 \\
\hline P2 & $08 / 18 / 78$ & -11 & -3.4 & 1110 & 13.5 & 127 & -- & .28 & 6.30 & 27 \\
\hline P2 & $08 / 18 / 78$ & -16 & -4.9 & 1115 & 13.2 & 155 & -- & .32 & 6.55 & 22 \\
\hline
\end{tabular}


Table 5.--Water-chemistry data collected from wells near Horsfall Beach, and from wells B5 and 44, 1978-82--Continued

\begin{tabular}{|c|c|c|c|c|c|c|c|c|c|c|}
\hline WELL & DATE & $\begin{array}{l}\text { ELEVAT } \\
\text { (FEET) }\end{array}$ & $\begin{array}{l}\text { TION } \\
\text { (METER) }\end{array}$ & TIME & 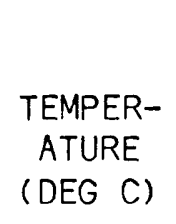 & $\begin{array}{l}\text { SPECIFIC } \\
\text { CONDUCT- } \\
\text { ANCE } \\
\text { (UMHOS) }\end{array}$ & $\begin{array}{c}\text { BICAR- } \\
\text { BONATE } \\
\text { (MG/L } \\
\text { AS } \\
\text { HCO3) }\end{array}$ & $\begin{array}{l}\text { IRON } \\
\text { DIS- } \\
\text { SOLVED } \\
\text { (MG/L) }\end{array}$ & $\begin{array}{c}\mathrm{PH} \\
(\mathrm{UNIT})\end{array}$ & $\begin{array}{l}\text { CHLO- } \\
\text { RIDE } \\
\text { DIS- } \\
\text { SOLVED } \\
(M G / L)\end{array}$ \\
\hline P2 & $08 / 18 / 78$ & -21 & -6.4 & 1122 & 12.9 & 260 & -- & .11 & 6.95 & 39 \\
\hline P2 & $08 / 18 / 78$ & -26 & -7.9 & 1130 & 12.8 & 225 & -- & .55 & 6.90 & 35 \\
\hline P2 & $08 / 18 / 78$ & -31 & -9.4 & 1135 & 12.6 & 137 & -- & 3.89 & 6.80 & 18 \\
\hline P2 & $08 / 18 / 78$ & -36 & -11.0 & 1140 & 12.5 & 148 & -- & 6.50 & 6.70 & 27 \\
\hline P2 & $08 / 18 / 78$ & -41 & -12.5 & 1150 & 12.6 & 175 & -- & 6.60 & 6.70 & 31 \\
\hline P2 & $09 / 14 / 78$ & -1 & -0.3 & 1015 & 14.7 & 164 & 17 & .86 & 6.10 & 34 \\
\hline $\mathrm{P} 2$ & $09 / 14 / 78$ & -6 & -1.8 & 1025 & 14.2 & 142 & 15 & .85 & 6.10 & 30 \\
\hline P2 & $09 / 14 / 78$ & -16 & -4.9 & 1050 & 13.4 & 137 & 35 & 1.50 & 6.50 & 21 \\
\hline P2 & $09 / 14 / 78$ & -21 & -6.4 & 1100 & 13.2 & 260 & 62 & .37 & 7.14 & 42 \\
\hline $\mathrm{P} 2$ & $09 / 14 / 78$ & -26 & -7.9 & 1110 & 13.2 & 205 & 52 & 1.20 & 7.10 & 28 \\
\hline P2 & $09 / 14 / 78$ & -31 & -9.4 & 1125 & 13.1 & 144 & 28 & 6.60 & 7.10 & 32 \\
\hline P2 & $09 / 14 / 78$ & -41 & -12.5 & 1150 & 13.6 & 165 & 24 & 8.40 & 7.14 & 26 \\
\hline P2 & $10 / 19 / 78$ & $\mathrm{P} \mid \mathrm{T}-1 /$ & -- & 1115 & 13.5 & 630 & 4 & 5.29 & 4.79 & 190 \\
\hline $\mathrm{P} 2$ & $10 / 19 / 78$ & 4 & 1.2 & 1130 & 15.1 & 182 & 37 & 3.60 & 5.85 & 33 \\
\hline P2 & $10 / 19 / 78$ & -1 & -0.3 & 1140 & 14.5 & 172 & 17 & 1.10 & 5.80 & 37 \\
\hline $\mathrm{P} 2$ & $10 / 19 / 78$ & -6 & -1.8 & 1150 & 14.0 & 154 & 20 & 1.20 & 5.95 & 32 \\
\hline $\mathrm{P} 2$ & $10 / 19 / 78$ & -11 & -3.4 & 1200 & 13.5 & 142 & 24 & 1.40 & 6.00 & 27 \\
\hline$P 2$ & $10 / 19 / 78$ & -16 & -4.9 & 1230 & 13.3 & 151 & 37 & 2.10 & 6.20 & 24 \\
\hline P2 & $10 / 19 / 78$ & -26 & -7.9 & 1315 & 12.8 & 177 & 52 & .95 & 6.65 & 24 \\
\hline $\mathrm{P} 2$ & $10 / 19 / 78$ & -31 & -9.4 & 1330 & 12.7 & 134 & 38 & 5.29 & 6.50 & 21 \\
\hline P2 & $10 / 19 / 78$ & -36 & -11.0 & 1340 & 12.7 & 136 & 37 & 6.30 & 6.50 & 21 \\
\hline P2 & $03 / 05 / 79$ & 4 & 1.2 & 1910 & 11.9 & 94 & -- & .36 & & 18 \\
\hline $\mathrm{P} 2$ & $03 / 05 / 79$ & -1 & -0.3 & 1920 & 9.8 & 105 & -- & .12 & 6.00 & 18 \\
\hline P2 & $03 / 05 / 79$ & -6 & -1.8 & 1930 & 9.0 & 110 & -- & .20 & 6.00 & 19 \\
\hline P2 & $03 / 05 / 79$ & -11 & -3.4 & 1940 & 9.0 & 110 & -- & .24 & 6.00 & 20 \\
\hline $\mathrm{P} 2$ & $03 / 05 / 79$ & -16 & -4.9 & 1950 & 10.4 & 134 & -- & .43 & 6.30 & 21 \\
\hline P2 & $03 / 05 / 79$ & -21 & -6.4 & 1955 & 11.2 & 163 & -- & 1.20 & 6.30 & 26 \\
\hline $\mathrm{P} 2$ & $03 / 05 / 79$ & -26 & -7.9 & 2005 & 11.4 & 165 & -- & 3.50 & 6.40 & 26 \\
\hline P2 & $03 / 05 / 79$ & -31 & -9.4 & 2010 & 11.4 & 165 & -- & 7.10 & 6.40 & 28 \\
\hline P2 & $03 / 05 / 79$ & -36 & -11.0 & 2020 & 11.3 & 155 & -- & 7.90 & 6.60 & 23 \\
\hline
\end{tabular}


Table 5.--Water-chemistry data collected from wells near Horsfall Beach, and from wells B5 and 44, 1978-82--Continued

\begin{tabular}{|c|c|c|c|c|c|c|c|c|c|c|}
\hline WELL & DATE & $\begin{array}{l}\text { ELEV } \\
\text { (FEET) }\end{array}$ & $\begin{array}{l}\text { ATION } \\
\text { (METER) }\end{array}$ & TIME & 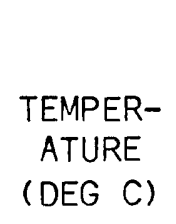 & $\begin{array}{l}\text { SPECIFIC } \\
\text { CONDUCT- } \\
\text { ANCE } \\
\text { (UMHOS) }\end{array}$ & $\begin{array}{c}\text { BICAR- } \\
\text { BONATE } \\
\text { (MG/L } \\
\text { AS } \\
\text { HCO3) }\end{array}$ & $\begin{array}{l}\text { IRON } \\
\text { DIS- } \\
\text { SOLVED } \\
(M G / L)\end{array}$ & $\begin{array}{c}P H \\
(U N \mid T)\end{array}$ & $\begin{array}{l}\text { CHLO- } \\
\text { RIDE } \\
\text { DIS- } \\
\text { SOLVED } \\
(M G / L)\end{array}$ \\
\hline P2 & $05 / 20 / 82$ & -6 & -1.8 & 1422 & 14.2 & 122 & 23 & .28 & 6.00 & -- \\
\hline P2 & $05 / 20 / 82$ & -21 & -6.4 & 1320 & 12.1 & 156 & 48 & 1.60 & 6.60 & -- \\
\hline P2 & $05 / 20 / 82$ & -41 & -12.5 & 1221 & 13.1 & 153 & 53 & 5.80 & 6.82 & -- \\
\hline P3 & $08 / 17 / 78$ & 13 & 4.0 & 1045 & 15.9 & 265 & -- & 7.20 & 5.70 & -- \\
\hline P3 & $08 / 17 / 78$ & 10 & 3.0 & 1105 & 15.2 & 285 & -- & 11.00 & 6.00 & -- \\
\hline P3 & $08 / 17 / 78$ & 7 & 2.1 & 1115 & 14.5 & 275 & -- & 9.40 & 6.10 & -- \\
\hline P3 & $08 / 17 / 78$ & 4 & 1.2 & 1125 & 14.2 & 207 & -- & 8.30 & 6.20 & -- \\
\hline P3 & $08 / 17 / 78$ & 1 & 0.3 & 1135 & 13.9 & 146 & -- & 6.00 & 6.30 & -- \\
\hline P3 & $08 / 17 / 78$ & -2 & -0.6 & 1145 & 13.4 & 220 & -- & 11.00 & 6.20 & -- \\
\hline P3 & $08 / 17 / 78$ & -7 & -2.1 & 1155 & 13.3 & 165 & -- & 13.00 & 6.30 & -- \\
\hline P3 & $08 / 17 / 78$ & -12 & -3.7 & 1205 & 13.3 & 235 & -- & 17.00 & 6.40 & -- \\
\hline P3 & $08 / 17 / 78$ & -17 & -5.2 & 1215 & 13.4 & 275 & -- & 26.00 & 6.40 & -- \\
\hline P3 & $08 / 17 / 78$ & -22 & -6.7 & 1225 & 13.7 & 310 & -- & 21.00 & 6.30 & -- \\
\hline P3 & $08 / 17 / 78$ & -27 & $-8 \cdot 2$ & 1235 & 13.6 & 315 & -- & 18.00 & 6.30 & -- \\
\hline P3 & $09 / 14 / 78$ & 13 & 4.0 & 1830 & 16.8 & 262 & 3 & 5.00 & 5.80 & 49 \\
\hline P3 & $09 / 14 / 78$ & 10 & 3.0 & 1840 & 15.8 & 235 & 1 & 9.70 & 6.15 & 33 \\
\hline P3 & $09 / 14 / 78$ & 7 & 2.1 & 1845 & 14.8 & 245 & 17 & 12.00 & 6.20 & 24 \\
\hline P3 & $09 / 14 / 78$ & 4 & 1.2 & 1850 & 14.2 & 213 & 8 & 12.00 & 6.20 & 20 \\
\hline P3 & $09 / 14 / 78$ & 1 & 0.3 & 1855 & 13.8 & 375 & 1 & 27.00 & 6.05 & 34 \\
\hline P3 & $09 / 14 / 78$ & -2 & -0.6 & 1900 & 13.5 & 215 & 34 & 14.00 & 6.15 & 35 \\
\hline P3 & $09 / 14 / 78$ & -7 & -2.1 & 1905 & 13.4 & 212 & 24 & 22.00 & 6.30 & 28 \\
\hline P3 & $09 / 14 / 78$ & -12 & -3.7 & 1910 & 13.3 & 280 & 31 & 31.00 & 6.30 & 31 \\
\hline P3 & $09 / 14 / 78$ & -17 & -5.2 & 1955 & 13.4 & 182 & 29 & 21.00 & 6.00 & 21 \\
\hline P3 & $09 / 14 / 78$ & -22 & -6.7 & 1920 & 13.3 & 144 & 29 & 9.30 & 6.20 & 28 \\
\hline P3 & $09 / 14 / 78$ & -27 & -8.2 & 1930 & 13.4 & 180 & 39 & 16.00 & 6.20 & 23 \\
\hline P3 & $10 / 17 / 78$ & 13 & 4.0 & 1700 & 16.2 & 225 & 20 & 3.60 & 5.65 & 35 \\
\hline P3 & $10 / 17 / 78$ & 10 & 3.0 & 1715 & 15.5 & 243 & 37 & 8.50 & 5.70 & 34 \\
\hline P3 & $10 / 17 / 78$ & 7 & 2.1 & 1725 & 14.9 & 349 & 41 & 12.00 & 5.80 & 21 \\
\hline P3 & $10 / 17 / 78$ & 4 & 1.2 & 1735 & 14.5 & 374 & 33 & 18.00 & 5.85 & 28 \\
\hline P3 & $10 / 17 / 78$ & 1 & 0.3 & 1745 & 14.0 & 318 & 38 & 20.00 & 5.80 & 34 \\
\hline P3 & $10 / 17 / 78$ & -2 & -0.6 & 1755 & 13.7 & 219 & 59 & 15.00 & 5.89 & 32 \\
\hline P3 & $10 / 17 / 78$ & -7 & -2.1 & 1805 & 13.4 & 416 & 60 & 50.00 & 6.00 & 37 \\
\hline P3 & $10 / 17 / 78$ & -12 & -3.7 & 1815 & 13.1 & 344 & 80 & 36.00 & 6.15 & 26 \\
\hline
\end{tabular}


Table 5.--Water-chem istry data collected from wells near Horsfall Beach, and from wells B5 and 44, 1978-82--Continued

\begin{tabular}{|c|c|c|c|c|c|c|c|c|c|c|}
\hline WELL & DATE & $\begin{array}{l}\text { ELEVA } \\
\text { (FEET) }\end{array}$ & $\begin{array}{l}\text { ATION } \\
\text { (METER) }\end{array}$ & TIME & $\begin{array}{c}\text { TEMPER- } \\
\text { ATURE } \\
\text { (DEG C) }\end{array}$ & $\begin{array}{l}\text { SPECIFIC } \\
\text { CONDUCT- } \\
\text { ANCE } \\
\text { (UMHOS) }\end{array}$ & $\begin{array}{c}\text { BICAR- } \\
\text { BONATE } \\
\text { (MG/L } \\
\text { AS } \\
\text { HCO3) }\end{array}$ & $\begin{array}{l}\text { IRON } \\
\text { DIS- } \\
\text { SOLVED } \\
\text { (MG/L) }\end{array}$ & $\begin{array}{c}\mathrm{PH} \\
\text { (UNIT) }\end{array}$ & $\begin{array}{l}\text { CHLO- } \\
\text { RIDE } \\
\text { DIS- } \\
\text { SOLVED } \\
(M G / L)\end{array}$ \\
\hline P3 & $10 / 17 / 78$ & -17 & -5.2 & 1820 & 13.1 & 166 & 71 & 25.00 & 6.25 & 21 \\
\hline P3 & $10 / 17 / 78$ & -22 & -6.7 & 1825 & 13.0 & 148 & 44 & 10.00 & 6.30 & 21 \\
\hline P3 & $10 / 17 / 78$ & -27 & -8.2 & 1830 & 12.9 & 187 & 77 & 23.00 & 6.35 & 20 \\
\hline P3 & $03 / 07 / 79$ & 13 & 4.0 & 1208 & 10.6 & 65 & -- & .53 & 5.50 & 10 \\
\hline P3 & $03 / 07 / 79$ & 10 & 3.0 & 1214 & 10.3 & 75 & -- & 1.70 & 5.89 & 11 \\
\hline P3 & $03 / 07 / 79$ & 7 & 2.1 & 1219 & 10.8 & 88 & -- & 2.30 & 6.30 & 9 \\
\hline P3 & $03 / 07 / 79$ & 4 & 1.2 & 1225 & 11.6 & 270 & -- & 12.00 & 6.10 & 22 \\
\hline P3 & $03 / 07 / 79$ & 1 & 0.3 & 1232 & 12.1 & 300 & -- & 17.00 & 6.10 & -- \\
\hline P3 & $03 / 07 / 79$ & -2 & -0.6 & 1232 & 12.2 & 215 & -- & 13.00 & 6.10 & 35 \\
\hline P3 & $03 / 07 / 79$ & -7 & -2.1 & 1244 & 12.8 & 285 & -- & 26.00 & 6.20 & 32 \\
\hline P3 & $03 / 07 / 79$ & -12 & -3.7 & 1251 & 12.8 & 215 & -- & 20.00 & 6.40 & 23 \\
\hline P3 & $03 / 07 / 79$ & -17 & -5.2 & 1258 & 12.9 & 210 & -- & 29.00 & 6.60 & 28 \\
\hline P3 & $03 / 07 / 79$ & -22 & -6.7 & 1304 & 12.7 & 117 & -- & 9.80 & 6.50 & 16 \\
\hline P3 & $03 / 07 / 79$ & -27 & -8.2 & 1310 & 12.6 & 185 & -- & 27.00 & 6.70 & 22 \\
\hline P4 & $08 / 17 / 78$ & 11 & 3.4 & 1840 & 14.0 & 175 & -- & 8.60 & 5.70 & -- \\
\hline P4 & $08 / 17 / 78$ & 8 & 2.4 & 1900 & 13.5 & 200 & -- & 38.00 & 5.90 & -- \\
\hline P4 & $08 / 17 / 78$ & 5 & 1.5 & 1915 & 13.1 & 220 & -- & 17.00 & 6.00 & -- \\
\hline P4 & $08 / 17 / 78$ & 2 & 0.6 & 1920 & 12.9 & 255 & -- & 21.00 & 6.00 & - \\
\hline P4 & $08 / 17 / 78$ & -1 & -0.3 & 1930 & 12.7 & 330 & -- & 28.00 & 6.00 & -- \\
\hline P4 & $08 / 17 / 78$ & -4 & -1.2 & 1940 & 12.4 & 205 & -- & 15.00 & 6.20 & -- \\
\hline P4 & $08 / 17 / 78$ & -9 & -2.7 & 1945 & 12.3 & 157 & -- & 11.00 & 6.30 & -- \\
\hline P4 & $08 / 17 / 78$ & -14 & -4.3 & 1950 & 12.8 & 210 & -- & 14.00 & 6.20 & -- \\
\hline P4 & $08 / 17 / 78$ & -19 & -5.8 & 1955 & 12.9 & 230 & -- & 17.00 & 6.30 & -- \\
\hline P4 & $08 / 17 / 78$ & -24 & -7.3 & 2000 & 12.9 & 240 & -- & 19.00 & 6.30 & - \\
\hline P4 & $08 / 17 / 78$ & -29 & -8.8 & 2010 & 12.9 & 245 & -- & 20.00 & 6.30 & -- \\
\hline P4 & $09 / 15 / 78$ & 11 & 3.4 & 0905 & 15.1 & 115 & -- & 9.00 & 5.50 & -- \\
\hline P4 & $09 / 15 / 78$ & 8 & 2.4 & 0915 & 14.8 & 190 & -- & 25.00 & 5.50 & -- \\
\hline P4 & $09 / 15 / 78$ & 5 & 1.5 & 0925 & 14.6 & 146 & -- & 17.00 & 5.60 & -- \\
\hline P4 & $09 / 15 / 78$ & 2 & 0.6 & 0930 & 14.2 & 180 & - & 20.00 & 5.70 & -- \\
\hline P4 & $09 / 15 / 78$ & -1 & -0.3 & 0935 & 13.7 & 182 & -- & 23.00 & 5.60 & -- \\
\hline P4 & $09 / 15 / 78$ & -4 & -1.2 & 0945 & 13.6 & 225 & -- & 22.00 & 5.40 & -- \\
\hline P4 & $09 / 15 / 78$ & -9 & -2.7 & 0950 & 13.4 & 187 & -- & 16.00 & 5.80 & -- \\
\hline P4 & $09 / 15 / 78$ & -14 & -4.3 & 0955 & 13.6 & 118 & -- & 11.00 & 6.00 & -- \\
\hline P4 & $09 / 15 / 78$ & -19 & -5.8 & 1000 & 13.5 & 118 & -- & 15.00 & 5.80 & - \\
\hline P4 & $09 / 15 / 78$ & -24 & -7.3 & 1005 & 13.7 & 124 & -- & 12.00 & 6.10 & -- \\
\hline P4 & $09 / 15 / 78$ & -29 & -8.8 & 1015 & 13.6 & 114 & -- & 11.00 & 6.10 & -- \\
\hline
\end{tabular}


Table 5.--Water-chem istry data collected from wells near Horsfall Beach, and from wells B5 and 44, 1978-82--Continued

\begin{tabular}{|c|c|c|c|c|c|c|c|c|c|c|}
\hline WELL & DATE & $\begin{array}{c}\text { ELEVA } \\
(\text { FEET) }\end{array}$ & $\begin{array}{l}\text { TION } \\
\text { (METER) }\end{array}$ & TIME & $\begin{array}{l}\text { TEMPER- } \\
\text { ATURE } \\
\left(\begin{array}{ll}\text { DEG } & \text { C) }\end{array}\right.\end{array}$ & $\begin{array}{l}\text { SPECIFIC } \\
\text { CONDUCT- } \\
\text { ANCE } \\
\text { (UMHOS) }\end{array}$ & $\begin{array}{c}\text { BICAR- } \\
\text { BONATE } \\
\text { (MG/L } \\
\text { AS } \\
\text { HCO3) }\end{array}$ & $\begin{array}{l}\text { IRON } \\
\text { DIS- } \\
\text { SOLVED } \\
\text { (MG/L) }\end{array}$ & $\begin{array}{c}\mathrm{PH} \\
(\mathrm{UNIT})\end{array}$ & $\begin{array}{l}\text { CHLO- } \\
\text { RIDE } \\
\text { DIS- } \\
\text { SOLVED } \\
(M G / L)\end{array}$ \\
\hline P4 & $10 / 17 / 78$ & $\mathrm{P} \mid \mathrm{T}^{1}$ & -- & 0930 & 15.6 & 232 & 85 & 14.00 & 5.50 & 10 \\
\hline P4 & $10 / 17 / 78$ & 11 & 3.4 & 1130 & 15.5 & 128 & 46 & 11.00 & 5.20 & 8 \\
\hline P4 & $10 / 17 / 78$ & 8 & 2.4 & 1200 & 15.4 & 155 & 63 & 17.00 & 5.45 & 6 \\
\hline P4 & $10 / 17 / 78$ & 5 & 1.5 & 1215 & 15.2 & 175 & 76 & 21.00 & 5.60 & 6 \\
\hline P4 & $10 / 17 / 78$ & 2 & 0.6 & 1230 & 14.7 & 180 & 77 & 19.00 & 5.65 & 6 \\
\hline P4 & $10 / 17 / 78$ & -1 & -0.3 & 1245 & 14.4 & 170 & 78 & 22.00 & 5.75 & 6 \\
\hline P4 & $10 / 17 / 78$ & -4 & -1.2 & 1300 & 14.0 & 180 & 52 & 14.00 & 5.89 & 15 \\
\hline P4 & $10 / 17 / 78$ & -9 & -2.7 & 1310 & 13.6 & 210 & 54 & 15.00 & 6.00 & 27 \\
\hline P4 & $10 / 17 / 78$ & -14 & $-4 \cdot 3$ & 1320 & 13.6 & 128 & 60 & 11.00 & 6.15 & 10 \\
\hline P4 & $10 / 17 / 78$ & -19 & -5.8 & 1330 & 13.5 & 165 & 52 & 17.00 & 6.15 & 20 \\
\hline P4 & $10 / 17 / 78$ & -24 & $-7 \cdot 3$ & 1345 & 13.5 & 136 & 60 & 14.00 & 6.20 & 9 \\
\hline P4 & $10 / 17 / 78$ & -29 & -8.8 & 1355 & 13.5 & 122 & 55 & 12.00 & 6.20 & 8 \\
\hline P4 & $03 / 07 / 79$ & 13 & 4.0 & 0950 & 10.8 & 32 & -- & .13 & 5.60 & 5 \\
\hline P4 & $03 / 07 / 79$ & 11 & 3.4 & 1000 & 9.8 & 64 & -- & 1.60 & 5.40 & 7 \\
\hline P4 & $03 / 07 / 79$ & 8 & 2.4 & 1007 & 10.2 & 114 & - & 13.00 & 5.40 & 9 \\
\hline P4 & 03/07/79 & 5 & 1.5 & 1013 & 9.6 & 118 & -- & 13.00 & 5.70 & 6 \\
\hline P4 & $03 / 07 / 79$ & 2 & 0.6 & 1018 & 9.9 & 132 & -- & 14.00 & 5.70 & 8 \\
\hline P4 & 03/07/79 & -1 & -0.3 & 1024 & 11.2 & 146 & -- & 17.00 & 5.60 & 6 \\
\hline P4 & 03/07/79 & -4 & -1.2 & 1031 & 11.8 & 148 & -- & 17.00 & 5.70 & 9 \\
\hline P4 & $03 / 07 / 79$ & -9 & -2.7 & 1039 & 12.3 & 170 & -- & 12.00 & 6.00 & 25 \\
\hline P4 & $03 / 07 / 79$ & -14 & $-4 \cdot 3$ & 1045 & 12.4 & 170 & -- & 16.00 & 6.10 & 15 \\
\hline P4 & 03/07/79 & -19 & -5.8 & 1050 & 12.4 & 142 & -- & 18.00 & 6.20 & 13 \\
\hline P4 & $03 / 07 / 79$ & -24 & -7.3 & 1055 & 12.4 & 117 & -- & 15.00 & 6.40 & 9 \\
\hline P4 & $03 / 07 / 79$ & -29 & -8.8 & 1100 & 12.3 & 114 & -- & 14.00 & 6.40 & 9 \\
\hline P5 & $09 / 13 / 78$ & 6 & 1.8 & 1015 & 15.5 & 195 & -- & 4.20 & 5.90 & -- \\
\hline P5 & $09 / 13 / 78$ & 2 & 0.6 & 1030 & 14.4 & 195 & -- & .61 & 5.80 & -- \\
\hline P5 & $09 / 13 / 78$ & -6 & -1.8 & 1050 & 12.7 & 152 & -- & 2.50 & 5.20 & -- \\
\hline P5 & $09 / 13 / 78$ & -10 & -3.0 & 1100 & 12.4 & 137 & -- & 5.80 & 4.80 & -- \\
\hline P5 & $09 / 13 / 78$ & -15 & -4.6 & 1110 & 12.0 & 145 & -- & 5.90 & 5.30 & -- \\
\hline P5 & $09 / 13 / 78$ & -20 & -6.1 & 1120 & 12.0 & 155 & -- & 7.90 & 5.30 & -- \\
\hline P5 & $09 / 13 / 78$ & -25 & -7.6 & 1130 & 12.5 & 168 & -- & 10.00 & 5.30 & -- \\
\hline P5 & $09 / 13 / 78$ & -30 & -9.1 & 1140 & 13.0 & 255 & -- & 17.00 & 5.00 & -- \\
\hline P5 & $09 / 13 / 78$ & -35 & -10.7 & 1150 & 12.5 & 315 & -- & 25.00 & 5.30 & -- \\
\hline
\end{tabular}


Table 5.--Water-chemistry data collected from well s near Horsfall Beach, and from wells B5 and 44, 1978-82--Continued

\begin{tabular}{|c|c|c|c|c|c|c|c|c|c|c|}
\hline WELL & DATE & $\begin{array}{l}\text { ELEVAT } \\
\text { (FEET) }\end{array}$ & $\begin{array}{l}\text { TION } \\
\text { (METER) }\end{array}$ & TIME & $\begin{array}{l}\text { TEMPER- } \\
\text { ATURE } \\
\text { (DEG C) }\end{array}$ & $\begin{array}{l}\text { SPECIFIC } \\
\text { CONDUCT- } \\
\text { ANCE } \\
\text { (UMHOS) }\end{array}$ & $\begin{array}{c}\text { BICAR- } \\
\text { BONATE } \\
\text { (MG/L } \\
\text { AS } \\
\text { HCO3) }\end{array}$ & $\begin{array}{l}\text { IRON } \\
\text { DIS- } \\
\text { SOLVED } \\
\text { (MG/L) }\end{array}$ & $\begin{array}{c}\mathrm{PH} \\
(\mathrm{UNIT})\end{array}$ & $\begin{array}{l}\text { CHLO- } \\
\text { RIDE } \\
\text { DIS- } \\
\text { SOLVED } \\
(M G / L)\end{array}$ \\
\hline P5 & $10 / 19 / 78$ & 6 & 1.8 & 1630 & 15.2 & 320 & 20 & 7.90 & 6.10 & -- \\
\hline P5 & $10 / 19 / 78$ & 2 & 0.6 & 1640 & 14.3 & 222 & 34 & 2.00 & 5.90 & -- \\
\hline P5 & $10 / 19 / 78$ & -2 & -0.6 & 1650 & 13.6 & 185 & 32 & 2.80 & 5.70 & -- \\
\hline P5 & $10 / 19 / 78$ & -6 & -1.8 & 1700 & 13.0 & 136 & 34 & 4.20 & 5.80 & -- \\
\hline P5 & $10 / 19 / 78$ & -10 & -3.0 & 1710 & 12.6 & 152 & 33 & 8.30 & 6.20 & -- \\
\hline P5 & $10 / 19 / 78$ & -15 & $-4 \cdot 6$ & 1720 & 12.3 & 134 & 40 & 6.80 & 6.40 & -- \\
\hline P5 & $10 / 19 / 78$ & -20 & -6.1 & 1730 & 12.1 & 210 & 30 & 14.00 & 6.40 & -- \\
\hline P5 & $10 / 19 / 78$ & -25 & -7.6 & 1735 & 12.0 & 250 & 30 & 19.00 & 6.40 & -- \\
\hline P5 & $10 / 19 / 78$ & -30 & -9.1 & 1740 & 12.0 & 288 & 89 & 27.00 & 6.40 & -- \\
\hline P5 & $10 / 19 / 78$ & -35 & -10.7 & 1750 & 12.3 & 299 & 104 & 26.00 & 6.50 & -- \\
\hline P5 & 03/06/79 & 6 & 1.8 & 1028 & 9.7 & 100 & -- & .10 & 5.50 & -- \\
\hline P5 & $03 / 06 / 70$ & 2 & 0.6 & 1034 & 9.7 & 100 & -- & .63 & 5.50 & -- \\
\hline P5 & $03 / 06 / 79$ & -2 & -0.6 & 1043 & 10.1 & 132 & -- & .90 & 6.00 & -- \\
\hline P5 & $03 / 46 / 79$ & -6 & -1.8 & 1054 & 11.3 & 155 & -- & 3.20 & 6.00 & -- \\
\hline P5 & $03 / 06 / 79$ & -10 & -3.0 & 1100 & 11.5 & 190 & -- & 8.90 & 6.20 & -- \\
\hline P5 & $03 / 06 / 79$ & -15 & -4.6 & 1106 & 11.8 & 165 & -- & 8.30 & 6.50 & -- \\
\hline P5 & $03 / 06 / 79$ & -20 & -6.1 & 1112 & 12.0 & 170 & -- & 9.40 & 6.50 & -- \\
\hline P5 & $03 / 06 / 79$ & -25 & -7.6 & 1119 & 11.8 & 173 & -- & 14.00 & 6.50 & -- \\
\hline P5 & $03 / 06 / 79$ & -30 & -9.1 & 1124 & 11.8 & 205 & -- & 15.00 & 6.60 & -- \\
\hline P5 & $03 / 06 / 79$ & -35 & -10.7 & 1128 & 11.9 & 370 & -- & 29.00 & 6.60 & -- \\
\hline P6 & $09 / 14 / 78$ & $\mathrm{PIT}$ & -- & 1130 & 17.6 & 345 & 4 & 14.00 & -- & 82 \\
\hline P6 & $09 / 14 / 78$ & 5 & 1.5 & 1210 & & 319 & 3 & 20.00 & 5.30 & 60 \\
\hline P6 & $09 / 14 / 78$ & 1 & 0.3 & 1224 & 16.1 & 309 & 12 & 17.00 & .80 & 57 \\
\hline P6 & $09 / 14 / 78$ & -3 & -0.9 & 1232 & 15.7 & 332 & 3 & 22.00 & 5.30 & 62 \\
\hline P6 & $09 / 14 / 78$ & -7 & -2.1 & 1240 & 15.0 & 368 & 8 & 15.00 & 6.00 & 51 \\
\hline P6 & $09 / 14 / 78$ & -11 & -3.4 & 1250 & 14.3 & 283 & 40 & 31.00 & 5.80 & 28 \\
\hline P6 & $09 / 14 / 78$ & -16 & -4.9 & 1310 & 14.8 & 330 & 13 & 37.00 & 5.80 & 40 \\
\hline P6 & $09 / 14 / 78$ & -21 & -6.4 & 1320 & 14.7 & 310 & 8 & 36.00 & 5.80 & 40 \\
\hline P6 & $09 / 14 / 78$ & -26 & -7.9 & 1330 & 13.8 & 370 & 8 & 39.00 & 5.80 & 57 \\
\hline P6 & $09 / 14 / 78$ & -31 & -9.4 & 1350 & 16.0 & 372 & 13 & 35.00 & 5.80 & 57 \\
\hline P6 & $10 / 18 / 78$ & $\mathrm{P} \mid \mathrm{T}^{1 / 1}$ & -- & 1810 & 14.6 & 358 & 43 & & & 82 \\
\hline P6 & $10 / 18 / 78$ & 5 & 1.5 & 1820 & 15.5 & 409 & 48 & 27.00 & & 93 \\
\hline P6 & $10 / 18 / 78$ & 1 & 0.3 & 1825 & 15.0 & 358 & 56 & 21.00 & 6.10 & 69 \\
\hline P6 & $10 / 18 / 78$ & -3 & -0.9 & 1830 & 14.4 & 350 & 74 & 26.00 & 6.00 & 64 \\
\hline P6 & $10 / 18 / 78$ & -7 & -2.1 & 1835 & 13.9 & 418 & 70 & 47.00 & 6.10 & 48 \\
\hline P6 & $10 / 18 / 78$ & -11 & -3.4 & 1840 & 13.3 & 402 & 102 & 55.00 & 6.25 & 37 \\
\hline P6 & $10 / 18 / 78$ & -16 & -4.9 & 1845 & 13.0 & 268 & 109 & 40.00 & 6.45 & 21 \\
\hline P6 & $10 / 18 / 78$ & -21 & -6.4 & 1855 & 12.8 & 197 & 83 & 28.00 & 6.40 & 21 \\
\hline
\end{tabular}


Table 5.--Water-chemistry data collected from wells near Horsfall Beach, and from wells B5 and 44, 1978-82--Continued

\begin{tabular}{|c|c|c|c|c|c|c|c|c|c|c|}
\hline WELL & DATE & $\begin{array}{l}\text { ELEVAT } \\
\text { (FEET) }\end{array}$ & $\begin{array}{l}\text { TION } \\
\text { (METER) }\end{array}$ & TIME & $\begin{array}{l}\text { TEMPER- } \\
\text { ATURE } \\
(\text { DEG C) }\end{array}$ & $\begin{array}{l}\text { SPECIFIC } \\
\text { CONDUCT- } \\
\text { ANCE } \\
\text { (UMHOS) }\end{array}$ & $\begin{array}{c}\text { BICAR- } \\
\text { BONATE } \\
\text { (MG/L } \\
\text { AS } \\
\text { HCO3) }\end{array}$ & $\begin{array}{l}\text { IRON } \\
\text { DIS- } \\
\text { SOLVED } \\
(M G / L)\end{array}$ & $\begin{array}{c}\mathrm{PH} \\
(\mathrm{UN} \mid \mathrm{T})\end{array}$ & $\begin{array}{l}\text { CHLO- } \\
\text { RIDE } \\
\text { DIS- } \\
\text { SOLVED } \\
(M G / L)\end{array}$ \\
\hline P6 & $10 / 18 / 78$ & -26 & -7.9 & 1905 & 12.7 & 148 & 70 & 18.00 & 6.30 & 15 \\
\hline P6 & $10 / 18 / 78$ & -31 & -9.4 & 1920 & 12.7 & 174 & 59 & 19.00 & 6.35 & 23 \\
\hline P6 & $03 / 07 / 79$ & 5 & 1.5 & 1505 & 12.2 & 94 & -- & 1.90 & 5.80 & 19 \\
\hline P6 & $03 / 07 / 79$ & 1 & 0.3 & 1512 & 9.8 & 110 & -- & 3.40 & 6.60 & 21 \\
\hline P6 & 03/07/79 & -3 & -0.9 & 1519 & 10.0 & 124 & -- & 5.50 & 6.60 & 20 \\
\hline $\mathrm{P} 6$ & $03 / 07 / 79$ & -7 & -2.1 & 1525 & 11.0 & 112 & -- & 6.40 & 6.60 & 17 \\
\hline P6 & 03/07/79 & -11 & -3.4 & 1533 & 11.8 & 378 & -- & 31.00 & 6.60 & 82 \\
\hline P6 & 03/07/79 & -16 & -4.9 & 1539 & 12.4 & $420^{\circ}$ & -- & 54.00 & 6.80 & 52 \\
\hline P6 & 03/07/79 & -21 & -6.4 & 1545 & 12.5 & 235 & -- & 30.00 & 6.80 & 22 \\
\hline P6 & 03/07/79 & -26 & -7.9 & 1551 & 12.5 & 180 & -- & 20.00 & 6.80 & 18 \\
\hline P6 & 03/07/79 & -31 & -9.4 & 1558 & 12.4 & 165 & -- & 13.00 & 6.90 & 17 \\
\hline P7 & $09 / 13 / 78$ & 8 & 2.4 & 1615 & 16.5 & 215 & 1 & 8.60 & 4.44 & 33 \\
\hline P7 & $09 / 13 / 78$ & 4 & 1.2 & 1625 & 15.8 & 215 & 1 & 8.60 & 4.75 & 31 \\
\hline P7 & $09 / 13 / 78$ & 0 & 0.0 & 1630 & 14.8 & 165 & 20 & 8.90 & 4.60 & 28 \\
\hline P7 & $09 / 13 / 78$ & -4 & -1.2 & 1635 & 14.3 & 155 & 34 & 14.00 & 4.40 & 24 \\
\hline P7 & $09 / 13 / 78$ & -8 & -2.4 & 1645 & 13.8 & 355 & 1 & 34.00 & 4.60 & 30 \\
\hline P7 & $09 / 13 / 78$ & -13 & -4.0 & 1650 & 13.8 & 295 & 1 & 25.00 & 4.55 & 33 \\
\hline P7 & $09 / 13 / 78$ & -18 & -5.5 & 1655 & 14.0 & 235 & 15 & 22.00 & 4.69 & 30 \\
\hline P7 & $10 / 18 / 78$ & PITII & -- & 1130 & 16.5 & 351 & 50 & 14.00 & 5.40 & 42 \\
\hline P7 & $10 / 18 / 78$ & 8 & 2.4 & 1145 & 16.3 & 211 & 37 & 13.00 & 5.70 & 35 \\
\hline P7 & $10 / 18 / 78$ & 4 & 1.2 & 1155 & 15.7 & 142 & 48 & 6.40 & 5.75 & 14 \\
\hline P7 & $10 / 18 / 78$ & 0 & 0.0 & 1205 & 15.3 & 193 & 41 & 11.00 & 5.60 & 27 \\
\hline P7 & $10 / 18 / 78$ & -4 & -1.2 & 1215 & 14.4 & 413 & 28 & 39.00 & 5.70 & 31 \\
\hline P7 & $10 / 18 / 78$ & -8 & -2.4 & 1225 & 14.0 & 308 & 48 & 26.00 & 5.89 & 44 \\
\hline P7 & $10 / 18 / 78$ & -13 & -4.0 & 1235 & 14.0 & 219 & 79 & 20.00 & 6.10 & 22 \\
\hline P7 & $10 / 18 / 78$ & -18 & -5.5 & 1245 & 13.5 & 249 & 70 & 23.00 & 6.20 & 32 \\
\hline P7 & $03 / 06 / 79$ & 8 & 2.4 & 1609 & 9.8 & 100 & -- & .12 & 5.40 & 17 \\
\hline P7 & $03 / 06 / 79$ & 4 & 1.2 & 1618 & 9.7 & 90 & -- & 3.70 & 6.00 & 10 \\
\hline P7 & $03 / 06 / 79$ & 0 & 0.0 & 1626 & 10.6 & 180 & -- & 5.50 & 6.00 & 33 \\
\hline$P 7$ & $03 / 06 / 79$ & -4 & -1.2 & 1633 & 11.4 & 175 & -- & 14.00 & 6.00 & 20 \\
\hline P7 & 03/06/79 & -8 & -2.4 & 1641 & 12.2 & 440 & -- & 45.00 & 6.20 & 38 \\
\hline P7 & $03 / 06 / 79$ & -13 & -4.0 & 1648 & 12.4 & 400 & -- & 46.00 & 6.30 & 42 \\
\hline $\mathrm{P7}$ & 03/06/79 & -18 & -5.5 & 1655 & 12.3 & 265 & -- & 25.00 & 6.40 & 23 \\
\hline
\end{tabular}

1/ Sampled from exposed water table near the well. 


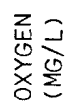

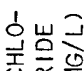

$\Upsilon 2$

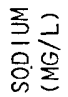

ड़

崫

岂崖

岁空言

产

它究市

깅

离崫志吉

工点

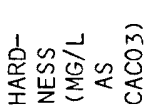

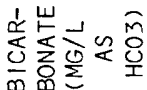

는

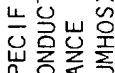

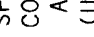

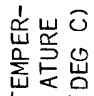

㠇

z을

崖

荘
1 1 in

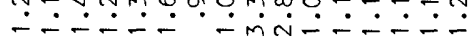

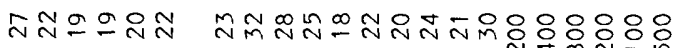

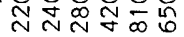

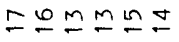

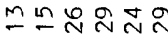

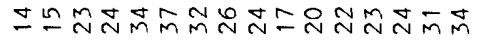

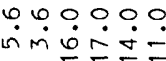

0000000000000000

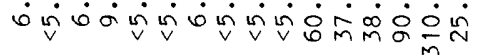

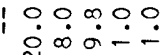

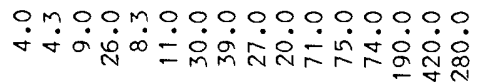

000000

inं

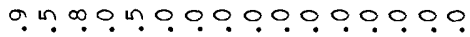

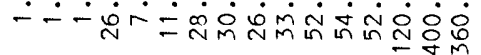

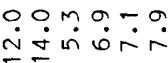

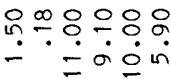

i 11 i

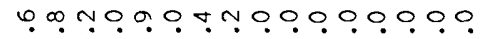

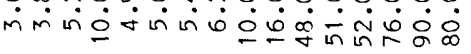

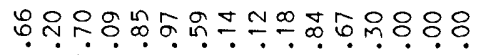

ì

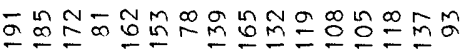

$\therefore=8800$

n் $\dot{0} \dot{0} \dot{0} \dot{0}$

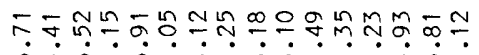

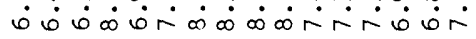

음ㅇㅇㅇ응

$\stackrel{\sim}{\sim} \stackrel{m}{m}=\stackrel{m}{=}=\underline{m}$

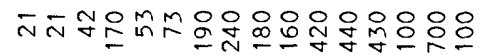

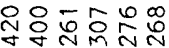

2

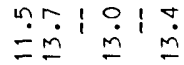

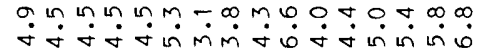
m̄m nn

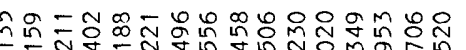

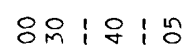

$\overline{\mathrm{N}} \overline{\mathrm{I}} \mathrm{g}$

तंज्नोन्नेन्ने

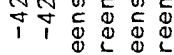

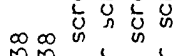

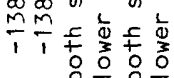

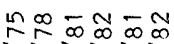

ㄱํㅇำ

으으으응

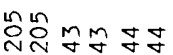

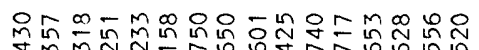

o- no armo in o on mr - 0

†은

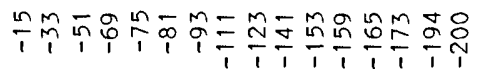

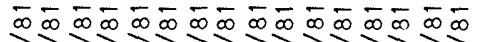

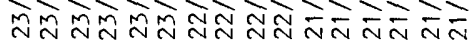

으으으으으으으으으으으으으으

$\bar{\infty} \bar{\infty} \bar{m} \bar{m} \bar{\infty} \bar{m} \bar{m} \bar{m} \bar{m} \bar{m} \bar{m} \bar{m} \bar{m} \bar{m} \bar{m} \bar{m}$

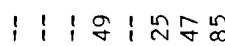

$\div \dot{-} \div$

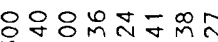

gNํ유용

ชชช์

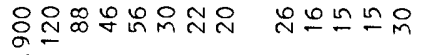

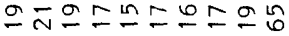

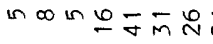

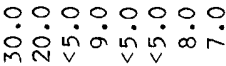

$\stackrel{0}{0} \begin{array}{lllll}1 & 1 & 1 & 1\end{array}$

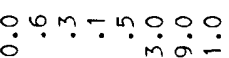

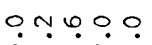

ำกㅊำ

$\ln 2-0.700,000$ กิ min

or $\infty 00$

om mNM Too

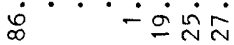

$\dot{x} \operatorname{co} \dot{m} \dot{\alpha}$

$0-0,0 N 00000$

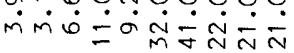

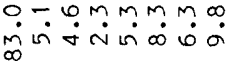

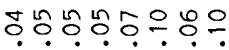

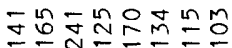

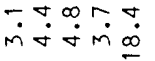

m.ง.า.

$888 \% \stackrel{\infty}{+}$

in

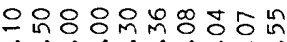

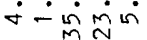

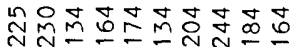

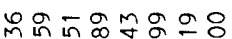

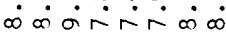

웅요융ㅇㅇ $\dot{\omega} \dot{0} \dot{\infty} \dot{r}$

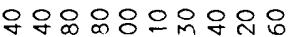

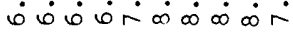

\&MN-In:

1 11111

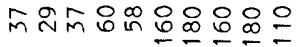

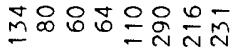

원스용ㅇㅇㅇ

ํำกำ

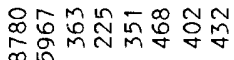

$\begin{array}{lllll}1 & 1 & 1 & 1 & 1\end{array}$

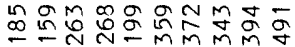

t.

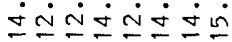

กํ. ㄴ. 9

$\dot{\underline{v}} \dot{\underline{I}} \dot{=} \dot{=}$

Un $\operatorname{co} r a-m N$ in $\forall a$

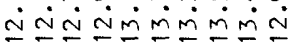

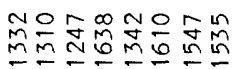

1 11111

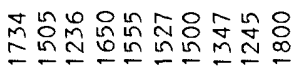

$\infty m-\infty-\infty-a$

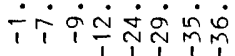

ํํำ

toun or nm m m

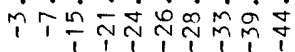

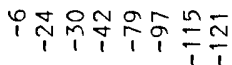

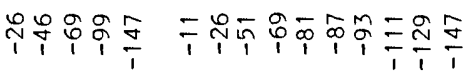

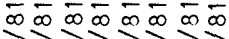

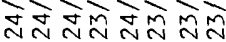

으으으으으응

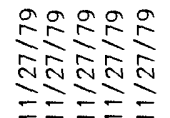

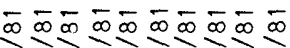

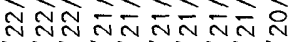

으으으으으으으으으으 mom $\bar{m} \tilde{m} \tilde{m} \tilde{m}$ $\tilde{m} \tilde{m} \tilde{m} \tilde{m} \tilde{m} \tilde{m} \tilde{m} \tilde{m} \tilde{m} \tilde{m}$ 


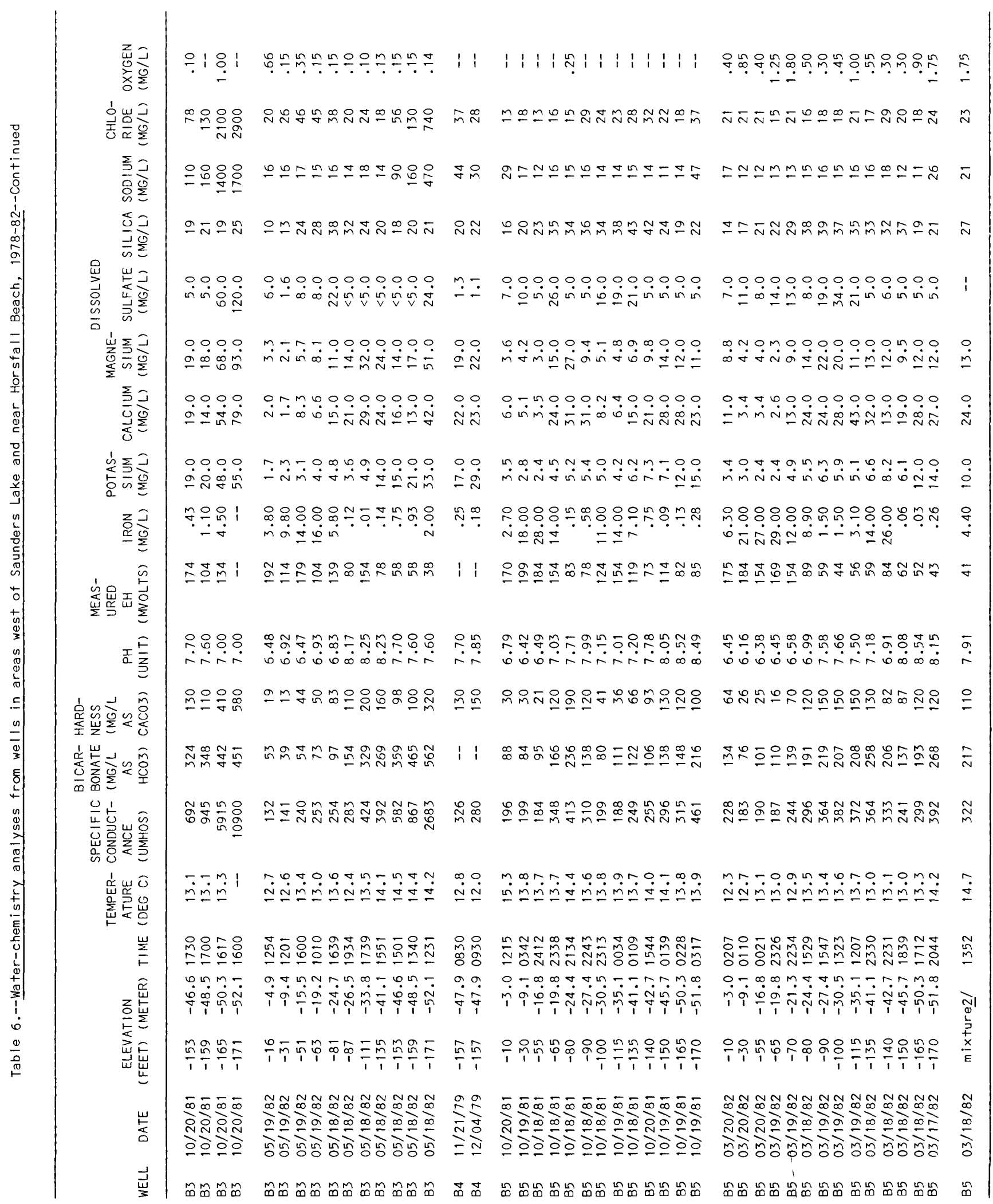




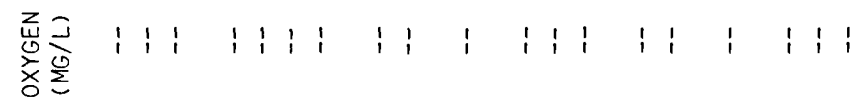

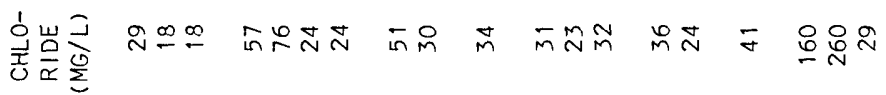

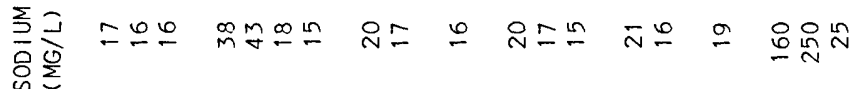

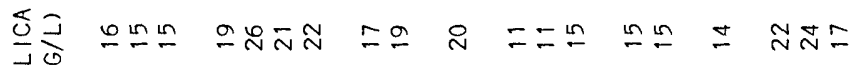

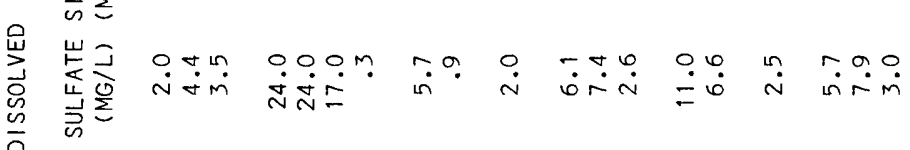

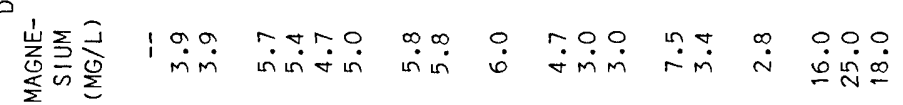

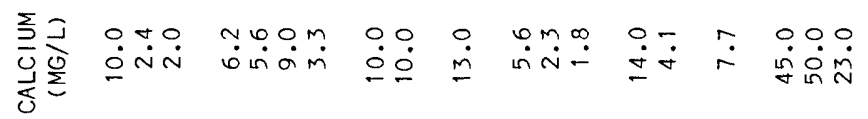

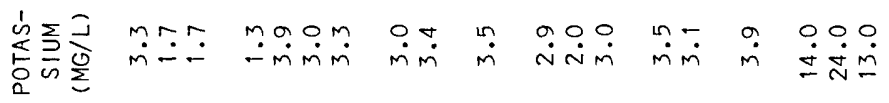
ż

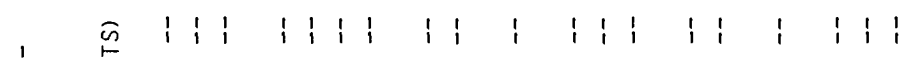
崖崖志索

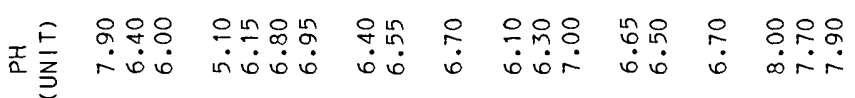

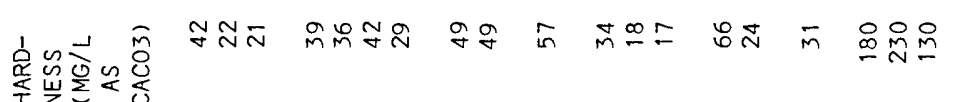

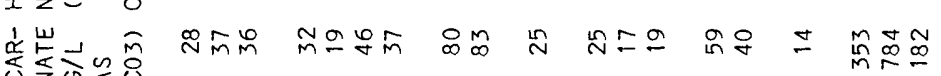

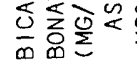

논

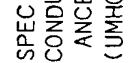

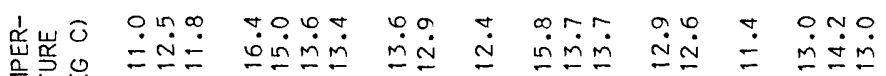
产点兽

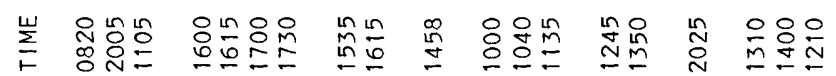
z䍃 䓌点

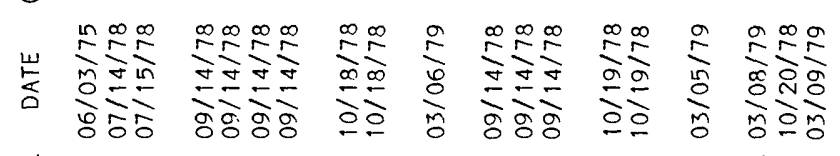

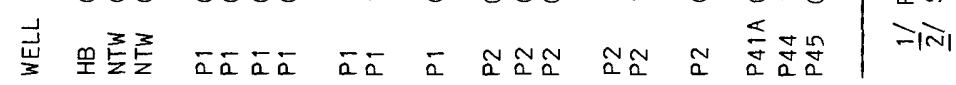


Table 7.--Water-chemistry data from

profile well B5, 1981-83

ALKA-

SPECIFIC LINITY IRON

TEMPER- CONDUCT- (MG/L DIS-

ELEVATION ATURE ANCE AS SOLVED PH

WELL DATE (FEET) (METER) (DEG C) (UMHOS) CACO3) (MG/L) (UNIT)

\begin{tabular}{llrrrrrrr}
\hline B5 & $08 / 27 / 81$ & 0 & 0.0 & 13.9 & 140 & 64 & .67 & 6.75 \\
B5 & $08 / 27 / 81$ & -10 & -3.0 & 13.9 & 220 & 64 & .55 & 7.00 \\
B5 & $08 / 27 / 81$ & -20 & -6.1 & 13.9 & 125 & 68 & 11.00 & 6.30 \\
B5 & $08 / 27 / 81$ & -30 & -9.1 & 14.9 & 140 & 94 & 16.25 & 6.41 \\
B5 & $08 / 27 / 81$ & -40 & -12.2 & 14.9 & 170 & 102 & 23.75 & 6.30 \\
B5 & $08 / 27 / 81$ & -45 & -13.7 & 14.8 & 170 & 88 & 19.50 & 6.40 \\
B5 & $08 / 27 / 81$ & -50 & -15.2 & 15.0 & 160 & 74 & 29.00 & 6.25 \\
B5 & $08 / 27 / 81$ & -55 & -16.8 & 15.1 & 170 & 98 & 34.75 & 6.49 \\
B5 & $08 / 27 / 81$ & -60 & -18.3 & 15.3 & 145 & 80 & 27.50 & 6.45 \\
B5 & $08 / 27 / 81$ & -65 & -19.8 & 15.3 & 210 & 130 & 15.00 & 6.80 \\
B5 & $08 / 27 / 81$ & -70 & -21.3 & 15.3 & 215 & 136 & 12.00 & 6.99 \\
B5 & $08 / 27 / 81$ & -75 & -22.9 & 13.8 & 170 & 130 & 12.00 & 7.10 \\
B5 & $08 / 27 / 81$ & -80 & -24.4 & 14.0 & 225 & 154 & 1.55 & 7.49 \\
B5 & $08 / 27 / 81$ & -85 & -25.9 & 14.3 & 220 & 148 & 1.25 & 7.60 \\
B5 & $08 / 27 / 81$ & -90 & -27.4 & 14.4 & 185 & 116 & .70 & 7.60 \\
B5 & $08 / 27 / 81$ & -100 & -30.5 & 13.8 & 130 & 64 & 10.00 & 7.20 \\
B5 & $08 / 27 / 81$ & -105 & -32.0 & 14.0 & 130 & 56 & 12.75 & 7.00 \\
B5 & $08 / 27 / 81$ & -115 & -35.1 & 14.4 & 135 & 60 & 13.50 & 6.82 \\
B5 & $08 / 27 / 81$ & -125 & -38.1 & 14.3 & 150 & 66 & 15.25 & 6.90 \\
B5 & $08 / 27 / 81$ & -135 & -41.1 & 14.7 & 140 & 60 & 11.25 & 7.00 \\
B5 & $08 / 27 / 81$ & -140 & -42.7 & 14.4 & 140 & 72 & 1.25 & 7.40 \\
B5 & $08 / 27 / 81$ & -145 & -44.2 & 14.2 & 175 & 104 & .32 & 7.80 \\
B5 & $08 / 27 / 81$ & -150 & -45.7 & 14.4 & 120 & 68 & .30 & 8.50 \\
B5 & $08 / 27 / 81$ & -155 & -47.2 & 14.7 & 180 & 114 & .15 & 8.40 \\
B5 & $08 / 27 / 81$ & -165 & -50.3 & 14.5 & 200 & 130 & .22 & 8.50 \\
B5 & $08 / 27 / 81$ & -170 & -51.8 & 14.8 & 270 & 164 & .20 & 8.70 \\
& & & & & & & & \\
\hline
\end{tabular}


Table 7.- Water-chem istry data from profile well B5, 1981-83--Continued

\begin{tabular}{|c|c|c|c|c|c|c|c|c|}
\hline WELL & DATE & $\begin{array}{l}\text { ELEVA } \\
\text { (FEET) }\end{array}$ & $\begin{array}{l}\text { ATION } \\
\text { (METER) }\end{array}$ & $\begin{array}{l}\text { TEMPER- } \\
\text { ATURE } \\
(D E G \quad C)\end{array}$ & $\begin{array}{l}\text { SPECIFIC } \\
\text { CONDUCT- } \\
\text { ANCE } \\
\text { (UMHOS) }\end{array}$ & $\begin{array}{c}\text { ALKA- } \\
\text { LINITY } \\
\text { (MG/L } \\
\text { AS } \\
\text { CACO3) }\end{array}$ & $\begin{array}{l}\text { IRON } \\
\text { DIS- } \\
\text { SOLVED } \\
(M G / L)\end{array}$ & $\begin{array}{c}\mathrm{PH} \\
(\mathrm{UN} \mid \mathrm{T})\end{array}$ \\
\hline B5 & $08 / 31 / 81$ & 0 & 0.0 & 14.9 & 110 & 70 & .22 & 6.52 \\
\hline B5 & $08 / 31 / 81$ & -10 & -3.0 & 13.9 & 130 & 100 & .82 & 6.81 \\
\hline B5 & $08 / 31 / 81$ & -20 & -6.1 & 14.3 & 80 & 60 & 10.50 & 6.35 \\
\hline B5 & $08 / 31 / 81$ & -30 & -9.1 & 14.1 & 90 & 70 & 13.50 & 6.40 \\
\hline B5 & $08 / 31 / 81$ & -40 & -12.2 & 14.2 & 115 & 88 & 26.00 & 6.35 \\
\hline B5 & $08 / 31 / 81$ & -45 & -13.7 & 14.3 & 145 & 110 & 33.00 & 6.50 \\
\hline B5 & $08 / 31 / 81$ & -50 & -15.2 & 14.5 & 130 & 78 & 30.00 & 6.50 \\
\hline B5 & $08 / 31 / 81$ & -55 & -16.8 & 14.8 & 130 & 86 & 30.25 & 6.40 \\
\hline B5 & $08 / 31 / 81$ & -60 & -18.3 & 14.8 & 125 & 86 & 33.00 & 6.50 \\
\hline B5 & $08 / 31 / 81$ & -65 & -19.8 & 14.9 & 135 & 142 & 15.25 & 6.71 \\
\hline B5 & $08 / 31 / 81$ & -70 & -21.3 & 14.9 & 185 & 138 & 12.25 & 6.95 \\
\hline B5 & $08 / 31 / 81$ & -75 & -22.9 & 15.1 & 170 & 140 & 12.00 & 6.90 \\
\hline B5 & $08 / 31 / 81$ & -80 & -24.4 & 15.2 & 210 & 150 & 2.00 & 7.45 \\
\hline B5 & $08 / 31 / 81$ & -85 & -25.9 & 15.3 & 205 & 152 & .71 & 7.61 \\
\hline B5 & $08 / 31 / 81$ & -90 & -27.4 & 15.1 & 175 & 114 & .62 & 7.80 \\
\hline B5 & $08 / 31 / 81$ & -100 & -30.5 & 15.1 & 120 & 70 & 10.50 & 7.25 \\
\hline B5 & $08 / 31 / 81$ & -105 & -32.0 & 15.1 & 120 & 62 & 12.50 & 7.00 \\
\hline B5 & $08 / 31 / 81$ & -115 & -35.1 & 15.1 & 135 & 60 & 18.75 & 6.90 \\
\hline B5 & $08 / 31 / 81$ & -125 & -38.1 & 15.2 & 130 & 60 & 19.25 & 6.88 \\
\hline B5 & $08 / 31 / 81$ & -135 & -41.1 & 15.7 & 145 & 66 & 9.00 & 7.05 \\
\hline B5 & $08 / 31 / 81$ & -140 & -42.7 & 15.4 & 130 & 72 & 5.25 & 7.45 \\
\hline B5 & $08 / 31 / 81$ & -145 & -44.2 & 15.5 & 210 & 126 & .32 & 7.80 \\
\hline B5 & $08 / 31 / 81$ & -150 & -45.7 & 15.8 & 150 & 80 & .11 & 8.15 \\
\hline B5 & $08 / 31 / 81$ & -155 & -47.2 & 16.1 & 185 & 110 & .12 & 8.11 \\
\hline B5 & $08 / 31 / 81$ & -165 & -50.3 & 15.5 & 200 & 130 & .14 & 8.40 \\
\hline B5 & $08 / 31 / 81$ & -170 & -51.8 & 15.8 & 295 & 166 & .05 & 8.50 \\
\hline
\end{tabular}


Table 7.--Water-chemistry data from profile well B5, 1981-83--Continued

\begin{tabular}{|c|c|c|c|c|c|c|c|c|}
\hline WELL & DATE & $\begin{array}{r}\text { ELEV } \\
\text { (FEET) }\end{array}$ & $\begin{array}{l}\text { ATION } \\
\text { (METER) }\end{array}$ & $\begin{array}{l}\text { TEMPER- } \\
\text { ATURE } \\
\text { (DEG C) }\end{array}$ & $\begin{array}{l}\text { SPECIFIC } \\
\text { CONDUCT- } \\
\text { ANCE } \\
\text { (UMHOS) }\end{array}$ & $\begin{array}{c}\text { ALKA- } \\
\text { LINITY } \\
\text { (MG/L } \\
\text { AS } \\
\text { CACO3) }\end{array}$ & $\begin{array}{l}\text { IRON } \\
\text { DIS- } \\
\text { SOLVED } \\
(M G / L)\end{array}$ & $\begin{array}{c}\mathrm{PH} \\
(U N \mid T)\end{array}$ \\
\hline B5 & $09 / 01 / 81$ & 0 & 0.0 & 15.4 & 120 & 70 & .16 & 6.50 \\
\hline B5 & $09 / 01 / 81$ & -10 & -3.0 & 14.5 & 150 & 96 & .86 & 6.90 \\
\hline B5 & $09 / 01 / 81$ & -20 & -6.1 & 14.4 & 100 & 60 & 11.60 & 6.51 \\
\hline B5 & $09 / 01 / 81$ & -30 & -9.1 & 14.4 & 115 & 70 & 16.75 & 6.50 \\
\hline B5 & $09 / 01 / 81$ & -40 & -12.2 & 14.4 & 125 & 86 & 27.75 & 6.49 \\
\hline B5 & $09 / 01 / 81$ & -45 & -13.7 & 14.6 & 150 & 110 & 33.75 & 6.49 \\
\hline B5 & $09 / 01 / 81$ & -50 & -15.2 & 14.7 & 145 & 74 & 32.25 & 6.31 \\
\hline B5 & $09 / 01 / 81$ & -55 & -16.8 & 14.7 & 130 & 80 & 32.50 & 6.50 \\
\hline B5 & $09 / 01 / 81$ & -60 & -18.3 & 14.7 & 135 & 86 & 35.00 & 6.51 \\
\hline B5 & $09 / 01 / 81$ & -65 & -19.8 & 14.8 & 200 & 142 & 15.50 & 6.81 \\
\hline B5 & $09 / 01 / 81$ & -70 & -21.3 & 14.9 & 195 & 136 & 15.25 & 6.99 \\
\hline B5 & $09 / 01 / 81$ & -75 & -22.9 & 15.1 & 190 & 140 & 14.50 & 6.92 \\
\hline B5 & $09 / 01 / 81$ & -80 & -24.4 & 15.1 & 225 & 146 & 1.65 & 7.50 \\
\hline B5 & $09 / 01 / 81$ & -85 & -25.9 & 15.2 & 230 & 148 & 1.15 & 7.70 \\
\hline B5 & $09 / 01 / 81$ & -90 & -27.4 & 15.2 & 175 & 116 & .59 & 7.90 \\
\hline B5 & $09 / 01 / 81$ & -100 & -30.5 & 15.0 & 180 & 66 & 12.00 & 7.20 \\
\hline B5 & $09 / 01 / 81$ & -105 & -32.0 & 14.9 & 130 & 60 & 12.50 & 7.00 \\
\hline B5 & $09 / 01 / 81$ & -115 & -35.1 & 15.1 & 140 & 58 & 15.75 & 6.73 \\
\hline B5 & $09 / 01 / 81$ & -125 & -38.1 & 15.2 & 140 & 60 & 17.00 & 6.85 \\
\hline B5 & $09 / 01 / 81$ & -135 & -41.1 & 15.3 & 160 & 68 & 9.25 & 6.99 \\
\hline B5 & $09 / 01 / 81$ & -140 & -42.7 & 15.3 & 140 & 76 & 1.10 & 7.51 \\
\hline B5 & $09 / 01 / 81$ & -145 & -44.2 & 15.3 & 200 & 124 & .26 & 3.05 \\
\hline B5 & $09 / 01 / 81$ & -150 & -45.7 & 15.4 & 160 & 86 & .09 & 8.20 \\
\hline B5 & $09 / 01 / 81$ & -155 & -47.2 & 15.4 & 185 & 110 & .07 & 8.41 \\
\hline B5 & $09 / 01 / 81$ & -165 & -50.3 & 15.4 & 210 & 128 & .13 & 8.50 \\
\hline B5 & $09 / 01 / 81$ & -170 & -51.8 & 15.4 & 290 & 168 & .15 & .87 \\
\hline
\end{tabular}


Table 7.--Water-chemistry data from profile well B5, 1981-83--Continued

\begin{tabular}{|c|c|c|c|c|c|c|c|c|}
\hline WELL & DATE & $\begin{array}{l}\text { ELEVA } \\
\text { (FEET) }\end{array}$ & $\begin{array}{l}\text { ATION } \\
\text { (METER) }\end{array}$ & $\begin{array}{c}\text { TEMPER- } \\
\text { ATURE } \\
\text { (DEG C) }\end{array}$ & $\begin{array}{l}\text { SPECIFIC } \\
\text { CONDUCT- } \\
\text { ANCE } \\
\text { (UMHOS) }\end{array}$ & $\begin{array}{c}\text { ALKA- } \\
\text { LINITY } \\
\text { (MG/L } \\
\text { AS } \\
\text { CACO3) }\end{array}$ & $\begin{array}{l}\text { IRON } \\
\text { DIS- } \\
\text { SOLVED } \\
\text { (MG/L) }\end{array}$ & $\begin{array}{c}\mathrm{PH} \\
(U N \mid T)\end{array}$ \\
\hline B5 & $09 / 02 / 81$ & 0 & 0.0 & 14.7 & 120 & 78 & .80 & - \\
\hline B5 & $09 / 02 / 81$ & -10 & -3.0 & 14.2 & 170 & 98 & 2.00 & - \\
\hline B5 & $09 / 02 / 81$ & -20 & -6.1 & 13.9 & 100 & 90 & 11.00 & 6.90 \\
\hline B5 & $09 / 02 / 81$ & -30 & -9.1 & 14.1 & 110 & 70 & 14.00 & 6.65 \\
\hline B5 & $09 / 02 / 81$ & -40 & -12.2 & 13.9 & 135 & 84 & 17.50 & 6.65 \\
\hline B5 & $09 / 02 / 81$ & -45 & -13.7 & 14.1 & 160 & 114 & 31.25 & 6.60 \\
\hline B5 & $09 / 02 / 81$ & -50 & $-15 \cdot 2$ & 14.4 & 145 & 80 & 35.00 & 6.50 \\
\hline B5 & $09 / 02 / 81$ & -55 & -16.8 & 14.3 & 140 & 78 & 32.50 & 6.60 \\
\hline B5 & $09 / 02 / 81$ & -60 & -18.3 & 14.5 & 140 & 86 & 33.50 & 6.55 \\
\hline B5 & $09 / 02 / 81$ & -65 & -19.8 & 14.6 & 220 & 142 & 13.75 & 6.90 \\
\hline B5 & $09 / 02 / 81$ & -70 & -21.3 & 14.7 & 135 & 130 & 12.00 & 6.99 \\
\hline B5 & $09 / 02 / 81$ & -75 & -22.9 & 14.7 & 125 & 144 & 13.00 & 7.00 \\
\hline B5 & $09 / 02 / 81$ & -80 & -24.4 & 14.8 & 255 & 152 & 1.75 & 7.45 \\
\hline B5 & $09 / 02 / 81$ & -85 & -25.9 & 14.6 & 255 & 150 & 1.45 & 7.65 \\
\hline B5 & $09 / 02 / 81$ & -90 & -27.4 & 14.6 & 220 & 118 & .65 & 7.70 \\
\hline B5 & $09 / 02 / 81$ & -100 & -30.5 & 14.5 & 150 & 68 & 11.00 & 7.00 \\
\hline B5 & $09 / 02 / 81$ & -105 & -32.0 & 14.7 & 140 & 60 & 11.50 & 6.90 \\
\hline B5 & $09 / 02 / 81$ & -115 & -35.1 & 14.7 & 150 & 60 & 13.75 & 6.90 \\
\hline B5 & $09 / 02 / 81$ & -125 & -38.1 & 14.8 & 150 & 60 & 14.00 & 6.85 \\
\hline B5 & $09 / 02 / 81$ & -135 & -41.1 & 15.3 & 180 & 68 & 7.60 & 6.95 \\
\hline B5 & $09 / 02 / 81$ & -140 & -42.7 & 14.8 & 160 & 80 & 1.00 & 7.30 \\
\hline B5 & $09 / 02 / 81$ & -145 & -44.2 & 15.3 & 230 & 130 & .35 & 7.75 \\
\hline B5 & $09 / 02 / 81$ & -150 & -45.7 & 15.3 & 175 & 90 & .25 & 7.70 \\
\hline B5 & $09 / 02 / 81$ & -155 & -47.2 & 15.3 & 205 & 118 & .04 & 8.10 \\
\hline B5 & $09 / 02 / 81$ & -165 & -50.3 & 15.3 & 230 & 130 & .05 & 8.15 \\
\hline B5 & $09 / 02 / 81$ & -170 & -51.8 & 15.3 & 320 & 170 & .21 & 8.30 \\
\hline
\end{tabular}


Table 7.--Water-chemistry data from profile well B5, 1981-83--Continued

\begin{tabular}{|c|c|c|c|c|c|c|c|c|}
\hline WELL & DATE & $\begin{array}{l}\text { ELEVA } \\
\text { (FEET) }\end{array}$ & $\begin{array}{l}\text { ATION } \\
\text { (METER) }\end{array}$ & $\begin{array}{l}\text { TEMPER- } \\
\text { ATURE } \\
(\text { DEG C) }\end{array}$ & $\begin{array}{l}\text { SPECIFIC } \\
\text { CONDUCT- } \\
\text { ANCE } \\
\text { (UMHOS) }\end{array}$ & $\begin{array}{c}\text { ALKA- } \\
\text { LINITY } \\
\text { (MG/L } \\
\text { AS } \\
\text { CACO3) }\end{array}$ & $\begin{array}{l}\text { IRON } \\
\text { DIS- } \\
\text { SOLVED } \\
\text { (MG/L) }\end{array}$ & $\begin{array}{c}\mathrm{PH} \\
(\mathrm{UN} \mid \mathrm{T})\end{array}$ \\
\hline B5 & $09 / 03 / 81$ & 0 & 0.0 & 14.7 & 155 & 64 & .10 & 6.50 \\
\hline B5 & $09 / 03 / 81$ & -10 & -3.0 & 13.7 & 185 & 90 & .98 & 6.80 \\
\hline B5 & $09 / 03 / 81$ & -20 & -6.1 & 13.8 & 110 & 60 & 12.50 & 6.40 \\
\hline B5 & $09 / 03 / 81$ & -30 & -9.1 & 13.8 & 130 & 70 & 17.25 & 6.70 \\
\hline B5 & $09 / 03 / 81$ & -40 & -12.2 & 13.8 & 150 & 80 & 27.00 & 6.52 \\
\hline B5 & $09 / 03 / 81$ & -45 & -13.7 & 13.9 & 180 & 104 & 31.00 & 6.51 \\
\hline B5 & $09 / 03 / 81$ & -50 & -15.2 & 13.9 & 165 & 80 & 32.00 & 6.30 \\
\hline B5 & $09 / 03 / 81$ & -55 & -16.8 & 14.3 & 160 & 80 & 31.25 & 6.40 \\
\hline B5 & $09 / 03 / 81$ & -60 & -18.3 & 14.3 & 160 & 84 & 33.75 & 6.41 \\
\hline B5 & $09 / 03 / 81$ & -65 & -19.8 & 14.7 & 250 & 148 & 16.00 & 6.71 \\
\hline B5 & $09 / 03 / 81$ & -70 & -21.3 & 14.7 & 250 & 130 & 14.75 & 6.99 \\
\hline B5 & $09 / 03 / 81$ & -75 & -22.9 & 14.8 & 250 & 146 & 15.00 & 6.90 \\
\hline B5 & $09 / 03 / 81$ & -80 & -24.4 & 14.8 & 275 & 152 & 1.50 & 7.50 \\
\hline B5 & $09 / 03 / 81$ & -85 & -25.9 & 14.9 & 275 & 148 & 1.25 & 7.65 \\
\hline B5 & $09 / 03 / 81$ & -90 & -27.4 & 14.6 & 230 & 116 & .61 & 7.75 \\
\hline B5 & $09 / 03 / 81$ & -100 & -30.5 & 14.4 & 150 & 66 & 11.75 & 7.10 \\
\hline B5 & $09 / 03 / 81$ & -105 & -32.0 & 14.6 & 145 & 56 & 13.75 & 6.90 \\
\hline B5 & $09 / 03 / 81$ & -115 & -35.1 & 14.6 & 155 & 60 & 17.00 & 6.89 \\
\hline B5 & $09 / 03 / 81$ & -125 & -38.1 & 14.7 & 160 & 60 & 16.00 & 6.80 \\
\hline B5 & $09 / 03 / 81$ & -135 & -41.1 & 15.0 & 180 & 68 & 9.50 & 6.95 \\
\hline B5 & $09 / 03 / 81$ & -140 & -42.7 & 14.6 & 160 & 76 & .75 & 7.40 \\
\hline B5 & $09 / 03 / 81$ & -145 & -44.2 & 15.2 & 240 & 116 & .27 & 7.90 \\
\hline B5 & $09 / 03 / 81$ & -150 & -45.7 & 15.3 & 180 & 90 & .15 & 8.05 \\
\hline B5 & $09 / 03 / 81$ & -155 & -47.2 & 15.4 & 200 & 112 & .08 & 8.30 \\
\hline B5 & $09 / 03 / 81$ & -165 & -50.3 & 15.2 & 235 & 130 & .18 & 8.35 \\
\hline B5 & $09 / 03 / 81$ & -170 & -51.8 & 15.3 & 325 & 160 & .13 & 8.42 \\
\hline
\end{tabular}


Table 7.- Water-chem istry data from profile well B5, 1981-83--Continued

\begin{tabular}{|c|c|c|c|c|c|c|c|c|}
\hline WELL & DATE & $\begin{array}{l}\text { ELEV } \\
\text { (FEET) }\end{array}$ & $\begin{array}{l}\text { ATION } \\
\text { (METER) }\end{array}$ & $\begin{array}{l}\text { TEMPER- } \\
\text { ATURE } \\
\text { (DEG C) }\end{array}$ & $\begin{array}{l}\text { SPECIFIC } \\
\text { CONDUCT- } \\
\text { ANCE } \\
\text { (UMHOS) }\end{array}$ & $\begin{array}{c}\text { ALKA- } \\
\text { LIN ITY } \\
\text { (MG/L } \\
\text { AS } \\
\text { CACO3) }\end{array}$ & $\begin{array}{l}\text { IRON } \\
\text { DIS- } \\
\text { SOLVED } \\
\text { (MG/L) }\end{array}$ & $\begin{array}{c}\mathrm{PH} \\
(\mathrm{UNIT})\end{array}$ \\
\hline B5 & $09 / 04 / 81$ & 0 & 0.0 & 14.8 & 160 & 68 & .12 & 6.40 \\
\hline B5 & $09 / 04 / 81$ & -10 & -3.0 & 13.8 & 170 & 88 & 1.60 & 6.90 \\
\hline B5 & $09 / 04 / 81$ & -20 & -6.1 & 13.7 & 110 & 58 & 13.75 & 6.43 \\
\hline B5 & $09 / 04 / 81$ & -30 & -9.1 & 13.8 & 125 & 68 & 17.50 & 6.20 \\
\hline B5 & $09 / 04 / 81$ & -40 & -12.2 & 13.8 & 145 & 84 & 26.25 & 6.30 \\
\hline B5 & $09 / 04 / 81$ & -45 & -13.7 & 13.9 & 170 & 98 & 30.50 & 6.40 \\
\hline B5 & $09 / 04 / 81$ & -50 & -15.2 & 14.1 & 155 & 78 & 30.75 & 6.32 \\
\hline B5 & $09 / 04 / 81$ & -55 & -16.8 & 14.1 & 155 & 80 & 33.00 & 6.40 \\
\hline B5 & $09 / 04 / 31$ & -60 & -18.3 & 14.3 & 155 & 88 & 34.50 & 6.40 \\
\hline B5 & $09 / 04 / 81$ & -65 & -19.8 & 14.7 & 245 & 146 & 16.25 & 6.85 \\
\hline B5 & $09 / 04 / 81$ & -70 & -21.3 & 14.7 & 240 & 134 & 14.50 & 6.93 \\
\hline B5 & $09 / 04 / 81$ & -75 & -22.9 & 14.6 & 245 & 150 & 15.00 & 6.85 \\
\hline B5 & $09 / 04 / 81$ & -80 & -24.4 & 14.5 & 265 & 168 & 1.32 & 7.50 \\
\hline B5 & $09 / 04 / 81$ & -85 & -25.9 & 14.8 & 270 & 150 & 1.25 & 7.61 \\
\hline B5 & $09 / 04 / 81$ & -90 & -27.4 & 14.8 & 230 & 140 & .64 & 7.90 \\
\hline B5 & $09 / 04 / 81$ & -100 & -30.5 & 14.6 & 150 & 64 & 15.25 & 7.20 \\
\hline B5 & $09 / 04 / 81$ & -105 & -32.0 & 14.7 & 150 & 60 & 14.50 & 6.85 \\
\hline B5 & $09 / 04 / 81$ & -115 & -35.1 & 14.3 & 155 & 58 & 16.50 & 6.85 \\
\hline B5 & $09 / 04 / 81$ & -125 & -38.1 & 14.4 & 160 & 58 & 17.00 & 6.80 \\
\hline B5 & $09 / 04 / 81$ & -135 & -41.1 & 14.8 & 180 & 68 & 9.00 & 6.85 \\
\hline B5 & $09 / 04 / 81$ & -140 & -42.7 & 14.5 & 160 & 76 & .78 & 7.45 \\
\hline B5 & $09 / 04 / 81$ & -145 & -44.2 & 14.7 & 230 & 120 & .31 & 7.71 \\
\hline B5 & $09 / 04 / 81$ & -150 & -45.7 & 14.7 & 180 & 90 & .17 & 8.05 \\
\hline B5 & $09 / 04 / 81$ & -155 & -47.2 & 14.9 & 200 & 112 & .09 & 8.40 \\
\hline B5 & $09 / 04 / 81$ & -165 & -50.3 & 14.9 & 225 & 134 & .20 & 8.31 \\
\hline B5 & $09 / 04 / 81$ & -170 & -51.8 & 14.9 & 330 & 170 & .23 & 8.40 \\
\hline
\end{tabular}


Table 7.--Water-chemistry data from profile well B5, 1981-83--Continued

\begin{tabular}{|c|c|c|c|c|c|c|c|c|}
\hline WELL & DATE & $\begin{array}{l}\text { ELEVA } \\
\text { (FEET) }\end{array}$ & $\begin{array}{l}\text { AT I ON } \\
\text { (METER) }\end{array}$ & $\begin{array}{l}\text { TEMPER- } \\
\text { ATURE } \\
\left(\begin{array}{ll}\text { DEG } & \text { C) }\end{array}\right.\end{array}$ & $\begin{array}{l}\text { SPECIFIC } \\
\text { CONDUCT- } \\
\text { ANCE } \\
\text { (UMHOS) }\end{array}$ & $\begin{array}{c}\text { ALKA- } \\
\text { LINITY } \\
\text { (MG/L } \\
\text { AS } \\
\text { CACO3) }\end{array}$ & $\begin{array}{l}\text { IRON } \\
\text { DIS- } \\
\text { SOLVED } \\
\text { (MG/L) }\end{array}$ & $\begin{array}{c}P H \\
(U N \mid T)\end{array}$ \\
\hline B5 & $09 / 09 / 81$ & 0 & 0.0 & 14.2 & 160 & 66 & .41 & 6.40 \\
\hline B5 & $09 / 09 / 81$ & -10 & -3.0 & 14.2 & 160 & 88 & 1.42 & 6.90 \\
\hline B5 & $09 / 09 / 81$ & -20 & -6.1 & 14.0 & 105 & 60 & 13.50 & 6.50 \\
\hline B5 & $09 / 09 / 81$ & -30 & -9.1 & 14.1 & 140 & 88 & 19.50 & 6.40 \\
\hline B5 & $09 / 09 / 81$ & -40 & -12.2 & 14.3 & 140 & 80 & 26.25 & 6.45 \\
\hline B5 & $09 / 09 / 81$ & -45 & -13.7 & 14.3 & 155 & 98 & 29.00 & 6.49 \\
\hline B5 & $09 / 09 / 81$ & -50 & -15.2 & 14.5 & 150 & 80 & 32.50 & 6.39 \\
\hline B5 & $09 / 09 / 81$ & -55 & -16.8 & 14.4 & 150 & 84 & 31.75 & 6.43 \\
\hline B5 & $09 / 09 / 81$ & -60 & -18.3 & 14.4 & 150 & 84 & 33.00 & 6.39 \\
\hline B5 & $09 / 09 / 81$ & -65 & -19.8 & 14.9 & 230 & 150 & 16.25 & 6.80 \\
\hline B5 & $09 / 09 / 81$ & -70 & -21.3 & 14.7 & 240 & 136 & 16.00 & 6.95 \\
\hline B5 & $09 / 09 / 81$ & -75 & -22.9 & 14.7 & 240 & 150 & 15.25 & 6.90 \\
\hline B5 & $09 / 09 / 81$ & -80 & -24.4 & 14.9 & 270 & 170 & 1.38 & 7.70 \\
\hline B5 & $09 / 09 / 81$ & -85 & -25.9 & 14.8 & 260 & 142 & 1.28 & 7.81 \\
\hline B5 & $09 / 09 / 81$ & -90 & -27.4 & 14.8 & 220 & 120 & .65 & 8.10 \\
\hline B5 & $09 / 09 / 81$ & -100 & -30.5 & 14.7 & 150 & 70 & 10.50 & 7.30 \\
\hline B5 & $09 / 09 / 81$ & -105 & -32.0 & 14.9 & 140 & 60 & 13.75 & 7.00 \\
\hline B5 & $09 / 09 / 81$ & -115 & -35.1 & 14.6 & 150 & 60 & 16.75 & 7.00 \\
\hline B5 & $09 / 09 / 81$ & -125 & -38.1 & 14.8 & 150 & 58 & 17.00 & 6.88 \\
\hline B5 & $09 / 09 / 81$ & -135 & -41.1 & 15.1 & 180 & 70 & 9.25 & 6.90 \\
\hline B5 & $09 / 09 / 81$ & -140 & -42.7 & 15.0 & 165 & 80 & 3.75 & 7.50 \\
\hline B5 & $09 / 09 / 81$ & -145 & -44.2 & 15.1 & 225 & 114 & .23 & 7.80 \\
\hline B5 & $09 / 09 / 81$ & -150 & -45.7 & 15.1 & 185 & 102 & .12 & 8.10 \\
\hline B5 & $09 / 09 / 81$ & -155 & -47.2 & 15.2 & 200 & 116 & .03 & 8.32 \\
\hline B5 & $09 / 09 / 81$ & -165 & -50.3 & 15.2 & 230 & 130 & .17 & 8.25 \\
\hline B5 & $09 / 09 / 81$ & -170 & -51.8 & 15.3 & 330 & 172 & .17 & 8.22 \\
\hline
\end{tabular}


Table 7.--Water-chemistry data from profile well B5, 1981-83--Continued

\begin{tabular}{|c|c|c|c|c|c|c|c|c|}
\hline WELL & DATE & $\begin{array}{c}\text { ELEV } \\
\text { (FEET) }\end{array}$ & $\begin{array}{l}\text { ATION } \\
\text { (METER) }\end{array}$ & $\begin{array}{l}\text { TEMPER- } \\
\text { ATURE } \\
(D E G \quad C)\end{array}$ & $\begin{array}{l}\text { SPECIFIC } \\
\text { CONDUCT- } \\
\text { ANCE } \\
\text { (UMHOS) }\end{array}$ & $\begin{array}{c}\text { ALKA- } \\
\text { LINITY } \\
(M G / L \\
\text { AS } \\
\text { CACO3) }\end{array}$ & $\begin{array}{l}\text { IRON } \\
\text { DIS- } \\
\text { SOLVED } \\
\text { (MG/L) }\end{array}$ & $\begin{array}{c}\text { PH } \\
\text { (UNIT) }\end{array}$ \\
\hline B5 & $09 / 10 / 81$ & 0 & 0.0 & 14.6 & 150 & 64 & .25 & 6.40 \\
\hline B5 & $09 / 10 / 81$ & -10 & -3.0 & 13.5 & 155 & 90 & 1.45 & 6.65 \\
\hline B5 & $09 / 10 / 81$ & -20 & -6.1 & 13.4 & 100 & 60 & 13.25 & 6.50 \\
\hline B5 & $09 / 10 / 81$ & -30 & -9.1 & 13.6 & 140 & 78 & 17.00 & 6.35 \\
\hline B5 & $09 / 10 \% 81$ & -40 & -12.2 & 13.8 & 140 & 84 & 27.25 & 6.42 \\
\hline B5 & $09 / 10 / 81$ & -45 & -13.7 & 13.9 & 155 & 90 & 27.75 & 6.55 \\
\hline B5 & $09 / 10 / 81$ & -50 & -15.2 & 14.1 & 155 & 86 & 32.25 & 6.45 \\
\hline B5 & $09 / 10 / 81$ & -55 & -16.8 & 14.2 & 150 & 80 & 32.50 & 6.52 \\
\hline B5 & $09 / 10 / 81$ & -60 & -18.3 & 14.4 & 150 & 80 & 32.25 & 6.42 \\
\hline B5 & $09 / 10 / 81$ & -65 & -19.8 & 14.6 & 240 & 144 & 15.25 & 6.80 \\
\hline B5 & $09 / 10 / 81$ & -70 & -21.3 & 14.8 & 245 & 138 & 15.75 & 6.90 \\
\hline B5 & $09 / 10 / 81$ & -75 & -22.9 & 14.7 & 240 & 146 & 15.50 & 6.95 \\
\hline B5 & $09 / 10 / 81$ & -80 & -24.4 & 15.0 & 270 & 162 & 3.25 & 7.75 \\
\hline B5 & $09 / 10 / 81$ & -85 & -25.9 & 14.7 & 260 & 150 & 1.24 & 7.81 \\
\hline B5 & $09 / 10 / 81$ & -90 & -27.4 & 14.8 & 210 & 112 & .61 & 8.12 \\
\hline B5 & $09 / 10 / 81$ & -100 & -30.5 & 14.9 & 150 & 66 & 13.25 & 7.80 \\
\hline B5 & $09 / 10 / 81$ & -105 & -32.0 & 14.8 & 140 & 60 & 13.75 & 6.92 \\
\hline B5 & $09 / 10 / 81$ & -115 & -35.1 & 14.9 & 155 & 58 & 16.75 & 6.75 \\
\hline B5 & $09 / 10 / 81$ & -125 & -38.1 & 15.1 & 155 & 58 & 16.25 & 6.78 \\
\hline B5 & $09 / 10 / 81$ & -135 & -41.1 & 15.4 & 175 & 120 & 9.25 & 6.85 \\
\hline B5 & $09 / 10 / 81$ & -140 & -42.7 & 14.9 & 160 & 76 & .72 & 7.55 \\
\hline B5 & $09 / 10 / 81$ & -145 & -44.2 & 15.3 & 220 & 116 & .23 & 7.90 \\
\hline B5 & $09 / 10 / 81$ & -150 & -45.7 & 15.4 & 190 & 94 & .05 & 8.05 \\
\hline B5 & $09 / 10 / 81$ & -155 & -47.2 & 15.4 & 210 & 120 & .04 & 8.21 \\
\hline B5 & $09 / 10 / 81$ & -165 & -50.3 & 15.2 & 230 & 128 & .17 & 8.32 \\
\hline B5 & $09 / 10 / 81$ & -170 & -51.8 & 15.8 & 340 & 198 & .17 & 8.25 \\
\hline
\end{tabular}


Table 7.--Water-chemistry data from profile well B5, 1981-83--Continued

\begin{tabular}{|c|c|c|c|c|c|c|c|c|}
\hline WELL & DATE & $\begin{array}{l}\text { ELEVA } \\
\text { (FEET) }\end{array}$ & $\begin{array}{l}\text { ATION } \\
\text { (METER) }\end{array}$ & $\begin{array}{l}\text { TEMPER- } \\
\text { ATURE } \\
\text { (DEG C) }\end{array}$ & $\begin{array}{l}\text { SPECIFIC } \\
\text { CONDUCT- } \\
\text { ANCE } \\
\text { (UMHOS) }\end{array}$ & $\begin{array}{c}\text { ALKA- } \\
\text { LINITY } \\
\text { (MG/L } \\
\text { AS } \\
\text { CACO3) }\end{array}$ & $\begin{array}{l}\text { IRON } \\
\text { DIS- } \\
\text { SOLVED } \\
\text { (MG/L) }\end{array}$ & $\begin{array}{c}\mathrm{PH} \\
\text { (UNIT) }\end{array}$ \\
\hline B5 & $09 / 11 / 81$ & 0 & 0.0 & 14.7 & 145 & 60 & .31 & 6.50 \\
\hline B5 & $09 / 11 / 81$ & -10 & -3.0 & 13.7 & 145 & 80 & 1.48 & 6.80 \\
\hline B5 & $09 / 11 / 81$ & -20 & -6.1 & 13.6 & 105 & 58 & 13.25 & 6.70 \\
\hline B5 & $09 / 11 / 81$ & -30 & -9.1 & 13.7 & 140 & 80 & 19.50 & 6.10 \\
\hline B5 & $09 / 11 / 81$ & -40 & -12.2 & 13.7 & 140 & 80 & 25.75 & 6.35 \\
\hline B5 & $09 / 11 / 81$ & -45 & -13.7 & 13.7 & 145 & 90 & 28.25 & 6.21 \\
\hline B5 & $09 / 11 / 81$ & -50 & -15.2 & 13.8 & 150 & 84 & 32.00 & 6.11 \\
\hline B5 & $09 / 11 / 81$ & -55 & -16.8 & 13.8 & 140 & 84 & 31.25 & 6.25 \\
\hline B5 & $09 / 11 / 81$ & -60 & -18.3 & 13.9 & 150 & 80 & 33.00 & 6.29 \\
\hline B5 & $09 / 11 / 81$ & -65 & -19.8 & 14.0 & 230 & 144 & 15.75 & 6.55 \\
\hline B5 & $09 / 11 / 81$ & -70 & -21.3 & 14.0 & 240 & 134 & 16.00 & 6.71 \\
\hline B5 & $09 / 11 / 81$ & -75 & -22.9 & 14.0 & 235 & 154 & 15.75 & 6.65 \\
\hline B5 & $09 / 11 / 81$ & -80 & -24.4 & 14.1 & 265 & 162 & 3.00 & 7.23 \\
\hline B5 & $09 / 11 / 81$ & -85 & -25.9 & 14.2 & 260 & 156 & 1.34 & 7.42 \\
\hline B5 & $09 / 11 / 81$ & -90 & -27.4 & 14.1 & 220 & 120 & .64 & 7.72 \\
\hline B5 & $09 / 11 / 81$ & -100 & -30.5 & 13.9 & 140 & 70 & 14.00 & 7.00 \\
\hline $\mathrm{B} 5$ & $09 / 11 / 81$ & -105 & -32.0 & 14.1 & 140 & 60 & 14.00 & 6.82 \\
\hline 85 & $09 / 11 / 81$ & -115 & -35.1 & 14.0 & 150 & 58 & 17.00 & 6.61 \\
\hline B5 & $09 / 11 / 81$ & -125 & -38.1 & 14.1 & 155 & 56 & 18.00 & 6.60 \\
\hline B5 & $09 / 11 / 81$ & -135 & -41.1 & 14.3 & 175 & 68 & 12.00 & 6.80 \\
\hline B5 & $09 / 11 / 81$ & -140 & -42.7 & 14.2 & 160 & 80 & .73 & 7.15 \\
\hline B5 & $09 / 11 / 81$ & -145 & -44.2 & 14.5 & 225 & 116 & .27 & 7.81 \\
\hline B5 & $09 / 11 / 81$ & -150 & -45.7 & 14.5 & 190 & 100 & .16 & 8.02 \\
\hline B5 & $09 / 11 / 81$ & -155 & -47.2 & 14.6 & 210 & 120 & .08 & 8.10 \\
\hline B5 & $09 / 11 / 81$ & -165 & -50.3 & 14.4 & 230 & 130 & .19 & 8.12 \\
\hline B5 & $09 / 11 / 81$ & -170 & -51.8 & 14.4 & 325 & 170 & .22 & 8.01 \\
\hline
\end{tabular}


Table 7.- Water-chem istry data from profile well B5, 1981-83--Continued

\begin{tabular}{|c|c|c|c|c|c|c|c|c|}
\hline WELL & DATE & $\begin{array}{l}\text { ELEVA } \\
\text { (FEET) }\end{array}$ & $\begin{array}{l}\text { ATION } \\
\text { (METER) }\end{array}$ & 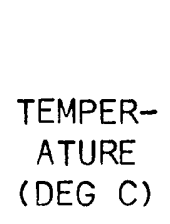 & $\begin{array}{l}\text { SPECIFIC } \\
\text { CONDUCT- } \\
\text { ANCE } \\
\text { (UMHOS) }\end{array}$ & $\begin{array}{c}\text { ALKA- } \\
\text { LINITY } \\
\text { (MG/L } \\
\text { AS } \\
\text { CACO3) }\end{array}$ & $\begin{array}{l}\text { IRON } \\
\text { DIS- } \\
\text { SOLVED } \\
\text { (MG/L) }\end{array}$ & $\begin{array}{c}\mathrm{PH} \\
(\mathrm{UNIT})\end{array}$ \\
\hline B5 & $09 / 14 / 81$ & 0 & 0.0 & 14.7 & 140 & 60 & .43 & 6.35 \\
\hline B5 & $09 / 14 / 81$ & -10 & -3.0 & 13.7 & 150 & 82 & 1.56 & 6.50 \\
\hline B5 & $09 / 14 / 81$ & -20 & -6.1 & 13.4 & 105 & 58 & 13.75 & 6.30 \\
\hline B5 & $09 / 14 / 81$ & -30 & -9.1 & 13.5 & 135 & 84 & 20.50 & 6.30 \\
\hline B5 & $09 / 14 / 81$ & -40 & -12.2 & 13.4 & 135 & 82 & 26.25 & 6.30 \\
\hline B5 & $09 / 14 / 81$ & -45 & -13.7 & 13.4 & 140 & 86 & 27.75 & 6.35 \\
\hline B5 & $09 / 14 / 81$ & -50 & -15.2 & 13.4 & 145 & 84 & 32.00 & 6.30 \\
\hline B5 & $09 / 14 / 81$ & -55 & -16.8 & 13.7 & 145 & 82 & 31.75 & 6.45 \\
\hline B5 & $09 / 14 / 81$ & -60 & -18.3 & 13.6 & 150 & 86 & 34.50 & 6.31 \\
\hline B5 & $09 / 14 / 81$ & -65 & -19.8 & 13.8 & 230 & 140 & 16.25 & 6.59 \\
\hline B5 & $09 / 14 / 81$ & -70 & -21.3 & 13.8 & 230 & 134 & 15.25 & 6.75 \\
\hline B5 & $09 / 14 / 81$ & -75 & -22.9 & 13.7 & 230 & 154 & 15.75 & 6.70 \\
\hline B5 & $09 / 14 / 81$ & -80 & -24.4 & 13.7 & 255 & 160 & 2.75 & 7.30 \\
\hline B5 & $09 / 14 / 81$ & -85 & -25.9 & 13.8 & 240 & 152 & 1.29 & 7.15 \\
\hline B5 & $09 / 14 / 81$ & -90 & -27.4 & 13.8 & 210 & 120 & .53 & 7.55 \\
\hline B5 & $09 / 14 / 81$ & -100 & -30.5 & 13.7 & 135 & 68 & 12.75 & 7.12 \\
\hline B5 & $09 / 14 / 81$ & -105 & -32.0 & 13.8 & 135 & 58 & 14.00 & 6.80 \\
\hline B5 & $09 / 14 / 81$ & -115 & -35.1 & 14.0 & 150 & 58 & 17.00 & 6.72 \\
\hline B5 & $09 / 14 / 81$ & -125 & -38.1 & 14.1 & 150 & 58 & 17.00 & 6.80 \\
\hline B5 & $09 / 14 / 81$ & -135 & -41.1 & 14.6 & 175 & 66 & 9.25 & 6.85 \\
\hline B5 & $09 / 14 / 81$ & -140 & -42.7 & 14.2 & 160 & 80 & .77 & 7.40 \\
\hline B5 & $09 / 14 / 81$ & -145 & $-44 \cdot 2$ & 14.7 & 210 & 116 & .23 & 7.65 \\
\hline B5 & $09 / 14 / 81$ & -150 & -45.7 & 14.8 & 190 & 106 & .13 & 8.05 \\
\hline B5 & $09 / 14 / 81$ & -155 & -47.2 & 15.1 & 200 & 120 & .07 & 8.30 \\
\hline B5 & $09 / 14 / 81$ & -165 & -50.3 & 14.9 & 225 & 134 & .11 & 8.35 \\
\hline B5 & $09 / 14 / 81$ & -170 & -51.8 & 15.3 & 310 & 176 & .08 & 8.12 \\
\hline
\end{tabular}



Table 7.- - Water-chemistry data from profile

\begin{tabular}{|c|c|c|c|c|c|c|c|c|}
\hline WELL & DATE & $\begin{array}{l}\text { ELEVA } \\
\text { (FEET) }\end{array}$ & $\begin{array}{l}\text { ATION } \\
\text { (METER) }\end{array}$ & $\begin{array}{l}\text { TEMPER- } \\
\text { ATURE } \\
\text { (DEG C) }\end{array}$ & $\begin{array}{l}\text { SPECIFIC } \\
\text { CONDUCT- } \\
\text { ANCE } \\
\text { (UMHOS) }\end{array}$ & $\begin{array}{c}\text { ALKA- } \\
\text { LINITY } \\
\text { (MG/L } \\
\text { AS } \\
\text { CACO3) }\end{array}$ & $\begin{array}{l}\text { IRON } \\
\text { DIS- } \\
\text { SOLVED } \\
\text { (MG/L) }\end{array}$ & $\begin{array}{c}\text { PH } \\
(\text { UNIT) }\end{array}$ \\
\hline B5 & $09 / 18 / 81$ & 0 & 0.0 & 14.7 & 130 & 58 & .53 & 6.35 \\
\hline B5 & $09 / 18 / 81$ & -10 & -3.0 & 13.7 & 140 & 88 & 1.68 & 6.55 \\
\hline B5 & $09 / 18 / 81$ & -20 & -6.1 & 13.6 & 100 & 60 & 14.00 & 6.60 \\
\hline B5 & $09 / 18 / 81$ & -30 & -9.1 & 13.6 & 115 & 76 & 17.75 & 6.40 \\
\hline B5 & $09 / 18 / 81$ & -40 & -12.2 & 13.7 & 130 & 80 & 25.00 & 6.19 \\
\hline B5 & $09 / 18 / 81$ & -45 & -13.7 & 13.7 & 155 & 94 & 30.50 & 6.15 \\
\hline B5 & $09 / 18 / 81$ & -50 & -15.2 & 13.9 & 150 & 90 & 33.00 & 6.18 \\
\hline 85 & $09 / 18 / 81$ & -55 & -16.8 & 14.1 & 140 & 78 & 31.25 & 6.40 \\
\hline B5 & $09 / 18 / 81$ & -60 & -18.3 & 14.4 & 140 & 86 & 32.75 & 6.23 \\
\hline B5 & $09 / 18 / 81$ & -65 & -19.8 & 14.5 & 215 & 140 & 16.00 & 6.61 \\
\hline B5 & $09 / 18 / 81$ & -70 & -21.3 & 14.5 & 240 & 134 & 14.50 & 6.69 \\
\hline B5 & $09 / 18 / 81$ & -75 & -22.9 & 14.9 & 220 & 156 & 18.50 & 6.80 \\
\hline B5 & $09 / 18 / 81$ & -80 & -24.4 & 15.3 & 255 & 170 & 1.22 & 7.50 \\
\hline B5 & $09 / 18 / 81$ & -85 & -25.9 & 15.2 & 260 & 160 & .78 & 7.70 \\
\hline B5 & $09 / 18 / 81$ & -90 & -27.4 & 15.2 & 220 & 120 & .58 & 7.75 \\
\hline B5 & $09 / 18 / 81$ & -100 & -30.5 & 14.9 & 150 & 70 & 14.25 & 7.05 \\
\hline B5 & $09 / 18 / 81$ & -105 & -32.0 & 15.1 & 135 & 66 & 14.50 & 6.75 \\
\hline B5 & $09 / 18 / 81$ & -115 & -35.1 & 14.7 & 145 & 60 & 16.75 & 6.62 \\
\hline B5 & $09 / 18 / 81$ & -125 & -38.1 & 14.8 & 150 & 58 & 17.50 & 6.61 \\
\hline B5 & $09 / 18 / 81$ & -135 & -41.1 & 15.0 & 160 & 66 & 10.00 & 6.60 \\
\hline B5 & $09 / 18 / 81$ & -140 & -42.7 & 14.7 & 160 & 78 & .86 & 6.95 \\
\hline B5 & $09 / 18 / 81$ & -145 & -44.2 & 15.0 & 210 & 120 & .25 & 7.30 \\
\hline B5 & $09 / 18 / 81$ & -150 & -45.7 & 15.0 & 190 & 104 & .08 & 7.69 \\
\hline B5 & $09 / 18 / 81$ & -155 & -47.2 & 15.2 & 200 & 116 & .03 & 7.98 \\
\hline B5 & $09 / 18 / 81$ & -165 & -50.3 & 14.9 & 220 & 134 & .15 & 7.92 \\
\hline B5 & $09 / 18 / 81$ & -170 & -51.8 & 15.0 & 310 & 168 & .18 & 7.95 \\
\hline
\end{tabular}


Table 7.--Water-chemistry data from profile well B5, 1981-83--Continued

\begin{tabular}{|c|c|c|c|c|c|c|c|c|}
\hline WELL & DATE & $\begin{array}{l}\text { ELEVA } \\
\text { (FEET) }\end{array}$ & $\begin{array}{l}\text { AT ION } \\
\text { (METER) }\end{array}$ & $\begin{array}{l}\text { TEMPER- } \\
\text { ATURE } \\
\text { (DEG C) }\end{array}$ & $\begin{array}{l}\text { SPECIFIC } \\
\text { CONDUCT- } \\
\text { ANCE } \\
\text { (UMHOS) }\end{array}$ & $\begin{array}{c}\text { ALKA- } \\
\text { LINITY } \\
\text { (MG/L } \\
\text { AS } \\
\text { CACO3) }\end{array}$ & $\begin{array}{c}\text { IRON } \\
\text { DIS- } \\
\text { SOLVED } \\
\text { (MG/L) }\end{array}$ & $\begin{array}{c}\mathrm{PH} \\
(\text { UNIT) }\end{array}$ \\
\hline B5 & $09 / 22 / 81$ & 0 & 0.0 & 14.7 & 135 & 46 & .65 & 6.20 \\
\hline B5 & $09 / 22 / 81$ & -10 & -3.0 & 13.5 & 140 & 78 & 1.67 & 6.35 \\
\hline B5 & $09 / 22 / 81$ & -20 & -6.1 & 13.4 & 100 & 56 & 13.25 & 6.22 \\
\hline B5 & $09 / 22 / 81$ & -30 & -9.1 & 13.6 & 130 & 76 & 19.25 & 6.20 \\
\hline B5 & $09 / 22 / 81$ & -40 & -12.2 & 13.4 & 130 & 80 & 26.25 & 6.31 \\
\hline B5 & $09 / 22 / 81$ & -45 & -13.7 & 13.6 & 135 & 82 & 26.50 & 6.40 \\
\hline B5 & $09 / 22 / 81$ & -50 & -15.2 & 13.6 & 150 & 86 & 32.25 & 6.40 \\
\hline B5 & $09 / 22 / 81$ & -55 & -16.8 & 13.7 & 135 & 80 & 30.50 & 6.49 \\
\hline B5 & $09 / 22 / 81$ & -60 & -18.3 & 13.9 & 140 & 80 & 33.00 & 6.50 \\
\hline B5 & $09 / 22 / 81$ & -65 & -19.8 & 14.1 & 220 & 142 & 15.50 & 6.70 \\
\hline B5 & $09 / 22 / 81$ & -70 & -21.3 & 14.2 & 220 & 134 & 15.75 & 6.90 \\
\hline B5 & $09 / 22 / 81$ & -75 & -22.9 & 14.3 & 220 & 144 & 15.25 & 6.98 \\
\hline B5 & $09 / 22 / 81$ & -80 & -24.4 & 14.4 & 250 & 168 & 2.75 & 7.41 \\
\hline B5 & $09 / 22: 81$ & -85 & -25.9 & 14.4 & 250 & 154 & 1.18 & 7.75 \\
\hline B5 & $09 / 22 / 81$ & -90 & -27.4 & 14.3 & 210 & 126 & .62 & 7.85 \\
\hline B5 & $09 / 22 / 81$ & -100 & -30.5 & 14.4 & 140 & 70 & 13.25 & 7.20 \\
\hline B5 & $09 / 22 / 81$ & -105 & -32.0 & 14.3 & 135 & 60 & 13.50 & 7.00 \\
\hline B5 & $09 / 22 / 81$ & -115 & -35.1 & 14.4 & 150 & 60 & 15.75 & 6.90 \\
\hline B5 & $09 / 22 / 81$ & -125 & -38.1 & 14.4 & 155 & 62 & 16.25 & 6.80 \\
\hline B5 & $09 / 22 / 81$ & -135 & -41.1 & 14.6 & 175 & 70 & 8.50 & 6.95 \\
\hline B5 & $09 / 22 / 81$ & -140 & -42.7 & 14.6 & 165 & 80 & .78 & 7.33 \\
\hline B5 & $09 / 22 / 81$ & -145 & -44.2 & 15.3 & 200 & 118 & .25 & 7.71 \\
\hline B5 & $09 / 22 / 81$ & -150 & -45.7 & 15.2 & 200 & 110 & .08 & 7.91 \\
\hline B5 & $09 / 22 / 81$ & -155 & -47.2 & 15.3 & 200 & 110 & .06 & 8.22 \\
\hline B5 & $09 / 22 / 81$ & -165 & -50.3 & 15.3 & 225 & 132 & .18 & 8.30 \\
\hline B5 & $09 / 22 / 81$ & -170 & -51.8 & 15.3 & 325 & 180 & .24 & 8.30 \\
\hline
\end{tabular}


Table 7.--Water-chem istry data from profile well B5, 1981-83--Continued

\begin{tabular}{|c|c|c|c|c|c|c|c|c|}
\hline WELL & DATE & $\begin{array}{l}\text { ELEV } \\
\text { (FEET) }\end{array}$ & $\begin{array}{l}\text { ITION } \\
\text { (METER) }\end{array}$ & $\begin{array}{l}\text { TEMPER- } \\
\text { ATURE } \\
(\text { DEG C) }\end{array}$ & $\begin{array}{l}\text { SPECIFIC } \\
\text { CONDUCT- } \\
\text { ANCE } \\
\text { (UMHOS) }\end{array}$ & $\begin{array}{c}\text { ALKA- } \\
\text { LINITY } \\
\text { (MG/L } \\
\text { AS } \\
\text { CACO3) }\end{array}$ & $\begin{array}{l}\text { IRON } \\
\text { DIS- } \\
\text { SOLVED } \\
\text { (MG/L) }\end{array}$ & $\begin{array}{c}\mathrm{PH} \\
(\mathrm{UNIT})\end{array}$ \\
\hline B5 & $09 / 25 / 81$ & 0 & 0.0 & 14.3 & 130 & 50 & .75 & 6.15 \\
\hline B5 & $09 / 25 / 81$ & -10 & -3.0 & 13.1 & 135 & 80 & 2.10 & 6.30 \\
\hline B5 & $09 / 25 / 81$ & -20 & -6.1 & 12.9 & 95 & 56 & 14.50 & 6.21 \\
\hline B5 & $09 / 25 / 81$ & -30 & -9.1 & 13.0 & 135 & 78 & 20.75 & 6.18 \\
\hline B5 & $09 / 25 / 81$ & -40 & -12.2 & 13.1 & 130 & 80 & 27.25 & 6.45 \\
\hline B5 & $09 / 25 / 81$ & -45 & -13.7 & 13.1 & 130 & 84 & 26.75 & 6.43 \\
\hline B5 & $09 / 25 / 81$ & -50 & -15.2 & 13.2 & 145 & 88 & 32.00 & 6.45 \\
\hline B5 & $09 / 25 / 81$ & -55 & -16.8 & 13.3 & 140 & 84 & 32.75 & 6.50 \\
\hline B5 & $09 / 25 / 81$ & -60 & -18.3 & 13.4 & 140 & 80 & 33.50 & 6.52 \\
\hline B5 & $09 / 25 / 81$ & -65 & -19.8 & 13.7 & 240 & 144 & 16.25 & 6.80 \\
\hline B5 & $09 / 25 / 81$ & -70 & -21.3 & 13.9 & 230 & 132 & 15.50 & 6.90 \\
\hline B5 & $09 / 25 / 81$ & -75 & -22.9 & 13.8 & 230 & 146 & 15.50 & 6.90 \\
\hline B5 & $09 / 25 / 81$ & -80 & -24.4 & 13.9 & 280 & 188 & 2.25 & 7.33 \\
\hline B5 & $09 / 25 / 81$ & -85 & -25.9 & 13.9 & 260 & 158 & 1.27 & 7.55 \\
\hline B5 & $09 / 25 / 81$ & -90 & -27.4 & 14.1 & 220 & 120 & .61 & 7.75 \\
\hline B5 & $09 / 25 / 81$ & -100 & -30.5 & 14.0 & 140 & 70 & 13.50 & 7.31 \\
\hline B5 & $09 / 25 / 81$ & -105 & -32.0 & 14.2 & 140 & 60 & 13.75 & 7.10 \\
\hline B5 & $09 / 25 / 81$ & -115 & -35.1 & 14.2 & 150 & 60 & 16.25 & 7.05 \\
\hline B5 & $09 / 25 / 81$ & -125 & -38.1 & 14.4 & 155 & 60 & 16.75 & 7.00 \\
\hline B5 & $09 / 25 / 81$ & -135 & -41.1 & 14.8 & 180 & 66 & 8.75 & 7.00 \\
\hline B5 & $09 / 25 / 81$ & -140 & -42.7 & 14.7 & 175 & 84 & .74 & 7.41 \\
\hline B5 & $09 / 25 / 81$ & -145 & -44.2 & 15.2 & 210 & 116 & .20 & 7.72 \\
\hline B5 & $09 / 25 / 81$ & -150 & -45.7 & 15.3 & 200 & 112 & .07 & 8.09 \\
\hline B5 & $09 / 25 / 81$ & -155 & -47.2 & 15.4 & 190 & 114 & .03 & 8.32 \\
\hline B5 & $09 / 25 / 81$ & -165 & -50.3 & 15.2 & 230 & 134 & .15 & 8.49 \\
\hline B5 & $09 / 25 / 81$ & -170 & -51.8 & 15.3 & 335 & 174 & .25 & 8.41 \\
\hline
\end{tabular}


Table 7.--Water-chemistry data from profile well B5, 1981-83--Continued

\begin{tabular}{|c|c|c|c|c|c|c|c|c|}
\hline WELL & DATE & $\begin{array}{l}\text { ELEVA } \\
\text { (FEET) }\end{array}$ & $\begin{array}{l}\text { ATION } \\
\text { (METER) }\end{array}$ & $\begin{array}{l}\text { TEMPER- } \\
\text { ATURE } \\
\left(\begin{array}{ll}\text { DEG } & \text { C) }\end{array}\right.\end{array}$ & $\begin{array}{l}\text { SPECIFIC } \\
\text { CONDUCT- } \\
\text { ANCE } \\
\text { (UMHOS) }\end{array}$ & $\begin{array}{l}\text { ALKA- } \\
\text { LINITY } \\
\text { (MG/L } \\
\text { AS } \\
\text { CACO3) }\end{array}$ & $\begin{array}{l}\text { IRON } \\
\text { DIS- } \\
\text { SOLVED } \\
\text { (MG/L) }\end{array}$ & $\begin{array}{c}\mathrm{PH} \\
(\mathrm{UNIT})\end{array}$ \\
\hline B5 & $09 / 28 / 81$ & 0 & 0.0 & 15.1 & 150 & 50 & .85 & 6.30 \\
\hline B5 & $09 / 28 / 81$ & -10 & -3.0 & 13.9 & 130 & 76 & 2.10 & 6.30 \\
\hline B5 & $09 / 28 / 81$ & -20 & -6.1 & 14.1 & 120 & 58 & 15.25 & 6.00 \\
\hline B5 & $09 / 28 / 81$ & -30 & -9.1 & 13.8 & 130 & 80 & 19.25 & 6.05 \\
\hline 85 & $09 / 28 / 81$ & -40 & -12.2 & 13.9 & 140 & 82 & 25.00 & 6.20 \\
\hline B5 & $09 / 28 / 81$ & -45 & -13.7 & 13.9 & 140 & 80 & 26.50 & 6.35 \\
\hline B5 & $09 / 28 / 81$ & -50 & -15.2 & 14.1 & 150 & 86 & 30.75 & 6.38 \\
\hline B5 & $09 / 28 / 81$ & -55 & -16.8 & 14.3 & 150 & 78 & 31.50 & 6.31 \\
\hline B5 & $09 / 28 / 81$ & -60 & $-18 \cdot 3$ & 14.4 & 145 & 80 & 32.00 & 6.39 \\
\hline B5 & $09 / 28 / 81$ & -65 & -19.8 & 14.5 & 245 & 140 & 16.75 & 6.80 \\
\hline B5 & $09 / 28 / 81$ & -70 & -21.3 & 14.4 & 240 & 132 & 14.25 & 6.99 \\
\hline B5 & $09 / 28 / 81$ & -75 & -22.9 & 14.6 & 230 & 142 & 15.00 & 6.92 \\
\hline B5 & $09 / 28 / 81$ & -80 & -24.4 & 14.9 & 275 & 190 & 1.51 & 7.60 \\
\hline B5 & $09 / 20 / 81$ & -85 & -25.9 & 14.8 & 255 & 154 & 1.21 & 7.65 \\
\hline 85 & $03 / 29 / 81$ & -90 & -27.4 & 14.8 & 210 & 114 & .60 & 7.73 \\
\hline B5 & $09 / 28 / 81$ & -100 & -30.5 & 14.6 & 150 & 72 & 12.75 & 7.12 \\
\hline B5 & $09 / 28 / 81$ & -105 & -32.0 & 14.8 & 130 & 60 & 13.50 & 6.85 \\
\hline B5 & $09 / 28 / 81$ & -115 & -35.1 & 14.8 & 140 & 58 & 15.75 & 6.80 \\
\hline B5 & $09 / 28 / 81$ & -125 & -38.1 & 14.7 & 155 & 60 & 16.25 & 6.80 \\
\hline B5 & $09 / 28 / 81$ & -135 & -41.1 & 15.4 & 175 & 64 & 8.75 & 6.90 \\
\hline B5 & $09 / 28 / 81$ & -140 & -42.7 & 14.9 & 180 & 86 & .69 & 7.33 \\
\hline B5 & $09 / 28 / 81$ & -145 & -44.2 & 15.2 & 220 & 116 & .22 & 7.85 \\
\hline 85 & $09 / 28 / 81$ & -150 & -45.7 & 15.3 & 205 & 108 & .08 & 8.01 \\
\hline B5 & $09 / 28 / 81$ & -155 & -47.2 & 15.4 & 185 & 106 & .02 & 8.40 \\
\hline B5 & $09 / 28 / 81$ & -165 & -50.3 & 15.2 & 225 & 132 & .15 & 8.32 \\
\hline 85 & $09 / 28 / 81$ & -170 & -51.8 & 15.2 & 330 & 170 & .48 & 7.71 \\
\hline
\end{tabular}


Table 7.--Water-chem istry data from profile well B5, 1981-83--Continued

\begin{tabular}{|c|c|c|c|c|c|c|c|c|}
\hline WELL & DATE & $\begin{array}{l}\text { ELEVA } \\
\text { (FEET) }\end{array}$ & $\begin{array}{l}\text { ATION } \\
\text { (METER) }\end{array}$ & $\begin{array}{l}\text { TEMPER- } \\
\text { ATURE } \\
\left(\begin{array}{ll}\text { DEG } & \text { C) }\end{array}\right.\end{array}$ & $\begin{array}{l}\text { SPECIFIC } \\
\text { CONDUCT- } \\
\text { ANCE } \\
\text { (UMHOS) }\end{array}$ & $\begin{array}{c}\text { ALKA- } \\
\text { LINITY } \\
\text { (MG/L } \\
\text { AS } \\
\text { CACO3) }\end{array}$ & $\begin{array}{l}\text { IRON } \\
\text { DIS- } \\
\text { SOLVED } \\
(M G / L)\end{array}$ & $\begin{array}{c}\mathrm{PH} \\
(\mathrm{UN} I T)\end{array}$ \\
\hline B5 & $10 / 02 / 81$ & 0 & 0.0 & 15.1 & 190 & 56 & 1.11 & 6.45 \\
\hline B5 & $10 / 02 / 81$ & -10 & -3.0 & 13.7 & 135 & 74 & 2.25 & 6.75 \\
\hline B5 & $10 / 02 / 81$ & -20 & -6.1 & 13.6 & 125 & 58 & 16.25 & 6.25 \\
\hline B5 & $10 / 02 / 81$ & -30 & -9.1 & 13.4 & 130 & 72 & 20.50 & 6.45 \\
\hline B5 & $10 / 02 / 81$ & -40 & -12.2 & 13.7 & 130 & 80 & 25.50 & 6.50 \\
\hline B5 & $10 / 02 / 81$ & -45 & -13.7 & 13.7 & 130 & 80 & 28.00 & 6.50 \\
\hline B5 & $10 / 02 / 81$ & -50 & -15.2 & 13.8 & 145 & 86 & 32.00 & 6.32 \\
\hline B5 & $10 / 02 / 81$ & -55 & -16.8 & 14.1 & 140 & 82 & 31.50 & 6.35 \\
\hline B5 & $10 / 02 / 81$ & -60 & -18.3 & 14.2 & 140 & 80 & 32.50 & 6.35 \\
\hline B5 & $10 / 02 / 81$ & -65 & -19.8 & 14.3 & 240 & 136 & 16.25 & 6.75 \\
\hline B5 & $10 / 02 / 81$ & -70 & -21.3 & 15.1 & 230 & 132 & 14.50 & 6.80 \\
\hline B5 & $10 / 02 / 81$ & -75 & -22.9 & 14.6 & 220 & 140 & 15.00 & 6.82 \\
\hline B5 & $10 / 05 / 81$ & 0 & 0.0 & 15.1 & 185 & 56 & 1.04 & 6.38 \\
\hline B5 & $10 / 05 / 81$ & -10 & -3.0 & 13.7 & 135 & 76 & 2.30 & 6.60 \\
\hline B5 & $10 / 05 / 81$ & -20 & -6.1 & 13.8 & 135 & 54 & 16.75 & 6.02 \\
\hline B5 & $10 / 05 / 81$ & -30 & -9.1 & 13.8 & 140 & 76 & 20.75 & 6.11 \\
\hline B5 & $10 / 05 / 81$ & -40 & -12.2 & 13.8 & 150 & 76 & 26.00 & 6.21 \\
\hline B5 & $10 / 05 / 81$ & -45 & -13.7 & 14.1 & 140 & 80 & 27.50 & 6.29 \\
\hline B5 & $10 / 05 / 81$ & -50 & -15.2 & 14.1 & 160 & 88 & 31.25 & 6.21 \\
\hline B5 & $10 / 05 / 81$ & -55 & -16.8 & 14.4 & 150 & 78 & 31.50 & 6.25 \\
\hline B5 & $10 / 05 / 81$ & -60 & -18.3 & 14.4 & 155 & 80 & 31.75 & 6.28 \\
\hline B5 & $10 / 05 / 81$ & -65 & -19.8 & 14.5 & 260 & 140 & 16.25 & 6.71 \\
\hline B5 & $10 / 05 / 81$ & -70 & -21.3 & 14.6 & 250 & 130 & 14.50 & 6.83 \\
\hline B5 & $10 / 05 / 81$ & -75 & -22.9 & 14.5 & 240 & 138 & 14.50 & 6.79 \\
\hline B5 & $10 / 05 / 81$ & -80 & -24.4 & 14.8 & 300 & 190 & 1.48 & 7.40 \\
\hline B5 & $10 / 05 / 81$ & -85 & -25.9 & 14.6 & 280 & 158 & 1.25 & 7.48 \\
\hline
\end{tabular}


Table 7.--Water-chemistry data from profile well B5, 1981-83--Continued

\begin{tabular}{|c|c|c|c|c|c|c|c|c|}
\hline WELL & DATE & $\begin{array}{l}\text { ELEVA } \\
\text { (FEET) }\end{array}$ & $\begin{array}{l}\text { ATI ON } \\
\text { (METER) }\end{array}$ & $\begin{array}{l}\text { TEMPER- } \\
\text { ATURE } \\
\text { (DEG C) }\end{array}$ & $\begin{array}{l}\text { SPECIFIC } \\
\text { CONDUCT- } \\
\text { ANCE } \\
\text { (UMHOS) }\end{array}$ & $\begin{array}{c}\text { ALKA- } \\
\text { LINITY } \\
\text { (MG/L } \\
\text { AS } \\
\text { CACO3) }\end{array}$ & $\begin{array}{l}\text { IRON } \\
\text { DIS- } \\
\text { SOLVED } \\
\text { (MG/L) }\end{array}$ & $\begin{array}{c}\mathrm{PH} \\
(U N \mid T)\end{array}$ \\
\hline B5 & $10 / 05 / 81$ & -90 & -27.4 & 14.6 & 235 & 114 & .62 & 7.59 \\
\hline B5 & $10 / 05 / 81$ & -100 & -30.5 & 14.5 & 145 & 70 & 12.75 & 6.85 \\
\hline B5 & $10 / 05 / 81$ & -105 & -32.0 & 14.7 & 150 & 60 & 14.00 & 6.71 \\
\hline B5 & $10 / 05 / 81$ & -115 & -35.1 & 14.6 & 145 & 56 & 15.50 & 6.68 \\
\hline 85 & $10 / 05 / 81$ & -125 & -38.1 & 14.8 & 160 & 52 & 16.50 & 6.65 \\
\hline B5 & $10 / 05 / 81$ & -135 & -41.1 & 15.0 & 190 & 66 & 9.25 & 6.81 \\
\hline B5 & $10 / 05 / 81$ & -140 & -42.7 & 14.8 & 195 & 88 & .80 & 7.39 \\
\hline B5 & $10 / 05 / 81$ & -145 & -44.2 & 15.1 & 220 & 114 & .27 & 7.72 \\
\hline B5 & $10 / 05 / 81$ & -150 & -45.7 & 15.1 & 220 & 114 & .07 & 7.81 \\
\hline B5 & $10 / 05 / 81$ & -155 & -47.2 & 15.1 & 185 & 98 & .03 & 8.21 \\
\hline B5 & $10 / 05 / 81$ & -165 & -50.3 & 14.9 & 235 & 130 & .13 & 8.22 \\
\hline B5 & $10 / 05 / 81$ & -170 & -51.8 & 15.1 & 365 & 180 & .27 & 8.13 \\
\hline B5 & $10 / 09 / 81$ & 0 & 0.0 & 15.2 & 220 & 54 & .98 & 6.35 \\
\hline B5 & $10 / 09 / 81$ & -10 & -3.0 & 13.9 & 140 & 74 & 2.30 & 6.60 \\
\hline B5 & $10 / 09 / 81$ & -20 & -6.1 & 13.8 & 145 & 54 & 17.00 & 6.12 \\
\hline B5 & $10 / 09 / 81$ & -30 & -9.1 & 13.9 & 145 & 76 & 20.00 & 6.19 \\
\hline B5 & $10 / 09 / 81$ & -40 & -12.2 & 14.0 & 155 & 76 & 25.75 & 6.29 \\
\hline B5 & $10 / 09 / 81$ & -45 & -13.7 & 14.1 & 145 & 80 & 28.25 & 6.32 \\
\hline B5 & $10 / 09 / 81$ & -50 & -15.2 & 14.1 & 160 & 90 & 31.25 & 6.39 \\
\hline B5 & $10 / 09 / 81$ & -55 & -16.8 & 14.3 & 150 & 78 & 31.50 & 6.40 \\
\hline B5 & $10 / 09 / 81$ & -60 & -18.3 & 14.3 & 170 & 78 & 34.25 & 6.41 \\
\hline B5 & $10 / 09 / 81$ & -65 & -19.8 & 14.4 & 275 & 132 & 16.75 & 6.81 \\
\hline B5 & $10 / 09 / 81$ & -70 & $-21 \cdot 3$ & 14.4 & 250 & 130 & 15.00 & 6.93 \\
\hline B5 & $10 / 09 / 81$ & -75 & -22.9 & 14.4 & 230 & 134 & 13.75 & 6.90 \\
\hline B5 & $10 / 09 / 81$ & -80 & -24.4 & 14.7 & 315 & 200 & 1.53 & 7.50 \\
\hline B5 & $10 / 09 / 81$ & -85 & -25.9 & 14.7 & 280 & 150 & 1.20 & 7.81 \\
\hline
\end{tabular}


Table 7.--Water-chemistry data from profile well $\mathrm{B} 5,1981-83$--Continued

\begin{tabular}{|c|c|c|c|c|c|c|c|c|}
\hline WELL & DATE & $\begin{array}{l}\text { ELEVA } \\
\text { (FEET) }\end{array}$ & $\begin{array}{l}\text { ATION } \\
\text { (METER) }\end{array}$ & $\begin{array}{l}\text { TEMPER- } \\
\text { ATURE } \\
\left(\begin{array}{lll}\text { DEG } & \text { C) }\end{array}\right.\end{array}$ & $\begin{array}{l}\text { SPECIFIC } \\
\text { CONDUCT- } \\
\text { ANCE } \\
\text { (UMHOS) }\end{array}$ & $\begin{array}{c}\text { ALKA- } \\
\text { LINITY } \\
\text { (MG/L } \\
\text { AS } \\
\text { CACO3) }\end{array}$ & $\begin{array}{l}\text { IRON } \\
\text { DIS- } \\
\text { SOLVED } \\
\text { (MG/L) }\end{array}$ & $\begin{array}{c}\text { PH } \\
(\text { UNIT) }\end{array}$ \\
\hline B5 & $10 / 09 / 81$ & -90 & -27.4 & 14.4 & 230 & 116 & .63 & 7.82 \\
\hline B5 & $10 / 09 / 81$ & -100 & -30.5 & 14.3 & 155 & 70 & 14.00 & 7.20 \\
\hline B5 & $10 / 09 / 81$ & -105 & -32.0 & 14.2 & 150 & 60 & 15.75 & 7.00 \\
\hline B5 & 10/09/81 & -115 & -35.1 & 14.3 & 150 & 56 & 15.75 & 6.92 \\
\hline B5 & $10 / 09 / 81$ & -125 & -38.1 & 14.4 & 160 & 60 & 16.50 & 6.89 \\
\hline B5 & $10 / 09 / 81$ & -135 & -41.1 & 14.6 & 195 & 66 & 9.50 & 6.60 \\
\hline B5 & $10 / 09 / 81$ & -140 & -42.7 & 14.4 & 200 & 90 & .88 & 7.49 \\
\hline B5 & $10 / 09 / 81$ & -145 & -44.2 & 14.7 & 220 & 120 & .23 & 7.90 \\
\hline B5 & $10 / 09 / 81$ & -150 & -45.7 & 14.6 & 230 & 120 & .12 & 8.02 \\
\hline B5 & $10 / 09 / 81$ & -155 & -47.2 & 14.6 & 190 & 98 & .06 & 8.31 \\
\hline B5 & $10 / 09 / 81$ & -165 & -50.3 & 14.6 & 230 & 136 & .17 & 8.35 \\
\hline B5 & $10 / 09 / 81$ & -170 & -51.8 & 14.8 & 360 & 188 & .35 & 8.40 \\
\hline B5 & $10 / 12 / 81$ & 0 & 0.0 & 14.3 & 250 & 64 & 1.06 & 6.40 \\
\hline $\begin{array}{l}\text { B5 } \\
\text { B5 }\end{array}$ & $\begin{array}{l}10 / 12 / 81 \\
10 / 12 / 81\end{array}$ & $\begin{array}{l}-10 \\
-20\end{array}$ & $\begin{array}{l}-3.0 \\
-6.1\end{array}$ & $\begin{array}{l}12.9 \\
12.9\end{array}$ & $\begin{array}{l}130 \\
135\end{array}$ & $\begin{array}{l}72 \\
50\end{array}$ & $\begin{array}{r}2.45 \\
16.25\end{array}$ & $\begin{array}{l}6.55 \\
6.12\end{array}$ \\
\hline B5 & $10 / 12 / 81$ & -30 & -9.1 & 12.9 & 140 & 68 & 21.00 & 6.14 \\
\hline B5 & $10 / 12 / 81$ & -40 & -12.2 & 12.9 & 155 & 72 & 27.75 & 6.22 \\
\hline B5 & $10 / 12 / 81$ & -45 & -13.7 & 12.9 & 150 & 82 & 28.25 & 6.30 \\
\hline B5 & $10 / 12 / 81$ & -50 & -15.2 & 13.2 & 160 & 88 & 31.25 & 6.31 \\
\hline B5 & $10 / 12 / 81$ & -55 & -16.8 & 13.4 & 160 & 80 & 31.00 & 6.32 \\
\hline B5 & $10 / 12 / 81$ & -60 & -18.3 & 13.7 & 180 & 72 & 37.00 & 6.31 \\
\hline B5 & $10 / 12 / 81$ & -65 & -19.8 & 13.7 & 255 & 130 & 17.00 & 6.90 \\
\hline B5 & $10 / 12 / 81$ & -70 & -21.3 & 14.0 & 260 & 132 & 14.50 & 6.99 \\
\hline B5 & $10 / 12 / 81$ & -75 & -22.9 & 13.9 & 230 & 132 & 13.25 & 7.00 \\
\hline B5 & $10 / 12 / 81$ & -80 & -24.4 & 14.1 & 320 & 200 & 1.58 & 7.55 \\
\hline B5 & $10 / 12 / 81$ & -85 & -25.9 & 14.1 & 270 & 152 & 1.23 & 7.60 \\
\hline B5 & $10 / 12 / 81$ & -90 & -27.4 & 14.1 & 235 & 116 & .59 & 7.70 \\
\hline
\end{tabular}


Table 7.--Water-chemistry data from profile well B5, 1981-83--Continued

\begin{tabular}{|c|c|c|c|c|c|c|c|c|}
\hline WELL & DATE & $\begin{array}{l}\text { ELEVA } \\
\text { (FEET) }\end{array}$ & $\begin{array}{l}\text { ATION } \\
\text { (METER) }\end{array}$ & $\begin{array}{l}\text { TEMPER- } \\
\text { ATURE } \\
(\text { DEG C) }\end{array}$ & $\begin{array}{l}\text { SPECIFIC } \\
\text { CONDUCT- } \\
\text { ANCE } \\
\text { (UMHOS) }\end{array}$ & $\begin{array}{l}\text { ALKA- } \\
\text { LINITY } \\
\text { (MG/L } \\
\text { AS } \\
\text { CACO3) }\end{array}$ & $\begin{array}{l}\text { IRON } \\
\text { DIS- } \\
\text { SOLVED } \\
\text { (MG/L) }\end{array}$ & $\begin{array}{c}\mathrm{PH} \\
(\mathrm{UNIT})\end{array}$ \\
\hline B5 & $10 / 12 / 81$ & -100 & -30.5 & 14.0 & 160 & 68 & 13.25 & 7.03 \\
\hline B5 & $10 / 12 / 81$ & -105 & -32.0 & 14.2 & 155 & 58 & 14.00 & 6.92 \\
\hline B5 & $10 / 12 / 81$ & -115 & -35.1 & 14.2 & 160 & 56 & 15.75 & 6.88 \\
\hline B5 & $10 / 12 / 81$ & -125 & -38.1 & 14.2 & 170 & 58 & 17.00 & 6.81 \\
\hline B5 & $10 / 12 / 81$ & -135 & -41.1 & 14.6 & 200 & 64 & 9.50 & 6.99 \\
\hline B5 & $10 / 12 / 81$ & -140 & -42.7 & 14.4 & 215 & 92 & .91 & 7.41 \\
\hline B5 & $10 / 12 / 81$ & -145 & -44.2 & 14.8 & 220 & 110 & .17 & 7.80 \\
\hline B5 & $10 / 12 / 81$ & -150 & -45.7 & 14.8 & 235 & 120 & .07 & 7.95 \\
\hline B5 & $10 / 12 / 81$ & -155 & -47.2 & 14.6 & 190 & 102 & .02 & 8.22 \\
\hline B5 & $10 / 12 / 81$ & -165 & -50.3 & 14.3 & 250 & 132 & .15 & 8.30 \\
\hline B5 & $10 / 12 / 81$ & -170 & -51.8 & 14.8 & 365 & 176 & .32 & 8.26 \\
\hline B5 & $10 / 16 / 81$ & 0 & 0.0 & 13.9 & 195 & 68 & .96 & 6.39 \\
\hline B5 & $10 / 16 / 81$ & -10 & -3.0 & 12.7 & 160 & 70 & 2.30 & 6.60 \\
\hline B5 & $10 / 16 / 81$ & -20 & -6.1 & 12.6 & 150 & 50 & 18.25 & 6.10 \\
\hline B5 & $10 / 16 / 81$ & -30 & -9.1 & 12.5 & 160 & 70 & 21.00 & 6.20 \\
\hline B5 & $10 / 16 / 81$ & -40 & -12.2 & 12.6 & 170 & 80 & 24.50 & 6.32 \\
\hline B5 & $10 / 16 / 81$ & -45 & -13.7 & 12.6 & 165 & 84 & 29.50 & 6.37 \\
\hline B5 & $10 / 16 / 81$ & -50 & -15.2 & 12.9 & 175 & 86 & 31.75 & 6.37 \\
\hline B5 & $10 / 16 / 81$ & -55 & -16.8 & 13.0 & 170 & 80 & 31.25 & 6.39 \\
\hline B5 & $10 / 16 / 81$ & -60 & -18.3 & 13.1 & 200 & 74 & 38.25 & 6.32 \\
\hline B5 & $10 / 16 / 81$ & -65 & -19.8 & 13.2 & 280 & 130 & 17.00 & 6.88 \\
\hline B5 & $10 / 16 / 81$ & -70 & -21.3 & 13.6 & 270 & 130 & 16.25 & 6.97 \\
\hline 85 & $10 / 16 / 81$ & -75 & -22.9 & 13.6 & 225 & 120 & 13.25 & 6.99 \\
\hline B5 & $10 / 16 / 81$ & -80 & -24.4 & 14.0 & 325 & 200 & 1.59 & 7.45 \\
\hline $\mathrm{B} 5$ & $10 / 16 / 81$ & -85 & -25.9 & 13.9 & 300 & 150 & 1.25 & 7.58 \\
\hline B5 & $10 / 16 / 81$ & -90 & -27.4 & 14.0 & 255 & 116 & .61 & 7.69 \\
\hline
\end{tabular}


Table 7.-- Water-chemistry data from profile
well B5, 1981-83--Continued

\begin{tabular}{|c|c|c|c|c|c|c|c|c|}
\hline WELL & DATE & $\begin{array}{l}\text { ELEVA } \\
\text { (FEET) }\end{array}$ & $\begin{array}{l}\text { ATION } \\
\text { (METER) }\end{array}$ & $\begin{array}{l}\text { TEMPER- } \\
\text { ATURE } \\
\text { (DEG C) }\end{array}$ & $\begin{array}{l}\text { SPECIFIC } \\
\text { CONDUCT- } \\
\text { ANCE } \\
\text { (UMHOS) }\end{array}$ & $\begin{array}{c}\text { ALKA- } \\
\text { LINITY } \\
\text { (MG/L } \\
\text { AS } \\
\text { CACO3) }\end{array}$ & $\begin{array}{l}\text { IRON } \\
\text { DIS- } \\
\text { SOLVED } \\
\text { (MG/L) }\end{array}$ & $\begin{array}{c}\text { PH } \\
(\text { UNIT) }\end{array}$ \\
\hline B5 & $10 / 16 / 81$ & -100 & -30.5 & 14.0 & 180 & 74 & 13.00 & 7.02 \\
\hline B5 & $10 / 16 / 81$ & -105 & -32.0 & 14.1 & 180 & 64 & 14.25 & 6.89 \\
\hline B5 & $10 / 16 / 81$ & -115 & -35.1 & 14.0 & 180 & 60 & 16.25 & 6.81 \\
\hline B5 & $10 / 16 / 81$ & -125 & -38.1 & 14.1 & 195 & 60 & 17.00 & 6.82 \\
\hline B5 & $10 / 16 / 81$ & -135 & -41.1 & 14.6 & 225 & 70 & 9.50 & 6.93 \\
\hline B5 & $10 / 16 / 81$ & -140 & -42.7 & 14.5 & 250 & 94 & .85 & 7.39 \\
\hline B5 & $10 / 16 / 81$ & -145 & -44.2 & 15.0 & 260 & 110 & .15 & 7.86 \\
\hline B5 & $10 / 16 / 81$ & -150 & -45.7 & 14.8 & 270 & 130 & .08 & 7.93 \\
\hline B5 & $10 / 16 / 81$ & -155 & -47.2 & 15.1 & 220 & 102 & .03 & 8.28 \\
\hline B5 & $10 / 16 / 81$ & -165 & -50.3 & 14.7 & 275 & 134 & .14 & 8.32 \\
\hline B5 & $10 / 16 / 81$ & -170 & -51.8 & 15.0 & 380 & 180 & .35 & 8.32 \\
\hline B5 & $10 / 27 / 81$ & 0 & 0.0 & 14.7 & 160 & 74 & .32 & 6.35 \\
\hline B5 & $10 / 27 / 81$ & -10 & -3.0 & 14.6 & 115 & 58 & 6.00 & 6.32 \\
\hline B5 & $10 / 27 / 81$ & -20 & -6.1 & 14.4 & 155 & 50 & 19.25 & 5.89 \\
\hline B5 & $10 / 27 / 81$ & -30 & -9.1 & 14.6 & 155 & 70 & 28.00 & 6.10 \\
\hline B5 & $10 / 27 / 81$ & -40 & -12.2 & 14.5 & 185 & 70 & 32.50 & 6.15 \\
\hline B5 & $10 / 27 / 81$ & -45 & -13.7 & 14.3 & 130 & 74 & 24.50 & 6.30 \\
\hline B5 & $10 / 27 / 81$ & -50 & -15.2 & 14.2 & 130 & 80 & 26.50 & 6.32 \\
\hline B5 & $10 / 27 / 81$ & -55 & -16.8 & 14.2 & 155 & 90 & 33.00 & 6.30 \\
\hline B5 & $10 / 27 / 81$ & -60 & -18.3 & 14.3 & 150 & 80 & 33.00 & 6.30 \\
\hline B5 & $10 / 27 / 81$ & -65 & -19.8 & 14.6 & 210 & 100 & 18.00 & 6.49 \\
\hline B5 & $10 / 27 / 81$ & -70 & -21.3 & 14.4 & 230 & 130 & 22.00 & 6.67 \\
\hline B5 & $10 / 27 / 81$ & -75 & -22.9 & 14.3 & 260 & 138 & 13.00 & 6.99 \\
\hline B5 & $10 / 27 / 81$ & -80 & -24.4 & 14.4 & 250 & 128 & 3.50 & 7.22 \\
\hline B5 & $10 / 27 / 81$ & -85 & -25.9 & 14.5 & 235 & 144 & 1.15 & 7.50 \\
\hline B5 & $10 / 27 / 81$ & -90 & -27.4 & 14.1 & 280 & 166 & 1.15 & 7.50 \\
\hline B5 & $10 / 27 / 81$ & -100 & -30.5 & 14.1 & 255 & 144 & 16.00 & 7.15 \\
\hline
\end{tabular}


Table 7.--Water-chemistry data from profile well B5, 1981-83--Continued

\begin{tabular}{|c|c|c|c|c|c|c|c|c|}
\hline WELL & DATE & $\begin{array}{l}\text { ELEVA } \\
\text { (FEET) }\end{array}$ & $\begin{array}{l}\text { TION } \\
\text { (METER) }\end{array}$ & $\begin{array}{c}\text { TEMPER- } \\
\text { ATURE } \\
(\text { DEG C) }\end{array}$ & $\begin{array}{l}\text { SPECIFIC } \\
\text { CONDUCT- } \\
\text { ANCE } \\
\text { (UMHOS) }\end{array}$ & $\begin{array}{c}\text { ALKA- } \\
\text { LINITY } \\
\text { (MG/L } \\
\text { AS } \\
\text { CACO3) }\end{array}$ & $\begin{array}{l}\text { IRON } \\
\text { DIS- } \\
\text { SOLVED } \\
\text { (MG/L) }\end{array}$ & $\begin{array}{c}\text { PH } \\
(\text { UNIT) }\end{array}$ \\
\hline B5 & $10 / 27 / 81$ & -105 & -32.0 & 13.1 & 220 & 122 & 24.25 & 6.62 \\
\hline B5 & $10 / 27 / 81$ & -115 & -35.1 & 13.0 & 200 & 106 & 23.50 & 6.71 \\
\hline B5 & $10 / 27 / 81$ & -125 & -38.1 & 13.1 & 180 & 90 & 21.25 & 6.72 \\
\hline 85 & $10 / 27 / 81$ & -135 & -41.1 & 13.2 & 150 & 76 & 11.75 & 6.88 \\
\hline 85 & $10 / 27 / 81$ & -140 & -42.7 & 13.1 & 155 & 80 & 1.55 & 7.25 \\
\hline B5 & $10 / 27 / 81$ & -145 & -44.2 & 13.7 & 210 & 100 & .17 & 7.56 \\
\hline$B 5$ & $10 / 27 / 81$ & -150 & -45.7 & 13.7 & 200 & 106 & .12 & 7.79 \\
\hline B5 & $10 / 27 / 81$ & -155 & -47.2 & 13.9 & 195 & 110 & .04 & 8.05 \\
\hline 85 & $10 / 27 / 81$ & -165 & -50.3 & 13.6 & 220 & 132 & .15 & 8.21 \\
\hline B5 & $10 / 27 / 81$ & -170 & -51.8 & 13.7 & 330 & 180 & .51 & 8.14 \\
\hline B5 & $11 / 04 / 81$ & -10 & -3.0 & 14.1 & 200 & 76 & 6.75 & 6.42 \\
\hline B5 & $11 / 06 / 81$ & 0 & 0.0 & 12.9 & 200 & 86 & .45 & 6.61 \\
\hline 85 & $11 / 06 / 81$ & -10 & -3.0 & 14.2 & 170 & 74 & 13.75 & 6.41 \\
\hline B5 & $11 / 06 / 81$ & -20 & -6.1 & 13.7 & 130 & 48 & 16.25 & 6.10 \\
\hline 85 & $11 / 06 / 81$ & -30 & -9.1 & 13.8 & 190 & 70 & 29.50 & 6.12 \\
\hline 85 & $11 / 06 / 81$ & -40 & -12.2 & 13.7 & 185 & 74 & 31.75 & 6.41 \\
\hline 85 & $11 / 06 / 81$ & -45 & -13.7 & 13.8 & 175 & 80 & 32.00 & 6.36 \\
\hline$B 5$ & $11 / 06 / 81$ & -50 & -15.2 & 13.8 & 190 & 78 & 36.75 & 6.30 \\
\hline B5 & $11 / 06 / 81$ & -55 & -16.8 & 14.0 & 180 & 78 & 33.50 & 6.31 \\
\hline B5 & $11 / 06 / 81$ & -60 & -18.3 & 14.2 & 160 & 80 & 30.00 & 6.31 \\
\hline 85 & $11 / 06 / 81$ & -65 & -19.8 & 14.4 & 200 & 94 & 19.00 & 6.52 \\
\hline B5 & $11 / 06 / 81$ & -70 & -21.3 & 14.7 & 245 & 122 & 20.75 & 6.62 \\
\hline B5 & $11 / 06 / 81$ & -75 & -22.9 & 14.6 & 290 & 132 & 11.25 & 6.99 \\
\hline B5 & $11 / 06 / 81$ & -80 & -24.4 & 14.8 & 270 & 144 & 2.75 & 7.12 \\
\hline B5 & $11 / 06 / 81$ & -85 & -25.9 & 14.9 & 280 & 166 & 1.27 & 7.40 \\
\hline B5 & $11 / 06 / 81$ & -90 & -27.4 & 14.8 & 265 & 154 & 1.17 & 7.48 \\
\hline
\end{tabular}


Table 7.--Water-chem istry data from profile well B5, 1981-83--Continued

\begin{tabular}{|c|c|c|c|c|c|c|c|c|}
\hline WELL & DATE & $\begin{array}{l}\text { ELEVA } \\
\text { (FEET) }\end{array}$ & $\begin{array}{l}\text { ITION } \\
\text { (METER) }\end{array}$ & $\begin{array}{l}\text { TEMPER- } \\
\text { ATURE } \\
\text { (DEG C) }\end{array}$ & $\begin{array}{l}\text { SPECIFIC } \\
\text { CONDUCT- } \\
\text { ANCE } \\
\text { (UMHOS) }\end{array}$ & $\begin{array}{l}\text { ALKA- } \\
\text { LINITY } \\
\text { (MG/L } \\
\text { AS } \\
\text { CACO3) }\end{array}$ & $\begin{array}{l}\text { IRON } \\
\text { DIS- } \\
\text { SOLVED } \\
\text { (MG/L) }\end{array}$ & $\begin{array}{c}\mathrm{PH} \\
(\mathrm{UN} \mid \mathrm{T})\end{array}$ \\
\hline B5 & $11 / 06 / 81$ & -100 & -30.5 & 14.6 & 280 & 158 & 9.25 & 7.23 \\
\hline B5 & $11 / 05 / 81$ & -105 & -32.0 & 14.6 & 265 & 144 & 21.25 & 6.89 \\
\hline B5 & $11 / 06 / 81$ & -115 & -35.1 & 14.7 & 250 & 126 & 26.25 & 6.71 \\
\hline B5 & $11 / 06 / 81$ & -125 & -38.1 & 14.8 & 210 & 100 & 23.25 & 6.72 \\
\hline B5 & $11 / 06 / 81$ & -135 & $-41 \cdot 1$ & 15.3 & 205 & 96 & 15.25 & 6.80 \\
\hline B5 & $11 / 06 / 81$ & -140 & -42.7 & 14.9 & 175 & 78 & 1.41 & 7.18 \\
\hline B5 & $11 / 06 / 81$ & -145 & -44.2 & 15.2 & 200 & 82 & .20 & 7.50 \\
\hline B5 & $11 / 06 / 81$ & -150 & -45.7 & 15.2 & 195 & 90 & .12 & 7.71 \\
\hline B5 & $11 / 06 / 81$ & -155 & -47.2 & 15.0 & 235 & 126 & .05 & 8.05 \\
\hline B5 & $11 / 06 / 81$ & -165 & -50.3 & 14.8 & 235 & 132 & .14 & 8.20 \\
\hline B5 & $11 / 06 / 81$ & -170 & -51.8 & 14.9 & 350 & 174 & .50 & 8.05 \\
\hline B5 & $11 / 09 / 81$ & 0 & 0.0 & 12.7 & 210 & 104 & .46 & 6.72 \\
\hline B5 & $11 / 09 / 81$ & -10 & -3.0 & 13.7 & 250 & 64 & 7.50 & 6.42 \\
\hline B5 & $11 / 09 / 81$ & -20 & -6.1 & 13.7 & 135 & 42 & 16.25 & 6.11 \\
\hline B5 & $11 / 09 / 81$ & -30 & -9.1 & 13.2 & 185 & 70 & 28.00 & 6.15 \\
\hline B5 & $11 / 09 / 81$ & -40 & -12.2 & 13.3 & 190 & 76 & 33.75 & 6.22 \\
\hline B5 & $11 / 09 / 81$ & -45 & -13.7 & 13.4 & 195 & 80 & 35.25 & 6.30 \\
\hline B5 & $11 / 09 / 81$ & -50 & -15.2 & 13.6 & 185 & 80 & 36.50 & 6.31 \\
\hline B5 & $11 / 09 / 81$ & -55 & -16.8 & 13.8 & 175 & 80 & 34.00 & 6.32 \\
\hline B5 & $11 / 09 / 81$ & -60 & -18.3 & 13.8 & 160 & 80 & 34.75 & 6.32 \\
\hline B5 & $11 / 09 / 81$ & -65 & -19.8 & 13.9 & 190 & 88 & 19.50 & 6.52 \\
\hline B5 & $11 / 09 / 81$ & -70 & -21.3 & 13.9 & 250 & 118 & 21.25 & 6.62 \\
\hline B5 & $11 / 09 / 81$ & -75 & -22.9 & 14.0 & 285 & 138 & 10.75 & 6.93 \\
\hline B5 & $11 / 09 / 81$ & -30 & -24.4 & 13.9 & 260 & 132 & 2.90 & 7.10 \\
\hline B5 & $11 / 09 / 81$ & -85 & -25.9 & 14.1 & 255 & 144 & 1.14 & 7.40 \\
\hline B5 & $11 / 09 / 81$ & -90 & -27.4 & 13.9 & 290 & 172 & 1.32 & 7.49 \\
\hline B5 & $11 / 09 / 81$ & -100 & -30.5 & 13.8 & 300 & 180 & 9.50 & 7.30 \\
\hline
\end{tabular}


Table 7.--Water-chemistry data from profile well B5, 1981-83--Continued

\begin{tabular}{|c|c|c|c|c|c|c|c|c|}
\hline WELL & DATE & $\begin{array}{l}\text { ELEVA } \\
\text { (FEET) }\end{array}$ & $\begin{array}{l}\text { ATION } \\
\text { (METER) }\end{array}$ & $\begin{array}{c}\text { TEMPER- } \\
\text { ATURE } \\
\text { (DEG C) }\end{array}$ & $\begin{array}{l}\text { SPECIFIC } \\
\text { CONDUCT- } \\
\text { ANCE } \\
\text { (UMHOS) }\end{array}$ & $\begin{array}{c}\text { ALKA- } \\
\text { LINITY } \\
\text { (MG/L } \\
\text { AS } \\
\text { CACO3) }\end{array}$ & $\begin{array}{l}\text { IRON } \\
\text { DIS- } \\
\text { SOLVED } \\
\text { (MG/L) }\end{array}$ & $\begin{array}{c}\text { PH } \\
\text { (UNIT) }\end{array}$ \\
\hline B5 & $11 / 09 / 81$ & -105 & -32.0 & 13.8 & 260 & 150 & 20.75 & 7.00 \\
\hline B5 & $11 / 09 / 81$ & -115 & -35.1 & 13.8 & 250 & 126 & 28.25 & 6.80 \\
\hline B5 & $11 / 09 / 81$ & -125 & -38.1 & 13.7 & 230 & 114 & 27.00 & 6.77 \\
\hline B5 & $11 / 09 / 81$ & -135 & -41.1 & 14.1 & 215 & 98 & 16.25 & 6.83 \\
\hline B5 & $11 / 09 / 81$ & -140 & -42.7 & 13.8 & 170 & 80 & 1.51 & 7.18 \\
\hline B5 & $11 / 09 / 81$ & -145 & -44.2 & 13.9 & 195 & 80 & .21 & 7.58 \\
\hline B5 & $11 / 09 / 81$ & -150 & -45.7 & 13.8 & 190 & 90 & .08 & 7.72 \\
\hline B5 & $11 / 09 / 81$ & -155 & -47.2 & 14.0 & 230 & 130 & .04 & 8.01 \\
\hline B5 & $11 / 09 / 81$ & -165 & -50.3 & 13.8 & 235 & 140 & .17 & 8.22 \\
\hline B5 & $11 / 09 / 81$ & -170 & -51.8 & 14.1 & 330 & 168 & .50 & 8.21 \\
\hline B5 & $11 / 13 / 81$ & 0 & 0.0 & 12.9 & 220 & 108 & .46 & 6.71 \\
\hline B5 & $11 / 13 / 81$ & -10 & -3.0 & 14.2 & 230 & 70 & 6.25 & 6.42 \\
\hline B5 & $11 / 13 / 81$ & -20 & -6.1 & 13.8 & 130 & 42 & 14.50 & 6.09 \\
\hline B5 & $11 / 13 / 81$ & -30 & -9.1 & 13.7 & 175 & 68 & 26.50 & 6.13 \\
\hline B5 & $11 / 13 / 81$ & -40 & -12.2 & 13.7 & 190 & 72 & 32.00 & 6.21 \\
\hline B5 & $11 / 13 / 81$ & -45 & -13.7 & 13.8 & 200 & 84 & 35.00 & 6.25 \\
\hline B5 & $11 / 13 / 81$ & -50 & -15.2 & 13.7 & 200 & 84 & 37.75 & 6.28 \\
\hline B5 & $11 / 13 / 81$ & -55 & -16.8 & 13.8 & 185 & 80 & 33.25 & 6.31 \\
\hline B5 & $11 / 13 / 81$ & -60 & -18.3 & 14.0 & 170 & 80 & 32.00 & 6.30 \\
\hline B5 & $11 / 13 / 81$ & -65 & -19.8 & 13.9 & 185 & 90 & 18.75 & 6.50 \\
\hline B5 & $11 / 13 / 81$ & -70 & -21.3 & 14.2 & 250 & 110 & 22.00 & 6.63 \\
\hline B5 & $11 / 13 / 81$ & -75 & -22.9 & 14.2 & 300 & 146 & 10.25 & 6.96 \\
\hline B5 & $11 / 13 / 81$ & -80 & -24.4 & 14.6 & 270 & 138 & 3.40 & 7.07 \\
\hline B5 & $11 / 13 / 81$ & -85 & -25.9 & 14.6 & 275 & 146 & 1.16 & 7.35 \\
\hline B5 & $11 / 13 / 81$ & -90 & -27.4 & 14.6 & 290 & 162 & 1.25 & 7.45 \\
\hline B5 & $11 / 13 / 81$ & -100 & -30.5 & 14.5 & 310 & 180 & 8.00 & 7.29 \\
\hline B5 & $11 / 13 / 81$ & -105 & -32.0 & 14.4 & 290 & 164 & 20.50 & 6.99 \\
\hline
\end{tabular}


Table 7.--Water-chemistry data from profile well B5, 1981-83--Continued

\begin{tabular}{|c|c|c|c|c|c|c|c|c|}
\hline WELL & DATE & $\begin{array}{l}\text { ELEVA } \\
\text { (FEET) }\end{array}$ & $\begin{array}{l}\text { ATION } \\
\text { (METER) }\end{array}$ & $\begin{array}{l}\text { TEMPER- } \\
\text { ATURE } \\
\text { (DEG C) }\end{array}$ & $\begin{array}{l}\text { SPECIFIC } \\
\text { CONDUCT- } \\
\text { ANCE } \\
\text { (UMHOS) }\end{array}$ & $\begin{array}{c}\text { ALKA- } \\
\text { LINITY } \\
\text { (MG/L } \\
\text { AS } \\
\text { CACO3) }\end{array}$ & $\begin{array}{l}\text { IRON } \\
\text { DIS- } \\
\text { SOLVED } \\
(M G / L)\end{array}$ & $\begin{array}{c}\mathrm{PH} \\
\text { (UNIT) }\end{array}$ \\
\hline B5 & $11 / 13 / 81$ & -115 & -35.1 & 14.8 & 260 & 130 & 27.75 & 6.80 \\
\hline B5 & $11 / 13 / 81$ & -125 & -38.1 & 14.4 & 260 & 124 & 28.75 & 6.72 \\
\hline B5 & $11 / 13 / 81$ & -135 & -41.1 & 14.5 & 230 & 100 & 17.00 & 6.73 \\
\hline B5 & $11 / 13 / 81$ & -140 & -42.7 & 14.3 & 185 & 80 & 1.74 & 7.30 \\
\hline B5 & $11 / 13 / 81$ & -145 & -44.2 & 14.7 & 200 & 82 & .19 & 7.58 \\
\hline B5 & $11 / 13 / 81$ & -150 & -45.7 & 14.3 & 200 & 90 & .07 & 7.73 \\
\hline B5 & $11 / 13 / 81$ & -155 & -47.2 & 14.5 & 245 & 130 & .03 & 8.02 \\
\hline B5 & $11 / 13 / 81$ & -165 & -50.3 & 14.2 & 240 & 130 & .12 & 8.20 \\
\hline B5 & $11 / 13 / 81$ & -170 & -51.8 & 14.2 & 340 & 174 & .49 & 8.13 \\
\hline B5 & $11 / 20 / 81$ & 0 & 0.0 & 13.1 & 220 & 104 & .43 & 6.75 \\
\hline B5 & $11 / 20 / 81$ & -10 & -3.0 & 14.1 & 200 & 78 & 4.30 & 6.50 \\
\hline B5 & $11 / 20 / 81$ & -20 & -6.1 & 14.2 & 135 & 42 & 14.25 & 6.23 \\
\hline B5 & $11 / 20 / 81$ & -30 & -9.1 & 13.8 & 160 & 56 & 21.00 & 6.29 \\
\hline B5 & $11 / 20 / 81$ & -40 & -12.2 & 13.9 & 180 & 68 & 26.75 & 6.32 \\
\hline B5 & $11 / 20 / 81$ & -45 & -13.7 & 13.8 & 185 & 80 & 31.50 & 6.38 \\
\hline B5 & $11 / 20 / 81$ & -50 & -15.2 & 13.7 & 210 & 84 & 38.75 & 6.37 \\
\hline B5 & $11 / 20 / 81$ & -55 & -16.8 & 13.7 & 185 & 76 & 33.25 & 6.39 \\
\hline B5 & $11 / 20 / 81$ & -60 & -18.3 & 13.6 & 165 & 80 & 30.50 & 6.40 \\
\hline B5 & $11 / 20 / 81$ & -65 & -19.8 & 14.1 & 195 & 88 & 19.75 & 6.54 \\
\hline B5 & $11 / 20 / 81$ & -70 & -21.3 & 13.9 & 220 & 116 & 19.25 & 6.75 \\
\hline B5 & $11 / 20 / 81$ & -75 & -22.9 & 13.8 & 300 & 152 & 9.25 & 6.90 \\
\hline 85 & $11 / 20 / 81$ & -80 & -24.4 & 13.9 & 275 & 146 & 3.90 & 7.00 \\
\hline B5 & $11 / 20 / 81$ & -85 & -25.9 & 13.8 & 300 & 162 & 1.27 & 7.32 \\
\hline B5 & $11 / 20 / 81$ & -90 & -27.4 & 13.8 & 285 & 152 & 1.32 & 7.50 \\
\hline B5 & $11 / 20 / 81$ & -100 & -30.5 & 13.8 & 290 & 160 & 6.00 & 7.40 \\
\hline B5 & $11 / 20 / 81$ & -105 & -32.0 & 13.8 & 300 & 180 & 21.50 & 7.10 \\
\hline
\end{tabular}


Table 7.--Water-chem istry data from profile well B5, 1981-83--Continued

\begin{tabular}{|c|c|c|c|c|c|c|c|c|}
\hline WELL & DATE & $\begin{array}{l}\text { ELEVA } \\
\text { (FEET) }\end{array}$ & $\begin{array}{l}\text { ATION } \\
\text { (METER) }\end{array}$ & $\begin{array}{l}\text { TEMPER- } \\
\text { ATURE } \\
\text { (DEG C) }\end{array}$ & $\begin{array}{l}\text { SPECIFIC } \\
\text { CONDUCT- } \\
\text { ANCE } \\
\text { (UMHOS) }\end{array}$ & $\begin{array}{c}\text { ALKA- } \\
\text { LINITY } \\
\text { (MG/L } \\
\text { AS } \\
\text { CACO3) }\end{array}$ & $\begin{array}{l}\text { IRON } \\
\text { DIS- } \\
\text { SOLVED } \\
\text { (MG/L) }\end{array}$ & $\begin{array}{c}\mathrm{PH} \\
(U N \mid T)\end{array}$ \\
\hline B5 & $11 / 20 / 81$ & -115 & -35.1 & 13.9 & 260 & 134 & 27.00 & 6.85 \\
\hline B5 & $11 / 20 / 81$ & -125 & -38.1 & 13.9 & 260 & 130 & 29.25 & 6.81 \\
\hline B5 & $11 / 20 / 81$ & -135 & -41.1 & 14.1 & 220 & 96 & 18.00 & 6.83 \\
\hline B5 & $11 / 20 / 81$ & -140 & -42.7 & 14.0 & 195 & 82 & 2.22 & 7.18 \\
\hline B5 & $11 / 20 / 81$ & -145 & -44.2 & 14.2 & 195 & 80 & .18 & 7.60 \\
\hline B5 & $11 / 20 / 81$ & -150 & -45.7 & 14.2 & 205 & 84 & .06 & 7.78 \\
\hline B5 & $11 / 20 / 81$ & -155 & -47.2 & 14.3 & 250 & 130 & .03 & 8.08 \\
\hline B5 & $11 / 20 / 81$ & -165 & -50.3 & 14.0 & 250 & 134 & .14 & 8.22 \\
\hline B5 & $11 / 20 / 81$ & -170 & -51.8 & 14.3 & 340 & 168 & .47 & 7.90 \\
\hline B5 & $11 / 23 / 81$ & 0 & 0.0 & 12.5 & 210 & 100 & .47 & 6.72 \\
\hline B5 & $11 / 23 / 81$ & -10 & -3.0 & 13.5 & 190 & 78 & 4.35 & 6.50 \\
\hline B5 & $11 / 23 / 81$ & -20 & -6.1 & 13.6 & 135 & 40 & 15.75 & 6.20 \\
\hline B5 & $11 / 23 / 81$ & -30 & -9.1 & 13.2 & 140 & 54 & 18.75 & 6.30 \\
\hline B5 & $11 / 23 / 81$ & -40 & -12.2 & 13.2 & 155 & 60 & 24.75 & 6.36 \\
\hline B5 & $11 / 23 / 81$ & -45 & -13.7 & 13.2 & 170 & 78 & 29.50 & 6.39 \\
\hline B5 & $11 / 23 / 81$ & -50 & -15.2 & 13.3 & 190 & 80 & 35.00 & 6.38 \\
\hline B5 & $11 / 23 / 81$ & -55 & -16.8 & 13.3 & 185 & 78 & 34.25 & 6.39 \\
\hline B5 & $11 / 23 / 81$ & -60 & -18.3 & 13.4 & 165 & 78 & 29.25 & 6.41 \\
\hline B5 & $11 / 23 / 81$ & -65 & -19.8 & 13.5 & 195 & 94 & 21.25 & 6.54 \\
\hline B5 & $11 / 23 / 81$ & -70 & -21.3 & 13.6 & 230 & 124 & 20.75 & 6.78 \\
\hline B5 & $11 / 23 / 81$ & -75 & -22.9 & 13.6 & 280 & 150 & 8.75 & 7.08 \\
\hline B5 & $11 / 23 / 81$ & -80 & -24.4 & 13.8 & 270 & 148 & 5.75 & 7.08 \\
\hline B5 & $11 / 23 / 81$ & -85 & -25.9 & 13.8 & 300 & 164 & 1.32 & 7.42 \\
\hline B5 & $11 / 23 / 81$ & -90 & -27.4 & 13.8 & 270 & 148 & 1.26 & 7.50 \\
\hline B5 & $11 / 23 / 81$ & -100 & -30.5 & 13.8 & 250 & 140 & 3.50 & 7.42 \\
\hline B5 & $11 / 23 / 81$ & -105 & -32.0 & 13.9 & 290 & 164 & 17.00 & 7.13 \\
\hline
\end{tabular}


Table 7.--Water-chemistry data from profile well B5, 1981-83--Continued

\begin{tabular}{|c|c|c|c|c|c|c|c|c|}
\hline WELL & DATE & $\begin{array}{l}\text { ELEVA } \\
\text { (FEET) }\end{array}$ & $\begin{array}{l}\text { ATION } \\
\text { (METER) }\end{array}$ & $\begin{array}{l}\text { TEMPER- } \\
\text { ATURE } \\
\left(\begin{array}{ll}\text { DEG } & \text { C) }\end{array}\right.\end{array}$ & $\begin{array}{l}\text { SPECIFIC } \\
\text { CONDUCT- } \\
\text { ANCE } \\
\text { (UMHOS) }\end{array}$ & $\begin{array}{c}\text { ALKA- } \\
\text { LINITY } \\
\text { (MG/L } \\
\text { AS } \\
\text { CACO3) }\end{array}$ & $\begin{array}{l}\text { IRON } \\
\text { DIS- } \\
\text { SOLVED } \\
(M G / L)\end{array}$ & $\begin{array}{c}\mathrm{PH} \\
(\mathrm{UN} \mid \mathrm{T})\end{array}$ \\
\hline B5 & $11 / 23 / 81$ & -115 & -35.1 & 13.9 & 270 & 150 & 31.00 & 6.84 \\
\hline 85 & $11 / 23 / 81$ & -125 & -38.1 & 13.9 & 260 & 130 & 29.25 & 6.85 \\
\hline B5 & $11 / 23 / 81$ & -135 & -41.1 & 13.9 & 225 & 104 & 19.25 & 6.88 \\
\hline B5 & $11 / 23 / 81$ & -140 & -42.7 & 13.7 & 205 & 84 & 2.90 & 7.13 \\
\hline B5 & $11 / 23 / 81$ & -145 & -44.2 & 13.7 & 190 & 74 & .22 & 7.60 \\
\hline B5 & $11 / 23 / 81$ & -150 & -45.7 & 13.8 & 215 & 90 & .07 & 7.73 \\
\hline B5 & $11 / 23 / 81$ & -155 & -47.2 & 13.8 & 250 & 132 & .03 & 8.04 \\
\hline B5 & $11 / 23 / 81$ & -165 & -50.3 & 13.6 & 240 & 130 & .13 & 8.22 \\
\hline B5 & $11 / 23 / 81$ & -170 & -51.8 & 13.8 & 335 & 166 & .46 & 8.20 \\
\hline B5 & $11 / 30 / 81$ & 0 & 0.0 & 12.1 & 250 & 82 & .68 & 6.72 \\
\hline B5 & $11 / 30 / 81$ & -10 & -3.0 & 12.9 & 185 & 80 & 4.65 & 6.49 \\
\hline B5 & $11 / 30 / 81$ & -20 & -6.1 & 13.1 & 135 & 46 & 16.75 & 6.15 \\
\hline B5 & $11 / 30 / 81$ & -30 & -9.1 & 13.0 & 150 & 56 & 21.00 & 6.25 \\
\hline B5 & $11 / 30 / 81$ & -40 & -12.2 & 12.9 & 155 & 60 & 24.25 & 6.33 \\
\hline B5 & $11 / 30 / 81$ & -45 & -13.7 & 12.9 & 160 & 60 & 25.75 & 6.38 \\
\hline B5 & $11 / 30 / 81$ & -50 & -15.2 & 12.8 & 160 & 64 & 29.00 & 6.39 \\
\hline B5 & $11 / 30 / 81$ & -55 & -16.8 & 12.7 & 175 & 80 & 32.25 & 6.39 \\
\hline B5 & $11 / 30 / 81$ & -60 & -18.3 & 12.8 & 165 & 80 & 30.75 & 6.40 \\
\hline B5 & $11 / 30 / 81$ & -65 & -19.8 & 12.8 & 190 & 102 & 22.00 & 6.52 \\
\hline B5 & $11 / 30 / 81$ & -70 & -21.3 & 12.8 & 210 & 120 & 20.50 & 6.78 \\
\hline B5 & $11 / 30 / 81$ & -75 & -22.9 & 12.8 & 275 & 150 & 8.00 & 7.09 \\
\hline B5 & $11 / 30 / 81$ & -80 & -24.4 & 12.8 & 260 & 146 & 6.50 & 7.03 \\
\hline B5 & $11 / 30 / 81$ & -85 & -25.9 & 12.8 & 280 & 162 & 1.24 & 7.40 \\
\hline B5 & $11 / 30 / 81$ & -90 & -27.4 & 12.8 & 260 & 144 & 1.34 & 7.48 \\
\hline B5 & $11 / 30 / 81$ & -100 & -30.5 & 12.8 & 235 & 130 & 4.25 & 7.45 \\
\hline B5 & $11 / 30 / 81$ & -105 & -32.0 & 12.8 & 240 & 130 & 11.00 & 7.22 \\
\hline
\end{tabular}


Table 7.--Water-chemistry data from profile well B5, 1981-83--Continued

\begin{tabular}{|c|c|c|c|c|c|c|c|c|}
\hline WELL & DATE & $\begin{array}{l}\text { ELEV } \\
\text { (FEET) }\end{array}$ & $\begin{array}{l}\text { ATION } \\
\text { (METER) }\end{array}$ & $\begin{array}{l}\text { TEMPER- } \\
\text { ATURE } \\
(\text { DEG C) }\end{array}$ & $\begin{array}{l}\text { SPECIFIC } \\
\text { CONDUCT- } \\
\text { ANCE } \\
\text { (UMHOS) }\end{array}$ & $\begin{array}{c}\text { ALKA- } \\
\text { LINITY } \\
\text { (MG/L } \\
\text { AS } \\
\text { CACO3) }\end{array}$ & $\begin{array}{l}\text { IRON } \\
\text { DIS- } \\
\text { SOLVED } \\
\text { (MG/L) }\end{array}$ & $\begin{array}{c}P H \\
(U N \mid T)\end{array}$ \\
\hline B5 & $11 / 30 / 81$ & -115 & -35.1 & 12.8 & 280 & 170 & 32.00 & 6.84 \\
\hline B5 & $11 / 30 / 81$ & -125 & -38.1 & 12.8 & 250 & 142 & 30.25 & 6.88 \\
\hline B5 & $11 / 30 / 81$ & -135 & -41.1 & 12.8 & 240 & 120 & 24.00 & 6.88 \\
\hline B5 & $11 / 30 / 81$ & -140 & -42.7 & 12.9 & 215 & 96 & 3.30 & 7.05 \\
\hline B5 & $11 / 30 / 81$ & -145 & -44.2 & 12.8 & 180 & 76 & .21 & 7.54 \\
\hline B5 & $11 / 30 / 81$ & -150 & -45.7 & 12.8 & 225 & 96 & .07 & 7.72 \\
\hline B5 & $11 / 30 / 81$ & -155 & -47.2 & 12.9 & 240 & 126 & .04 & 8.01 \\
\hline B5 & $11 / 30 / 81$ & -165 & -50.3 & 12.8 & 240 & 134 & .12 & 8.18 \\
\hline B5 & $11 / 30 / 81$ & -170 & -51.8 & 12.8 & 335 & 168 & .48 & 8.15 \\
\hline B5 & $12 / 04 / 81$ & 0 & 0.0 & 12.8 & 220 & 84 & .73 & 6.64 \\
\hline B5 & $12 / 04 / 81$ & -20 & -6.1 & 14.1 & 140 & 44 & 16.50 & 6.08 \\
\hline B5 & $12 / 04 / 81$ & -30 & -9.1 & 13.8 & 160 & 58 & 21.50 & 6.22 \\
\hline B5 & $12 / 04 / 81$ & -40 & -12.2 & 13.8 & 170 & 60 & 24.50 & 6.29 \\
\hline B5 & $12 / 04 / 81$ & -45 & -13.7 & 13.8 & 170 & 64 & 27.00 & 6.32 \\
\hline B5 & $12 / 04 / 81$ & -50 & -15.2 & 13.7 & 180 & 66 & 30.00 & 6.35 \\
\hline B5 & $12 / 04 / 81$ & -55 & -16.8 & 14.1 & 180 & 76 & 31.25 & 6.32 \\
\hline B5 & $12 / 04 / 81$ & -60 & -18.3 & 13.9 & 170 & 78 & 28.75 & 6.35 \\
\hline B5 & $12 / 04 / 81$ & -65 & -19.8 & 14.1 & 175 & 80 & 18.50 & 6.50 \\
\hline B5 & $12 / 04 / 81$ & -70 & -21.3 & 14.6 & 225 & 118 & 20.00 & 6.68 \\
\hline B5 & $12 / 04 / 81$ & -75 & -22.9 & 14.1 & 285 & 148 & 8.00 & 7.03 \\
\hline B5 & $12 / 04 / 81$ & -80 & -24.4 & 14.2 & 275 & 148 & 6.50 & 7.00 \\
\hline B5 & $12 / 04 / 81$ & -85 & -25.9 & 14.3 & 300 & 162 & 1.23 & 7.43 \\
\hline B5 & $12 / 04 / 81$ & -90 & -27.4 & 14.1 & 275 & 144 & 1.32 & 7.49 \\
\hline B5 & $12 / 04 / 81$ & -100 & -30.5 & 14.2 & 240 & 122 & 3.75 & 7.45 \\
\hline B5 & $12 / 04 / 81$ & -105 & -32.0 & 14.1 & 250 & 126 & 9.25 & 7.19 \\
\hline B5 & $12 / 04 / 81$ & -115 & -35.1 & 14.2 & 300 & 178 & 31.00 & 6.80 \\
\hline
\end{tabular}


Table 7.--Water-chem istry data from profile well B5, 1981-83--Continued

\begin{tabular}{|c|c|c|c|c|c|c|c|c|}
\hline WELL & DATE & $\begin{array}{l}\text { ELEVA } \\
\text { (FEET) }\end{array}$ & $\begin{array}{l}\text { ATION } \\
\text { (METER) }\end{array}$ & 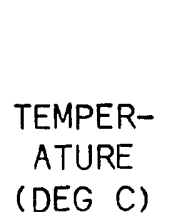 & $\begin{array}{l}\text { SPECIFIC } \\
\text { CONDUCT- } \\
\text { ANCE } \\
\text { (UMHOS) }\end{array}$ & $\begin{array}{c}\text { ALKA- } \\
\text { LINITY } \\
\text { (MG/L } \\
\text { AS } \\
\text { CACO3) }\end{array}$ & $\begin{array}{l}\text { IRON } \\
\text { DIS- } \\
\text { SOLVED } \\
\text { (MG/L) }\end{array}$ & $\begin{array}{c}\mathrm{PH} \\
(\mathrm{UN} \mid \mathrm{T})\end{array}$ \\
\hline B5 & $12 / 04 / 81$ & -125 & -38.1 & 14.2 & 270 & 146 & 29.25 & 6.80 \\
\hline B5 & $12 / 04 / 81$ & -135 & -41.1 & 14.1 & 255 & 120 & 24.25 & 6.79 \\
\hline B5 & $12 / 04 / 81$ & -140 & -42.7 & 13.9 & 230 & 100 & 6.25 & 7.01 \\
\hline B5 & $12 / 04 / 81$ & -145 & -44.2 & 14.2 & 195 & 76 & .16 & 7.50 \\
\hline B5 & $12 / 04 / 81$ & -150 & -45.7 & 14.1 & 245 & 98 & .09 & 7.71 \\
\hline B5 & $12 / 04 / 81$ & -155 & -47.2 & 14.2 & 255 & 130 & .03 & 8.01 \\
\hline B5 & $12 / 04 / 81$ & -165 & -50.3 & 13.9 & 250 & 136 & .08 & 8.19 \\
\hline B5 & $12 / 04 / 81$ & -170 & -51.8 & 13.9 & 340 & 170 & .45 & 8.14 \\
\hline B5 & $12 / 07 / 81$ & 0 & 0.0 & 12.4 & 215 & 100 & .66 & 6.70 \\
\hline B5 & $12 / 07 / 81$ & -10 & -3.0 & 13.2 & 195 & 80 & 7.50 & 6.42 \\
\hline B5 & $12 / 07 / 81$ & -20 & -6.1 & 13.6 & 140 & 42 & 16.50 & 6.10 \\
\hline B5 & $12 / 07 / 81$ & -30 & -9.1 & 13.4 & 160 & $: 58$ & 21.00 & 6.25 \\
\hline B5 & $12 / 07 / 81$ & -40 & -12.2 & 13.2 & 165 & 62 & 25.50 & 6.32 \\
\hline B5 & $12 / 07 / 81$ & -45 & -13.7 & 13.2 & 170 & 66 & 26.50 & 6.35 \\
\hline B5 & $12 / 07 / 81$ & -50 & -15.2 & 13.1 & 180 & 66 & 31.25 & 6.37 \\
\hline B5 & $12 / 07 / 81$ & -55 & -16.8 & 13.2 & 180 & 72 & 31.25 & 6.39 \\
\hline B5 & $12 / 07 / 81$ & -60 & -18.3 & 13.1 & 165 & 74 & 28.75 & 6.38 \\
\hline B5 & $12 / 07 / 81$ & -65 & -19.8 & 13.2 & 180 & 88 & 22.00 & 6.49 \\
\hline B5 & $12 / 07 / 81$ & -70 & -21.3 & 13.1 & 220 & 114 & 21.00 & 6.82 \\
\hline B5 & $12 / 07 / 81$ & -75 & -22.9 & 13.1 & 280 & 152 & 8.25 & 7.02 \\
\hline B5 & $12 / 07 / 81$ & -80 & -24.4 & 13.1 & 270 & 152 & 7.00 & 7.00 \\
\hline B5 & $12 / 07 / 81$ & -85 & -25.9 & 12.7 & 280 & 162 & 1.26 & 7.01 \\
\hline B5 & $12 / 07 / 81$ & -90 & -27.4 & 12.8 & 255 & 142 & 1.36 & 7.42 \\
\hline B5 & $12 / 07 / 81$ & -100 & -30.5 & 12.9 & 225 & 124 & 3.50 & 7.42 \\
\hline B5 & $12 / 07 / 81$ & -105 & -32.0 & 12.9 & 245 & 128 & 14.25 & 7.16 \\
\hline B5 & $12 / 07 / 81$ & -115 & -35.1 & 13.0 & 280 & 164 & 29.75 & 6.88 \\
\hline
\end{tabular}


Table 7.--Water-chemistry data from profile well B5, 1981-83--Continued

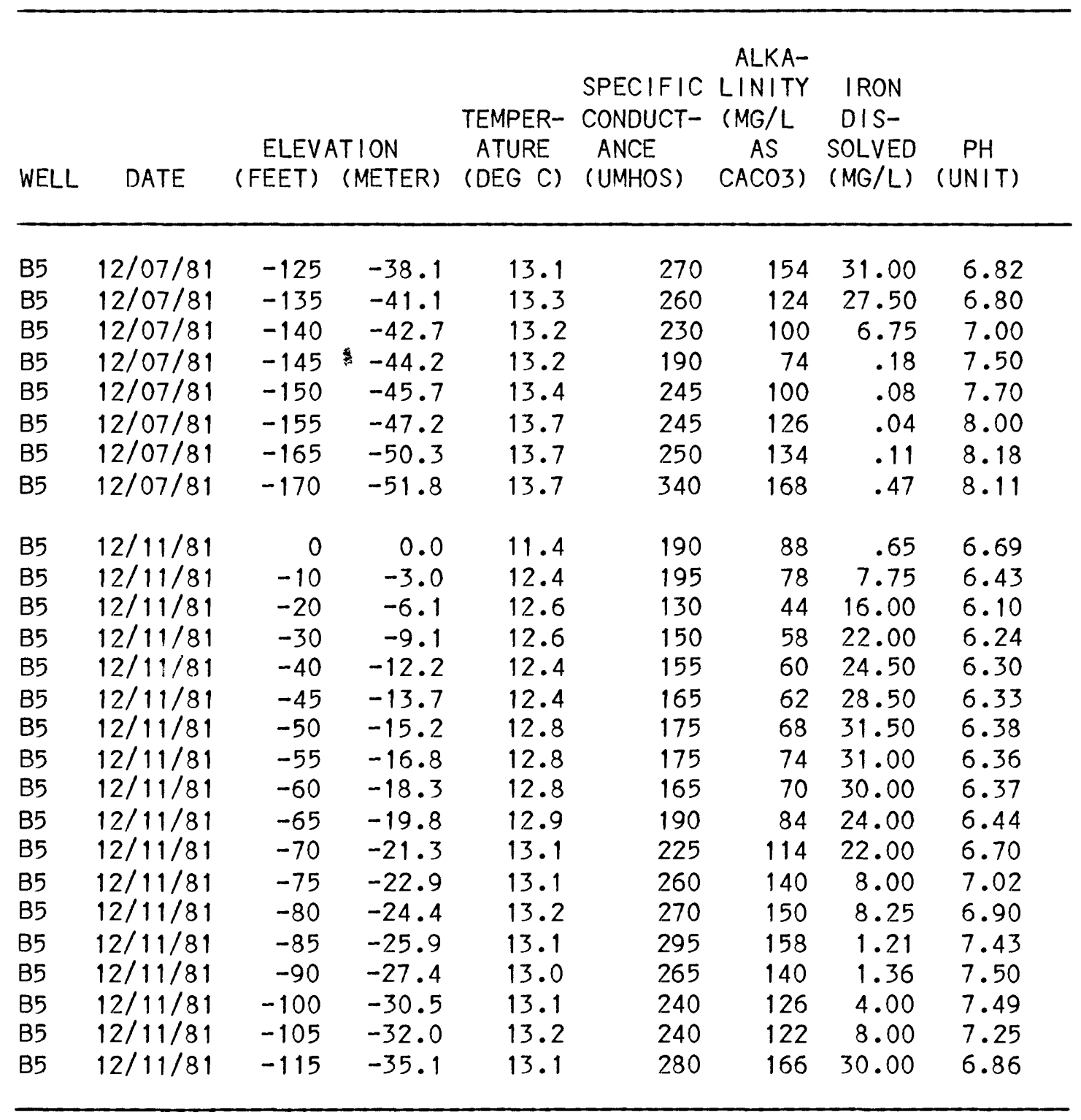


Table 7.--Water-chemistry data from profile well B5, 1981-83--Continued

\begin{tabular}{|c|c|c|c|c|c|c|c|c|}
\hline WELL & DATE & $\begin{array}{l}\text { ELEVA } \\
\text { (FEET) }\end{array}$ & $\begin{array}{l}\text { ATION } \\
\text { (METER) }\end{array}$ & $\begin{array}{l}\text { TEMPER- } \\
\text { ATURE } \\
(\text { DEG C) }\end{array}$ & $\begin{array}{l}\text { SPECIFIC } \\
\text { CONDUCT- } \\
\text { ANCE } \\
\text { (UMHOS) }\end{array}$ & $\begin{array}{c}\text { ALKA- } \\
\text { LINITY } \\
\text { (MG/L } \\
\text { AS } \\
\text { CACO3) }\end{array}$ & $\begin{array}{l}\text { IRON } \\
\text { DIS- } \\
\text { SOLVED } \\
\text { (MG/L) }\end{array}$ & $\begin{array}{c}\text { PH } \\
(U N I T)\end{array}$ \\
\hline B5 & $12 / 11 / 81$ & -125 & -38.1 & 13.2 & 275 & 154 & 31.50 & 6.80 \\
\hline B5 & $12 / 11 / 81$ & -135 & -41.1 & 13.2 & 260 & 128 & 27.75 & 6.75 \\
\hline B5 & $12 / 11 / 81$ & -140 & -42.7 & 13.3 & 240 & 102 & 7.50 & 6.93 \\
\hline B5 & $12 / 11 / 81$ & -145 & -44.2 & 13.4 & 195 & 74 & .21 & 7.50 \\
\hline B5 & $12 / 11 / 81$ & -150 & -45.7 & 13.6 & 250 & 98 & .08 & 7.69 \\
\hline B5 & $12 / 11 / 81$ & -155 & -47.2 & 13.5 & 250 & 126 & .07 & 8.00 \\
\hline B5 & $12 / 11 / 81$ & -165 & -50.3 & 13.3 & 250 & 134 & .12 & 8.20 \\
\hline B5 & $12 / 11 / 81$ & -170 & -51.8 & 13.4 & 330 & 164 & .45 & 8.15 \\
\hline B5 & $01 / 04 / 82$ & 0 & 0.0 & 11.1 & 200 & 100 & 1.39 & -- \\
\hline B5 & $01 / 04 / 82$ & -10 & -3.0 & 12.1 & 180 & 78 & 8.25 & -- \\
\hline B5 & $01 / 04 / 82$ & -20 & -6.1 & 12.6 & 130 & 44 & 16.50 & -- \\
\hline B5 & $01 / 04 / 82$ & -30 & -9.1 & 12.6 & 150 & 58 & 21.00 & -- \\
\hline B5 & $01 / 04 / 82$ & -40 & -12.2 & 12.6 & 160 & 66 & 24.00 & -- \\
\hline B5 & $01 / 04 / 82$ & -45 & -13.7 & 12.6 & 160 & 64 & 26.00 & -- \\
\hline B5 & $01 / 04 / 82$ & -50 & -15.2 & 12.7 & 160 & 70 & 29.25 & -- \\
\hline 85 & $01 / 04 / 82$ & -55 & -16.8 & 12.7 & 170 & 70 & 30.00 & -- \\
\hline B5 & $01 / 04 / 82$ & -60 & -18.3 & 12.7 & 160 & 68 & 29.50 & -- \\
\hline B5 & $01 / 04 / 82$ & -65 & -19.8 & 12.8 & 170 & 74 & 27.25 & -- \\
\hline B5 & $01 / 04 / 82$ & -70 & -21.3 & 12.8 & 220 & 108 & 22.00 & -- \\
\hline B5 & $01 / 04 / 82$ & -75 & -22.9 & 12.8 & 260 & 140 & 8.50 & -- \\
\hline B5 & $01 / 04 / 82$ & -80 & -24.4 & 12.8 & 265 & 150 & 9.50 & -- \\
\hline B5 & $01 / 04 / 82$ & -85 & -25.9 & 12.8 & 290 & 160 & 1.26 & -- \\
\hline B5 & $01 / 04 / 82$ & -90 & -27.4 & 12.8 & 285 & 160 & 1.53 & -- \\
\hline B5 & $01 / 04 / 82$ & -100 & -30.5 & 12.9 & 250 & 132 & 3.25 & -- \\
\hline B5 & $01 / 04 / 82$ & -105 & -32.0 & 12.9 & 250 & 134 & 5.00 & -- \\
\hline B5 & $01 / 04 / 82$ & -115 & -35.1 & 12.9 & 240 & 130 & 20.50 & -- \\
\hline
\end{tabular}


Table 7.--Water-chemistry data from profile well B5, 1981-83--Continued

\begin{tabular}{|c|c|c|c|c|c|c|c|c|}
\hline WELL & DATE & $\begin{array}{l}\text { ELEV } \\
\text { (FEET) }\end{array}$ & $\begin{array}{l}\text { ATION } \\
\text { (METER) }\end{array}$ & $\begin{array}{l}\text { TEMPER- } \\
\text { ATURE } \\
\left(\begin{array}{ll}\text { DEG } & \text { C) }\end{array}\right.\end{array}$ & $\begin{array}{l}\text { SPECIFIC } \\
\text { CONDUCT- } \\
\text { ANCE } \\
\text { (UMHOS) }\end{array}$ & $\begin{array}{c}\text { ALKA- } \\
\text { LINITY } \\
\text { (MG/L } \\
\text { AS } \\
\text { CACO3) }\end{array}$ & $\begin{array}{l}\text { IRON } \\
\text { DIS- } \\
\text { SOLVED } \\
(M G / L)\end{array}$ & $\begin{array}{c}P H \\
(U N \mid T)\end{array}$ \\
\hline B5 & $01 / 04 / 82$ & -125 & -38.1 & 12.9 & 250 & 136 & 25.25 & -- \\
\hline B5 & $01 / 04 / 82$ & -135 & -41.1 & 12.9 & 270 & 154 & 31.75 & -- \\
\hline B5 & $01 / 04 / 82$ & -140 & -42.7 & 12.9 & 250 & 116 & 12.50 & -- \\
\hline B5 & $01 / 04 / 82$ & -145 & -44.2 & 12.9 & 180 & 78 & .18 & -- \\
\hline B5 & $01 / 04 / 82$ & -150 & -45.7 & 12.8 & 245 & 104 & .06 & -- \\
\hline B5 & $01 / 04 / 82$ & -155 & -47.2 & 12.8 & 220 & 116 & .03 & -- \\
\hline B5 & $01 / 04 / 82$ & -165 & -50.3 & 12.4 & 230 & 134 & .08 & -- \\
\hline B5 & $01 / 04 / 82$ & -170 & -51.8 & 12.4 & 305 & 160 & .42 & -- \\
\hline B5 & $01 / 26 / 82$ & 0 & 0.0 & 11.4 & 220 & 108 & 1.26 & -- \\
\hline B5 & $01 / 26 / 82$ & -10 & -3.0 & 12.3 & 185 & 80 & 6.20 & -- \\
\hline B5 & $01 / 26 / 82$ & -20 & -6.1 & 12.8 & 140 & 40 & 17.25 & -- \\
\hline B5 & $01 / 26 / 82$ & -30 & -9.1 & 12.8 & 160 & 60 & 22.50 & -- \\
\hline B5 & $01 / 20 / 82$ & -40 & -12.2 & 12.9 & 165 & 64 & 24.75 & -- \\
\hline 85 & $01 / 26 / 82$ & -45 & -13.7 & 12.9 & 160 & 66 & 25.00 & -- \\
\hline B5 & $01 / 26 / 82$ & -50 & -15.2 & 12.9 & 165 & 68 & 28.50 & - \\
\hline B5 & $01 / 26 / 82$ & -55 & -16.8 & 12.9 & 170 & 70 & 31.25 & -- \\
\hline B5 & $01 / 26 / 82$ & -60 & -18.3 & 12.8 & 170 & 68 & 30.25 & -- \\
\hline 85 & $01 / 26 / 82$ & -65 & -19.8 & 12.6 & 170 & 70 & 30.00 & -- \\
\hline B5 & $01 / 26 / 82$ & -70 & -21.3 & 12.8 & 220 & 108 & 19.00 & -- \\
\hline 85 & $01 / 26 / 82$ & -75 & -22.9 & 12.7 & 235 & 128 & 8.75 & -- \\
\hline B5 & $01 / 26 / 82$ & -80 & -24.4 & 12.7 & 280 & 144 & 11.75 & - \\
\hline B5 & $01 / 26 / 82$ & -85 & -25.9 & 12.6 & 270 & 160 & 2.20 & -- \\
\hline B5 & $01 / 26 / 82$ & -90 & -27.4 & 12.6 & 280 & 160 & 1.45 & -- \\
\hline B5 & $01 / 26 / 82$ & -100 & -30.5 & 12.6 & 280 & 150 & 1.40 & -- \\
\hline B5 & $01 / 26 / 82$ & -105 & -32.0 & 12.4 & 260 & 130 & 1.95 & -- \\
\hline B5 & $01 / 26 / 82$ & -115 & -35.1 & 12.5 & 245 & 140 & 13.25 & -- \\
\hline
\end{tabular}


Table 7.--Water-chemistry data from profile well B5, 1981-83--Continued

\begin{tabular}{|c|c|c|c|c|c|c|c|c|}
\hline WELL & DATE & $\begin{array}{l}\text { ELEVA } \\
\text { (FEET) }\end{array}$ & $\begin{array}{l}\text { ATION } \\
\text { (METER) }\end{array}$ & $\begin{array}{l}\text { TEMPER- } \\
\text { ATURE } \\
\text { (DEG C) }\end{array}$ & $\begin{array}{l}\text { SPECIFIC } \\
\text { CONDUCT- } \\
\text { ANCE } \\
\text { (UMHOS) }\end{array}$ & $\begin{array}{c}\text { ALKA- } \\
\text { LINITY } \\
\text { (MG/L } \\
\text { AS } \\
\text { CACO3) }\end{array}$ & $\begin{array}{l}\text { IRON } \\
\text { DIS- } \\
\text { SOLVED } \\
\text { (MG/L) }\end{array}$ & $\begin{array}{c}P H \\
(U N \mid T)\end{array}$ \\
\hline B5 & $01 / 26 / 82$ & -125 & -38.1 & 12.6 & 230 & 122 & 18.25 & -- \\
\hline B5 & $01 / 26 / 82$ & -135 & -41.1 & 12.6 & 270 & 164 & 33.00 & -- \\
\hline B5 & $01 / 26 / 82$ & -140 & -42.7 & 12.5 & 270 & 132 & 15.75 & -- \\
\hline B5 & $01 / 26 / 82$ & -145 & -44.2 & 12.3 & 185 & 80 & .27 & -- \\
\hline B5 & $01 / 26 / 82$ & -150 & -45.7 & 12.6 & 230 & 104 & .09 & - \\
\hline B5 & $01 / 26 / 82$ & -155 & -47.2 & 12.5 & 220 & 118 & .03 & -- \\
\hline B5 & $01 / 26 / 82$ & -165 & -50.3 & 12.5 & 235 & 138 & .07 & -- \\
\hline B5 & $01 / 26 / 82$ & -170 & -51.8 & 12.5 & 310 & 160 & .41 & -- \\
\hline B5 & $02 / 16 / 82$ & 0 & 0.0 & 11.9 & 235 & 114 & 1.54 & 6.92 \\
\hline B5 & $02 / 16 / 82$ & -10 & -3.0 & 12.6 & 200 & 84 & 5.70 & 6.47 \\
\hline B5 & $02 / 16 / 82$ & -20 & -6.1 & 13.1 & 140 & 40 & 16.75 & 6.00 \\
\hline B5 & $02 / 16 / 82$ & -30 & -9.1 & 13.3 & 170 & 56 & 20.00 & 6.20 \\
\hline B5 & $02 / 16 / 82$ & -40 & -12.2 & 13.3 & 170 & 64 & 23.25 & 6.25 \\
\hline B5 & $02 / 16 / 82$ & -45 & -13.7 & 13.3 & 175 & 68 & 24.50 & 6.29 \\
\hline B5 & $02 / 16 / 82$ & -50 & -15.2 & 13.7 & 175 & 70 & 26.50 & 6.15 \\
\hline B5 & $02 / 16 / 82$ & -55 & -16.8 & 13.6 & 180 & 76 & 30.50 & 6.19 \\
\hline B5 & $02 / 16 / 82$ & -60 & -18.3 & 13.7 & 170 & 72 & 28.00 & 6.18 \\
\hline B5 & $02 / 16 / 82$ & -65 & -19.8 & 13.7 & 180 & 72 & 30.50 & 6.21 \\
\hline 85 & $02 / 16 / 82$ & -70 & $-21 \cdot 3$ & 13.7 & 220 & 104 & 16.50 & 6.62 \\
\hline 85 & $02 / 16 / 82$ & -75 & -22.9 & 13.7 & 230 & 108 & 10.00 & 6.75 \\
\hline 85 & $02 / 16 / 82$ & -80 & -24.4 & 13.7 & 270 & 140 & 12.00 & 6.89 \\
\hline B5 & $02 / 16 / 82$ & -85 & -25.9 & 13.6 & 290 & 158 & 2.80 & 6.90 \\
\hline 85 & $02 / 16 / 82$ & -90 & -27.4 & 13.3 & 300 & 158 & 1.46 & 7.41 \\
\hline B5 & $02 / 16 / 82$ & -100 & -30.5 & 13.4 & 315 & 160 & 1.40 & 7.48 \\
\hline B5 & $02 / 16 / 82$ & -105 & -32.0 & 13.4 & 310 & 146 & 1.74 & 7.49 \\
\hline B5 & $02 / 16 / 82$ & -115 & -35.1 & 13.4 & 270 & 130 & 6.75 & 7.30 \\
\hline
\end{tabular}


Table 7.--Water-chemistry data from profile well B5, 1981-83--Continued

\begin{tabular}{|c|c|c|c|c|c|c|c|c|}
\hline WELL & DATE & $\begin{array}{l}\text { ELEVA } \\
\text { (FEET) }\end{array}$ & $\begin{array}{l}\text { ATION } \\
\text { (METER) }\end{array}$ & $\begin{array}{l}\text { TEMPER- } \\
\text { ATURE } \\
(D E G \quad C)\end{array}$ & $\begin{array}{l}\text { SPECIFIC } \\
\text { CONDUCT- } \\
\text { ANCE } \\
\text { (UMHOS) }\end{array}$ & $\begin{array}{c}\text { ALKA- } \\
\text { LINITY } \\
\text { (MG/L } \\
\text { AS } \\
\text { CACO3) }\end{array}$ & $\begin{array}{l}\text { IRON } \\
\text { DIS- } \\
\text { SOLVED } \\
\text { (MG/L) }\end{array}$ & $\begin{array}{c}P H \\
(U N \mid T)\end{array}$ \\
\hline B5 & $02 / 16 / 82$ & -125 & -38.1 & 13.3 & 230 & 116 & 14.25 & 7.10 \\
\hline B5 & $02 / 16 / 82$ & -135 & -41.1 & 13.3 & 290 & 170 & 25.50 & 6.78 \\
\hline B5 & $02 / 16 / 82$ & -140 & -42.7 & 13.2 & 280 & 136 & 24.00 & 6.65 \\
\hline B5 & $02 / 16 / 82$ & -145 & $-44 \cdot 2$ & 13.3 & 200 & 86 & .22 & 7.52 \\
\hline B5 & $02 / 16 / 82$ & -150 & -45.7 & 13.2 & 220 & 98 & .08 & 7.68 \\
\hline B5 & $02 / 16 / 82$ & -155 & -47.2 & 13.2 & 230 & 118 & .05 & 8.00 \\
\hline B5 & $02 / 16 / 82$ & -165 & -50.3 & 13.1 & 240 & 134 & .08 & 8.13 \\
\hline B5 & $02 / 16 / 82$ & -170 & -51.8 & 13.2 & 310 & 160 & .38 & 7.95 \\
\hline B5 & $03 / 08 / 82$ & 0 & 0.0 & 12.9 & 220 & 100 & .91 & 6.79 \\
\hline 85 & $03 / 08 / 82$ & -10 & -3.0 & 12.5 & 200 & 90 & 6.00 & 6.43 \\
\hline B5 & $03 / 08 / 82$ & -20 & -6.1 & 13.0 & 145 & 40 & 14.50 & 5.90 \\
\hline B5 & $03 / 08 / 82$ & -30 & -9.1 & 13.6 & 170 & 54 & 20.50 & 6.10 \\
\hline B5 & $03 / 08 / 82$ & -40 & -12.2 & 13.8 & 175 & 62 & 22.25 & 6.20 \\
\hline B5 & $03 / 08 / 82$ & -45 & -13.7 & 13.7 & 180 & 68 & 25.75 & 6.29 \\
\hline B5 & $03 / 08 / 82$ & -50 & -15.2 & 13.7 & 175 & 64 & 23.50 & 6.25 \\
\hline B5 & $03 / 08 / 82$ & -55 & -16.8 & 14.0 & 180 & 72 & 28.75 & 6.30 \\
\hline B5 & $03 / 08 / 82$ & -60 & -18.3 & 14.3 & 175 & 78 & 29.50 & 6.28 \\
\hline B5 & $03 / 08 / 82$ & -65 & -19.8 & 14.2 & 180 & 76 & 31.00 & 6.31 \\
\hline B5 & $03 / 08 / 82$ & -70 & -21.3 & 14.1 & 215 & 98 & 15.00 & 6.68 \\
\hline B5 & $03 / 08 / 82$ & -75 & -22.9 & 14.1 & 215 & 108 & 10.25 & 6.90 \\
\hline B5 & $03 / 08 / 82$ & -80 & -24.4 & 13.8 & 260 & 138 & 11.25 & 7.12 \\
\hline B5 & $03 / 08 / 82$ & -85 & -25.9 & 13.7 & 300 & 160 & 3.20 & 7.23 \\
\hline B5 & $03 / 08 / 82$ & -90 & -27.4 & 13.8 & 310 & 154 & 1.45 & 7.50 \\
\hline B5 & $03 / 08 / 82$ & -100 & -30.5 & 13.7 & 320 & 144 & 1.54 & 7.50 \\
\hline B5 & $03 / 08 / 82$ & -105 & -32.0 & 13.7 & 340 & 140 & 1.70 & 7.51 \\
\hline B5 & $03 / 08 / 82$ & -115 & -35.1 & 13.7 & 300 & 142 & 5.50 & 7.35 \\
\hline
\end{tabular}



Table 7.-- Water-chem istry data from profile

\begin{tabular}{|c|c|c|c|c|c|c|c|c|}
\hline WELL & DATE & $\begin{array}{l}\text { ELEVA } \\
\text { (FEET) }\end{array}$ & $\begin{array}{l}\text { ATION } \\
\text { (METER) }\end{array}$ & $\begin{array}{l}\text { TEMPER- } \\
\text { ATURE } \\
\text { (DEG C) }\end{array}$ & $\begin{array}{l}\text { SPECIFIC } \\
\text { CONDUCT- } \\
\text { ANCE } \\
\text { (UMHOS) }\end{array}$ & $\begin{array}{c}\text { ALKA- } \\
\text { LINITY } \\
\text { (MG/L } \\
\text { AS } \\
\text { CACO3) }\end{array}$ & $\begin{array}{l}\text { IRON } \\
\text { DIS- } \\
\text { SOLVED } \\
\text { (MG/L) }\end{array}$ & $\begin{array}{c}\mathrm{PH} \\
(U N I T)\end{array}$ \\
\hline B5 & $03 / 08 / 82$ & -125 & -38.1 & 13.7 & 245 & 120 & 9.00 & 7.18 \\
\hline B5 & $03 / 08 / 82$ & -135 & -41.1 & 13.9 & 290 & 164 & 19.00 & 6.91 \\
\hline B5 & $03 / 08 / 82$ & -140 & -42.7 & 13.5 & 280 & 140 & 26.25 & 6.70 \\
\hline B5 & $03 / 08 / 82$ & -145 & -44.2 & 13.6 & 200 & 86 & .24 & 7.54 \\
\hline B5 & $03 / 08 / 82$ & -150 & -45.7 & 13.6 & 210 & 90 & .08 & 7.92 \\
\hline B5 & $03 / 08 / 82$ & -155 & -47.2 & 13.7 & 220 & 114 & .04 & 8.22 \\
\hline B5 & $03 / 08 / 82$ & -165 & -50.3 & 13.6 & 250 & 130 & .08 & 8.23 \\
\hline B5 & $03 / 08 / 82$ & -170 & -51.8 & 13.7 & 310 & 160 & .39 & 8.12 \\
\hline B5 & $03 / 28 / 82$ & 0 & 0.0 & 12.9 & -- & 84 & 1.26 & 6.72 \\
\hline B5 & $03 / 28 / 82$ & -10 & -3.0 & 12.3 & -- & 84 & 11.50 & 6.32 \\
\hline B5 & $03 / 28 / 82$ & -20 & -6.1 & 12.0 & -- & 42 & 12.50 & 5.94 \\
\hline B5 & $03 / 28 / 82$ & -30 & -9.1 & 11.9 & -- & 64 & 22.00 & 6.21 \\
\hline B5 & $03 / 28 / 82$ & -40 & -12.2 & 11.8 & -- & 56 & 20.70 & 6.15 \\
\hline $\begin{array}{l}\text { B5 } \\
\text { B5 }\end{array}$ & $\begin{array}{l}03 / 28 / 82 \\
03 / 28 / 82\end{array}$ & $\begin{array}{l}-45 \\
-50\end{array}$ & $\begin{array}{l}-13.7 \\
-15.2\end{array}$ & $\begin{array}{l}11.9 \\
12.2\end{array}$ & -- & $\begin{array}{l}70 \\
68\end{array}$ & $\begin{array}{l}22.50 \\
25.75\end{array}$ & $\begin{array}{l}6.30 \\
6.33\end{array}$ \\
\hline B5 & $03 / 28 / 82$ & -55 & -16.8 & 12.4 & -- & 46 & 22.00 & 6.40 \\
\hline B5 & $03 / 28 / 82$ & -60 & -18.3 & 12.6 & -- & 58 & 22.50 & 6.42 \\
\hline B5 & $03 / 28 / 82$ & -65 & -19.8 & 12.6 & -- & 40 & 16.00 & 6.50 \\
\hline B5 & $03 / 28 / 82$ & -70 & -21.3 & 12.8 & -- & 42 & 6.75 & 6.65 \\
\hline B5 & $03 / 28 / 82$ & -75 & -22.9 & 12.9 & -- & 92 & 10.75 & 6.80 \\
\hline B5 & $03 / 28 / 82$ & -80 & -24.4 & 13.0 & -- & 114 & 7.50 & 6.89 \\
\hline B5 & $03 / 28 / 82$ & -85 & -25.9 & 13.2 & -- & 140 & 4.00 & 7.06 \\
\hline B5 & $03 / 28 / 82$ & -90 & -27.4 & 13.2 & -- & 150 & 3.40 & 7.12 \\
\hline B5 & $03 / 28 / 82$ & -100 & -30.5 & 13.2 & -- & 154 & 1.40 & 7.43 \\
\hline B5 & $03 / 28 / 82$ & -105 & -32.0 & 13.2 & -- & 152 & 1.65 & 7.55 \\
\hline B5 & $03 / 28 / 82$ & -115 & -35.1 & 13.2 & -- & 146 & 1.36 & 7.55 \\
\hline
\end{tabular}



Table 7.-- Water-chemistry data from profile

\begin{tabular}{|c|c|c|c|c|c|c|c|c|}
\hline WELL & DATE & $\begin{array}{l}\text { ELEVA } \\
\text { (FEET) }\end{array}$ & $\begin{array}{l}\text { ATION } \\
\text { (METER) }\end{array}$ & $\begin{array}{l}\text { TEMPER- } \\
\text { ATURE } \\
(\text { DEG C) }\end{array}$ & $\begin{array}{l}\text { SPECIFIC } \\
\text { CONDUCT- } \\
\text { ANCE } \\
\text { (UMHOS) }\end{array}$ & $\begin{array}{c}\text { ALKA- } \\
\text { LINITY } \\
\text { (MG/L } \\
\text { AS } \\
\text { CACO3) }\end{array}$ & $\begin{array}{l}\text { IRON } \\
\text { DIS- } \\
\text { SOLVED } \\
(M G / L)\end{array}$ & $\begin{array}{c}\mathrm{PH} \\
(\mathrm{UN} \mid \mathrm{T})\end{array}$ \\
\hline B5 & $03 / 28 / 82$ & -125 & -38.1 & 13.2 & -- & 140 & 2.70 & 7.43 \\
\hline B5 & $03 / 28 / 82$ & -135 & -41.1 & 13.4 & -- & 138 & 3.30 & 7.40 \\
\hline B5 & $03 / 28 / 82$ & -140 & -42.7 & 13.2 & -- & 140 & 25.50 & 6.90 \\
\hline B5 & $03 / 28 / 82$ & -145 & -44.2 & 13.4 & -- & 90 & .74 & 7.10 \\
\hline B5 & $03 / 28 / 82$ & -150 & -45.7 & 13.4 & -- & 72 & .08 & 7.70 \\
\hline B5 & $03 / 28 / 82$ & -155 & -47.2 & 13.4 & -- & 110 & .02 & 8.00 \\
\hline B5 & $03 / 28 / 82$ & -165 & -50.3 & 13.6 & -- & 132 & .03 & 8.25 \\
\hline B5 & $03 / 28 / 82$ & -170 & -51.8 & 13.7 & -- & 154 & .28 & 8.18 \\
\hline B5 & $04 / 06 / 82$ & 0 & 0.0 & 11.5 & 200 & 108 & 1.64 & 6.31 \\
\hline B5 & $04 / 06 / 82$ & -10 & -3.0 & 11.5 & 175 & 86 & 10.75 & 6.29 \\
\hline B5 & $04 / 06 / 82$ & -20 & -6.1 & 11.7 & 130 & 46 & 15.75 & 5.84 \\
\hline B5 & $04 / 06 / 82$ & -30 & -9.1 & 11.8 & 135 & 54 & 22.00 & 6.00 \\
\hline B5 & $04 / 06 / 82$ & -40 & -12.2 & 12.0 & 125 & 52 & 22.00 & 6.12 \\
\hline B5 & $04 / 06 / 82$ & -45 & -13.7 & 12.1 & 130 & 54 & 21.75 & 6.14 \\
\hline B5 & $04 / 06 / 82$ & -50 & -15.2 & 12.4 & 135 & 60 & 24.50 & 6.11 \\
\hline B5 & $04 / 06 / 82$ & -55 & -16.8 & 12.5 & 160 & 70 & 33.00 & 6.18 \\
\hline B5 & $04 / 06 / 82$ & -65 & -19.8 & 12.7 & 140 & 70 & 31.50 & 6.18 \\
\hline B5 & $04 / 06 / 82$ & -70 & -21.3 & 12.8 & 190 & 90 & 15.75 & 6.42 \\
\hline B5 & $04 / 06 / 82$ & -75 & -22.9 & 12.7 & 200 & 104 & 14.25 & 6.61 \\
\hline B5 & $04 / 06 / 82$ & -80 & -24.4 & 12.8 & 235 & 132 & 12.00 & 6.73 \\
\hline $\mathrm{B} 5$ & $04 / 06 / 82$ & -85 & -25.9 & 12.8 & 240 & 140 & 3.20 & 6.85 \\
\hline 85 & $04 / 06 / 82$ & -90 & -27.4 & 12.9 & 260 & 156 & 1.73 & 7.16 \\
\hline B5 & $04 / 06 / 82$ & -100 & -30.5 & 12.9 & 270 & 152 & 1.55 & 7.38 \\
\hline B5 & $04 / 06 / 82$ & -105 & -32.0 & 12.7 & 280 & 150 & 1.59 & 7.32 \\
\hline B5 & $04 / 06 / 82$ & -115 & -35.1 & 12.7 & 260 & 146 & 4.25 & 7.29 \\
\hline B5 & $04 / 06 / 82$ & -125 & -38.1 & 12.9 & 235 & 130 & 6.75 & 7.19 \\
\hline
\end{tabular}


Table 7.- Water-chem istry data from profile well B5, 1981-83--Continued

\begin{tabular}{|c|c|c|c|c|c|c|c|c|}
\hline WELL & DATE & $\begin{array}{l}\text { ELEVA } \\
\text { (FEET) }\end{array}$ & $\begin{array}{l}\text { TIION } \\
\text { (METER) }\end{array}$ & $\begin{array}{l}\text { TEMPER- } \\
\text { ATURE } \\
\left(\begin{array}{ll}\text { DEG } & \text { C) }\end{array}\right.\end{array}$ & $\begin{array}{l}\text { SPECIFIC } \\
\text { CONDUCT- } \\
\text { ANCE } \\
\text { (UMHOS) }\end{array}$ & $\begin{array}{c}\text { ALKA- } \\
\text { LINITY } \\
(M G / L \\
\text { AS } \\
\text { CACO3) }\end{array}$ & $\begin{array}{l}\text { IRON } \\
\text { DIS- } \\
\text { SOLVED } \\
\text { (MG/L) }\end{array}$ & $\begin{array}{c}\mathrm{PH} \\
(\mathrm{UN} \mid \mathrm{T})\end{array}$ \\
\hline B5 & $04 / 06 / 82$ & -135 & -41.1 & 12.9 & 290 & 180 & 14.75 & 6.99 \\
\hline B5 & $04 / 06 / 82$ & -140 & -42.7 & 12.9 & 250 & 138 & 28.75 & 6.61 \\
\hline B5 & $04 / 06 / 82$ & -145 & -44.2 & 12.9 & 180 & 90 & .30 & 7.10 \\
\hline B5 & $04 / 06 / 82$ & -150 & -45.7 & 12.9 & 190 & 90 & .07 & 7.49 \\
\hline B5 & $04 / 06 / 82$ & -165 & -50.3 & 12.9 & 220 & 130 & .06 & 7.99 \\
\hline B5 & $04 / 06 / 82$ & -170 & -51.8 & 13.1 & 285 & 156 & .38 & 8.00 \\
\hline B5 & $04 / 26 / 82$ & 0 & 0.0 & 11.7 & 175 & 90 & 1.28 & 6.56 \\
\hline B5 & $04 / 26 / 82$ & -10 & -3.0 & 11.8 & 200 & 88 & 9.50 & 6.41 \\
\hline B5 & $04 / 26 / 82$ & -20 & -6.1 & 11.8 & 135 & 46 & 15.00 & 6.00 \\
\hline B5 & $04 / 26 / 82$ & -30 & -9.1 & 12.0 & 150 & 58 & 21.75 & 6.15 \\
\hline B5 & $04 / 26 / 82$ & -40 & -12.2 & 12.2 & 160 & 62 & 22.50 & 6.25 \\
\hline B5 & $04 / 26 / 82$ & -45 & -13.7 & 12.3 & 160 & 68 & 24.50 & 6.32 \\
\hline B5 & $04 / 26 / 82$ & -50 & -15.2 & 12.6 & 150 & 60 & 24.00 & 6.37 \\
\hline $\begin{array}{l}\text { B5 } \\
\text { B5 }\end{array}$ & $\begin{array}{l}04 / 26 / 82 \\
04 / 26 / 82\end{array}$ & $\begin{array}{l}-55 \\
-60\end{array}$ & $\begin{array}{l}-16.8 \\
-18.3\end{array}$ & $\begin{array}{l}12.8 \\
12.8\end{array}$ & $\begin{array}{l}140 \\
150\end{array}$ & $\begin{array}{l}58 \\
66\end{array}$ & $\begin{array}{l}24.50 \\
29.50\end{array}$ & $\begin{array}{l}6.41 \\
6.38\end{array}$ \\
\hline B5 & $04 / 26 / 82$ & -65 & -19.8 & 12.8 & 150 & 66 & 31.00 & 6.40 \\
\hline B5 & $04 / 26 / 82$ & -70 & -21.3 & 13.0 & 165 & 88 & 13.25 & 6.62 \\
\hline B5 & $04 / 26 / 82$ & -75 & -22.9 & 13.0 & 210 & 108 & 9.25 & 6.81 \\
\hline B5 & $04 / 26 / 82$ & -80 & -24.4 & 13.2 & 240 & 130 & 10.50 & 7.00 \\
\hline $\begin{array}{l}\text { B5 } \\
\text { B5 }\end{array}$ & $\begin{array}{l}04 / 26 / 82 \\
04 / 26 / 82\end{array}$ & $\begin{array}{l}-85 \\
-90\end{array}$ & $\begin{array}{l}-25.9 \\
-27.4\end{array}$ & $\begin{array}{l}13.0 \\
13.1\end{array}$ & $\begin{array}{l}260 \\
250\end{array}$ & $\begin{array}{l}150 \\
152\end{array}$ & $\begin{array}{l}4.50 \\
2.30\end{array}$ & $\begin{array}{l}7.11 \\
7.28\end{array}$ \\
\hline B5 & $04 / 26 / 82$ & -100 & -30.5 & 13.0 & 270 & 150 & 1.56 & 7.53 \\
\hline B5 & $04 / 26 / 82$ & -105 & -32.0 & 13.1 & 290 & 154 & 1.70 & 7.62 \\
\hline B5 & $04 / 26 / 82$ & -115 & -35.1 & 13.1 & 280 & 140 & 4.50 & 7.53 \\
\hline B5 & $04 / 26 / 82$ & -125 & -38.1 & 13.1 & 255 & 132 & 5.75 & 7.42 \\
\hline B5 & $04 / 26 / 82$ & -135 & -41.1 & 13.1 & 285 & 172 & 8.25 & 7.34 \\
\hline
\end{tabular}


Table 7.--Water-chemistry data from profile well B5, 1981-83--Continued

\begin{tabular}{|c|c|c|c|c|c|c|c|c|}
\hline WELL & DATE & $\begin{array}{l}\text { ELEVA } \\
\text { (FEET) }\end{array}$ & $\begin{array}{l}\text { ATION } \\
\text { (METER) }\end{array}$ & $\begin{array}{l}\text { TEMPER- } \\
\text { ATURE } \\
(\text { DEG C) }\end{array}$ & $\begin{array}{l}\text { SPECIFIC } \\
\text { CONDUCT- } \\
\text { ANCE } \\
\text { (UMHOS) }\end{array}$ & $\begin{array}{c}\text { ALKA- } \\
\text { LINITY } \\
\text { (MG/L } \\
\text { AS } \\
\text { CACO3) }\end{array}$ & $\begin{array}{l}\text { IRON } \\
\text { DIS- } \\
\text { SOLVED } \\
(M G / L)\end{array}$ & $\begin{array}{c}P H \\
(U N \mid T)\end{array}$ \\
\hline B5 & $04 / 26 / 82$ & -140 & -42.7 & 13.1 & 260 & 144 & 28.25 & 6.91 \\
\hline B5 & $04 / 26 / 82$ & -145 & -44.2 & 12.9 & 185 & 92 & .42 & 7.32 \\
\hline B5 & $04 / 26 / 82$ & -150 & -45.7 & 13.1 & 190 & 90 & .09 & 7.75 \\
\hline B5 & $04 / 26 / 82$ & -155 & -47.2 & 13.0 & 205 & 110 & .03 & 8.12 \\
\hline B5 & $04 / 26 / 82$ & -165 & -50.3 & 13.1 & 230 & 130 & .04 & 8.35 \\
\hline B5 & $04 / 26 / 82$ & -170 & -51.8 & 13.1 & 300 & 162 & .36 & 8.22 \\
\hline B5 & $05 / 28 / 82$ & 0 & 0.0 & 12.9 & -- & 84 & 1.26 & 6.72 \\
\hline B5 & $05 / 28 / 82$ & -10 & -3.0 & 12.3 & - & 84 & 11.50 & 6.32 \\
\hline B5 & $05 / 28 / 82$ & -20 & -6.1 & 12.0 & -- & 42 & 12.50 & 5.94 \\
\hline B5 & $05 / 28 / 82$ & -30 & -9.1 & 11.9 & -- & 64 & 22.00 & 6.21 \\
\hline B5 & $05 / 28 / 82$ & -40 & -12.2 & 11.8 & -- & 56 & 20.75 & 6.15 \\
\hline B5 & $05 / 28 / 82$ & -45 & -13.7 & 11.9 & -- & 70 & 22.50 & 6.30 \\
\hline B5 & $05 / 28 / 82$ & -50 & -15.2 & 12.2 & -- & 68 & 25.75 & 6.33 \\
\hline B5 & $05 / 28 / 82$ & -55 & -16.8 & 12.4 & -- & 46 & 22.00 & 6.40 \\
\hline B5 & $05 / 28 / 82$ & -60 & -18.3 & 12.6 & - & 58 & 22.50 & 6.42 \\
\hline 85 & $05 / 28 / 82$ & -65 & -19.8 & 12.6 & -- & 40 & 16.00 & 6.50 \\
\hline B5 & $05 / 28 / 82$ & -70 & $-21 \cdot 3$ & 12.8 & -- & 42 & 6.75 & 6.65 \\
\hline B5 & $05 / 28 / 82$ & -75 & -22.9 & 12.9 & -- & 92 & 10.75 & 6.80 \\
\hline B5 & $05 / 28 / 82$ & -80 & -24.4 & 13.0 & -- & 114 & 7.50 & 6.89 \\
\hline B5 & $05 / 28 / 82$ & -85 & -25.9 & 13.2 & -- & 140 & 4.00 & 7.06 \\
\hline B5 & $05 / 28 / 82$ & -90 & -27.4 & 13.2 & -- & 150 & 3.40 & 7.12 \\
\hline B5 & $05 / 28 / 82$ & -100 & -30.5 & 13.2 & - & 154 & 1.40 & 7.43 \\
\hline B5 & $05 / 28 / 82$ & -105 & -32.0 & 13.2 & -- & 152 & 1.65 & 7.55 \\
\hline B5 & $05 / 28 / 82$ & -115 & -35.1 & 13.2 & -- & 146 & 1.36 & 7.55 \\
\hline B5 & $05 / 28 / 82$ & -125 & -38.1 & 13.2 & - & 140 & 2.70 & 7.43 \\
\hline B5 & $05 / 28 / 82$ & -135 & -41.1 & 13.4 & -- & 138 & 3.30 & 7.40 \\
\hline
\end{tabular}


Table 7.--Water-chem istry data from profile well B5, 1981-83--Continued

\begin{tabular}{|c|c|c|c|c|c|c|c|c|}
\hline WELL & DATE & $\begin{array}{l}\text { ELEV } \\
\text { (FEET) }\end{array}$ & $\begin{array}{l}\text { ATION } \\
\text { (METER) }\end{array}$ & $\begin{array}{l}\text { TEMPER- } \\
\text { ATURE } \\
\text { (DEG C) }\end{array}$ & $\begin{array}{l}\text { SPECIFIC } \\
\text { CONDUCT- } \\
\text { ANCE } \\
\text { (UMHOS) }\end{array}$ & $\begin{array}{c}\text { ALKA- } \\
\text { LINITY } \\
\text { (MG/L } \\
\text { AS } \\
\text { CACO3) }\end{array}$ & $\begin{array}{l}\text { IRON } \\
\text { DIS- } \\
\text { SOLVED } \\
\text { (MG/L) }\end{array}$ & $\begin{array}{c}\text { PH } \\
(\text { UNIT) }\end{array}$ \\
\hline B5 & $05 / 28 / 82$ & -140 & -42.7 & 13.2 & -- & 140 & 25.50 & 6.90 \\
\hline B5 & $05 / 28 / 82$ & -145 & -44.2 & 13.4 & -- & 90 & .74 & 7.10 \\
\hline B5 & $05 / 28 / 82$ & -150 & -45.7 & 13.4 & -- & 72 & .08 & 7.70 \\
\hline B5 & $05 / 28 / 82$ & -155 & -47.2 & 13.4 & -- & 110 & .02 & 8.00 \\
\hline B5 & $05 / 28 / 82$ & -165 & -50.3 & 13.6 & -- & 132 & .03 & 8.25 \\
\hline B5 & $05 / 28 / 82$ & -170 & -51.8 & 13.7 & - & 154 & .28 & 8.18 \\
\hline B5 & $06 / 21 / 82$ & 0 & 0.0 & 13.1 & -- & 106 & 3.10 & 6.80 \\
\hline B5 & $06 / 21 / 82$ & -10 & -3.0 & 12.7 & -- & 80 & 11.25 & 6.42 \\
\hline B5 & $06 / 21 / 82$ & -20 & -6.1 & 12.3 & -- & 44 & 13.20 & 5.99 \\
\hline B5 & $06 / 21 / 82$ & -30 & -9.1 & 12.0 & -- & 48 & 18.50 & 6.16 \\
\hline B5 & $06 / 21 / 82$ & -40 & -12.2 & 12.1 & -- & 54 & 19.00 & 6.12 \\
\hline B5 & $06 / 21 / 82$ & -45 & -13.7 & 12.2 & -- & 44 & 16.50 & 6.22 \\
\hline B5 & $06 / 21 / 82$ & -50 & -15.2 & 12.4 & -- & 44 & 18.50 & 6.26 \\
\hline B5 & $06 / 21 / 82$ & -55 & -16.8 & 12.4 & -- & 76 & 25.70 & 6.22 \\
\hline B5 & $06 / 21 / 82$ & -60 & -18.3 & 12.6 & -- & 72 & 30.50 & 6.23 \\
\hline B5 & $06 / 21 / 82$ & -65 & -19.8 & 12.6 & -- & 52 & 24.00 & 6.28 \\
\hline B5 & $06 / 21 / 82$ & -70 & -21.3 & 12.8 & -- & 50 & 9.50 & 6.50 \\
\hline B5 & $06 / 21 / 82$ & -75 & -22.9 & 13.0 & -- & 86 & 13.25 & 6.69 \\
\hline B5 & $06 / 21 / 82$ & -80 & -24.4 & 13.1 & -- & 120 & 8.50 & 6.81 \\
\hline B5 & $06 / 21 / 82$ & -85 & -25.9 & 13.2 & -- & 140 & 6.50 & 6.93 \\
\hline B5 & $06 / 21 / 82$ & -90 & -27.4 & 13.2 & -- & 144 & 5.25 & 6.95 \\
\hline B5 & $06 / 21 / 82$ & -100 & -30.5 & 13.3 & -- & 160 & 1.39 & 7.25 \\
\hline B5 & $06 / 21 / 82$ & -105 & -32.0 & 13.3 & -- & 154 & 1.65 & 7.32 \\
\hline B5 & $06 / 21 / 82$ & -115 & -35.1 & 13.3 & -- & 164 & 1.86 & 7.34 \\
\hline B5 & $06 / 21 / 82$ & -125 & -38.1 & 13.6 & -- & 140 & 2.30 & 7.33 \\
\hline B5 & $06 / 21 / 82$ & -135 & -41.1 & 13.7 & -- & 136 & 3.40 & 7.30 \\
\hline
\end{tabular}


Table 7.--Water-chemistry data from profile well B5, 1981-83--Continued

\begin{tabular}{|c|c|c|c|c|c|c|c|c|}
\hline WELL & DATE & $\begin{array}{l}\text { ELEVA } \\
\text { (FEET) }\end{array}$ & $\begin{array}{l}\text { ATION } \\
\text { (METER) }\end{array}$ & $\begin{array}{l}\text { TEMPER- } \\
\text { ATURE } \\
\left(\begin{array}{ll}\text { DEG } & \text { C) }\end{array}\right.\end{array}$ & $\begin{array}{l}\text { SPECIFIC } \\
\text { CONDUCT- } \\
\text { ANCE } \\
\text { (UMHOS) }\end{array}$ & $\begin{array}{c}\text { ALKA- } \\
\text { LINITY } \\
\text { (MG/L } \\
\text { AS } \\
\text { CACO3) }\end{array}$ & $\begin{array}{l}\text { IRON } \\
\text { DIS- } \\
\text { SOLVED } \\
(M G / L)\end{array}$ & $\begin{array}{c}\text { PH } \\
\text { (UNIT) }\end{array}$ \\
\hline B5 & $06 / 21 / 82$ & -140 & -42.7 & 13.4 & -- & 134 & 24.50 & 6.69 \\
\hline B5 & $06 / 21 / 82$ & -145 & -44.2 & 13.8 & -- & 90 & 1.12 & 6.88 \\
\hline B5 & $06 / 21 / 82$ & -150 & -45.7 & 13.5 & -- & 72 & .08 & 7.48 \\
\hline B5 & $06 / 21 / 82$ & -155 & -47.2 & 13.6 & -- & 114 & .03 & 7.73 \\
\hline B5 & $06 / 21 / 82$ & -165 & -50.3 & 13.5 & -- & 136 & .04 & 8.10 \\
\hline B5 & $06 / 21 / 82$ & -170 & -51.8 & 13.7 & -- & 150 & .33 & 7.92 \\
\hline B5 & $07 / 19 / 82$ & 0 & 0 & 14.3 & -- & 52 & 2.25 & 6.72 \\
\hline B5 & $07 / 19 / 82$ & -10 & -3.0 & 13.9 & -- & 80 & 11.75 & 6.49 \\
\hline B5 & $07 / 19 / 82$ & -20 & -6.1 & 13.8 & -- & 44 & 13.00 & 6.02 \\
\hline B5 & $07 / 19 / 82$ & -30 & -9.1 & 13.1 & -- & 62 & 21.00 & 6.41 \\
\hline B5 & $07 / 19 / 82$ & -40 & -12.2 & 13.2 & -- & 64 & 19.00 & 6.42 \\
\hline B5 & $07 / 19 / 82$ & -45 & -13.7 & 13.3 & - & 66 & 23.25 & 6.62 \\
\hline B5 & $07 / 19 / 82$ & -50 & -15.2 & 13.2 & -- & 68 & 23.75 & 6.32 \\
\hline B5 & $07 / 19 / 82$ & -55 & -16.8 & 13.4 & -- & 62 & 21.50 & 6.48 \\
\hline B5 & $07 / 19 / 82$ & -60 & -18.3 & 13.4 & -- & 70 & 30.25 & 6.51 \\
\hline B5 & $07 / 19 / 82$ & -65 & -19.8 & 13.4 & -- & 68 & 30.50 & 6.62 \\
\hline B5 & $07 / 19 / 82$ & -70 & -21.3 & 13.5 & -- & 62 & 11.25 & 7.02 \\
\hline B5 & $07 / 19 / 82$ & -75 & -22.9 & 13.9 & -- & 120 & 14.25 & 7.20 \\
\hline B5 & $07 / 19 / 82$ & -80 & -24.4 & 14.1 & -- & 118 & 8.00 & 7.42 \\
\hline B5 & $07 / 19 / 82$ & -85 & -25.9 & 14.2 & -- & 156 & 8.00 & 6.79 \\
\hline B5 & $07 / 19 / 82$ & -90 & -27.4 & 14.2 & -- & 152 & 6.50 & 6.69 \\
\hline B5 & $07 / 19 / 82$ & -100 & -30.5 & 14.2 & -- & 150 & 1.52 & 7.00 \\
\hline B5 & $07 / 19 / 82$ & -105 & -32.0 & 14.3 & -- & 182 & 1.75 & 7.22 \\
\hline B5 & $07 / 19 / 82$ & -115 & -35.1 & 14.4 & - & 174 & 1.92 & 7.60 \\
\hline B5 & $07 / 19 / 82$ & -125 & -38.1 & 14.4 & -- & 170 & 2.21 & 7.73 \\
\hline B5 & $07 / 19 / 82$ & -135 & -41.1 & 14.8 & -- & 152 & 3.20 & 7.75 \\
\hline
\end{tabular}


Table 7.--Water-chemistry data from profile well B5, 1981-83--Continued

\begin{tabular}{|c|c|c|c|c|c|c|c|c|}
\hline WELL & DATE & $\begin{array}{l}\text { ELEVA } \\
\text { (FEET) }\end{array}$ & $\begin{array}{l}\text { ITION } \\
\text { (METER) }\end{array}$ & $\begin{array}{l}\text { TEMPER- } \\
\text { ATURE } \\
\left(\begin{array}{lll}\text { DEG } & \text { C) }\end{array}\right.\end{array}$ & $\begin{array}{l}\text { SPECIFIC } \\
\text { CONDUCT- } \\
\text { ANCE } \\
\text { (UMHOS) }\end{array}$ & $\begin{array}{c}\text { ALKA- } \\
\text { LINITY } \\
\text { (MG/L } \\
\text { AS } \\
\text { CACO3) }\end{array}$ & $\begin{array}{l}\text { IRON } \\
\text { DIS- } \\
\text { SOLVED } \\
\text { (MG/L) }\end{array}$ & $\begin{array}{c}\mathrm{PH} \\
(\mathrm{UN} \mid \mathrm{T})\end{array}$ \\
\hline B5 & $07 / 19 / 82$ & -140 & -42.7 & 14.7 & -- & 124 & 21.50 & 7.23 \\
\hline B5 & $07 / 19 / 82$ & -145 & -44.2 & 14.7 & - & 82 & 1.37 & 7.56 \\
\hline B5 & $07 / 19 / 82$ & -150 & -45.7 & 14.6 & -- & 72 & .08 & 8.10 \\
\hline B5 & $07 / 19 / 82$ & -155 & -47.2 & 14.7 & -- & 134 & .03 & 8.50 \\
\hline B5 & $07 / 19 / 82$ & -165 & -50.3 & 14.7 & -- & 134 & .07 & 8.83 \\
\hline B5 & $07 / 19 / 82$ & -170 & -51.8 & 15.0 & -- & 158 & .28 & 8.72 \\
\hline B5 & $08 / 17 / 82$ & 0 & 0.0 & 14.2 & 210 & 100 & 3.40 & 6.43 \\
\hline B5 & $08 / 17 / 82$ & -10 & -3.0 & 14.4 & 190 & 86 & 11.00 & 6.40 \\
\hline B5 & $08 / 17 / 82$ & -20 & -6.1 & 14.1 & 130 & 46 & 12.75 & 6.03 \\
\hline B5 & $08 / 17 / 82$ & -30 & -9.1 & 14.0 & 155 & 64 & 19.50 & 6.25 \\
\hline B5 & $08 / 17 / 82$ & -40 & -12.2 & 14.1 & 150 & 58 & 18.25 & 6.30 \\
\hline B5 & $08 / 17 / 82$ & -45 & -13.7 & 14.3 & 160 & 66 & 21.50 & 6.32 \\
\hline B5 & $08 / 17 / 82$ & -50 & -15.2 & 14.3 & 165 & 70 & 23.50 & 6.46 \\
\hline B5 & $08 / 17 / 82$ & -55 & -16.8 & 13.8 & 160 & 68 & 24.00 & 6.47 \\
\hline B5 & $08 / 17 / 82$ & -60 & -18.3 & 13.8 & 170 & 72 & 29.00 & 6.38 \\
\hline B5 & $08 / 17 / 82$ & -65 & -19.8 & 13.8 & 155 & 60 & 29.50 & 6.31 \\
\hline B5 & $08 / 17 / 82$ & -70 & -21.3 & 14.1 & 140 & 62 & 11.75 & 6.50 \\
\hline B5 & $08 / 17 / 82$ & -75 & -22.9 & 14.3 & 230 & 124 & 14.25 & 6.75 \\
\hline B5 & $08 / 17 / 82$ & -80 & -24.4 & 14.6 & 230 & 120 & 7.50 & 6.82 \\
\hline $\begin{array}{l}\text { B5 } \\
\text { B5 }\end{array}$ & $\begin{array}{l}08 / 17 / 82 \\
08 / 17 / 82\end{array}$ & $\begin{array}{l}-85 \\
-90\end{array}$ & $\begin{array}{l}-25.9 \\
-27.4\end{array}$ & $\begin{array}{l}14.6 \\
14.7\end{array}$ & $\begin{array}{l}250 \\
275\end{array}$ & $\begin{array}{l}134 \\
144\end{array}$ & $\begin{array}{l}6.75 \\
5.50\end{array}$ & $\begin{array}{l}6.80 \\
6.99\end{array}$ \\
\hline B5 & $08 / 17 / 82$ & -100 & -30.5 & 14.8 & 275 & 150 & 1.90 & 7.37 \\
\hline B5 & $08 / 17 / 82$ & -105 & -32.0 & 14.8 & 290 & 158 & 1.50 & 7.62 \\
\hline B5 & $08 / 17 / 82$ & -115 & -35.1 & 15.0 & 310 & 170 & 1.85 & 7.54 \\
\hline B5 & $08 / 17 / 82$ & -125 & -38.1 & 15.4 & 310 & 158 & 2.40 & 7.54 \\
\hline B5 & $08 / 17 / 82$ & -135 & -41.1 & 15.6 & 280 & 152 & 3.25 & 7.50 \\
\hline
\end{tabular}


Table 7.-- Water-chem istry data from profile
well B5, 1981-83--Continued

\begin{tabular}{|c|c|c|c|c|c|c|c|c|}
\hline WELL & DATE & $\begin{array}{l}\text { ELEVA } \\
\text { (FEET) }\end{array}$ & $\begin{array}{l}\text { ATION } \\
\text { (METER) }\end{array}$ & $\begin{array}{l}\text { TEMPER- } \\
\text { ATURE } \\
(D E G \quad C)\end{array}$ & $\begin{array}{l}\text { SPECIFIC } \\
\text { CONDUCT- } \\
\text { ANCE } \\
\text { (UMHOS) }\end{array}$ & $\begin{array}{c}\text { ALKA- } \\
\text { LINITY } \\
\text { (MG/L } \\
\text { AS } \\
\text { CACO3) }\end{array}$ & $\begin{array}{l}\text { IRON } \\
\text { DIS- } \\
\text { SOLVED } \\
\text { (MG/L) }\end{array}$ & $\begin{array}{c}\mathrm{PH} \\
(\mathrm{UN} \mid \mathrm{T})\end{array}$ \\
\hline B5 & $08 / 17 / 82$ & -140 & -42.7 & 15.3 & 270 & 132 & 18.75 & 7.00 \\
\hline B5 & $08 / 17 / 82$ & -145 & -44.2 & 15.5 & 185 & 72 & 1.90 & 7.20 \\
\hline B5 & $08 / 17 / 82$ & -150 & -45.7 & 15.7 & 205 & 74 & .22 & 7.85 \\
\hline B5 & $08 / 17 / 82$ & -155 & -47.2 & 15.4 & 230 & 124 & .03 & 8.00 \\
\hline B5 & $08 / 17 / 82$ & -165 & -50.3 & 15.6 & 255 & 136 & .02 & 8.39 \\
\hline B5 & $08 / 17 / 82$ & -170 & -51.8 & 15.9 & 335 & 158 & .29 & 8.19 \\
\hline B5 & $09 / 17 / 82$ & 0 & 0.0 & 14.1 & 135 & 72 & 3.30 & 6.65 \\
\hline B5 & $09 / 17 / 82$ & -10 & -3.0 & 13.6 & 150 & 80 & 9.00 & 6.54 \\
\hline B5 & $09 / 17 / 82$ & -20 & -6.1 & 13.3 & 130 & 56 & 13.75 & 6.05 \\
\hline B5 & $09 / 17 / 82$ & -30 & -9.1 & 13.0 & 150 & 62 & 21.25 & 6.22 \\
\hline B5 & $09 / 17 / 82$ & -40 & -12.2 & 13.2 & 150 & 58 & 19.75 & 6.31 \\
\hline B5 & $09 / 17 / 82$ & -45 & -13.7 & 13.2 & 155 & 66 & 22.25 & 6.39 \\
\hline B5 & $09 / 17 / 82$ & -50 & -15.2 & 13.1 & 160 & 68 & 24.00 & 6.38 \\
\hline $\begin{array}{l}\text { B5 } \\
\text { B5 }\end{array}$ & $\begin{array}{l}09 / 17 / 82 \\
09 / 17 / 82\end{array}$ & $\begin{array}{l}-55 \\
-60\end{array}$ & $\begin{array}{l}-16.8 \\
-18.3\end{array}$ & $\begin{array}{l}13.1 \\
13.2\end{array}$ & $\begin{array}{l}150 \\
160\end{array}$ & $\begin{array}{l}66 \\
68\end{array}$ & $\begin{array}{l}25.25 \\
28.00\end{array}$ & $\begin{array}{l}6.42 \\
6.40\end{array}$ \\
\hline B5 & $09 / 17 / 82$ & -65 & -19.8 & 13.2 & 160 & 72 & 33.75 & 6.46 \\
\hline B5 & $09 / 17 / 82$ & -70 & $-21 \cdot 3$ & 13.6 & 140 & 60 & 13.25 & 6.64 \\
\hline B5 & $09 / 17 / 82$ & -75 & -22.9 & 13.8 & 200 & 112 & 13.25 & 6.88 \\
\hline B5 & $09 / 17 / 82$ & -80 & -24.4 & 13.9 & 225 & 126 & 7.50 & 7.00 \\
\hline B5 & $09 / 17 / 82$ & -85 & -25.9 & 14.0 & 255 & 144 & 9.25 & 7.10 \\
\hline B5 & $09 / 17 / 82$ & -90 & -27.4 & 13.9 & 250 & 138 & 6.50 & 7.10 \\
\hline B5 & $09 / 17 / 82$ & -100 & -30.5 & 14.1 & 295 & 160 & 4.40 & 7.18 \\
\hline B5 & $09 / 17 / 82$ & -105 & -32.0 & 14.2 & 270 & 160 & 1.82 & 7.44 \\
\hline B5 & $09 / 17 / 82$ & -115 & -35.1 & 14.3 & 290 & 170 & 1.90 & 7.55 \\
\hline B5 & $09 / 17 / 82$ & -125 & -38.1 & 14.4 & 295 & 170 & 2.35 & 7.20 \\
\hline B5 & $09 / 17 / 82$ & -135 & -41.1 & 15.1 & 305 & 172 & 2.90 & 7.32 \\
\hline B5 & $09 / 17 / 82$ & -140 & -42.7 & 14.8 & 265 & 128 & 18.50 & 6.85 \\
\hline
\end{tabular}


Table 7.--Water-chemistry data from profile well B5, 1981-83--Continued

\begin{tabular}{|c|c|c|c|c|c|c|c|c|}
\hline WELL & DATE & $\begin{array}{l}\text { ELEVA } \\
\text { (FEET) }\end{array}$ & $\begin{array}{l}\text { AT ION } \\
\text { (METER) }\end{array}$ & $\begin{array}{l}\text { TEMPER- } \\
\text { ATURE } \\
(D E G \quad C)\end{array}$ & $\begin{array}{l}\text { SPECIFIC } \\
\text { CONDUCT- } \\
\text { ANCE } \\
\text { (UMHOS) }\end{array}$ & $\begin{array}{c}\text { ALKA- } \\
\text { LINITY } \\
\text { (MG/L } \\
\text { AS } \\
\text { CACO3) }\end{array}$ & $\begin{array}{l}\text { IRON } \\
\text { DIS- } \\
\text { SOLVED } \\
\text { (MG/L) }\end{array}$ & $\begin{array}{c}\mathrm{PH} \\
(\mathrm{UN} \mid \mathrm{T})\end{array}$ \\
\hline B5 & $09 / 17 / 82$ & -145 & -44.2 & 14.9 & 175 & 64 & 2.95 & 7.10 \\
\hline B5 & $09 / 17 / 82$ & -150 & -45.7 & 15.1 & 200 & 68 & .12 & 7.68 \\
\hline B5 & $09 / 17 / 82$ & -155 & -47.2 & 14.9 & 250 & 130 & .06 & 8.00 \\
\hline B5 & $09 / 17 / 82$ & -165 & -50.3 & 14.7 & 245 & 130 & .05 & 8.10 \\
\hline B5 & $09 / 17 / 82$ & -170 & -51.8 & 15.1 & 325 & 164 & .33 & 7.90 \\
\hline B5 & $10 / 18 / 82$ & 0 & 0 & 13.5 & 155 & 70 & 3.90 & 6.60 \\
\hline B5 & $10 / 18 / 82$ & -10 & -3.0 & 13.1 & 110 & 56 & 6.75 & 6.45 \\
\hline B5 & $10 / 18 / 82$ & -20 & -6.1 & 12.8 & 135 & 56 & 16.00 & 5.85 \\
\hline B5 & $10 / 18 / 82$ & -30 & -9.1 & 12.7 & 150 & 64 & 23.50 & 6.10 \\
\hline B5 & $10 / 18 / 82$ & -40 & -12.2 & 12.9 & 150 & 60 & 21.00 & 6.12 \\
\hline B5 & $10 / 18 / 82$ & -45 & -13.7 & 12.9 & 150 & 62 & 25.75 & 6.09 \\
\hline B5 & $10 / 18 / 82$ & -50 & -15.2 & 13.0 & 130 & 56 & 20.25 & 6.20 \\
\hline B5 & $10 / 18 / 82$ & -55 & -16.8 & 13.1 & 130 & 56 & 21.75 & 6.32 \\
\hline B5 & $10 / 18 / 82$ & -60 & -18.3 & 13.1 & 150 & 66 & 27.25 & 6.32 \\
\hline B5 & $10 / 18 / 82$ & -65 & -19.8 & 13.2 & 160 & 74 & 33.00 & 6.46 \\
\hline B5 & $10 / 18 / 82$ & -70 & -21.3 & 13.5 & 170 & 90 & 18.00 & 6.62 \\
\hline B5 & $10 / 18 / 82$ & -75 & -22.9 & 13.7 & 195 & 106 & 12.50 & 6.85 \\
\hline B5 & $10 / 18 / 82$ & -80 & -24.4 & 14.0 & 250 & 140 & 9.25 & 7.02 \\
\hline B5 & $10 / 18 / 82$ & -85 & -25.9 & 14.1 & 290 & 152 & 10.75 & 7.09 \\
\hline B5 & $10 / 18 / 82$ & -90 & -27.4 & 14.2 & 285 & 134 & 7.25 & 6.99 \\
\hline B5 & $10 / 18 / 82$ & -100 & -30.5 & 14.8 & 260 & 140 & 6.00 & 7.19 \\
\hline B5 & $10 / 18 / 82$ & -105 & -32.0 & 14.9 & 265 & 152 & 2.90 & 7.32 \\
\hline B5 & $10 / 18 / 82$ & -115 & -35.1 & 14.9 & 280 & 160 & 1.84 & 7.54 \\
\hline B5 & $10 / 18 / 82$ & -125 & -38.1 & 15.2 & 305 & 172 & 2.10 & 7.25 \\
\hline B5 & $10 / 18 / 82$ & -135 & -41.1 & 15.8 & 280 & 148 & 2.40 & 7.59 \\
\hline B5 & $10 / 18 / 82$ & -140 & -42.7 & 15.7 & 270 & 124 & 16.25 & 6.78 \\
\hline
\end{tabular}


Table 7.-- Water-chemistry data from profile well B5, 1981-83--Continued

\begin{tabular}{|c|c|c|c|c|c|c|c|c|}
\hline WELL & DATE & $\begin{array}{c}\text { ELEV } \\
\text { (FEET) }\end{array}$ & $\begin{array}{l}\text { TION } \\
\text { (METER) }\end{array}$ & $\begin{array}{l}\text { TEMPER- } \\
\text { ATURE } \\
\text { (DEG C) }\end{array}$ & $\begin{array}{l}\text { SPECIFIC } \\
\text { CONDUCT- } \\
\text { ANCE } \\
\text { (UMHOS) }\end{array}$ & $\begin{array}{c}\text { ALKA- } \\
\text { LINITY } \\
\text { (MG/L } \\
\text { AS } \\
\text { CACO3) }\end{array}$ & $\begin{array}{l}\text { IRON } \\
\text { DIS- } \\
\text { SOLVED } \\
\text { (MG/L) }\end{array}$ & $\begin{array}{c}\mathrm{PH} \\
(\mathrm{UN} I T)\end{array}$ \\
\hline B5 & $10 / 18 / 82$ & -145 & -44.2 & 15.6 & 170 & 64 & 3.05 & 7.00 \\
\hline B5 & $10 / 18 / 82$ & -150 & -45.7 & 15.9 & 205 & 74 & .11 & 7.47 \\
\hline B5 & $10 / 18 / 82$ & -155 & -47.2 & 15.9 & 260 & 134 & .06 & 7.56 \\
\hline B5 & $10 / 18 / 82$ & -165 & -50.3 & 16.2 & 250 & 130 & .07 & 8.00 \\
\hline B5 & $10 / 18 / 82$ & -170 & -51.8 & 16.2 & 340 & 168 & .35 & 8.01 \\
\hline B5 & $11 / 23 / 82$ & 0 & 0 & 13.1 & 140 & 52 & 4.10 & 6.50 \\
\hline B5 & $11 / 23 / 82$ & -10 & -3.0 & 12.9 & 130 & 62 & 7.75 & 6.40 \\
\hline B5 & $11 / 23 / 82$ & -20 & -6.1 & 12.8 & 130 & 60 & 15.25 & 6.05 \\
\hline B5 & $11 / 23 / 82$ & -30 & -9.1 & 12.4 & 150 & 60 & 20.75 & 6.12 \\
\hline B5 & $11 / 23 / 82$ & -40 & -12.2 & 12.6 & 140 & 60 & 18.00 & 6.09 \\
\hline B5 & $11 / 23 / 82$ & -45 & -13.7 & 12.8 & 150 & 68 & 23.50 & 5.62 \\
\hline B5 & $11 / 23 / 82$ & -50 & -15.2 & 12.7 & 155 & 68 & 25.00 & 5.83 \\
\hline B5 & $11 / 23 / 82$ & -55 & -16.8 & 12.9 & 135 & 66 & 25.50 & 6.00 \\
\hline B5 & $11 / 30 / 82$ & -60 & -18.3 & 12.8 & 140 & 66 & 28.50 & 6.03 \\
\hline B5 & $11 / 30 / 82$ & -65 & -19.8 & 13.0 & 140 & 72 & 33.75 & 6.13 \\
\hline B5 & $11 / 30 / 82$ & -70 & -21.3 & 13.1 & 165 & 80 & 19.00 & 6.28 \\
\hline B5 & $11 / 30 / 82$ & -75 & -22.9 & 12.7 & 140 & 86 & 12.25 & 6.30 \\
\hline B5 & $11 / 30 / 82$ & -80 & -24.4 & 12.9 & 180 & 114 & 8.25 & 6.71 \\
\hline B5 & $11 / 30 / 82$ & -85 & -25.9 & 12.9 & 190 & 128 & 8.25 & 6.85 \\
\hline B5 & $11 / 30 / 82$ & -90 & -27.4 & 12.9 & 200 & 132 & 6.50 & 6.89 \\
\hline B5 & $11 / 30 / 82$ & -100 & -30.5 & 13.1 & 270 & 150 & 7.00 & 6.82 \\
\hline B5 & $11 / 30 / 82$ & -105 & -32.0 & 13.0 & 285 & 144 & 5.25 & 6.99 \\
\hline B5 & $11 / 30 / 82$ & -115 & -35.1 & 13.1 & 310 & 152 & 2.25 & 7.15 \\
\hline B5 & $11 / 30 / 82$ & -125 & -38.1 & 13.1 & 260 & 150 & 1.76 & 7.14 \\
\hline B5 & $11 / 30 / 82$ & -135 & -41.1 & 13.6 & 280 & 174 & 2.05 & 7.15 \\
\hline
\end{tabular}


Table 7.--Water-chemistry data from profile well B5, 1981-83--Continued

\begin{tabular}{|c|c|c|c|c|c|c|c|c|}
\hline WELL & DATE & $\begin{array}{l}\text { ELEV } \\
\text { (FEET) }\end{array}$ & $\begin{array}{l}\text { TION } \\
\text { (METER) }\end{array}$ & $\begin{array}{l}\text { TEMPER- } \\
\text { ATURE } \\
\text { (DEG C) }\end{array}$ & $\begin{array}{l}\text { SPECIFIC } \\
\text { CONDUCT- } \\
\text { ANCE } \\
\text { (UMHOS) }\end{array}$ & $\begin{array}{c}\text { ALKA- } \\
\text { LINITY } \\
\text { (MG/L } \\
\text { AS } \\
\text { CACO3) }\end{array}$ & $\begin{array}{l}\text { IRON } \\
\text { DIS- } \\
\text { SOLVED } \\
\text { (MG/L) }\end{array}$ & $\begin{array}{c}\text { PH } \\
(U N I T)\end{array}$ \\
\hline B5 & $11 / 30 / 82$ & -140 & -42.7 & 13.2 & 280 & 148 & 11.00 & 6.72 \\
\hline B5 & $11 / 30 / 82$ & -145 & $-44 \cdot 2$ & 13.1 & 165 & 68 & 5.50 & 6.60 \\
\hline B5 & $11 / 30 / 82$ & -150 & -45.7 & 13.3 & 190 & 72 & .13 & 7.00 \\
\hline B5 & $11 / 30 / 82$ & -155 & -47.2 & 13.3 & 200 & 110 & .05 & 7.32 \\
\hline B5 & $11 / 30 / 82$ & -165 & -50.3 & 13.1 & 230 & 140 & .05 & 7.60 \\
\hline B5 & $11 / 30 / 82$ & -170 & -51.8 & 13.6 & 315 & 162 & .32 & 7.41 \\
\hline B5 & $01 / 10 / 83$ & 0 & 0 & 12.5 & 180 & 88 & 6.50 & 6.46 \\
\hline B5 & $01 / 10 / 83$ & -10 & -3.0 & 13.8 & 150 & 74 & 8.25 & 6.45 \\
\hline B5 & $01 / 10 / 83$ & -20 & -6.1 & 13.6 & 130 & 62 & 14.50 & 6.25 \\
\hline B5 & $01 / 10 / 83$ & -30 & -9.1 & 13.0 & 145 & 62 & 19.00 & 6.30 \\
\hline B5 & $01 / 10 / 83$ & -40 & -12.2 & 13.1 & 130 & 58 & 14.45 & 6.29 \\
\hline B5 & $01 / 10 / 83$ & -45 & -13.7 & 13.0 & 160 & 72 & 23.50 & 6.32 \\
\hline B5 & $01 / 10 / 83$ & -50 & -15.2 & 13.1 & 160 & 74 & 26.00 & 6.45 \\
\hline B5 & $01 / 10 / 83$ & -55 & -16.8 & 13.1 & 170 & 80 & 27.50 & 6.42 \\
\hline B5 & $01 / 10 / 83$ & -60 & -18.3 & 12.9 & 155 & 68 & 27.25 & 6.43 \\
\hline B5 & $01 / 10 / 83$ & -65 & -19.8 & 13.0 & 160 & 76 & 30.25 & 6.48 \\
\hline B5 & $01 / 10 / 83$ & -70 & -21.3 & 13.1 & 180 & 92 & 22.50 & 6.58 \\
\hline B5 & $01 / 10 / 83$ & -75 & -22.9 & 12.9 & 200 & 112 & 15.50 & 6.82 \\
\hline B5 & $01 / 10 / 83$ & -80 & -24.4 & 13.1 & 200 & 110 & 7.75 & 7.00 \\
\hline B5 & $01 / 10 / 83$ & -85 & -25.9 & 13.1 & 275 & 156 & 10.30 & 7.02 \\
\hline B5 & $01 / 10 / 83$ & -90 & -27.4 & 13.1 & 280 & 158 & 8.00 & 7.11 \\
\hline B5 & $01 / 10 / 83$ & -100 & -30.5 & 13.1 & 270 & 154 & 7.00 & 7.13 \\
\hline B5 & $01 / 10 / 83$ & -105 & -32.0 & 13.4 & 260 & 150 & 6.25 & 7.15 \\
\hline B5 & $01 / 10 / 83$ & -115 & -35.1 & 13.4 & 280 & 166 & 6.75 & 7.30 \\
\hline B5 & $01 / 10 / 83$ & -125 & -38.1 & 13.3 & 300 & 170 & 1.95 & 7.40 \\
\hline B5 & $01 / 10 / 83$ & -135 & -41.1 & 13.7 & 280 & 164 & 1.70 & 7.52 \\
\hline
\end{tabular}


Table 7.--Water-chemistry data from profile well B5, 1981-83--Continued

\begin{tabular}{|c|c|c|c|c|c|c|c|c|}
\hline WELL & DATE & $\begin{array}{l}\text { ELEVA } \\
\text { (FEET) }\end{array}$ & $\begin{array}{l}\text { ATION } \\
\text { (METER) }\end{array}$ & $\begin{array}{l}\text { TEMPER- } \\
\text { ATURE } \\
(D E G \quad C)\end{array}$ & $\begin{array}{l}\text { SPECIFIC } \\
\text { CONDUCT- } \\
\text { ANCE } \\
\text { (UMHOS) }\end{array}$ & $\begin{array}{c}\text { ALKA- } \\
\text { LINITY } \\
\text { (MG/L } \\
\text { AS } \\
\text { CACO3) }\end{array}$ & $\begin{array}{l}\text { IRON } \\
\text { DIS- } \\
\text { SOLVED } \\
\text { (MG/L) }\end{array}$ & $\begin{array}{c}\mathrm{PH} \\
(\mathrm{UNIT})\end{array}$ \\
\hline B5 & $01 / 10 / 83$ & -140 & -42.7 & 13.4 & 280 & 138 & 8.25 & 7.30 \\
\hline B5 & $01 / 10 / 83$ & -145 & -44.2 & 13.4 & 175 & 78 & 6.75 & 7.10 \\
\hline B5 & $01 / 10 / 83$ & -150 & -45.7 & 13.7 & 190 & 66 & .27 & 7.80 \\
\hline B5 & $01 / 10 / 83$ & -155 & -47.2 & 13.8 & 215 & 118 & .06 & 7.99 \\
\hline B5 & $01 / 10 / 83$ & -165 & -50.3 & 13.6 & 245 & 136 & .05 & 8.30 \\
\hline B5 & $01 / 10 / 83$ & -170 & -51.8 & 14.1 & 310 & 162 & .29 & 8.20 \\
\hline B5 & $02 / 15 / 83$ & 0 & 0.0 & 11.8 & 125 & 56 & 4.50 & 6.20 \\
\hline B5 & $02 / 15 / 83$ & -10 & -3.0 & 13.4 & 160 & 74 & 7.75 & 6.35 \\
\hline B5 & $02 / 15 / 83$ & -20 & -6.1 & 14.2 & 160 & 66 & 16.00 & 6.28 \\
\hline B5 & $02 / 15 / 83$ & -30 & -9.1 & 13.7 & 140 & 66 & 17.75 & 6.19 \\
\hline B5 & $02 / 15 / 83$ & -40 & -12.2 & 13.8 & 140 & 62 & 16.00 & 6.15 \\
\hline B5 & $02 / 15 / 83$ & -45 & -13.7 & 13.6 & 150 & 72 & 20.00 & 6.24 \\
\hline B5 & $02 /: 5 / 83$ & -50 & -15.2 & 13.5 & 155 & 80 & 21.25 & 6.12 \\
\hline B5 & $02 / 15 / 83$ & -55 & -16.8 & 13.4 & 155 & 78 & 22.50 & 6.22 \\
\hline 85 & $02 / 15 / 83$ & -60 & -18.3 & 13.2 & 150 & 68 & 27.25 & 6.20 \\
\hline B5 & $02 / 15 / 83$ & -65 & -19.8 & 13.7 & 150 & 70 & 25.75 & 6.22 \\
\hline B5 & $02 / 15 / 83$ & -70 & -21.3 & 13.6 & 150 & 66 & 16.75 & 6.33 \\
\hline B5 & $02 / 15 / 83$ & -75 & -22.9 & 13.4 & 210 & 116 & 17.00 & 6.53 \\
\hline 85 & $02 / 15 / 83$ & -80 & -24.4 & 13.5 & 220 & 126 & 13.25 & 6.73 \\
\hline B5 & $02 / 15 / 83$ & -85 & -25.9 & 13.4 & 220 & 124 & 7.75 & 6.90 \\
\hline B5 & $02 / 15 / 83$ & -90 & -27.4 & 13.4 & 250 & 148 & 6.50 & 7.00 \\
\hline B5 & $02 / 15 / 83$ & -100 & -30.5 & 13.4 & 280 & 148 & 6.75 & 6.91 \\
\hline B5 & $02 / 15 / 83$ & -105 & -32.0 & 13.5 & 280 & 144 & 6.00 & 6.85 \\
\hline B5 & $02 / 15 / 83$ & -115 & -35.1 & 13.5 & 285 & 150 & 4.25 & 7.06 \\
\hline B5 & $02 / 15 / 83$ & -125 & -38.1 & 13.7 & 300 & 140 & 1.74 & 7.17 \\
\hline B5 & $02 / 15 / 83$ & -135 & -41.1 & 13.6 & 280 & 162 & 1.56 & 7.12 \\
\hline
\end{tabular}


Table 7.--Water-chemistry data from profile well B5, 1981-83--Continued

\begin{tabular}{|c|c|c|c|c|c|c|c|c|}
\hline WELL & DATE & $\begin{array}{l}\text { ELEVA } \\
\text { (FEET) }\end{array}$ & $\begin{array}{l}\text { ATION } \\
\text { (METER) }\end{array}$ & 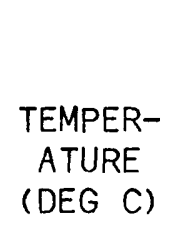 & $\begin{array}{l}\text { SPECIFIC } \\
\text { CONDUCT- } \\
\text { ANCE } \\
\text { (UMHOS) }\end{array}$ & $\begin{array}{c}\text { ALKA- } \\
\text { LINITY } \\
\text { (MG/L } \\
\text { AS } \\
\text { CACO3) }\end{array}$ & $\begin{array}{l}\text { IRON } \\
\text { DIS- } \\
\text { SOLVED } \\
(M G / L)\end{array}$ & $\begin{array}{c}\mathrm{PH} \\
(\mathrm{UN} \mid \mathrm{T})\end{array}$ \\
\hline B5 & $02 / 15 / 83$ & -140 & -42.7 & 13.4 & 280 & 158 & 5.25 & 6.90 \\
\hline B5 & $02 / 15 / 83$ & -145 & -44.2 & 13.3 & 180 & 80 & 8.00 & 6.55 \\
\hline 85 & $02 / 15 / 83$ & -150 & -45.7 & 13.5 & 155 & 62 & .12 & 7.12 \\
\hline B5 & $02 / 15 / 83$ & -155 & -47.2 & 13.4 & 200 & 96 & .02 & 7.36 \\
\hline B5 & $02 / 15 / 83$ & -165 & -50.3 & 13.2 & 235 & 140 & .03 & 7.71 \\
\hline B5 & $02 / 15 / 83$ & -170 & -51.8 & 13.6 & 330 & 170 & .25 & 7.60 \\
\hline 85 & $03 / 15 / 83$ & 0 & 0 & 11.7 & 80 & 46 & 2.00 & 6.25 \\
\hline B5 & $03 / 15 / 83$ & -10 & -3.0 & 12.9 & 145 & 64 & 5.75 & 6.19 \\
\hline B5 & $03 / 15 / 83$ & -20 & -6.1 & 13.5 & 160 & 66 & 16.25 & 6.12 \\
\hline B5 & $03 / 15 / 83$ & -30 & -9.1 & 13.3 & 145 & 56 & 18.00 & 6.07 \\
\hline B5 & $03 / 15 / 83$ & -40 & -12.2 & 13.6 & 140 & 52 & 16.75 & 6.04 \\
\hline B5 & $03 / 15 / 83$ & -45 & -13.7 & 13.6 & 145 & 58 & 19.00 & 6.13 \\
\hline B5 & $03 / 15 / 83$ & -50 & -15.2 & 13.6 & 140 & 56 & 19.50 & 6.15 \\
\hline B5 & $03 / 15 / 83$ & -55 & -16.8 & 13.7 & 150 & 68 & 22.25 & 6.23 \\
\hline B5 & $03 / 15 / 83$ & -60 & -18.3 & 13.6 & 150 & 76 & 27.75 & 6.25 \\
\hline B5 & $03 / 15 / 83$ & -65 & -19.8 & 13.6 & 160 & 76 & 31.25 & 6.22 \\
\hline B5 & $03 / 15 / 83$ & -70 & -21.3 & 13.8 & 155 & 74 & 20.25 & 6.32 \\
\hline B5 & $03 / 15 / 83$ & -75 & -22.9 & 13.9 & 185 & 94 & 17.00 & 6.54 \\
\hline B5 & $03 / 15 / 83$ & -80 & -24.4 & 13.8 & 220 & 130 & 13.75 & 6.70 \\
\hline B5 & $03 / 15 / 83$ & -85 & -25.9 & 13.8 & 250 & 130 & 9.50 & 6.81 \\
\hline B5 & $03 / 15 / 83$ & -90 & -27.4 & 13.7 & 275 & 142 & 9.50 & 6.91 \\
\hline B5 & $03 / 15 / 83$ & -100 & -30.5 & 13.8 & 255 & 140 & 6.50 & 7.00 \\
\hline B5 & $03 / 15 / 83$ & -105 & -32.0 & 13.9 & 280 & 152 & 6.50 & 7.00 \\
\hline 85 & $03 / 15 / 83$ & -115 & -35.1 & 14.2 & 300 & 142 & 6.50 & 7.01 \\
\hline 85 & $03 / 15 / 83$ & -125 & -38.1 & 14.1 & 330 & 158 & 3.25 & 7.32 \\
\hline B5 & $03 / 15 / 83$ & -135 & -41.1 & 14.3 & 260 & 148 & 1.43 & 7.62 \\
\hline B5 & $03 / 15 / 83$ & -140 & -42.7 & 14.2 & 290 & 152 & 5.50 & 7.27 \\
\hline
\end{tabular}


Table 7.--Water-chemistry data from profile well B5, 1981-83--Continued

\begin{tabular}{|c|c|c|c|c|c|c|c|c|}
\hline WELL & DATE & $\begin{array}{l}\text { ELEVA } \\
\text { (FEET) }\end{array}$ & $\begin{array}{l}\text { ATION } \\
\text { (METER) }\end{array}$ & $\begin{array}{l}\text { TEMPER- } \\
\text { ATURE } \\
\text { (DEG } \\
\text { (D) }\end{array}$ & $\begin{array}{l}\text { SPECIFIC } \\
\text { CONDUCT- } \\
\text { ANCE } \\
\text { (UMHOS) }\end{array}$ & $\begin{array}{c}\text { ALKA- } \\
\text { LIN ITY } \\
\text { (MG/L } \\
\text { AS } \\
\text { CACO3) }\end{array}$ & $\begin{array}{l}\text { IRON } \\
\text { DIS- } \\
\text { SOLVED } \\
\text { (MG/L) }\end{array}$ & $\begin{array}{c}\text { PH } \\
(\text { UNIT) }\end{array}$ \\
\hline B5 & $03 / 15 / 83$ & -145 & -44.2 & 14.3 & 185 & 82 & 9.50 & 6.82 \\
\hline B5 & $03 / 15 / 83$ & -150 & -45.7 & 14.3 & 160 & 64 & .11 & 7.40 \\
\hline B5 & $03 / 15 / 83$ & -155 & -47.2 & 14.4 & 200 & 100 & .03 & 7.76 \\
\hline B5 & $03 / 15 / 83$ & -165 & -50.3 & 14.4 & 250 & 138 & .06 & 8.20 \\
\hline B5 & $03 / 15 / 83$ & -170 & -51.8 & 14.8 & 330 & 164 & .28 & 8.09 \\
\hline B5 & $04 / 23 / 83$ & 0 & 0 & 12.1 & 60 & 32 & 1.35 & 6.55 \\
\hline B5 & $04 / 28 / 83$ & -10 & -3.0 & 12.5 & 120 & 58 & 6.25 & 6.32 \\
\hline B5 & $04 / 28 / 83$ & -20 & -6.1 & 12.7 & 120 & 62 & 14.00 & 6.22 \\
\hline B5 & $04 / 28 / 83$ & -30 & -9.1 & 13.2 & 100 & 48 & 12.25 & 6.15 \\
\hline B5 & $04 / 28 / 83$ & -40 & -12.2 & 13.2 & 80 & 40 & 9.25 & 6.21 \\
\hline B5 & $04 / 28 / 83$ & -45 & -13.7 & 13.2 & 90 & 40 & 11.25 & 6.22 \\
\hline B5 & $04 / 28 / 83$ & -50 & -15.2 & 13.6 & 100 & 50 & 13.75 & 6.28 \\
\hline B5 & $04 / 2^{\urcorner / 83}$ & -55 & -16.8 & 13.7 & 145 & 70 & 21.25 & 6.30 \\
\hline B5 & $04 / 28 / 83$ & -60 & -18.3 & 13.6 & 160 & 72 & 28.00 & 6.35 \\
\hline B5 & $04 / 28 / 83$ & -65 & -19.8 & 13.7 & 140 & 60 & 23.75 & 6.30 \\
\hline B5 & $04 / 28 / 83$ & -70 & -21.3 & 13.8 & 155 & 76 & 24.00 & 6.40 \\
\hline B5 & $04 / 28 / 83$ & -75 & -22.9 & 14.3 & 200 & 98 & 17.50 & 6.59 \\
\hline B5 & $04 / 28 / 83$ & -80 & -24.4 & 13.9 & 250 & 142 & 19.75 & 6.75 \\
\hline B5 & $04 / 28 / 83$ & -85 & -25.9 & 14.1 & 240 & 132 & 8.75 & 6.92 \\
\hline B5 & $04 / 28 / 83$ & -90 & -27.4 & 13.8 & 260 & 138 & 8.50 & 7.05 \\
\hline B5 & $04 / 28 / 83$ & -100 & -30.5 & 14.1 & 290 & 142 & 5.75 & 6.95 \\
\hline B5 & $04 / 28 / 83$ & -105 & -32.0 & 14.1 & 280 & 140 & 6.50 & 7.10 \\
\hline B5 & $04 / 28 / 83$ & -115 & -35.1 & 14.3 & 275 & 148 & 6.00 & 7.00 \\
\hline B5 & $04 / 28 / 83$ & -125 & -38.1 & 14.3 & 290 & 160 & 2.75 & 7.25 \\
\hline B5 & $04 / 28 / 83$ & -135 & -41.1 & 14.4 & 265 & 156 & 1.50 & 7.50 \\
\hline B5 & $04 / 28 / 83$ & -140 & -42.7 & 14.3 & 280 & 158 & 4.00 & 7.40 \\
\hline B5 & $04 / 28 / 83$ & -145 & -44.2 & 14.1 & 190 & 86 & 10.75 & 6.74 \\
\hline
\end{tabular}


Table 7.--Water-chemistry data from profile well B5, 1981-83--Continued

\begin{tabular}{|c|c|c|c|c|c|c|c|c|}
\hline WELL & DATE & $\begin{array}{l}\text { ELEVA } \\
\text { (FEET) }\end{array}$ & $\begin{array}{l}\text { ATION } \\
\text { (METER) }\end{array}$ & $\begin{array}{l}\text { TEMPER- } \\
\text { ATURE } \\
\text { (DEG C) }\end{array}$ & $\begin{array}{l}\text { SPECIFIC } \\
\text { CONDUCT- } \\
\text { ANCE } \\
\text { (UMHOS) }\end{array}$ & $\begin{array}{c}\text { ALKA- } \\
\text { LINITY } \\
\text { (MG/L } \\
\text { AS } \\
\text { CACO3) }\end{array}$ & $\begin{array}{l}\text { IRON } \\
\text { DIS- } \\
\text { SOLVED } \\
(M G / L)\end{array}$ & $\begin{array}{c}\mathrm{PH} \\
(U N \mid T)\end{array}$ \\
\hline B5 & $04 / 28 / 83$ & -150 & -45.7 & 14.4 & 165 & 70 & .14 & 7.50 \\
\hline B5 & $04 / 28 / 83$ & -155 & -47.2 & 14.4 & 200 & 96 & .06 & 7.71 \\
\hline B5 & $04 / 28 / 83$ & -165 & -50.3 & 14.2 & 250 & 140 & .08 & 8.10 \\
\hline B5 & $04 / 28 / 83$ & -170 & -51.8 & 14.4 & 325 & 166 & .25 & 8.00 \\
\hline B5 & $06 / 06 / 83$ & 0 & 0 & 13.0 & 50 & 20 & 1.22 & 7.05 \\
\hline B5 & $06 / 06 / 83$ & -10 & -3.0 & 12.7 & 80 & 40 & 4.00 & 6.62 \\
\hline B5 & $06 / 06 / 83$ & -20 & -6.1 & 12.8 & 105 & 58 & 11.75 & 6.58 \\
\hline B5 & $06 / 06 / 83$ & -30 & -9.1 & - & 60 & 34 & .7 .00 & 6.50 \\
\hline B5 & $06 / 06 / 83$ & -40 & -12.2 & 12.8 & 55 & 30 & 7.00 & 6.43 \\
\hline B5 & $06 / 06 / 83$ & -45 & -13.7 & 13.1 & 60 & 32 & 7.50 & 6.50 \\
\hline B5 & $06 / 06 / 83$ & -50 & -15.2 & 13.1 & 65 & 34 & 8.25 & 6.49 \\
\hline B5 & $06 / 06 / 83$ & -55 & -16.8 & 13.5 & 70 & .34 & 9.50 & 6.55 \\
\hline B5 & $06 / 06 / 83$ & -60 & -18.3 & 13.7 & 125 & 64 & 24.00 & 6.50 \\
\hline B5 & $06 / 06 / 83$ & -65 & -19.8 & 13.6 & 110 & 54 & 20.75 & 6.43 \\
\hline B5 & $06 / 06 / 83$ & -70 & -21.3 & 13.7 & 120 & 62 & 19.50 & 6.45 \\
\hline B5 & $06 / 06 / 83$ & -75 & -22.9 & 13.8 & 185 & 94 & 17.00 & 6.55 \\
\hline B5 & $06 / 06 / 83$ & -80 & -24.4 & 13.9 & 195 & 104 & 16.75 & 6.70 \\
\hline B5 & $06 / 06 / 83$ & -85 & -25.9 & 13.8 & 250 & 134 & 9.00 & 6.92 \\
\hline B5 & $06 / 06 / 83$ & -90 & -27.4 & 13.9 & 240 & 128 & 7.50 & 7.04 \\
\hline B5 & $06 / 06 / 83$ & -100 & -30.5 & 13.8 & 280 & 152 & 6.25 & 7.10 \\
\hline B5 & $06 / 06 / 83$ & -105 & -32.0 & 14.1 & 290 & 150 & 6.25 & 7.10 \\
\hline B5 & $06 / 06 / 83$ & -115 & -35.1 & 14.1 & 280 & 156 & 6.50 & 7.10 \\
\hline B5 & $06 / 06 / 83$ & -125 & -38.1 & 14.2 & 280 & 160 & 4.50 & 7.25 \\
\hline B5 & $06 / 06 / 83$ & -135 & -41.1 & 14.6 & 255 & 150 & 1.22 & 7.50 \\
\hline B5 & $06 / 06 / 83$ & -140 & -42.7 & 14.3 & 280 & 150 & 3.25 & 7.40 \\
\hline B5 & $06 / 06 / 83$ & -145 & -44.2 & 14.6 & 185 & 82 & 11.00 & 6.81 \\
\hline B5 & $06 / 06 / 83$ & -150 & -45.7 & 14.6 & 160 & 68 & .11 & 7.62 \\
\hline B5 & $06 / 06 / 83$ & -155 & -47.2 & 14.6 & 195 & 98 & .05 & 7.75 \\
\hline B5 & $06 / 06 / 83$ & -165 & -50.3 & 14.8 & 250 & 138 & .03 & 8.11 \\
\hline B5 & $06 / 06 / 83$ & -170 & -51.8 & 14.4 & 310 & 168 & .12 & 8.05 \\
\hline
\end{tabular}


Table 8.--Water-chemistry data from production wells, 1971-83

[Samples collected and analyzed by personnel of the Coos Bay-North Bend Water Board]

\begin{tabular}{|c|c|c|c|c|c|c|c|c|}
\hline WELL & DATE & $\begin{array}{l}\text { ALKA- } \\
\text { LINITY } \\
\text { (MG/L } \\
\text { AS } \\
\text { CACO3) }\end{array}$ & $\begin{array}{l}\text { HARD- } \\
\text { NESS } \\
\text { (MG/L } \\
\text { AS } \\
\text { CACO3) }\end{array}$ & $\begin{array}{l}\text { IRON } \\
\text { DIS- } \\
\text { SOLVED } \\
\text { (MG/L) }\end{array}$ & $\begin{array}{c}\mathrm{PH} \\
(\mathrm{UNIT})\end{array}$ & $\begin{array}{l}\text { CARBON } \\
\text { DIOXIDE } \\
(M G / L)\end{array}$ & $\begin{array}{l}\text { FLUO- } \\
\text { RIDE } \\
\text { DIS- } \\
\text { SOLVED } \\
(M G / L)\end{array}$ & $\begin{array}{l}\text { CHLO- } \\
\text { RIDE } \\
\text { DIS- } \\
\text { SOLVED } \\
(M G / L)\end{array}$ \\
\hline 41 & $03 / 08 / 71$ & 76 & 60 & 1.80 & 6.80 & 25.0 & .01 & 25 \\
\hline 41 & $04 / 21 / 71$ & 78 & 60 & 1.60 & 7.00 & 16.0 & .01 & 25 \\
\hline 41 & $05 / 10 / 71$ & 84 & 72 & 4.40 & 6.60 & 44.0 & .01 & 25 \\
\hline 41 & $06 / 11 / 71$ & 70 & 44 & 3.80 & 6.80 & 23.0 & .02 & 32 \\
\hline 41 & $07 / 16 / 71$ & 84 & 54 & 7.00 & 6.80 & 28.0 & .01 & 18 \\
\hline 41 & $08 / 17 / 71$ & 85 & 56 & 6.40 & 6.70 & 34.0 & -- & 20 \\
\hline 41 & $09 / 15 / 71$ & 56 & 56 & 3.80 & 6.60 & 28.0 & .01 & 16 \\
\hline 41 & $10 / 22 / 71$ & 58 & 54 & 3.60 & 6.00 & 28.0 & .01 & 18 \\
\hline 41 & $12 / 21 / 71$ & 78 & 82 & 3.00 & 7.00 & 16.0 & .01 & 18 \\
\hline 41 & $01 / 25 / 72$ & 76 & 86 & 2.60 & 7.00 & 17.0 & .01 & 18 \\
\hline 41 & $02 / 16 / 72$ & 70 & 42 & 2.60 & 6.80 & 23.0 & .01 & 22 \\
\hline 41 & $03 / 24 / 72$ & 72 & 50 & 3.80 & 6.50 & 48.0 & .17 & 18 \\
\hline 41 & $04 / 28 / 72$ & 78 & 56 & 4.00 & 6.50 & 52.0 & -- & 18 \\
\hline 41 & $05 / 18 / 72$ & 88 & 58 & 4.40 & 6.80 & 26.0 & .02 & 17 \\
\hline 41 & $08 / 30 / 72$ & 92 & 62 & 3.80 & 6.60 & 47.0 & .02 & 17 \\
\hline 41 & $09 / 26 / 72$ & 100 & 74 & 7.00 & 6.40 & 64.0 & .01 & 19 \\
\hline 41 & $11 / 28 / 72$ & 70 & 60 & 6.50 & 6.50 & 45.0 & .01 & 16 \\
\hline 41 & $12 / 26 / 72$ & 78 & 60 & 7.00 & 6.60 & 41.0 & .01 & 18 \\
\hline 41 & $02 / 27 / 73$ & 120 & 112 & 5.00 & 7.10 & 19.0 & .01 & 28 \\
\hline 41 & $03 / 30 / 73$ & 118 & 116 & 5.40 & 7.00 & 27.0 & .01 & 26 \\
\hline 41 & $04 / 27 / 73$ & 58 & 54 & 3.00 & 6.80 & 19.0 & .01 & 20 \\
\hline 41 & $05 / 24 / 73$ & 114 & 72 & 3.20 & 7.00 & 23.0 & .01 & 30 \\
\hline 41 & $06 / 28 / 73$ & 106 & 90 & 3.40 & 6.80 & 34.0 & .01 & 31 \\
\hline
\end{tabular}


Table 8.--Water-chemistry data from production wells, 1971-83--Continued

[Samples collected and analyzed by personnel of the Coos Bay-North Bend Water Board]

\begin{tabular}{|c|c|c|c|c|c|c|c|c|}
\hline WELL & DATE & $\begin{array}{c}\text { ALKA- } \\
\text { LINITY } \\
\text { (MG/L } \\
\text { AS } \\
\text { CACO3) }\end{array}$ & $\begin{array}{l}\text { HARD- } \\
\text { NESS } \\
(M G / L \\
\text { AS } \\
\text { CACO3) }\end{array}$ & $\begin{array}{l}\text { IRON } \\
\text { DIS- } \\
\text { SOLVED } \\
\text { (MG/L) }\end{array}$ & $\begin{array}{c}\mathrm{PH} \\
(\mathrm{UNIT})\end{array}$ & $\begin{array}{l}\text { CARBON } \\
\text { DIOXIDE } \\
(M G / L)\end{array}$ & $\begin{array}{l}\text { FLUO- } \\
\text { RIDE } \\
\text { DIS- } \\
\text { SOLVED } \\
(M G / L)\end{array}$ & $\begin{array}{l}\text { CHLO- } \\
\text { RIDE } \\
\text { DIS- } \\
\text { SOLVED } \\
(M G / L)\end{array}$ \\
\hline 41 & $08 / 30 / 73$ & 106 & 82 & 5.00 & 6.60 & 50.0 & .01 & 22 \\
\hline 41 & $10 / 09 / 73$ & 116 & 96 & 5.60 & 6.60 & 55.0 & .01 & 24 \\
\hline 41 & $11 / 06 / 73$ & 108 & 102 & 5.60 & 6.80 & 36.0 & .01 & 26 \\
\hline 41 & $12 / 20 / 73$ & 124 & 88 & 3.00 & 6.40 & -- & .01 & 24 \\
\hline 41 & $01 / 29 / 74$ & 110 & 82 & 3.20 & 6.60 & 58.0 & .01 & 28 \\
\hline 41 & $02 / 26 / 74$ & 108 & 76 & 4.00 & 6.80 & 35.0 & .01 & 22 \\
\hline 41 & $03 / 28 / 74$ & 110 & 76 & 4.20 & 6.90 & 28.0 & .01 & 24 \\
\hline 41 & $04 / 30 / 74$ & 104 & 80 & 4.40 & 6.90 & 27.0 & .01 & 22 \\
\hline 41 & $07 / 24 / 74$ & 114 & 90 & 6.20 & 7.10 & 19.0 & .10 & 24 \\
\hline 41 & $08 / 28 / 74$ & 102 & 98 & 5.20 & 7.00 & 22.0 & .10 & 26 \\
\hline 41 & $09 / 27 / 74$ & 100 & 84 & 5.60 & 7.00 & 20.0 & .10 & 28 \\
\hline 41 & $10 / 24 / 74$ & 96 & 96 & 5.40 & 7.00 & 18.0 & .10 & 28 \\
\hline 41 & $11 / 21 / 74$ & 100 & 96 & 4.80 & 7.00 & 21.0 & .10 & 29 \\
\hline 41 & $12 / 31 / 74$ & 90 & 94 & 3.50 & 7.00 & 18.0 & .10 & 30 \\
\hline 41 & $02 / 28 / 75$ & 94 & 86 & 4.20 & 7.00 & 19.0 & .10 & 30 \\
\hline 41 & $03 / 26 / 75$ & 104 & 76 & 4.00 & 7.00 & 21.0 & .10 & 31 \\
\hline 41 & $04 / 23 / 75$ & 106 & 80 & 4.20 & .7 .00 & 31.0 & .10 & 30 \\
\hline 41 & $05 / 27 / 75$ & 118 & 96 & 3.80 & 6.50 & 76.0 & .10 & 28 \\
\hline 41 & $07 / 29 / 75$ & 112 & 96 & 3.20 & 6.70 & 45.0 & .10 & 28 \\
\hline 41 & $09 / 25 / 75$ & 108 & 100 & 3.20 & 6.50 & 36.0 & .10 & 28 \\
\hline 41 & $11 / 25 / 75$ & 104 & 94 & 3.20 & 6.90 & 27.0 & .10 & 26 \\
\hline 41 & $12 / 30 / 75$ & 116 & 100 & 3.80 & 6.90 & 29.0 & .10 & 22 \\
\hline
\end{tabular}


Table 8.--Water-chemistry data from production wells, 1971-83--Continued

[Samples collected and analyzed by personnel of the Coos Bay-North Bend Water Board]

\begin{tabular}{|c|c|c|c|c|c|c|c|c|}
\hline WELL & DATE & $\begin{array}{c}\text { ALKA- } \\
\text { LINITY } \\
\text { (MG/L } \\
\text { AS } \\
\text { CACO3) }\end{array}$ & $\begin{array}{l}\text { HARD- } \\
\text { NESS } \\
\text { (MG/L } \\
\text { AS } \\
\text { CACO3) }\end{array}$ & $\begin{array}{l}\text { IRON } \\
\text { DIS- } \\
\text { SOLVED } \\
\text { (MG/L) }\end{array}$ & $\begin{array}{c}\text { PH } \\
(\text { UNIT) }\end{array}$ & $\begin{array}{l}\text { CARBON } \\
\text { DIOXIDE } \\
(M G / L)\end{array}$ & $\begin{array}{l}\text { FLUO- } \\
\text { RIDE } \\
\text { DIS- } \\
\text { SOLVED } \\
(M G / L)\end{array}$ & $\begin{array}{l}\text { CHLO- } \\
\text { RIDE } \\
\text { DIS- } \\
\text { SOLVED } \\
\text { (MG/L) }\end{array}$ \\
\hline 41 & $02 / 19 / 76$ & 110 & 104 & 4.00 & 6.80 & 35.0 & .10 & 27 \\
\hline 41 & $03 / 30 / 76$ & 112 & 108 & 6.50 & 6.40 & 90.0 & .20 & 20 \\
\hline 41 & $04 / 23 / 76$ & 110 & 100 & 7.00 & 6.50 & 75.0 & .20 & 22 \\
\hline 41 & $06 / 17 / 76$ & 105 & 110 & 7.00 & 6.60 & 57.0 & .15 & 22 \\
\hline 41 & $07 / 19 / 76$ & 98 & 90 & 6.20 & 6.80 & 30.0 & .15 & 28 \\
\hline 41 & $08 / 20 / 76$ & 96 & 86 & 6.40 & 6.90 & 25.0 & .20 & 28 \\
\hline 41 & $09 / 21 / 76$ & 97 & 85 & 6.50 & 6.80 & 26.0 & .20 & 30 \\
\hline 41 & $10 / 21 / 76$ & 102 & 110 & 6.60 & 6.70 & -- & .15 & 24 \\
\hline 41 & $11 / 18 / 76$ & 95 & 66 & 7.60 & 6.90 & 24.0 & .20 & 30 \\
\hline 41 & $02 / 15 / 77$ & 11.0 & 100 & 4.00 & 6.80 & 36.0 & .10 & 26 \\
\hline 41 & $03 / 15 / 77$ & 110 & 104 & 7.00 & 6.70 & 45.0 & .20 & 20 \\
\hline 41 & $04 / 15 / 77$ & 10 & 110 & 6.60 & 6.60 & 55.0 & .20 & 20 \\
\hline 41 & $05 / 16 / 77$ & 108 & 100 & 6.80 & 6.80 & 35.0 & .20 & 20 \\
\hline 41 & $06 / 21 / 77$ & 110 & 100 & 6.80 & 6.60 & 55.0 & .20 & 25 \\
\hline 41 & $07 / 21 / 77$ & 120 & 110 & 7.30 & 6.50 & 80.0 & -- & 20 \\
\hline 41 & $08 / 19 / 77$ & 118 & 112 & 6.50 & 6.50 & 80.0 & - & 20 \\
\hline 41 & $09 / 15 / 77$ & 120 & 104 & 6.50 & 6.60 & 62.0 & -- & 12 \\
\hline 41 & $10 / 20 / 77$ & 112 & 90 & 7.20 & 6.50 & 80.0 & -- & 19 \\
\hline 41 & $12 / 20 / 77$ & 110 & 98 & 4.20 & 6.60 & 55.0 & -- & 23 \\
\hline 41 & $01 / 16 / 78$ & 118 & 100 & 5.20 & 6.60 & 60.0 & -- & 10 \\
\hline 41 & $02 / 15 / 78$ & 100 & 110 & 4.60 & 6.50 & 65.0 & -- & 25 \\
\hline 41 & $03 / 14 / 78$ & 120 & 90 & 6.00 & 6.60 & 65.0 & -- & 22 \\
\hline 41 & $04 / 12 / 78$ & 100 & 90 & 5.00 & 6.40 & -- & -- & 25 \\
\hline
\end{tabular}


Table 8.- - Water-chem istry data from production

[Samples collected and analyzed by personnel of the Coos Bay-North Bend Water Board]

\begin{tabular}{|c|c|c|c|c|c|c|c|c|}
\hline WELL & DATE & $\begin{array}{c}\text { ALKA- } \\
\text { LINITY } \\
\text { (MG/L } \\
\text { AS } \\
\text { CACO3) }\end{array}$ & $\begin{array}{l}\text { HARD- } \\
\text { NESS } \\
\text { (MG/L } \\
\text { AS } \\
\text { CACO3) }\end{array}$ & $\begin{array}{l}\text { IRON } \\
\text { DIS- } \\
\text { SOLVED } \\
\text { (MG/L) }\end{array}$ & $\begin{array}{c}\text { PH } \\
(U N \mid T)\end{array}$ & $\begin{array}{l}\text { CARBON } \\
D I O X I D E \\
(M G / L)\end{array}$ & $\begin{array}{l}\text { FLUO- } \\
\text { RIDE } \\
\text { DIS- } \\
\text { SOLVED } \\
(M G / L)\end{array}$ & $\begin{array}{l}\text { CHLO- } \\
\text { RIDE } \\
\text { DIS- } \\
\text { SOLVED } \\
(M G / L)\end{array}$ \\
\hline 41 & $05 / 11 / 78$ & 120 & 104 & 6.00 & 6.70 & 48.0 & -- & 23 \\
\hline 41 & $06 / 12 / 78$ & 120 & 98 & 7.00 & 6.70 & 48.0 & -- & 22 \\
\hline 41 & $07 / 17 / 78$ & 102 & 92 & 5.40 & 6.70 & 40.0 & -- & 25 \\
\hline 41 & $08 / 11 / 78$ & 104 & 100 & 5.40 & 6.40 & -- & -- & 16 \\
\hline 41 & $09 / 15 / 78$ & 110 & 84 & 5.10 & 6.60 & 60.0 & -- & 22 \\
\hline 41 & $10 / 25 / 78$ & 106 & 90 & 7.00 & 6.40 & - & -- & 22 \\
\hline 41 & $11 / 15 / 78$ & 110 & 110 & 8.00 & 6.60 & 58.0 & -- & 23 \\
\hline 41 & $12 / 13 / 78$ & 108 & 104 & 10.00 & 6.80 & 35.0 & -- & 25 \\
\hline 41 & $01 / 22 / 79$ & 118 & 100 & 10.00 & 6.80 & 38.0 & -- & 25 \\
\hline 41 & $02 / 22 / 79$ & 100 & 100 & 9.50 & 6.50 & 65.0 & -- & 25 \\
\hline 41 & $03 / 20 / 79$ & 96 & 100 & 10.00 & 6.50 & 85.0 & -- & 30 \\
\hline 41 & $04 / 19 / 79$ & 120 & 110 & 9.00 & 6.90 & 30.0 & -- & 22 \\
\hline 41 & $05 / 21 / 79$ & 104 & 94 & 14.50 & 6.60 & 55.0 & .12 & 22 \\
\hline 41 & $06 / 13 / 79$ & 100 & 100 & 14.50 & 6.80 & 32.0 & .12 & 22 \\
\hline 41 & $08 / 16 / 79$ & 108 & 90 & 14.00 & 6.50 & 70.0 & -- & 25 \\
\hline 41 & $10 / 23 / 79$ & 120 & 96 & 15.00 & 6.60 & 60.0 & -- & 23 \\
\hline 41 & $11 / 20 / 79$ & 118 & 94 & 15.00 & 6.70 & 48.0 & - & 24 \\
\hline 41 & $12 / 17 / 79$ & 118 & 100 & 15.25 & 6.70 & 48.0 & -- & 24 \\
\hline 41 & $01 / 13 / 80$ & 96 & 100 & 15.00 & 6.70 & 40.0 & -- & 20 \\
\hline 41 & $02 / 14 / 80$ & 100 & 100 & 12.50 & 6.40 & -- & -- & 30 \\
\hline 41 & $03 / 14 / 80$ & 118 & 90 & 15.00 & 6.70 & 47.0 & -- & 25 \\
\hline 41 & $04 / 15 / 80$ & 104 & 90 & 15.00 & 6.70 & 42.0 & -- & 20 \\
\hline 41 & $05 / 13 / 80$ & 116 & 92 & 16.25 & 6.60 & 60.0 & -- & 20 \\
\hline 41 & $06 / 16 / 80$ & 104 & 90 & 15.00 & 6.70 & 42.0 & -- & 23 \\
\hline
\end{tabular}


Table 8.--Water-chemistry data from production wells, 1971-83--Continued

[Samples collected and analyzed by personnel of the Coos Bay-North Bend Water Board]

\begin{tabular}{|c|c|c|c|c|c|c|c|c|}
\hline WELL & DATE & $\begin{array}{c}\text { ALKA- } \\
\text { LINITY } \\
\text { (MG/L } \\
\text { AS } \\
\text { CACO3) }\end{array}$ & $\begin{array}{l}\text { HARD- } \\
\text { NESS } \\
\text { (MG/L } \\
\text { AS } \\
\text { CACO3) }\end{array}$ & $\begin{array}{l}\text { IRON } \\
\text { DIS- } \\
\text { SOLVED } \\
\text { (MG/L) }\end{array}$ & $\begin{array}{c}\mathrm{PH} \\
(\mathrm{UN} \mid \mathrm{T})\end{array}$ & $\begin{array}{l}\text { CARBON } \\
\text { DIOXIDE } \\
(M G / L)\end{array}$ & $\begin{array}{l}\text { FLUO- } \\
\text { RIDE } \\
\text { DIS- } \\
\text { SOLVED } \\
(M G / L)\end{array}$ & $\begin{array}{l}\text { CHLO- } \\
\text { RIDE } \\
\text { DIS- } \\
\text { SOLVED } \\
(M G / L)\end{array}$ \\
\hline 41 & $07 / 11 / 80$ & 110 & 102 & 16.00 & 6.60 & 55.0 & -- & 22 \\
\hline 41 & $08 / 12 / 80$ & 100 & 100 & 16.25 & 6.60 & 51.0 & -- & 25 \\
\hline 41 & $09 / 15 / 80$ & 100 & 90 & 15.00 & 6.50 & 66.0 & .13 & 25 \\
\hline 41 & $10 / 21 / 80$ & 118 & 100 & 15.00 & 6.70 & 48.0 & - & 25 \\
\hline 41 & $11 / 14 / 80$ & 100 & 90 & 15.00 & 6.70 & 40.0 & -- & 25 \\
\hline 41 & $12 / 16 / 80$ & 128 & 90 & 15.00 & 6.80 & 40.0 & -- & 30 \\
\hline 41 & $01 / 15 / 81$ & 118 & -- & 15.00 & 6.60 & 39.0 & .11 & 30 \\
\hline 41 & $02 / 16 / 81$ & 104 & 100 & 12.00 & 6.50 & 68.0 & -- & 30 \\
\hline 41 & $03 / 16 / 81$ & 110 & 100 & 15.00 & 6.60 & 58.0 & -- & 25 \\
\hline 41 & $04 / 15 / 81$ & 124 & 94 & 15.00 & 6.70 & 50.0 & .40 & 31 \\
\hline 41 & $05 / 15 / 81$ & 105 & 100 & 15.00 & 6.70 & 44.0 & -- & 30 \\
\hline 41 & $06 / 11 / 81$ & 122 & 102 & 14.00 & 6.50 & 80.0 & -- & 28 \\
\hline 41 & $07 / 14 / 81$ & 118 & 90 & 12.50 & 6.50 & 75.0 & .03 & 35 \\
\hline 41 & $08 / 28 / 81$ & 120 & 92 & 15.00 & 6.70 & 50.0 & - & 25 \\
\hline 41 & $09 / 17 / 81$ & 118 & 90 & 15.00 & 6.50 & 75.0 & -- & 25 \\
\hline 41 & $10 / 27 / 81$ & 120 & 100 & 15.50 & 6.50 & 77.0 & -- & 28 \\
\hline 41 & $11 / 19 / 81$ & 96 & 62 & 15.00 & 6.30 & -- & -- & 15 \\
\hline 41 & $12 / 15 / 81$ & 120 & 96 & 15.50 & 6.60 & 64.0 & -- & 15 \\
\hline 41 & $01 / 22 / 82$ & 110 & 120 & 14.50 & 6.70 & 45.0 & -- & 30 \\
\hline 41 & $09 / 23 / 82$ & 118 & 90 & 14.50 & 6.60 & 62.0 & -- & 30 \\
\hline 41 & $10 / 20 / 82$ & 110 & 86 & 14.00 & 6.60 & 56.0 & -- & 21 \\
\hline 41 & $11 / 19 / 82$ & 120 & 90 & 15.00 & 6.60 & 62.0 & -- & 25 \\
\hline 41 & $12 / 20 / 82$ & 78 & 64 & 15.00 & 6.70 & 32.0 & -- & 25 \\
\hline
\end{tabular}


Table 8.--Water-chemistry data from production wells, 1971-83--Continued

[Samples collected and analyzed by personnel of the Coos Bay-North Bend Water Board]

\begin{tabular}{|c|c|c|c|c|c|c|c|c|}
\hline WELL & DATE & $\begin{array}{l}\text { ALKA- } \\
\text { LINITY } \\
\text { (MG/L } \\
\text { AS } \\
\text { CACO3) }\end{array}$ & $\begin{array}{l}\text { HARD- } \\
\text { NESS } \\
\text { (MG/L } \\
\text { AS } \\
\text { CACO3) }\end{array}$ & $\begin{array}{l}\text { IRON } \\
\text { DIS- } \\
\text { SOLVED } \\
\text { (MG/L) }\end{array}$ & $\begin{array}{c}\text { PH } \\
(U N \mid T)\end{array}$ & $\begin{array}{l}\text { CARBON } \\
D I O X I D E \\
(M G / L)\end{array}$ & $\begin{array}{l}\text { FLUO- } \\
\text { RIDE } \\
\text { DIS- } \\
\text { SOLVED } \\
(M G / L)\end{array}$ & $\begin{array}{l}\text { CHLO- } \\
\text { RIDE } \\
\text { DIS- } \\
\text { SOLVED } \\
(M G / L)\end{array}$ \\
\hline 41 & $01 / 17 / 83$ & 110 & 92 & 13.00 & 6.50 & 72.0 & -- & 25 \\
\hline 41 & $02 / 16 / 83$ & 80 & 74 & 11.00 & 6.60 & 42.0 & -- & 25 \\
\hline 41 & $03 / 18 / 83$ & 78 & 68 & 10.00 & 6.70 & 32.0 & - & 25 \\
\hline 41 & $04 / 26 / 83$ & 76 & 66 & 15.00 & 6.60 & 40.0 & -- & 20 \\
\hline 41 & $05 / 16 / 83$ & 118 & 100 & 10.00 & 6.70 & 48.0 & -- & 30 \\
\hline 41 & $06 / 22 / 83$ & 72 & 70 & 9.20 & 6.60 & 37.0 & -- & 23 \\
\hline 42 & $03 / 08 / 71$ & 60 & 50 & 4.00 & 6.40 & 45.0 & .01 & 29 \\
\hline 42 & $04 / 21 / 71$ & 58 & 54 & 3.50 & 6.30 & 40.0 & .01 & 30 \\
\hline 42 & $05 / 10 / 71$ & 40 & 40 & 6.50 & 6.40 & 26.0 & .01 & 28 \\
\hline 42 & $06 / 11 / 71$ & 70 & 42 & 7.00 & 6.60 & 36.0 & .02 & 28 \\
\hline 42 & $07 / 16 / 71$ & 102 & 72 & 7.00 & 6.80 & 34.0 & .01 & 23 \\
\hline 42 & $08 / 17 / 71$ & 100 & 72 & 3.60 & 6.40 & 32.0 & - & 23 \\
\hline 42 & $09 / 15 / 71$ & 72 & 68 & 5.00 & 6.40 & 47.0 & .01 & 18 \\
\hline 42 & $10 / 22 / 71$ & 68 & 68 & 5.20 & 6.40 & 55.0 & .01 & 18 \\
\hline 42 & $12 / 21 / 71$ & 78 & 112 & 4.40 & 6.60 & 40.0 & .01 & 22 \\
\hline 42 & $01 / 25 / 72$ & 78 & 108 & 3.20 & 6.80 & 38.0 & .02 & 20 \\
\hline 42 & $02 / 16 / 72$ & 68 & 52 & 2.20 & 6.70 & 21.0 & .02 & 18 \\
\hline 42 & $03 / 24 / 72$ & 60 & 42 & 4.80 & 6.70 & 38.0 & .20 & 18 \\
\hline 42 & $04 / 28 / 72$ & 60 & 50 & 4.60 & 6.90 & 16.0 & - & 15 \\
\hline 42 & $05 / 18 / 72$ & 84 & 54 & 4.00 & 6.60 & 47.0 & .03 & 20 \\
\hline 42 & $08 / 30 / 72$ & 86 & 58 & 4.80 & 6.60 & 27.0 & .02 & 19 \\
\hline 42 & $09 / 26 / 72$ & 74 & 54 & 4.80 & 6.60 & 37.0 & .01 & 22 \\
\hline 42 & $11 / 28 / 72$ & 78 & 56 & 5.00 & 6.80 & 24.0 & .02 & 18 \\
\hline
\end{tabular}


Table 8.--Water-chem istry data from production

[Samples collected and analyzed by personnel of the Coos Bay-North Bend Water Board]

\begin{tabular}{|c|c|c|c|c|c|c|c|c|}
\hline WELL & DATE & $\begin{array}{c}\text { ALKA- } \\
\text { LIN|TY } \\
\text { (MG/L } \\
\text { AS } \\
\text { CACO3) }\end{array}$ & $\begin{array}{l}\text { HARD- } \\
\text { NESS } \\
\text { (MG/L } \\
\text { AS } \\
\text { CACO3) }\end{array}$ & $\begin{array}{l}\text { IRON } \\
\text { DIS- } \\
\text { SOLVED } \\
\text { (MG/L) }\end{array}$ & $\begin{array}{c}\mathrm{PH} \\
(\mathrm{UN} / \mathrm{T})\end{array}$ & $\begin{array}{l}\text { CARBON } \\
\text { DIOXIDE } \\
(M G / L)\end{array}$ & $\begin{array}{l}\text { FLUO- } \\
\text { RIDE } \\
\text { DIS- } \\
\text { SOLVED } \\
\text { (MG/L) }\end{array}$ & $\begin{array}{l}\text { CHLO- } \\
\text { RIDE } \\
\text { DIS- } \\
\text { SOLVED } \\
(M G / L)\end{array}$ \\
\hline 42 & $12 / 26 / 72$ & 76 & 54 & 5.00 & 6.80 & 35.0 & .01 & 22 \\
\hline 42 & $02 / 27 / 73$ & 116 & 122 & 5.20 & 6.60 & 60.0 & .02 & 27 \\
\hline 42 & $03 / 30 / 73$ & 114 & 108 & 5.40 & 6.80 & 37.0 & .01 & 28 \\
\hline 42 & $04 / 27 / 73$ & 80 & 56 & 4.20 & 6.70 & 32.0 & .01 & 24 \\
\hline 42 & $05 / 24 / 73$ & 80 & 54 & 2.80 & 6.40 & 60.0 & .01 & 25 \\
\hline 42 & $06 / 28 / 73$ & 70 & 52 & 2.80 & 6.60 & 37.0 & .01 & 29 \\
\hline 42 & $08 / 30 / 73$ & 88 & 50 & 4.80 & 6.30 & 63.0 & .01 & 22 \\
\hline 42 & $10 / 09 / 73$ & 92 & 64 & 5.40 & 6.50 & 60.0 & .01 & 20 \\
\hline 42 & $11 / 06 / 73$ & 84 & 68 & 5.20 & 6.90 & 21.0 & .01 & 20 \\
\hline 42 & $12 / 20 / 73$ & 84 & 76 & 4.20 & 6.40 & -- & .02 & 28 \\
\hline 42 & $01 / 29 / 74$ & 80 & 52 & 4.20 & 6.50 & 52.0 & .01 & 24 \\
\hline 42 & $02 / 26 / 74$ & 80 & 70 & 3.60 & 6.40 & -- & .01 & 20 \\
\hline 42 & $03 / 28 / 74$ & 86 & 66 & 3.40 & 6.60 & 45.0 & .01 & 20 \\
\hline 42 & $04 / 30 / 74$ & 98 & 82 & 3.60 & 6.80 & 33.0 & .01 & 22 \\
\hline 42 & $07 / 24 / 74$ & 70 & 54 & 5.00 & 6.40 & 50.0 & .10 & 23 \\
\hline 42 & $08 / 28 / 74$ & 84 & 64 & 4.80 & 6.60 & 36.0 & .10 & 28 \\
\hline 42 & $09 / 27 / 74$ & 86 & 68 & 5.00 & 6.80 & 28.0 & .10 & 28 \\
\hline 42 & $10 / 24 / 74$ & 92 & 84 & 5.20 & 6.90 & 23.0 & .10 & 26 \\
\hline 42 & $11 / 21 / 74$ & 92 & 66 & 5.00 & 6.90 & 25.0 & .10 & 28 \\
\hline 42 & $12 / 31 / 74$ & 54 & 58 & 6.00 & 6.90 & 16.0 & .10 & 28 \\
\hline 42 & $02 / 28 / 75$ & 58 & 64 & 3.50 & 7.00 & 13.0 & .10 & 26 \\
\hline 42 & $03 / 26 / 75$ & 92 & 90 & 3.20 & 7.00 & 18.0 & .10 & 24 \\
\hline
\end{tabular}


Table 8.--Water-chemistry data from production wells, 1971-83--Continued

[Samples collected and analyzed by personnel of the Coos Bay-North Bend Water Board]

\begin{tabular}{|c|c|c|c|c|c|c|c|c|}
\hline WELL & DATE & $\begin{array}{c}\text { ALKA- } \\
\text { LINITY } \\
(M G / L \\
\text { AS } \\
\text { CACO3) }\end{array}$ & $\begin{array}{l}\text { HARD- } \\
\text { NESS } \\
\text { (MG/L } \\
\text { AS } \\
\text { CACO3) }\end{array}$ & $\begin{array}{l}\text { IRON } \\
\text { DIS- } \\
\text { SOLVED } \\
\text { (MG/L) }\end{array}$ & $\begin{array}{c}\mathrm{PH} \\
(\mathrm{UN} \mid \mathrm{T})\end{array}$ & $\begin{array}{l}\text { CARBON } \\
\text { DIOXIDE } \\
(M G / L)\end{array}$ & $\begin{array}{l}\text { FLUO- } \\
\text { RIDE } \\
\text { DIS- } \\
\text { SOLVED } \\
(M G / L)\end{array}$ & $\begin{array}{l}\text { CHLO- } \\
\text { RIDE } \\
\text { DIS- } \\
\text { SOLVED } \\
(M G / L)\end{array}$ \\
\hline 42 & $04 / 23 / 75$ & 98 & 96 & 3.60 & 7.10 & 16.0 & .10 & 24 \\
\hline 42 & $05 / 27 / 75$ & 78 & 74 & 3.60 & 6.60 & 42.0 & .10 & 24 \\
\hline 42 & $07 / 29 / 75$ & 78 & 82 & 3.60 & 6.90 & 20.0 & .10 & 20 \\
\hline 42 & $09 / 25 / 75$ & 86 & 98 & 3.40 & 6.90 & 22.0 & .10 & 30 \\
\hline 42 & $11 / 25 / 75$ & 92 & 96 & 3.40 & 6.90 & 24.0 & .10 & 30 \\
\hline 42 & $12 / 30 / 75$ & 80 & 90 & 3.00 & 6.70 & 33.0 & .10 & 20 \\
\hline 42 & $02 / 29 / 76$ & 78 & 70 & 4.00 & 6.70 & 35.0 & .10 & 25 \\
\hline 42 & $03 / 30 / 76$ & 80 & 70 & 6.40 & 6.40 & 65.0 & .20 & 24 \\
\hline 42 & $04 / 23 / 76$ & 80 & 80 & 6.80 & 6.50 & 42.0 & .20 & 22 \\
\hline 42 & $06 / 17 / 76$ & 80 & 70 & 6.80 & 6.40 & 60.0 & .15 & 20 \\
\hline 42 & $07 / 19 / 76$ & 80 & 70 & 6.00 & 6.50 & 55.0 & .20 & 24 \\
\hline 42 & $08 / 20 / 76$ & 82 & 70 & 6.00 & 6.50 & 55.0 & .18 & 22 \\
\hline 42 & $09 / 21 / 76$ & 83 & 70 & 6.10 & 6.60 & 54.0 & .18 & 24 \\
\hline 42 & $10 / 21 / 76$ & 80 & 70 & 6.40 & 6.50 & -- & .20 & 22 \\
\hline 42 & $11 / 18 / 76$ & 86 & 64 & 7.20 & 6.60 & 45.0 & .20 & 23 \\
\hline 42 & $02 / 15 / 77$ & 74 & 120 & 4.30 & 6.60 & 36.0 & .10 & 24 \\
\hline 42 & $03 / 15 / 77$ & 80 & 70 & 6.00 & 6.70 & 32.0 & .20 & 20 \\
\hline 42 & $04 / 15 / 77$ & 80 & 74 & 6.50 & 6.50 & 50.0 & .20 & 20 \\
\hline 42 & $05 / 16 / 77$ & 78 & 60 & 6.80 & 6.50 & 54.0 & .20 & 22 \\
\hline 42 & $06 / 21 / 77$ & 80 & 60 & 6.20 & 6.50 & 54.0 & .20 & 20 \\
\hline 42 & $07 / 21 / 77$ & 80 & 90 & 7.00 & 6.40 & -- & -- & 20 \\
\hline 42 & $08 / 19 / 77$ & 84 & 92 & 7.00 & 6.30 & -- & -- & 18 \\
\hline 42 & $09 / 15 / 77$ & 90 & 90 & 7.50 & 6.50 & 60.0 & -- & 15 \\
\hline
\end{tabular}


Table 8.--Water-chemistry data from production wells, 1971-83--Continued

[Samples collected and analyzed by personnel of the Coos Bay-North Bend Water Board]

\begin{tabular}{|c|c|c|c|c|c|c|c|c|}
\hline WELL & DATE & $\begin{array}{c}\text { ALKA- } \\
\text { LIN ITY } \\
\text { (MG/L } \\
\text { AS } \\
\text { CACO3) }\end{array}$ & $\begin{array}{l}\text { HARD- } \\
\text { NESS } \\
\text { (MG/L } \\
\text { AS } \\
\text { CACO3) }\end{array}$ & $\begin{array}{l}\text { IRON } \\
\text { DIS- } \\
\text { SOLVED } \\
\text { (MG/L) }\end{array}$ & $\begin{array}{c}\mathrm{PH} \\
(U N \mid T)\end{array}$ & $\begin{array}{l}\text { CARBON } \\
\text { DIOXIDE } \\
(M G / L)\end{array}$ & $\begin{array}{l}\text { FLUO- } \\
\text { RIDE } \\
\text { DIS- } \\
\text { SOLVED } \\
\text { (MG/L) }\end{array}$ & $\begin{array}{l}\text { CHLO- } \\
\text { RIDE } \\
\text { DIS- } \\
\text { SOLVED } \\
(M G / L)\end{array}$ \\
\hline 42 & $10 / 20 / 77$ & 80 & 68 & 6.40 & 6.60 & 40.0 & - & 17 \\
\hline 42 & $12 / 20 / 77$ & 70 & 90 & 5.20 & 6.40 & 55.0 & -- & 28 \\
\hline 42 & $01 / 16 / 78$ & 84 & 70 & 5.00 & 6.40 & -- & -- & 20 \\
\hline 42 & $02 / 15 / 78$ & 70 & 90 & 4.80 & 6.40 & 55.0 & -- & 20 \\
\hline 42 & $03 / 14 / 78$ & 84 & 64 & 6.20 & 6.60 & 45.0 & -- & 22 \\
\hline 42 & $04 / 12 / 78$ & 70 & 75 & 5.50 & 6.40 & -- & -- & 25 \\
\hline 42 & $05 / 11 / 78$ & 80 & 70 & 4.00 & 6.70 & 34.0 & -- & 23 \\
\hline 42 & $06 / 12 / 78$ & 94 & 64 & 7.00 & 6.50 & 60.0 & -- & 21 \\
\hline 42 & $07 / 17 / 78$ & 88 & 68 & 5.40 & 6.50 & 60.0 & -- & 20 \\
\hline 42 & $08 / 11 / 78$ & 80 & 70 & 6.00 & 6.40 & -- & -- & 20 \\
\hline 42 & $09 / 15 / 78$ & 76 & 60 & 5.00 & 6.40 & -- & -- & 20 \\
\hline 42 & $10 / 25 / 78$ & 76 & 70 & 7.20 & 6.30 & -- & -- & 20 \\
\hline 42 & $11 / 15 / 78$ & 86 & 70 & 10.00 & 6.40 & -- & -- & 20 \\
\hline 42 & $12 / 13 / 78$ & 80 & 90 & 10.00 & 6.70 & 32.0 & -- & 25 \\
\hline 42 & $01 / 22 / 79$ & 84 & 80 & 10.00 & 6.70 & 35.0 & -- & 25 \\
\hline 42 & $02 / 22 / 79$ & 80 & 84 & 9.80 & 6.40 & -- & -- & 25 \\
\hline 42 & $03 / 20 / 79$ & 70 & 70 & 10.00 & 6.40 & -- & -- & 30 \\
\hline 42 & $04 / 19 / 79$ & 94 & 100 & 9.00 & 6.70 & 38.0 & -- & 24 \\
\hline 42 & $05 / 21 / 79$ & 80 & 64 & 12.50 & 6.50 & 54.0 & .10 & 22 \\
\hline 42 & $06 / 13 / 79$ & 82 & 82 & 13.00 & 6.60 & 43.0 & .11 & 22 \\
\hline 42 & $08 / 16 / 79$ & 78 & 80 & 13.00 & 6.50 & 52.0 & -- & 25 \\
\hline 42 & $10 / 23 / 79$ & 90 & 60 & 14.00 & 6.50 & 60.0 & -- & 23 \\
\hline 42 & $11 / 20 / 79$ & 90 & 76 & 15.00 & 6.60 & 46.0 & -- & 22 \\
\hline
\end{tabular}


Table 8.--Water-chem istry data from production wells, 1971-83--Continued

[Samples collected and analyzed by personnel of the Coos Bay-North Bend Water Board]

\begin{tabular}{|c|c|c|c|c|c|c|c|c|}
\hline WELL & DATE & $\begin{array}{c}\text { ALKA- } \\
\text { LINITY } \\
\text { (MG/L } \\
\text { AS } \\
\text { CACO3) }\end{array}$ & $\begin{array}{l}\text { HARD- } \\
\text { NESS } \\
\text { (MG/L } \\
\text { AS } \\
\text { CACO3) }\end{array}$ & $\begin{array}{l}\text { IRON } \\
\text { DIS- } \\
\text { SOLVED } \\
\text { (MG/L) }\end{array}$ & $\begin{array}{c}\text { PH } \\
(U N \mid T)\end{array}$ & $\begin{array}{l}\text { CARBON } \\
\text { DIOXIDE } \\
(M G / L)\end{array}$ & $\begin{array}{l}\text { FLUO- } \\
\text { RIDE } \\
\text { DIS- } \\
\text { SOLVED } \\
(M G / L)\end{array}$ & $\begin{array}{l}\text { CHLO- } \\
\text { RIDE } \\
\text { DIS- } \\
\text { SOLVED } \\
(M G / L)\end{array}$ \\
\hline 42 & $12 / 17 / 79$ & 90 & 80 & 14.75 & 6.70 & 37.0 & -- & 20 \\
\hline 42 & $01 / 13 / 80$ & 70 & 80 & 20.00 & 6.50 & 45.0 & -- & 25 \\
\hline 42 & $02 / 14 / 80$ & 70 & 94 & 12.50 & 6.40 & -- & -- & 25 \\
\hline 42 & $03 / 14 / 80$ & 80 & 64 & 15.00 & 6.60 & 41.0 & -- & 25 \\
\hline 42 & $04 / 15 / 80$ & 80 & 70 & 20.00 & 6.60 & 40.0 & -- & 25 \\
\hline 42 & $05 / 13 / 80$ & 80 & 70 & 17.50 & 6.50 & 54.0 & -- & 25 \\
\hline 42 & $06 / 16 / 80$ & 90 & 70 & 14.00 & 6.50 & 59.0 & -- & 25 \\
\hline 42 & $07 / 11 / 80$ & 88 & 76 & 15.50 & 6.40 & 65.0 & -- & 25 \\
\hline 42 & $08 / 12 / 80$ & 98 & 62 & 16.25 & 6.50 & 64.0 & -- & 20 \\
\hline 42 & $09 / 15 / 80$ & 100 & 64 & 15.00 & 6.50 & 66.0 & -- & 20 \\
\hline 42 & $10 / 21 / 80$ & 90 & 60 & 15.00 & 6.50 & 52.0 & -- & 25 \\
\hline 42 & $11 / 14 / 80$ & 90 & 70 & 15.00 & 6.40 & 55.0 & -- & 25 \\
\hline 42 & $12 / 16 / 80$ & 90 & 74 & 13.00 & 6.50 & 60.0 & -- & 28 \\
\hline 42 & $01 / 15 / 81$ & 66 & -- & 15.00 & 6.50 & 44.0 & -- & 30 \\
\hline 42 & $02 / 16 / 81$ & 70 & 65 & 12.50 & 6.40 & -- & -- & 30 \\
\hline 42 & $03 / 16 / 81$ & 106 & 90 & 15.00 & 6.50 & 70.0 & -- & 25 \\
\hline 42 & $04 / 15 / 81$ & 94 & 80 & 15.00 & 6.30 & - & -- & 32 \\
\hline 42 & $05 / 15 / 81$ & 90 & 70 & 14.50 & 6.50 & 58.0 & -- & 25 \\
\hline 42 & $06 / 11 / 81$ & 88 & 74 & 16.00 & 6.30 & 78.0 & -- & 26 \\
\hline 42 & $07 / 14 / 81$ & 90 & 80 & 15.00 & 6.50 & 56.0 & -- & 35 \\
\hline 42 & $08 / 28 / 81$ & 92 & 80 & 14.50 & 6.60 & 48.0 & -- & 30 \\
\hline 42 & $09 / 17 / 81$ & 100 & 80 & 14.50 & 6.60 & 52.0 & -- & 30 \\
\hline 42 & $10 / 27 / 81$ & 86 & 68 & 16.00 & 6.30 & 95.0 & -- & 26 \\
\hline
\end{tabular}


Table 8.--Water-chemistry data from production wells, 1971-83--Continued

[Samples collected and analyzed by personnel of the Coos Bay-North Bend Water Board]

\begin{tabular}{|c|c|c|c|c|c|c|c|c|}
\hline WELL & DATE & $\begin{array}{c}\text { ALKA- } \\
\text { LIN ITY } \\
\text { (MG/L } \\
\text { AS } \\
\text { CACO3) }\end{array}$ & $\begin{array}{l}\text { HARD- } \\
\text { NESS } \\
\text { (MG/L } \\
\text { AS } \\
\text { CACO3) }\end{array}$ & $\begin{array}{l}\text { IRON } \\
\text { DIS- } \\
\text { SOLVED } \\
\text { (MG/L) }\end{array}$ & $\begin{array}{c}\text { PH } \\
(\text { UNIT) }\end{array}$ & $\begin{array}{l}\text { CARBON } \\
\text { DIOXIDE } \\
(M G / L)\end{array}$ & $\begin{array}{l}\text { FLUO- } \\
\text { RIDE } \\
\text { DIS- } \\
\text { SOLVED } \\
\text { (MG/L) }\end{array}$ & $\begin{array}{l}\text { CHLO- } \\
\text { RIDE } \\
\text { DIS- } \\
\text { SOLVED } \\
\text { (MG/L) }\end{array}$ \\
\hline 42 & $11 / 19 / 81$ & 120 & 100 & 15.00 & 6.60 & 62.0 & - & 15 \\
\hline 42 & $12 / 15 / 81$ & 96 & 80 & 15.00 & 6.40 & -- & -- & 20 \\
\hline 42 & $01 / 22 / 82$ & 90 & 100 & 15.00 & 5.50 & 58.0 & -- & 35 \\
\hline 42 & $05 / 20 / 82$ & 86 & 60 & 15.00 & 6.50 & 56.0 & -- & 34 \\
\hline 42 & $06 / 15 / 82$ & 88 & 70 & 15.00 & 6.20 & - & -- & 28 \\
\hline 42 & $07 / 15 / 82$ & 92 & 65 & 15.00 & 6.50 & 59.0 & -- & 35 \\
\hline 42 & $08 / 11 / 82$ & 90 & 70 & 12.50 & 6.60 & 45.0 & -- & 35 \\
\hline 42 & $09 / 23 / 82$ & 120 & 108 & 15.00 & 6.90 & 30.0 & -- & 22 \\
\hline 42 & $10 / 20 / 82$ & 82 & 62 & 14.50 & 6.40 & 66.0 & -- & 23 \\
\hline 42 & $11 / 19 / 82$ & 90 & 70 & 15.00 & 6.50 & 58.0 & -- & 25 \\
\hline 42 & $12 / 20 / 82$ & 90 & 68 & 14.80 & 6.70 & 36.0 & -- & 25 \\
\hline 42 & $01 / 17 / 83$ & 80 & 50 & 15.00 & 6.50 & 54.0 & -- & 25 \\
\hline 42 & $02 / 16 / 83$ & 84 & 56 & 15.00 & 6.50 & 56.0 & -- & 25 \\
\hline 42 & $03 / 18 / 83$ & 84 & 80 & 15.00 & 6.50 & 54.0 & -- & 18 \\
\hline 42 & $04 / 26 / 83$ & 88 & 82 & 14.00 & 6.50 & 57.0 & -- & 20 \\
\hline 42 & $05 / 16 / 83$ & 78 & 50 & 7.50 & 6.60 & 40.0 & -- & 32 \\
\hline 42 & $06 / 22 / 83$ & 86 & 58 & 15.50 & 6.50 & 56.0 & -- & 25 \\
\hline 43 & $03 / 08 / 71$ & 60 & 36 & 3.60 & 6.30 & 45.0 & .01 & 28 \\
\hline 43 & $04 / 21 / 71$ & 56 & 40 & 3.20 & 6.40 & 30.0 & -- & 30 \\
\hline
\end{tabular}


Table 8.- Water-chemistry data from production wells, 1971-83--Continued

[Samples collected and analyzed by personnel of the Coos Bay-North Bend Water Board]

\begin{tabular}{|c|c|c|c|c|c|c|c|c|}
\hline WELL & DATE & $\begin{array}{c}\text { ALKA- } \\
\text { LINITY } \\
\text { (MG/L } \\
\text { AS } \\
\text { CACO3) }\end{array}$ & $\begin{array}{l}\text { HARD- } \\
\text { NESS } \\
\text { (MG/L } \\
\text { AS } \\
\text { CACO3) }\end{array}$ & $\begin{array}{l}\text { IRON } \\
\text { DIS- } \\
\text { SOLVED } \\
(M G / L)\end{array}$ & $\begin{array}{c}\text { PH } \\
(U N I T)\end{array}$ & $\begin{array}{l}\text { CARBON } \\
\text { DIOXIDE } \\
(M G / L)\end{array}$ & $\begin{array}{l}\text { FLUO- } \\
\text { RIDE } \\
\text { DIS- } \\
\text { SOLVED } \\
\text { (MG/L) }\end{array}$ & $\begin{array}{l}\text { CHLO- } \\
\text { RIDE } \\
\text { DIS- } \\
\text { SOLVED } \\
(M G / L)\end{array}$ \\
\hline 43 & $05 / 10 / 71$ & 112 & 116 & 3.60 & 6.80 & 47.0 & -- & 28 \\
\hline 43 & $06 / 11 / 71$ & 68 & 42 & 7.00 & 6.50 & 45.0 & .02 & 26 \\
\hline 43 & $07 / 16 / 71$ & 60 & 66 & 6.20 & 6.50 & 40.0 & .01 & 20 \\
\hline 43 & $08 / 17 / 71$ & 64 & 68 & 5.80 & 6.20 & 40.0 & -- & 22 \\
\hline 43 & $09 / 15 / 71$ & 122 & 102 & 6.80 & 6.90 & 32.0 & .01 & 15 \\
\hline 43 & $10 / 22 / 71$ & 118 & 110 & 6.60 & 6.80 & 38.0 & .01 & 14 \\
\hline 43 & $12 / 21 / 71$ & 112 & 150 & 4.00 & 6.80 & 36.0 & .03 & 16 \\
\hline 43 & $01 / 25 / 72$ & 108 & 136 & 3.40 & 6.80 & 36.0 & .01 & 16 \\
\hline 43 & $02 / 16 / 72$ & 116 & 104 & 2.80 & 7.00 & 24.0 & .02 & 23 \\
\hline 43 & $03 / 24 / 72$ & 116 & 90 & 2.60 & 7.00 & 18.5 & .14 & 13 \\
\hline 43 & $04 / 28 / 72$ & 117 & 98 & 2.40 & 7.00 & 13.0 & -- & 12 \\
\hline 43 & $05 / 18 / 72$ & 112 & 76 & 3.40 & 6.90 & 28.0 & .03 & 15 \\
\hline 43 & $08 / 30 / 72$ & 114 & 78 & 3.60 & 7.00 & 43.0 & .03 & 15 \\
\hline 43 & $09 / 26 / 72$ & 34 & 32 & 5.80 & 6.30 & 30.0 & .03 & 18 \\
\hline 43 & $11 / 28 / 72$ & 114 & 96 & 4.60 & 6.00 & 100.0 & .01 & 18 \\
\hline 43 & $12 / 26 / 72$ & 110 & 104 & 4.40 & 6.00 & 100.0 & .01 & 20 \\
\hline 43 & $02 / 27 / 73$ & 96 & 124 & 3.60 & 7.10 & 17.0 & .01 & 26 \\
\hline 43 & $03 / 30 / 73$ & 92 & 126 & 3.40 & 7.10 & 16.0 & .02 & 24 \\
\hline 43 & $04 / 27 / 73$ & 112 & 74 & 4.00 & 6.50 & 73.0 & .02 & 22 \\
\hline 43 & $05 / 24 / 73$ & 68 & 48 & 3.00 & 6.20 & 70.0 & .01 & 30 \\
\hline 43 & $06 / 28 / 73$ & 64 & 46 & 3.40 & 6.60 & 35.0 & .01 & 30 \\
\hline 43 & $08 / 30 / 73$ & 108 & 78 & 4.20 & 6.20 & -- & .02 & 18 \\
\hline 43 & $10 / 09 / 73$ & 112 & 70 & 4.40 & 6.20 & 100.0 & .01 & 22 \\
\hline
\end{tabular}


Table 8.- - Water-chemistry data from production wells, 1971-83--Continued

[Samples collected and analyzed by personnel of the Coos Bay-North Bend Water Board]

\begin{tabular}{|c|c|c|c|c|c|c|c|c|}
\hline WELL & DATE & $\begin{array}{l}\text { ALKA- } \\
\text { LINITY } \\
\text { (MG/L } \\
\text { AS } \\
\text { CACO3) }\end{array}$ & $\begin{array}{l}\text { HARD- } \\
\text { NESS } \\
\text { (MG/L } \\
\text { AS } \\
\text { CACO3) }\end{array}$ & $\begin{array}{l}\text { IRON } \\
\text { DIS- } \\
\text { SOLVED } \\
\text { (MG/L) }\end{array}$ & $\begin{array}{c}\mathrm{PH} \\
(\mathrm{UN} \mid \mathrm{T})\end{array}$ & $\begin{array}{l}\text { CARBON } \\
\text { DIOXIDE } \\
(M G / L)\end{array}$ & $\begin{array}{l}\text { FLUO- } \\
\text { RIDE } \\
\text { DIS- } \\
\text { SOLVED } \\
\text { (MG/L) }\end{array}$ & $\begin{array}{l}\text { CHLO- } \\
\text { RIDE } \\
\text { DIS- } \\
\text { SOLVED } \\
\text { (MG/L) }\end{array}$ \\
\hline 43 & $11 / 06 / 73$ & 114 & 74 & 4.60 & 6.60 & 59.0 & .01 & 24 \\
\hline 43 & $12 / 20 / 73$ & 112 & 98 & 4.00 & 6.60 & 57.0 & .01 & 26 \\
\hline 43 & $01 / 29 / 74$ & 108 & 100 & 4.60 & 6.40 & -- & .01 & 22 \\
\hline 43 & $02 / 26 / 74$ & 110 & 94 & 3.60 & 6.50 & 70.0 & .02 & 17 \\
\hline 43 & $03 / 28 / 74$ & 116 & 98 & 3.60 & 6.60 & 59.0 & .02 & 18 \\
\hline 43 & $04 / 30 / 74$ & 120 & 104 & 3.60 & 6.90 & 32.0 & .02 & 18 \\
\hline 43 & $07 / 24 / 74$ & 114 & 114 & 3.00 & 6.80 & 34.0 & .20 & 18 \\
\hline 43 & $08 / 28 / 74$ & 108 & 108 & 3.40 & 6.80 & 39.0 & .20 & 20 \\
\hline 43 & $09 / 27 / 74$ & 110 & 102 & 3.20 & 6.80 & 35.0 & .10 & 24 \\
\hline 43 & $10 / 24 / 74$ & 116 & 108 & 3.60 & 6.80 & 38.0 & .10 & 20 \\
\hline 43 & $11 / 21 / 74$ & 108 & 104 & 3.60 & 6.80 & 36.0 & .20 & 20 \\
\hline 43 & $12 / 31 / 74$ & 78 & 64 & 3.50 & 6.50 & 62.0 & .10 & 25 \\
\hline 43 & $02 / 28 / 75$ & 84 & 84 & 3.00 & 6.80 & 28.0 & .10 & 24 \\
\hline 43 & $03 / 26 / 75$ & 118 & 88 & 3.00 & 6.80 & 40.0 & .10 & 24 \\
\hline 43 & $04 / 23 / 75$ & 112 & 84 & 3.40 & 6.90 & 26.0 & .20 & 26 \\
\hline 43 & $05 / 27 / 75$ & 115 & 98 & 4.20 & 6.70 & 46.0 & .10 & 26 \\
\hline 43 & $07 / 29 / 75$ & 114 & 102 & 3.80 & 6.80 & 37.0 & .10 & 28 \\
\hline 43 & $09 / 25 / 75$ & 110 & 108 & 4.00 & 6.90 & 28.0 & .10 & 28 \\
\hline 43 & $11 / 25 / 75$ & 108 & 102 & 3.80 & 7.10 & 17.5 & .10 & 28 \\
\hline 43 & $12 / 30 / 75$ & 108 & 115 & 4.20 & 7.00 & 22.0 & .20 & 18 \\
\hline 43 & $02 / 19 / 76$ & 104 & 92 & 4.00 & 6.70 & 42.0 & .10 & 17 \\
\hline 43 & $03 / 30 / 76$ & 110 & 112 & 6.20 & 6.50 & 72.0 & .10 & 18 \\
\hline
\end{tabular}


Table 8.-- Water-chem istry data from production

[Samples collected and analyzed by personnel of the Coos Bay-North Bend Water Board]

\begin{tabular}{|c|c|c|c|c|c|c|c|c|}
\hline WELL & DATE & $\begin{array}{l}\text { ALKA- } \\
\text { LINITY } \\
\text { (MG/L } \\
\text { AS } \\
\text { CACO3) }\end{array}$ & $\begin{array}{l}\text { HARD- } \\
\text { NESS } \\
\text { (MG/L } \\
\text { AS } \\
\text { CACO3) }\end{array}$ & $\begin{array}{l}\text { IRON } \\
\text { DIS- } \\
\text { SOLVED } \\
(M G / L)\end{array}$ & $\begin{array}{c}\text { PH } \\
(\text { UNIT) }\end{array}$ & $\begin{array}{l}\text { CARBON } \\
\text { DIOXIDE } \\
(M G / L)\end{array}$ & $\begin{array}{l}\text { FLUO- } \\
\text { RIDE } \\
\text { DIS- } \\
\text { SOLVED } \\
\text { (MG/L) }\end{array}$ & $\begin{array}{l}\text { CHLO- } \\
\text { RIDE } \\
\text { DIS- } \\
\text { SOLVED } \\
\text { (MG/L) }\end{array}$ \\
\hline 43 & $04 / 23 / 76$ & 104 & 100 & 6.20 & 6.70 & 42.0 & .10 & 18 \\
\hline 43 & $06 / 17 / 76$ & 110 & 90 & 7.00 & 6.50 & 78.0 & .10 & 18 \\
\hline 43 & $07 / 19 / 76$ & 96 & 120 & 6.20 & 6.80 & 32.0 & .18 & 18 \\
\hline 43 & $08 / 20 / 76$ & 94 & 118 & 6.20 & 6.70 & 38.0 & .15 & 18 \\
\hline 43 & $09 / 21 / 76$ & 90 & 120 & 6.20 & 6.70 & 40.0 & .20 & 18 \\
\hline 43 & $10 / 21 / 76$ & 104 & 110 & 7.00 & 6.60 & -- & .15 & 18 \\
\hline 43 & $11 / 18 / 76$ & 90 & 100 & 6.60 & 6.80 & 25.0 & .17 & 19 \\
\hline 43 & $02 / 15 / 77$ & 96 & 96 & 4.20 & 6.80 & 32.0 & .15 & 18 \\
\hline 43 & $03 / 15 / 77$ & 118 & 110 & 7.00 & 6.70 & 50.0 & .30 & 10 \\
\hline 43 & $04 / 15 / 77$ & 110 & 100 & 6.40 & 6.60 & 58.0 & .15 & 15 \\
\hline 43 & $05 / 16 / 77$ & 108 & 100 & 6.20 & 6.70 & 45.0 & .22 & 18 \\
\hline 43 & $06 / 21 / 77$ & 108 & 90 & 5.80 & 6.60 & 54.0 & .30 & 18 \\
\hline 43 & $07 / 21 / 77$ & 104 & 90 & 7.00 & 6.60 & 50.0 & - & 15 \\
\hline 43 & $08 / 19 / 77$ & 104 & 94 & 7.00 & 6.50 & 50.0 & -- & 16 \\
\hline 43 & $09 / 15 / 77$ & 110 & 110 & 7.00 & 6.60 & 56.0 & -- & 10 \\
\hline 43 & $10 / 20 / 77$ & 106 & 94 & 8.20 & 6.60 & 55.0 & -- & 14 \\
\hline 43 & $12 / 20 / 77$ & 90 & 100 & 5.40 & 6.70 & 36.0 & -- & 20 \\
\hline 43 & $01 / 16 / 78$ & 106 & 98 & 5.40 & 6.50 & 75.0 & -- & 18 \\
\hline 43 & $02 / 15 / 78$ & 104 & 100 & 5.80 & 6.40 & 75.0 & -- & 20 \\
\hline 43 & $03 / 14 / 78$ & 90 & 90 & 5.00 & 6.80 & 28.0 & -- & 18 \\
\hline 43 & $04 / 12 / 78$ & 102 & 110 & 4.50 & 6.40 & -- & -- & 20 \\
\hline 43 & $05 / 11 / 78$ & 110 & 90 & 5.80 & 6.80 & 36.0 & -- & 20 \\
\hline 43 & $06 / 12 / 78$ & 114 & 92 & 6.00 & 6.70 & 45.0 & -- & 17 \\
\hline
\end{tabular}


Table 8.--Water-chemistry data from production

[Samples collected and analyzed by personnel of the Coos Bay-North Bend Water Board]

\begin{tabular}{|c|c|c|c|c|c|c|c|c|}
\hline WELL & DATE & $\begin{array}{c}\text { ALKA- } \\
\text { LINITY } \\
\text { (MG/L } \\
\text { AS } \\
\text { CACO3) }\end{array}$ & $\begin{array}{l}\text { HARD- } \\
\text { NESS } \\
\text { (MG/L } \\
\text { AS } \\
\text { CAC03) }\end{array}$ & $\begin{array}{l}\text { IRON } \\
\text { DIS- } \\
\text { SOLVED } \\
\text { (MG/L) }\end{array}$ & $\begin{array}{c}\text { PH } \\
(\text { UNIT) }\end{array}$ & $\begin{array}{l}\text { CARBON } \\
\text { DIOXIDE } \\
(M G / L)\end{array}$ & $\begin{array}{l}\text { FLUO- } \\
\text { RIDE } \\
\text { DIS- } \\
\text { SOLVED } \\
(M G / L)\end{array}$ & $\begin{array}{l}\text { CHLO- } \\
\text { RIDE } \\
\text { DIS- } \\
\text { SOLVED } \\
(M G / L)\end{array}$ \\
\hline 43 & $07 / 17 / 78$ & 110 & 90 & 4.80 & 6.70 & 45.0 & -- & 18 \\
\hline 43 & $08 / 11 / 78$ & 102 & 104 & 6.00 & 6.50 & 67.0 & -- & 18 \\
\hline 43 & $09 / 15 / 78$ & 110 & 92 & 4.30 & 6.60 & 60.0 & -- & 15 \\
\hline 43 & $10 / 25 / 78$ & 108 & 100 & 7.00 & 6.40 & -- & -- & 16 \\
\hline 43 & $11 / 15 / 78$ & 110 & 100 & 9.00 & 6.60 & 58.0 & -- & 22 \\
\hline 43 & $12 / 13 / 78$ & 100 & 100 & 10.00 & 6.80 & 31.0 & -- & 20 \\
\hline 43 & $01 / 22 / 79$ & 118 & 100 & 9.60 & 6.80 & 38.0 & -- & 20 \\
\hline 43 & $02 / 22 / 79$ & 100 & 90 & 9.50 & 6.60 & 55.0 & -- & 22 \\
\hline 43 & $n 3 / 20 / 79$ & 100 & 100 & 10.00 & 6.60 & 54.0 & -- & 20 \\
\hline 43 & $-4 / 19 / 79$ & 110 & 104 & 8.50 & 6.70 & 45.0 & -- & 20 \\
\hline 43 & $05 / 21 / 79$ & 106 & 92 & 9.50 & 6.70 & 44.0 & .12 & 18 \\
\hline 43 & $06 / 13 / 79$ & 110 & 100 & 9.50 & 6.80 & 36.0 & .12 & 20 \\
\hline 43 & $08 / 16 / 79$ & 100 & 94 & 9.00 & 6.60 & 54.0 & -- & 20 \\
\hline 43 & $10 / 23 / 79$ & 110 & 92 & 10.00 & 6.50 & 70.0 & -- & 19 \\
\hline 43 & $11 / 20 / 79$ & 110 & 80 & 12.50 & 6.80 & 35.0 & -- & 20 \\
\hline 43 & $12 / 17 / 79$ & 92 & 90 & 12.50 & 6.70 & 38.0 & -- & 18 \\
\hline 43 & $01 / 13 / 80$ & 100 & 105 & 12.50 & 6.60 & 52.0 & -- & 20 \\
\hline 43 & $02 / 14 / 80$ & 100 & 90 & 9.00 & 6.50 & 65.0 & -- & 20 \\
\hline 43 & $03 / 14 / 80$ & 108 & 94 & 15.00 & 6.60 & 55.0 & -- & 20 \\
\hline 43 & $04 / 15 / 80$ & 108 & 80 & 15.00 & 6.60 & 55.0 & -- & 20 \\
\hline 43 & $05 / 13 / 80$ & 100 & 100 & 12.50 & 6.50 & 65.0 & -- & 20 \\
\hline 43 & $06 / 16 / 80$ & 110 & 94 & 9.00 & 6.60 & 55.0 & -- & 20 \\
\hline 43 & $07 / 11 / 80$ & 112 & 94 & 10.00 & 6.60 & 55.0 & - & 20 \\
\hline
\end{tabular}


Table 8.--Water-chemistry data from production wells, 1971-83--Continued

[Samples collected and analyzed by personnel of the Coos Bay-North Bend Water Board]

\begin{tabular}{|c|c|c|c|c|c|c|c|c|}
\hline WELL & DATE & $\begin{array}{c}\text { ALKA- } \\
\text { LINITY } \\
\text { (MG/L } \\
\text { AS } \\
\text { CACO3) }\end{array}$ & $\begin{array}{l}\text { HARD- } \\
\text { NESS } \\
\text { (MG/L } \\
\text { AS } \\
\text { CACO3) }\end{array}$ & $\begin{array}{l}\text { IRON } \\
\text { DIS- } \\
\text { SOLVED } \\
\text { (MG/L) }\end{array}$ & $\begin{array}{c}\text { PH } \\
(U N I T)\end{array}$ & $\begin{array}{l}\text { CARBON } \\
\text { DIOXIDE } \\
(M G / L)\end{array}$ & $\begin{array}{l}\text { FLUO- } \\
\text { RIDE } \\
\text { DIS- } \\
\text { SOLVED } \\
(M G / L)\end{array}$ & $\begin{array}{l}\text { CHLO- } \\
\text { RIDE } \\
\text { DIS- } \\
\text { SOLVED } \\
(M G / L)\end{array}$ \\
\hline 43 & $08 / 12 / 80$ & 108 & 82 & 13.00 & 6.60 & 56.0 & -- & 20 \\
\hline 43 & $09 / 15 / 80$ & 105 & 90 & 11.00 & 6.60 & 55.0 & -- & 18 \\
\hline 43 & $10 / 21 / 80$ & 100 & 100 & 11.50 & 6.60 & 52.0 & -- & 20 \\
\hline 43 & $11 / 14 / 80$ & 104 & 90 & 11.00 & 6.60 & 55.0 & -- & 25 \\
\hline 43 & $12 / 16 / 80$ & 110 & 98 & 10.50 & 6.70 & 45.0 & -- & 25 \\
\hline 43 & $01 / 15 / 81$ & 90 & -- & 10.00 & 6.50 & 58.0 & -- & 20 \\
\hline 43 & $02 / 16 / 81$ & 100 & 90 & 8.50 & 6.50 & 65.0 & -- & 25 \\
\hline 43 & $03 / 16 / 81$ & 90 & 78 & 11.00 & 6.50 & 59.0 & -- & 30 \\
\hline 43 & $04 / 15 / 81$ & 108 & 82 & 10.50 & 6.50 & 74.0 & -- & 22 \\
\hline 43 & $05 / 15 / 81$ & 96 & 100 & 10.00 & 6.60 & 48.0 & -- & 25 \\
\hline 43 & $06 / 11 / 81$ & 114 & 96 & 10.50 & 6.60 & 57.0 & -- & 21 \\
\hline 43 & $07 / 14 / 81$ & 100 & 84 & 10.00 & 6.50 & 65.0 & -- & 30 \\
\hline 43 & $08 / 28 / 81$ & 104 & 90 & 10.50 & 6.60 & 54.0 & -- & 25 \\
\hline 43 & $09 / 17 / 81$ & 90 & 90 & 10.00 & 6.50 & 58.0 & -- & 25 \\
\hline 43,1 & $10 / 27 / 81$ & 110 & 90 & 11.00 & 6.30 & 100.0 & -- & 21 \\
\hline $43 \frac{11}{11}$ & $11 / 19 / 81$ & 120 & 108 & 5.50 & 7.00 & 24.0 & -- & 18 \\
\hline $43-$ & $12 / 15 / 81$ & 125 & 102 & 4.00 & 6.90 & 33.0 & -- & 15 \\
\hline $43 \frac{11}{1}$ & $01 / 22 / 82$ & 130 & 104 & 4.00 & 7.00 & 26.0 & - & 25 \\
\hline $43 \frac{11}{11}$ & $05 / 20 / 82$ & 136 & 118 & 4.50 & 6.90 & 35.0 & - & 30 \\
\hline $43 \frac{11}{11}$ & $06 / 15 / 82$ & 132 & 110 & 4.37 & 6.70 & 54.0 & -- & 20 \\
\hline $43 \frac{11}{11}$ & $07 / 15 / 82$ & 130 & 108 & 4.37 & 6.90 & 32.0 & -- & 25 \\
\hline $43 \frac{11}{11}$ & $08 / 11 / 82$ & 136 & 110 & 4.00 & 7.00 & 29.0 & -- & 30 \\
\hline $43-1$ & $09 / 23 / 82$ & 90 & 68 & 4.50 & 6.60 & 47.0 & -- & 30 \\
\hline
\end{tabular}

1/ Well pumping from lower screen only. 
Table 8.- Water-chemistry data from production wells, 1971-83--Continued

[Samples collected and analyzed by personnel of the Coos Bay-North Bend Water Board]

$\begin{array}{ccccccc}\text { ALKA- } & \text { HARD- } & & & & \text { FLUO- } & \text { CHLO- } \\ \text { LINITY } & \text { NESS } & \text { IRON } & & \text { RIDE } & \text { RIDE } \\ \text { (MG/L } & (M G / L & \text { DIS- } & & \text { CARBON } & \text { DIS- } & \text { DIS- } \\ \text { AS } & \text { AS } & \text { SOLVED } & \text { PH } & \text { DIOXIDE } & \text { SOLVED } & \text { SOLVED } \\ \text { CACO3) } & \text { CACO3) } & (M G / L) & (U N I T) & (M G / L) & (M G / L) & (M G / L)\end{array}$

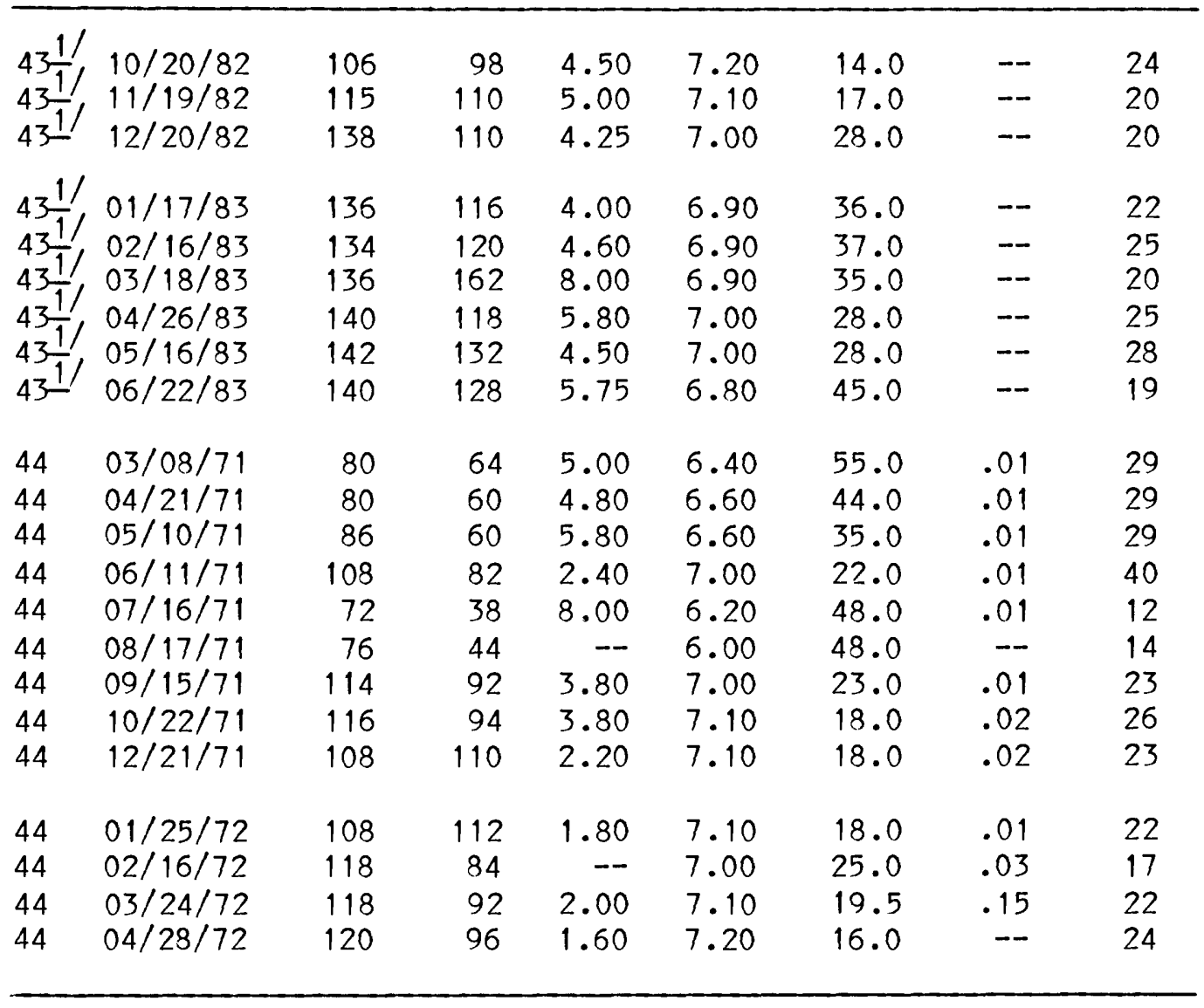

1/ Well pumping from lower screen only. 
Table 8.--Water-chemistry data from production wells, 1971-83--Continued

ГSamples collected and analyzed by personnel of the Coos Bay-North Bend Water Board]

\begin{tabular}{|c|c|c|c|c|c|c|c|c|}
\hline WELL & DATE & $\begin{array}{c}\text { ALKA- } \\
\text { LINITY } \\
\text { (MG/L } \\
\text { AS } \\
\text { CACO3) }\end{array}$ & $\begin{array}{l}\text { HARD- } \\
\text { NESS } \\
\text { (MG/L } \\
\text { AS } \\
\text { CACO3) }\end{array}$ & $\begin{array}{l}\text { IRON } \\
\text { DIS- } \\
\text { SOLVED } \\
\text { (MG/L) }\end{array}$ & $\begin{array}{c}\text { PH } \\
(U N \mid T)\end{array}$ & $\begin{array}{l}\text { CARBON } \\
\text { DIOXIDE } \\
(M G / L)\end{array}$ & $\begin{array}{l}\text { FLUO- } \\
\text { RIDE } \\
\text { DIS- } \\
\text { SOLVED } \\
\text { (MG/L) }\end{array}$ & $\begin{array}{l}\text { CHLO- } \\
\text { RIDE } \\
\text { DIS- } \\
\text { SOLVED } \\
(M G / L)\end{array}$ \\
\hline 44 & $05 / 18 / 72$ & 104 & 84 & 2.60 & 7.00 & 21.0 & .04 & 22 \\
\hline 44 & $08 / 30 / 72$ & 108 & 78 & 3.00 & 7.20 & 24.0 & .03 & 22 \\
\hline 44 & $09 / 26 / 72$ & 110 & 80 & 5.20 & 6.90 & 28.0 & .03 & 23 \\
\hline 44 & $11 / 28 / 72$ & 104 & 88 & 4.20 & 7.00 & 22.0 & .01 & 22 \\
\hline 44 & $12 / 26 / 72$ & 108 & 92 & 3.60 & 7.20 & 14.0 & .02 & 18 \\
\hline 44 & $02 / 27 / 73$ & 120 & 114 & 3.20 & 7.00 & 23.0 & .01 & 18 \\
\hline 44 & $03 / 30 / 73$ & 124 & 112 & 3.00 & 7.10 & 19.5 & .01 & 18 \\
\hline 44 & $04 / 27 / 73$ & 106 & 88 & 2.00 & 6.90 & 28.0 & .02 & 20 \\
\hline 44 & $05 / 24 / 73$ & 118 & 80 & 3.60 & 6.90 & 30.0 & .01 & 31 \\
\hline 44 & $06 / 28 / 73$ & 94 & 96 & 3.80 & 6.80 & 30.0 & .01 & 30 \\
\hline 44 & $08 / 30 / 73$ & 116 & 88 & 4.00 & 6.70 & 47.0 & .01 & 22 \\
\hline 44 & $10 / 09 / 73$ & 116 & 96 & 4.00 & 6.90 & 29.0 & .01 & 26 \\
\hline 44 & $11 / 06 / 73$ & 116 & 84 & 4.20 & 7.10 & 18.0 & .04 & 26 \\
\hline 44 & $12 / 20 / 73$ & 112 & 46 & 4.00 & 6.80 & 35.0 & .01 & 22 \\
\hline 44 & $01 / 29 / 74$ & 106 & 88 & 4.80 & 6.90 & 28.0 & .02 & 22 \\
\hline 44 & $02 / 26 / 74$ & 104 & 92 & 3.20 & 6.50 & 68.0 & .01 & 21 \\
\hline 44 & $03 / 28 / 74$ & 108 & 90 & 3.80 & 6.80 & 36.0 & .02 & 20 \\
\hline 44 & $04 / 30 / 74$ & 112 & 110 & 4.00 & 6.80 & 37.0 & .02 & 24 \\
\hline 44 & $07 / 24 / 74$ & 114 & 102 & 2.20 & 7.00 & 23.0 & .10 & 23 \\
\hline 44 & $08 / 28 / 74$ & 108 & 100 & 2.80 & 7.10 & 17.0 & .10 & 28 \\
\hline 44 & $09 / 27 / 74$ & 112 & 106 & 3.40 & 7.10 & 18.0 & .10 & 30 \\
\hline
\end{tabular}


Table 8.--Water-chemistry data from production wells, 1971-83--Continued

[Samples collected and analyzed by personnel of the Coos Bay-North Bend Water Board]

\begin{tabular}{|c|c|c|c|c|c|c|c|c|}
\hline WELL & DATE & $\begin{array}{c}\text { ALKA- } \\
\text { LIN ITY } \\
\text { (MG/L } \\
\text { AS } \\
\text { CACO3) }\end{array}$ & $\begin{array}{l}\text { HARD- } \\
\text { NESS } \\
\text { (MG/L } \\
\text { AS } \\
\text { CACO3) }\end{array}$ & $\begin{array}{l}\text { IRON } \\
\text { DIS- } \\
\text { SOLVED } \\
\text { (MG/L) }\end{array}$ & $\begin{array}{c}\mathrm{PH} \\
(\mathrm{UN} \mid \mathrm{T})\end{array}$ & $\begin{array}{l}\text { CARBON } \\
\text { DIOXIDE } \\
(M G / L)\end{array}$ & $\begin{array}{l}\text { FLUO- } \\
\text { RIDE } \\
\text { DIS- } \\
\text { SOLVED } \\
\text { (MG/L) }\end{array}$ & $\begin{array}{l}\text { CHLO- } \\
\text { RIDE } \\
\text { DIS- } \\
\text { SOLVED } \\
(M G / L)\end{array}$ \\
\hline 44 & $10 / 24 / 74$ & 112 & 100 & 3.00 & 7.00 & 23.0 & .10 & 28 \\
\hline 44 & $11 / 21 / 74$ & 108 & 106 & 3.20 & 7.10 & 17.5 & .20 & 31 \\
\hline 44 & $12 / 31 / 74$ & 106 & 88 & 1.50 & 6.90 & 27.0 & .20 & 32 \\
\hline 44 & $02 / 28 / 75$ & 102 & 96 & 3.20 & 7.00 & 21.0 & .10 & 30 \\
\hline $\begin{array}{l}44 \\
44\end{array}$ & $\begin{array}{l}03 / 26 / 75 \\
04 / 23 / 75\end{array}$ & $\begin{array}{l}110 \\
108\end{array}$ & $\begin{array}{l}100 \\
102\end{array}$ & $\begin{array}{l}3.20 \\
3.20\end{array}$ & $\begin{array}{l}6.80 \\
6.80\end{array}$ & $\begin{array}{l}36.0 \\
36.0\end{array}$ & $\begin{array}{l}.10 \\
.20\end{array}$ & $\begin{array}{l}32 \\
32\end{array}$ \\
\hline 44 & $05 / 27 / 75$ & 112 & 94 & 3.20 & 6.80 & 37.0 & .20 & 22 \\
\hline 44 & $07 / 29 / 75$ & 110 & 90 & 2.80 & 6.80 & 35.0 & .20 & 26 \\
\hline 44 & $09 / 25 / 75$ & 110 & 110 & 2.80 & 7.00 & 23.0 & .20 & 24 \\
\hline 44 & $11 / 25 / 75$ & 116 & 112 & 3.00 & 7.00 & 23.0 & .20 & 22 \\
\hline 44 & $12 / 30 / 75$ & 104 & 100 & 3.00 & 7.00 & 21.0 & .10 & 17 \\
\hline 44 & $02 / 19 / 76$ & 100 & 98 & 3.80 & 6.90 & 27.0 & .10 & 20 \\
\hline 44 & $03 / 30 / 76$ & 106 & 84 & 6.20 & 6.90 & 27.0 & .15 & 22 \\
\hline 44 & $04 / 23 / 76$ & 100 & 102 & 6.20 & 6.90 & 26.0 & .15 & 20 \\
\hline 44 & $06 / 17 / 76$ & 100 & 90 & 7.00 & 6.60 & 50.0 & .20 & 20 \\
\hline 44 & $07 / 19 / 76$ & 100 & 86 & 6.20 & 6.90 & 26.0 & .20 & 20 \\
\hline 44 & $08 / 20 / 76$ & 100 & 80 & -- & 6.90 & 26.0 & .20 & 24 \\
\hline 44 & $09 / 21 / 76$ & 100 & 90 & 2.00 & 6.90 & 28.0 & .20 & 22 \\
\hline 44 & $10 / 21 / 76$ & 100 & 86 & 7.00 & 6.90 & -- & .20 & 20 \\
\hline 44 & $11 / 18 / 76$ & 100 & 90 & 5.00 & 7.00 & 20.0 & .20 & 24 \\
\hline 44 & $02 / 15 / 77$ & 100 & 100 & 4.00 & 7.00 & 20.0 & .15 & 24 \\
\hline 44 & $03 / 15 / 77$ & 104 & 86 & 6.00 & 6.70 & 42.0 & .20 & 20 \\
\hline
\end{tabular}


Table 8.--Water-chemistry data from production wells, 1971-83--Continued

[Samples collected and analyzed by personnel of the Coos Bay-North Bend Water Board]

\begin{tabular}{|c|c|c|c|c|c|c|c|c|}
\hline WELL & DATE & $\begin{array}{c}\text { ALKA- } \\
\text { LINITY } \\
\text { (MG/L } \\
\text { AS } \\
\text { CACO3) }\end{array}$ & $\begin{array}{l}\text { HARD- } \\
\text { NESS } \\
(M G / L \\
\text { AS } \\
\text { CACO3) }\end{array}$ & $\begin{array}{l}\text { IRON } \\
\text { DIS- } \\
\text { SOLVED } \\
(M G / L)\end{array}$ & $\begin{array}{c}P H \\
(U N I T)\end{array}$ & $\begin{array}{l}\text { CARBON } \\
\text { DIOXIDE } \\
(M G / L)\end{array}$ & $\begin{array}{l}\text { FLUO- } \\
\text { RIDE } \\
\text { DIS- } \\
\text { SOLVED } \\
(M G / L)\end{array}$ & $\begin{array}{l}\text { CHLO- } \\
\text { RIDE } \\
\text { DIS- } \\
\text { SOLVED } \\
(M G / L)\end{array}$ \\
\hline 44 & $04 / 15 / 77$ & 108 & 90 & 6.40 & 6.70 & 45.0 & .20 & 20 \\
\hline 44 & $05 / 16 / 77$ & 100 & 110 & 6.20 & 6.80 & 33.0 & .20 & 20 \\
\hline 44 & $06 / 21 / 77$ & 104 & 84 & 7.50 & 6.70 & 43.0 & .20 & 24 \\
\hline 44 & $07 / 21 / 77$ & 104 & 104 & 6.00 & 6.70 & 42.0 & -- & 20 \\
\hline 44 & $08 / 19 / 77$ & 100 & 104 & 6.80 & 6.70 & 42.0 & -- & 20 \\
\hline 44 & $09 / 15 / 77$ & 104 & 120 & 7.00 & 6.60 & 54.0 & -- & 10 \\
\hline 44 & $10 / 20 / 77$ & 104 & 92 & 5.60 & 5.60 & 52.0 & -- & 16 \\
\hline 44 & $12 / 20 / 77$ & 100 & 80 & 5.60 & 6.60 & 50.0 & -- & 20 \\
\hline 44 & $01 / 16 / 78$ & 106 & 100 & 5.40 & 6.80 & 35.0 & - & 20 \\
\hline 44 & $02 / 15 / 78$ & 102 & 100 & 5.00 & 6.60 & 55.0 & -- & 20 \\
\hline 44 & $03 / 14 / 78$ & 104 & 98 & 5.00 & 6.80 & 34.0 & -- & 20 \\
\hline 44 & $04 / 12 / 78$ & 100 & 100 & 4.50 & 6.70 & 45.0 & -- & 24 \\
\hline 44 & $05 / 11 / 78$ & 110 & 100 & 4.00 & 7.00 & 23.0 & -- & 20 \\
\hline 44 & $06 / 12 / 78$ & 110 & 92 & 6.00 & 6.90 & 28.0 & -- & 21 \\
\hline 44 & $07 / 17 / 78$ & 104 & 94 & 4.80 & 6.70 & 45.0 & -- & 20 \\
\hline 44 & $08 / 11 / 78$ & 100 & 100 & 5.80 & 6.60 & 50.0 & -- & 22 \\
\hline 44 & $09 / 15 / 78$ & 100 & 100 & 4.20 & 6.60 & 52.0 & -- & 22 \\
\hline 44 & $10 / 25 / 78$ & 104 & 88 & 7.00 & 6.50 & 65.0 & -- & 20 \\
\hline 44 & $11 / 15 / 78$ & 104 & 110 & 8.00 & 6.60 & 55.0 & -- & 20 \\
\hline 44 & $12 / 13 / 78$ & 104 & 100 & 9.00 & 6.80 & 33.0 & -- & 25 \\
\hline 44 & $01 / 22 / 79$ & 110 & 100 & 8.40 & 6.90 & 29.0 & -- & 20 \\
\hline 44 & $02 / 22 / 79$ & 100 & 100 & 5.20 & 6.60 & 55.0 & -- & 22 \\
\hline 44 & $03 / 20 / 79$ & 100 & 105 & 8.00 & 6.70 & 40.0 & -- & 30 \\
\hline
\end{tabular}


Table 8.--Water-chemistry data from production wells, 1971-83--Continued

[Samples collected and analyzed by personnel of the Coos Bay-North Bend Water Board]

\begin{tabular}{|c|c|c|c|c|c|c|c|c|}
\hline WELL & DATE & $\begin{array}{c}\text { ALKA- } \\
\text { LINITY } \\
\text { (MG/L } \\
\text { AS } \\
\text { CACO3) }\end{array}$ & $\begin{array}{l}\text { HARD- } \\
\text { NESS } \\
\text { (MG/L } \\
\text { AS } \\
\text { CACO3) }\end{array}$ & $\begin{array}{l}\text { IRON } \\
\text { DIS- } \\
\text { SOLVED } \\
\text { (MG/L) }\end{array}$ & $\begin{array}{c}\mathrm{PH} \\
(\mathrm{UN} \mid \mathrm{T})\end{array}$ & $\begin{array}{l}\text { CARBON } \\
\text { DIOXIDE } \\
(M G / L)\end{array}$ & $\begin{array}{l}\text { FLUO- } \\
\text { RIDE } \\
\text { DIS- } \\
\text { SOLVED } \\
\text { (MG/L) }\end{array}$ & $\begin{array}{l}\text { CHLO- } \\
\text { RIDE } \\
\text { DIS- } \\
\text { SOLVED } \\
\text { (MG/L) }\end{array}$ \\
\hline 44 & $04 / 19 / 79$ & 104 & 96 & 8.50 & 6.90 & 27.0 & -- & 24 \\
\hline 44 & $05 / 21 / 79$ & 100 & 88 & 9.00 & 6.80 & 32.0 & .10 & 21 \\
\hline 44 & $06 / 13 / 79$ & 104 & 90 & 9.50 & 6.90 & 28.0 & .10 & 20 \\
\hline 44 & $08 / 16 / 79$ & 100 & 84 & 9.00 & 6.80 & 33.0 & -- & 20 \\
\hline 44 & $10 / 23 / 79$ & 104 & 86 & 10.00 & 6.80 & 34.0 & -- & 21 \\
\hline 44 & $11 / 20 / 79$ & 110 & 84 & 11.25 & 6.90 & 28.0 & -- & 22 \\
\hline 44 & $12 / 17 / 79$ & 102 & 92 & 12.25 & 6.90 & 28.0 & -- & 20 \\
\hline 44 & $01 / 13 / 80$ & 100 & 105 & 12.50 & 6.70 & 42.0 & -- & 25 \\
\hline 44 & $02 / 14 / 80$ & 96 & 100 & 8.00 & 6.70 & 40.0 & -- & 25 \\
\hline 44 & $03 / 14 / 80$ & 100 & 90 & 12.50 & 6.70 & 40.0 & -- & 25 \\
\hline 44 & $04 / 15 / 80$ & 104 & 90 & 15.00 & 6.70 & 42.0 & -- & 25 \\
\hline 44 & $05 / 13 / 80$ & 100 & 90 & 12.50 & 6.70 & 41.0 & -- & 22 \\
\hline 44 & $06 / 16 / 80$ & 100 & 94 & .90 & 6.60 & 52.0 & -- & 23 \\
\hline 44 & $07 / 11 / 80$ & 100 & 100 & 10.00 & 6.60 & 49.0 & -- & 22 \\
\hline 44 & $08 / 12 / 80$ & 100 & 82 & 13.00 & 6.70 & 40.0 & -- & 20 \\
\hline 44 & $09 / 15 / 80$ & 100 & 94 & 9.00 & 6.60 & 52.0 & -- & 25 \\
\hline 44 & $10 / 21 / 80$ & 100 & 90 & 10.00 & 6.70 & 41.0 & -- & 25 \\
\hline 44 & $11 / 14 / 80$ & 100 & 100 & 10.00 & 6.80 & 32.0 & -- & 25 \\
\hline 44 & $12 / 16 / 80$ & 100 & 100 & 10.00 & 7.30 & 10.0 & -- & 25 \\
\hline 44 & $01 / 15 / 81$ & 94 & -- & 9.50 & 6.70 & 38.0 & -- & 25 \\
\hline 44 & $02 / 16 / 81$ & 100 & 104 & 8.00 & 6.60 & 52.0 & -- & 25 \\
\hline 44 & $03 / 16 / 81$ & 104 & 90 & 10.00 & 6.70 & 43.0 & -- & 25 \\
\hline 44 & $04 / 15 / 81$ & 104 & 90 & 10.50 & 6.70 & 42.0 & -- & 30 \\
\hline
\end{tabular}


Table 8.- - Water-chemistry data from production

[Samples collected and analyzed by personnel of the Coos Bay-North Bend Water Board]

\begin{tabular}{|c|c|c|c|c|c|c|c|c|}
\hline WELL & DATE & $\begin{array}{c}\text { ALKA- } \\
\text { LINITY } \\
\text { (MG/L } \\
\text { AS } \\
\text { CACO3) }\end{array}$ & $\begin{array}{l}\text { HARD- } \\
\text { NESS } \\
\text { (MG/L } \\
\text { AS } \\
\text { CACO3) }\end{array}$ & $\begin{array}{l}\text { IRON } \\
\text { DIS- } \\
\text { SOLVED } \\
\text { (MG/L) }\end{array}$ & $\begin{array}{c}\text { PH } \\
(U N \mid T)\end{array}$ & $\begin{array}{l}\text { CARBON } \\
\text { DIOXIDE } \\
(M G / L)\end{array}$ & $\begin{array}{l}\text { FLUO- } \\
\text { RIDE } \\
\text { DIS- } \\
\text { SOLVED } \\
\text { (MG/L) }\end{array}$ & $\begin{array}{l}\text { CHLO- } \\
\text { RIDE } \\
\text { DIS- } \\
\text { SOLVED } \\
\text { (MG/L) }\end{array}$ \\
\hline 44 & $05 / 15 / 81$ & 100 & 96 & 9.50 & 6.70 & 41.0 & -- & 25 \\
\hline 44 & $06 / 11 / 81$ & 110 & 90 & 9.50 & 6.60 & 55.0 & - & 26 \\
\hline 44 & $07 / 14 / 81$ & 100 & 90 & 9.50 & 6.60 & 52.0 & - & 32 \\
\hline 44 & $08 / 28 / 81$ & 110 & 94 & 9.50 & 6.90 & 28.0 & - & 25 \\
\hline $44 \frac{11}{11}$ & $\begin{array}{l}09 / 17 / 81 \\
10 / 22 / 81\end{array}$ & $\begin{array}{l}102 \\
120\end{array}$ & $\begin{array}{r}90 \\
100\end{array}$ & $\begin{array}{r}10.50 \\
3.10\end{array}$ & $\begin{array}{l}6.60 \\
7.20\end{array}$ & $\begin{array}{l}53.0 \\
16.0\end{array}$ & - & $\begin{array}{l}25 \\
32\end{array}$ \\
\hline $44 \frac{1}{1 /}$ & $10 / 27 / 81$ & 108 & 94 & 10.50 & 6.50 & 70.0 & -- & 26 \\
\hline $44 \frac{11}{1 /}$ & $11 / 19 / 81$ & 118 & 108 & 5.25 & 7.00 & 23.0 & - & 15 \\
\hline $44^{11}$ & $12 / 15 / 81$ & 118 & 104 & 5.50 & 6.90 & 31.0 & - & 22 \\
\hline $44 \frac{1 /}{1 /}$ & $01 / 22 / 82$ & 116 & 105 & 5.20 & 6.90 & 30.0 & $\cdots$ & 30 \\
\hline $44 \frac{11}{1 /}$ & $06 / 15 / 82$ & 110 & 92 & 6.25 & 6.70 & 45.0 & - & 23 \\
\hline $44 \frac{11}{1 /}$ & $07 / 15 / 82$ & 110 & 96 & 7.50 & 7.00 & 23.0 & -- & 27 \\
\hline $44 \frac{11}{11}$ & $08 / 11 / 82$ & 106 & 100 & 6.50 & 6.90 & 29.0 & -- & 26 \\
\hline $44 \frac{17}{11}$ & $09 / 23 / 82$ & 100 & 100 & 7.50 & 6.80 & 32.0 & -- & 25 \\
\hline $44 \frac{1}{11}$ & $10 / 20 / 82$ & 114 & 102 & 7.25 & 6.80 & 36.0 & -- & 25 \\
\hline $44 \frac{11}{11}$ & $11 / 19 / 82$ & 110 & 100 & 7.25 & 6.90 & 28.0 & -- & 25 \\
\hline 44 & $12 / 20 / 82$ & 100 & 90 & 7.25 & 6.80 & 32.0 & -- & 25 \\
\hline $44 \frac{1 /}{1 /}$ & $01 / 17 / 83$ & 110 & 90 & 6.50 & 6.80 & 37.0 & - & 25 \\
\hline $44 \frac{1 /}{1 /}$ & $02 / 16 / 83$ & 108 & 96 & 6.75 & 6.80 & 34.0 & - & 20 \\
\hline $44 \frac{11}{11}$ & $03 / 18 / 83$ & 112 & 120 & 6.80 & 6.90 & 29.0 & -- & 23 \\
\hline $44 \frac{11}{11}$ & $04 / 26 / 83$ & 112 & 94 & 6.60 & 7.00 & 24.0 & -- & 28 \\
\hline $44 \frac{11}{11}$ & $05 / 16 / 83$ & 108 & 102 & 6.50 & 6.90 & 29.0 & -- & 30 \\
\hline $44-$ & $06 / 22 / 83$ & 116 & 110 & 6.25 & 6.90 & 30.0 & -- & 23 \\
\hline
\end{tabular}

1/ Well pumping from lower screen only. 
Table 8.--Water-chemistry data from production wells, 1971-83--Continued

[Samples collected and analyzed by personnel of the Coos Bay-North Bend Water Board]

\begin{tabular}{|c|c|c|c|c|c|c|c|c|}
\hline WELL & DATE & $\begin{array}{c}\text { ALKA- } \\
\text { LINITY } \\
\text { (MG/L } \\
\text { AS } \\
\text { CACO3) }\end{array}$ & $\begin{array}{l}\text { HARD- } \\
\text { NESS } \\
\text { (MG/L } \\
\text { AS } \\
\text { CACO3) }\end{array}$ & $\begin{array}{l}\text { IRON } \\
\text { DIS- } \\
\text { SOLVED } \\
\text { (MG/L) }\end{array}$ & $\begin{array}{c}\mathrm{PH} \\
(\mathrm{UN} \mid \mathrm{T})\end{array}$ & $\begin{array}{l}\text { CARBON } \\
\text { DIOXIDE } \\
(M G / L)\end{array}$ & $\begin{array}{l}\text { FLUO- } \\
\text { RIDE } \\
\text { DIS- } \\
\text { SOLVED } \\
\text { (MG/L) }\end{array}$ & $\begin{array}{l}\text { CHLO- } \\
\text { RIDE } \\
\text { DIS- } \\
\text { SOLVED } \\
\text { (MG/L) }\end{array}$ \\
\hline 45 & $03 / 08 / 71$ & 100 & 90 & .01 & 7.40 & 8.0 & .01 & 30 \\
\hline 45 & $04 / 21 / 71$ & 102 & 108 & - & 7.40 & 5.0 & .01 & 30 \\
\hline 45 & $05 / 10 / 71$ & 82 & 80 & .50 & 7.20 & 8.0 & .02 & 31 \\
\hline 45 & $06 / 11 / 71$ & 100 & 88 & .04 & 7.40 & 8.0 & .01 & 38 \\
\hline 45 & $07 / 16 / 71$ & 100 & 94 & .08 & 7.20 & 13.0 & .03 & 26 \\
\hline 45 & $08 / 17 / 71$ & 106 & 98 & .05 & 7.00 & 22.0 & -- & 22 \\
\hline 45 & $09 / 15 / 71$ & 100 & 104 & .01 & 7.40 & 8.0 & .03 & 24 \\
\hline 45 & $10 / 22 / 71$ & 108 & 106 & -- & 7.60 & 6.0 & .03 & 26 \\
\hline 45 & $12 / 21 / 71$ & 104 & 111 & .02 & 7.40 & 8.0 & .04 & 22 \\
\hline 45 & $01 / 25 / 72$ & 106 & 108 & -- & 7.20 & 19.0 & .01 & 20 \\
\hline 45 & $02 / 16 / 72$ & 96 & 86 & -- & 7.30 & 9.5 & .02 & 18 \\
\hline 45 & $03 / 24 / 72$ & 86 & 80 & -- & 7.10 & 14.0 & .22 & 20 \\
\hline 45 & $04 / 28 \% / 72$ & 82 & 76 & -- & 7.20 & 11.0 & -- & 18 \\
\hline 45 & $05 / 18 / 72$ & 102 & 74 & .04 & 7.30 & 11.0 & .04 & 22 \\
\hline 45 & $08 / 30 / 72$ & 112 & 88 & -- & 7.60 & 11.0 & .04 & 20 \\
\hline 45 & $09 / 26 / 72$ & 114 & 82 & -- & 7.10 & 18.0 & .03 & 24 \\
\hline 45 & $11 / 28 / 72$ & 108 & 90 & -- & 7.30 & 13.0 & .03 & 26 \\
\hline 45 & $12 / 26 / 72$ & 102 & 92 & -- & 7.50 & 7.0 & .02 & 24 \\
\hline 45 & $02 / 27 / 73$ & 108 & 128 & .01 & 7.40 & 8.5 & .01 & 19 \\
\hline 45 & $03 / 30 / 73$ & 114 & 124 & .01 & 7.40 & 9.0 & .01 & 22 \\
\hline 45 & $04 / 27 / 73$ & 68 & 52 & .01 & 7.00 & 14.0 & .02 & 26 \\
\hline 45 & $05 / 24 / 73$ & 93 & 78 & -- & 7.30 & 9.0 & .02 & 22 \\
\hline 45 & $06 / 28 / 73$ & 104 & 102 & -- & 7.20 & 13.0 & .02 & 24 \\
\hline
\end{tabular}


Table 8.- - Water-chemistry data from production

[Samples collected and analyzed by personnel of the Coos Bay-North Bend Water Board]

\begin{tabular}{|c|c|c|c|c|c|c|c|c|}
\hline WELL & DATE & $\begin{array}{c}\text { ALKA- } \\
\text { LINITY } \\
\text { (MG/L } \\
\text { AS } \\
\text { CACO3) }\end{array}$ & $\begin{array}{l}\text { HARD- } \\
\text { NESS } \\
\text { (MG/L } \\
\text { AS } \\
\text { CACO3) }\end{array}$ & $\begin{array}{l}\text { IRON } \\
\text { DIS- } \\
\text { SOLVED } \\
\text { (MG/L) }\end{array}$ & $\begin{array}{c}\mathrm{PH} \\
(\mathrm{UN} \mid \mathrm{T})\end{array}$ & $\begin{array}{l}\text { CARBON } \\
\text { DIOXIDE } \\
(M G / L)\end{array}$ & $\begin{array}{l}\text { FLUO- } \\
\text { RIDE } \\
\text { DIS- } \\
\text { SOLVED } \\
\text { (MG/L) }\end{array}$ & $\begin{array}{l}\text { CHLO- } \\
\text { RIDE } \\
\text { DIS- } \\
\text { SOLVED } \\
(M G / L)\end{array}$ \\
\hline 45 & $08 / 30 / 73$ & 116 & 98 & .01 & 7.40 & 9.0 & .03 & 26 \\
\hline 45 & $10 / 09 / 73$ & 132 & 112 & .02 & 7.00 & 46.0 & .04 & 26 \\
\hline 45 & $11 / 06 / 73$ & 136 & 116 & -- & 7.10 & 22.0 & .04 & 26 \\
\hline 45 & $12 / 20 / 73$ & 112 & 92 & .01 & 7.10 & 18.0 & .02 & 20 \\
\hline 45 & $01 / 29 / 74$ & 106 & 86 & .01 & 7.10 & 17.0 & .02 & 20 \\
\hline 45 & $02 / 26 / 74$ & 108 & 88 & -- & 7.10 & 17.0 & .02 & 24 \\
\hline 45 & $03 / 30 / 74$ & 108 & 104 & .01 & 7.00 & 23.0 & .02 & 26 \\
\hline 45 & $04 / 30 / 74$ & 110 & 110 & .01 & 7.10 & 18.0 & .01 & 28 \\
\hline 45 & $07 / 24 / 74$ & 110 & 88 & .03 & 7.40 & 8.5 & .30 & 21 \\
\hline 45 & $08 / 28 / 74$ & 112 & 96 & .02 & 7.30 & 14.0 & .30 & 26 \\
\hline 45 & $09 / 27 / 74$ & 122 & 104 & .02 & 7.20 & 16.0 & .30 & 30 \\
\hline 45 & $10 / 24 / 74$ & 120 & 110 & - & 7.20 & 15.0 & .20 & 28 \\
\hline 45 & $11 / 21 / 74$ & 116 & 106 & .02 & 7.10 & 18.5 & .40 & 30 \\
\hline 45 & $12 / 31 / 74$ & 104 & 96 & -- & 7.10 & 17.0 & .40 & 26 \\
\hline 45 & $02 / 28 / 75$ & 106 & 110 & .01 & 7.10 & 17.0 & .20 & 22 \\
\hline 45 & $03 / 26 / 75$ & 100 & 96 & -- & 7.10 & 16.0 & .20 & 24 \\
\hline 45 & $04 / 23 / 75$ & 106 & 100 & -- & 7.10 & 17.0 & .40 & 26 \\
\hline 45 & $05 / 27 / 75$ & 112 & 90 & -- & 7.20 & 14.0 & .20 & 18 \\
\hline 45 & $07 / 29 / 75$ & 112 & 90 & -- & 7.10 & 18.0 & .20 & 18 \\
\hline 45 & $09 / 25 / 75$ & 114 & 88 & -- & 7.10 & 17.0 & .20 & 22 \\
\hline 45 & $11 / 25 / 75$ & 114 & 94 & -- & 7.10 & 18.5 & .10 & 22 \\
\hline 45 & $12 / 30 / 75$ & 104 & 94 & .01 & 7.40 & 8.5 & .30 & 20 \\
\hline
\end{tabular}


Table 8.-- Water-chem istry data from production

[Samples collected and analyzed by personnel of the Coos Bay-North Bend Water Board]

\begin{tabular}{|c|c|c|c|c|c|c|c|c|}
\hline WELL & DATE & $\begin{array}{c}\text { ALKA- } \\
\text { LINITY } \\
\text { (MG/L } \\
\text { AS } \\
\text { CACO3) }\end{array}$ & $\begin{array}{l}\text { HARD- } \\
\text { NESS } \\
\text { (MG/L } \\
\text { AS } \\
\text { CACO3) }\end{array}$ & $\begin{array}{l}\text { IRON } \\
\text { DIS- } \\
\text { SOLVED } \\
(M G / L)\end{array}$ & $\begin{array}{c}\text { PH } \\
(U N I T)\end{array}$ & $\begin{array}{l}\text { CARBON } \\
\text { DIOXIDE } \\
(M G / L)\end{array}$ & $\begin{array}{l}\text { FLUO- } \\
\text { RIDE } \\
\text { DIS- } \\
\text { SOLVED } \\
(M G / L)\end{array}$ & $\begin{array}{l}\text { CHLO- } \\
\text { RIDE } \\
\text { DIS- } \\
\text { SOLVED } \\
(M G / L)\end{array}$ \\
\hline 45 & $02 / 19 / 76$ & 100 & 92 & .08 & 7.30 & 10.0 & .10 & 20 \\
\hline 45 & $03 / 30 / 76$ & 104 & 95 & .08 & 7.40 & 8.5 & .10 & 22 \\
\hline 45 & $04 / 23 / 76$ & 100 & 95 & .10 & 7.30 & 10.0 & .10 & 20 \\
\hline 45 & $06 / 17 / 76$ & 100 & 96 & .40 & 7.40 & 8.0 & .10 & 20 \\
\hline 45 & $07 / 19 / 76$ & 102 & 90 & .05 & 7.30 & 10.0 & .15 & 24 \\
\hline 45 & $08 / 20 / 76$ & 104 & 94 & .01 & 7.30 & 11.0 & .18 & 22 \\
\hline 45 & $09 / 21 / 76$ & 102 & 94 & .02 & 7.30 & 12.0 & .18 & 24 \\
\hline 45 & $10 / 21 / 76$ & 108 & 128 & .02 & 7.30 & 18.0 & .30 & 21 \\
\hline 45 & $11 / 18 / 76$ & 100 & 95 & .03 & 7.20 & 14.0 & .22 & 25 \\
\hline 45 & $02 / 15 / 77$ & 100 & 120 & .30 & 7.30 & 10.0 & .12 & 24 \\
\hline 45 & $03 / 15 / 77$ & 110 & 88 & .15 & 7.30 & 12.0 & .20 & 20 \\
\hline 45 & $04 / 15 / 77$ & 98 & 110 & .40 & 7.30 & 10.0 & .20 & 20 \\
\hline 45 & $05 / 16 / 77$ & 102 & 100 & .30 & 7.40 & 9.0 & .20 & 20 \\
\hline 45 & $06 / 21 / 77$ & 102 & 110 & .80 & 7.40 & 9.0 & .25 & 20 \\
\hline 45 & $07 / 21 / 77$ & 102 & 112 & 1.00 & 7.20 & 16.0 & -- & 20 \\
\hline 45 & $08 / 19 / 77$ & 102 & 110 & 1.00 & 7.20 & 16.0 & -- & 22 \\
\hline 45 & $09 / 15 / 77$ & 110 & 104 & 1.00 & 7.10 & 16.0 & -- & 15 \\
\hline 45 & $10 / 20 / 77$ & 106 & 86 & .80 & 7.10 & 17.5 & -- & 17 \\
\hline 45 & $12 / 20 / 77$ & 110 & 90 & -- & 7.10 & 16.0 & -- & 20 \\
\hline 45 & $01 / 16 / 78$ & 104 & 100 & .03 & 7.10 & 17.0 & -- & 20 \\
\hline 45 & $02 / 15 / 78$ & 108 & 100 & .05 & 7.10 & 15.0 & -- & 18 \\
\hline 45 & $03 / 14 / 78$ & 104 & 98 & 1.00 & 7.30 & 11.0 & -- & 18 \\
\hline 45 & $04 / 12 / 78$ & 102 & 100 & 1.00 & 7.30 & 12.0 & -- & 20 \\
\hline
\end{tabular}



Table 3.-- Water-chemistry data from production

[Samples collected and analyzed by personnel of the Coos Bay-North Bend Water Board]

\begin{tabular}{|c|c|c|c|c|c|c|c|c|}
\hline WELL & DATE & $\begin{array}{l}\text { ALKA- } \\
\text { LINITY } \\
\text { (MG/L } \\
\text { AS } \\
\text { CACO3) }\end{array}$ & $\begin{array}{l}\text { HARD- } \\
\text { NESS } \\
\text { (MG/L } \\
\text { AS } \\
\text { CACO3) }\end{array}$ & $\begin{array}{l}\text { IRON } \\
\text { DIS- } \\
\text { SOLVED } \\
\text { (MG/L) }\end{array}$ & $\begin{array}{c}\mathrm{PH} \\
(\mathrm{UN} \mid \mathrm{T})\end{array}$ & $\begin{array}{l}\text { CARBON } \\
\text { DIOXIDE } \\
(M G / L)\end{array}$ & $\begin{array}{l}\text { FLUO- } \\
\text { RIDE } \\
\text { DIS- } \\
\text { SOLVED } \\
(M G / L)\end{array}$ & $\begin{array}{l}\text { CHLO- } \\
\text { RIDE } \\
\text { DIS- } \\
\text { SOLVED } \\
(M G / L)\end{array}$ \\
\hline 45 & $05 / 11 / 78$ & 100 & 100 & 1.00 & 7.30 & 10.0 & -- & 20 \\
\hline 45 & $06 / 12 / 78$ & 106 & 94 & .20 & 7.20 & 13.0 & -- & 20 \\
\hline 45 & $07 / 17 / 78$ & 100 & 84 & .80 & 7.30 & 50.0 & -- & 20 \\
\hline 45 & $08 / 11 / 78$ & 102 & 90 & 1.00 & 7.10 & 16.0 & -- & 20 \\
\hline 45 & $09 / 15 / 78$ & 100 & 90 & .50 & 7.10 & 17.0 & -- & 20 \\
\hline 45 & $10 / 25 / 78$ & 104 & 90 & 2.30 & 7.10 & 17.0 & -- & 18 \\
\hline 45 & $11 / 15 / 78$ & 104 & 100 & .80 & 7.10 & 17.0 & -- & 20 \\
\hline 45 & $12 / 13 / 78$ & 102 & 90 & 1.00 & 7.40 & 8.0 & -- & 20 \\
\hline 45 & $01 / 22 / 79$ & 104 & 90 & 1.10 & 7.30 & 10.0 & -- & 25 \\
\hline 45 & $02 / 22 / 79$ & 100 & 104 & 2.00 & 7.30 & 10.0 & -- & 25 \\
\hline 45 & $03 / 20 / 79$ & 105 & 100 & .20 & 7.30 & 12.0 & -- & 20 \\
\hline 45 & $04 / 19 / 79$ & 100 & 96 & .60 & 7.30 & 10.0 & -- & 20 \\
\hline 45 & $05 / 21 / 79$ & 104 & 90 & .30 & 7.30 & 11.0 & .10 & 20 \\
\hline 45 & $06 / 13 / 79$ & 104 & 90 & .30 & 7.40 & 8.5 & .10 & 22 \\
\hline 45 & $08 / 16 / 79$ & 104 & 96 & .40 & 7.40 & 8.0 & -- & 20 \\
\hline 45 & $10 / 23 / 79$ & 106 & 104 & .28 & 7.30 & 11.0 & -- & 21 \\
\hline 45 & $11 / 20 / 79$ & 104 & 94 & .30 & 7.40 & 8.5 & -- & 20 \\
\hline 45 & $12 / 17 / 79$ & 104 & 90 & .29 & 7.40 & 8.2 & -- & 18 \\
\hline 45 & $01 / 13 / 80$ & 104 & 110 & .38 & 7.30 & 11.0 & - & 30 \\
\hline 45 & $02 / 14 / 80$ & 102 & 96 & .20 & 7.30 & 12.0 & -- & 20 \\
\hline 45 & $03 / 14 / 80$ & 104 & 98 & .30 & 7.30 & 11.0 & -- & 25 \\
\hline 45 & $04 / 15 / 80$ & 100 & 90 & .20 & 7.30 & 10.0 & -- & 20 \\
\hline 45 & $05 / 13 / 80$ & 102 & 90 & .40 & 7.30 & 11.0 & -- & 22 \\
\hline
\end{tabular}


Table 8.- - Water-chemistry data from production

[Samples collected and analyzed by personnel of the Coos Bay-North Bend Water Board]

\begin{tabular}{|c|c|c|c|c|c|c|}
\hline $\begin{array}{l}\text { ALKA- } \\
\text { LINITY }\end{array}$ & $\begin{array}{l}\text { HARD- } \\
\text { NESS }\end{array}$ & IRON & & & $\begin{array}{l}\text { FLUO- } \\
\text { RIDE }\end{array}$ & $\begin{array}{l}\text { CHLO- } \\
\text { RIDE }\end{array}$ \\
\hline (MG/L & (MG/L & DIS- & & CARBON & DIS- & DIS- \\
\hline AS & AS & SOLVED & $\mathrm{PH}$ & DIOXIDE & SOLVED & SOLVED \\
\hline
\end{tabular}
WELL DATE CACO3) CACO3) (MG/L) (UNIT) (MG/L) (MG/L) (MG/L)

\begin{tabular}{|c|c|c|c|c|c|c|c|c|}
\hline 45 & $06 / 16 / 80$ & 104 & 90 & .50 & 7.20 & 13.0 & -- & 23 \\
\hline 45 & $07 / 11 / 80$ & 108 & 92 & .60 & 7.50 & 68.0 & -- & 23 \\
\hline 45 & $08 / 12 / 80$ & 104 & 86 & .20 & 7.30 & 11.0 & .10 & 20 \\
\hline 45 & $09 / 15 / 80$ & 100 & 100 & .45 & 7.30 & 10.0 & -- & 18 \\
\hline 45 & $10 / 21 / 80$ & 102 & 108 & .25 & 7.30 & 10.5 & -- & 20 \\
\hline 45 & $11 / 14 / 80$ & 100 & 100 & .35 & 7.30 & 10.0 & -- & 30 \\
\hline 45 & $12 / 16 / 80$ & 104 & 104 & .10 & 7.40 & 8.2 & -- & 25 \\
\hline 45 & $01 / 15 / 81$ & 104 & -- & .39 & 7.40 & 8.4 & -- & 25 \\
\hline 45 & $02 / 16 / 81$ & 104 & 100 & .08 & 7.30 & 10.0 & -- & 25 \\
\hline 45 & $03 / 16 / 81$ & 100 & 100 & .50 & 7.20 & 13.0 & -- & 25 \\
\hline 45 & $04 / 15 / 81$ & 108 & 96 & .46 & 7.30 & 12.0 & -- & 25 \\
\hline 45 & $05 / 15 / 81$ & 100 & 100 & .19 & 7.30 & 10.0 & -- & 25 \\
\hline 45 & $06 / 11 / 81$ & 114 & 94 & 2.30 & 7.10 & 18.5 & -- & 27 \\
\hline 45 & $07 / 14 / 81$ & 104 & 94 & .20 & 7.30 & 11.0 & -- & 32 \\
\hline 45 & $08 / 28 / 81$ & 110 & 96 & .20 & 7.20 & 14.0 & -- & 28 \\
\hline 45 & $09 / 17 / 81$ & 104 & 94 & .22 & 7.20 & 13.0 & -- & 25 \\
\hline 45 & $10 / 27 / 81$ & 112 & 90 & 1.70 & 7.10 & 18.0 & -- & 25 \\
\hline 45 & $11 / 19 / 81$ & 100 & 100 & .20 & 7.30 & 10.0 & -- & 20 \\
\hline 45 & $12 / 15 / 81$ & 100 & 100 & .25 & 7.20 & 13.0 & -- & 25 \\
\hline 45 & $01 / 22 / 82$ & 102 & 95 & .35 & 7.20 & 13.0 & -- & 30 \\
\hline 45 & $05 / 20 / 82$ & 100 & 92 & .18 & 7.30 & 10.0 & -- & 30 \\
\hline 45 & $06 / 15 / 82$ & 104 & 104 & .18 & 7.30 & 11.0 & -- & 25 \\
\hline 45 & $07 / 15 / 82$ & 100 & 90 & .38 & 7.20 & 13.0 & -- & 30 \\
\hline
\end{tabular}


Table 8.--Water-chemistry data from production

[Samples collected and analyzed by personnel of the Coos Bay-North Bend Water Board]

\begin{tabular}{|c|c|c|c|c|c|c|c|c|}
\hline WELL & DATE & $\begin{array}{l}\text { ALKA- } \\
\text { LINITY } \\
\text { (MG/L } \\
\text { AS } \\
\text { CACO3) }\end{array}$ & $\begin{array}{l}\text { HARD- } \\
\text { NESS } \\
\text { (MG/L } \\
\text { AS } \\
\text { CACO3) }\end{array}$ & $\begin{array}{l}\text { IRON } \\
\text { DIS- } \\
\text { SOLVED } \\
\text { (MG/L) }\end{array}$ & $\begin{array}{c}\mathrm{PH} \\
(\mathrm{UN} \mid \mathrm{T})\end{array}$ & $\begin{array}{l}\text { CARBON } \\
\text { DIOXIDE } \\
(M G / L)\end{array}$ & $\begin{array}{l}\text { FLUO- } \\
\text { RIDE } \\
\text { DIS- } \\
\text { SOLVED } \\
\text { (MG/L) }\end{array}$ & $\begin{array}{l}\text { CHLO- } \\
\text { RIDE } \\
\text { DIS- } \\
\text { SOLVED } \\
(M G / L)\end{array}$ \\
\hline 45 & $08 / 11 / 82$ & 104 & 92 & .15 & 7.30 & 13.0 & -- & 32 \\
\hline 45 & $09 / 23 / 82$ & 90 & 90 & .18 & 7.30 & 9.2 & -- & 25 \\
\hline 45 & $10 / 20 / 82$ & 108 & 94 & .18 & 7.30 & 1.1 & -- & 24 \\
\hline 45 & $11 / 19 / 82$ & 100 & 84 & .28 & 7.30 & 10.0 & -- & 25 \\
\hline 45 & $12 / 20 / 82$ & 102 & 94 & .28 & 7.30 & 10.0 & -- & 25 \\
\hline 45 & $01 / 17 / 83$ & 100 & 90 & .60 & 7.30 & 10.0 & -- & 25 \\
\hline 45 & $02 / 16 / 83$ & 108 & 90 & .15 & 7.30 & 11.0 & -- & 25 \\
\hline 45 & $03 / 18 / 83$ & 102 & 108 & .18 & 7.30 & 10.0 & -- & 22 \\
\hline 45 & $04 / 26 / 83$ & 116 & 94 & .40 & 7.40 & 9.20 & -- & 28 \\
\hline 45 & $05 / 16 / 83$ & 104 & 100 & .18 & 7.30 & 11.0 & -- & 30 \\
\hline 45 & $06 / 22 / 83$ & 108 & 98 & .35 & 7.30 & 11.0 & -- & 25 \\
\hline 46 & $03 / 08 / 71$ & 122 & 110 & .01 & 7.50 & 8.0 & .01 & 32 \\
\hline $\begin{array}{l}46 \\
46\end{array}$ & $\begin{array}{l}04 / 21 / 71 \\
05 / 10 / 71\end{array}$ & $\begin{array}{l}130 \\
126\end{array}$ & $\begin{array}{l}114 \\
114\end{array}$ &.$\overline{-0}$ & $\begin{array}{l}7.50 \\
7.40\end{array}$ & $\begin{array}{r}8.0 \\
14.0\end{array}$ & $\begin{array}{l}.01 \\
.01\end{array}$ & $\begin{array}{l}31 \\
31\end{array}$ \\
\hline 46 & $06 / 11 / 71$ & 122 & 116 & .02 & 7.50 & 8.0 & .01 & 40 \\
\hline 46 & $07 / 16 / 71$ & 170 & 122 & .01 & 7.50 & 11.0 & .02 & 28 \\
\hline 46 & $08 / 17 / 71$ & 168 & 96 & .10 & 7.10 & 28.0 & -- & 31 \\
\hline 46 & $09 / 15 / 71$ & 124 & 102 & 1.20 & 7.60 & 6.0 & .03 & 24 \\
\hline 46 & $10 / 22 / 71$ & 120 & 100 & .04 & 7.60 & 6.0 & -- & 26 \\
\hline 46 & $12 / 21 / 71$ & 124 & 118 & .01 & 7.60 & 7.5 & -- & 26 \\
\hline 46 & $01 / 25 / 72$ & 118 & 118 & -- & 7.40 & 9.0 & .01 & 26 \\
\hline 46 & $02 / 16 / 72$ & 130 & 110 & -- & 7.50 & 8.0 & .04 & 16 \\
\hline
\end{tabular}


Table 8.--Water-chemistry data from production wells, 1971-83--Continued

[Samples collected and analyzed by personnel of the Coos Bay-North Bend Water Board]

\begin{tabular}{|c|c|c|c|c|c|c|c|c|}
\hline WELL & DATE & $\begin{array}{c}\text { ALKA- } \\
\text { LINITY } \\
\text { (MG/L } \\
\text { AS } \\
\text { CACO3) }\end{array}$ & $\begin{array}{l}\text { HARD- } \\
\text { NESS } \\
\text { (MG/L } \\
\text { AS } \\
\text { CACO3) }\end{array}$ & $\begin{array}{l}\text { IRON } \\
\text { DIS- } \\
\text { SOLVED } \\
\text { (MG/L) }\end{array}$ & $\begin{array}{c}\mathrm{PH} \\
(\mathrm{UN} I T)\end{array}$ & $\begin{array}{l}\text { CARBON } \\
\text { DIOXIDE } \\
(M G / L)\end{array}$ & $\begin{array}{l}\text { FLUO- } \\
\text { RIDE } \\
\text { DIS- } \\
\text { SOLVED } \\
(M G / L)\end{array}$ & $\begin{array}{l}\text { CHLO- } \\
\text { RIDE } \\
\text { DIS- } \\
\text { SOLVED } \\
(M G / L)\end{array}$ \\
\hline 46 & $03 / 24 / 72$ & 126 & 126 & -- & 7.40 & 21.0 & .23 & 14 \\
\hline 46 & $04 / 28 / 72$ & 130 & 118 & -- & 7.30 & 13.5 & -- & -- \\
\hline 46 & $05 / 18 / 72$ & 136 & 104 & .01 & 7.60 & 7.5 & .04 & 25 \\
\hline 46 & $08 / 30 / 72$ & 124 & 114 & -- & 7.60 & 14.0 & .04 & 27 \\
\hline 46 & $09 / 26 / 72$ & 114 & 86 & -- & 7.20 & 14.0 & .04 & 26 \\
\hline 46 & $11 / 28 / 72$ & 124 & 114 & -- & 7.00 & 26.0 & .04 & 28 \\
\hline 46 & $12 / 26 / 72$ & 126 & 116 & -- & 7.10 & 21.0 & .04 & 26 \\
\hline 46 & $02 / 27 / 73$ & 110 & 132 & -- & 7.50 & 65.0 & .01 & 26 \\
\hline 46 & $0: / 30 / 73$ & 120 & 128 & -- & 7.60 & 8.0 & .04 & 30 \\
\hline 46 & $04 / 27 / 73$ & 120 & 100 & -- & 7.50 & 7.5 & .04 & 29 \\
\hline 46 & $05 / 24 / 73$ & 130 & 98 & -- & 7.50 & 8.5 & .04 & 24 \\
\hline 46 & $06 / 28 / 73$ & 126 & 116 & -- & 7.40 & 11.0 & .04 & 26 \\
\hline 46 & $08 / 30 / 73$ & 134 & 108 & -- & 7.40 & 11.0 & .04 & 24 \\
\hline 46 & $10 / 09 / 73$ & 128 & 110 & -- & 7.10 & 21.0 & .04 & 24 \\
\hline 46 & $11 / 06 / 73$ & 130 & 126 & -- & 7.00 & 27.0 & .04 & 22 \\
\hline 46 & $12 / 20 / 73$ & 132 & 106 & .01 & 7.30 & 16.0 & .04 & 30 \\
\hline 46 & $01 / 29 / 74$ & 132 & 114 & -- & 7.30 & 14.0 & .04 & 31 \\
\hline 46 & $02 / 26 / 74$ & 125 & 112 & -- & 7.20 & 16.0 & .04 & 27 \\
\hline 46 & $03 / 28 / 74$ & 120 & 110 & .01 & 7.20 & 15.0 & .04 & 28 \\
\hline 46 & $04 / 30 / 74$ & 116 & 114 & .04 & 7.20 & 15.0 & .04 & 28 \\
\hline 46 & $07 / 24 / 74$ & 124 & 114 & .01 & 7.40 & 9.5 & .40 & 25 \\
\hline 46 & $08 / 28 / 74$ & 120 & 112 & -- & 7.40 & 10.0 & .40 & 30 \\
\hline 46 & $09 / 27 / 74$ & 124 & 120 & -- & 7.30 & 14.0 & .40 & 28 \\
\hline
\end{tabular}


Table 8.- - Water-chemistry data from production

[Samples collected and analyzed by personnel of the Coos Bay-North Bend Water Board]

\begin{tabular}{|c|c|c|c|c|c|c|c|c|}
\hline WELL & DATE & $\begin{array}{l}\text { ALKA- } \\
\text { LINITY } \\
\text { (MG/L } \\
\text { AS } \\
\text { CACO3) }\end{array}$ & $\begin{array}{l}\text { HARD- } \\
\text { NESS } \\
\text { (MG/L } \\
\text { AS } \\
\text { CACO3) }\end{array}$ & $\begin{array}{l}\text { IRON } \\
\text { DIS- } \\
\text { SOLVED } \\
\text { (MG/L) }\end{array}$ & $\begin{array}{c}\mathrm{PH} \\
(\mathrm{UN} \mid \mathrm{T})\end{array}$ & $\begin{array}{l}\text { CARBON } \\
\text { DIOXIDE } \\
(M G / L)\end{array}$ & $\begin{array}{l}\text { FLUO- } \\
\text { RIDE } \\
\text { DIS- } \\
\text { SOLVED } \\
(M G / L)\end{array}$ & $\begin{array}{l}\text { CHLO- } \\
\text { RIDE } \\
\text { DIS- } \\
\text { SOLVED } \\
(M G / L)\end{array}$ \\
\hline 46 & $10 / 24 / 74$ & 126 & 132 & -- & 7.40 & 20.0 & .40 & 26 \\
\hline 46 & $11 / 21 / 74$ & 120 & 124 & - & 7.20 & 15.0 & .20 & 26 \\
\hline 46 & $12 / 31 / 74$ & 128 & 122 & - & 7.30 & 13.0 & .40 & 21 \\
\hline 46 & $02 / 28 / 75$ & 132 & 124 & -- & 7.30 & 8.0 & .40 & 24 \\
\hline 46 & $03 / 26 / 75$ & 116 & 130 & -- & 7.10 & 18.5 & .40 & 16 \\
\hline 46 & $04 / 23 / 75$ & 116 & 126 & -- & 7.00 & 23.0 & .40 & 14 \\
\hline 46 & $05 / 27 / 75$ & 130 & 118 & -- & 7.40 & 11.0 & .40 & 24 \\
\hline 46 & $07 / 29 / 75$ & 132 & 106 & -- & 7.40 & 11.0 & .40 & 22 \\
\hline 46 & $09 / 25 / 75$ & 128 & 108 & - & 7.50 & 8.0 & .40 & 22 \\
\hline 46 & $11 / 25 / 75$ & 124 & 108 & -- & 7.30 & 13.0 & .40 & 28 \\
\hline 46 & $12 / 30 / 75$ & 116 & 110 & -- & 7.60 & 6.0 & .40 & 25 \\
\hline 46 & $02 / 19 / 76$ & 110 & 116 & .07 & 7.40 & 90.0 & .10 & 25 \\
\hline 46 & $03 / 30 / 76$ & 115 & 120 & -- & 7.50 & 7.5 & .15 & 28 \\
\hline 46 & $04 / 23 / 76$ & 120 & 120 & .02 & 7.40 & 9.0 & .20 & 25 \\
\hline 46 & $06 / 17 / 76$ & 118 & 110 & .10 & 7.50 & 7.0 & .15 & 30 \\
\hline 46 & $07 / 19 / 76$ & 112 & 120 & -- & 7.40 & 9.0 & .15 & 30 \\
\hline 46 & $08 / 20 / 76$ & 112 & 116 & 2.00 & 7.40 & 9.0 & .15 & 32 \\
\hline 46 & $09 / 21 / 76$ & 110 & 112 & .06 & 7.40 & 10.0 & .15 & 32 \\
\hline 46 & $10 / 21 / 76$ & 114 & 116 & .10 & 7.40 & - & .15 & 30 \\
\hline 46 & $11 / 18 / 76$ & 108 & 108 & .08 & 7.40 & 8.0 & .20 & 30 \\
\hline
\end{tabular}


Table 8.--Water-chemistry data from production wells, 1971-83--Continued

[Samples collected and analyzed by personnel of the Coos Bay-North Bend Water Board]

\begin{tabular}{|c|c|c|c|c|c|c|}
\hline ALKA- & HARD- & & & & FLUO- & CHLO- \\
\hline LINITY & NESS & IRON & & & RIDE & RIDE \\
\hline (MG/L & (MG/L & DIS- & & CARBON & DIS- & DIS- \\
\hline AS & AS & SOLVED & $\mathrm{PH}$ & DIOXI1)E & SOLVED & SOLVEL \\
\hline
\end{tabular}
WELL DATE CACO3) CACO3) (MG/L) (UNIT) (MG/L) (MG/L) (MG/L)

\begin{tabular}{lllllllll}
\hline 46 & $02 / 15 / 77$ & 110 & 120 & .10 & 7.40 & 9.0 & .15 & 28 \\
46 & $03 / 15 / 77$ & 120 & 114 & -- & 7.50 & 7.0 & .25 & 25 \\
46 & $04 / 15 / 77$ & 110 & 120 & .10 & 7.40 & 9.0 & .10 & 30 \\
46 & $05 / 16 / 77$ & 120 & 118 & .10 & 7.50 & 80.0 & .20 & 30 \\
46 & $06 / 21 / 77$ & 120 & 118 & .60 & 7.40 & 9.0 & .20 & 28 \\
46 & $07 / 21 / 77$ & 112 & 120 & .04 & 7.30 & 11.0 & -- & 25 \\
46 & $08 / 19 / 77$ & 110 & 118 & .20 & 7.30 & 11.0 & -- & 25 \\
46 & $09 / 15 / 77$ & 120 & 120 & .01 & 7.20 & 15.0 & -- & 20 \\
46 & $10 / 20 / 77$ & 118 & 112 & 1.20 & 7.20 & 15.0 & -- & 20 \\
46 & $12 / 20 / 77$ & 112 & 110 & 4.80 & 7.10 & 18.0 & -- & 22 \\
& & & & & & & & \\
45 & $11 / 16 / 78$ & 120 & 104 & .01 & 7.20 & 15.0 & -- & 25 \\
46 & $02 / 15 / 78$ & 130 & 105 & -- & 7.20 & 16.0 & -- & 25 \\
46 & $03 / 14 / 78$ & 116 & 110 & -- & 7.50 & 65.0 & -- & 25 \\
46 & $04 / 12 / 78$ & 120 & 120 & .05 & 7.30 & 17.0 & -- & 25 \\
46 & $05 / 11 / 78$ & 120 & 118 & -- & 7.50 & 8.0 & -- & 22 \\
46 & $06 / 12 / 78$ & 118 & 116 & .13 & 7.50 & 8.0 & -- & 26 \\
46 & $07 / 17 / 78$ & 120 & 104 & .50 & 7.40 & 10.0 & -- & 25 \\
46 & $08 / 11 / 78$ & 120 & 120 & .10 & 7.20 & 10.0 & -- & 25 \\
46 & $09 / 15 / 78$ & 110 & 110 & -- & 7.30 & 10.0 & -- & 25 \\
46 & $10 / 25 / 78$ & 118 & 106 & .16 & 7.20 & 14.0 & -- & 22 \\
46 & $11 / 15 / 78$ & 120 & 110 & .10 & 7.30 & 13.0 & -- & 25 \\
46 & $12 / 13 / 78$ & 118 & 118 & .20 & 7.40 & 10.0 & -- & 25 \\
& & & & & & & & \\
46 & $01 / 22 / 79$ & 118 & 104 & .30 & 7.40 & 10.0 & -- & 25 \\
46 & $02 / 22 / 79$ & 120 & 110 & .40 & 7.40 & 10.0 & -- & 25 \\
& & & & & & & & \\
\hline
\end{tabular}


Table 8.- -Water-chemistry data from production wells, 1971-83--Continued

[Samples collected and analyzed by personnel of the Coos Bay-North Bend Water Board]

\begin{tabular}{|c|c|c|c|c|c|c|c|c|}
\hline WELL & DATE & $\begin{array}{c}\text { ALKA- } \\
\text { LINITY } \\
\text { (MG/L } \\
\text { AS } \\
\text { CACO3) }\end{array}$ & $\begin{array}{l}\text { HARD- } \\
\text { NESS } \\
\text { (MG/L } \\
\text { AS } \\
\text { CACO3) }\end{array}$ & $\begin{array}{l}\text { IRON } \\
\text { DIS- } \\
\text { SOLVED } \\
(M G / L)\end{array}$ & $\begin{array}{c}\mathrm{PH} \\
(\mathrm{UNIT})\end{array}$ & $\begin{array}{c}\text { CARBON } \\
\text { DIOXIDE } \\
(M G / L)\end{array}$ & $\begin{array}{l}\text { FLUO- } \\
\text { RIDE } \\
\text { DIS- } \\
\text { SOLVED } \\
\text { (MG/L) }\end{array}$ & $\begin{array}{l}\text { CHLO- } \\
\text { RIDE } \\
\text { DIS- } \\
\text { SOLVED } \\
(M G / L)\end{array}$ \\
\hline 46 & $03 / 20 / 79$ & 120 & 118 & .10 & 7.40 & 8.0 & -- & 25 \\
\hline 46 & $04 / 19 / 79$ & 120 & 110 & .10 & 7.50 & 7.0 & -- & 20 \\
\hline 46 & $05 / 21 / 79$ & 110 & 108 & .09 & 7.50 & 7.0 & .10 & 25 \\
\hline 46 & $06 / 13 / 79$ & 118 & 104 & .10 & 7.50 & 8.0 & .11 & 25 \\
\hline 46 & $08 / 16 / 79$ & 116 & 110 & .05 & 7.60 & 1.0 & -- & 20 \\
\hline 46 & $10 / 23 / 79$ & 124 & 116 & .05 & 7.60 & 7.0 & -- & 25 \\
\hline 46 & $11 / 20 / 79$ & 120 & 108 & .06 & 7.60 & 6.0 & -- & 25 \\
\hline 46 & $12 / 17 / 79$ & 120 & 110 & .05 & 7.60 & 6.0 & -- & 20 \\
\hline 46 & $01 / 13 / 80$ & 118 & 118 & .05 & 7.50 & 7.2 & -- & 30 \\
\hline 46 & $02 / 14 / 80$ & 120 & 106 & .08 & 7.60 & 6.0 & -- & 25 \\
\hline 46 & $03 / 14 / 80$ & 118 & 118 & .10 & 7.60 & 6.0 & -- & 25 \\
\hline 46 & $04 / 15 / 80$ & 120 & 104 & .15 & 7.50 & 8.0 & -- & 25 \\
\hline 46 & $05 / 13 / 80$ & 120 & 108 & .10 & 7.50 & 7.0 & -- & 25 \\
\hline 46 & $06 / 16 / 80$ & 120 & 118 & .02 & 7.50 & 8.0 & .01 & 25 \\
\hline 46 & $07 / 11 / 80$ & 118 & 102 & .04 & 7.60 & 6.0 & -- & 25 \\
\hline 46 & $08 / 12 / 80$ & 118 & 108 & .08 & 7.60 & 6.0 & -- & 20 \\
\hline 46 & $09 / 15 / 80$ & 118 & 110 & .06 & 7.50 & 7.5 & -- & 25 \\
\hline 46 & $10 / 21 / 80$ & 118 & 118 & .10 & 7.50 & 7.5 & -- & 25 \\
\hline 46 & $11 / 14 / 80$ & 120 & 110 & .02 & 7.50 & 7.0 & -- & 30 \\
\hline 46 & $12 / 16 / 80$ & 120 & 110 & .05 & 7.50 & 7.2 & -- & 30 \\
\hline 46 & $01 / 15 / 81$ & 110 & -- & .05 & 7.40 & 7.0 & -- & 30 \\
\hline 46 & $02 / 16 / 81$ & 118 & 108 & .01 & 7.50 & 7.2 & -- & 30 \\
\hline 46 & $03 / 16 / 81$ & 120 & 118 & .09 & 7.50 & 8.0 & -- & 30 \\
\hline
\end{tabular}


Table 8.--Water-chemistry data from production wells, 1971-83--Continued

[Samples collected and analyzed by personnel of the Coos Bay-North Bend Water Board]

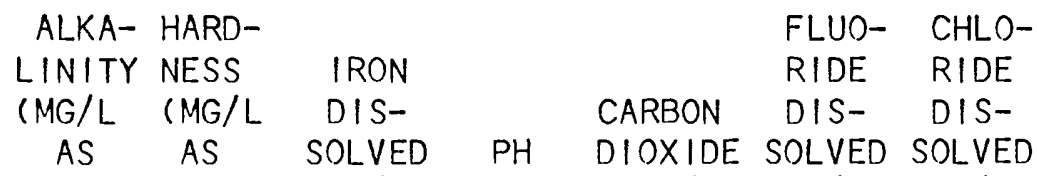
WELL DATE CACO3) CACO3) $(M G / L)(U N I T)(M G / L)(M G / L)(M G / L)$

\begin{tabular}{lllllllll}
\hline 46 & $04 / 15 / 81$ & 120 & 108 & .04 & 7.50 & 7.5 & -- & 30 \\
46 & $05 / 15 / 81$ & 120 & 120 & -- & 7.50 & 8.0 & -- & 30 \\
46 & $06 / 11 / 81$ & 124 & 112 & .07 & 7.30 & 12.0 & -- & 30 \\
46 & $07 / 14 / 81$ & 120 & 110 & .10 & 7.50 & 7.5 & -- & 35 \\
46 & $08 / 28 / 81$ & 122 & 110 & .02 & 7.60 & 6.0 & -- & 30 \\
46 & $09 / 17 / 81$ & 120 & 118 & .08 & 7.50 & 7.6 & -- & 30 \\
46 & $10 / 27 / 81$ & 126 & 100 & .15 & 7.30 & 16.0 & -- & 30 \\
46 & $11 / 19 / 81$ & 118 & 110 & .20 & 7.50 & 7.5 & -- & 25 \\
46 & $12 / 15 / 81$ & 120 & 130 & .05 & 7.50 & 75.0 & -- & 25 \\
46 & $01 / 22 / 82$ & 120 & 110 & .02 & 7.50 & 7.5 & -- & 35 \\
46 & $05 / 20 / 82$ & 120 & 114 & .12 & 7.50 & 7.6 & -- & 30 \\
46 & $06 / 15 / 82$ & 122 & 110 & .05 & 7.50 & 7.8 & -- & 25 \\
46 & $07 / 15 / 82$ & 120 & 102 & .12 & 7.50 & 7.5 & -- & 32 \\
46 & $08 / 11 / 82$ & 120 & 110 & .12 & 7.50 & 7.5 & -- & 34 \\
46 & $09 / 23 / 82$ & 120 & 118 & .05 & 7.50 & 7.5 & -- & 30 \\
46 & $10 / 20 / 82$ & 120 & 112 & .04 & 7.30 & 1.2 & -- & 28 \\
46 & $11 / 19 / 82$ & 120 & 110 & .20 & 7.50 & 7.6 & -- & 30 \\
46 & $12 / 20 / 82$ & 120 & 118 & .05 & 7.50 & 7.6 & -- & 30 \\
& & & & & & & & \\
46 & $01 / 17 / 83$ & 120 & 112 & .11 & 7.50 & 7.6 & -- & 25 \\
46 & $02 / 16 / 83$ & 120 & 104 & .01 & 7.50 & 7.5 & -- & 29 \\
46 & $03 / 18 / 83$ & 122 & 120 & .04 & 7.50 & 7.6 & -- & 28 \\
46 & $04 / 26 / 83$ & 124 & 138 & .09 & 7.50 & 80.0 & -- & 28 \\
46 & $05 / 16 / 83$ & 118 & 104 & .06 & 7.50 & 7.5 & -- & 32 \\
46 & $06 / 22 / 83$ & 122 & 110 & .07 & 7.50 & 7.8 & -- & 29 \\
& & & & & & & & \\
\hline
\end{tabular}


Table 8.--Water-chemistry data from production

[Samples collected and analyzed by personnel of the Coos Bay-North Bend Water Board]

\begin{tabular}{|c|c|c|c|c|c|c|c|c|}
\hline WELL & DATE & $\begin{array}{c}\text { ALKA- } \\
\text { LINITY } \\
\text { (MG/L } \\
\text { AS } \\
\text { CACO3) }\end{array}$ & $\begin{array}{l}\text { HARD- } \\
\text { NESS } \\
\text { (MG/L } \\
\text { AS } \\
\text { CACO3) }\end{array}$ & $\begin{array}{l}\text { IRON } \\
\text { DIS- } \\
\text { SOLVED } \\
(M G / L)\end{array}$ & $\begin{array}{c}\text { PH } \\
\text { (UNIT) }\end{array}$ & $\begin{array}{l}\text { CARBON } \\
\text { DIOXIDE } \\
(M G / L)\end{array}$ & $\begin{array}{l}\text { FLUO- } \\
\text { RIDE } \\
\text { DIS- } \\
\text { SOLVED } \\
\text { (MG/L) }\end{array}$ & $\begin{array}{l}\text { CHLO- } \\
\text { RIDE } \\
\text { DIS- } \\
\text { SOLVED } \\
(M G / L)\end{array}$ \\
\hline 47 & $03 / 08 / 71$ & 112 & 102 & .02 & 7.30 & 12.0 & .01 & 31 \\
\hline 47 & $04 / 21 / 71$ & 112 & 106 & .02 & 7.20 & 12.0 & .01 & 31 \\
\hline 47 & $05 / 10 / 71$ & 122 & 118 & .06 & 7.10 & 18.0 & -- & 31 \\
\hline 47 & $06 / 11 / 71$ & 120 & 114 & .08 & 7.00 & 24.0 & -- & 32 \\
\hline 47 & $07 / 16 / 71$ & 120 & 102 & 4.00 & 7.10 & 19.0 & .04 & 31 \\
\hline 47 & $08 / 17 / 71$ & 170 & 106 & 2.02 & 7.00 & 35.0 & .02 & 31 \\
\hline 47 & $09 / 15 / 71$ & 120 & 116 & .06 & 7.00 & 24.0 & -- & 28 \\
\hline 47 & $10 / 22 / 71$ & 120 & 116 & .04 & 7.10 & 19.0 & -- & 28 \\
\hline 47 & $12 / 21 / 71$ & 118 & 114 & .04 & 7.10 & 19.0 & -- & 28 \\
\hline 47 & $01 / 25 / 72$ & 118 & 110 & .04 & 7.00 & 32.0 & .01 & 26 \\
\hline 47 & $02 / 16 / 72$ & 182 & 104 & .02 & 7.20 & 22.0 & -- & 26 \\
\hline 47 & $03 / 24 / 72$ & 116 & 110 & .01 & 6.80 & 36.0 & .02 & 18 \\
\hline 47 & $05 / 18 / 72$ & 106 & 118 & .04 & 6.60 & 58.0 & .20 & 18 \\
\hline 47 & $08 / 30 / 72$ & 114 & 122 & .04 & 7.00 & 23.0 & .04 & 22 \\
\hline 47 & $09 / 26 / 72$ & 126 & 112 & .08 & 7.00 & 25.0 & .01 & 30 \\
\hline 47 & $11 / 28 / 72$ & 118 & 108 & .06 & 6.80 & 38.0 & .01 & 26 \\
\hline 47 & $12 / 26 / 72$ & 116 & 122 & .04 & 6.60 & 60.0 & .01 & 28 \\
\hline 47 & $02 / 27 / 73$ & 124 & 122 & .08 & 6.80 & 36.0 & -- & 27 \\
\hline 47 & $03 / 30 / 73$ & 132 & 112 & .07 & 7.00 & 27.0 & .02 & 26 \\
\hline 47 & $04 / 27 / 73$ & 96 & 96 & .06 & 7.10 & 16.0 & .01 & 26 \\
\hline 47 & $05 / 24 / 73$ & 114 & 84 & .08 & 6.90 & 29.0 & .01 & 26 \\
\hline 47 & $06 / 28 / 73$ & -- & - & -- & 7.00 & -- & -- & -- \\
\hline 47 & $08 / 30 / 73$ & 124 & 100 & .08 & 6.60 & 64.0 & .01 & 25 \\
\hline 47 & $10 / 09 / 73$ & 110 & 122 & .07 & 6.80 & 35.0 & .01 & 30 \\
\hline
\end{tabular}


Table 8.--Water-chemistry data from production wells, 1971-83--Continued

[Samples collected and analyzed by personnel of the Coos Bay-North Bend Water Board]

\begin{tabular}{|c|c|c|c|c|c|c|c|c|}
\hline WELL & DATE & $\begin{array}{l}\text { ALKA- } \\
\text { LINITY } \\
\text { (MG/L } \\
\text { AS } \\
\text { CACO3) }\end{array}$ & $\begin{array}{l}\text { HARD- } \\
\text { NESS } \\
\text { (MG/L } \\
\text { AS } \\
\text { CACO3) }\end{array}$ & $\begin{array}{l}\text { IRON } \\
\text { DIS- } \\
\text { SOLVED } \\
\text { (MG/L) }\end{array}$ & $\begin{array}{c}\mathrm{PH} \\
(\mathrm{UNIT})\end{array}$ & $\begin{array}{l}\text { CARBON } \\
\text { DIOXIDE } \\
(M G / L)\end{array}$ & $\begin{array}{l}\text { FLUO- } \\
\text { RIDE } \\
\text { DIS- } \\
\text { SOLVED } \\
\text { (MG/L) }\end{array}$ & $\begin{array}{l}\text { CHLO- } \\
\text { RIDE } \\
\text { DIS- } \\
\text { SOLVED } \\
(M G / L)\end{array}$ \\
\hline 47 & $11 / 06 / 73$ & 98 & 120 & .06 & 6.90 & 25.0 & .02 & 28 \\
\hline 47 & $12 / 20 / 73$ & 110 & 86 & .06 & 6.90 & 28.0 & .01 & 26 \\
\hline 47 & $01 / 29 / 74$ & 116 & 108 & .07 & 7.00 & 22.0 & .09 & 28 \\
\hline 47 & $02 / 26 / 74$ & 112 & 108 & .06 & 6.90 & 28.0 & .02 & 28 \\
\hline 47 & $04 / 30 / 74$ & 108 & 120 & .08 & 6.80 & 35.0 & .01 & 30 \\
\hline 47 & $08 / 28 / 74$ & 98 & 120 & .01 & 6.60 & 51.0 & .10 & 22 \\
\hline 47 & $09 / 27 / 74$ & 102 & 132 & .08 & 6.60 & 75.0 & .20 & 26 \\
\hline 47 & $11 / 21 / 74$ & 110 & 122 & .08 & 6.80 & 36.0 & .10 & 20 \\
\hline 47 & $12 / 31 / 74$ & 174 & 154 & .02 & 7.30 & 34.0 & .40 & 17 \\
\hline 47 & $02 / 28 / 75$ & 122 & 126 & .04 & 6.90 & 21.0 & .40 & 18 \\
\hline 47 & $03 / 26 / 75$ & 114 & 134 & .04 & 7.00 & 24.0 & .20 & 20 \\
\hline 47 & $04 / 23 / 75$ & 112 & 130 & .04 & 7.00 & 22.0 & .20 & 20 \\
\hline 47 & $05 / 27 / 75$ & 106 & 120 & .04 & 6.90 & 28.0 & .20 & 20 \\
\hline 47 & $07 / 29 / 75$ & 100 & 108 & .04 & 7.00 & 20.0 & .10 & 20 \\
\hline 47 & $09 / 25 / 75$ & 104 & 112 & .04 & 7.00 & 23.0 & .10 & 26 \\
\hline 47 & $11 / 25 / 75$ & 96 & 100 & .04 & 7.10 & 15.5 & .10 & 30 \\
\hline 47 & $12 / 30 / 75$ & 180 & 140 & .15 & 7.50 & 12.0 & .40 & 7 \\
\hline 47 & $02 / 19 / 76$ & 170 & 164 & .50 & 7.40 & 14.0 & .10 & 20 \\
\hline 47 & $03 / 30 / 76$ & 160 & 160 & 1.50 & 7.40 & 14.0 & .20 & 20 \\
\hline 47 & $04 / 23 / 76$ & 98 & 150 & 7.00 & 7.40 & 8.0 & .20 & 20 \\
\hline 47 & $06 / 17 / 76$ & 90 & 160 & 1.40 & 7.30 & 8.0 & .15 & - \\
\hline 47 & $07 / 19 / 76$ & 90 & 164 & 1.30 & 7.20 & 8.0 & .20 & 20 \\
\hline
\end{tabular}


Table 8.--Water-chemistry data from production wells, 1971-83--Continued

[Samples collected and analyzed by personnel of the Coos Bay-North Bend Water Board]

\begin{tabular}{|c|c|c|c|c|c|c|c|c|}
\hline WELL & DATE & $\begin{array}{c}\text { ALKA- } \\
\text { LINITY } \\
\text { (MG/L } \\
\text { AS } \\
\text { CACO3) }\end{array}$ & $\begin{array}{l}\text { HARD- } \\
\text { NESS } \\
\text { (MG/L } \\
\text { AS } \\
\text { CACO3) }\end{array}$ & $\begin{array}{l}\text { IRON } \\
\text { DIS- } \\
\text { SOLVED } \\
\text { (MG/L) }\end{array}$ & $\begin{array}{c}\text { PH } \\
(\text { UNIT) }\end{array}$ & $\begin{array}{l}\text { CARBON } \\
\text { DIOXIDE } \\
(M G / L)\end{array}$ & $\begin{array}{l}\text { FLUO- } \\
\text { RIDE } \\
\text { DIS- } \\
\text { SOLVED } \\
\text { (MG/L) }\end{array}$ & $\begin{array}{l}\text { CHLO- } \\
\text { RIDE } \\
\text { DIS- } \\
\text { SOLVED } \\
(M G / L)\end{array}$ \\
\hline 47 & $08 / 20 / 76$ & 86 & 150 & 5.60 & 7.30 & 9.0 & .20 & 22 \\
\hline 47 & $09 / 21 / 76$ & 84 & 145 & 5.60 & 7.20 & 90.0 & .20 & 20 \\
\hline 47 & $10 / 21 / 76$ & 84 & 148 & 5.50 & 7.30 & 9.0 & .20 & 22 \\
\hline 47 & $11 / 18 / 76$ & 180 & 125 & .06 & 7.30 & 25.0 & .18 & 15 \\
\hline 47 & $02 / 15 / 77$ & 180 & 140 & .15 & 7.50 & 11.0 & .20 & 10 \\
\hline 47 & $03 / 15 / 77$ & 100 & 96 & .20 & 7.00 & 16.0 & .08 & 20 \\
\hline 47 & $04 / 15 / 77$ & 182 & 118 & .10 & 7.50 & 12.0 & .26 & 8 \\
\hline 47 & $05 / 16 / 77$ & 114 & 84 & 1.00 & 6.90 & 29.0 & .10 & 20 \\
\hline 47 & $06 / 21 / 77$ & 180 & 160 & 1.80 & 7.40 & 14.0 & .20 & 15 \\
\hline 47 & $07 / 21 / 77$ & 160 & 140 & 1.20 & 7.30 & 16.0 & -- & 15 \\
\hline 47 & $08 / 19 / 77$ & 162 & 136 & 1.00 & 7.30 & 16.0 & -- & 8 \\
\hline 47 & $09 / 15 / 77$ & 164 & 140 & -- & 7.30 & 16.0 & -- & 5 \\
\hline 47 & $10 / 20 / 77$ & 140 & 132 & 1.40 & 7.20 & 17.5 & -- & 15 \\
\hline 47 & $12 / 20 / 77$ & 122 & 140 & 2.00 & 7.10 & 19.0 & -- & 15 \\
\hline 47 & $01 / 16 / 78$ & 92 & 80 & 2.00 & 7.10 & 10.0 & -- & 15 \\
\hline 47 & $02 / 16 / 78$ & 94 & 80 & 2.00 & 7.20 & 14.0 & -- & 16 \\
\hline 47 & $03 / 14 / 78$ & 90 & 85 & 2.00 & 7.10 & 10.0 & -- & 12 \\
\hline 47 & $04 / 12 / 78$ & 100 & 104 & 4.00 & -- & 17.0 & -- & 8 \\
\hline 47 & $05 / 11 / 78$ & 140 & 120 & 6.00 & 7.30 & 11.0 & -- & 20 \\
\hline 47 & $06 / 12 / 78$ & 82 & 120 & 5.80 & 7.40 & 60.0 & -- & 20 \\
\hline 47 & $07 / 17 / 78$ & 80 & 118 & 5.60 & 7.30 & 8.0 & -- & 25 \\
\hline 47 & $08 / 11 / 78$ & 150 & 140 & 1.50 & 7.10 & 24.0 & -- & 15 \\
\hline 47 & $09 / 15 / 78$ & 140 & 130 & 2.50 & 7.10 & 22.0 & -- & 18 \\
\hline
\end{tabular}


Table 8.--Water-chemistry data from production wells, 1971-83--Continued

[Samples collected and analyzed by personnel of the Coos Bay-North Bend Water Board]

\begin{tabular}{|c|c|c|c|c|c|c|}
\hline ALKA- & HARD- & & & & FLUO- & CHLO- \\
\hline LINITY & NESS & IRON & & & RIDE & RIDE \\
\hline (MG/L & (MG/L & DIS- & & CARBON & DIS- & DIS- \\
\hline AS & AS & SOLVED & $\mathrm{PH}$ & DIOXIDE & SOLVED & Sol $\mathrm{V}$ \\
\hline
\end{tabular}
WELL DATE CAC03) CACO3) $(M G / L) \quad(U N I T) \quad(M G / L) \quad(M G / L) \quad(M G / L)$

\begin{tabular}{rrrrrrrrr}
\hline 47 & $10 / 25 / 78$ & 140 & 135 & 2.00 & 7.10 & 22.0 & -- & 16 \\
47 & $11 / 15 / 78$ & 140 & 135 & 1.50 & 7.20 & 18.0 & -- & 21 \\
47 & $12 / 13 / 78$ & 135 & 130 & 2.00 & 7.30 & 13.0 & -- & 13 \\
& & & & & & & & \\
47 & $01 / 22 / 79$ & 140 & 120 & 1.80 & 7.20 & 13.0 & -- & 15 \\
47 & $02 / 22 / 79$ & 160 & 140 & 1.00 & 7.50 & 10.0 & -- & 10 \\
47 & $03 / 20 / 79$ & 158 & 138 & 1.10 & 7.50 & 8.0 & -- & 10 \\
47 & $04 / 19 / 79$ & 125 & 118 & 1.40 & 7.30 & 13.0 & -- & 12 \\
47 & $05 / 21 / 79$ & 120 & 104 & 1.40 & 7.30 & 12.0 & .11 & 12 \\
47 & $06 / 13 / 79$ & 124 & 120 & 1.50 & 7.30 & 13.0 & .12 & 15 \\
47 & $08 / 16 / 79$ & 140 & 130 & .25 & 7.50 & 9.0 & -- & 15 \\
47 & $10 / 23 / 79$ & 146 & 126 & .39 & 7.50 & 9.0 & -- & 15 \\
47 & $11 / 20 / 79$ & 144 & 119 & .06 & 7.60 & 7.0 & -- & 18 \\
47 & $12 / 17 / 79$ & 145 & 124 & .38 & 7.50 & 10.0 & -- & 20 \\
& & & & & & & & \\
47 & $01 / 13 / 80$ & 130 & 120 & .15 & 7.50 & 8.0 & -- & 20 \\
47 & $02 / 14 / 80$ & 126 & 118 & .12 & 7.40 & 10.0 & -- & 20 \\
47 & $03 / 14 / 80$ & 127 & 116 & .10 & 7.50 & 8.0 & -- & 20 \\
47 & $04 / 15 / 80$ & 126 & 120 & .18 & 7.50 & 8.0 & -- & 20 \\
47 & $05 / 13 / 80$ & 100 & 98 & 1.20 & 7.30 & 10.0 & -- & 15 \\
47 & $06 / 16 / 80$ & 140 & 130 & .20 & 7.50 & 9.0 & -- & 12 \\
47 & $07 / 11 / 80$ & 144 & 122 & 1.40 & 7.60 & 8.0 & -- & 14 \\
47 & $08 / 12 / 80$ & 140 & 120 & .30 & 7.50 & 15.0 & -- & 12 \\
47 & $09 / 15 / 80$ & 140 & 120 & .20 & 7.50 & 8.5 & -- & 15 \\
47 & $10 / 21 / 80$ & 140 & 130 & .20 & 7.50 & 9.1 & -- & 10 \\
& & & & & & & & \\
\hline & & & & & & & & \\
\hline
\end{tabular}


Table 8.--Water-chemistry data from production wells, 1971-83--Continued

[Samples collected and analyzed by personnel of the Coos Bay-North Bend Water Board]

ALKA- HARD-

LINITY NESS

(MG/L $\quad(M G / L$

SOLVED
FLUO- CHLO-

RIDE RIDE

CARBON DIS- DIS-

DIOXIDE SOLVED SOLVED

WELL DATE CACO3) CACO3) $(M G / L)(U N I T) \quad(M G / L)(M G / L) \quad(M G / L)$

\begin{tabular}{rrrrrrrrr}
\hline 47 & $11 / 14 / 80$ & 120 & 112 & 1.00 & 7.40 & 10.0 & -- & 20 \\
47 & $12 / 16 / 80$ & 122 & 110 & .65 & 7.40 & 9.4 & -- & 20 \\
47 & $01 / 15 / 81$ & 120 & -- & 1.60 & 7.50 & 9.6 & -- & 20 \\
47 & $02 / 16 / 81$ & 118 & 108 & 1.50 & 7.60 & 6.0 & .11 & 20 \\
47 & $03 / 16 / 81$ & 120 & 104 & .90 & 7.30 & 12.0 & -- & 20 \\
47 & $04 / 15 / 81$ & 124 & 110 & .30 & 7.30 & 14.0 & -- & 15 \\
47 & $05 / 15 / 81$ & 150 & 120 & .40 & 7.40 & 12.0 & -- & 30 \\
47 & $06 / 11 / 81$ & 144 & 130 & 1.44 & 7.10 & 24.0 & -- & 22 \\
47 & $07 / 14 / 81$ & 140 & 120 & .70 & 7.40 & 11.0 & -- & 30 \\
47 & $08 / 28 / 81$ & 135 & 110 & .68 & 7.30 & 13.5 & -- & 32 \\
47 & $09 / 17 / 81$ & 120 & 110 & .35 & 7.50 & 8.0 & -- & 25 \\
47 & $10 / 27 / 81$ & 140 & 118 & .42 & 7.50 & 8.7 & -- & 15 \\
47 & $11 / 19 / 81$ & 135 & 116 & .50 & 7.40 & 11.0 & -- & 14 \\
47 & $12 / 15 / 81$ & 118 & 110 & 3.25 & 7.10 & 18.0 & -- & 20 \\
& & & & & & & & \\
47 & $01 / 22 / 82$ & 116 & 104 & 2.60 & 7.10 & 18.0 & -- & 32 \\
47 & $05 / 20 / 82$ & 116 & 68 & 2.30 & 7.10 & 18.0 & -- & 20 \\
47 & $06 / 15 / 82$ & 142 & 120 & .80 & 7.50 & 9.0 & -- & 20 \\
47 & $07 / 15 / 82$ & 135 & 120 & 2.00 & 7.30 & 13.0 & -- & 30 \\
47 & $08 / 11 / 82$ & 140 & 120 & 1.40 & 7.40 & 11.0 & -- & 32 \\
47 & $09 / 23 / 82$ & 130 & 108 & 1.50 & 7.40 & 11.0 & -- & 20 \\
47 & $10 / 20 / 82$ & 136 & 120 & .32 & 7.30 & 14.0 & -- & 30 \\
47 & $11 / 19 / 82$ & 70 & 100 & 1.80 & 7.10 & 12.0 & -- & 28 \\
47 & $12 / 20 / 82$ & 128 & 108 & 1.60 & 7.30 & 13.0 & -- & 20 \\
& & & & & & & & \\
\hline
\end{tabular}


Table 8.--Water-chemistry data from production wells, 1971-83--Continued

[Samples collected and analyzed by personnel of the Coos Bay-North Bend Water Board]

\begin{tabular}{|c|c|c|c|c|c|c|c|c|}
\hline WELL & DATE & $\begin{array}{c}\text { ALKA- } \\
\text { LIN ITY } \\
\text { (MG/L } \\
\text { AS } \\
\text { CACO3) }\end{array}$ & $\begin{array}{l}\text { HARD- } \\
\text { NESS } \\
\text { (MG/L } \\
\text { AS } \\
\text { CACO3) }\end{array}$ & $\begin{array}{l}\text { IRON } \\
\text { DIS- } \\
\text { SOLVED } \\
\text { (MG/L) }\end{array}$ & $\begin{array}{c}\mathrm{PH} \\
(\mathrm{UN} \mid \mathrm{T})\end{array}$ & $\begin{array}{l}\text { CARBON } \\
\text { DIOXIDE } \\
(M G / L)\end{array}$ & $\begin{array}{l}\text { FLUO- } \\
\text { RIDE } \\
\text { DIS- } \\
\text { SOLVED } \\
(M G / L)\end{array}$ & $\begin{array}{l}\text { CHLO- } \\
\text { RIDE } \\
\text { DIS- } \\
\text { SOLVED } \\
(M G / L)\end{array}$ \\
\hline 47 & $01 / 17 / 83$ & 136 & 106 & 3.25 & 7.10 & 23.0 & -- & 30 \\
\hline 47 & $02 / 16 / 83$ & 140 & 110 & 3.20 & 7.10 & 22.0 & -- & 28 \\
\hline 47 & $03 / 18 / 83$ & 136 & 112 & 3.00 & 7.00 & 29.0 & -- & 25 \\
\hline 47 & $04 / 26 / 83$ & 140 & 110 & 3.10 & 7.10 & 22.0 & -- & 24 \\
\hline 47 & $05 / 16 / 83$ & 120 & 116 & .90 & 7.10 & 18.0 & -- & 35 \\
\hline 47 & $06 / 22 / 83$ & 130 & 112 & 2.65 & 7.10 & 21.0 & -- & 24 \\
\hline 48 & $03 / 08 / 71$ & 72 & 56 & 3.80 & 7.20 & 10.0 & .01 & 29 \\
\hline 48 & $04 / 21 / 71$ & 70 & 56 & 3.60 & 7.20 & 9.0 & -- & 30 \\
\hline 48 & $05 / 10 / 71$ & 78 & 70 & 2.40 & 7.00 & 16.0 & .01 & 31 \\
\hline 48 & $06 / 11 / 71$ & 72 & 70 & 2.50 & 7.00 & 15.0 & .01 & 32 \\
\hline 48 & $07 / 16 / 71$ & 76 & 92 & 2.00 & 7.20 & 10.0 & .02 & 26 \\
\hline 48 & $08 / 17 / 71$ & 82 & 84 & 4.20 & 7.10 & 13.0 & .03 & 24 \\
\hline 48 & $09 / 15 / 71$ & 78 & 70 & 2.40 & 6.80 & 26.0 & .01 & 26 \\
\hline 48 & $10 / 22 / 71$ & 84 & 72 & 2.00 & 6.80 & 38.0 & .01 & 31 \\
\hline 48 & $12 / 21 / 71$ & 86 & 82 & 1.60 & 7.40 & 6.0 & .01 & 24 \\
\hline 48 & $01 / 25 / 72$ & 92 & 85 & 1.20 & 7.20 & 23.0 & .03 & 22 \\
\hline 48 & $02 / 16 / 72$ & 114 & 110 & 2.00 & 6.80 & 36.0 & -- & 20 \\
\hline 48 & $03 / 24 / 72$ & 80 & 62 & 3.60 & 7.00 & 16.0 & .15 & 22 \\
\hline 48 & $05 / 18 / 72$ & 86 & 86 & 3.40 & 6.80 & 22.0 & .04 & 24 \\
\hline 48 & $08 / 30 / 72$ & 90 & 104 & 4.90 & 7.10 & 21.0 & .04 & 26 \\
\hline 48 & $09 / 26 / 72$ & 86 & 80 & 3.80 & 6.80 & 27.0 & .01 & 31 \\
\hline 48 & $11 / 28 / 72$ & 88 & 74 & 3.20 & 7.00 & 14.0 & .01 & 30 \\
\hline 48 & $12 / 26 / 72$ & 96 & 84 & 3.40 & 7.20 & 15.0 & .02 & 30 \\
\hline
\end{tabular}


Table 8.--Water-chemistry data from production wells, 1971-83--Continued

[Samples collected and analyzed by personnel of the Coos Bay-North Bend Water Board]

\begin{tabular}{|c|c|c|c|c|c|c|c|c|}
\hline WELL & DATE & $\begin{array}{c}\text { ALKA- } \\
\text { LINITY } \\
\text { (MG/L } \\
\text { AS } \\
\text { CACO3) }\end{array}$ & $\begin{array}{l}\text { HARD- } \\
\text { NESS } \\
\text { (MG/L } \\
\text { AS } \\
\text { CACO3) }\end{array}$ & $\begin{array}{l}\text { IRON } \\
\text { DIS- } \\
\text { SOLVED } \\
\text { (MG/L) }\end{array}$ & $\begin{array}{c}\mathrm{PH} \\
(\mathrm{UN} \mid \mathrm{T})\end{array}$ & $\begin{array}{l}\text { CARBON } \\
\text { DIOXIDE } \\
(M G / L)\end{array}$ & $\begin{array}{l}\text { FLUO- } \\
\text { RIDE } \\
\text { DIS- } \\
\text { SOLVED } \\
(M G / L)\end{array}$ & $\begin{array}{l}\text { CHLO- } \\
\text { RIDE } \\
\text { DIS- } \\
\text { SOLVED } \\
(M G / L)\end{array}$ \\
\hline 48 & $02 / 27 / 73$ & 112 & 96 & 3.20 & 7.20 & 14.0 & .02 & 29 \\
\hline 48 & $03 / 30 / 73$ & 116 & 104 & 3.40 & 6.90 & 30.0 & .02 & 30 \\
\hline 48 & $04 / 27 / 73$ & 108 & 84 & 3.00 & 6.80 & 35.0 & .01 & 29 \\
\hline 48 & $05 / 24 / 73$ & 110 & 98 & 3.20 & 6.60 & 56.0 & .01 & 24 \\
\hline 48 & $06 / 28 / 73$ & -- & -- & - & 6.80 & - & - & -- \\
\hline 48 & $08 / 30 / 73$ & 116 & 116 & 3.50 & 6.80 & 38.0 & .01 & 28 \\
\hline 48 & $10 / 09 / 73$ & 102 & 122 & 4.80 & 6.40 & 25.0 & .01 & 31 \\
\hline 48 & $11 / 06 / 73$ & 106 & 84 & 5.00 & 6.60 & 5.5 & .01 & 29 \\
\hline 48 & $12 / 20 / 73$ & 88 & 94 & 4.40 & 6.40 & -- & .01 & 24 \\
\hline 48 & $01 / 29 / 74$ & 94 & 98 & 4.80 & 6.80 & 31.0 & .01 & 30 \\
\hline 48 & $02 / 26 / 74$ & 120 & 84 & 4.20 & 6.80 & 40.0 & .01 & 30 \\
\hline 48 & $04 / 30 / 74$ & 126 & 106 & 4.60 & 6.80 & 46.0 & .01 & 26 \\
\hline 48 & $08 / 28 / 74$ & 106 & 118 & 2.00 & 7.00 & 22.0 & .10 & 26 \\
\hline 48 & $09 / 27 / 74$ & 110 & 126 & 3.00 & 7.10 & 18.0 & .20 & 26 \\
\hline 48 & $11 / 21 / 74$ & 90 & 112 & 4.80 & 6.90 & 25.0 & .10 & 24 \\
\hline 48 & $12 / 31 / 74$ & 134 & 152 & 3.50 & 6.90 & 35.0 & .20 & 34 \\
\hline 48 & $02 / 28 / 75$ & 96 & 116 & 4.20 & 6.90 & 25.0 & .20 & 22 \\
\hline 48 & $03 / 26 / 75$ & 106 & 108 & 4.00 & 6.90 & 27.0 & .10 & 28 \\
\hline 48 & $04 / 23 / 75$ & 114 & 114 & 4.60 & 6.90 & 29.0 & .20 & 26 \\
\hline 48 & $05 / 27 / 75$ & 104 & 106 & 3.60 & 6.60 & 55.0 & .20 & 24 \\
\hline 48 & $07 / 29 / 75$ & 102 & 106 & 3.80 & 6.50 & 65.0 & .10 & 24 \\
\hline 48 & $09 / 25 / 75$ & 102 & 100 & 3.40 & 6.80 & 33.0 & .20 & 24 \\
\hline 48 & $11 / 25 / 75$ & 84 & 102 & 3.20 & 7.00 & 15.0 & .10 & 31 \\
\hline 48 & $12 / 30 / 75$ & 104 & 112 & 2.00 & 6.90 & 27.0 & .10 & 15 \\
\hline
\end{tabular}


Table 8.- - Water-chemistry data from production

[Samples collected and analyzed by personnel of the Coos Bay-North Bend Water Board]

\begin{tabular}{|c|c|c|c|c|c|c|c|c|}
\hline WELL & DATE & $\begin{array}{l}\text { ALKA- } \\
\text { LINITY } \\
\text { (MG/L } \\
\text { AS } \\
\text { CACO3) }\end{array}$ & $\begin{array}{l}\text { HARD- } \\
\text { NESS } \\
\text { (MG/L } \\
\text { AS } \\
\text { CACO3) }\end{array}$ & $\begin{array}{l}\text { IRON } \\
\text { DIS- } \\
\text { SOLVED } \\
\text { (MG/L) }\end{array}$ & $\begin{array}{c}\mathrm{PH} \\
(\mathrm{UNIT})\end{array}$ & $\begin{array}{l}\text { CARBON } \\
\text { DIOXIDE } \\
(M G / L)\end{array}$ & $\begin{array}{l}\text { FLUO- } \\
\text { RIDE } \\
\text { DIS- } \\
\text { SOLVED } \\
\text { (MG/L) }\end{array}$ & $\begin{array}{l}\text { CHLO- } \\
\text { RIDE } \\
\text { DIS- } \\
\text { SOLVED } \\
\text { (MG/L) }\end{array}$ \\
\hline 48 & $02 / 29 / 76$ & 124 & 112 & 4.20 & 6.90 & 32.0 & .20 & 25 \\
\hline 48 & $03 / 30 / 76$ & 96 & 100 & 6.80 & 6.90 & 26.0 & .15 & 20 \\
\hline 48 & $04 / 23 / 76$ & 36 & 102 & 6.60 & 6.90 & 9.0 & .10 & 20 \\
\hline 48 & $06 / 17 / 76$ & 38 & 104 & 5.70 & 6.80 & 13.0 & .15 & 20 \\
\hline 48 & $07 / 19 / 76$ & 38 & 100 & 5.80 & 6.90 & 12.0 & .15 & 24 \\
\hline 48 & $08 / 20 / 76$ & 42 & 104 & 7.00 & 6.90 & 12.0 & .18 & 26 \\
\hline 48 & $09 / 21 / 76$ & 42 & 100 & 7.10 & 6.80 & 16.0 & .18 & 24 \\
\hline 48 & $10 / 21 / 76$ & 42 & 102 & 7.00 & 6.90 & 12.0 & .18 & 25 \\
\hline 48 & $11 / 18 / 76$ & 120 & 104 & 6.50 & 7.10 & 18.0 & .22 & 17 \\
\hline 48 & $02 / 15 / 77$ & 100 & 100 & 2.80 & 7.00 & 20.0 & .15 & 16 \\
\hline 48 & $03 / 15 / 77$ & 108 & 84 & -- & 6.80 & 35.0 & .10 & 29 \\
\hline 48 & $04 / 15 / 77$ & 106 & 120 & 2.60 & 7.00 & 27.0 & .10 & 18 \\
\hline 48 & $05 / 16 / 77$ & 110 & 98 & 3.20 & 6.60 & 56.0 & .10 & 24 \\
\hline 48 & $06 / 21 / 77$ & 70 & 80 & 2.20 & 7.30 & 9.0 & .10 & 10 \\
\hline 48 & $07 / 21 / 77$ & 74 & 64 & 1.40 & 7.10 & 13.0 & -- & 10 \\
\hline 48 & $08 / 19 / 77$ & 76 & 66 & 1.50 & 7.10 & 13.0 & -- & 10 \\
\hline 48 & $09 / 15 / 77$ & 74 & 66 & 1.30 & 7.10 & 13.0 & -- & 10 \\
\hline 48 & $10 / 20 / 77$ & 74 & 62 & 1.60 & 7.00 & 15.0 & -- & 10 \\
\hline 48 & $12 / 20 / 77$ & 80 & 100 & 2.00 & 7.00 & 17.0 & -- & 15 \\
\hline 48 & $01 / 16 / 78$ & 80 & 70 & 2.20 & 7.10 & 13.0 & -- & 11 \\
\hline 48 & $02 / 16 / 78$ & 80 & 74 & 2.30 & 7.10 & 13.0 & -- & 15 \\
\hline 48 & $03 / 14 / 78$ & 80 & 74 & 2.30 & 7.10 & 13.0 & -- & 10 \\
\hline 48 & $04 / 12 / 78$ & 80 & 70 & 3.00 & -- & 13.0 & -- & 13 \\
\hline
\end{tabular}


Table 8.--Water-chemistry data from production wells, 1971-83--Continued

[Samples collected and analyzed by personnel of the Coos Bay-North Bend Water Board]

\begin{tabular}{|c|c|c|c|c|c|c|c|c|}
\hline WELL & DATE & $\begin{array}{c}\text { ALKA- } \\
\text { LINITY } \\
\text { (MG/L } \\
\text { AS } \\
\text { CACO3) }\end{array}$ & $\begin{array}{l}\text { HARD- } \\
\text { NESS } \\
\text { (MG/L } \\
\text { AS } \\
\text { CACO3) }\end{array}$ & $\begin{array}{l}\text { IRON } \\
\text { DIS- } \\
\text { SOLVED } \\
(M G / L)\end{array}$ & $\begin{array}{c}\mathrm{PH} \\
(\mathrm{UN} / \mathrm{T})\end{array}$ & $\begin{array}{l}\text { CARBON } \\
\text { DIOXIDE } \\
(M G / L)\end{array}$ & $\begin{array}{l}\text { FLUO- } \\
\text { RIDE } \\
\text { DIS- } \\
\text { SOLVED } \\
\text { (MG/L) }\end{array}$ & $\begin{array}{l}\text { CHLO- } \\
\text { RIDE } \\
\text { DIS- } \\
\text { SOLVED } \\
(M G / L)\end{array}$ \\
\hline 48 & $05 / 11 / 78$ & 80 & 84 & 2.50 & 7.20 & 10.0 & - & 13 \\
\hline 48 & $06 / 12 / 78$ & 34 & 80 & 7.60 & 7.10 & 6.0 & -- & 13 \\
\hline 48 & $07 / 17 / 78$ & 32 & 84 & 3.00 & 6.80 & 11.0 & -- & 15 \\
\hline 48 & $08 / 11 / 78$ & 70 & 60 & 2.80 & 6.90 & 18.0 & -- & 10 \\
\hline 48 & $09 / 15 / 78$ & 70 & 64 & 2.90 & 6.80 & 24.0 & -- & 12 \\
\hline 48 & $10 / 25 / 78$ & 72 & 60 & 2.80 & 6.90 & 19.0 & -- & 10 \\
\hline 48 & $11 / 15 / 78$ & 78 & 70 & 5.10 & 6.90 & 20.0 & -- & 13 \\
\hline 48 & $12 / 13 / 78$ & 70 & 70 & 3.00 & 7.10 & 11.0 & -- & 15 \\
\hline 48 & $01 / 22 / 79$ & 60 & 80 & 3.00 & 7.00 & 12.0 & -- & 12 \\
\hline 48 & $02 / 22 / 79$ & 90 & 70 & 2.20 & 7.20 & 13.0 & -- & 13 \\
\hline 48 & $03 / 20 / 79$ & 94 & 74 & 2.40 & 7.10 & 15.0 & -- & 14 \\
\hline 48 & $04 / 19 / 79$ & 70 & 70 & 1.60 & 7.10 & 12.0 & -- & 15 \\
\hline 48 & $05 / 21 / 79$ & 70 & 80 & 4.00 & 7.10 & 11.0 & .12 & 15 \\
\hline 48 & $06 / 13 / 79$ & 70 & 62 & 3.60 & 7.10 & 12.0 & .12 & 15 \\
\hline 48 & $08 / 16 / 79$ & 80 & 70 & 1.00 & 7.30 & 8.2 & - & 15 \\
\hline 48 & $10 / 23 / 79$ & 84 & 78 & 1.30 & 7.30 & 8.0 & -- & 12 \\
\hline 48 & $11 / 20 / 79$ & 90 & 76 & .38 & 7.30 & 9.0 & -- & 20 \\
\hline 48 & $12 / 17 / 79$ & 80 & 70 & 1.20 & 7.30 & 8.0 & -- & 15 \\
\hline 48 & $01 / 13 / 80$ & 80 & 80 & 1.50 & 7.20 & 10.0 & - & 15 \\
\hline 48 & $02 / 14 / 80$ & 84 & 82 & 1.60 & 7.10 & 17.0 & -- & 18 \\
\hline 48 & $03 / 14 / 80$ & 89 & 90 & 1.60 & 7.20 & 11.0 & -- & 20 \\
\hline 48 & $04 / 15 / 80$ & 84 & 84 & 1.40 & 7.30 & 9.0 & -- & 20 \\
\hline 48 & $05 / 13 / 80$ & 92 & 80 & 1.60 & 7.10 & 10.0 & -- & 15 \\
\hline
\end{tabular}


Table 3.- $\frac{\text { Water-chem istry data from production }}{\text { wells, 1971-83--Continued }}$

[Samples collected and analyzed by personnel of the Coos Bay-North Bend Water Board]

\begin{tabular}{|c|c|c|c|c|c|c|c|c|}
\hline WELL & DATE & $\begin{array}{c}\text { ALKA- } \\
\text { LINITY } \\
\text { (MG/L } \\
\text { AS } \\
\text { CACO3) }\end{array}$ & $\begin{array}{l}\text { HARD- } \\
\text { NESS } \\
\text { (MG/L } \\
\text { AS } \\
\text { CACO3) }\end{array}$ & $\begin{array}{l}\text { IRON } \\
\text { DIS- } \\
\text { SOLVED } \\
\text { (MG/L) }\end{array}$ & $\begin{array}{c}\text { PH } \\
(U N \mid T)\end{array}$ & $\begin{array}{l}\text { CARBON } \\
\text { DIOXIDE } \\
(M G / L)\end{array}$ & $\begin{array}{l}\text { FLUO- } \\
\text { RIDE } \\
\text { DIS- } \\
\text { SOLVED } \\
\text { (MG/L) }\end{array}$ & $\begin{array}{l}\text { CHLO- } \\
\text { RIDE } \\
\text { DIS- } \\
\text { SOLVED } \\
(M G / L)\end{array}$ \\
\hline 48 & $06 / 16 / 80$ & 90 & 80 & 1.60 & 7.10 & 14.0 & -- & 12 \\
\hline 48 & $07 / 11 / 80$ & 92 & 90 & 2.00 & 7.20 & 14.0 & -- & 13 \\
\hline 48 & $08 / 12 / 80$ & 80 & 80 & 1.30 & 7.20 & 10.0 & -- & 15 \\
\hline 48 & $09 / 15 / 80$ & 82 & 80 & 1.50 & 7.10 & 13.0 & -- & 15 \\
\hline 48 & $10 / 21 / 80$ & 80 & 90 & 1.60 & 7.20 & 10.0 & -- & 15 \\
\hline 48 & $11 / 14 / 80$ & 84 & 80 & 1.50 & 7.10 & 14.0 & -- & 15 \\
\hline 48 & $12 / 16 / 80$ & 80 & 70 & 1.60 & 7.10 & 12.0 & - & 18 \\
\hline 48 & $01 / 15 / 81$ & 80 & -- & 2.80 & 7.10 & 13.0 & -- & 18 \\
\hline 48 & $02 / 16 / 81$ & 80 & 70 & 2.90 & 7.10 & 13.0 & - & 15 \\
\hline 48 & $03 / 16 / 81$ & 78 & 78 & 1.40 & 7.00 & 16.0 & -- & 18 \\
\hline 48 & $04 / 15 / 81$ & 90 & 82 & 2.40 & 7.10 & 14.0 & -- & 18 \\
\hline 48 & $05 / 15 / 81$ & 100 & 100 & 2.00 & 7.10 & 16.0 & -- & 20 \\
\hline 48 & $06 / 11 / 81$ & 104 & 88 & 2.60 & 6.90 & 27.0 & -- & 15 \\
\hline 48 & $07 / 14 / 81$ & 100 & 100 & 3.60 & 7.10 & 16.0 & -- & 28 \\
\hline 48 & $08 / 28 / 81$ & 100 & 96 & 3.60 & 7.00 & 20.0 & -- & 27 \\
\hline 48 & $09 / 17 / 81$ & 80 & 70 & 1.80 & 7.20 & 10.0 & -- & 18 \\
\hline 48 & $10 / 27 / 81$ & 90 & 76 & 1.60 & 7.20 & 11.5 & -- & 10 \\
\hline 48 & $11 / 19 / 81$ & 90 & 70 & 1.80 & 7.10 & 14.5 & -- & 12 \\
\hline 48 & $12 / 15 / 81$ & 70 & 70 & 4.75 & 7.10 & 12.0 & -- & 18 \\
\hline 48 & $01 / 22 / 82$ & 88 & 80 & 3.20 & 7.00 & 18.0 & -- & 20 \\
\hline 48 & $05 / 20 / 82$ & 62 & 64 & 3.80 & 6.90 & 16.0 & -- & 20 \\
\hline 48 & $06 / 15 / 82$ & 70 & 60 & 2.00 & 7.10 & 11.0 & -- & 10 \\
\hline 48 & $07 / 15 / 82$ & 46 & 40 & 3.20 & 6.90 & 38.0 & -- & 15 \\
\hline
\end{tabular}


Table 8.--Water-chem istry data from production wells, 1971-83--Continued

[Samples collected and analyzed by personnel of the Coos Bay-North Bend Water Board]

ALKA- HARD-

LINITY NESS IRON

(MG/L (MG/L DIS-

AS AS SOLVED

WELL DATE CACO3) CACO3) (MG/L
FLUO- CHLO-

RIDE RIDE

CARBON DIS- DIS-

PH DIOXIDE SOLVED SOLVED

\begin{tabular}{rrrrrrrrr}
\hline 48 & $08 / 11 / 82$ & 70 & 60 & 2.50 & 7.00 & 14.0 & -- & 20 \\
48 & $09 / 23 / 82$ & 60 & 50 & 2.50 & 7.10 & 9.5 & -- & 20 \\
48 & $10 / 20 / 82$ & 76 & 64 & 2.80 & 7.00 & 16.0 & -- & 25 \\
48 & $11 / 19 / 82$ & 110 & 60 & 1.15 & 7.10 & 18.0 & -- & 25 \\
48 & $12 / 20 / 82$ & 64 & 60 & 2.40 & 7.10 & 11.0 & -- & 24 \\
48 & $01 / 17 / 83$ & 58 & 50 & 2.50 & 6.90 & 11.0 & -- & 16 \\
48 & $02 / 16 / 83$ & 70 & 70 & 1.75 & 7.10 & 12.0 & -- & 12 \\
48 & $03 / 18 / 83$ & 62 & 60 & 3.20 & 7.00 & 13.0 & -- & 15 \\
48 & $04 / 26 / 83$ & 64 & 64 & 3.20 & 7.00 & 14.0 & -- & 20 \\
48 & $05 / 16 / 83$ & 76 & 70 & 1.00 & 7.00 & 14.0 & -- & 18 \\
48 & $06 / 22 / 83$ & 60 & 56 & 2.70 & 6.90 & 15.5 & -- & 12 \\
& & & & & & & & \\
49 & $03 / 08 / 71$ & 142 & 122 & 3.40 & 6.60 & 75.0 & .02 & 29 \\
49 & $04 / 21 / 71$ & 144 & 120 & 3.40 & 6.50 & 90.0 & .02 & 30 \\
49 & $05 / 10 / 71$ & 62 & 40 & 3.00 & 7.40 & 5.0 & .02 & 31 \\
49 & $06 / 11 / 71$ & 68 & 80 & 3.20 & 7.30 & 7.0 & .02 & 33 \\
49 & $07 / 16 / 71$ & 60 & 78 & 2.20 & 7.20 & 8.0 & .02 & 28 \\
49 & $08 / 17 / 71$ & 70 & 74 & 2.50 & 7.40 & 5.5 & .01 & 26 \\
49 & $09 / 15 / 71$ & 64 & 56 & 3.00 & 7.60 & 32.0 & .01 & 30 \\
49 & $10 / 22 / 71$ & 84 & 48 & 2.40 & 6.80 & 38.0 & .01 & 30 \\
49 & $12 / 21 / 71$ & 82 & 65 & 2.00 & 7.00 & 13.0 & .01 & 22 \\
49 & $01 / 25 / 72$ & 86 & 67 & 2.40 & 7.00 & 17.0 & .01 & 28 \\
49 & $02 / 16 / 72$ & 72 & 96 & 1.80 & 6.60 & 38.0 & -- & 17 \\
& & & & & & & & \\
\hline & & & & & & & & \\
\hline
\end{tabular}


Table 8.-- Water-chemistry data from production

[Samples collected and analyzed by personnel of the Coos Bay-North Bend Water Board]

$\begin{array}{ccccccc}\text { ALKA- } & \text { HARD- } & & & & \text { FLUO- } & \text { CHLO- } \\ \text { LINITY } & \text { NESS } & \text { IRON } & & \text { RIDE } & \text { RIDE } \\ \text { (MG/L } & (M G / L & \text { DIS- } & & \text { CARBON } & \text { DIS- } & \text { DIS- } \\ \text { AS } & \text { AS } & \text { SOLVED } & \text { PH } & \text { DIOXIDE } & \text { SOLVED } & \text { SOLVED } \\ \text { CACO3) } & \text { CACO3) } & (M G / L) & (U N I T) & (M G / L) & (M G / L) & (M G / L)\end{array}$

WELL DATE CACO3) CACO3) (MG/L) (UNIT) (MG/L) (MG/L) (MG/L)

\begin{tabular}{rrrrrrrrr}
\hline 49 & $03 / 24 / 72$ & 136 & 140 & 3.10 & 7.40 & 12.0 & .20 & 26 \\
49 & $05 / 18 / 72$ & 124 & 156 & 4.00 & 7.60 & 6.5 & .04 & 22 \\
49 & $08 / 30 / 72$ & 118 & 166 & 4.60 & 6.90 & 30.0 & .03 & 20 \\
49 & $09 / 26 / 72$ & 102 & 64 & 4.60 & 7.10 & 13.0 & .01 & 28 \\
49 & $11 / 28 / 72$ & 112 & 76 & 4.00 & 7.00 & 23.0 & .02 & 22 \\
49 & $12 / 26 / 72$ & 114 & 66 & 4.40 & 7.00 & 23.0 & .02 & 30 \\
49 & $02 / 27 / 73$ & 118 & 122 & 4.20 & 6.60 & 66.0 & .01 & 30 \\
49 & $03 / 30 / 73$ & 130 & 108 & 4.80 & 6.80 & 43.0 & .01 & 30 \\
49 & $04 / 27 / 73$ & 120 & 102 & 4.40 & 7.00 & 24.0 & .02 & 28 \\
49 & $05 / 24 / 73$ & 96 & 92 & 3.80 & 7.00 & 19.0 & .01 & 29 \\
49 & $06 / 28 / 73$ & -- & -- & -1 & 7.20 & -- & -- & -- \\
49 & $08 / 30 / 73$ & 84 & 122 & 4.20 & 7.10 & 13.0 & .01 & 30 \\
49 & $10 / 09 / 73$ & 120 & 96 & 5.40 & 7.00 & 24.0 & .01 & 30 \\
49 & $11 / 06 / 73$ & 116 & 86 & 5.60 & 7.00 & 22.0 & .01 & 30 \\
49 & $12 / 20 / 73$ & 116 & 84 & 5.00 & 7.00 & 23.0 & .01 & 22 \\
& & & & & & & & \\
49 & $01 / 29 / 74$ & 118 & 114 & 4.80 & 7.10 & 19.0 & .01 & 30 \\
49 & $02 / 26 / 74$ & 124 & 110 & 4.80 & 7.10 & 19.5 & .01 & 24 \\
49 & $04 / 30 / 74$ & 122 & 118 & 5.00 & 7.10 & 19.5 & .01 & 22 \\
49 & $08 / 28 / 74$ & 100 & 108 & 5.20 & 7.10 & 16.0 & .20 & 20 \\
49 & $09 / 27 / 74$ & 104 & 90 & 5.00 & 7.10 & 16.0 & .20 & 18 \\
49 & $11 / 21 / 74$ & 102 & 108 & 5.00 & 7.10 & 16.0 & .20 & 18 \\
49 & $12 / 31 / 74$ & 82 & 160 & 2.50 & 7.10 & 10.0 & .20 & 26 \\
& & & & & & & & \\
\hline
\end{tabular}


Table 8.--Water-chemistry data from production wells, 1971-83--Continued

[Samples collected and analyzed by personnel of the Coos Bay-North Bend Water Board]

\begin{tabular}{|c|c|c|c|c|c|c|c|c|}
\hline WELL & DATE & $\begin{array}{c}\text { ALKA- } \\
\text { LINITY } \\
\text { (MG/L } \\
\text { AS } \\
\text { CACO3) }\end{array}$ & $\begin{array}{l}\text { HARD- } \\
\text { NESS } \\
(M G / L \\
\text { AS } \\
\text { CACO3) }\end{array}$ & $\begin{array}{l}\text { IRON } \\
\text { DIS- } \\
\text { SOLVED } \\
\text { (MG/L) }\end{array}$ & $\begin{array}{c}\mathrm{PH} \\
(\mathrm{UN} \mid \mathrm{T})\end{array}$ & $\begin{array}{l}\text { CARBON } \\
\text { DIOXIDE } \\
(M G / L)\end{array}$ & $\begin{array}{l}\text { FLUO- } \\
\text { RIDE } \\
\text { DIS- } \\
\text { SOLVED } \\
(M G / L)\end{array}$ & $\begin{array}{l}\text { CHLO- } \\
\text { RIDE } \\
\text { DIS- } \\
\text { SOLVED } \\
(M G / L)\end{array}$ \\
\hline 49 & $02 / 28 / 75$ & 112 & 104 & 4.60 & 7.10 & 18.0 & .10 & 20 \\
\hline 49 & $03 / 26 / 75$ & 110 & 110 & 4.00 & 7.00 & 22.0 & .10 & 24 \\
\hline 49 & $04 / 23 / 75$ & 108 & 110 & 3.80 & 7.10 & 17.0 & .10 & 26 \\
\hline 49 & $05 / 27 / 75$ & 96 & 108 & 3.80 & 7.00 & 20.0 & .10 & 28 \\
\hline 49 & $07 / 29 / 75$ & 98 & 110 & 4.20 & 6.90 & 25.0 & .10 & 24 \\
\hline 49 & $09 / 25 / 75$ & 100 & 104 & 4.00 & 6.90 & 26.5 & .20 & 26 \\
\hline 49 & $11 / 25 / 75$ & 102 & 104 & 3.60 & 6.90 & 26.0 & .20 & 26 \\
\hline 49 & $12 / 30 / 75$ & 64 & 70 & 2.80 & 7.10 & 11.0 & .10 & 17 \\
\hline 49 & $02 / 19 / 76$ & 60 & 64 & 1.50 & 7.00 & 12.0 & .15 & 12 \\
\hline 49 & $03 / 30 / 76$ & 36 & 60 & 7.00 & 7.10 & 6.0 & .15 & 12 \\
\hline 49 & $04 / 23 / 76$ & 64 & 60 & 5.80 & 7.10 & 11.0 & .30 & 14 \\
\hline 49 & $06 / 17 / 76$ & 62 & 68 & 7.00 & 7.00 & 11.0 & .20 & 18 \\
\hline 49 & $07 / 19 / 76$ & 62 & 74 & 7.10 & 7.00 & 14.0 & .18 & 26 \\
\hline 49 & $08 / 20 / 76$ & 68 & 76 & 5.40 & 7.00 & 9.5 & .15 & 26 \\
\hline 49 & $09 / 21 / 76$ & 68 & 76 & 5.20 & 7.00 & 94.0 & .16 & 26 \\
\hline 49 & $10 / 21 / 76$ & 68 & 78 & 5.40 & 7.00 & 14.0 & .15 & 26 \\
\hline 49 & $11 / 18 / 76$ & 50 & 58 & 5.00 & 7.10 & 8.0 & .20 & 18 \\
\hline 49 & $02 / 15 / 77$ & 70 & 90 & 3.00 & 7.00 & 14.0 & .14 & 20 \\
\hline 49 & $03 / 15 / 77$ & 120 & 102 & 3.00 & 7.00 & 24.0 & .10 & 28 \\
\hline 49 & $04 / 15 / 77$ & 62 & 108 & 3.00 & 7.00 & 12.0 & .12 & 16 \\
\hline
\end{tabular}


Table 8.- Water-chemistry data from production wells, 1971-83--Continued

[Samples collected and analyzed by personnel of the Coos Bay-North Bend Water Board]

\begin{tabular}{|c|c|c|c|c|c|c|c|c|}
\hline WELL & DATE & $\begin{array}{c}\text { ALKA- } \\
\text { LINITY } \\
\text { (MG/L } \\
\text { AS } \\
\text { CACO3) }\end{array}$ & $\begin{array}{l}\text { HARD- } \\
\text { NESS } \\
\text { (MG/L } \\
\text { AS } \\
\text { CACO3) }\end{array}$ & $\begin{array}{l}\text { IRON } \\
\text { DIS- } \\
\text { SOLVED } \\
\text { (MG/L) }\end{array}$ & $\begin{array}{c}\text { PH } \\
(U N \mid T)\end{array}$ & $\begin{array}{l}\text { CARBON } \\
\text { DIOXIDE } \\
(M G / L)\end{array}$ & $\begin{array}{l}\text { FLUO- } \\
\text { RIDE } \\
\text { DIS- } \\
\text { SOLVED } \\
(M G / L)\end{array}$ & $\begin{array}{l}\text { CHLO- } \\
\text { RIDE } \\
\text { DIS- } \\
\text { SOLVED } \\
(M G / L)\end{array}$ \\
\hline 49 & $05 / 16 / 77$ & 96 & 92 & 4.20 & 7.00 & 19.0 & .20 & 30 \\
\hline 49 & $06 / 21 / 77$ & 64 & 70 & 2.20 & 7.20 & 80.0 & .20 & 12 \\
\hline 49 & $07 / 21 / 77$ & 60 & 60 & 2.20 & 7.00 & 12.0 & -- & 10 \\
\hline 49 & $08 / 19 / 77$ & 62 & 62 & 2.20 & 7.00 & 12.0 & -- & 10 \\
\hline 49 & $09 / 15 / 77$ & 60 & 62 & 1.50 & 7.00 & 12.0 & -- & 12 \\
\hline 49 & $10 / 20 / 77$ & 78 & 60 & 2.60 & 6.90 & 20.0 & - & 10 \\
\hline 49 & $12 / 20 / 77$ & 62 & 70 & 3.00 & 6.90 & 11.0 & -- & 25 \\
\hline 49 & $01 / 16 / 78$ & 60 & 60 & 3.40 & 6.90 & 15.0 & - & 15 \\
\hline 49 & $02 / 16 / 78$ & 70 & 68 & 3.20 & 6.90 & 18.0 & -- & 15 \\
\hline 49 & $13 / 14 / 78$ & 62 & 60 & 3.50 & 6.80 & 16.0 & -- & 15 \\
\hline 19 & $j 4 / 12 / 78$ & 50 & 60 & 5.00 & -- & 8.0 & -- & 15 \\
\hline 49 & $05 / 11 / 78$ & 38 & 30 & 4.00 & 6.90 & 10.0 & -- & 13 \\
\hline 49 & $06 / 12 / 78$ & 90 & 28 & 3.80 & 6.90 & 24.0 & -- & 13 \\
\hline 49 & $07 / 17 / 78$ & 90 & 30 & 3.90 & 6.90 & 23.0 & -- & 14 \\
\hline 49 & $08 / 11 / 78$ & 60 & 60 & 2.50 & 6.90 & 16.0 & -- & 10 \\
\hline 49 & $09 / 15 / 78$ & 64 & 60 & 2.60 & 6.80 & 22.0 & -- & 10 \\
\hline 49 & $10 / 25 / 78$ & 62 & -- & 2.40 & 6.80 & 20.0 & -- & 12 \\
\hline 49 & $11 / 15 / 78$ & 60 & 60 & 3.20 & 7.00 & 14.0 & -- & 13 \\
\hline 49 & $12 / 13 / 78$ & 60 & 60 & 2.20 & 7.10 & 10.0 & -- & 15 \\
\hline 49 & $01 / 22 / 79$ & 58 & 60 & 2.50 & 7.10 & 10.0 & -- & 12 \\
\hline 49 & $02 / 22 / 79$ & 50 & 52 & 2.20 & 7.10 & 8.0 & -- & 15 \\
\hline 49 & $03 / 20 / 79$ & 52 & 60 & 2.20 & 7.10 & 8.0 & -- & 15 \\
\hline
\end{tabular}


Table 8.--Water-chem istry data from production wells, 1971-83--Continued

[Samples collected and analyzed by personnel of the Coos Bay-North Bend Water Board]

\begin{tabular}{|c|c|c|c|c|c|c|c|c|}
\hline WELL & DATE & $\begin{array}{c}\text { ALKA- } \\
\text { LINITY } \\
\text { (MG/L } \\
\text { AS } \\
\text { CACO3) }\end{array}$ & $\begin{array}{l}\text { HARD- } \\
\text { NESS } \\
\text { (MG/L } \\
\text { AS } \\
\text { CACO3) }\end{array}$ & $\begin{array}{l}\text { IRON } \\
\text { DIS- } \\
\text { SOLVED } \\
\text { (MG/L) }\end{array}$ & $\begin{array}{c}\text { PH } \\
(U N \mid T)\end{array}$ & $\begin{array}{l}\text { CARBON } \\
\text { DIOXIDE } \\
(M G / L)\end{array}$ & $\begin{array}{l}\text { FLUO- } \\
\text { RIDE } \\
\text { DIS- } \\
\text { SOLVED } \\
(M G / L)\end{array}$ & $\begin{array}{l}\text { CHLO- } \\
\text { RIDE } \\
\text { DIS- } \\
\text { SOLVED } \\
\text { (MG/L) }\end{array}$ \\
\hline 49 & $04 / 19 / 79$ & 54 & 60 & 1.60 & 7.20 & 7.0 & -- & 12 \\
\hline 49 & $05 / 21 / 79$ & 50 & 46 & 3.00 & 7.10 & 8.0 & .10 & 18 \\
\hline 49 & $06 / 13 / 79$ & 84 & 80 & 5.00 & 6.90 & 22.0 & .10 & 20 \\
\hline 49 & $08 / 16 / 79$ & 104 & 90 & 1.20 & 7.30 & 10.0 & -- & 15 \\
\hline 49 & $10 / 23 / 79$ & 52 & 68 & 1.55 & 7.10 & 8.0 & -- & 10 \\
\hline 49 & $11 / 20 / 79$ & 58 & 70 & 1.25 & 7.10 & 10.0 & -- & 12 \\
\hline 49 & $12 / 17 / 79$ & 40 & 40 & 3.00 & 7.10 & 7.0 & -- & 15 \\
\hline 49 & $01 / 13 / 80$ & 50 & 50 & 5.00 & 7.10 & 8.0 & -- & 15 \\
\hline 49 & $02 / 14 / 80$ & 56 & 56 & 4.50 & 7.10 & 9.0 & -- & 15 \\
\hline 49 & $03 / 14 / 80$ & 60 & 58 & 4.20 & 7.10 & 9.0 & -- & 18 \\
\hline 49 & $04 / 15 / 80$ & 55 & 56 & 4.20 & 7.10 & 9.0 & -- & 15 \\
\hline 49 & $05 / 13 / 80$ & 50 & 50 & 2.50 & 7.10 & 8.0 & -- & 18 \\
\hline 49 & $06 / 16 / 80$ & 45 & 50 & 2.40 & 7.00 & 9.0 & -- & 13 \\
\hline 49 & $07 / 11 / 80$ & 56 & 52 & 3.00 & 7.10 & 9.0 & -- & 15 \\
\hline 49 & $08 / 12 / 80$ & 50 & 50 & 1.40 & 7.10 & 8.0 & -- & 15 \\
\hline 49 & $09 / 15 / 80$ & 50 & 60 & .90 & 7.10 & 8.0 & -- & 15 \\
\hline 49 & $10 / 21 / 80$ & 45 & 50 & 2.40 & 7.00 & 9.1 & -- & 13 \\
\hline 49 & $11 / 14 / 80$ & 80 & 80 & 2.80 & 7.10 & 13.0 & -- & 20 \\
\hline 49 & $12 / 16 / 80$ & 100 & 100 & 4.20 & 7.10 & 16.0 & -- & 20 \\
\hline 49 & $01 / 15 / 81$ & 76 & -- & 3.20 & 7.00 & 16.0 & -- & 20 \\
\hline 49 & $02 / 16 / 81$ & 74 & 96 & 3.00 & 7.00 & 15.0 & -- & 20 \\
\hline 49 & $03 / 16 / 81$ & 80 & 90 & 3.40 & 7.10 & 13.0 & -- & 20 \\
\hline 49 & $04 / 15 / 81$ & 50 & 42 & 2.40 & 7.10 & 8.0 & -- & 18 \\
\hline
\end{tabular}


Table 8.--Water-chemistry data from production wells, 1971-83--Continued

[Samples collected and analyzed by personnel of the Coos Bay-North Bend Water Board]

\begin{tabular}{|c|c|c|c|c|c|c|c|c|}
\hline WELL & DATE & $\begin{array}{c}\text { ALKA- } \\
\text { LIN ITY } \\
\text { (MG/L } \\
\text { AS } \\
\text { CACO3) }\end{array}$ & $\begin{array}{l}\text { HARD- } \\
\text { NESS } \\
\text { (MG/L } \\
\text { AS } \\
\text { CACO3) }\end{array}$ & $\begin{array}{l}\text { IRON } \\
\text { DIS- } \\
\text { SOLVED } \\
\text { (MG/L) }\end{array}$ & $\begin{array}{c}\mathrm{PH} \\
(\mathrm{UN} \mid \mathrm{T})\end{array}$ & $\begin{array}{l}\text { CARBON } \\
\text { DIOXIDE } \\
(M G / L)\end{array}$ & $\begin{array}{l}\text { FLUO- } \\
\text { RIDE } \\
\text { DIS- } \\
\text { SOLVED } \\
\text { (MG/L) }\end{array}$ & $\begin{array}{l}\text { CHLO- } \\
\text { RIDE } \\
\text { DIS- } \\
\text { SOLVED } \\
(M G / L)\end{array}$ \\
\hline 49 & $05 / 15 / 81$ & 60 & 50 & 1.20 & 7.10 & 9.6 & -- & 15 \\
\hline 49 & $06 / 11 / 81$ & 80 & 76 & 4.00 & 7.00 & 17.0 & -- & 17 \\
\hline 49 & $07 / 14 / 81$ & 70 & 70 & 3.60 & 7.10 & 11.5 & - & 30 \\
\hline 49 & $08 / 28 / 81$ & 72 & 74 & 3.40 & 7.10 & 12.0 & -- & 28 \\
\hline 49 & $09 / 17 / 81$ & 54 & 50 & 1.50 & 7.20 & 6.8 & -- & 18 \\
\hline 49 & $10 / 27 / 81$ & 60 & 62 & 1.40 & 7.10 & 9.7 & -- & 10 \\
\hline 49 & $11 / 19 / 81$ & 68 & 64 & 2.00 & 7.10 & 11.0 & -- & 10 \\
\hline 49 & $12 / 15 / 81$ & 80 & 80 & 5.50 & 7.10 & 13.0 & -- & 20 \\
\hline 49 & $01 / 22 / 82$ & 82 & 80 & 4.40 & 7.10 & 14.0 & -- & 30 \\
\hline 49 & $05 / 20 / 82$ & 58 & 58 & 3.60 & 7.00 & 12.0 & -- & 30 \\
\hline 49 & $06 / 15 / 82$ & 90 & 70 & 3.80 & 6.90 & 22.0 & - & 20 \\
\hline 49 & $07 / 15 / 82$ & 118 & 100 & 5.20 & 7.10 & 19.0 & -- & 35 \\
\hline 49 & $08 / 11 / 82$ & 82 & 70 & 2.50 & 7.20 & 10.50 & - & 30 \\
\hline 49 & $09 / 23 / 82$ & 70 & 64 & 3.00 & 7.30 & 7.1 & -- & 25 \\
\hline 49 & $10 / 20 / 82$ & 80 & 68 & 3.00 & 7.10 & 13.0 & - & 32 \\
\hline 49 & $11 / 19 / 82$ & 80 & 65 & 1.60 & 7.20 & 10.0 & - & 30 \\
\hline 49 & $12 / 20 / 82$ & 72 & 64 & 3.20 & 7.20 & 9.0 & -- & 25 \\
\hline 49 & $01 / 17 / 83$ & 128 & 110 & 7.50 & 7.10 & 22.0 & - & 30 \\
\hline 49 & $02 / 16 / 83$ & 124 & 104 & 4.00 & 7.10 & 20.0 & -- & 29 \\
\hline 49 & $03 / 18 / 83$ & 90 & 80 & 3.20 & 7.20 & 14.0 & -- & 20 \\
\hline 49 & $04 / 26 / 83$ & 92 & 82 & 3.40 & 7.10 & 15.0 & -- & 18 \\
\hline 49 & $05 / 16 / 83$ & 120 & 120 & 1.20 & 7.10 & 18.0 & -- & 35 \\
\hline 49 & $06 / 22 / 83$ & 128 & 102 & 4.70 & 7.10 & 21.0 & -- & 23 \\
\hline
\end{tabular}


Table 8.--Water-chemistry data from production wells, 1971-83--Continued

[Samples collected and analyzed by personnel of the Coos Bay-North Bend Water Board]

\begin{tabular}{|c|c|c|c|c|c|c|c|c|}
\hline WELL & DATE & $\begin{array}{c}\text { ALKA- } \\
\text { LINITY } \\
\text { (MG/L } \\
\text { AS } \\
\text { CACO3) }\end{array}$ & $\begin{array}{l}\text { HARD- } \\
\text { NESS } \\
\text { (MG/L } \\
\text { AS } \\
\text { CACO3) }\end{array}$ & $\begin{array}{l}\text { IRON } \\
\text { DIS- } \\
\text { SOLVED } \\
\text { (MG/L) }\end{array}$ & $\begin{array}{c}P H \\
(U N \mid T)\end{array}$ & $\begin{array}{l}\text { CARBON } \\
D I O X I D E \\
(M G / L)\end{array}$ & $\begin{array}{l}\text { FLUO- } \\
\text { RIDE } \\
\text { DIS- } \\
\text { SOLVED } \\
\text { (MG/L) }\end{array}$ & $\begin{array}{l}\text { CHLO- } \\
\text { RIDE } \\
\text { DIS- } \\
\text { SOLVED } \\
(M G / L)\end{array}$ \\
\hline 50 & $03 / 08 / 71$ & 52 & 52 & 4.00 & 7.00 & 11.0 & .01 & 31 \\
\hline 50 & $04 / 21 / 71$ & 56 & 52 & 3.80 & 7.00 & 12.0 & .02 & 31 \\
\hline 50 & $05 / 10 / 71$ & 88 & 82 & 3.80 & 6.80 & 21.0 & .01 & 31 \\
\hline 50 & $06 / 11 / 71$ & 88 & 64 & 4.00 & 6.80 & 28.0 & .01 & 31 \\
\hline 50 & $07 / 16 / 71$ & 102 & 96 & 4.00 & 7.10 & 18.0 & .02 & 22 \\
\hline 50 & $08 / 17 / 71$ & 96 & 102 & 4.40 & 7.10 & 16.0 & .02 & 28 \\
\hline 50 & $09 / 15 / 71$ & 86 & 86 & 3.60 & 6.80 & 28.0 & .01 & 32 \\
\hline 50 & $10 / 22 / 71$ & 70 & 80 & 3.00 & 7.10 & 13.0 & .01 & 31 \\
\hline 50 & $12 / 21 / 71$ & 64 & 84 & 2.60 & 6.80 & 36.0 & .01 & 28 \\
\hline 50 & $01 / 25 / 72$ & 68 & 88 & 2.40 & 6.90 & 22.0 & .02 & 24 \\
\hline 50 & $02 / 16 / 72$ & 112 & 128 & 2.40 & 7.00 & 23.0 & - & 24 \\
\hline 50 & $03 / 24 / 72$ & 60 & 56 & 4.00 & 6.60 & 32.0 & .15 & 22 \\
\hline 50 & $05 / 18 / 72$ & 66 & 65 & 4.40 & 6.80 & 34.0 & .03 & 26 \\
\hline 50 & $08 / 30 / 72$ & 76 & 70 & 5.00 & 7.20 & 12.0 & .02 & 28 \\
\hline 50 & $09 / 26 / 72$ & 88 & 102 & 5.00 & 7.10 & 14.5 & .02 & 32 \\
\hline 50 & $11 / 28 / 72$ & 92 & 92 & 4.60 & 7.10 & 15.0 & .02 & 28 \\
\hline 50 & $12 / 26 / 72$ & 86 & 86 & 5.00 & 7.10 & 14.0 & .02 & 32 \\
\hline 50 & $02 / 27 / 73$ & 126 & 112 & 5.80 & 7.20 & 16.5 & .02 & 30 \\
\hline 50 & $03 / 30 / 73$ & 132 & 112 & 5.40 & 7.10 & 23.0 & .02 & 30 \\
\hline 50 & $04 / 27 / 73$ & 114 & 76 & 5.20 & 7.10 & 17.0 & .01 & 32 \\
\hline 50 & $05 / 24 / 73$ & 104 & 76 & 5.00 & 7.00 & 21.0 & .01 & 30 \\
\hline 50 & $08 / 30 / 73$ & 108 & 84 & 4.80 & 7.00 & 22.0 & .01 & 31 \\
\hline 50 & $10 / 09 / 73$ & 120 & 78 & 5.80 & 7.00 & 24.0 & .01 & 31 \\
\hline
\end{tabular}


Table 8.-- Water-chemistry data from production

[Samples collected and analyzed by personnel of the Coos Bay-North Bend Water Board]

\begin{tabular}{|c|c|c|c|c|c|c|c|c|}
\hline WELL & DATE & $\begin{array}{c}\text { ALKA- } \\
\text { LINITY } \\
\text { (MG/L } \\
\text { AS } \\
\text { CACO3) }\end{array}$ & $\begin{array}{l}\text { HARD- } \\
\text { NESS } \\
\text { (MG/L } \\
\text { AS } \\
\text { CACO3) }\end{array}$ & $\begin{array}{l}\text { IRON } \\
\text { DIS- } \\
\text { SOLVED } \\
\text { (MG/L) }\end{array}$ & $\begin{array}{c}\text { PH } \\
(U N I T)\end{array}$ & $\begin{array}{l}\text { CARBON } \\
\text { DIOXIDE } \\
(M G / L)\end{array}$ & $\begin{array}{l}\text { FLUO- } \\
\text { RIDE } \\
\text { DIS- } \\
\text { SOLVED } \\
\text { (MG/L) }\end{array}$ & $\begin{array}{l}\text { CHLO- } \\
\text { RIDE } \\
\text { DIS- } \\
\text { SOLVED } \\
(M G / L)\end{array}$ \\
\hline $\begin{array}{l}50 \\
50\end{array}$ & $\begin{array}{l}11 / 06 / 73 \\
12 / 20 / 73\end{array}$ & $\begin{array}{l}124 \\
120\end{array}$ & $\begin{array}{l}98 \\
92\end{array}$ & $\begin{array}{l}5.60 \\
4.80\end{array}$ & $\begin{array}{l}7.10 \\
7.00\end{array}$ & $\begin{array}{l}19.5 \\
24.0\end{array}$ & $\begin{array}{l}.01 \\
.01\end{array}$ & $\begin{array}{l}31 \\
30\end{array}$ \\
\hline 50 & $01 / 29 / 74$ & 126 & 110 & 5.20 & 6.90 & 33.0 & .01 & 24 \\
\hline 50 & $02 / 26 / 74$ & 118 & 96 & 5.00 & 7.00 & 25.0 & .01 & 26 \\
\hline 50 & $04 / 30 / 74$ & 124 & 124 & 5.00 & 6.90 & 34.0 & .01 & 22 \\
\hline 50 & $08 / 28 / 74$ & 106 & 90 & 5.20 & 7.20 & 17.0 & .10 & 28 \\
\hline 50 & $09 / 27 / 74$ & 112 & 108 & 5.20 & 7.40 & 8.0 & .10 & 22 \\
\hline 50 & $11 / 21 / 74$ & 102 & 96 & 4.60 & 7.00 & 22.0 & .20 & 22 \\
\hline 50 & $12 / 31 / 74$ & 92 & 96 & 3.50 & 6.90 & 32.0 & .10 & 32 \\
\hline 50 & $02 / 28 / 75$ & 108 & 92 & 5.20 & 7.10 & 17.5 & .10 & 22 \\
\hline 50 & $03 / 26 / 75$ & 104 & 104 & 4.60 & 7.10 & 17.0 & .20 & 28 \\
\hline 50 & $04 / 23 / 75$ & 108 & 108 & 4.60 & 7.10 & 17.0 & .20 & 30 \\
\hline 50 & $05 / 27 / 75$ & 98 & 100 & 5.00 & 7.10 & 16.0 & .10 & 26 \\
\hline 50 & $07 / 29 / 75$ & 98 & 98 & 4.40 & 6.90 & 25.0 & .10 & 26 \\
\hline 50 & $09 / 25 / 75$ & 102 & 102 & 4.00 & 6.90 & 26.5 & .10 & 30 \\
\hline 50 & $11 / 25 / 75$ & 106 & 100 & 3.80 & 6.90 & 27.0 & .20 & 24 \\
\hline 50 & $12 / 30 / 75$ & 94 & 90 & 3.20 & 6.90 & 24.0 & .10 & 18 \\
\hline 50 & $02 / 19 / 76$ & 70 & 70 & 3.50 & 6.70 & 28.0 & .10 & 17 \\
\hline 50 & $03 / 30 / 76$ & 60 & 50 & 1.00 & 6.60 & 35.0 & .30 & 20 \\
\hline 50 & $04 / 23 / 76$ & 126 & 120 & 6.90 & 6.70 & 54.0 & .15 & 20 \\
\hline 50 & $06 / 17 / 76$ & 130 & 112 & 5.80 & 6.50 & 85.0 & .15 & 25 \\
\hline 50 & $07 / 19 / 76$ & 130 & 116 & 5.60 & 6.40 & 100.0 & .20 & 26 \\
\hline
\end{tabular}


Table 8.- $\frac{\text { Water-chemistry data from production }}{\text { wells, 1971-83--Continued }}$

[Samples collected and analyzed by personnel of the Coos Bay-North Bend Water Board]

\begin{tabular}{|c|c|c|c|c|c|c|c|c|}
\hline WELL & DATE & $\begin{array}{c}\text { ALKA- } \\
\text { LINITY } \\
\text { (MG/L } \\
\text { AS } \\
\text { CACO3) }\end{array}$ & $\begin{array}{l}\text { HARD- } \\
\text { NESS } \\
\text { (MG/L } \\
\text { AS } \\
\text { CACO3) }\end{array}$ & $\begin{array}{l}\text { IRON } \\
\text { DIS- } \\
\text { SOLVED } \\
\text { (MG/L) }\end{array}$ & $\begin{array}{c}\mathrm{PH} \\
(\mathrm{UN} \mid \mathrm{T})\end{array}$ & $\begin{array}{l}\text { CARBON } \\
\text { DIOXIDE } \\
(M G / L)\end{array}$ & $\begin{array}{l}\text { FLUO- } \\
\text { RIDE } \\
\text { DIS- } \\
\text { SOLVED } \\
\text { (MG/L) }\end{array}$ & $\begin{array}{l}\text { CHLO- } \\
\text { RIDE } \\
\text { DIS- } \\
\text { SOLVED } \\
\text { (MG/L) }\end{array}$ \\
\hline 50 & $08 / 20 / 76$ & 115 & 116 & 6.30 & 6.50 & 75.0 & .25 & 24 \\
\hline 50 & $09 / 21 / 76$ & 110 & 116 & 6.20 & 6.50 & 70.0 & .22 & 24 \\
\hline 50 & $10 / 21 / 76$ & 112 & 114 & 6.20 & 6.50 & 72.0 & .25 & 24 \\
\hline 50 & $11 / 18 / 76$ & 64 & 69 & 6.50 & 7.10 & 50.0 & .24 & 20 \\
\hline 50 & $02 / 15 / 77$ & 100 & 90 & 3.60 & 6.80 & 32.0 & .10 & 20 \\
\hline 50 & $03 / 15 / 77$ & 114 & 70 & 4.40 & 6.40 & 17.0 & .20 & 32 \\
\hline 50 & $04 / 15 / 77$ & 90 & 80 & 3.40 & 7.00 & 26.0 & .10 & 20 \\
\hline 50 & $05 / 16 / 77$ & 104 & 76 & 6.20 & 7.00 & 21.0 & .18 & 29 \\
\hline 50 & $06 / 21 / 77$ & 104 & 98 & 3.80 & 7.10 & 16.0 & .15 & 18 \\
\hline 50 & $07 / 21 / 77$ & 100 & 100 & 3.40 & 6.90 & 25.0 & -- & 15 \\
\hline 50 & $08 / 19 / 77$ & 100 & 108 & 4.20 & 6.90 & 26.0 & -- & 14 \\
\hline 50 & $09 / 15 / 77$ & 100 & 100 & 2.30 & 6.90 & 25.0 & -- & 14 \\
\hline 50 & $10 / 20 / 77$ & 102 & 98 & 3.80 & 6.90 & 26.0 & -- & 15 \\
\hline 50 & $12 / 20 / 77$ & 102 & 102 & 3.50 & 6.80 & 32.0 & -- & 20 \\
\hline 50 & $01 / 16 / 78$ & 100 & 98 & 3.80 & 6.90 & 25.0 & -- & 18 \\
\hline 50 & $02 / 16 / 78$ & 102 & 100 & 4.00 & 6.80 & 32.0 & -- & 20 \\
\hline 50 & $03 / 14 / 78$ & 100 & 100 & 4.00 & 6.80 & 24.0 & -- & 18 \\
\hline 50 & $04 / 12 / 78$ & 90 & 104 & 5.20 & -- & 18.0 & -- & 20 \\
\hline 50 & $05 / 11 / 78$ & 90 & 80 & 4.00 & 6.90 & 22.0 & -- & 18 \\
\hline 50 & $06 / 12 / 78$ & 90 & 80 & 4.00 & 6.80 & 28.0 & -- & 18 \\
\hline 50 & $07 / 17 / 78$ & 92 & 82 & 4.20 & 6.90 & 25.0 & -- & 18 \\
\hline 50 & $08 / 11 / 78$ & 80 & 80 & 6.00 & 6.80 & 26.0 & -- & 15 \\
\hline 50 & $09 / 15 / 78$ & 70 & 84 & 6.20 & 6.70 & 28.0 & -- & 15 \\
\hline
\end{tabular}


Table 8.- - Water-chemistry data from production

[Samples collected and analyzed by personnel of the Coos Bay-North Bend Water Board]

\begin{tabular}{|c|c|c|c|c|c|c|c|c|}
\hline WELL & DATE & $\begin{array}{c}\text { ALKA- } \\
\text { LINITY } \\
(M G / L \\
\text { AS } \\
\text { CACO3) }\end{array}$ & $\begin{array}{l}\text { HARD- } \\
\text { NESS } \\
\text { (MG/L } \\
\text { AS } \\
\text { CACO3) }\end{array}$ & $\begin{array}{l}\text { IRON } \\
\text { DIS- } \\
\text { SOLVED } \\
\text { (MG/L) }\end{array}$ & $\begin{array}{c}\mathrm{PH} \\
(\mathrm{UN} / \mathrm{T})\end{array}$ & $\begin{array}{l}\text { CARBON } \\
\text { DIOXIDE } \\
(M G / L)\end{array}$ & $\begin{array}{l}\text { FLUO- } \\
\text { RIDE } \\
\text { DIS- } \\
\text { SOLVED } \\
\text { (MG/L) }\end{array}$ & $\begin{array}{l}\text { CHLO- } \\
\text { RIDE } \\
\text { DIS- } \\
\text { SOLVED } \\
(M G / L)\end{array}$ \\
\hline 50 & $10 / 25 / 78$ & 75 & 82 & 6.00 & 6.70 & 30.0 & -- & 15 \\
\hline 50 & $11 / 15 / 78$ & 70 & 75 & 6.00 & 6.80 & 23.0 & -- & 21 \\
\hline 50 & $12 / 13 / 78$ & 80 & 70 & 5.00 & 6.90 & 20.0 & -- & 23 \\
\hline 50 & $01 / 22 / 79$ & 60 & 80 & 3.80 & 6.90 & 16.0 & -- & 22 \\
\hline 50 & $02 / 22 / 79$ & 90 & 100 & 3.20 & 7.10 & 9.0 & -- & 18 \\
\hline 50 & $03 / 20 / 79$ & 96 & 100 & 3.80 & 7.00 & 20.0 & -- & 18 \\
\hline 50 & $04 / 19 / 79$ & 76 & 80 & 3.80 & 6.90 & 20.0 & -- & 15 \\
\hline 50 & $05 / 21 / 79$ & 70 & 70 & 5.00 & 6.90 & 18.0 & .10 & 15 \\
\hline 50 & $06 / 13 / 79$ & 96 & 100 & 4.50 & 7.00 & 20.0 & .10 & 20 \\
\hline 50 & $08 / 16 / 79$ & 100 & 96 & 3.40 & 7.10 & 16.0 & - & 20 \\
\hline 50 & $10 / 23 / 79$ & 108 & 96 & 3.60 & 7.00 & 22.0 & -- & 17 \\
\hline 50 & $11 / 20 / 79$ & 100 & 94 & 1.60 & 7.00 & 20.0 & - & 18 \\
\hline 50 & $12 / 17 / 79$ & 100 & 90 & 4.00 & 7.10 & 16.0 & -- & 18 \\
\hline 50 & $01 / 13 / 80$ & 90 & 86 & 5.80 & 6.90 & 25.0 & -- & 18 \\
\hline 50 & $02 / 14 / 80$ & 100 & 92 & 3.40 & 7.10 & 16.0 & -- & 20 \\
\hline 50 & $03 / 14 / 80$ & 102 & 94 & 3.60 & 7.10 & 16.0 & -- & 20 \\
\hline 50 & $04 / 15 / 80$ & 90 & 80 & 5.80 & 6.80 & 28.0 & -- & 18 \\
\hline 50 & $05 / 13 / 80$ & 102 & 82 & 3.80 & 7.00 & 21.0 & -- & 18 \\
\hline 50 & $06 / 16 / 80$ & 100 & 90 & 3.40 & 7.00 & 21.0 & -- & 15 \\
\hline 50 & $07 / 11 / 80$ & 102 & 96 & 3.20 & 7.00 & 22.0 & -- & 16 \\
\hline 50 & $08 / 12 / 80$ & 104 & 90 & 2.80 & 7.10 & 17.0 & -- & 15 \\
\hline 50 & $09 / 15 / 80$ & 104 & 90 & 6.20 & 6.90 & 26.0 & -- & 25 \\
\hline 50 & $10 / 21 / 80$ & 100 & 90 & 3.40 & 7.00 & 21.0 & -- & 15 \\
\hline
\end{tabular}


Table 8.- $\frac{\text { Water-chemistry data from production }}{\text { wells, 1971-83--Continued }}$

[Samples collected and analyzed by personnel of the Coos Bay-North Bend Water Board]

\begin{tabular}{|c|c|c|c|c|c|c|c|c|}
\hline WELL & DATE & $\begin{array}{c}\text { ALKA- } \\
\text { LINITY } \\
\text { (MG/L } \\
\text { AS } \\
\text { CACO3) }\end{array}$ & $\begin{array}{l}\text { HARD- } \\
\text { NESS } \\
(M G / L \\
\text { AS } \\
\text { CACO3) }\end{array}$ & $\begin{array}{l}\text { IRON } \\
\text { DIS- } \\
\text { SOLVED } \\
\text { (MG/L) }\end{array}$ & $\begin{array}{c}\mathrm{PH} \\
(\mathrm{UN} / \mathrm{T})\end{array}$ & $\begin{array}{l}\text { CARBON } \\
\text { DIOXIDE } \\
(M G / L)\end{array}$ & $\begin{array}{l}\text { FLUO- } \\
\text { RIDE } \\
\text { DIS- } \\
\text { SOLVED } \\
\text { (MG/L) }\end{array}$ & $\begin{array}{l}\text { CHLO- } \\
\text { RIDE } \\
\text { DIS- } \\
\text { SOLVED } \\
(M G / L)\end{array}$ \\
\hline 50 & $11 / 14 / 80$ & 100 & 100 & 3.40 & 7.00 & 20.0 & -- & 20 \\
\hline 50 & $12 / 16 / 80$ & 90 & 80 & 4.00 & 7.00 & 17.0 & -- & 18 \\
\hline 50 & $01 / 15 / 81$ & 80 & -- & 6.20 & 6.70 & 32.0 & -- & 20 \\
\hline 50 & $02 / 16 / 81$ & 80 & 80 & 6.00 & 6.80 & 26.0 & -- & 20 \\
\hline 50 & $03 / 16 / 81$ & 70 & 54 & 6.00 & 6.70 & 29.0 & -- & 20 \\
\hline 50 & $04 / 15 / 81$ & 80 & 80 & 4.00 & 6.90 & 20.0 & -- & 22 \\
\hline 50 & $05 / 15 / 81$ & 100 & 96 & 2.80 & 6.70 & 41.0 & -- & 22 \\
\hline 50 & $06 / 11 / 81$ & 108 & 98 & 3.54 & 6.90 & 28.0 & -- & 20 \\
\hline 50 & $07 / 14 / 81$ & 100 & 90 & 3.60 & 7.00 & 20.0 & -- & 32 \\
\hline 50 & $08 / 28 / 81$ & 104 & 90 & 3.50 & 7.00 & 22.0 & -- & 34 \\
\hline 50 & $09 / 17 / 81$ & 96 & 86 & 3.60 & 7.00 & 20.0 & - & 20 \\
\hline 50 & $10 / 27 / 81$ & 110 & 98 & 1.40 & 7.10 & 18.0 & -- & 14 \\
\hline 50 & $11 / 19 / 81$ & 100 & 100 & 2.20 & 7.00 & 10.0 & -- & 15 \\
\hline 50 & $12 / 15 / 81$ & 80 & 80 & 4.50 & 6.90 & 20.0 & -- & 20 \\
\hline 50 & $01 / 22 / 82$ & 76 & 80 & 6.00 & 6.70 & 31.0 & -- & 32 \\
\hline 50 & $05 / 20 / 82$ & 104 & 92 & 3.40 & 7.10 & 17.0 & -- & 20 \\
\hline 50 & $06 / 15 / 82$ & 100 & 84 & 3.40 & 6.90 & 26.0 & -- & 20 \\
\hline 50 & $07 / 15 / 82$ & 80 & 64 & 5.20 & 6.90 & 20.0 & -- & 25 \\
\hline 50 & $08 / 11 / 82$ & 82 & 70 & 4.00 & 6.90 & 21.0 & -- & 25 \\
\hline 50 & $09 / 23 / 82$ & 64 & 60 & 7.00 & 6.40 & 45.0 & -- & 25 \\
\hline 50 & $10 / 20 / 82$ & 82 & 72 & 4.50 & 6.80 & 26.0 & -- & 25 \\
\hline 50 & $11 / 19 / 82$ & 80 & 68 & 4.50 & 6.70 & 32.0 & - & 25 \\
\hline 50 & $12 / 20 / 82$ & 66 & 62 & 6.80 & 6.80 & 22.0 & -- & 25 \\
\hline
\end{tabular}


Table 8.--Water-chemistry data from production wells, 1971-83--Continued

[Samples collected and analyzed by personnel of the Coos Bay-North Bend Water Board]

\begin{tabular}{|c|c|c|c|c|c|c|c|c|}
\hline WELL & DATE & $\begin{array}{c}\text { ALKA- } \\
\text { LINITY } \\
\text { (MG/L } \\
\text { AS } \\
\text { CACO3) }\end{array}$ & $\begin{array}{l}\text { HARD- } \\
\text { NESS } \\
\text { (MG/L } \\
\text { AS } \\
\text { CACO3) }\end{array}$ & $\begin{array}{l}\text { IRON } \\
\text { DIS- } \\
\text { SOLVED } \\
\text { (MG/L) }\end{array}$ & $\begin{array}{c}\mathrm{PH} \\
(\mathrm{UN} \mid \mathrm{T})\end{array}$ & $\begin{array}{l}\text { CARBON } \\
\text { DIOXIDE } \\
(M G / L)\end{array}$ & $\begin{array}{l}\text { FLUO- } \\
\text { RIDE } \\
\text { DIS- } \\
\text { SOLVED } \\
\text { (MG/L) }\end{array}$ & $\begin{array}{l}\text { CHLO- } \\
\text { RIDE } \\
\text { DIS- } \\
\text { SOLVED } \\
(M G / L)\end{array}$ \\
\hline 50 & $01 / 17 / 83$ & 90 & 70 & 3.50 & 6.90 & 23.0 & -- & 20 \\
\hline 50 & $02 / 16 / 83$ & 90 & 80 & 3.60 & 6.90 & 23.0 & -- & 20 \\
\hline 50 & $03 / 18 / 83$ & 80 & 86 & 5.00 & 6.80 & 26.0 & -- & 18 \\
\hline 50 & $04 / 26 / 83$ & 80 & 84 & 4.80 & 6.90 & 22.0 & -- & 18 \\
\hline 50 & $05 / 16 / 83$ & 90 & 100 & 2.00 & 7.00 & 17.0 & -- & 20 \\
\hline 50 & $05 / 20 / 83$ & 96 & 80 & 3.85 & 7.00 & 20.0 & -- & 19 \\
\hline 51 & $03 / 08 / 71$ & 104 & 92 & 4.80 & 6.60 & 54.0 & -- & 32 \\
\hline 51 & $04 / 21 / 71$ & 102 & 102 & 4.20 & 6.60 & 50.0 & -- & 32 \\
\hline 51 & $05 / 10 / 71$ & 56 & 50 & 8.00 & 6.60 & 18.0 & -- & 32 \\
\hline 51 & $06 / 11 / 71$ & 75 & 40 & 7.80 & 6.50 & 48.0 & .01 & 31 \\
\hline 51 & $07 / 16 / 71$ & 94 & 88 & 5.80 & 6.60 & 42.0 & .01 & 20 \\
\hline 51 & $08 / 17 / 71$ & 102 & 86 & 5.60 & 6.00 & 100.0 & .02 & 22 \\
\hline 51 & $09 / 15 / 71$ & 70 & 56 & 8.40 & 6.80 & 23.0 & .01 & 30 \\
\hline 51 & $10 / 22 / 71$ & 104 & 48 & 5.20 & 6.40 & 80.0 & .01 & 30 \\
\hline 51 & $12 / 21 / 71$ & 88 & 64 & 4.00 & 6.80 & 30.0 & .01 & 26 \\
\hline 51 & $01 / 25 / 72$ & 84 & 60 & 3.20 & 6.80 & 27.0 & .01 & 24 \\
\hline 51 & $02 / 16 / 72$ & 120 & 104 & 2.60 & 7.10 & 19.0 & -- & 28 \\
\hline 51 & $03 / 24 / 72$ & 100 & 136 & 4.80 & 6.90 & 34.0 & .15 & 15 \\
\hline 51 & $05 / 18 / 72$ & 112 & 78 & 5.00 & 7.00 & 22.0 & .04 & 24 \\
\hline 51 & $08 / 30 / 72$ & 120 & 112 & 5.60 & 7.10 & 30.0 & .04 & 26 \\
\hline 51 & $09 / 26 / 72$ & 110 & 62 & 5.80 & 7.00 & 21.0 & .01 & 30 \\
\hline 51 & $11 / 28 / 72$ & 104 & 68 & 4.60 & 7.10 & 17.0 & .02 & 30 \\
\hline 51 & $12 / 26 / 72$ & 110 & 66 & 5.20 & 7.00 & 21.0 & .02 & 31 \\
\hline
\end{tabular}


Table 8.-- Water-chemistry data from production

[Samples collected and analyzed by personnel of the Coos Bay-North Bend Water Board]

\begin{tabular}{|c|c|c|c|c|c|c|c|c|}
\hline WELL & DATE & $\begin{array}{c}\text { ALKA- } \\
\text { LINITY } \\
\text { (MG/L } \\
\text { AS } \\
\text { CACO3) }\end{array}$ & $\begin{array}{l}\text { HARD- } \\
\text { NESS } \\
\text { (MG/L } \\
\text { AS } \\
\text { CACO3) }\end{array}$ & $\begin{array}{l}\text { IRON } \\
\text { DIS- } \\
\text { SOLVED } \\
\text { (MG/L) }\end{array}$ & $\begin{array}{c}\mathrm{PH} \\
(\mathrm{UN} \mid \mathrm{T})\end{array}$ & $\begin{array}{l}\text { CARBON } \\
\text { DIOXIDE } \\
(M G / L)\end{array}$ & $\begin{array}{l}\text { FLUO- } \\
\text { RIDE } \\
\text { DIS- } \\
\text { SOLVED } \\
\text { (MG/L) }\end{array}$ & $\begin{array}{l}\text { CHLO- } \\
\text { RIDE } \\
\text { DIS- } \\
\text { SOLVED } \\
(M G / L)\end{array}$ \\
\hline 51 & $02 / 27 / 73$ & 122 & 118 & 6.00 & 6.80 & 40.0 & .02 & 32 \\
\hline 51 & $03 / 30 / 73$ & 126 & 108 & 6.00 & 6.60 & 65.0 & .02 & 36 \\
\hline 51 & $04 / 27 / 73$ & 106 & 68 & 5.60 & 6.60 & 55.0 & .01 & 34 \\
\hline 51 & $05 / 24 / 73$ & 118 & 78 & 5.60 & 6.80 & 38.0 & .01 & 30 \\
\hline 51 & $08 / 30 / 73$ & 116 & 90 & 5.80 & 6.80 & 38.0 & .01 & 30 \\
\hline 51 & $10 / 09 / 73$ & 120 & 102 & 6.00 & 7.10 & 19.0 & .01 & 30 \\
\hline 51 & $11 / 06 / 73$ & 122 & 108 & 6.20 & 7.10 & 19.5 & .01 & 31 \\
\hline 51 & $12 / 20 / 73$ & 120 & 100 & 5.60 & 6.90 & 31.0 & .01 & 30 \\
\hline 51 & $01 / 29 / 74$ & 120 & 106 & 5.20 & 6.60 & 62.0 & .01 & 31 \\
\hline 51 & $02 / 26 / 74$ & 124 & 104 & 5.00 & 6.80 & 41.0 & .01 & 27 \\
\hline 51 & $04 / 30 / 74$ & 112 & 108 & 5.60 & 6.90 & 28.0 & .01 & 24 \\
\hline 51 & $08 / 28 / 74$ & 110 & 106 & 4.40 & 6.80 & 37.0 & .10 & 30 \\
\hline 51 & $09 / 27 / 74$ & 116 & 104 & 5.60 & 6.90 & 28.0 & .10 & 30 \\
\hline 51 & $11 / 21 / 74$ & 108 & 90 & 5.20 & 6.90 & 27.0 & .10 & 30 \\
\hline 51 & $12 / 31 / 74$ & 132 & 112 & 5.00 & 6.80 & 45.0 & .10 & 36 \\
\hline 51 & $02 / 28 / 75$ & 108 & 96 & 5.60 & 6.80 & 35.0 & .10 & 24 \\
\hline 51 & $07 / 29 / 75$ & 100 & 114 & 4.00 & 7.00 & 21.0 & .10 & 29 \\
\hline 51 & $09 / 25 / 75$ & 106 & 112 & 4.20 & 7.00 & 22.0 & .20 & 30 \\
\hline 51 & $11 / 25 / 75$ & 106 & 106 & 4.00 & 7.10 & 17.5 & .20 & 30 \\
\hline 51 & $12 / 30 / 75$ & 140 & 100 & 3.80 & 6.90 & 36.0 & .20 & 20 \\
\hline 51 & $02 / 19 / 76$ & 130 & 130 & 4.00 & 6.90 & 32.0 & .15 & 20 \\
\hline 51 & $03 / 30 / 76$ & 120 & 120 & 7.00 & 6.70 & 50.0 & .15 & 22 \\
\hline
\end{tabular}


Table 8.--Water-chemistry data from production wells, 1971-83--Continued

[Samples collected and analyzed by personnel of the Coos Bay-North Bend Water Board]

\begin{tabular}{|c|c|c|c|c|c|c|c|c|}
\hline WELL & DATE & $\begin{array}{l}\text { ALKA- } \\
\text { LINITY } \\
\text { (MG/L } \\
\text { AS } \\
\text { CACO3) }\end{array}$ & $\begin{array}{l}\text { HARD- } \\
\text { NESS } \\
\text { (MG/L } \\
\text { AS } \\
\text { CACO3) }\end{array}$ & $\begin{array}{l}\text { IRON } \\
\text { DIS- } \\
\text { SOLVED } \\
\text { (MG/L) }\end{array}$ & $\begin{array}{c}\mathrm{PH} \\
(\mathrm{UN} \mid \mathrm{T})\end{array}$ & $\begin{array}{l}\text { CARBON } \\
\text { DIOXIDE } \\
(M G / L)\end{array}$ & $\begin{array}{l}\text { FLUO- } \\
\text { RIDE } \\
\text { DIS- } \\
\text { SOLVED } \\
(M G / L)\end{array}$ & $\begin{array}{l}\text { CHLO- } \\
\text { RIDE } \\
\text { DIS- } \\
\text { SOLVED } \\
\text { (MG/L) }\end{array}$ \\
\hline $\begin{array}{l}51 \\
51\end{array}$ & $\begin{array}{l}04 / 23 / 76 \\
06 / 17 / 76\end{array}$ & $\begin{array}{l}118 \\
120\end{array}$ & $\begin{array}{l}84 \\
90\end{array}$ & $\begin{array}{l}5.60 \\
6.40\end{array}$ & $\begin{array}{l}6.80 \\
6.60\end{array}$ & $\begin{array}{l}70.0 \\
60.0\end{array}$ & $\begin{array}{l}.15 \\
.20\end{array}$ & $\begin{array}{l}23 \\
25\end{array}$ \\
\hline 51 & $07 / 19 / 76$ & 124 & 96 & 6.20 & 6.50 & 82.0 & .20 & 25 \\
\hline 51 & $08 / 20 / 76$ & 120 & 90 & 4.00 & 6.50 & 80.0 & .20 & 24 \\
\hline 51 & $09 / 21 / 76$ & 115 & 90 & 4.00 & 6.60 & 78.0 & .22 & 24 \\
\hline 51 & $10 / 21 / 76$ & 116 & 90 & 4.20 & 6.60 & 60.0 & .22 & 24 \\
\hline 51 & $11 / 18 / 76$ & 100 & 90 & 6.50 & 6.80 & 32.0 & .18 & 20 \\
\hline 51 & $02 / 15 / 77$ & 128 & 105 & 3.80 & 6.60 & 65.0 & .22 & 20 \\
\hline 51 & $03 / 15 / 77$ & 106 & 68 & 5.20 & 6.60 & 55.0 & .10 & 30 \\
\hline 51 & $04 / 15 / 77$ & 136 & 90 & 4.20 & 6.80 & 32.0 & .20 & 20 \\
\hline 51 & $05 / 16 / 77$ & 118 & 78 & 6.20 & 6.80 & 38.0 & .18 & 30 \\
\hline 51 & $06 / 21 / 77$ & 140 & 110 & 5.60 & 6.90 & 40.0 & .15 & 10 \\
\hline 51 & $07 / 21 / 77$ & 140 & 120 & 7.20 & 6.90 & 63.0 & - & 18 \\
\hline 51 & $08 / 19 / 77$ & 142 & 118 & 5.20 & 7.00 & 64.0 & -- & 20 \\
\hline 51 & $09 / 15 / 77$ & 140 & 120 & 3.40 & 6.90 & 64.0 & -- & 18 \\
\hline 51 & $10 / 20 / 77$ & 136 & 124 & 6.00 & 6.80 & 43.0 & -- & 15 \\
\hline 51 & $12 / 20 / 77$ & 140 & 124 & 5.20 & 6.80 & 45.0 & -- & 20 \\
\hline 51 & $01 / 16 / 78$ & 130 & 122 & 4.20 & 6.80 & 42.0 & -- & 20 \\
\hline 51 & $02 / 16 / 78$ & 125 & 118 & 4.20 & 6.80 & 40.0 & -- & 20 \\
\hline 51 & $03 / 14 / 78$ & 128 & 122 & 4.20 & 6.80 & 43.0 & -- & 20 \\
\hline 51 & $04 / 12 / 78$ & 104 & 120 & 5.00 & - & 28.0 & -- & 15 \\
\hline 51 & $05 / 11 / 78$ & 90 & 80 & 4.20 & 6.80 & 22.0 & -- & 20 \\
\hline 51 & $06 / 12 / 78$ & 80 & 78 & 4.20 & 6.80 & 25.0 & -- & 20 \\
\hline
\end{tabular}


Table 8.- $\frac{\text { Water-chem istry data from production }}{\text { wells, 1971-83--Continued }}$

[Samples collected and analyzed by personnel of the Coos Bay-North Bend Water Board]

\begin{tabular}{|c|c|c|c|c|c|c|c|c|}
\hline WELL & DATE & $\begin{array}{c}\text { ALKA- } \\
\text { LINITY } \\
\text { (MG/L } \\
\text { AS } \\
\text { CACO3) }\end{array}$ & $\begin{array}{l}\text { HARD- } \\
\text { NESS } \\
\text { (MG/L } \\
\text { AS } \\
\text { CACO3) }\end{array}$ & $\begin{array}{l}\text { IRON } \\
\text { DIS- } \\
\text { SOLVED } \\
\text { (MG/L) }\end{array}$ & $\begin{array}{c}\mathrm{PH} \\
(\mathrm{UNIT})\end{array}$ & $\begin{array}{l}\text { CARBON } \\
\text { DIOXIDE } \\
(M G / L)\end{array}$ & $\begin{array}{l}\text { FLUO- } \\
\text { RIDE } \\
\text { DIS- } \\
\text { SOLVED } \\
\text { (MG/L) }\end{array}$ & $\begin{array}{l}\text { CHLO- } \\
\text { RIDE } \\
\text { DIS- } \\
\text { SOLVED } \\
(M G / L)\end{array}$ \\
\hline 51 & $07 / 17 / 78$ & 84 & 80 & 4.40 & 6.70 & 32.0 & -- & 20 \\
\hline 51 & $08 / 11 / 78$ & 110 & 110 & 6.00 & 6.70 & 45.0 & -- & 15 \\
\hline 51 & $09 / 15 / 78$ & 80 & 110 & 6.00 & 6.60 & 42.0 & -- & 18 \\
\hline 51 & $10 / 25 / 78$ & 100 & 112 & 6.00 & 6.60 & 50.0 & -- & 17 \\
\hline 51 & $11 / 15 / 78$ & 100 & 100 & 8.00 & 6.80 & 31.0 & -- & 21 \\
\hline 51 & $12 / 13 / 78$ & 70 & 70 & 5.20 & 6.80 & 21.0 & -- & 23 \\
\hline 51 & $01 / 22 / 79$ & 80 & 100 & 5.80 & 6.80 & 24.0 & -- & 20 \\
\hline 51 & $02 / 22 / 79$ & 100 & 100 & 7.50 & 6.80 & 32.0 & -- & 18 \\
\hline 51 & $03 / 20 / 79$ & 104 & 104 & 7.40 & 6.70 & 44.0 & -- & 18 \\
\hline 51 & $04 / 19 / 79$ & 66 & 60 & 6.00 & 6.80 & 21.0 & -- & 18 \\
\hline 51 & $05 / 21 / 79$ & 80 & 68 & 7.50 & 6.80 & 27.0 & .10 & 15 \\
\hline 51 & $06 / 13 / 79$ & 90 & 80 & 7.00 & 7.00 & 18.0 & .10 & 15 \\
\hline 51 & $08 / 16 / 79$ & 76 & 60 & 5.00 & 6.90 & 4.0 & -- & 20 \\
\hline 51 & $10 / 23 / 79$ & 144 & 134 & 6.20 & 7.00 & 28.0 & -- & 16 \\
\hline 51 & $11 / 20 / 79$ & 140 & 130 & 4.80 & 7.00 & 28.0 & -- & 16 \\
\hline 51 & $12 / 17 / 79$ & 140 & 118 & 6.00 & 7.10 & 23.0 & -- & 18 \\
\hline 51 & $01 / 13 / 80$ & 140 & 130 & 6.00 & 7.10 & 24.0 & -- & 18 \\
\hline 51 & $02 / 14 / 80$ & 128 & 120 & 6.10 & 7.00 & 26.0 & -- & 18 \\
\hline 51 & $03 / 14 / 80$ & 130 & 118 & 6.20 & 6.90 & 33.0 & -- & 20 \\
\hline 51 & $04 / 15 / 80$ & 142 & 128 & 6.20 & 7.00 & 28.0 & -- & 20 \\
\hline 51 & $05 / 13 / 80$ & 140 & 120 & 6.20 & 6.90 & 33.0 & -- & 18 \\
\hline 51 & $06 / 16 / 80$ & 140 & 120 & 6.00 & 7.00 & 29.0 & -- & 15 \\
\hline 51 & $07 / 11 / 80$ & 146 & 120 & 6.20 & 7.00 & 30.0 & -- & 18 \\
\hline
\end{tabular}


Table 8.- $\frac{\text { Water-chemistry data from production }}{\text { wells, 1971-83--Continued }}$

[Samples collected and analyzed by personnel of the Coos Bay-North Bend Water Board]

\begin{tabular}{|c|c|c|c|c|c|c|c|c|}
\hline WELL & DATE & $\begin{array}{l}\text { ALKA- } \\
\text { LINITY } \\
\text { (MG/L } \\
\text { AS } \\
\text { CACO3) }\end{array}$ & $\begin{array}{l}\text { HARD- } \\
\text { NESS } \\
\text { (MG/L } \\
\text { AS } \\
\text { CACO3) }\end{array}$ & $\begin{array}{l}\text { IRON } \\
\text { DIS- } \\
\text { SOLVED } \\
\text { (MG/L) }\end{array}$ & $\begin{array}{c}\mathrm{PH} \\
(\mathrm{UN} \mid \mathrm{T})\end{array}$ & $\begin{array}{l}\text { CARBON } \\
\text { DIOXIDE } \\
(M G / L)\end{array}$ & $\begin{array}{l}\text { FLUO- } \\
\text { RIDE } \\
\text { DIS- } \\
\text { SOLVED } \\
\text { (MG/L) }\end{array}$ & $\begin{array}{l}\text { CHLO- } \\
\text { RIDE } \\
\text { DIS- } \\
\text { SOLVED } \\
\text { (MG/L) }\end{array}$ \\
\hline 51 & $08 / 12 / 80$ & 140 & 120 & 6.20 & 7.10 & 22.0 & -- & 18 \\
\hline 51 & $09 / 15 / 80$ & 140 & 120 & 6.00 & 6.90 & 36.0 & -- & 20 \\
\hline 51 & $10 / 21 / 80$ & 140 & 120 & 6.20 & 7.00 & 28.0 & -- & 20 \\
\hline 51 & $11 / 14 / 80$ & 120 & 120 & 6.00 & 7.10 & 18.0 & -- & 20 \\
\hline 51 & $12 / 16 / 80$ & 118 & 104 & 6.20 & 6.90 & 30.0 & -- & 20 \\
\hline 51 & $01 / 15 / 81$ & 140 & -- & 6.20 & 7.00 & 29.0 & -- & 20 \\
\hline 51 & $02 / 16 / 81$ & 140 & 102 & 5.00 & 7.10 & 23.0 & -- & 22 \\
\hline 51 & $03 / 16 / 81$ & 116 & 100 & 6.10 & 6.70 & 48.0 & -- & 20 \\
\hline 51 & $04 / 15 / 81$ & 140 & 118 & 3.40 & 6.90 & 36.0 & -- & 22 \\
\hline 51 & $05 / 15 / 81$ & 130 & 120 & 5.00 & 6.90 & 34.0 & -- & 22 \\
\hline 51 & $06 / 11 / 81$ & 130 & 122 & 8.85 & 6.70 & 55.0 & -- & 18 \\
\hline 51 & $07 / 14 / 81$ & 140 & 120 & 6.00 & 7.00 & 28.0 & -- & 30 \\
\hline 51 & $08 / 28 / 81$ & 135 & 116 & 6.20 & 6.80 & 44.0 & -- & 28 \\
\hline 51 & $09 / 17 / 81$ & 136 & 118 & 6.00 & 6.90 & 35.0 & -- & 25 \\
\hline 51 & $10 / 27 / 81$ & 142 & 120 & 6.40 & 7.00 & 29.5 & -- & 14 \\
\hline 51 & $11 / 19 / 81$ & 118 & 118 & 6.60 & 6.90 & 30.0 & -- & 16 \\
\hline 51 & $12 / 15 / 81$ & 110 & 110 & 8.00 & 6.90 & 28.0 & -- & 20 \\
\hline 51 & $01 / 22 / 82$ & 120 & 110 & 6.40 & 6.80 & 38.0 & - & 30 \\
\hline 51 & $05 / 20 / 82$ & 118 & 62 & 6.20 & 6.60 & 61.0 & -- & 25 \\
\hline 51 & $06 / 15 / 82$ & 140 & 120 & 6.20 & 6.90 & 36.0 & -- & 18 \\
\hline 51 & $07 / 15 / 82$ & 136 & 118 & 6.40 & 6.80 & 44.0 & -- & 20 \\
\hline 51 & $08 / 11 / 82$ & 124 & 104 & 7.60 & 6.90 & 32.0 & -- & 30 \\
\hline 51 & $09 / 23 / 82$ & 140 & 120 & 7.50 & 6.60 & 72.0 & -- & 25 \\
\hline
\end{tabular}


Table 8.-- Water-chemistry data from production

[Samples collected and analyzed by personnel of the Coos Bay-North Bend Water Board]

\begin{tabular}{|c|c|c|c|c|c|c|c|c|}
\hline WELL & DATE & $\begin{array}{c}\text { ALKA- } \\
\text { LINITY } \\
\text { (MG/L } \\
\text { AS } \\
\text { CACO3) }\end{array}$ & $\begin{array}{l}\text { HARD- } \\
\text { NESS } \\
\text { (MG/L } \\
\text { AS } \\
\text { CACO3) }\end{array}$ & $\begin{array}{l}\text { IRON } \\
\text { DIS- } \\
\text { SOLVED } \\
\text { (MG/L) }\end{array}$ & $\begin{array}{c}\mathrm{PH} \\
(\mathrm{UN} \mid \mathrm{T})\end{array}$ & $\begin{array}{l}\text { CARBON } \\
\text { DIOXIDE } \\
(M G / L)\end{array}$ & $\begin{array}{l}\text { FLUO- } \\
\text { RIDE } \\
\text { DIS- } \\
\text { SOLVED } \\
(M G / L)\end{array}$ & $\begin{array}{l}\text { CHLO- } \\
\text { RIDE } \\
\text { DIS- } \\
\text { SOLVED } \\
(M G / L)\end{array}$ \\
\hline 51 & $10 / 20 / 82$ & 138 & 100 & 7.00 & 6.80 & 45.0 & -- & 25 \\
\hline 51 & $11 / 19 / 82$ & 130 & 110 & 8.25 & 6.80 & 41.0 & -- & 26 \\
\hline 51 & $12 / 20 / 82$ & 66 & 118 & 7.40 & 6.60 & 34.0 & -- & 26 \\
\hline 51 & $01 / 17 / 83$ & 138 & 120 & 7.50 & 6.80 & 46.0 & -- & 20 \\
\hline 51 & $02 / 16 / 83$ & 122 & 110 & 8.50 & 6.70 & 51.0 & -- & 20 \\
\hline 51 & $05 / 16 / 83$ & 98 & 90 & 4.80 & 6.90 & 26.0 & -- & 22 \\
\hline 51 & $06 / 22 / 83$ & 132 & 114 & 13.5 & 6.70 & 54.0 & -- & 19 \\
\hline 52 & $03 / 08 / 71$ & 64 & 58 & 4.60 & 6.90 & 14.0 & -- & 28 \\
\hline 52 & $04 / 21 / 71$ & 60 & 56 & 4.20 & 6.80 & 20.0 & -- & 28 \\
\hline 52 & $05 / 10 / 71$ & 94 & 80 & 6.80 & 6.60 & 55.0 & -- & 29 \\
\hline 52 & $06 / 11 / 71$ & 98 & 68 & 7.00 & 6.60 & 50.0 & .01 & 32 \\
\hline 52 & $07 / 16 / 71$ & 52 & 42 & 7.20 & 7.20 & 7.0 & .01 & 28 \\
\hline 52 & $08 / 17 / 71$ & 60 & 74 & 7.00 & 7.00 & 17.0 & .02 & 30 \\
\hline 52 & $09 / 15 / 71$ & 100 & 144 & 6.40 & 6.60 & 57.0 & .01 & 28 \\
\hline 52 & $10 / 22 / 71$ & 150 & 136 & 5.00 & 6.80 & 50.0 & .01 & 28 \\
\hline 52 & $12 / 21 / 71$ & 144 & 138 & 4.60 & 7.10 & 24.0 & .02 & 28 \\
\hline 52 & $01 / 25 / 72$ & 132 & 124 & 3.00 & 7.10 & 22.0 & .01 & 18 \\
\hline 52 & $02 / 16 / 72$ & 124 & 118 & 2.40 & 7.10 & 20.0 & -- & 26 \\
\hline 52 & $03 / 24 / 72$ & 54 & 144 & 4.60 & 6.80 & 18.0 & .04 & 15 \\
\hline 52 & $05 / 18 / 72$ & 68 & 90 & 5.00 & 7.00 & 14.0 & .04 & 20 \\
\hline 52 & $08 / 30 / 72$ & 84 & 108 & 5.40 & 7.10 & 17.0 & .04 & 24 \\
\hline 52 & $09 / 26 / 72$ & 144 & 130 & 7.00 & 6.60 & 74.0 & .01 & 24 \\
\hline
\end{tabular}


Table 8.-- Water-chem istry data from production

[Samples collected and analyzed by personnel of the Coos Bay-North Bend Water Board]

\begin{tabular}{|c|c|c|c|c|c|c|c|c|}
\hline WELL & DATE & $\begin{array}{l}\text { ALKA- } \\
\text { LINITY } \\
\text { (MG/L } \\
\text { AS } \\
\text { CACO3) }\end{array}$ & $\begin{array}{l}\text { HARD- } \\
\text { NESS } \\
\text { (MG/L } \\
\text { AS } \\
\text { CACO3) }\end{array}$ & $\begin{array}{l}\text { IRON } \\
\text { DIS- } \\
\text { SOLVED } \\
\text { (MG/L) }\end{array}$ & $\begin{array}{c}\mathrm{PH} \\
(U N \mid T)\end{array}$ & $\begin{array}{l}\text { CARBON } \\
\text { DIOXIDE } \\
(M G / L)\end{array}$ & $\begin{array}{l}\text { FLUO- } \\
\text { RIDE } \\
\text { DIS- } \\
\text { SOLVED } \\
(M G / L)\end{array}$ & $\begin{array}{l}\text { CHLO- } \\
\text { RIDE } \\
\text { DIS- } \\
\text { SOLVED } \\
(M G / L)\end{array}$ \\
\hline 52 & $11 / 28 / 72$ & 132 & 122 & 5.80 & 7.00 & 27.0 & .02 & 26 \\
\hline 52 & $12 / 26 / 72$ & 128 & 112 & 6.00 & 7.10 & 22.0 & .02 & 24 \\
\hline 52 & $02 / 27 / 73$ & 126 & 112 & 7.20 & 6.90 & 31.5 & .02 & 30 \\
\hline 52 & $03 / 30 / 73$ & 126 & 114 & 6.80 & 6.80 & 41.0 & .02 & 32 \\
\hline 52 & $04 / 27 / 73$ & 116 & 84 & 6.00 & 7.40 & 9.5 & .01 & 31 \\
\hline 52 & $05 / 24 / 73$ & 130 & 110 & 5.60 & 6.90 & 33.0 & .01 & 31 \\
\hline 52 & $08 / 30 / 73$ & 124 & 118 & 5.60 & 7.10 & 19.0 & .01 & 32 \\
\hline 52 & $10 / 09 / 73$ & 132 & 114 & 5.40 & 7.00 & 26.0 & .01 & 31 \\
\hline 52 & $11 / 06 / 73$ & 126 & 116 & 5.80 & 7.00 & 26.0 & .01 & 30 \\
\hline 52 & $12 / 20 / 73$ & 122 & 108 & 5.00 & 6.90 & 31.0 & .01 & 31 \\
\hline 52 & $01 / 29 / 74$ & 118 & 116 & 5.00 & 6.90 & 30.0 & .01 & 26 \\
\hline 52 & $02 / 26 / 74$ & 108 & 110 & 5.20 & 6.90 & 38.0 & .01 & 30 \\
\hline 52 & $04 / 30 / 74$ & 108 & 116 & 5.20 & 7.10 & 17.5 & .01 & 24 \\
\hline 52 & $08 / 28 / 74$ & 124 & 86 & 4.60 & 7.10 & 19.0 & -- & 30 \\
\hline 52 & $09 / 27 / 74$ & 120 & 98 & 4.80 & 7.00 & 25.0 & .20 & 30 \\
\hline 52 & $11 / 21 / 74$ & 84 & 102 & 5.40 & 6.90 & 22.0 & .10 & 31 \\
\hline 52 & $02 / 28 / 75$ & 82 & 104 & 6.20 & 6.90 & 21.0 & .20 & 28 \\
\hline 52 & $03 / 26 / 75$ & 96 & 116 & 5.20 & 6.90 & 25.0 & .20 & 30 \\
\hline 52 & $04 / 23 / 75$ & 100 & 108 & 4.80 & 6.90 & 26.0 & .20 & 30 \\
\hline 52 & $05 / 27 / 75$ & 88 & 96 & 4.20 & 6.90 & 23.0 & .10 & 30 \\
\hline 52 & $07 / 29 / 75$ & 96 & 102 & 3.80 & 7.00 & 20.0 & .20 & 30 \\
\hline 52 & $09 / 25 / 75$ & 96 & 106 & 3.20 & 7.10 & 12.0 & .20 & 24 \\
\hline
\end{tabular}


Table 8.-- Water-chemistry data from production

[Samples collected and analyzed by personnel of the Coos Bay-North Bend Water Board]

\begin{tabular}{|c|c|c|c|c|c|c|c|c|}
\hline WELL & DATE & $\begin{array}{c}\text { ALKA- } \\
\text { LINITY } \\
\text { (MG/L } \\
\text { AS } \\
\text { CACO3) }\end{array}$ & $\begin{array}{l}\text { HARD- } \\
\text { NESS } \\
\text { (MG/L } \\
\text { AS } \\
\text { CACO3) }\end{array}$ & $\begin{array}{l}\text { IRON } \\
\text { DIS- } \\
\text { SOLVED } \\
(M G / L)\end{array}$ & $\begin{array}{c}\mathrm{PH} \\
\text { (UNIT) }\end{array}$ & $\begin{array}{l}\text { CARBON } \\
\text { DIOXIDE } \\
(M G / L)\end{array}$ & $\begin{array}{l}\text { FLUO- } \\
\text { RIDE } \\
\text { DIS- } \\
\text { SOLVED } \\
\text { (MG/L) }\end{array}$ & $\begin{array}{l}\text { CHLO- } \\
\text { RIDE } \\
\text { DIS- } \\
\text { SOLVED } \\
(M G / L)\end{array}$ \\
\hline 52 & $11 / 25 / 75$ & 98 & 102 & 3.40 & 7.00 & 20.0 & .10 & 28 \\
\hline 52 & $12 / 30 / 75$ & 80 & 80 & 4.20 & 6.80 & 36.0 & .20 & 20 \\
\hline 52 & $02 / 19 / 76$ & 80 & 94 & 4.00 & 6.70 & 31.0 & .20 & 20 \\
\hline 52 & $03 / 30 / 76$ & 86 & 80 & 6.50 & 6.70 & 36.0 & .15 & 24 \\
\hline 52 & $04 / 23 / 76$ & 90 & 120 & 6.80 & 6.80 & 30.0 & .15 & 24 \\
\hline 52 & $06 / 17 / 76$ & 96 & 118 & 4.00 & 6.80 & 31.0 & .22 & 26 \\
\hline 52 & $07 / 19 / 76$ & 98 & 112 & 4.40 & 6.90 & 25.0 & .22 & 28 \\
\hline 52 & $08 / 20 / 76$ & 96 & 108 & 4.20 & 7.00 & 20.0 & .20 & 28 \\
\hline 52 & $09 / 21 / 76$ & 96 & 106 & 4.20 & 6.50 & 20.0 & .20 & 28 \\
\hline 52 & $10 / 21 / 76$ & 96 & 108 & 4.00 & 7.00 & 19.5 & .20 & 28 \\
\hline 52 & $11 / 18 / 76$ & 190 & 130 & -- & 6.70 & 78.0 & .20 & 18 \\
\hline 52 & $02 / 15 / 77$ & 90 & 80 & 4.30 & 6.90 & 22.0 & -- & 22 \\
\hline 52 & $03 / 15 / 77$ & 116 & 84 & 5.60 & 7.30 & 95.0 & .10 & 31 \\
\hline 52 & $04 / 15 / 77$ & 80 & 100 & 4.20 & 6.90 & 38.0 & .22 & 22 \\
\hline 52 & $05 / 16 / 77$ & 130 & 78 & 6.20 & 6.90 & 33.0 & .20 & 30 \\
\hline 52 & $06 / 21 / 77$ & 110 & 100 & 5.20 & 7.10 & 16.0 & .10 & 15 \\
\hline 52 & $07 / 21 / 77$ & 125 & 110 & 7.00 & 7.00 & 26.0 & -- & 18 \\
\hline 52 & $08 / 19 / 77$ & 125 & 112 & 7.00 & 6.90 & 25.0 & -- & 20 \\
\hline 52 & $09 / 15 / 77$ & 125 & 110 & 7.00 & 7.00 & 28.0 & -- & 18 \\
\hline 52 & $10 / 20 / 77$ & 138 & 112 & 6.80 & 6.80 & 48.0 & -- & 15 \\
\hline 52 & $12 / 20 / 77$ & 120 & 104 & 5.40 & 6.80 & 40.0 & -- & 20 \\
\hline
\end{tabular}


Table 8.- - Water-chemistry data from production

[Samples collected and analyzed by personnel of the Coos Bay-North Bend Water Board]

ALKA- HARD-

LINITY NESS IRON

(MG/L $\quad(M G / L \quad D I S-$

AS AS SOLVED

FLUO- CHLO-

RIDE RIDE

SOLVED PH DIOXIDE SOLVED SOLVED

WELL DATE CACO3) CACO3) (MG/L) (UNIT) (MG/L) (MG/L) (MG/L)

\begin{tabular}{rrrrrrrrr}
\hline 52 & $01 / 16 / 78$ & 50 & 50 & 4.80 & 6.60 & 25.0 & -- & 18 \\
52 & $02 / 16 / 78$ & 60 & 60 & 4.60 & 6.70 & 24.0 & -- & 18 \\
52 & $03 / 14 / 78$ & 56 & 60 & 5.00 & 6.50 & 26.0 & -- & 17 \\
52 & $04 / 12 / 78$ & 90 & 108 & 5.40 & -- & 30.0 & -- & 18 \\
52 & $05 / 11 / 78$ & 80 & 70 & 5.00 & 6.80 & 26.0 & -- & 20 \\
52 & $06 / 12 / 78$ & 135 & 75 & 5.80 & 6.80 & 40.0 & -- & 22 \\
52 & $07 / 17 / 78$ & 130 & 75 & 5.60 & 6.60 & 68.0 & -- & 24 \\
52 & $08 / 11 / 78$ & 108 & 82 & 6.40 & 6.70 & 45.0 & -- & 15 \\
52 & $09 / 15 / 78$ & 100 & 80 & 6.20 & 6.70 & 40.0 & -- & 18 \\
52 & $10 / 25 / 78$ & 104 & 80 & 6.40 & 6.70 & 42.0 & -- & 16 \\
52 & $11 / 15 / 78$ & 104 & 130 & 8.50 & 6.90 & 28.0 & -- & 23 \\
52 & $12 / 13 / 78$ & 90 & 100 & 5.80 & 6.90 & 22.0 & -- & 23 \\
& & & & & & & & \\
52 & $01 / 22 / 79$ & 84 & 90 & 6.00 & 6.90 & 21.0 & -- & 20 \\
52 & $02 / 22 / 79$ & 100 & 80 & 7.80 & 6.80 & 32.0 & -- & 18 \\
52 & $03 / 20 / 79$ & 98 & 80 & 7.60 & 6.60 & 54.0 & -- & 20 \\
52 & $04 / 19 / 79$ & 90 & 70 & 6.40 & 6.90 & 22.0 & -- & 15 \\
52 & $05 / 21 / 79$ & 78 & 80 & 11.00 & 6.60 & -- & .10 & 18 \\
52 & $06 / 13 / 79$ & 80 & 70 & 11.00 & 6.90 & 21.0 & .11 & 22 \\
52 & $08 / 16 / 79$ & 94 & 80 & 4.40 & 6.90 & 25.0 & -- & 20 \\
52 & $10 / 23 / 79$ & 138 & 110 & 6.20 & 7.00 & 26.0 & -- & 18 \\
52 & $11 / 20 / 79$ & 136 & 108 & 5.80 & 7.00 & 28.0 & -- & 18 \\
52 & $12 / 17 / 79$ & 90 & 100 & 6.20 & 6.90 & 23.0 & -- & 20 \\
& & & & & & & & \\
\hline & & & & & & & \\
\hline
\end{tabular}


Table 8.--Water-chemistry data from production wells, 1971-83--Continued

[Samples collected and analyzed by personnel of the Coos Bay-North Bend Water Board]

\begin{tabular}{|c|c|c|c|c|c|c|c|c|}
\hline WELL & DATE & $\begin{array}{c}\text { ALKA- } \\
\text { LINITY } \\
\text { (MG/L } \\
\text { AS } \\
\text { CACO3) }\end{array}$ & $\begin{array}{l}\text { HARD- } \\
\text { NESS } \\
\text { (MG/L } \\
\text { AS } \\
\text { CACO3) }\end{array}$ & $\begin{array}{l}\text { IRON } \\
\text { DIS- } \\
\text { SOLVED } \\
\text { (MG/L) }\end{array}$ & $\begin{array}{c}\text { PH } \\
(\text { UNIT) }\end{array}$ & $\begin{array}{l}\text { CARBON } \\
\text { DIOXIDE } \\
(M G / L)\end{array}$ & $\begin{array}{l}\text { FLUO- } \\
\text { RIDE } \\
\text { DIS- } \\
\text { SOLVED } \\
(M G / L)\end{array}$ & $\begin{array}{l}\text { CHLO- } \\
\text { RIDE } \\
\text { DIS- } \\
\text { SOLVED } \\
(M G / L)\end{array}$ \\
\hline 52 & $01 / 13 / 80$ & 80 & 82 & 3.00 & 6.90 & 21.0 & -- & 20 \\
\hline 52 & $02 / 14 / 80$ & 82 & 84 & 7.40 & 6.80 & 26.0 & -- & 20 \\
\hline 52 & $03 / 14 / 80$ & 84 & 82 & 6.80 & 6.80 & 22.0 & -- & 20 \\
\hline 52 & $04 / 15 / 80$ & 90 & 86 & 7.80 & 7.00 & 18.0 & -- & 18 \\
\hline 52 & $05 / 13 / 80$ & 88 & 80 & 7.00 & 6.70 & 33.0 & - & 25 \\
\hline 52 & $06 / 16 / 80$ & 120 & 100 & 5.60 & 6.90 & 31.0 & -- & 18 \\
\hline 52 & $07 / 11 / 80$ & 122 & 100 & 5.80 & 7.00 & 25.0 & -- & 18 \\
\hline 52 & $08 / 12 / 80$ & 120 & 102 & 6.20 & 7.00 & 24.0 & - & 20 \\
\hline 52 & $09 / 15 / 80$ & 104 & 100 & 6.10 & 7.00 & 21.0 & -- & 22 \\
\hline 52 & $10 / 21 / 80$ & 120 & 100 & 5.60 & 6.90 & 31.0 & -- & 18 \\
\hline 52 & $11 / 14 / 80$ & 104 & 110 & 6.00 & 7.10 & 17.0 & - & 20 \\
\hline 52 & $12 / 16 / 80$ & 120 & 100 & 6.00 & 7.10 & 18.0 & .11 & 20 \\
\hline 52 & $01 / 15 / 81$ & 120 & - & 6.00 & 7.10 & 19.0 & - & 25 \\
\hline 52 & $02 / 16 / 81$ & 120 & 100 & 3.60 & 7.10 & 19.0 & -- & 32 \\
\hline 52 & $03 / 16 / 81$ & 60 & 70 & 6.20 & 6.50 & 39.0 & -- & 20 \\
\hline 52 & $04 / 15 / 81$ & 124 & 100 & 5.00 & 6.90 & 32.0 & - & 20 \\
\hline 52 & $05 / 15 / 81$ & 120 & 104 & 5.00 & 7.00 & 24.0 & - & 20 \\
\hline 52 & $06 / 11 / 81$ & 90 & 76 & 9.35 & 6.50 & 57.0 & -- & 21 \\
\hline 52 & $07 / 14 / 81$ & 90 & 90 & 6.20 & 6.70 & 37.0 & - & 32 \\
\hline 52 & $08 / 28 / 81$ & 96 & 92 & 6.20 & 6.80 & 31.0 & - & 30 \\
\hline 52 & $09 / 17 / 81$ & 130 & 96 & 6.00 & 6.90 & 34.0 & - & 25 \\
\hline 52 & $10 / 27 / 81$ & 126 & 94 & 6.00 & 7.00 & 28.0 & - & 15 \\
\hline 52 & $11 / 19 / 81$ & 120 & 96 & 6.50 & 6.90 & 30.0 & - & 16 \\
\hline 52 & $12 / 15 / 81$ & 54 & 45 & 7.50 & 6.70 & 22.0 & -- & 15 \\
\hline
\end{tabular}


Table 8.--Water-chemistry data from production wells, 1971-83--Continued

[Samples collected and analyzed by personnel of the Coos Bay-North Bend Water Board]

\begin{tabular}{|c|c|c|c|c|c|c|c|c|}
\hline WELL & DATE & $\begin{array}{c}\text { ALKA- } \\
\text { LINITY } \\
\text { (MG/L } \\
\text { AS } \\
\text { CACO3) }\end{array}$ & $\begin{array}{l}\text { HARD- } \\
\text { NESS } \\
\text { (MG/L } \\
\text { AS } \\
\text { CACO3) }\end{array}$ & $\begin{array}{l}\text { IRON } \\
\text { DIS- } \\
\text { SOLVED } \\
\text { (MG/L) }\end{array}$ & $\begin{array}{c}\mathrm{PH} \\
(\mathrm{UN} \mid \mathrm{T})\end{array}$ & $\begin{array}{l}\text { CARBON } \\
\text { DIOXIDE } \\
(M G / L)\end{array}$ & $\begin{array}{l}\text { FLUO- } \\
\text { RIDE } \\
\text { DIS- } \\
\text { SOLVED } \\
\text { (MG/L) }\end{array}$ & $\begin{array}{l}\text { CHLO- } \\
\text { RIDE } \\
\text { DIS- } \\
\text { SOLVED } \\
(M G / L)\end{array}$ \\
\hline 52 & $01 / 22 / 82$ & 78 & 68 & 6.40 & 6.70 & 32.0 & -- & 30 \\
\hline 52 & $05 / 20 / 82$ & 56 & 82 & 6.00 & 7.00 & 11.0 & -- & 30 \\
\hline 52 & $06 / 15 / 82$ & 125 & 100 & 5.00 & 6.90 & 32.0 & -- & 20 \\
\hline 52 & $07 / 15 / 82$ & 80 & 62 & 6.60 & 6.80 & 26.0 & -- & 25 \\
\hline 52 & $08 / 11 / 82$ & 90 & 64 & 7.60 & 6.80 & 28.0 & -- & 30 \\
\hline 52 & $09 / 23 / 82$ & 80 & 72 & 8.00 & 6.60 & 42.0 & - & 25 \\
\hline 52 & $10 / 20 / 82$ & 94 & 94 & 7.00 & 6.80 & 31.0 & -- & 25 \\
\hline 52 & $11 / 19 / 82$ & 80 & 68 & 7.50 & 6.80 & 26.0 & -- & 25 \\
\hline 52 & $12 / 20 / 82$ & 136 & 120 & 7.80 & 6.50 & 90.0 & - & 20 \\
\hline 52 & $01 / 17 / 83$ & 100 & 82 & 5.75 & 6.80 & 33.0 & -- & 24 \\
\hline 52 & $02 / 16 / 83$ & 80 & 70 & 6.50 & 6.70 & 33.0 & -- & 18 \\
\hline 52 & $06 / 22 / 83$ & 128 & 100 & 5.50 & 7.00 & 26.0 & -- & 24 \\
\hline 53 & $03 / 08 / 71$ & 152 & 140 & 3.50 & 7.10 & 21.0 & .01 & 29 \\
\hline 53 & $04 / 21 / 71$ & 146 & 134 & 3.20 & 7.10 & 24.0 & .02 & 20 \\
\hline 53 & $05 / 10 / 71$ & 160 & 142 & 4.20 & 7.10 & 26.0 & .02 & 28 \\
\hline 53 & $06 / 11 / 71$ & 146 & 124 & 1.60 & 6.90 & 38.0 & .01 & 32 \\
\hline 53 & $07 / 16 / 71$ & 148 & 126 & 4.20 & 7.00 & 27.0 & .04 & 21 \\
\hline 53 & $08 / 17 / 71$ & 144 & 122 & 2.20 & 7.00 & 39.0 & .04 & 18 \\
\hline 53 & $09 / 15 / 71$ & 152 & 130 & 2.80 & 6.90 & 35.0 & .04 & 22 \\
\hline 53 & $10 / 22 / 71$ & 166 & 124 & 2.00 & 7.00 & 34.0 & .04 & 26 \\
\hline 53 & $12 / 21 / 71$ & 152 & 141 & 2.00 & 7.40 & 12.0 & .02 & 18 \\
\hline
\end{tabular}


Table 8.--Water-chemistry data from production wells, 1971-83--Continued

[Samples collected and analyzed by personnel of the Coos Bay-North Bend Water Board]

\begin{tabular}{|c|c|c|c|c|c|c|c|c|}
\hline WELL & DATE & $\begin{array}{c}\text { ALKA- } \\
\text { LINITY } \\
\text { (MG/L } \\
\text { AS } \\
\text { CACO3) }\end{array}$ & $\begin{array}{l}\text { HARD- } \\
\text { NESS } \\
\text { (MG/L } \\
\text { AS } \\
\text { CACO3) }\end{array}$ & $\begin{array}{l}\text { IRON } \\
\text { DIS- } \\
\text { SOLVED } \\
(M G / L)\end{array}$ & $\begin{array}{c}\mathrm{PH} \\
(\mathrm{UN} I T)\end{array}$ & $\begin{array}{l}\text { CARBON } \\
\text { DIOXIDE } \\
(M G / L)\end{array}$ & $\begin{array}{l}\text { FLUO- } \\
\text { RIDE } \\
\text { DIS- } \\
\text { SOLVED } \\
\text { (MG/L) }\end{array}$ & $\begin{array}{l}\text { CHLO- } \\
\text { RIDE } \\
\text { DIS- } \\
\text { SOLVED } \\
(M G / L)\end{array}$ \\
\hline 53 & $01 / 25 / 72$ & 156 & 115 & 2.80 & 7.10 & 25.0 & .02 & 17 \\
\hline 53 & $02 / 16 / 72$ & 158 & 130 & 2.00 & 7.10 & 26.0 & -- & 26 \\
\hline 53 & $03 / 24 / 72$ & 140 & 112 & 3.20 & 7.00 & 28.0 & .15 & 13 \\
\hline 53 & $04 / 28 / 72$ & 144 & 114 & 4.40 & 7.10 & 13.0 & -- & 12 \\
\hline 53 & $05 / 18 / 72$ & 152 & 120 & 2.60 & 7.00 & 32.0 & .04 & 19 \\
\hline 53 & $08 / 30 / 72$ & 144 & 118 & 2.80 & 6.80 & 46.0 & .02 & 20 \\
\hline 53 & $09 / 26 / 72$ & 148 & 136 & 4.60 & 7.00 & 30.0 & .02 & 30 \\
\hline 53 & $11 / 28 / 72$ & 150 & 92 & 3.20 & 7.10 & 23.0 & .02 & 30 \\
\hline 53 & $12 / 26 / 72$ & 144 & 86 & 2.80 & 7.10 & 24.0 & .01 & 32 \\
\hline 53 & $02 / 27 / 73$ & 132 & 108 & 3.20 & 7.00 & 27.0 & .01 & 27 \\
\hline 53 & $03 / 30 / 73$ & 136 & 108 & 3.60 & 7.10 & 22.0 & .01 & 28 \\
\hline 53 & $04 / 27 / 73$ & 148 & 116 & 2.80 & 7.30 & 15.0 & .01 & 24 \\
\hline 53 & $05 / 24 / 73$ & 144 & 110 & 3.60 & 6.80 & 46.0 & .01 & 30 \\
\hline 53 & $06 / 28 / 73$ & 148 & 122 & 3.80 & 6.80 & 49.0 & .02 & 31 \\
\hline 53 & $08 / 30 / 73$ & 160 & 122 & 4.00 & 7.00 & 33.0 & .02 & 30 \\
\hline 53 & $10 / 09 / 73$ & 156 & 128 & 4.20 & 7.00 & 32.0 & .01 & 28 \\
\hline 53 & $11 / 06 / 73$ & 142 & 122 & 5.60 & 7.10 & 23.0 & .01 & 30 \\
\hline 53 & $12 / 20 / 73$ & 148 & 128 & 3.60 & 7.10 & 38.0 & .01 & 24 \\
\hline 53 & $01 / 29 / 74$ & 132 & 118 & 3.60 & 7.10 & 22.0 & .01 & 28 \\
\hline 53 & $02 / 26 / 74$ & 126 & 80 & 4.20 & 6.90 & 33.0 & .01 & 28 \\
\hline 53 & $03 / 28 / 74$ & 110 & 88 & 4.00 & 7.00 & 22.0 & .01 & 28 \\
\hline 53 & $04 / 30 / 74$ & 108 & 96 & 4.40 & 7.00 & 22.0 & .01 & 30 \\
\hline 53 & $07 / 24 / 74$ & 142 & 140 & 4.80 & 7.00 & 30.0 & .10 & 18 \\
\hline
\end{tabular}


Table 8.--Water-chemistry data from production wells, 1971-83--Continued

[Samples collected and analyzed by personnel of the Coos Bay-North Bend Water Board]

\begin{tabular}{|c|c|c|c|c|c|c|c|c|}
\hline WELL & DATE & $\begin{array}{l}\text { ALKA- } \\
\text { LINITY } \\
\text { (MG/L } \\
\text { AS } \\
\text { CACO3) }\end{array}$ & $\begin{array}{l}\text { HARD- } \\
\text { NESS } \\
\text { (MG/L } \\
\text { AS } \\
\text { CACO3) }\end{array}$ & $\begin{array}{l}\text { IRON } \\
\text { DIS- } \\
\text { SOLVED } \\
\text { (MG/L) }\end{array}$ & $\begin{array}{c}\mathrm{PH} \\
(\mathrm{UN} \mid \mathrm{T})\end{array}$ & $\begin{array}{l}\text { CARBON } \\
\text { DIOXIDE } \\
(M G / L)\end{array}$ & $\begin{array}{l}\text { FLUO- } \\
\text { RIDE } \\
\text { DIS- } \\
\text { SOLVED } \\
\text { (MG/L) }\end{array}$ & $\begin{array}{l}\text { CHLO- } \\
\text { RIDE } \\
\text { DIS- } \\
\text { SOLVED } \\
\text { (MG/L) }\end{array}$ \\
\hline 53 & $08 / 28 / 74$ & 140 & 148 & 4.40 & 6.90 & 37.0 & .10 & 18 \\
\hline 53 & $09 / 27 / 74$ & 154 & 134 & 4.20 & 6.90 & 40.0 & .20 & 20 \\
\hline 53 & $11 / 21 / 74$ & 104 & 122 & 4.00 & 7.00 & 22.0 & .10 & 22 \\
\hline 53 & $12 / 31 / 74$ & 152 & 74 & 3.00 & 7.00 & 31.0 & .10 & 22 \\
\hline 53 & $02 / 28 / 75$ & 100 & 118 & 3.80 & 7.10 & 16.0 & .10 & 32 \\
\hline 53 & $03 / 26 / 75$ & 156 & 144 & 3.80 & 6.90 & 40.0 & .10 & 28 \\
\hline 53 & $04 / 23 / 75$ & 136 & 132 & 3.80 & 6.80 & 44.0 & .10 & 31 \\
\hline 53 & $05 / 27 / 75$ & 144 & 130 & 4.00 & 7.00 & 27.0 & .20 & 22 \\
\hline 53 & $07 / 29 / 75$ & 122 & 120 & 3.20 & 6.60 & 64.0 & .10 & 28 \\
\hline 53 & $09 / 25 / 75$ & 112 & 122 & 3.80 & 6.80 & 36.0 & .10 & 24 \\
\hline 53 & $11 / 25 / 75$ & 100 & 110 & 3.40 & 6.80 & 33.0 & .10 & 24 \\
\hline 53 & $12 / 30 / 75$ & 140 & 140 & 3.20 & 6.90 & 36.0 & .10 & 20 \\
\hline 53 & $02 / 19 / 76$ & 150 & 130 & 3.50 & 6.90 & 39.0 & .20 & 20 \\
\hline 53 & $03 / 30 / 76$ & 140 & 140 & 5.00 & 7.10 & 22.0 & .25 & 24 \\
\hline 53 & $04 / 23 / 76$ & 144 & 135 & 4.20 & 7.10 & 20.0 & .25 & 20 \\
\hline 53 & $06 / 17 / 76$ & 140 & 116 & 4.00 & 7.00 & 28.0 & .20 & 30 \\
\hline 53 & $07 / 19 / 76$ & 160 & 125 & 4.20 & 7.00 & 32.0 & .25 & 25 \\
\hline 53 & $08 / 20 / 76$ & 150 & 118 & 4.20 & 7.20 & 20.0 & .25 & 25 \\
\hline 53 & $09 / 21 / 76$ & 148 & 144 & 5.00 & 7.10 & 21.8 & .20 & 22 \\
\hline 53 & $10 / 21 / 76$ & 146 & 142 & 4.20 & 7.00 & 30.0 & .20 & 21 \\
\hline 53 & $11 / 18 / 76$ & 146 & 140 & 5.00 & 7.00 & 28.0 & .20 & 24 \\
\hline
\end{tabular}


Table 8.--Water-chem istry data from production wells, 1971-83--Continued

[Samples collected and analyzed by personnel of the Coos Bay-North Bend Water Board]

\begin{tabular}{|c|c|c|c|c|c|c|c|c|}
\hline WELL & DATE & $\begin{array}{c}\text { ALKA- } \\
\text { LINITY } \\
\text { (MG/L } \\
\text { AS } \\
\text { CACO3) }\end{array}$ & $\begin{array}{l}\text { HARD- } \\
\text { NESS } \\
\text { (MG/L } \\
\text { AS } \\
\text { CACO3) }\end{array}$ & $\begin{array}{l}\text { IRON } \\
\text { DIS- } \\
\text { SOLVED } \\
\text { (MG/L) }\end{array}$ & $\begin{array}{c}\mathrm{PH} \\
(\mathrm{UN} \mid \mathrm{T})\end{array}$ & $\begin{array}{l}\text { CARBON } \\
\text { DIOXIDE } \\
(M G / L)\end{array}$ & $\begin{array}{l}\text { FLUO- } \\
\text { RIDE } \\
\text { DIS- } \\
\text { SOLVED } \\
\text { (MG/L) }\end{array}$ & $\begin{array}{l}\text { CHLO- } \\
\text { RIDE } \\
\text { DIS- } \\
\text { SOLVED } \\
(M G / L)\end{array}$ \\
\hline 53 & $02 / 15 / 77$ & 128 & 118 & 3.40 & 7.00 & 28.0 & .20 & 26 \\
\hline 53 & $03 / 15 / 77$ & 140 & 120 & 4.20 & 7.00 & 28.0 & .10 & 20 \\
\hline 53 & $04 / 15 / 77$ & 140 & 120 & 4.20 & 7.00 & 29.0 & .20 & 22 \\
\hline 53 & $05 / 16 / 77$ & 135 & 130 & 4.40 & 7.10 & 22.0 & .20 & 20 \\
\hline 53 & $06 / 21 / 77$ & 140 & 140 & 4.20 & 7.10 & 22.0 & .10 & 20 \\
\hline 53 & $07 / 21 / 77$ & 140 & 130 & 6.00 & 7.00 & 30.0 & -- & 20 \\
\hline 53 & $08 / 19 / 77$ & 138 & 120 & 5.00 & 7.10 & 29.0 & -- & 20 \\
\hline 53 & $09 / 15 / 77$ & 135 & 130 & 5.00 & 7.00 & 27.0 & -- & 20 \\
\hline 53 & $10 / 20 / 77$ & 140 & 156 & 5.40 & 6.80 & 45.0 & -- & 16 \\
\hline 53 & $12 / 20 / 77$ & 160 & 140 & 3.50 & 6.80 & 52.0 & -- & 36 \\
\hline 53 & $01 / 16 / 78$ & 140 & 135 & 4.30 & 6.80 & 45.0 & -- & 20 \\
\hline 53 & $02 / 14 / 78$ & 144 & 120 & 4.10 & 6.90 & 37.0 & -- & 20 \\
\hline 53 & $03 / 14 / 78$ & 100 & 100 & 4.00 & 7.10 & 38.0 & -- & 18 \\
\hline 53 & $04 / 12 / 78$ & 140 & 140 & 5.20 & 6.90 & 36.0 & -- & 13 \\
\hline 53 & $05 / 11 / 78$ & 140 & 130 & 4.00 & 7.10 & 23.0 & -- & 20 \\
\hline 53 & $06 / 12 / 78$ & 146 & 136 & 5.40 & 7.10 & 24.0 & -- & 20 \\
\hline 53 & $07 / 17 / 78$ & 140 & 130 & 4.10 & 7.00 & 28.0 & -- & 20 \\
\hline 53 & $08 / 11 / 78$ & 140 & 120 & 4.00 & 6.80 & 45.0 & -- & 20 \\
\hline 53 & $09 / 15 / 78$ & 135 & 120 & 4.00 & 6.80 & 45.0 & -- & 20 \\
\hline 53 & $10 / 25 / 78$ & 142 & 122 & 4.40 & 6.80 & 45.0 & -- & 19 \\
\hline 53 & $11 / 15 / 78$ & 138 & 160 & 4.20 & 6.80 & 63.0 & -- & 24 \\
\hline 53 & $12 / 13 / 78$ & 130 & 130 & 4.00 & 6.90 & 32.0 & -- & 25 \\
\hline
\end{tabular}


Table 8.- $\frac{\text { Water-chem istry data from production }}{\text { well } 5,1971-83-- \text { Continued }}$

[Samples collected and analyzed by personnel of the Coos Bay-North Bend Water Board]

\begin{tabular}{|c|c|c|c|c|c|c|c|c|}
\hline WELL & DATE & $\begin{array}{c}\text { ALKA- } \\
\text { LINITY } \\
\text { (MG/L } \\
\text { AS } \\
\text { CACO3) }\end{array}$ & $\begin{array}{l}\text { HARD- } \\
\text { NESS } \\
\text { (MG/L } \\
\text { AS } \\
\text { CACO3) }\end{array}$ & $\begin{array}{l}\text { IRON } \\
\text { DIS- } \\
\text { SOLVED } \\
\text { (MG/L) }\end{array}$ & $\begin{array}{c}\mathrm{PH} \\
(\mathrm{UN} \mid \mathrm{T})\end{array}$ & $\begin{array}{l}\text { CARBON } \\
\text { DIOXIDE } \\
(M G / L)\end{array}$ & $\begin{array}{l}\text { FLUO- } \\
\text { RIDE } \\
\text { DIS- } \\
\text { SOLVED } \\
\text { (MG/L) }\end{array}$ & $\begin{array}{l}\text { CHLO- } \\
\text { RIDE } \\
\text { DIS- } \\
\text { SOLVED } \\
(M G / L)\end{array}$ \\
\hline 53 & $01 / 22 / 79$ & 130 & 130 & 4.20 & 7.10 & 27.0 & -- & 20 \\
\hline 53 & $02 / 22 / 79$ & 140 & 140 & 4.00 & 7.10 & 22.0 & -- & 20 \\
\hline 53 & $03 / 20 / 79$ & 135 & 140 & 4.20 & 7.10 & 22.0 & -- & 20 \\
\hline 53 & $04 / 19 / 79$ & 140 & 120 & 5.60 & 7.10 & 22.0 & - & 20 \\
\hline 53 & $05 / 21 / 79$ & 135 & 120 & 4.20 & 7.10 & 22.0 & .18 & 20 \\
\hline 53 & $06 / 13 / 79$ & 135 & 120 & 5.00 & 7.10 & 22.0 & .12 & 22 \\
\hline 53 & $08 / 16 / 79$ & 135 & 120 & 4.50 & 7.10 & 22.0 & - & 20 \\
\hline 53 & $10 / 23 / 79$ & 140 & 126 & 3.50 & 7.10 & 22.0 & -- & 19 \\
\hline 53 & $11 / 20 / 79$ & 140 & 120 & 4.20 & 7.10 & 23.0 & -- & 20 \\
\hline 53 & $12 / 17 / 79$ & 140 & 120 & 4.40 & 7.10 & 23.0 & -- & 20 \\
\hline 53 & $01 / 13 / 80$ & 130 & 120 & 4.00 & 7.10 & 21.0 & -- & 20 \\
\hline 53 & $02 / 14 / 80$ & 130 & 120 & 4.00 & 7.10 & 21.0 & - & 20 \\
\hline 53 & $03 / 14 / 80$ & 135 & 118 & 3.80 & 7.10 & 22.0 & - & 20 \\
\hline 53 & $04 / 15 / 80$ & 130 & 120 & 5.00 & 7.10 & 21.0 & -- & 20 \\
\hline 53 & $05 / 13 / 80$ & 124 & 118 & 4.40 & 7.00 & 25.0 & -- & 20 \\
\hline 53 & $06 / 16 / 80$ & 120 & 104 & 4.00 & 7.00 & 23.0 & -- & 20 \\
\hline 53 & $07 / 11 / 80$ & 136 & 120 & 4.00 & 7.10 & 22.0 & -- & 20 \\
\hline 53 & $08 / 12 / 80$ & 122 & 120 & 4.10 & 7.10 & 20.0 & -- & 20 \\
\hline 53 & $09 / 15 / 80$ & 130 & 120 & 5.00 & 7.10 & 22.0 & -- & 20 \\
\hline 53 & $10 / 21 / 80$ & 130 & 120 & 4.20 & 7.10 & 21.0 & -- & 22 \\
\hline 53 & $11 / 14 / 80$ & 130 & 118 & 4.40 & 7.10 & 21.0 & -- & 22 \\
\hline 53 & $12 / 16 / 80$ & 124 & 112 & 4.00 & 6.90 & 32.0 & -- & 30 \\
\hline
\end{tabular}


Table 8.--Water-chem istry data from production wells, 1971-83--Continued

[Samples collected and analyzed by personnel of the Coos Bay-North Bend Water Board]

\begin{tabular}{|c|c|c|c|c|c|c|c|c|}
\hline WELL & DATE & $\begin{array}{c}\text { ALKA- } \\
\text { LINITY } \\
\text { (MG/L } \\
\text { AS } \\
\text { CACO3) }\end{array}$ & $\begin{array}{l}\text { HARD- } \\
\text { NESS } \\
\text { (MG/L } \\
\text { AS } \\
\text { CACO3) }\end{array}$ & $\begin{array}{l}\text { IRON } \\
\text { DIS- } \\
\text { SOLVED } \\
\text { (MG/L) }\end{array}$ & $\begin{array}{c}\text { PH } \\
(U N \mid T)\end{array}$ & $\begin{array}{l}\text { CARBON } \\
\text { DIOXIDE } \\
(M G / L)\end{array}$ & $\begin{array}{l}\text { FLUO- } \\
\text { RIDE } \\
\text { DIS- } \\
\text { SOLVED } \\
(M G / L)\end{array}$ & $\begin{array}{l}\text { CHLO- } \\
\text { RIDE } \\
\text { DIS- } \\
\text { SOLVED } \\
(M G / L)\end{array}$ \\
\hline 53 & $01 / 15 / 81$ & 120 & -- & 4.20 & 7.10 & 19.0 & -- & 25 \\
\hline 53 & $02 / 16 / 81$ & 135 & 118 & 3.80 & 7.10 & 22.0 & -- & 42 \\
\hline 53 & $03 / 16 / 81$ & 120 & 120 & 1.80 & 7.10 & 19.0 & .10 & 25 \\
\hline 53 & $04 / 15 / 81$ & 126 & 118 & 4.00 & 6.90 & 34.0 & -- & 25 \\
\hline 53 & $05 / 15 / 81$ & 135 & 104 & 3.20 & 6.90 & 35.0 & -- & 25 \\
\hline 53 & $06 / 11 / 81$ & 138 & 124 & 4.35 & 7.10 & 22.0 & - & 23 \\
\hline 53 & $07 / 14 / 81$ & 122 & 118 & 4.40 & 7.10 & 19.0 & -- & 30 \\
\hline 53 & $08 / 28 / 81$ & 130 & 110 & 4.40 & 7.00 & 28.0 & -- & 20 \\
\hline 53 & $09 / 17 / 81$ & 136 & 114 & 4.50 & 7.00 & 28.0 & -- & 24 \\
\hline 53 & $10 / 27 / 81$ & 136 & 120 & 4.70 & 6.90 & 36.0 & -- & 24 \\
\hline 53 & $11 / 19 / 81$ & 130 & 120 & 3.80 & 7.00 & 26.0 & -- & 10 \\
\hline 53 & $12 / 15 / 81$ & 130 & 120 & 4.20 & 7.10 & 21.0 & -- & 20 \\
\hline 53 & $01 / 22 / 82$ & 130 & 120 & 4.60 & 7.10 & 22.0 & -- & 30 \\
\hline 53 & $05 / 20 / 82$ & 125 & 112 & 3.60 & 6.90 & 31.0 & -- & 25 \\
\hline 53 & $06 / 15 / 82$ & 130 & 100 & 5.80 & 7.10 & 21.0 & -- & 20 \\
\hline 53 & $07 / 21 / 82$ & 118 & 104 & 3.60 & 7.10 & 18.0 & -- & 28 \\
\hline 53 & $08 / 11 / 82$ & 130 & 100 & 3.80 & 7.10 & 21.0 & -- & 30 \\
\hline 53 & $09 / 23 / 82$ & 120 & 110 & 3.80 & 7.10 & 19.0 & -- & 23 \\
\hline 53 & $10 / 20 / 82$ & 130 & 112 & 3.80 & 7.10 & 21.0 & -- & 22 \\
\hline 53 & $11 / 19 / 82$ & 126 & 110 & 3.50 & 7.00 & 26.0 & -- & 18 \\
\hline 53 & $12 / 20 / 82$ & 128 & 100 & 3.60 & 7.10 & 21.0 & -- & 30 \\
\hline 53 & $01 / 17 / 83$ & 124 & 106 & 3.40 & 7.10 & 20.0 & -- & 20 \\
\hline 53 & $02 / 16 / 83$ & 122 & 108 & 3.60 & 7.10 & 20.0 & -- & 22 \\
\hline
\end{tabular}


Table 8.--Water-chemistry data from production wells, 1971-83--Continued

[Samples collected and analyzed by personnel of the Coos Bay-North Bend Water Board]

\begin{tabular}{|c|c|c|c|c|c|c|c|c|}
\hline WELL & DATE & $\begin{array}{c}\text { ALKA- } \\
\text { LINITY } \\
\text { (MG/L } \\
\text { AS } \\
\text { CACO3) }\end{array}$ & $\begin{array}{l}\text { HARD- } \\
\text { NESS } \\
\text { (MG/L } \\
\text { AS } \\
\text { CACO3) }\end{array}$ & $\begin{array}{l}\text { IRON } \\
\text { DIS- } \\
\text { SOLVED } \\
\text { (MG/L) }\end{array}$ & $\begin{array}{c}\mathrm{PH} \\
(U N \mid T)\end{array}$ & $\begin{array}{l}\text { CARBON } \\
D I O X \mid D E \\
(M G / L)\end{array}$ & $\begin{array}{l}\text { FLUO- } \\
\text { RIDE } \\
\text { DIS- } \\
\text { SOLVED } \\
\text { (MG/L) }\end{array}$ & $\begin{array}{l}\text { CHLO- } \\
\text { RIDE } \\
\text { DIS- } \\
\text { SOLVED } \\
(M G / L)\end{array}$ \\
\hline $\begin{array}{l}53 \\
53\end{array}$ & $\begin{array}{l}04 / 26 / 83 \\
05 / 16 / 83\end{array}$ & $\begin{array}{l}122 \\
120\end{array}$ & $\begin{array}{l}110 \\
120\end{array}$ & $\begin{array}{l}3.60 \\
3.40\end{array}$ & $\begin{array}{l}7.10 \\
7.00\end{array}$ & $\begin{array}{l}19.0 \\
24.0\end{array}$ & - & $\begin{array}{l}28 \\
25\end{array}$ \\
\hline 53 & $06 / 22 / 83$ & 126 & 116 & 4.00 & 7.00 & 26.0 & -- & 21 \\
\hline 54 & $03 / 08 / 71$ & 170 & 150 & 3.20 & 6.80 & 55.0 & -- & 30 \\
\hline 54 & $04 / 21 / 71$ & 170 & 150 & 2.80 & 7.10 & 28.0 & -- & 32 \\
\hline 54 & $05 / 10 / 71$ & 180 & 160 & 2.20 & 7.10 & 29.0 & -- & 31 \\
\hline 54 & $06 / 11 / 71$ & 172 & 152 & 2.20 & 7.10 & 27.0 & .01 & 58 \\
\hline 54 & $07 / 16 / 71$ & 172 & 138 & 4.60 & 7.10 & 29.0 & .02 & 36 \\
\hline 54 & $08 / 17 / 71$ & 102 & 90 & 2.80 & 6.60 & 54.0 & .02 & 25 \\
\hline 54 & $09 / 15 / 71$ & 172 & 154 & 3.40 & 7.10 & 25.0 & .01 & 32 \\
\hline 54 & $10 / 22 / 71$ & 126 & 148 & 2.60 & 7.20 & 16.0 & .01 & 31 \\
\hline 54 & $12 / 21 / 71$ & 144 & 146 & 2.20 & 7.50 & 9.0 & .01 & 27 \\
\hline 54 & $01 / 25 / 72$ & 176 & 136 & 3.00 & 7.10 & 28.0 & .03 & 32 \\
\hline 54 & $02 / 16 / 72$ & 180 & 142 & 2.40 & 7.20 & 23.0 & - & 22 \\
\hline 54 & $03 / 24 / 72$ & 154 & 120 & 3.80 & 6.80 & 54.0 & .14 & 20 \\
\hline 54 & $04 / 28 / 72$ & 148 & 126 & 3.20 & 7.00 & 30.0 & -- & 22 \\
\hline 54 & $05 / 18 / 72$ & 195 & 142 & 3.20 & 6.90 & 50.0 & .01 & 35 \\
\hline 54 & $08 / 30 / 72$ & 126 & 136 & 3.00 & 7.00 & 25.0 & .02 & 32 \\
\hline 54 & $09 / 26 / 72$ & 182 & 142 & 5.00 & 7.10 & 29.0 & .01 & 24 \\
\hline 54 & $11 / 28 / 72$ & 180 & 128 & 3.60 & 7.00 & 32.0 & .01 & 20 \\
\hline 54 & $12 / 26 / 72$ & 162 & 118 & 3.50 & 7.10 & 26.0 & .01 & 24 \\
\hline 54 & $02 / 27 / 73$ & 124 & 112 & 3.60 & 7.00 & 25.0 & .01 & 31 \\
\hline 54 & $03 / 30 / 73$ & 122 & 114 & 3.80 & 7.00 & 25.0 & .01 & 32 \\
\hline
\end{tabular}


Table 8.--Water-chemistry data from production wells, 1971-83--Continued

[Samples collected and analyzed by personnel of the Coos Bay-North Bend Water Board]

\begin{tabular}{|c|c|c|c|c|c|c|c|c|}
\hline WELL & DATE & $\begin{array}{c}\text { ALKA- } \\
\text { LINITY } \\
\text { (MG/L } \\
\text { AS } \\
\text { CACO3) }\end{array}$ & $\begin{array}{l}\text { HARD- } \\
\text { NESS } \\
\text { (MG/L } \\
\text { AS } \\
\text { CACO3) }\end{array}$ & $\begin{array}{l}\text { IRON } \\
\text { DIS- } \\
\text { SOLVED } \\
\text { (MG/L) }\end{array}$ & $\begin{array}{c}\mathrm{PH} \\
(\mathrm{UN} \mid \mathrm{T})\end{array}$ & $\begin{array}{l}\text { CARBON } \\
\text { DIOXIDE } \\
(M G / L)\end{array}$ & $\begin{array}{l}\text { FLUO- } \\
\text { RIDE } \\
\text { DIS- } \\
\text { SOLVED } \\
\text { (MG/L) }\end{array}$ & $\begin{array}{l}\text { CHLO- } \\
\text { RIDE } \\
\text { DIS- } \\
\text { SOLVED } \\
(M G / L)\end{array}$ \\
\hline 54 & $04 / 27 / 73$ & 170 & 144 & 2.00 & 7.30 & 17.0 & .01 & 20 \\
\hline 54 & $05 / 24 / 73$ & 192 & 136 & 2.40 & 6.90 & 50.0 & .01 & 27 \\
\hline 54 & $06 / 28 / 73$ & 180 & 134 & 3.00 & 7.20 & 10.0 & .01 & 28 \\
\hline 54 & $08 / 30 / 73$ & 174 & 150 & 3.80 & 7.10 & 29.0 & .02 & 28 \\
\hline 54 & $10 / 09 / 73$ & 176 & 158 & 3.80 & 6.80 & 56.0 & .01 & 28 \\
\hline 54 & $11 / 06 / 73$ & 152 & 146 & 3.20 & 6.90 & 39.0 & .01 & 29 \\
\hline 54 & $12 / 20 / 73$ & 182 & 174 & 3.20 & 6.90 & 30.0 & .01 & 22 \\
\hline 54 & $01 / 29 / 74$ & 156 & 160 & 3.00 & 7.00 & 32.0 & .01 & 30 \\
\hline 54 & $02 / 26 / 74$ & 124 & 86 & 3.80 & 6.90 & 29.0 & .01 & 26 \\
\hline 54 & $03 / 28 / 74$ & 118 & 92 & 3.60 & 7.10 & 19.0 & .01 & 30 \\
\hline 54 & $04 / 30 / 74$ & 114 & 108 & 3.80 & 7.10 & 18.5 & .01 & 30 \\
\hline 54 & $07 / 24 / 74$ & 144 & 130 & 5.00 & 7.10 & 23.0 & .10 & 24 \\
\hline 54 & $08 / 28 / 74$ & 154 & 128 & 5.00 & 7.00 & 31.0 & .10 & 28 \\
\hline 54 & $09 / 27 / 74$ & 150 & 144 & 5.00 & 7.10 & 24.0 & .10 & 28 \\
\hline 54 & $11 / 21 / 74$ & 112 & 134 & 3.60 & 6.80 & 37.0 & .10 & 24 \\
\hline 54 & $12 / 31 / 74$ & 132 & 122 & 3.50 & 6.80 & 44.0 & .20 & 26 \\
\hline 54 & $02 / 28 / 75$ & 106 & 136 & 3.20 & 6.90 & 27.0 & .20 & 20 \\
\hline 54 & $03 / 26 / 75$ & 104 & 92 & 3.20 & 6.50 & 70.0 & .20 & 26 \\
\hline 54 & $04 / 23 / 75$ & 104 & 96 & 3.00 & 6.60 & 24.0 & .20 & 30 \\
\hline 54 & $05 / 27 / 75$ & 104 & 102 & 3.20 & 6.80 & 33.0 & .10 & 26 \\
\hline 54 & $07 / 29 / 75$ & 100 & 94 & 2.60 & 6.90 & 21.0 & .10 & 26 \\
\hline 54 & $09 / 25 / 75$ & 100 & 98 & 3.00 & 6.90 & 28.0 & .10 & 30 \\
\hline 54 & $11 / 25 / 75$ & 102 & 96 & 3.00 & 7.00 & 20.0 & .20 & 28 \\
\hline 54 & $12 / 30 / 75$ & 150 & 140 & 2.80 & 7.00 & 31.0 & .10 & 30 \\
\hline
\end{tabular}


Table 8.- - Water-chem istry data from production

[Samples collected and analyzed by personnel of the Coos Bay-North Bend Water Board]

\begin{tabular}{|c|c|c|c|c|c|c|c|c|}
\hline WELL & DATE & $\begin{array}{c}\text { ALKA- } \\
\text { LINITY } \\
\text { (MG/L } \\
\text { AS } \\
\text { CACO3) }\end{array}$ & $\begin{array}{l}\text { HARD- } \\
\text { NESS } \\
\text { (MG/L } \\
\text { AS } \\
\text { CACO3) }\end{array}$ & $\begin{array}{l}\text { IRON } \\
\text { DIS- } \\
\text { SOLVED } \\
\text { (MG/L) }\end{array}$ & $\begin{array}{c}P H \\
(U N \mid T)\end{array}$ & $\begin{array}{l}\text { CARBON } \\
\text { DIOXIDE } \\
(M G / L)\end{array}$ & $\begin{array}{l}\text { FLUO- } \\
\text { RIDE } \\
\text { DIS- } \\
\text { SOLVED } \\
\text { (MG/L) }\end{array}$ & $\begin{array}{l}\text { CHLO- } \\
\text { RIDE } \\
\text { DIS- } \\
\text { SOLVED } \\
\text { (MG/L) }\end{array}$ \\
\hline 54 & $02 / 19 / 76$ & 120 & 110 & 4.10 & 6.90 & 31.0 & .20 & 26 \\
\hline 54 & $03 / 30 / 76$ & 100 & 104 & 6.80 & 6.80 & 31.0 & .30 & 30 \\
\hline 54 & $04 / 23 / 76$ & 150 & 135 & 6.00 & 7.10 & 24.0 & .22 & 24 \\
\hline 54 & $06 / 17 / 76$ & 145 & 120 & 5.80 & 7.10 & 7.0 & .25 & 28 \\
\hline 54 & $07 / 19 / 76$ & 170 & 140 & 6.80 & 7.10 & 28.0 & .30 & 34 \\
\hline 54 & $08 / 20 / 76$ & 160 & 135 & 3.60 & 6.50 & -- & .25 & 35 \\
\hline 54 & $09 / 21 / 76$ & 164 & 146 & 4.00 & 7.10 & 26.0 & .15 & 30 \\
\hline 54 & $10 / 21 / 76$ & 164 & 144 & 3.80 & 7.10 & 23.5 & .15 & 31 \\
\hline 54 & $11 / 18 / 76$ & 166 & 135 & 4.00 & 7.00 & 35.0 & .15 & 30 \\
\hline 54 & $02 / 15 / 77$ & 136 & 140 & 3.60 & 7.10 & 22.0 & .18 & 30 \\
\hline 54 & $03 / 15 / 77$ & 150 & 140 & 3.80 & 7.20 & 19.0 & .25 & 32 \\
\hline 54 & $04 / 15 / 77$ & 150 & 135 & 3.90 & 7.10 & 18.0 & .10 & 30 \\
\hline 54 & $05 / 16 / 77$ & 150 & 140 & 4.20 & 7.10 & 26.0 & .15 & 30 \\
\hline 54 & $06 / 21 / 77$ & 160 & 145 & 4.40 & 7.10 & 26.0 & .15 & 10 \\
\hline 54 & $07 / 21 / 77$ & 160 & 150 & 5.00 & 7.00 & 34.0 & - & 32 \\
\hline 54 & $08 / 19 / 77$ & 162 & 145 & 4.20 & 7.00 & 35.0 & -- & 30 \\
\hline 54 & $09 / 15 / 77$ & 148 & 145 & 4.00 & 7.10 & 22.0 & -- & 22 \\
\hline 54 & $10 / 20 / 77$ & 164 & 148 & 5.00 & 7.00 & 33.0 & -- & 28 \\
\hline 54 & $12 / 20 / 77$ & 140 & 130 & 4.30 & 6.90 & 36.0 & -- & 24 \\
\hline 54 & $01 / 16 / 78$ & 160 & 140 & 3.90 & 6.90 & 42.0 & -- & 32 \\
\hline 54 & $02 / 14 / 78$ & 160 & 160 & 4.20 & 7.00 & 32.0 & -- & 33 \\
\hline 54 & $03 / 14 / 78$ & 140 & 126 & 4.80 & 7.10 & -- & -- & 26 \\
\hline 54 & $04 / 12 / 78$ & 120 & 120 & 5.20 & 7.20 & 15.0 & -- & 25 \\
\hline
\end{tabular}


Table 8.-- Water-chemistry data from production

[Samples collected and analyzed by personnel of the Coos Bay-North Bend Water Board]

\begin{tabular}{|c|c|c|c|c|c|c|c|c|}
\hline WELL & DATE & $\begin{array}{c}\text { ALKA- } \\
\text { LINITY } \\
\text { (MG/L } \\
\text { AS } \\
\text { CACO3) }\end{array}$ & $\begin{array}{l}\text { HARD- } \\
\text { NESS } \\
\text { (MG/L } \\
\text { AS } \\
\text { CACO3) }\end{array}$ & $\begin{array}{l}\text { IRON } \\
\text { DIS- } \\
\text { SOLVED } \\
\text { (MG/L) }\end{array}$ & $\begin{array}{c}\mathrm{PH} \\
(U N \mid T)\end{array}$ & $\begin{array}{l}\text { CARBON } \\
\text { DIOXIDE } \\
(M G / L)\end{array}$ & $\begin{array}{l}\text { FLUO- } \\
\text { RIDE } \\
\text { DIS- } \\
\text { SOLVED } \\
\text { (MG/L) }\end{array}$ & $\begin{array}{l}\text { CHLO- } \\
\text { RIDE } \\
\text { DIS- } \\
\text { SOLVED } \\
(M G / L)\end{array}$ \\
\hline 54 & $05 / 11 / 78$ & 140 & 140 & 4.20 & 7.10 & 23.0 & - & 30 \\
\hline 54 & $06 / 12 / 78$ & 170 & 148 & 4.80 & 7.10 & 27.0 & -- & 32 \\
\hline 54 & $07 / 17 / 78$ & 160 & 145 & 4.00 & 7.10 & 26.0 & -- & 30 \\
\hline 54 & $08 / 11 / 78$ & 160 & 140 & 3.80 & 6.90 & 40.0 & -- & 30 \\
\hline 54 & $09 / 15 / 78$ & 160 & 140 & 4.00 & 6.90 & 40.0 & -- & 32 \\
\hline 54 & $10 / 25 / 78$ & 162 & 148 & 4.00 & 6.90 & 40.0 & -- & 33 \\
\hline 54 & $11 / 15 / 78$ & 160 & 150 & 4.20 & 7.00 & 32.0 & -- & 35 \\
\hline 54 & $12 / 13 / 78$ & 160 & 140 & 4.00 & 7.00 & 32.0 & -- & 35 \\
\hline 54 & $01 / 22 / 79$ & 150 & 140 & 3.50 & 7.10 & 25.0 & -- & 35 \\
\hline 54 & $02 / 22 / 79$ & 160 & 150 & 4.00 & 7.10 & 26.0 & - & 35 \\
\hline 54 & $03 / 20 / 79$ & 160 & 150 & 2.20 & 7.20 & 20.0 & - & 30 \\
\hline 54 & $04 / 19 / 79$ & 160 & 135 & 3.80 & 7.10 & 28.0 & -- & 34 \\
\hline 54 & $05 / 21 / 79$ & 140 & 104 & 3.80 & 7.10 & 23.0 & .12 & 35 \\
\hline 54 & $06 / 13 / 79$ & 160 & 140 & 4.00 & 7.20 & 20.0 & .11 & 35 \\
\hline 54 & $08 / 16 / 79$ & 160 & 130 & 3.50 & 7.10 & 26.0 & -- & 35 \\
\hline 54 & $10 / 23 / 79$ & 160 & 148 & 3.40 & 7.10 & 26.0 & -- & 33 \\
\hline 54 & $11 / 20 / 79$ & 158. & 140 & 3.80 & 7.10 & 26.0 & -- & 30 \\
\hline 54 & $12 / 17 / 79$ & 160 & 135 & 3.60 & 7.20 & 20.0 & -- & 32 \\
\hline 54 & $01 / 13 / 80$ & 160 & 140 & 3.80 & 7.10 & 26.0 & -- & 45 \\
\hline 54 & $02 / 14 / 80$ & 140 & 140 & 3.20 & 7.20 & 18.0 & -- & 30 \\
\hline 54 & $03 / 14 / 80$ & 140 & 135 & 3.60 & 7.10 & 23.0 & -- & 30 \\
\hline 54 & $04 / 15 / 80$ & 140 & 140 & 3.60 & 7.10 & 22.0 & -- & 40 \\
\hline 54 & $05 / 13 / 80$ & 160 & 140 & 3.20 & 7.10 & 26.0 & -- & 35 \\
\hline
\end{tabular}


Table 8.- - Water-chemistry data from production

[Samples collected and analyzed by personnel of the Coos Bay-North Bend Water Board]

\begin{tabular}{|c|c|c|c|c|c|c|c|c|}
\hline WELL & DATE & $\begin{array}{c}\text { ALKA- } \\
\text { LINITY } \\
\text { (MG/L } \\
\text { AS } \\
\text { CACO3) }\end{array}$ & $\begin{array}{l}\text { HARD- } \\
\text { NESS } \\
\text { (MG/L } \\
\text { AS } \\
\text { CACO3) }\end{array}$ & $\begin{array}{l}\text { IRON } \\
\text { DIS- } \\
\text { SOLVED } \\
\text { (MG/L) }\end{array}$ & $\begin{array}{c}P H \\
(U N \mid T)\end{array}$ & $\begin{array}{l}\text { CARBON } \\
\text { DIOXIDE } \\
(M G / L)\end{array}$ & $\begin{array}{l}\text { FLUO- } \\
\text { RIDE } \\
\text { DIS- } \\
\text { SOLVED } \\
\text { (MG/L) }\end{array}$ & $\begin{array}{l}\text { CHLO- } \\
\text { RIDE } \\
\text { DIS- } \\
\text { SOLVED } \\
(M G / L)\end{array}$ \\
\hline 54 & $06 / 16 / 80$ & 140 & 140 & 3.20 & 7.10 & 22.0 & -- & 30 \\
\hline 54 & $07 / 11 / 80$ & 156 & 140 & 3.40 & 7.10 & 25.0 & -- & 33 \\
\hline 54 & $08 / 12 / 80$ & 138 & 152 & 3.40 & 7.10 & 23.0 & -- & 31 \\
\hline 54 & $09 / 15 / 80$ & 150 & 140 & 3.60 & 7.10 & 24.0 & -- & 35 \\
\hline 54 & $10 / 21 / 80$ & 160 & 140 & 3.60 & 7.10 & 26.0 & -- & 30 \\
\hline 54 & $11 / 14 / 80$ & 140 & 140 & 3.40 & 7.10 & 22.0 & -- & 36 \\
\hline 54 & $12 / 16 / 80$ & 150 & 140 & 3.80 & 7.00 & 30.0 & -- & 40 \\
\hline 54 & $01 / 15 / 81$ & 135 & -- & 3.60 & 7.10 & 24.0 & -- & 35 \\
\hline 54 & $02 / 16 / 81$ & 160 & 140 & 3.60 & 7.10 & 26.0 & -- & 32 \\
\hline 54 & $03 / 16 / 81$ & 140 & 140 & 3.20 & 7.10 & 22.0 & -- & 35 \\
\hline 54 & $04 / 15 / 81$ & 140 & 130 & 3.60 & 7.00 & 28.0 & -- & 40 \\
\hline 54 & $05 / 15 / 81$ & 140 & 140 & 3.00 & 7.10 & 23.0 & -- & 38 \\
\hline 54 & $06 / 11 / 81$ & 172 & 144 & 3.30 & 7.10 & 28.0 & -- & 39 \\
\hline 54 & $07 / 14 / 81$ & 158 & 130 & 3.80 & 7.10 & 26.0 & -- & 40 \\
\hline 54 & $08 / 28 / 81$ & 160 & 130 & 3.70 & 7.10 & 23.0 & -- & 40 \\
\hline 54 & $09 / 17 / 81$ & 168 & 134 & 3.80 & 7.10 & 28.0 & -- & 41 \\
\hline 54 & $10 / 27 / 81$ & 164 & 130 & 4.00 & 7.10 & 14.0 & -- & 38 \\
\hline 54 & $11 / 19 / 81$ & 160 & 140 & 3.60 & 7.10 & 26.0 & -- & 40 \\
\hline 54 & $12 / 15 / 81$ & 144 & 140 & 3.60 & 7.10 & 23.0 & -- & 35 \\
\hline 54 & $01 / 22 / 82$ & 145 & 130 & 2.10 & 7.10 & 24.0 & -- & 40 \\
\hline 54 & $05 / 20 / 82$ & 155 & 140 & 2.60 & 7.00 & 32.0 & -- & 40 \\
\hline 54 & $06 / 15 / 82$ & 160 & 140 & 3.60 & 7.20 & 20.0 & -- & 36 \\
\hline 54 & $07 / 21 / 82$ & 150 & 130 & 3.40 & 7.10 & 24.0 & -- & 38 \\
\hline
\end{tabular}


Table 8.-- Water-chemistry data from production

[Samples collected and analyzed by personnel of the Coos Bay-North Bend Water Board]

\begin{tabular}{|c|c|c|c|c|c|c|c|c|}
\hline WELL & DATE & $\begin{array}{l}\text { ALKA- } \\
\text { LINITY } \\
\text { (MG/L } \\
\text { AS } \\
\text { CACO3) }\end{array}$ & $\begin{array}{l}\text { HARD- } \\
\text { NESS } \\
\text { (MG/L } \\
\text { AS } \\
\text { CACO3) }\end{array}$ & $\begin{array}{l}\text { IRON } \\
\text { DIS- } \\
\text { SOLVED } \\
\text { (MG/L) }\end{array}$ & $\begin{array}{c}\mathrm{PH} \\
(\mathrm{UN} \mid T)\end{array}$ & $\begin{array}{l}\text { CARBON } \\
D I O X I D E \\
(M G / L)\end{array}$ & $\begin{array}{l}\text { FLUO- } \\
\text { RIDE } \\
\text { DIS- } \\
\text { SOLVED } \\
\text { (MG/L) }\end{array}$ & $\begin{array}{l}\text { CHLO- } \\
\text { RIDE } \\
\text { DIS- } \\
\text { SOLVED } \\
(M G / L)\end{array}$ \\
\hline 54 & $08 / 11 / 82$ & 150 & 130 & 3.40 & 7.10 & 23.0 & -- & 45 \\
\hline 54 & $09 / 23 / 82$ & 146 & 130 & 3.40 & 7.10 & 24.0 & -- & 38 \\
\hline 54 & $10 / 20 / 82$ & 150 & 134 & 3.50 & 7.10 & 24.0 & -- & 37 \\
\hline 54 & $11 / 19 / 82$ & 150 & 136 & 3.30 & 7.10 & 24.0 & -- & 32 \\
\hline 54 & $12 / 20 / 82$ & 145 & 128 & 3.40 & 7.10 & 24.0 & -- & 46 \\
\hline 54 & $01 / 17 / 83$ & 156 & 134 & 3.20 & 7.10 & 26.0 & -- & 32 \\
\hline 54 & $02 / 16 / 83$ & 145 & 130 & 3.40 & 7.10 & 24.0 & -- & 35 \\
\hline 54 & $04 / 26 / 83$ & 150 & 130 & 1.12 & 7.10 & 24.0 & -- & 35 \\
\hline 54 & $05 / 16 / 83$ & 144 & 138 & 3.20 & 7.10 & 23.0 & -- & 40 \\
\hline 54 & $06 / 22 / 83$ & 152 & 134 & 3.90 & 7.10 & 24.0 & -- & 38 \\
\hline 55 & $03 / 08 / 71$ & 108 & 90 & 3.00 & 6.90 & 28.0 & .01 & 32 \\
\hline 55 & $04 / 21 / 71$ & 104 & 90 & 3.40 & 7.00 & 22.0 & .05 & 26 \\
\hline 55 & $05 / 10 / 71$ & 108 & 92 & 2.40 & 7.00 & 21.0 & .04 & 28 \\
\hline 55 & $06 / 11 / 71$ & 108 & 94 & 4.00 & 7.00 & 22.0 & .01 & 50 \\
\hline 55 & $07 / 16 / 71$ & 108 & 82 & 3.20 & 6.90 & 28.0 & .02 & 26 \\
\hline 55 & $08 / 17 / 71$ & 148 & 96 & 4.80 & 7.00 & 30.0 & .02 & 30 \\
\hline 55 & $09 / 15 / 71$ & 100 & 84 & 6.20 & 6.80 & 32.0 & .01 & 25 \\
\hline 55 & $10 / 22 / 71$ & 126 & 76 & 3.00 & 7.00 & 16.0 & .01 & 27 \\
\hline 55 & $12 / 21 / 71$ & 84 & 84 & 4.40 & 7.10 & 14.0 & .01 & 20 \\
\hline 55 & $01 / 25 / 72$ & 102 & 80 & 3.20 & 7.10 & 14.0 & .02 & 23 \\
\hline 55 & $02 / 16 / 72$ & 110 & 102 & 1.80 & 6.90 & 28.0 & -- & 28 \\
\hline 55 & $03 / 24 / 72$ & 102 & 84 & 4.60 & 7.00 & 21.0 & .20 & 18 \\
\hline
\end{tabular}


Table 3.--Water-chemistry data from production wells, 1971-83--Continued

[Samples collected and analyzed by personnel of the Coos Bay-North Bend Water Board]

\begin{tabular}{|c|c|c|c|c|c|c|c|c|}
\hline WELL & DATE & $\begin{array}{c}\text { ALKA- } \\
\text { LINITY } \\
\text { (MG/L } \\
\text { AS } \\
\text { CACO3) }\end{array}$ & $\begin{array}{l}\text { HARD- } \\
\text { NESS } \\
\text { (MG/L } \\
\text { AS } \\
\text { CACO3) }\end{array}$ & $\begin{array}{l}\text { IRON } \\
\text { DIS- } \\
\text { SOLVED } \\
\text { (MG/L) }\end{array}$ & $\begin{array}{c}\mathrm{PH} \\
(\mathrm{UN} \mid \mathrm{T})\end{array}$ & $\begin{array}{l}\text { CARBON } \\
\text { DIOXIDE } \\
(M G / L)\end{array}$ & $\begin{array}{l}\text { FLUO- } \\
\text { RIDE } \\
\text { DIS- } \\
\text { SOLVED } \\
\text { (MG/L) }\end{array}$ & $\begin{array}{l}\text { CHLO- } \\
\text { RIDE } \\
\text { DIS- } \\
\text { SOLVED } \\
(M G / L)\end{array}$ \\
\hline 55 & $04 / 28 / 72$ & 106 & 80 & 4.00 & 7.00 & 12.0 & - & 18 \\
\hline 55 & $05 / 18 / 72$ & 106 & 68 & 3.20 & 6.70 & 44.0 & -- & 20 \\
\hline 55 & $08 / 30 / 72$ & 134 & 72 & 3.20 & 7.00 & 27.0 & .01 & 26 \\
\hline 55 & $09 / 26 / 72$ & 102 & 66 & 5.00 & 6.60 & 53.0 & .03 & 31 \\
\hline 55 & $11 / 28 / 72$ & 100 & 72 & 4.00 & 6.80 & 34.0 & .01 & 28 \\
\hline 55 & $12 / 26 / 72$ & 96 & 68 & 4.00 & 6.60 & 50.0 & .01 & 30 \\
\hline 55 & $02 / 27 / 73$ & 98 & 112 & 3.20 & 6.80 & 33.0 & .02 & 30 \\
\hline 55 & $03 / 30 / 73$ & 96 & 120 & 3.60 & 6.90 & 25.0 & .02 & 32 \\
\hline 55 & $04 / 27 / 73$ & 64 & 72 & 4.40 & 6.60 & 44.0 & .01 & 28 \\
\hline 55 & $05 / 24 / 73$ & 72 & 42 & 2.60 & 6.40 & 56.0 & .01 & 24 \\
\hline 55 & $06 / 28 / 73$ & 60 & 44 & 3.80 & 6.70 & 25.0 & .02 & 22 \\
\hline 55 & $08 / 30 / 73$ & 156 & 126 & 3.20 & 7.00 & 31.0 & .02 & 24 \\
\hline 55 & $10 / 09 / 73$ & 144 & 134 & 2.80 & 6.90 & 37.0 & .01 & 26 \\
\hline 55 & $11 / 06 / 73$ & 124 & 130 & 3.60 & 6.90 & 31.0 & .01 & 24 \\
\hline 55 & $12 / 20 / 73$ & 58 & 58 & 4.00 & 6.90 & 16.0 & .01 & 20 \\
\hline 55 & $01 / 29 / 74$ & 64 & 56 & 4.20 & 7.00 & 13.0 & .01 & 26 \\
\hline 55 & $02 / 26 / 74$ & 108 & 90 & 4.40 & 6.80 & 35.0 & .02 & 21 \\
\hline 55 & $04 / 30 / 74$ & 106 & 104 & 4.60 & 7.00 & 21.0 & .02 & 18 \\
\hline 55 & $07 / 24 / 74$ & 96 & 84 & 4.20 & 6.90 & 25.0 & .10 & 20 \\
\hline 55 & $08 / 28 / 74$ & 92 & 92 & 4.00 & 7.00 & 17.0 & .10 & 26 \\
\hline 55 & $09 / 27 / 74$ & 86 & 86 & 4.00 & 7.00 & 15.0 & .10 & 30 \\
\hline 55 & $11 / 21 / 74$ & 86 & 80 & 3.20 & 7.10 & 12.0 & .20 & 30 \\
\hline 55 & $12 / 31 / 74$ & 104 & 94 & 2.50 & 7.00 & 21.0 & .10 & 34 \\
\hline
\end{tabular}


Table 8.-- Water-chem istry data from production

[Samples collected and analyzed by personnel of the Coos Bay-North Bend Water Board]

\begin{tabular}{|c|c|c|c|c|c|c|c|c|}
\hline WELL & DATE & $\begin{array}{l}\text { ALKA- } \\
\text { LINITY } \\
\text { (MG/L } \\
\text { AS } \\
\text { CACO3) }\end{array}$ & $\begin{array}{l}\text { HARD- } \\
\text { NESS } \\
\text { (MG/L } \\
\text { AS } \\
\text { CACO3) }\end{array}$ & $\begin{array}{l}\text { IRON } \\
\text { DIS- } \\
\text { SOLVED } \\
\text { (MG/L) }\end{array}$ & $\begin{array}{c}\text { PH } \\
(U N I T)\end{array}$ & $\begin{array}{l}\text { CARBON } \\
\text { DIOXIDE } \\
(M G / L)\end{array}$ & $\begin{array}{l}\text { FLUO- } \\
\text { RIDE } \\
\text { DIS- } \\
\text { SOLVED } \\
\text { (MG/L) }\end{array}$ & $\begin{array}{l}\text { CHLO- } \\
\text { RIDE } \\
\text { DIS- } \\
\text { SOLVED } \\
(M G / L)\end{array}$ \\
\hline 55 & $02 / 28 / 75$ & 90 & 74 & 4.20 & 7.10 & 15.0 & .10 & 28 \\
\hline 55 & $03 / 26 / 75$ & 64 & 52 & 4.00 & 6.50 & 54.0 & .20 & 36 \\
\hline 55 & $04 / 23 / 75$ & 72 & 56 & 4.00 & 6.80 & 23.0 & .20 & 30 \\
\hline 55 & $05 / 27 / 75$ & 102 & 88 & 2.00 & 6.90 & 26.0 & .10 & 29 \\
\hline 55 & $07 / 29 / 75$ & 106 & 92 & 2.60 & 6.80 & 34.0 & .20 & 30 \\
\hline 55 & $09 / 25 / 75$ & 104 & 90 & 2.40 & 7.00 & 21.0 & .10 & 30 \\
\hline 55 & $11 / 25 / 75$ & 104 & 96 & 2.60 & 7.00 & 23.0 & .10 & 26 \\
\hline 55 & $12 / 30 / 75$ & 100 & 100 & 3.20 & 7.00 & 20.0 & .10 & 30 \\
\hline 55 & $02 / 19 / 76$ & 98 & 96 & 3.50 & 6.90 & 25.0 & .20 & 27 \\
\hline 55 & $03 / 30 / 76$ & 100 & 90 & 5.00 & 6.90 & 26.0 & .30 & 28 \\
\hline 55 & $04 / 23 / 76$ & 100 & 108 & 3.60 & 6.90 & 26.0 & .25 & 32 \\
\hline 55 & $06 / 17 / 76$ & 58 & 50 & 4.40 & 6.50 & 32.0 & .30 & 18 \\
\hline 55 & $07 / 19 / 76$ & 60 & 64 & 4.50 & 6.60 & 30.0 & .25 & 20 \\
\hline 55 & $08 / 20 / 76$ & 80 & 66 & 4.00 & 6.60 & 40.0 & .30 & 30 \\
\hline 55 & $09 / 21 / 76$ & 86 & 104 & 4.00 & 6.80 & 28.0 & .15 & 26 \\
\hline 55 & $10 / 21 / 76$ & 116 & 108 & 4.20 & 6.90 & 29.0 & .15 & 25 \\
\hline 55 & $11 / 18 / 76$ & 90 & 104 & 4.20 & 6.90 & 45.0 & .20 & 28 \\
\hline 55 & $02 / 15 / 77$ & 100 & 100 & 3.90 & 7.10 & 16.0 & .20 & 28 \\
\hline 55 & $03 / 15 / 77$ & 60 & 64 & 4.20 & 6.80 & 20.0 & .15 & 18 \\
\hline 55 & $04 / 15 / 77$ & 65 & 68 & 4.00 & 6.80 & 20.0 & .20 & 20 \\
\hline 55 & $05 / 16 / 77$ & 100 & 84 & 4.40 & 7.00 & 20.0 & .18 & 27 \\
\hline 55 & $06 / 21 / 77$ & 100 & 90 & 5.20 & 7.10 & 14.0 & .18 & 25 \\
\hline 55 & $07 / 21 / 77$ & 104 & 90 & 5.00 & 6.90 & 27.0 & - & 26 \\
\hline
\end{tabular}


Table 8.--Water-chemistry data from production

[Samples collected and analyzed by personnel of the Coos Bay-North Bend Water Board]

\begin{tabular}{|c|c|c|c|c|c|c|c|c|}
\hline WELL & DATE & $\begin{array}{c}\text { ALKA- } \\
\text { LINITY } \\
\text { (MG/L } \\
\text { AS } \\
\text { CACO3) }\end{array}$ & $\begin{array}{l}\text { HARD- } \\
\text { NESS } \\
\text { (MG/L } \\
\text { AS } \\
\text { CACO3) }\end{array}$ & $\begin{array}{l}\text { IRON } \\
\text { DIS- } \\
\text { SOLVED } \\
\text { (MG/L) }\end{array}$ & $\begin{array}{c}\mathrm{PH} \\
(\mathrm{UN} I T)\end{array}$ & $\begin{array}{l}\text { CARBON } \\
\text { DIOXIDE } \\
(M G / L)\end{array}$ & $\begin{array}{l}\text { FLUO- } \\
\text { RIDE } \\
\text { DIS- } \\
\text { SOLVED } \\
(M G / L)\end{array}$ & $\begin{array}{l}\text { CHLO- } \\
\text { RIDE } \\
\text { DIS- } \\
\text { SOLVED } \\
(M G / L)\end{array}$ \\
\hline 55 & $08 / 19 / 77$ & 108 & 90 & 4.20 & 6.90 & 28.0 & - & 24 \\
\hline 55 & $09 / 15 / 77$ & 100 & 85 & 4.00 & 7.00 & 21.0 & -- & 25 \\
\hline 55 & $10 / 20 / 77$ & 104 & 102 & 5.00 & 6.80 & 34.0 & -- & 24 \\
\hline 55 & $12 / 20 / 77$ & 105 & 90 & 3.80 & 6.70 & 42.0 & -- & 28 \\
\hline 55 & $01 / 16 / 78$ & 104 & 90 & 4.20 & 6.90 & 26.0 & -- & 28 \\
\hline 55 & $02 / 14 / 78$ & 100 & 100 & 4.00 & 6.80 & 33.0 & -- & 26 \\
\hline 55 & $03 / 14 / 78$ & 100 & 90 & 4.20 & 7.00 & -- & -- & 28 \\
\hline 55 & $04 / 12 / 78$ & 110 & 104 & 4.60 & 6.90 & 29.0 & -- & 25 \\
\hline 55 & $05 / 11 / 78$ & 104 & 120 & 4.20 & 7.00 & 22.0 & -- & 25 \\
\hline 55 & $v_{0} / 12 / 78$ & 114 & 104 & 4.80 & 7.00 & 24.0 & -- & 26 \\
\hline 55 & $07 / 17 / 78$ & 104 & 86 & 4.00 & 7.10 & 17.0 & -- & 25 \\
\hline 55 & $08 / 11 / 78$ & 94 & 100 & 4.00 & 6.80 & 30.0 & -- & 28 \\
\hline 55 & $09 / 15 / 78$ & 84 & 70 & 4.40 & 6.80 & 28.0 & -- & 25 \\
\hline 55 & $10 / 25 / 78$ & 120 & 118 & 2.10 & 6.90 & 30.0 & -- & 24 \\
\hline 55 & $11 / 15 / 78$ & 118 & 100 & 4.00 & 6.90 & 30.0 & -- & 25 \\
\hline 55 & $12 / 13 / 78$ & 100 & 85 & 4.00 & 6.80 & 31.0 & -- & 30 \\
\hline 55 & $01 / 22 / 79$ & 100 & 100 & 4.20 & 7.10 & 12.0 & - & 30 \\
\hline 55 & $02 / 22 / 79$ & 110 & 100 & 5.20 & 6.90 & 29.0 & -- & 25 \\
\hline 55 & $03 / 20 / 79$ & 104 & 120 & 4.00 & 7.00 & 22.0 & -- & 29 \\
\hline 55 & $04 / 19 / 79$ & 100 & 90 & 4.40 & 6.90 & 26.0 & -- & 30 \\
\hline 55 & $05 / 21 / 79$ & 100 & 80 & 4.50 & 7.00 & 20.0 & .10 & 30 \\
\hline 55 & $06 / 13 / 79$ & 104 & 90 & 4.50 & 7.00 & 21.0 & .12 & 26 \\
\hline 55 & $08 / 16 / 79$ & 100 & 100 & 5.40 & 7.00 & 21.0 & -- & 26 \\
\hline
\end{tabular}


Table 8.--Water-chemistry data from production wells, 1971-83--Continued

[Samples collected and analyzed by personnel of the Coos Bay-North Bend Water Board]

\begin{tabular}{|c|c|c|c|c|c|c|c|c|}
\hline WELL & DATE & $\begin{array}{c}\text { ALKA- } \\
\text { LINITY } \\
\text { (MG/L } \\
\text { AS } \\
\text { CACO3) }\end{array}$ & $\begin{array}{l}\text { HARD- } \\
\text { NESS } \\
(M G / L \\
\text { AS } \\
\text { CACO3) }\end{array}$ & $\begin{array}{l}\text { IRON } \\
\text { DIS- } \\
\text { SOLVED } \\
\text { (MG/L) }\end{array}$ & $\begin{array}{c}\text { PH } \\
(\text { UNIT) }\end{array}$ & $\begin{array}{l}\text { CARBON } \\
\text { DIOXIDE } \\
(M G / L)\end{array}$ & $\begin{array}{l}\text { FLUO- } \\
\text { RIDE } \\
\text { DIS- } \\
\text { SOLVED } \\
(M G / L)\end{array}$ & $\begin{array}{l}\text { CHLO- } \\
\text { RIDE } \\
\text { DIS- } \\
\text { SOLVED } \\
\text { (MG/L) }\end{array}$ \\
\hline 55 & $10 / 23 / 79$ & 118 & 102 & 3.20 & 6.90 & 30.0 & -- & 26 \\
\hline 55 & $11 / 20 / 79$ & 100 & 100 & 4.00 & 6.90 & 26.0 & -- & 30 \\
\hline 55 & $12 / 17 / 79$ & 100 & 90 & 5.00 & 7.10 & 16.0 & -- & 30 \\
\hline 55 & $01 / 13 / 80$ & 100 & 84 & 4.20 & 6.90 & 26.0 & - & 30 \\
\hline 55 & $02 / 14 / 80$ & 100 & 84 & 4.00 & 7.00 & 20.0 & -- & 30 \\
\hline 55 & $03 / 14 / 80$ & 100 & 100 & 4.20 & 6.90 & 26.0 & - & 30 \\
\hline 55 & $04 / 15 / 80$ & 102 & 90 & 4.20 & 7.00 & 21.0 & -- & 25 \\
\hline 55 & $05 / 13 / 80$ & 100 & 90 & 5.00 & 6.90 & 26.0 & -- & 30 \\
\hline 55 & $06 / 16 / 80$ & 100 & 92 & 4.10 & 6.90 & 31.0 & -- & 30 \\
\hline 55 & $07 / 11 / 80$ & 106 & 88 & 3.60 & 7.00 & 22.0 & -- & 30 \\
\hline 55 & $08 / 12 / 80$ & 104 & 104 & 4.00 & 6.80 & 33.0 & -- & 30 \\
\hline 55 & $09 / 15 / 80$ & 100 & 90 & 4.00 & 6.90 & 26.0 & -- & 30 \\
\hline 55 & $10 / 21 / 80$ & 100 & 90 & 5.20 & 6.90 & 26.0 & -- & 32 \\
\hline 55 & $11 / 14 / 80$ & 100 & 100 & 4.00 & 6.90 & 26.0 & -- & 20 \\
\hline 55 & $12 / 16 / 80$ & 100 & 86 & 4.00 & 6.90 & 26.0 & -- & 32 \\
\hline 55 & $01 / 15 / 81$ & 94 & -- & 4.00 & 6.90 & 24.0 & - & 30 \\
\hline 55 & $02 / 16 / 81$ & 102 & 86 & 4.00 & 6.90 & 26.0 & -- & 32 \\
\hline 55 & $03 / 16 / 81$ & 100 & 90 & 2.80 & 6.90 & 26.0 & -- & 32 \\
\hline 55 & $04 / 15 / 81$ & 100 & 100 & 3.80 & 6.90 & 26.0 & -- & 35 \\
\hline 55 & $05 / 15 / 81$ & 106 & 90 & 3.60 & 6.90 & 27.0 & -- & 35 \\
\hline 55 & $06 / 11 / 81$ & 104 & 94 & 4.25 & 6.90 & 27.0 & -- & - \\
\hline 55 & $07 / 14 / 81$ & 104 & 90 & 4.40 & 6.90 & 27.0 & - & 38 \\
\hline 55 & $08 / 28 / 81$ & 102 & 92 & 5.00 & 6.60 & 52.0 & -- & 34 \\
\hline 55 & $09 / 17 / 81$ & 130 & 98 & 2.75 & 7.10 & 20.0 & -- & 37 \\
\hline
\end{tabular}


Table 8.--Water-chemistry data from production wells, 1971-83--Continued

[Samples collected and analyzed by personnel of the Coos Bay-North Bend Water Board]

\begin{tabular}{|c|c|c|c|c|c|c|c|c|}
\hline WELL & DATE & $\begin{array}{c}\text { ALKA- } \\
\text { LINITY } \\
\text { (MG/L } \\
\text { AS } \\
\text { CACO3) }\end{array}$ & $\begin{array}{l}\text { HARD- } \\
\text { NESS } \\
\text { (MG/L } \\
\text { AS } \\
\text { CACO3) }\end{array}$ & $\begin{array}{l}\text { IRON } \\
\text { DIS- } \\
\text { SOLVED } \\
\text { (MG/L) }\end{array}$ & $\begin{array}{c}\mathrm{PH} \\
(\mathrm{UN} I T)\end{array}$ & $\begin{array}{l}\text { CARBON } \\
\text { DIOXIDE } \\
(M G / L)\end{array}$ & $\begin{array}{l}\text { FLUO- } \\
\text { RIDE } \\
\text { DIS- } \\
\text { SOLVED } \\
(M G / L)\end{array}$ & $\begin{array}{l}\text { CHLO- } \\
\text { RIDE } \\
\text { DIS- } \\
\text { SOLVED } \\
(M G / L)\end{array}$ \\
\hline 55 & $10 / 27 / 81$ & 124 & 86 & 3.25 & 7.20 & 16.0 & -- & 32 \\
\hline 55 & $11 / 19 / 81$ & 100 & 90 & 4.40 & 7.00 & 10.0 & -- & 25 \\
\hline 55 & $12 / 15 / 81$ & 90 & 90 & 3.60 & 6.90 & 22.0 & -- & 30 \\
\hline 55 & $01 / 22 / 82$ & 96 & 100 & 4.40 & 6.90 & 26.0 & - & 35 \\
\hline 55 & $05 / 20 / 82$ & 100 & 90 & 4.20 & 6.80 & 32.0 & -- & 35 \\
\hline 55 & $06 / 15 / 82$ & 96 & 90 & 4.20 & 6.90 & 25.0 & -- & 30 \\
\hline 55 & $07 / 21 / 82$ & 98 & 82 & 3.80 & 6.90 & 25.0 & -- & 35 \\
\hline 55 & $08 / 11 / 82$ & 100 & 80 & 4.00 & 6.90 & 26.0 & -- & 40 \\
\hline 55 & $09 / 23 / 82$ & 100 & 90 & 4.40 & 6.70 & 41.0 & -- & 33 \\
\hline 55 & $10 / 20 / 82$ & 104 & 90 & 4.40 & 6.90 & 26.0 & -- & 30 \\
\hline 55 & $11 / 19 / 82$ & 102 & 94 & 4.40 & 6.80 & 32.0 & -- & 26 \\
\hline 55 & $12 / 20 / 82$ & 100 & 85 & 3.90 & 7.10 & 16.0 & -- & 42 \\
\hline 55 & $01 / 17 / 83$ & 100 & 92 & 4.60 & 6.80 & 32.0 & -- & 30 \\
\hline 55 & $02 / 16 / 83$ & 100 & 96 & 4.40 & 6.90 & 26.0 & - & 30 \\
\hline 55 & $03 / 18 / 83$ & 98 & 90 & 4.40 & 6.70 & 40.0 & -- & 25 \\
\hline 55 & $04 / 26 / 83$ & 102 & 94 & 6.40 & 6.80 & 32.0 & - & 35 \\
\hline 55 & $05 / 16 / 83$ & 100 & 105 & 4.30 & 6.90 & 26.0 & -- & 30 \\
\hline 55 & $06 / 22 / 83$ & 100 & 82 & 4.20 & 6.90 & 26.0 & - & 30 \\
\hline 56 & $03 / 08 / 71$ & 128 & 112 & 2.80 & 7.10 & 20.0 & .02 & 31 \\
\hline 56 & $04 / 21 / 71$ & 124 & 104 & 2.40 & 6.90 & 34.0 & -- & 26 \\
\hline 56 & $05 / 10 / 71$ & 124 & 112 & 4.00 & 6.90 & 32.0 & .01 & 26 \\
\hline 56 & $06 / 11 / 71$ & 128 & 114 & 5.00 & 6.90 & 33.0 & .01 & 42 \\
\hline
\end{tabular}


Table 8.--Water-chem istry data from production wells, 1971-83--Continued

[Samples collected and analyzed by personnel of the Coos Bay-North Bend Water Board]

\begin{tabular}{|c|c|c|c|c|c|c|c|c|}
\hline WELL & DATE & $\begin{array}{c}\text { ALKA- } \\
\text { LINITY } \\
\text { (MG/L } \\
\text { AS } \\
\text { CACO3) }\end{array}$ & $\begin{array}{l}\text { HARD- } \\
\text { NESS } \\
\text { (MG/L } \\
\text { AS } \\
\text { CACO3) }\end{array}$ & $\begin{array}{l}\text { IRON } \\
\text { DIS- } \\
\text { SOLVED } \\
\text { (MG/L) }\end{array}$ & $\begin{array}{c}P H \\
(U N \mid T)\end{array}$ & $\begin{array}{l}\text { CARBON } \\
\text { DIOXIDE } \\
(M G / L)\end{array}$ & $\begin{array}{l}\text { FLUO- } \\
\text { RIDE } \\
\text { DIS- } \\
\text { SOLVED } \\
\text { (MG/L) }\end{array}$ & $\begin{array}{l}\text { CHLO- } \\
\text { RIDE } \\
\text { DIS- } \\
\text { SOLVED } \\
(M G / L)\end{array}$ \\
\hline 56 & $07 / 16 / 71$ & 130 & 104 & 6.00 & 6.80 & 44.0 & .02 & 24 \\
\hline 56 & $08 / 17 / 71$ & 132 & 110 & 5.00 & 6.80 & 44.0 & .04 & 26 \\
\hline 56 & $09 / 15 / 71$ & 126 & 120 & 5.40 & 6.90 & 33.0 & .01 & 26 \\
\hline 56 & $10 / 22 / 71$ & 134 & 118 & 5.00 & 7.00 & 28.0 & .01 & 26 \\
\hline 56 & $12 / 21 / 71$ & 124 & 102 & 4.20 & 7.40 & 10.0 & .02 & 27 \\
\hline 56 & $01 / 25 / 72$ & 130 & 88 & 2.50 & 7.00 & 21.0 & .02 & 24 \\
\hline 56 & $02 / 16 / 72$ & 138 & 124 & 1.80 & 7.00 & 28.0 & -- & 22 \\
\hline 56 & $03 / 24 / 72$ & 80 & 64 & 4.20 & 6.60 & 43.0 & .15 & 22 \\
\hline 56 & $04 / 28 / 72$ & 78 & 68 & 3.60 & 6.50 & 49.0 & -- & 22 \\
\hline 56 & $05 / 18 / 72$ & 70 & 52 & 2.40 & 6.60 & 35.0 & .01 & 17 \\
\hline 56 & $08 / 30 / 72$ & 82 & 44 & 2.40 & 6.40 & 60.0 & .01 & 18 \\
\hline 56 & $09 / 26 / 72$ & 142 & 110 & 4.40 & 6.80 & 36.0 & .01 & 26 \\
\hline 56 & $11 / 28 / 72$ & 132 & 102 & 3.20 & 7.00 & 28.0 & .01 & 22 \\
\hline 56 & $12 / 26 / 72$ & 122 & 108 & 2.60 & 7.10 & 19.5 & .01 & 22 \\
\hline 56 & $02 / 27 / 73$ & 122 & 102 & 2.80 & 7.10 & 19.5 & .01 & 27 \\
\hline 56 & $03 / 30 / 73$ & 118 & 106 & 2.40 & 7.00 & 24.0 & .01 & 24 \\
\hline 56 & $04 / 27 / 73$ & 122 & 98 & 4.20 & 6.80 & 40.0 & .02 & 33 \\
\hline 56 & $05 / 24 / 73$ & 130 & 114 & 2.00 & 6.60 & 68.0 & .02 & 18 \\
\hline 56 & $06 / 28 / 73$ & 122 & 92 & 2.40 & 7.00 & 26.0 & .01 & 20 \\
\hline 56 & $08 / 30 / 73$ & 172 & 106 & 3.80 & 6.80 & 56.0 & .01 & 31 \\
\hline 56 & $10 / 09 / 73$ & 150 & 116 & 4.00 & 6.60 & 64.0 & .01 & 30 \\
\hline 56 & $11 / 06 / 73$ & 136 & 120 & 4.00 & 7.00 & 29.0 & .01 & 30 \\
\hline 56 & $12 / 20 / 73$ & 124 & 116 & 3.80 & 6.80 & 31.0 & .01 & 18 \\
\hline
\end{tabular}


Table 8.--Water-chemistry data from production wells, 1971-83--Continued

[Samples collected and analyzed by personnel of the Coos Bay-North Bend Water Board]

\begin{tabular}{|c|c|c|c|c|c|c|c|c|}
\hline WELL & DATE & $\begin{array}{l}\text { ALKA- } \\
\text { LINITY } \\
\text { (MG/L } \\
\text { AS } \\
\text { CACO3) }\end{array}$ & $\begin{array}{l}\text { HARD- } \\
\text { NESS } \\
\text { (MG/L } \\
\text { AS } \\
\text { CACO3) }\end{array}$ & $\begin{array}{l}\text { IRON } \\
\text { DIS- } \\
\text { SOLVED } \\
\text { (MG/L) }\end{array}$ & $\begin{array}{c}\text { PH } \\
(U N \mid T)\end{array}$ & $\begin{array}{l}\text { CARBON } \\
\text { DIOXIDE } \\
(M G / L)\end{array}$ & $\begin{array}{l}\text { FLUO- } \\
\text { RIDE } \\
\text { DIS- } \\
\text { SOLVED } \\
\text { (MG/L) }\end{array}$ & $\begin{array}{l}\text { CHLO- } \\
\text { RIDE } \\
\text { DIS- } \\
\text { SOLVED } \\
\text { (MG/L) }\end{array}$ \\
\hline 56 & $01 / 29 / 74$ & 120 & 108 & 4.40 & 7.20 & 16.0 & .01 & 31 \\
\hline 56 & $02 / 26 / 74$ & 126 & 126 & 3.20 & 6.90 & 33.0 & .02 & 21 \\
\hline 56 & $04 / 30 / 74$ & 120 & 116 & 3.80 & 6.90 & 30.0 & .02 & 20 \\
\hline 56 & $07 / 24 / 74$ & 102 & 82 & 4.20 & 6.80 & 36.0 & .20 & 22 \\
\hline 56 & $08 / 28 / 74$ & 94 & 98 & 4.20 & 6.90 & 15.0 & .20 & 30 \\
\hline 56 & $09 / 27 / 74$ & 90 & 106 & 3.60 & 7.10 & 15.0 & .20 & 31 \\
\hline 56 & $11 / 21 / 74$ & 94 & 92 & 3.80 & 7.10 & 16.0 & .10 & 30 \\
\hline 56 & $12 / 31 / 74$ & 118 & 104 & 3.20 & 6.90 & 30.0 & .20 & 24 \\
\hline 56 & $02 / 28 / 75$ & 96 & 102 & 3.80 & 7.10 & 15.0 & .10 & 30 \\
\hline 56 & $03 / 26 / 75$ & 118 & 90 & 4.00 & 6.80 & 35.0 & .10 & 24 \\
\hline 56 & $04 / 23 / 75$ & 114 & 98 & 4.20 & 6.80 & 35.0 & .20 & 26 \\
\hline 56 & $05 / 27 / 75$ & 120 & 100 & 2.20 & 6.90 & 31.0 & .20 & 30 \\
\hline 56 & $07 / 29 / 75$ & 116 & 104 & 2.20 & 7.10 & 18.5 & .20 & 30 \\
\hline 56 & $09 / 25 / 75$ & 112 & 100 & 2.60 & 7.10 & 17.0 & .20 & 31 \\
\hline 56 & $11 / 25 / 75$ & 96 & 100 & 2.60 & 7.10 & 15.5 & .20 & 30 \\
\hline 56 & $12 / 30 / 75$ & 110 & 108 & 3.80 & 7.00 & 22.0 & .20 & 30 \\
\hline 56 & $02 / 19 / 76$ & 96 & 100 & 4.20 & 6.90 & 24.0 & .35 & 27 \\
\hline 56 & $03 / 30 / 76$ & 80 & 80 & 7.40 & 6.60 & 43.0 & .30 & 22 \\
\hline 56 & $04 / 23 / 76$ & 90 & 90 & 7.00 & 6.60 & 48.0 & .25 & 20 \\
\hline 56 & $06 / 17 / 76$ & 100 & 90 & 5.80 & 6.70 & 42.0 & .20 & 26 \\
\hline 56 & $07 / 19 / 76$ & 120 & 120 & 4.20 & 6.70 & 48.0 & .18 & 30 \\
\hline 56 & $08 / 20 / 76$ & 100 & 118 & 4.50 & 6.70 & 42.0 & .18 & 22 \\
\hline 56 & $09 / 21 / 76$ & 110 & 108 & 5.00 & 6.80 & 35.0 & .22 & 24 \\
\hline
\end{tabular}


Table 8.--Water-chemistry data from production wells, 1971-83--Continued

[Samples collected and analyzed by personnel of the Coos Bay-North Bend Water Board]

\begin{tabular}{|c|c|c|c|c|c|c|c|c|}
\hline WELL & DATE & $\begin{array}{l}\text { ALKA- } \\
\text { LINITY } \\
\text { (MG/L } \\
\text { AS } \\
\text { CACO3) }\end{array}$ & $\begin{array}{l}\text { HARD- } \\
\text { NESS } \\
\text { (MG/L } \\
\text { AS } \\
\text { CACO3) }\end{array}$ & $\begin{array}{l}\text { IRON } \\
\text { DIS- } \\
\text { SOLVED } \\
\text { (MG/L) }\end{array}$ & $\begin{array}{c}\text { PH } \\
(U N I T)\end{array}$ & $\begin{array}{l}\text { CARBON } \\
\text { DIOXIDE } \\
(M G / L)\end{array}$ & $\begin{array}{l}\text { FLUO- } \\
\text { RIDE } \\
\text { DIS- } \\
\text { SOLVED } \\
\text { (MG/L) }\end{array}$ & $\begin{array}{l}\text { CHLO- } \\
\text { RIDE } \\
\text { DIS- } \\
\text { SOLVED } \\
(M G / L)\end{array}$ \\
\hline 56 & $10 / 21 / 76$ & 112 & 98 & 5.20 & 6.90 & 26.0 & .20 & 26 \\
\hline 56 & $11 / 18 / 76$ & 108 & 108 & 5.00 & 6.80 & 35.0 & .20 & 26 \\
\hline 56 & $02 / 15 / 77$ & 110 & 100 & 3.80 & 7.00 & 22.0 & .20 & 30 \\
\hline 56 & $03 / 15 / 77$ & 110 & 120 & 4.00 & 7.00 & 22.0 & .20 & 28 \\
\hline 56 & $04 / 15 / 77$ & 112 & 112 & 4.10 & 7.10 & 23.0 & .15 & 26 \\
\hline 56 & $05 / 16 / 77$ & 104 & 100 & 5.00 & 7.00 & 22.0 & .20 & 25 \\
\hline 56 & $06 / 21 / 77$ & 110 & 120 & 5.60 & 7.10 & 18.0 & .15 & 22 \\
\hline 56 & $07 / 21 / 77$ & 110 & 100 & 5.30 & 6.80 & 36.0 & -- & 25 \\
\hline 56 & $08 / 19 / 77$ & 108 & 104 & 4.20 & 6.90 & 35.0 & -- & 24 \\
\hline 56 & $09 / 15 / 77$ & 109 & 100 & 5.40 & 6.80 & 35.0 & -- & 25 \\
\hline 56 & $10 / 20 / 77$ & 110 & 104 & 5.00 & 6.70 & 45.0 & -- & 22 \\
\hline 56 & $12 / 20 / 77$ & 100 & 100 & 4.20 & 6.80 & 32.0 & -- & 25 \\
\hline 56 & $01 / 16 / 78$ & 108 & 102 & 4.80 & 6.80 & 35.0 & -- & 25 \\
\hline 56 & $02 / 14 / 78$ & 104 & 100 & 4.20 & 6.80 & 34.0 & -- & 25 \\
\hline 56 & $03 / 14 / 78$ & 104 & 100 & 5.30 & 7.00 & - & -- & 25 \\
\hline 56 & $04 / 12 / 78$ & 120 & 100 & 5.00 & 6.90 & 30.0 & -- & 28 \\
\hline 56 & $05 / 11 / 78$ & 120 & 120 & 6.00 & 7.00 & 25.0 & -- & 28 \\
\hline 56 & $06 / 12 / 78$ & 120 & 104 & 5.60 & 6.90 & 30.0 & -- & 23 \\
\hline 56 & $07 / 17 / 78$ & 104 & 100 & 4.20 & 6.90 & 28.0 & -- & 25 \\
\hline 56 & $08 / 11 / 78$ & 110 & 100 & 3.80 & 6.80 & 36.0 & -- & 25 \\
\hline 56 & $09 / 15 / 78$ & 100 & 104 & 4.10 & 6.90 & 26.0 & -- & 25 \\
\hline 56 & $10 / 25 / 78$ & 114 & 106 & 5.60 & 6.70 & 45.0 & -- & 24 \\
\hline 56 & $11 / 15 / 78$ & 120 & 110 & 7.00 & 6.80 & 58.0 & -- & 25 \\
\hline 56 & $12 / 13 / 78$ & 110 & 100 & 5.00 & 6.90 & 28.0 & -- & 30 \\
\hline
\end{tabular}


Table 8.--Water-chemistry data from production wells, 1971-83--Continued

[Samples collected and analyzed by personnel of the Coos Bay-North Bend Water Board]

\begin{tabular}{|c|c|c|c|c|c|c|c|c|}
\hline WELL & DATE & $\begin{array}{l}\text { ALKA- } \\
\text { LINITY } \\
\text { (MG/L } \\
\text { AS } \\
\text { CACO3) }\end{array}$ & $\begin{array}{l}\text { HARD- } \\
\text { NESS } \\
\text { (MG/L } \\
\text { AS } \\
\text { CACO3) }\end{array}$ & $\begin{array}{l}\text { IRON } \\
\text { DIS- } \\
\text { SOLVED } \\
\text { (MG/L) }\end{array}$ & $\begin{array}{c}\text { PH } \\
(\text { UNIT) }\end{array}$ & $\begin{array}{l}\text { CARBON } \\
\text { DIOXIDE } \\
(M G / L)\end{array}$ & $\begin{array}{l}\text { FLUO- } \\
\text { RIDE } \\
\text { DIS- } \\
\text { SOLVED } \\
\text { (MG/L) }\end{array}$ & $\begin{array}{l}\text { CHLO- } \\
\text { RIDE } \\
\text { DIS- } \\
\text { SOLVED } \\
(M G / L)\end{array}$ \\
\hline 56 & $01 / 22 / 79$ & 104 & 104 & 5.80 & 7.00 & 22.0 & -- & 25 \\
\hline 56 & $02 / 22 / 79$ & 110 & 100 & 4.00 & 7.00 & 22.0 & -- & 28 \\
\hline 56 & $03 / 20 / 79$ & 110 & 100 & 6.00 & 6.90 & 28.0 & -- & 30 \\
\hline 56 & $04 / 19 / 79$ & 110 & 100 & 5.00 & 6.90 & 28.0 & -- & 25 \\
\hline 56 & $05 / 21 / 79$ & 110 & 100 & 5.00 & 6.90 & 28.0 & .10 & 25 \\
\hline 56 & $06 / 13 / 79$ & 110 & 100 & 6.00 & 6.90 & 28.0 & .10 & 26 \\
\hline 56 & $08 / 16 / 79$ & 100 & 100 & 5.60 & 6.90 & 26.0 & - & 25 \\
\hline 56 & $10 / 23 / 79$ & 114 & 106 & 3.80 & 6.90 & 29.0 & -- & 26 \\
\hline 56 & $11 / 20 / 79$ & 110 & 104 & 5.10 & 6.90 & 28.0 & -- & 25 \\
\hline 56 & $12 / 17 / 79$ & 110 & 100 & 6.00 & 7.00 & 17.0 & -- & 25 \\
\hline 56 & $01 / 13 / 80$ & 118 & 100 & 5.00 & 6.90 & 31.0 & -- & 25 \\
\hline 56 & $02 / 14 / 80$ & 105 & 92 & 5.20 & 6.90 & 24.0 & -- & 30 \\
\hline 56 & $03 / 14 / 80$ & 110 & 100 & 5.00 & 6.90 & 28.0 & -- & 30 \\
\hline 56 & $04 / 15 / 80$ & 112 & 100 & 5.80 & 6.90 & 28.0 & -- & 25 \\
\hline 56 & $05 / 13 / 80$ & 108 & 104 & 5.00 & 6.90 & 28.0 & -- & 25 \\
\hline 56 & $06 / 16 / 80$ & 100 & 92 & 5.80 & 6.90 & 26.0 & -- & 25 \\
\hline 56 & $07 / 11 / 80$ & 114 & 102 & 4.80 & 6.90 & 31.0 & -- & 26 \\
\hline 56 & $08 / 12 / 80$ & 100 & 90 & 5.00 & 7.00 & 20.0 & -- & 25 \\
\hline 56 & $09 / 15 / 80$ & 100 & 100 & 7.50 & 6.90 & 25.0 & -- & 25 \\
\hline 56 & $10 / 21 / 80$ & 118 & 100 & 5.80 & 7.00 & 24.0 & -- & 32 \\
\hline 56 & $11 / 14 / 80$ & 118 & 110 & 3.00 & 6.90 & 30.0 & -- & 30 \\
\hline 56 & $12 / 16 / 80$ & 120 & 98 & 5.00 & 6.80 & 39.0 & -- & 30 \\
\hline
\end{tabular}


Table 8.--Water-chem istry data from production wells, 1971-83--Continued

[Samples collected and analyzed by personnel of the Coos Bay-North Bend Water Board]

\begin{tabular}{|c|c|c|c|c|c|c|c|c|}
\hline WELL & DATE & $\begin{array}{c}\text { ALKA- } \\
\text { LINITY } \\
\text { (MG/L } \\
\text { AS } \\
\text { CACO3) }\end{array}$ & $\begin{array}{l}\text { HARD- } \\
\text { NESS } \\
\text { (MG/L } \\
\text { AS } \\
\text { CACO3) }\end{array}$ & $\begin{array}{l}\text { IRON } \\
\text { DIS- } \\
\text { SOLVED } \\
\text { (MG/L) }\end{array}$ & $\begin{array}{c}\mathrm{PH} \\
(\mathrm{UN} \mid \mathrm{T})\end{array}$ & $\begin{array}{l}\text { CARBON } \\
\text { DIOXIDE } \\
(M G / L)\end{array}$ & $\begin{array}{l}\text { FLUO- } \\
\text { RIDE } \\
\text { DIS- } \\
\text { SOLVED } \\
\text { (MG/L) }\end{array}$ & $\begin{array}{l}\text { CHLO- } \\
\text { RIDE } \\
\text { DIS- } \\
\text { SOLVED } \\
(M G / L)\end{array}$ \\
\hline 56 & $01 / 15 / 81$ & 110 & -- & 4.80 & 6.90 & 29.0 & -- & 30 \\
\hline 56 & $02 / 16 / 81$ & 120 & 98 & 5.00 & 6.90 & 32.0 & -- & 30 \\
\hline 56 & $03 / 16 / 81$ & 118 & 100 & 5.00 & 6.90 & 30.0 & - & 30 \\
\hline 56 & $04 / 15 / 81$ & 110 & 100 & 5.00 & 6.90 & 28.0 & -- & 32 \\
\hline 56 & $05 / 15 / 81$ & 110 & 100 & 4.00 & 6.80 & 35.0 & -- & 30 \\
\hline 56 & $06 / 11 / 81$ & 120 & 104 & 5.00 & 6.90 & 30.0 & -- & 30 \\
\hline 56 & $07 / 14 / 81$ & 120 & 100 & 5.00 & 6.90 & 30.0 & -- & 35 \\
\hline 56 & $08 / 28 / 81$ & 110 & 100 & 5.00 & 6.90 & 29.0 & -- & 30 \\
\hline 56 & $09 / 17 / 81$ & 122 & 100 & 5.00 & 6.90 & 31.0 & -- & 29 \\
\hline 56 & $10 / 27 / 81$ & 120 & 106 & 6.00 & 6.90 & 30.0 & - & 23 \\
\hline 56 & $11 / 19 / 81$ & 120 & 104 & 5.20 & 7.00 & 24.0 & -- & 25 \\
\hline 56 & $12 / 15 / 81$ & 120 & 102 & 5.40 & 7.00 & 25.0 & - & 25 \\
\hline 56 & $01 / 22 / 82$ & 112 & 100 & 5.40 & 6.90 & 29.0 & -- & 35 \\
\hline 56 & $05 / 20 / 82$ & 118 & 118 & 4.80 & 6.80 & 38.0 & -- & 35 \\
\hline 56 & $06 / 15 / 82$ & 100 & 104 & 4.80 & 6.90 & 26.0 & -- & 30 \\
\hline 56 & $07 / 21 / 82$ & 108 & 92 & 4.60 & 6.90 & 28.0 & -- & 32 \\
\hline 56 & $08 / 11 / 82$ & 104 & 90 & 4.80 & 6.90 & 27.0 & -- & 35 \\
\hline 56 & $09 / 23 / 82$ & 108 & 100 & 4.80 & 6.90 & 28.0 & -- & 30 \\
\hline 56 & $10 / 20 / 82$ & 114 & 104 & 4.80 & 6.90 & 29.0 & -- & 29 \\
\hline 56 & $11 / 19 / 82$ & 116 & 106 & 4.80 & 6.90 & 28.0 & - & 24 \\
\hline 56 & $12 / 20 / 82$ & 100 & 90 & 4.70 & 6.90 & 26.0 & -- & 30 \\
\hline
\end{tabular}


Table 8.--Water-chemistry data from production wells, 1971-83--Continued

[Samples collected and analyzed by personnel of the Coos Bay-North Bend Water Board]

\begin{tabular}{|c|c|c|c|c|c|c|c|c|}
\hline WELL & DATE & $\begin{array}{c}\text { ALKA- } \\
\text { LINITY } \\
\text { (MG/L } \\
\text { AS } \\
\text { CACO3) }\end{array}$ & $\begin{array}{l}\text { HARD- } \\
\text { NESS } \\
(M G / L \\
\text { AS } \\
\text { CACO3) }\end{array}$ & $\begin{array}{l}\text { IRON } \\
\text { DIS- } \\
\text { SOLVED } \\
\text { (MG/L) }\end{array}$ & $\begin{array}{c}\mathrm{PH} \\
(\mathrm{UN} \mid \mathrm{T})\end{array}$ & $\begin{array}{l}\text { CARBON } \\
\text { DIOXIDE } \\
(M G / L)\end{array}$ & $\begin{array}{l}\text { FLUO- } \\
\text { RIDE } \\
\text { DIS- } \\
\text { SOLVED } \\
\text { (MG/L) }\end{array}$ & $\begin{array}{l}\text { CHLO- } \\
\text { RIDE } \\
\text { DIS- } \\
\text { SOLVED } \\
(M G / L)\end{array}$ \\
\hline 56 & $01 / 17 / 83$ & 110 & 96 & 4.80 & 6.90 & 28.0 & - & 27 \\
\hline 56 & $02 / 16 / 83$ & 112 & 100 & 4.40 & 6.90 & 28.0 & -- & 26 \\
\hline 56 & $03 / 18 / 83$ & 110 & 104 & 4.40 & 6.90 & 28.0 & - & 25 \\
\hline 56 & $04 / 26 / 83$ & 110 & 96 & 4.20 & 7.00 & 23.0 & - & 30 \\
\hline 56 & $05 / 16 / 83$ & 110 & 100 & 4.20 & 6.80 & 36.0 & -- & 30 \\
\hline 56 & $06 / 22 / 83$ & 92 & 96 & 4.65 & 6.90 & 23.0 & - & 24 \\
\hline 57 & $03 / 08 / 71$ & 132 & 112 & .02 & 7.40 & 11.0 & - & 31 \\
\hline 57 & $04 / 21 / 71$ & 126 & 118 & .02 & 7.50 & 8.0 & .03 & 29 \\
\hline 57 & $05 / 10 / 71$ & 132 & 114 & .80 & 7.50 & 8.0 & .02 & 30 \\
\hline 57 & $06 / 11 / 71$ & 138 & 110 & .30 & 7.40 & 13.0 & .04 & 50 \\
\hline 57 & $07 / 16 / 71$ & 130 & 160 & 1.10 & 7.30 & 14.0 & .04 & 23 \\
\hline 57 & $08 / 17 / 71$ & 138 & 112 & 1.03 & 7.20 & 18.0 & .04 & 25 \\
\hline 57 & $09 / 15 / 71$ & 140 & 142 & 1.10 & 7.50 & 9.0 & .02 & 28 \\
\hline 57 & $10 / 22 / 71$ & 142 & 136 & .04 & 7.60 & 7.0 & .02 & 29 \\
\hline 57 & $12 / 21 / 71$ & 138 & 128 & 1.00 & 7.60 & 7.0 & .02 & 28 \\
\hline 57 & $01 / 25 / 72$ & 132 & 100 & .04 & 7.30 & 21.0 & .04 & 26 \\
\hline 57 & $02 / 16 / 72$ & 94 & 104 & .02 & 7.40 & 7.5 & -- & 18 \\
\hline 57 & $03 / 24 / 72$ & 88 & 60 & .04 & 7.40 & 7.0 & .40 & 28 \\
\hline 57 & $04 / 28 / 72$ & 88 & 68 & .03 & 7.60 & 4.5 & -- & 26 \\
\hline 57 & $05 / 18 / 72$ & 138 & 112 & .02 & 7.40 & 12.0 & .03 & 26 \\
\hline 57 & $08 / 30 / 72$ & 140 & 140 & .20 & 7.20 & 26.0 & .04 & 30 \\
\hline 57 & $09 / 26 / 72$ & 138 & 110 & 1.30 & 7.10 & 23.0 & .01 & 28 \\
\hline 57 & $11 / 28 / 72$ & 132 & 114 & .04 & 7.40 & 12.0 & .01 & 27 \\
\hline
\end{tabular}


Table 8.- -Water-chemistry data from production wells, 1971-83--Continued

[Samples collected and analyzed by personnel of the Coos Bay-North Bend Water Board]

\begin{tabular}{|c|c|c|c|c|c|c|c|c|}
\hline WELL & DATE & $\begin{array}{c}\text { ALKA- } \\
\text { LINITY } \\
\text { (MG/L } \\
\text { AS } \\
\text { CACO3) }\end{array}$ & $\begin{array}{l}\text { HARD- } \\
\text { NESS } \\
\text { (MG/L } \\
\text { AS } \\
\text { CACO3) }\end{array}$ & $\begin{array}{l}\text { IRON } \\
\text { DIS- } \\
\text { SOLVED } \\
\text { (MG/L) }\end{array}$ & $\begin{array}{c}\text { PH } \\
(U N \mid T)\end{array}$ & $\begin{array}{l}\text { CARBON } \\
\text { DIOXIDE } \\
(M G / L)\end{array}$ & $\begin{array}{l}\text { FLUO- } \\
\text { RIDE } \\
\text { DIS- } \\
\text { SOLVED } \\
(M G / L)\end{array}$ & $\begin{array}{l}\text { CHLO- } \\
\text { RIDE } \\
\text { DIS- } \\
\text { SOLVED } \\
(M G / L)\end{array}$ \\
\hline 57 & $12 / 26 / 72$ & 124 & 126 & .02 & 7.60 & 8.0 & .01 & 24 \\
\hline 57 & $02 / 27 / 73$ & 136 & 128 & .06 & 7.40 & 8.0 & .04 & 24 \\
\hline 57 & $03 / 30 / 73$ & 136 & 114 & .04 & 7.60 & 7.0 & .04 & 24 \\
\hline 57 & $04 / 27 / 73$ & 130 & 98 & .06 & 7.20 & 16.5 & .04 & 30 \\
\hline 57 & $05 / 24 / 73$ & 132 & 112 & .04 & 7.20 & 17.0 & .04 & 30 \\
\hline 57 & $06 / 28 / 73$ & 128 & 108 & .04 & 7.40 & 11.0 & .04 & 29 \\
\hline 57 & $08 / 30 / 73$ & 140 & 116 & .04 & 7.30 & 14.0 & .02 & 32 \\
\hline 57 & $10 / 09 / 73$ & 132 & 144 & .04 & 7.10 & 21.0 & .04 & 30 \\
\hline 57 & $11 / 06 / 73$ & 120 & 156 & .04 & 7.10 & 19.0 & .04 & 31 \\
\hline 57 & $12 / 20 / 73$ & 122 & 118 & .02 & 7.30 & 13.0 & .04 & 24 \\
\hline 57 & $01 / 29 / 74$ & 122 & 108 & .02 & 7.30 & 14.0 & .04 & 24 \\
\hline 57 & $02 / 26 / 74$ & 130 & 122 & .04 & 7.10 & 21.0 & .04 & 28 \\
\hline 57 & $04 / 30 / 74$ & 132 & 120 & .04 & 7.10 & 21.0 & .04 & 22 \\
\hline 57 & $07 / 24 / 74$ & 108 & 110 & .80 & 7.30 & 12.0 & .40 & 22 \\
\hline 57 & $08 / 28 / 74$ & 106 & 108 & .07 & 7.10 & 17.0 & .30 & 30 \\
\hline 57 & $09 / 27 / 74$ & 104 & 110 & .08 & 7.10 & 16.0 & .40 & 30 \\
\hline 57 & $11 / 21 / 74$ & 102 & 102 & .08 & 7.00 & 22.0 & .20 & 29 \\
\hline 57 & $12 / 31 / 74$ & 120 & 120 & .04 & 7.20 & 16.0 & .40 & 28 \\
\hline 57 & $02 / 28 / 75$ & 110 & 106 & .04 & 6.90 & 28.0 & .20 & 28 \\
\hline 57 & $03 / 26 / 75$ & 122 & 112 & .04 & 7.20 & 16.0 & .40 & 26 \\
\hline 57 & $04 / 23 / 75$ & 118 & 102 & .04 & 7.20 & 15.0 & .40 & 26 \\
\hline 57 & $05 / 27 / 75$ & 128 & 102 & .02 & 7.20 & 22.0 & .30 & 28 \\
\hline
\end{tabular}


Table 8.--Water-chemistry data from production wells, 1971-83--Continued

[Samples collected and analyzed by personnel of the Coos Bay-North Bend Water Board]

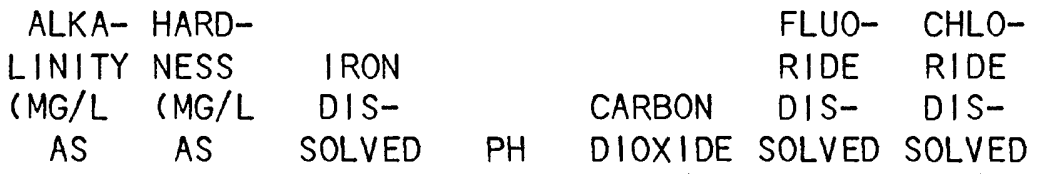
WELL DATE CACO3) CACO3) (MG/L) (UNIT) (MG/L) (MG/L) (MG/L)

\begin{tabular}{lllllllll}
\hline 57 & $07 / 29 / 75$ & 118 & 100 & .02 & 7.10 & 19.0 & .40 & 30 \\
57 & $09 / 25 / 75$ & 114 & 102 & .02 & 7.10 & 18.5 & .40 & 30 \\
57 & $11 / 25 / 75$ & 110 & 104 & .02 & 7.10 & 18.0 & .40 & 30 \\
57 & $12 / 30 / 75$ & 120 & 104 & 1.80 & 7.20 & 16.0 & .40 & 30 \\
57 & $02 / 19 / 76$ & 120 & 104 & .90 & 7.20 & 15.0 & .20 & 27 \\
57 & $03 / 30 / 76$ & 110 & 108 & 1.24 & 7.30 & 12.0 & .30 & 28 \\
57 & $04 / 23 / 76$ & 120 & 108 & 1.40 & 7.30 & 14.0 & .25 & 16 \\
57 & $06 / 17 / 76$ & 118 & 100 & 1.20 & 7.20 & 15.0 & .18 & 30 \\
57 & $07 / 19 / 76$ & 120 & 118 & 1.60 & 7.20 & 15.0 & .20 & 30 \\
57 & $08 / 20 / 76$ & 120 & 120 & 1.20 & 7.30 & 7.0 & .20 & 28 \\
57 & $09 / 21 / 76$ & 122 & 126 & 1.20 & 7.20 & 15.5 & .15 & 25 \\
57 & $10 / 21 / 76$ & 120 & 114 & 1.40 & 7.20 & 15.0 & .15 & 27 \\
57 & $11 / 18 / 76$ & 118 & 120 & 1.00 & 7.10 & 18.0 & .18 & 25 \\
& & & & & & & & \\
57 & $02 / 15 / 77$ & 104 & 110 & .48 & 7.20 & 8.0 & .18 & 30 \\
57 & $03 / 15 / 77$ & 120 & 120 & 1.20 & 7.20 & 12.0 & .20 & 30 \\
57 & $04 / 15 / 77$ & 118 & 110 & 1.20 & 7.20 & 12.0 & .20 & 30 \\
57 & $05 / 16 / 77$ & 112 & 110 & 2.20 & 7.30 & 11.0 & .20 & 25 \\
57 & $06 / 21 / 77$ & 118 & 110 & 2.20 & 7.20 & 15.0 & .20 & 10 \\
57 & $07 / 21 / 77$ & 120 & 104 & 2.70 & 7.10 & 20.0 & -- & 25 \\
57 & $08 / 19 / 77$ & 118 & 102 & 2.50 & 7.10 & 18.0 & -- & 25 \\
57 & $09 / 15 / 77$ & 118 & 104 & 3.00 & 7.10 & 18.0 & -- & 30 \\
57 & $10 / 20 / 77$ & 118 & 112 & 2.40 & 7.10 & 19.0 & -- & 22 \\
57 & $12 / 20 / 77$ & 120 & 102 & 2.00 & 7.00 & 25.0 & -- & 28 \\
& & & & & & & & \\
\hline & & & & & & & & \\
\hline
\end{tabular}


Table 8.- - Water-chemistry data from production

[Samples collected and analyzed by personnel of the Coos Bay-North Bend Water Board]

\begin{tabular}{|c|c|c|c|c|c|c|c|c|}
\hline WELL & DATE & $\begin{array}{l}\text { ALKA- } \\
\text { LINITY } \\
\text { (MG/L } \\
\text { AS } \\
\text { CACO3) }\end{array}$ & $\begin{array}{l}\text { HARD- } \\
\text { NESS } \\
\text { (MG/L } \\
\text { AS } \\
\text { CACO3) }\end{array}$ & $\begin{array}{l}\text { IRON } \\
\text { DIS- } \\
\text { SOLVED } \\
\text { (MG/L) }\end{array}$ & $\begin{array}{c}\mathrm{PH} \\
(\mathrm{UN} \mid \mathrm{T})\end{array}$ & $\begin{array}{l}\text { CARBON } \\
\text { DIOXIDE } \\
(M G / L)\end{array}$ & $\begin{array}{l}\text { FLUO- } \\
\text { RIDE } \\
\text { DIS- } \\
\text { SOLVED } \\
\text { (MG/L) }\end{array}$ & $\begin{array}{l}\text { CHLO- } \\
\text { RIDE } \\
\text { DIS- } \\
\text { SOLVED } \\
\text { (MG/L) }\end{array}$ \\
\hline 57 & $01 / 16 / 78$ & 120 & 102 & 2.50 & 7.00 & 25.0 & -- & 25 \\
\hline 57 & $02 / 14 / 78$ & 120 & 104 & 2.50 & 7.10 & 18.0 & -- & 26 \\
\hline 57 & $03 / 14 / 78$ & 120 & 100 & 2.20 & 7.20 & -- & -- & 25 \\
\hline 57 & $04 / 12 / 78$ & 120 & 118 & 2.50 & 7.10 & 25.0 & -- & 30 \\
\hline 57 & $05 / 11 / 78$ & 120 & 100 & 2.00 & 7.20 & 15.0 & -- & 30 \\
\hline 57 & $06 / 12 / 78$ & 120 & 118 & 2.60 & 7.30 & 12.0 & -- & 25 \\
\hline 57 & $07 / 17 / 78$ & 120 & 104 & 2.80 & 7.20 & 15.0 & -- & 25 \\
\hline 57 & $08 / 11 / 78$ & 120 & 120 & 2.30 & 7.10 & 18.0 & -- & 25 \\
\hline 57 & $09 / 15 / 78$ & 120 & 108 & 2.50 & 7.10 & 19.0 & -- & 30 \\
\hline 57 & $10 / 25 / 78$ & 120 & 112 & 1.80 & 7.00 & 25.0 & -- & 25 \\
\hline 57 & $11 / 15 / 78$ & 120 & 116 & 2.50 & 7.10 & 19.0 & -- & 28 \\
\hline 57 & $12 / 13 / 78$ & 110 & 110 & 2.40 & 7.10 & 17.0 & -- & 30 \\
\hline 57 & $01 / 22 / 79$ & 120 & 102 & 2.20 & 7.20 & 15.0 & - & 28 \\
\hline 57 & $02 / 22 / 79$ & 115 & 110 & 2.50 & 7.20 & 14.0 & -- & 30 \\
\hline 57 & $03 / 20 / 79$ & 120 & 110 & 1.50 & 7.30 & 12.0 & -- & 28 \\
\hline 57 & $04 / 19 / 79$ & 120 & 104 & 1.60 & 7.20 & 15.0 & -- & 28 \\
\hline $\begin{array}{l}57 \\
57\end{array}$ & $\begin{array}{l}05 / 21 / 79 \\
06 / 13 / 79\end{array}$ & $\begin{array}{l}118 \\
120\end{array}$ & $\begin{array}{l}120 \\
104\end{array}$ & 1.80 & 7.30 & 20.0 & .10 & 25 \\
\hline $\begin{array}{l}51 \\
57\end{array}$ & $08 / 16 / 79$ & $\begin{array}{l}120 \\
118\end{array}$ & $\begin{array}{l}104 \\
106\end{array}$ & $\begin{array}{l}1.60 \\
2.20\end{array}$ & $\begin{array}{l}7.30 \\
7.30\end{array}$ & $\begin{array}{l}12.0 \\
14.0\end{array}$ & $\begin{array}{l}.10 \\
--\end{array}$ & $\begin{array}{l}28 \\
30\end{array}$ \\
\hline 57 & $10 / 23 / 79$ & 120 & 112 & 1.55 & 7.20 & 15.0 & -- & 26 \\
\hline 57 & $11 / 20 / 79$ & 120 & 100 & 1.60 & 7.20 & 16.0 & -- & 25 \\
\hline 57 & $12 / 17 / 79$ & 118 & 105 & 1.70 & 7.30 & 13.0 & - & 30 \\
\hline
\end{tabular}


Table 8.--Water-chemistry data from production wells, 1971-83--Continued

[Samples collected and analyzed by personnel of the Coos Bay-North Bend Water Board]

\begin{tabular}{|c|c|c|c|c|c|c|c|c|}
\hline WELL & DATE & $\begin{array}{l}\text { ALKA- } \\
\text { LINITY } \\
\text { (MG/L } \\
\text { AS } \\
\text { CACO3) }\end{array}$ & $\begin{array}{l}\text { HARD- } \\
\text { NESS } \\
\text { (MG/L } \\
\text { AS } \\
\text { CACO3) }\end{array}$ & $\begin{array}{l}\text { IRON } \\
\text { DIS- } \\
\text { SOLVED } \\
\text { (MG/L) }\end{array}$ & $\begin{array}{c}\text { PH } \\
(\text { UNIT) }\end{array}$ & $\begin{array}{l}\text { CARBON } \\
\text { DIOXIDE } \\
(M G / L)\end{array}$ & $\begin{array}{l}\text { FLUO- } \\
\text { RIDE } \\
\text { DIS- } \\
\text { SOLVED } \\
(M G / L)\end{array}$ & $\begin{array}{l}\text { CHLO- } \\
\text { RIDE } \\
\text { DIS- } \\
\text { SOLVED } \\
\text { (MG/L) }\end{array}$ \\
\hline 57 & $01 / 13 / 80$ & 118 & 100 & 1.60 & 7.20 & 16.0 & -- & 25 \\
\hline 57 & $02 / 14 / 80$ & 110 & 100 & 1.60 & 7.30 & 11.0 & -- & 30 \\
\hline 57 & $03 / 14 / 80$ & 118 & 110 & 1.70 & 7.20 & 15.0 & - & 30 \\
\hline 57 & $04 / 15 / 80$ & 118 & 110 & 3.40 & 7.20 & 15.0 & -- & 30 \\
\hline 57 & $05 / 13 / 80$ & 116 & 120 & 3.20 & 7.20 & 15.0 & -- & 30 \\
\hline 57 & $06 / 16 / 80$ & 120 & 104 & 1.80 & 7.20 & 15.0 & -- & 25 \\
\hline 57 & $07 / 11 / 80$ & 118 & 104 & 2.60 & 7.20 & 14.0 & -- & 28 \\
\hline 57 & $08 / 12 / 80$ & 118 & 108 & 1.60 & 7.30 & 13.0 & -- & 25 \\
\hline 57 & $09 / 15 / 80$ & 118 & 100 & 1.90 & 7.20 & 15.0 & -- & 30 \\
\hline 57 & $10 / 21 / 80$ & 120 & 100 & 1.60 & 7.30 & 12.0 & -- & 32 \\
\hline 57 & $11 / 14 / 80$ & 120 & 100 & 2.00 & 7.20 & 15.0 & -- & 30 \\
\hline 57 & $12 / 16 / 80$ & 120 & 100 & 4.20 & 7.10 & 19.0 & -- & 30 \\
\hline 57 & $01 / 15 / 81$ & 118 & -- & 1.60 & 7.30 & 12.0 & -- & 32 \\
\hline 57 & $02 / 16 / 81$ & 116 & 104 & 4.00 & 7.20 & 14.0 & -- & 30 \\
\hline 57 & $03 / 16 / 81$ & 115 & 110 & 1.80 & 7.10 & 18.0 & -- & 35 \\
\hline 57 & $04 / 15 / 81$ & 118 & 104 & 1.50 & 7.10 & 18.0 & -- & 32 \\
\hline 57 & $05 / 15 / 81$ & 120 & 118 & 1.20 & 7.10 & 19.0 & -- & 32 \\
\hline 57 & $06 / 11 / 81$ & 126 & 108 & 1.85 & 7.20 & 16.0 & -- & 31 \\
\hline 57 & $07 / 14 / 81$ & 110 & 120 & .70 & 7.10 & 18.0 & -- & 35 \\
\hline 57 & $08 / 28 / 81$ & 120 & 100 & 1.65 & 7.20 & 15.0 & -- & 30 \\
\hline 57 & $09 / 17 / 81$ & 122 & 114 & 2.35 & 7.20 & 17.0 & -- & 29 \\
\hline 57 & $10 / 27 / 81$ & 118 & 116 & 1.80 & 7.20 & 15.0 & -- & 24 \\
\hline 57 & $11 / 19 / 81$ & 110 & 100 & 3.20 & 7.20 & 14.0 & -- & 30 \\
\hline 57 & $12 / 15 / 81$ & 110 & 104 & 1.80 & 7.30 & 11.0 & -- & 30 \\
\hline
\end{tabular}


Table 8.-- Water-chemistry data from production

[Samples collected and analyzed by personnel of the Coos Bay-North Bend Water Board]

\begin{tabular}{|c|c|c|c|c|c|c|c|c|}
\hline WELL & DATE & $\begin{array}{c}\text { ALKA- } \\
\text { LINITY } \\
\text { (MG/L } \\
\text { AS } \\
\text { CACO3) }\end{array}$ & $\begin{array}{l}\text { HARD- } \\
\text { NESS } \\
\text { (MG/L } \\
\text { AS } \\
\text { CACO3) }\end{array}$ & $\begin{array}{l}\text { IRON } \\
\text { DIS- } \\
\text { SOLVED } \\
\text { (MG/L) }\end{array}$ & $\begin{array}{c}\mathrm{PH} \\
(\mathrm{UNIT})\end{array}$ & $\begin{array}{l}\text { CARBON } \\
\text { DIOXIDE } \\
(M G / L)\end{array}$ & $\begin{array}{l}\text { FLUO- } \\
\text { RIDE } \\
\text { DIS- } \\
\text { SOLVED } \\
\text { (MG/L) }\end{array}$ & $\begin{array}{l}\text { CHLO- } \\
\text { RIDE } \\
\text { DIS- } \\
\text { SOLVED } \\
(M G / L)\end{array}$ \\
\hline 57 & $01 / 22 / 82$ & 118 & 100 & 2.00 & 7.20 & 15.0 & - & 35 \\
\hline 57 & $05 / 20 / 82$ & 118 & 118 & 1.90 & 7.20 & 15.0 & -- & 35 \\
\hline 57 & $06 / 15 / 82$ & 118 & 100 & 1.80 & 7.20 & 15.0 & -- & 30 \\
\hline 57 & $07 / 21 / 82$ & 118 & 104 & 1.80 & 7.20 & 15.0 & -- & 35 \\
\hline 57 & $08 / 11 / 82$ & 118 & 100 & 1.80 & 7.20 & 15.0 & -- & 35 \\
\hline 57 & $09 / 23 / 82$ & 118 & 108 & 1.80 & 7.30 & 12.0 & -- & 32 \\
\hline 57 & $10 / 20 / 82$ & 122 & 102 & 1.80 & 7.20 & 16.0 & -- & 29 \\
\hline 57 & $11 / 19 / 82$ & 120 & 118 & 1.80 & 7.20 & 19.0 & -- & 25 \\
\hline 57 & $12 / 20 / 82$ & 116 & 104 & 7.00 & 7.20 & 15.0 & -- & 36 \\
\hline 57 & $01 / 17 / 83$ & 116 & 102 & 2.00 & 7.20 & 14.0 & -- & 28 \\
\hline 57 & $02 / 16 / 83$ & 118 & 100 & 2.00 & 7.20 & 15.0 & -- & 28 \\
\hline 57 & $03 / 18 / 83$ & 118 & 110 & 1.80 & 7.10 & 18.0 & -- & 28 \\
\hline 57 & $04 / 26 / 83$ & 124 & 108 & 2.20 & 7.20 & 16.0 & -- & 32 \\
\hline 57 & $05 / 16 / 83$ & 116 & 112 & 1.80 & 7.20 & 14.0 & -- & 32 \\
\hline 57 & $06 / 22 / 83$ & 94 & 108 & 2.60 & 7.10 & 15.0 & -- & 27 \\
\hline 58 & $03 / 08 / 71$ & 120 & 104 & 2.40 & 7.00 & 24.0 & -- & 31 \\
\hline 58 & $04 / 21 / 71$ & 118 & 104 & 2.00 & 7.10 & 19.0 & .05 & 28 \\
\hline 58 & $05 / 10 / 71$ & 120 & 112 & 1.80 & 6.90 & 31.0 & .04 & 30 \\
\hline 58 & $06 / 11 / 71$ & 122 & 100 & 2.80 & 7.00 & 25.0 & .01 & 52 \\
\hline 58 & $07 / 16 / 71$ & 120 & 94 & 1.60 & 6.90 & 32.0 & .03 & 27 \\
\hline 58 & $08 / 17 / 71$ & 118 & 104 & 1.20 & 6.60 & 63.0 & .04 & 27 \\
\hline 58 & $09 / 15 / 71$ & 122 & 122 & 4.80 & 7.00 & 26.0 & .03 & 30 \\
\hline 58 & $10 / 22 / 71$ & 126 & 120 & 4.40 & 7.00 & 16.0 & .03 & 31 \\
\hline 58 & $12 / 21 / 71$ & 120 & 118 & 3.60 & 7.10 & 19.0 & .02 & 30 \\
\hline
\end{tabular}


Table 8.--Water-chem istry data from production wells, 1971-83--Continued

[Samples collected and analyzed by personnel of the Coos Bay-North Bend Water Board]

\begin{tabular}{|c|c|c|c|c|c|c|c|c|}
\hline WELL & DATE & $\begin{array}{c}\text { ALKA- } \\
\text { LINITY } \\
\text { (MG/L } \\
\text { AS } \\
\text { CACO3) }\end{array}$ & $\begin{array}{l}\text { HARD- } \\
\text { NESS } \\
\text { (MG/L } \\
\text { AS } \\
\text { CACO3) }\end{array}$ & $\begin{array}{l}\text { IRON } \\
\text { DIS- } \\
\text { SOLVED } \\
\text { (MG/L) }\end{array}$ & $\begin{array}{c}\text { PH } \\
(U N \mid T)\end{array}$ & $\begin{array}{l}\text { CARBON } \\
\text { DIOXIDE } \\
(M G / L)\end{array}$ & $\begin{array}{l}\text { FLUO- } \\
\text { RIDE } \\
\text { DIS- } \\
\text { SOLVED } \\
\text { (MG/L) }\end{array}$ & $\begin{array}{l}\text { CHLO- } \\
\text { RIDE } \\
\text { DIS- } \\
\text { SOLVED } \\
(M G / L)\end{array}$ \\
\hline 58 & $01 / 25 / 72$ & 115 & 100 & 2.80 & 7.00 & 18.0 & .02 & 25 \\
\hline 58 & $02 / 16 / 72$ & 124 & 94 & 2.40 & 7.00 & 25.0 & - & 28 \\
\hline 58 & $03 / 24 / 72$ & 118 & 92 & 3.40 & 7.00 & 24.0 & .22 & 36 \\
\hline 58 & $04 / 28 / 72$ & 122 & 104 & 3.00 & 7.00 & 24.0 & -- & 30 \\
\hline 58 & $05 / 18 / 72$ & 118 & 92 & 2.80 & 7.10 & 19.5 & .01 & 27 \\
\hline 58 & $08 / 30 / 72$ & 106 & 104 & 3.00 & 7.10 & 17.5 & .01 & 30 \\
\hline 58 & $09 / 26 / 72$ & 118 & 102 & 4.20 & 6.80 & 60.0 & .01 & 28 \\
\hline 58 & $11 / 28 / 72$ & 118 & 116 & 3.00 & 7.00 & 26.0 & .01 & 29 \\
\hline 58 & $12 / 26 / 72$ & 112 & 112 & 2.40 & 7.00 & 33.0 & .01 & 28 \\
\hline 58 & $02 / 27 / 73$ & 120 & 114 & 2.80 & 7.00 & 24.0 & .02 & 30 \\
\hline 58 & $03 / 30 / 73$ & 122 & 108 & 3.00 & 7.10 & 19.0 & .02 & 30 \\
\hline 58 & $04 / 27 / 73$ & 112 & 88 & 6.20 & 6.90 & 29.0 & .01 & 34 \\
\hline 58 & $05 / 24 / 73$ & 120 & 96 & 3.00 & 6.80 & 38.0 & .01 & 31 \\
\hline 58 & $06 / 28 / 73$ & 110 & 98 & 2.80 & 7.10 & 18.0 & .01 & 30 \\
\hline 58 & $08 / 30 / 73$ & 122 & 94 & 5.00 & 6.90 & 32.0 & .01 & 32 \\
\hline 58 & $10 / 09 / 73$ & 118 & 102 & 5.60 & 6.90 & 39.0 & .01 & 31 \\
\hline 58 & $11 / 06 / 73$ & 112 & 108 & 5.20 & 7.00 & 23.0 & .01 & 32 \\
\hline 58 & $12 / 20 / 73$ & 110 & 100 & 3.20 & 6.90 & 28.0 & .01 & 28 \\
\hline 58 & $01 / 29 / 74$ & 108 & 92 & 4.00 & 6.80 & 37.0 & .01 & 30 \\
\hline 58 & $02 / 26 / 74$ & 120 & 102 & 3.20 & 6.90 & 31.0 & .01 & 22 \\
\hline 58 & $04 / 30 / 74$ & 124 & 108 & 3.60 & 6.80 & 40.0 & .02 & 24 \\
\hline 58 & $07 / 24 / 74$ & 104 & 90 & 4.00 & 6.90 & 28.0 & .10 & 20 \\
\hline 58 & $08 / 28 / 74$ & 100 & 94 & 3.60 & 6.90 & 25.0 & .10 & 28 \\
\hline
\end{tabular}


Table 8.--Water-chemistry data from production

[Samples collected and analyzed by personnel of the Coos Bay-North Bend Water Board]

$\begin{array}{ccccccc}\text { ALKA- HARD- } & & & & \text { FLUO- } & \text { CHLO- } \\ \text { LINITY } & \text { NESS } & \text { IRON } & & \text { RIDE } & \text { RIDE } \\ (M G / L & (M G / L & \text { DIS- } & & \text { CARBON } & \text { DIS- } & \text { DIS- } \\ \text { AS } & \text { AS } & \text { SOLVED } & \text { PH } & \text { DIOXIDE } & \text { SOLVED } & \text { SOLVED } \\ \text { CACO3) } & \text { CACO3) } & (M G / L) & (U N I T) & (M G / L) & (M G / L) & (M G / L)\end{array}$

\begin{tabular}{|c|c|c|c|c|c|c|c|c|}
\hline 58 & $09 / 27 / 74$ & 104 & 108 & 4.20 & 6.80 & 34.0 & .20 & 30 \\
\hline 58 & $11 / 21 / 74$ & 104 & 104 & 4.20 & 6.90 & 27.0 & .10 & 29 \\
\hline 58 & $12 / 31 / 74$ & 112 & 98 & 3.50 & 6.80 & 36.0 & .20 & 34 \\
\hline 58 & $02 / 28 / 75$ & 102 & 104 & 3.80 & 7.00 & 20.0 & .10 & 28 \\
\hline 58 & $03 / 26 / 75$ & 112 & 96 & 4.80 & 6.80 & 34.0 & .10 & 28 \\
\hline 58 & $04 / 23 / 75$ & 114 & 98 & 4.40 & 6.80 & 35.0 & .10 & 30 \\
\hline 58 & $05 / 27 / 75$ & 116 & 88 & 4.00 & 6.80 & 38.0 & .40 & 24 \\
\hline 58 & $07 / 29 / 75$ & 110 & 98 & 3.60 & 7.00 & 22.0 & .26 & 31 \\
\hline 58 & $09 / 25 / 75$ & 106 & 96 & 3.40 & 7.10 & 17.0 & .20 & 30 \\
\hline 58 & $11 / 25 / 75$ & 100 & 100 & 3.20 & 7.10 & 16.0 & .10 & 28 \\
\hline 58 & $12 / 30 / 75$ & 126 & 110 & 5.00 & 6.90 & 33.0 & .10 & 30 \\
\hline 58 & $02 / 19 / 76$ & 110 & 100 & 4.00 & 6.90 & 17.0 & .25 & 27 \\
\hline 58 & $03 / 30 / 76$ & 120 & 108 & 5.00 & 6.90 & 31.0 & .30 & 28 \\
\hline 58 & $04 / 23 / 76$ & 104 & 90 & 4.50 & 7.00 & 22.0 & .20 & 30 \\
\hline 58 & $06 / 17 / 76$ & 108 & 102 & 5.00 & 6.90 & 28.0 & .25 & 30 \\
\hline 58 & $07 / 19 / 76$ & 90 & 100 & 4.20 & 6.80 & 28.0 & .25 & 30 \\
\hline 58 & $08 / 20 / 76$ & 104 & 96 & 4.50 & 6.70 & 42.0 & .25 & 30 \\
\hline 58 & $09 / 21 / 76$ & 112 & 110 & 4.00 & 6.90 & 28.0 & .25 & 24 \\
\hline 58 & $10 / 21 / 76$ & 108 & 118 & 4.60 & 6.90 & 28.0 & .25 & 24 \\
\hline 58 & $11 / 18 / 76$ & 115 & 108 & 4.00 & 6.80 & 38.0 & .25 & 24 \\
\hline
\end{tabular}


Table 8.--Water-chem istry data from production wells, 1971-83--Continued

[Samples collected and analyzed by personnel of the Coos Bay-North Bend Water Board]

\begin{tabular}{|c|c|c|c|c|c|c|c|c|}
\hline WELL & DATE & $\begin{array}{c}\text { ALKA- } \\
\text { LINITY } \\
\text { (MG/L } \\
\text { AS } \\
\text { CACO3) }\end{array}$ & $\begin{array}{l}\text { HARD- } \\
\text { NESS } \\
\text { (MG/L } \\
\text { AS } \\
\text { CACO3) }\end{array}$ & $\begin{array}{l}\text { IRON } \\
\text { DIS- } \\
\text { SOLVED } \\
\text { (MG/L) }\end{array}$ & $\begin{array}{c}P H \\
(U N \mid T)\end{array}$ & $\begin{array}{l}\text { CARBON } \\
\text { DIOXIDE } \\
(M G / L)\end{array}$ & $\begin{array}{l}\text { FLUO- } \\
\text { RIDE } \\
\text { DIS- } \\
\text { SOLVED } \\
\text { (MG/L) }\end{array}$ & $\begin{array}{l}\text { CHLO- } \\
\text { RIDE } \\
\text { DIS- } \\
\text { SOLVED } \\
(M G / L)\end{array}$ \\
\hline 58 & $02 / 15 / 77$ & 100 & 90 & 4.20 & 7.00 & 20.0 & .18 & 30 \\
\hline 58 & $03 / 15 / 77$ & 110 & 90 & 3.80 & 7.00 & 22.0 & .20 & 30 \\
\hline 58 & $04 / 15 / 77$ & 110 & 94 & 3.80 & 6.90 & 21.0 & .20 & 32 \\
\hline 58 & $05 / 16 / 77$ & 104 & 100 & 5.50 & 7.10 & 14.0 & .20 & 25 \\
\hline 58 & $06 / 21 / 77$ & 110 & 90 & 4.60 & 7.10 & 17.0 & .20 & 25 \\
\hline 58 & $07 / 21 / 77$ & 104 & 90 & 5.00 & 6.90 & 28.0 & - & 25 \\
\hline 58 & $08 / 19 / 77$ & 104 & 96 & 5.40 & 7.00 & 28.0 & -- & 25 \\
\hline 58 & $09 / 15 / 77$ & 100 & 94 & 5.30 & 6.90 & 26.0 & -- & 30 \\
\hline 58 & $10 / 20 / 77$ & 112 & 100 & 5.00 & 6.80 & 36.0 & -- & 22 \\
\hline 58 & $12 / 20 / 77$ & 105 & 90 & 4.20 & 6.80 & 34.0 & -- & 25 \\
\hline 58 & $01 / 16 / 78$ & 108 & 96 & 4.60 & 6.90 & 28.0 & -- & 25 \\
\hline 58 & $02 / 14 / 78$ & 104 & 96 & 4.20 & 6.80 & 34.0 & -- & 25 \\
\hline 58 & $03 / 14 / 78$ & 100 & 96 & 4.20 & 7.10 & - & -- & 25 \\
\hline 58 & $04 / 12 / 78$ & 108 & 94 & 4.30 & 6.90 & 28.0 & -- & 28 \\
\hline 58 & $05 / 11 / 78$ & 104 & 90 & 5.80 & 7.00 & 22.0 & -- & 25 \\
\hline 58 & $06 / 12 / 78$ & 110 & 98 & 5.40 & 7.00 & 22.0 & -- & 23 \\
\hline 58 & $07 / 17 / 78$ & 100 & 90 & 4.00 & 6.90 & 27.0 & - & 26 \\
\hline 58 & $08 / 11 / 78$ & 104 & 100 & 4.00 & 6.80 & 32.0 & -- & 25 \\
\hline 58 & $09 / 15 / 78$ & 100 & 85 & 4.20 & 6.80 & 32.0 & -- & 28 \\
\hline 58 & $10 / 25 / 78$ & 108 & 106 & 5.00 & 6.80 & 34.0 & -- & 24 \\
\hline 58 & $11 / 15 / 78$ & 108 & 104 & 4.00 & 7.00 & 22.0 & -- & 25 \\
\hline 58 & $12 / 13 / 78$ & 100 & 100 & 4.20 & 6.80 & 31.0 & -- & 30 \\
\hline
\end{tabular}


Table 8.-- Water-chemistry data from production

[Samples collected and analyzed by personnel of the Coos Bay-North Bend Water Board]

\begin{tabular}{|c|c|c|c|c|c|c|c|c|}
\hline WELL & DATE & $\begin{array}{c}\text { ALKA- } \\
\text { LINITY } \\
\text { (MG/L } \\
\text { AS } \\
\text { CACO3) }\end{array}$ & $\begin{array}{l}\text { HARD- } \\
\text { NESS } \\
\text { (MG/L } \\
\text { AS } \\
\text { CACO3) }\end{array}$ & $\begin{array}{l}\text { IRON } \\
\text { DIS- } \\
\text { SOLVED } \\
\text { (MG/L) }\end{array}$ & $\begin{array}{c}P H \\
(U N \mid T)\end{array}$ & $\begin{array}{l}\text { CARBON } \\
D I O X I D E \\
(M G / L)\end{array}$ & $\begin{array}{l}\text { FLUO- } \\
\text { RIDE } \\
\text { DIS- } \\
\text { SOLVED } \\
\text { (MG/L) }\end{array}$ & $\begin{array}{l}\text { CHLO- } \\
\text { RIDE } \\
\text { DIS- } \\
\text { SOLVED } \\
(M G / L)\end{array}$ \\
\hline 58 & $01 / 22 / 79$ & 100 & 90 & 4.60 & 7.00 & 20.0 & -- & 25 \\
\hline 58 & $02 / 22 / 79$ & 100 & 100 & 4.00 & 7.00 & 20.0 & - & 25 \\
\hline 58 & $03 / 20 / 79$ & 100 & 100 & 5.00 & 7.00 & 20.0 & -- & 30 \\
\hline 58 & $04 / 19 / 79$ & 100 & 100 & 5.00 & 7.10 & 16.0 & -- & 28 \\
\hline 58 & $05 / 21 / 79$ & 104 & 90 & 5.00 & 7.10 & -- & .10 & 25 \\
\hline 58 & $06 / 13 / 79$ & 104 & 90 & 5.00 & 6.90 & 26.0 & .10 & 25 \\
\hline 58 & $08 / 16 / 79$ & 104 & 90 & 4.40 & 7.00 & 22.0 & -- & 25 \\
\hline 58 & $10 / 23 / 79$ & 110 & 90 & 3.60 & 6.90 & 28.0 & - & 25 \\
\hline 58 & $11 / 20 / 79$ & 100 & 100 & 5.20 & 6.90 & 26.0 & -- & 25 \\
\hline 58 & $12 / 17 / 79$ & 110 & 90 & 5.00 & 7.10 & 18.0 & - & 32 \\
\hline 58 & $01 / 13 / 80$ & 104 & 84 & 4.80 & 7.00 & 22.0 & -- & 25 \\
\hline 58 & $02 / 14 / 80$ & 100 & 100 & 4.60 & 7.00 & 20.0 & - & 30 \\
\hline 58 & $03 / 14 / 80$ & 100 & 108 & 4.40 & 7.00 & 20.0 & -- & 30 \\
\hline 58 & $04 / 15 / 80$ & 100 & 112 & 5.00 & 6.90 & 26.0 & -- & 25 \\
\hline 58 & $05 / 13 / 80$ & 104 & 90 & 6.00 & 6.90 & 26.0 & -- & 25 \\
\hline 58 & $06 / 16 / 80$ & 100 & 90 & 4.20 & 6.90 & 26.0 & -- & 25 \\
\hline 58 & $07 / 11 / 80$ & 102 & 90 & 4.40 & 6.90 & 26.0 & - & 25 \\
\hline 58 & $08 / 12 / 80$ & 96 & 90 & 4.00 & 6.90 & 24.0 & -- & 25 \\
\hline 58 & $09 / 15 / 80$ & 100 & 100 & 4.40 & 6.90 & 26.0 & -- & 25 \\
\hline 58 & $10 / 21 / 80$ & 104 & 90 & 4.20 & 7.00 & 22.0 & - & 30 \\
\hline 58 & $11 / 14 / 80$ & 100 & 90 & 4.40 & 6.80 & 26.0 & -- & 30 \\
\hline 58 & $12 / 16 / 80$ & 104 & 84 & 4.20 & 6.80 & 34.0 & - & 30 \\
\hline
\end{tabular}


Table 8.--Water-chemistry data from production wells, 1971-83--Continued

[Samples collected and analyzed by personnel of the Coos Bay-North Bend Water Board]

\begin{tabular}{|c|c|c|c|c|c|c|c|c|}
\hline WELL & DATE & $\begin{array}{c}\text { ALKA- } \\
\text { LINITY } \\
\text { (MG/L } \\
\text { AS } \\
\text { CACO3) }\end{array}$ & $\begin{array}{l}\text { HARD- } \\
\text { NESS } \\
\text { (MG/L } \\
\text { AS } \\
\text { CACO3) }\end{array}$ & $\begin{array}{l}\text { IRON } \\
\text { DIS- } \\
\text { SOLVED } \\
\text { (MG/L) }\end{array}$ & $\begin{array}{c}\text { PH } \\
(U N I T)\end{array}$ & $\begin{array}{l}\text { CARBON } \\
\text { DIOXIDE } \\
(M G / L)\end{array}$ & $\begin{array}{l}\text { FLUO- } \\
\text { RIDE } \\
\text { DIS- } \\
\text { SOLVED } \\
(M G / L)\end{array}$ & $\begin{array}{l}\text { CHLO- } \\
\text { RIDE } \\
\text { DIS- } \\
\text { SOLVED } \\
(M G / L)\end{array}$ \\
\hline 58 & $01 / 15 / 81$ & 100 & -- & 4.40 & 6.90 & 26.0 & -- & 25 \\
\hline 58 & $02 / 16 / 81$ & 104 & 90 & 4.60 & 6.90 & 27.0 & -- & 28 \\
\hline 58 & $03 / 16 / 81$ & 104 & 90 & 4.20 & 6.90 & 26.0 & - & 30 \\
\hline 58 & $04 / 15 / 81$ & 100 & 90 & 3.60 & 6.90 & 26.0 & -- & 30 \\
\hline 58 & $05 / 15 / 81$ & 96 & 96 & 4.00 & 6.90 & 22.0 & .10 & 32 \\
\hline 58 & $06 / 11 / 81$ & 102 & 88 & 4.20 & 6.80 & 32.0 & -- & 29 \\
\hline 58 & $07 / 14 / 81$ & 110 & 96 & 4.80 & 6.90 & 28.0 & -- & 35 \\
\hline 58 & $08 / 28 / 81$ & 102 & 96 & 4.80 & 7.00 & 21.0 & -- & 25 \\
\hline 58 & $09 / 17 / 81$ & 130 & 92 & 2.45 & 7.30 & 13.0 & -- & 35 \\
\hline 58 & $10 / 27 / 81$ & 130 & 100 & 2.20 & 7.20 & 16.5 & -- & 29 \\
\hline 58 & $11 / 19 / 81$ & 102 & 94 & 4.60 & 6.90 & 26.0 & -- & 30 \\
\hline 58 & $12 / 15 / 81$ & 100 & 90 & 4.60 & 7.10 & 16.0 & - & 26 \\
\hline 58 & $01 / 22 / 82$ & 100 & 100 & 4.60 & 6.90 & 26.0 & -- & 35 \\
\hline 58 & $05 / 20 / 82$ & 100 & 90 & 4.40 & 6.90 & 26.0 & -- & 30 \\
\hline 58 & $06 / 15 / 82$ & 100 & 84 & 5.60 & 7.00 & 21.0 & -- & 30 \\
\hline 58 & $07 / 21 / 82$ & 100 & 100 & 4.20 & 6.80 & 32.0 & -- & 35 \\
\hline 58 & $08 / 11 / 82$ & 100 & 86 & 4.40 & 6.90 & 26.0 & -- & 35 \\
\hline 58 & $09 / 23 / 82$ & 100 & 90 & 4.20 & 6.90 & 26.0 & -- & 35 \\
\hline 58 & $10 / 20 / 82$ & 106 & 106 & 4.40 & 6.90 & 27.0 & -- & 28 \\
\hline 58 & $11 / 19 / 82$ & 104 & 96 & 4.10 & 6.90 & 26.0 & -- & 25 \\
\hline 58 & $12 / 20 / 82$ & 104 & 88 & 4.30 & 6.80 & 33.0 & -- & 35 \\
\hline 58 & $01 / 17 / 83$ & 102 & 90 & 4.40 & 6.90 & 27.0 & - & 30 \\
\hline 58 & $02 / 16 / 83$ & 102 & 80 & 4.20 & 6.90 & 26.0 & -- & 27 \\
\hline
\end{tabular}


Table 8.--Water-chemistry data from production wells, 1971-83--Continued

[Samples collected and analyzed by personnel of the Coos Bay-North Bend Water Board]

\begin{tabular}{|c|c|c|c|c|c|c|c|c|}
\hline WELL & DATE & $\begin{array}{l}\text { ALKA- } \\
\text { LINITY } \\
\text { (MG/L } \\
\text { AS } \\
\text { CACO3) }\end{array}$ & $\begin{array}{l}\text { HARD- } \\
\text { NESS } \\
\text { (MG/L } \\
\text { AS } \\
\text { CACO3) }\end{array}$ & $\begin{array}{l}\text { IRON } \\
\text { DIS- } \\
\text { SOLVED } \\
\text { (MG/L) }\end{array}$ & $\begin{array}{c}\mathrm{PH} \\
(\mathrm{UNIT})\end{array}$ & $\begin{array}{l}\text { CARBON } \\
\text { DIOXIDE } \\
(M G / L)\end{array}$ & $\begin{array}{l}\text { FLUO- } \\
\text { RIDE } \\
\text { DIS- } \\
\text { SOLVED } \\
(M G / L)\end{array}$ & $\begin{array}{l}\text { CHLO- } \\
\text { RIDE } \\
\text { DIS- } \\
\text { SOLVED } \\
(M G / L)\end{array}$ \\
\hline $\begin{array}{l}58 \\
58\end{array}$ & $\begin{array}{l}03 / 18 / 83 \\
04 / 26 / 83\end{array}$ & $\begin{array}{l}104 \\
104\end{array}$ & $\begin{array}{r}90 \\
112\end{array}$ & $\begin{array}{l}4.20 \\
4.00\end{array}$ & $\begin{array}{l}6.90 \\
6.90\end{array}$ & $\begin{array}{l}26.0 \\
26.0\end{array}$ & -- & $\begin{array}{l}28 \\
32\end{array}$ \\
\hline 58 & $05 / 16 / 83$ & 102 & 100 & 3.00 & 6.90 & 26.0 & -- & 35 \\
\hline 58 & $06 / 22 / 83$ & 106 & 90 & 4.75 & 6.90 & 27.0 & -- & 26 \\
\hline 59 & $05 / 15 / 81$ & 120 & 110 & .35 & 7.30 & 12.0 & -- & 35 \\
\hline 59 & $06 / 11 / 81$ & 114 & 94 & 2.30 & 7.10 & 18.0 & .40 & 23 \\
\hline 59 & $07 / 14 / 81$ & 100 & 90 & 3.00 & 7.10 & 16.0 & - & 30 \\
\hline 59 & $08 / 28 / 81$ & 96 & 80 & 3.00 & 7.20 & 22.0 & -- & 25 \\
\hline 59 & $09 / 17 / 81$ & 104 & 40 & 3.50 & 7.10 & 14.0 & -- & 22 \\
\hline 59 & $10 / 27 / 81$ & 104 & 82 & 3.70 & 7.10 & 17.0 & -- & 18 \\
\hline 59 & $11 / 19 / 81$ & 90 & 72 & 3.70 & 7.00 & 16.0 & -- & 20 \\
\hline 59 & $12 / 15 / 81$ & 92 & 84 & 3.20 & 7.10 & 14.5 & -- & 20 \\
\hline 59 & $01 / 22 / 82$ & 90 & 80 & 3.40 & 6.90 & 24.0 & -- & 30 \\
\hline 59 & $05 / 20 / 82$ & 84 & 70 & 3.40 & 6.90 & 22.0 & -- & 20 \\
\hline 59 & $06 / 15 / 82$ & 84 & 80 & 3.40 & 7.10 & 14.0 & -- & 20 \\
\hline 59 & $07 / 21 / 82$ & 82 & 70 & 3.20 & 7.00 & 11.0 & -- & 26 \\
\hline 59 & $08 / 11 / 82$ & 82 & 68 & 3.00 & 7.00 & 17.0 & -- & 26 \\
\hline 59 & $09 / 23 / 82$ & 80 & 74 & 3.20 & 7.00 & 16.0 & -- & 25 \\
\hline 59 & $10 / 20 / 82$ & 80 & 68 & 3.20 & 6.90 & 20.0 & -- & 18 \\
\hline 59 & $11 / 19 / 82$ & 86 & 76 & 3.20 & 6.90 & 22.0 & -- & 21 \\
\hline 59 & $12 / 20 / 82$ & 86 & 69 & 2.90 & 7.00 & 17.0 & -- & 24 \\
\hline 59 & $01 / 17 / 83$ & 84 & 70 & 3.20 & 7.00 & 17.0 & -- & 20 \\
\hline 59 & $02 / 16 / 83$ & 84 & 72 & 3.20 & 7.10 & 12.0 & -- & 20 \\
\hline 59 & $03 / 18 / 83$ & 84 & 92 & 3.00 & 7.00 & 17.0 & -- & 20 \\
\hline
\end{tabular}


Table 8.--Water-chemistry data from production wells, 1971-83--Continued

[Samples collected and analyzed by personnel of the Coos Bay-North Bend Water Board]

\begin{tabular}{|c|c|c|c|c|c|c|c|c|}
\hline WELL & DATE & $\begin{array}{c}\text { ALKA- } \\
\text { LINITY } \\
\text { (MG/L } \\
\text { AS } \\
\text { CACO3) }\end{array}$ & $\begin{array}{l}\text { HARD- } \\
\text { NESS } \\
(M G / L \\
\text { AS } \\
\text { CACO3) }\end{array}$ & $\begin{array}{l}\text { IRON } \\
\text { DIS- } \\
\text { SOLVED } \\
\text { (MG/L) }\end{array}$ & $\begin{array}{c}\text { PH } \\
(\text { UNIT) }\end{array}$ & $\begin{array}{l}\text { CARBON } \\
D I O X I D E \\
(M G / L)\end{array}$ & $\begin{array}{l}\text { FLUO- } \\
\text { RIDE } \\
\text { DIS- } \\
\text { SOLVED } \\
\text { (MG/L) }\end{array}$ & $\begin{array}{l}\text { CHLO- } \\
\text { RIDE } \\
\text { DIS- } \\
\text { SOLVED } \\
(M G / L)\end{array}$ \\
\hline 59 & $04 / 26 / 83$ & 84 & 80 & 3.00 & 6.90 & 21.0 & -- & 25 \\
\hline 59 & $05 / 16 / 83$ & 84 & 88 & 2.00 & 7.00 & 18.0 & -- & 25 \\
\hline 59 & $06 / 22 / 83$ & 90 & 76 & 3.60 & 7.00 & 18.0 & - & 17 \\
\hline 60 & $02 / 27 / 80$ & 120 & 90 & .25 & 7.60 & 6.0 & .12 & 37 \\
\hline $\begin{array}{l}60 \\
60\end{array}$ & $\begin{array}{l}05 / 15 / 81 \\
06 / 11 / 81\end{array}$ & $\begin{array}{l}122 \\
142\end{array}$ & $\begin{array}{r}96 \\
100\end{array}$ & $\begin{array}{l}.15 \\
.23\end{array}$ & $\begin{array}{l}7.50 \\
7.30\end{array}$ & $\begin{array}{r}8.0 \\
14.0\end{array}$ & $\begin{array}{r}.12 \\
--\end{array}$ & $\begin{array}{l}45 \\
50\end{array}$ \\
\hline 60 & $07 / 14 / 81$ & 145 & 140 & .30 & 7.50 & 9.2 & -- & 55 \\
\hline 60 & $08 / 28 / 81$ & 140 & 104 & .30 & 7.50 & 9.0 & - & 45 \\
\hline 60 & $09 / 17 / 81$ & 160 & 104 & .05 & 7.50 & 10.0 & -- & 46 \\
\hline 60 & $10 / 27 / 81$ & 164 & 100 & .28 & 7.50 & 10.6 & -- & 39 \\
\hline 60 & $11 / 19 / 81$ & 150 & 108 & .45 & 7.50 & 9.2 & -- & 40 \\
\hline 60 & $12 / 15 / 81$ & 142 & 102 & .32 & 7.50 & 9.0 & -- & 45 \\
\hline 60 & $01 / 22 / 82$ & 145 & 120 & .40 & 6.90 & 9.1 & -- & 45 \\
\hline 60 & $05 / 20 / 82$ & 145 & 96 & .32 & 7.50 & 9.0 & - & 45 \\
\hline 60 & $06 / 15 / 82$ & 140 & 100 & 3.00 & 7.50 & 9.0 & - & 40 \\
\hline 60 & $07 / 21 / 82$ & 142 & 90 & 1.70 & 7.50 & 9.0 & -- & 45 \\
\hline 60 & $08 / 11 / 82$ & 140 & 90 & .38 & 7.50 & 8.6 & - & 46 \\
\hline 60 & $09 / 23 / 82$ & 140 & 96 & .35 & 7.50 & 8.5 & -- & 40 \\
\hline 60 & $10 / 20 / 82$ & 150 & 88 & .39 & 7.50 & 9.5 & -- & 36 \\
\hline 60 & $11 / 19 / 82$ & 154 & 92 & .22 & 7.40 & 12.0 & -- & 36 \\
\hline 60 & $12 / 20 / 82$ & 145 & 90 & .40 & 7.50 & 9.0 & -- & 45 \\
\hline 60 & $01 / 17 / 83$ & 142 & 94 & .35 & 7.50 & 9.0 & -- & 38 \\
\hline 60 & $02 / 16 / 83$ & 140 & 90 & .80 & 7.50 & 9.0 & - & 38 \\
\hline 60 & $03 / 18 / 83$ & 138 & 96 & .32 & 7.50 & 8.80 & -- & 35 \\
\hline 60 & $04 / 26 / 83$ & 138 & 100 & .32 & 7.30 & 14.0 & -- & 40 \\
\hline 60 & $05 / 16 / 83$ & 138 & 124 & .28 & 7.50 & 8.9 & -- & 40 \\
\hline 60 & $06 / 22 / 83$ & 138 & 86 & .44 & 7.50 & 8.9 & - & 37 \\
\hline
\end{tabular}


Table 9.--Miscellaneous water-chemistry data from wells in the study area.

\begin{tabular}{|c|c|c|c|c|c|c|c|c|c|c|c|}
\hline \multirow[b]{3}{*}{ WELL } & \multirow[b]{3}{*}{ DATE } & \multirow[b]{3}{*}{ TIME } & \multirow{2}{*}{\multicolumn{2}{|c|}{ ELEVATION }} & \multirow{3}{*}{$\begin{array}{r}\text { ARSENIC } \\
(M G / L)\end{array}$} & \multirow{3}{*}{$\begin{array}{l}\text { BORON } \\
(M G / L)\end{array}$} & \multicolumn{2}{|c|}{ DISSOLVED } & \multirow{3}{*}{$\begin{array}{c}\text { BROMIDE } \\
(M G / L)\end{array}$} & \multirow{3}{*}{$\begin{array}{l}\text { STRON } \\
\text { TIUM } \\
\text { (MG/L) }\end{array}$} & \multirow{3}{*}{$\begin{array}{r}\text { ORGAN } \\
\text { CARB } \\
\text { (MG/ }\end{array}$} \\
\hline & & & & & & & $\begin{array}{l}\text { MANGA- } \\
\text { NESE }\end{array}$ & & & & \\
\hline & & & (FEET) & (METER) & & & $(M G / L)$ & $(M G / L)$ & & & \\
\hline 3 & $08 / 11 / 77$ & -- & $191 /$ & -- & .015 & .030 & .040 & -- & -- & -- & -- \\
\hline 7 & $08 / 10 / 77$ & -- & $21 \overline{1} /$ & -- & .001 & $<.020$ & $<.010$ & -- & -- & -- & -- \\
\hline 10 & $08 / 11 / 77$ & -- & 2011 & -- & .031 & .040 & .090 & -- & -- & -- & -- \\
\hline 11 & $08 / 11 / 77$ & -- & $20 \overline{1} /$ & -- & .019 & .040 & .070 & -- & -- & -- & $\ldots$ \\
\hline 15 & $08 / 12 / 77$ & -- & $19 \overline{1 /}$ & -- & .020 & .030 & .080 & -- & -- & -- & -- \\
\hline 21 & $08 / 12 / 77$ & -- & $20 \overline{1 /}$ & -- & .019 & $<.020$ & .060 & -- & -- & -- & -- \\
\hline 26 & $08 / 15 / 77$ & -- & $19 \overline{1 /}$ & -- & .007 & .030 & .030 & -- & -- & -- & -- \\
\hline 32 & $08 / 15 / 77$ & -- & $20 \overline{1} /$ & -- & .021 & $<.020$ & .040 & -- & -- & -- & -- \\
\hline 33 & $08 / 16 / 77$ & -- & $20 \overline{11}$ & -- & $<.001$ & .030 & $<.010$ & -- & -- & -- & -- \\
\hline 418 & $08 / 05 / 77$ & 1440 & $19 \overline{1} /$ & -- & .028 & $<.020$ & .040 & -- & -- & -- & -- \\
\hline 42 & $08 / 17 / 77$ & 1205 & 19 II & -- & .005 & .030 & .200 & -- & -- & -- & -- \\
\hline 44 & $08 / 18 / 77$ & 1130 & $181 /$ & -- & .002 & .030 & .080 & -- & -- & -- & -- \\
\hline 45 & $08 / 18 / 77$ & 1435 & $20 \overline{11}$ & -- & .034 & .030 & .040 & -- & -- & -- & -- \\
\hline 46 & $08 / 18 / 77$ & 1555 & $19 \overline{1} /$ & -- & .020 & $<.020$ & .080 & -- & -- & -- & -- \\
\hline 47 & $08 / 18 / 77$ & 1330 & $19 \overline{1 /}$ & -- & .033 & .030 & .040 & -- & -- & -- & -- \\
\hline 48 & $08 / 22 / 77$ & 1450 & $21 \overline{1} /$ & -- & .050 & $<.020$ & .080 & -- & -- & -- & -- \\
\hline 49 & $08 / 23 / 77$ & 1315 & $19 \overline{1 /}$ & -- & .016 & $<.020$ & .060 & -- & -- & -- & -- \\
\hline 50 & $08 / 23 / 77$ & 1605 & $20 \overline{11}$ & -- & .010 & $<.020$ & .060 & -- & -- & -- & -- \\
\hline $51 \mathrm{~A}$ & $08 / 24 / 77$ & 1130 & $15 \overline{1 /}$ & -- & .017 & .030 & .050 & -- & -- & -- & -- \\
\hline $51 \mathrm{~B}$ & $08 / 24 / 77$ & 1320 & $18 \overline{1} /$ & -- & .019 & $<.020$ & .040 & -- & -- & -- & -- \\
\hline 52 & $08 / 29 / 77$ & 1035 & $19 \overline{1} /$ & -- & .003 & $<.020$ & .030 & -- & -- & -- & -- \\
\hline B1 & $10 / 21 / 81$ & 1717 & -159 & -48.5 & -- & .810 & -- & -- & 7.60 & -- & -- \\
\hline B3 & $05 / 19 / 82$ & 1254 & -16 & -4.9 & -- & -- & - & -- & -- & .070 & -- \\
\hline B3 & $05 / 19 / 82$ & 1201 & -31 & -9.4 & -- & -- & -- & -- & -- & .060 & -- \\
\hline B3 & $05 / 19 / 82$ & 1600 & -51 & -15.5 & -- & -- & -- & -- & -- & .110 & -- \\
\hline B3 & $05 / 19 / 82$ & 1010 & -63 & -19.2 & -- & -- & -- & -- & -- & .130 & -- \\
\hline B3 & $05 / 18 / 82$ & 1639 & -81 & -24.7 & -- & -- & -- & -- & -- & .160 & -- \\
\hline B3 & $05 / 18 / 82$ & 1934 & -87 & -26.5 & -- & -- & -- & - & -- & .160 & -- \\
\hline B3 & $05 / 18 / 82$ & 1739 & -110 & -33.5 & -- & -- & -- & -- & -- & .160 & -- \\
\hline B3 & $05 / 18 / 82$ & 1551 & -135 & -41.1 & -- & -- & -- & -- & -- & .140 & -- \\
\hline B3 & $05 / 18 / 82$ & 1501 & -153 & -46.6 & -- & -- & -- & -- & -- & .160 & -- \\
\hline B3 & $05 / 18 / 82$ & 1340 & -159 & -48.5 & -- & -- & -- & -- & -- & .170 & -- \\
\hline B3 & $05 / 18 / 82$ & 1231 & -171 & -52.1 & -- & -- & -- & -- & -- & .460 & -- \\
\hline B4 & $12 / 04 / 79$ & 0930 & -- & -- & .010 & .090 & .007 & -- & -- & -- & -- \\
\hline B4 & $11 / 21 / 79$ & 0830 & -157 & -- & .010 & .120 & .007 & -- & -- & - & -- \\
\hline HB & $06 / 03 / 75$ & 0820 & -- & -- & -- & .004 & .050 & -- & -- & -- & -- \\
\hline$H B$ & $06 / 02 / 75$ & 2100 & -- & -- & -- & $<.020$ & .040 & -- & -- & -- & -- \\
\hline P1 & $10 / 18 / 78$ & 1450 & 6 & 1.8 & -- & -- & -- & -- & .30 & -- & -- \\
\hline P1 & $10 / 18 / 78$ & 1505 & 2 & 0.6 & -- & -- & -- & -- & .10 & -- & -- \\
\hline P1 & $10 / 18 / 78$ & 1515 & -2 & -0.6 & -- & -- & -- & - & .10 & -- & -- \\
\hline P1 & $10 / 18 / 78$ & 1525 & -6 & -1.8 & -- & -- & -- & -- & .10 & -- & -- \\
\hline$P 1$ & $10 / 18 / 78$ & 1535 & -10 & -3.0 & -- & -- & -- & .020 & .20 & -- & -- \\
\hline P1 & $10 / 18 / 78$ & 1545 & -14 & -4.3 & -- & -- & -- & -- & .10 & -- & -- \\
\hline P1 & $10 / 18 / 78$ & 1555 & -18 & -5.5 & -- & -- & -- & -- & .10 & -- & -- \\
\hline $\mathrm{P} 1$ & $10 / 18 / 78$ & 1605 & -22 & -6.7 & -- & -- & -- & -- & .10 & -- & -- \\
\hline P1 & $10 / 18 / 78$ & 1458 & -26 & -7.9 & -- & -- & .070 & .010 & .10 & -- & 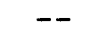 \\
\hline
\end{tabular}


Table 9.--Miscel laneous water-chemistry data from wells in the study area.--Continued

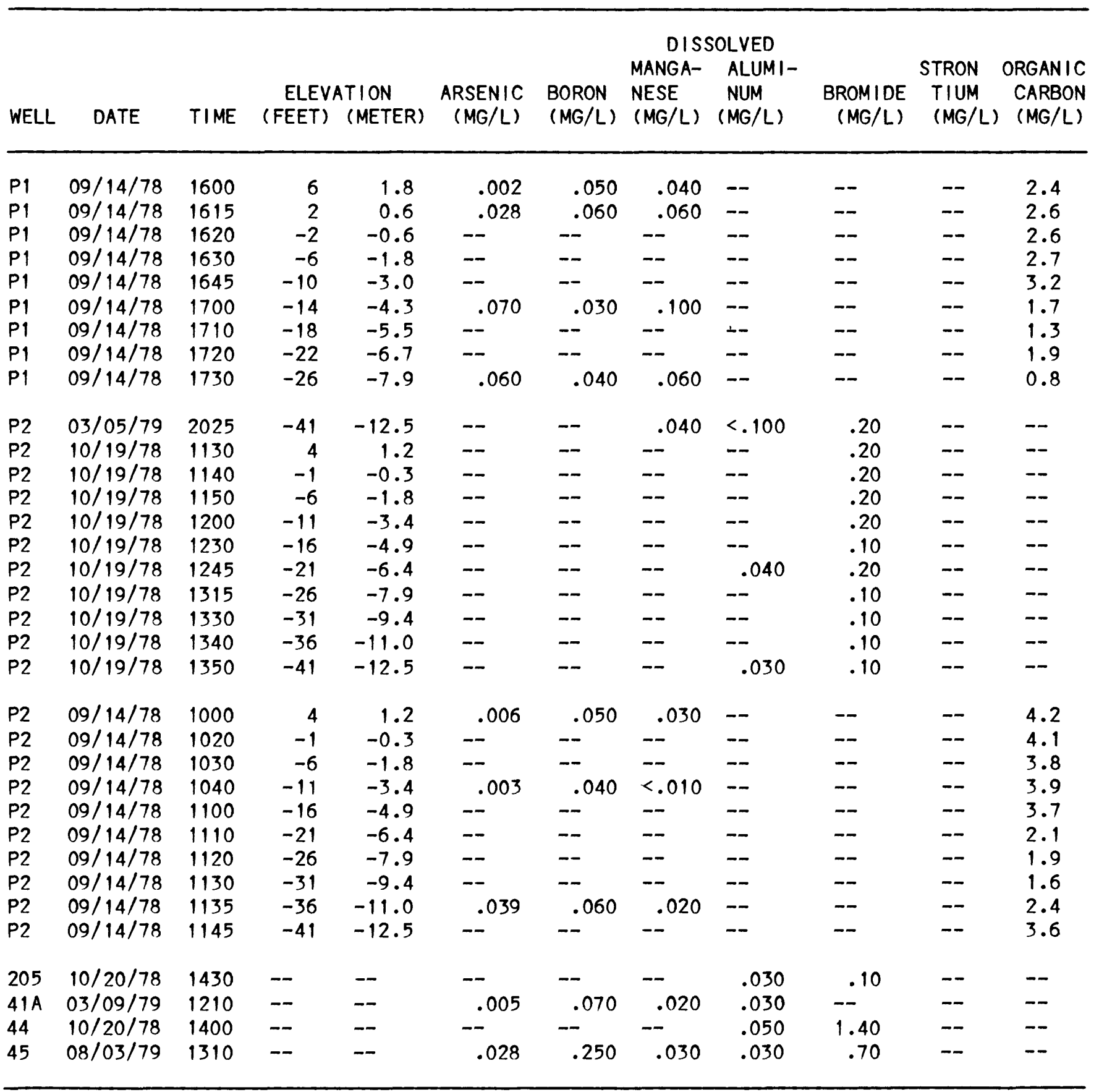

1/ Sampling depth (elevations not available). 
r.Updated from Robison, 1973, table 67

\begin{tabular}{|c|c|c|c|c|c|c|c|c|c|c|}
\hline \multirow[b]{2}{*}{$\begin{array}{l}\text { Well } \\
\text { number }\end{array}$} & \multicolumn{2}{|c|}{ Year } & \multirow[b]{2}{*}{$\begin{array}{l}\text { Alti- } \\
\text { tude } \\
(f+)\end{array}$} & \multicolumn{2}{|c|}{$\begin{array}{c}\text { Well screens } \\
\text { interval }\end{array}$} & \multirow[b]{2}{*}{$\begin{array}{l}\text { Total } \\
\text { length } \\
\text { (feet) }\end{array}$} & \multirow{2}{*}{$\begin{array}{c}\text { Total } \\
\text { aquifer } \\
\text { thickness } \\
\text { (feet) }\end{array}$} & \multicolumn{3}{|c|}{$\begin{array}{l}\text { Conducted or } \\
\text { reported test }\end{array}$} \\
\hline & Drilled & $\begin{array}{l}\text { Routine } \\
\text { opera- } \\
\text { tion }\end{array}$ & & $\begin{array}{l}\text { Depth } \\
(\text { feet) }\end{array}$ & $\begin{array}{c}\text { Alti tude } \\
(f+\text { below } \\
\text { msl) }\end{array}$ & & & $\begin{array}{l}\text { Rate } \\
\text { gpm }\end{array}$ & $\begin{array}{l}\text { Draw- } \\
\text { down } \\
(f+)\end{array}$ & $\begin{array}{l}\text { Specific } \\
\text { capacity } \\
\text { gpm }(f+)\end{array}$ \\
\hline 41 & 1968 & 1970 & 24 & $57-104$ & $33-80$ & 47 & 98 & 485 & 20.9 & 23 \\
\hline 42 & 1968 & & 22 & $52-100$ & $30-78$ & 48 & 125 & 350 & 19.2 & 18 \\
\hline 43 & 1968 & 1970 & 20 & $\begin{array}{r}71-108 \\
150-171\end{array}$ & $\begin{array}{c}51-88 \\
130-151\end{array}$ & $\begin{array}{l}58^{*} \\
21^{* *}\end{array}$ & $\begin{array}{l}170 \\
170\end{array}$ & $\begin{array}{l}466 \\
391\end{array}$ & $\begin{array}{l}17.1 \\
35.3\end{array}$ & $\begin{array}{l}27^{*} \\
11^{* *}\end{array}$ \\
\hline 44 & 1968 & 1970 & 22 & $\begin{array}{r}87-119 \\
159-185\end{array}$ & $\begin{array}{c}65-97 \\
137-163\end{array}$ & $\begin{array}{l}58^{*} \\
26^{* *}\end{array}$ & $\begin{array}{l}170 \\
170\end{array}$ & $\begin{array}{l}438 \\
426\end{array}$ & $\begin{array}{l}12.6 \\
21.4\end{array}$ & $\begin{array}{l}34.9^{*} \\
20^{* *}\end{array}$ \\
\hline 45 & 1968 & 1970 & 18 & $\begin{array}{c}69-84 \\
108-130 \\
154-176\end{array}$ & $\begin{array}{c}51-66 \\
90-112 \\
136-159\end{array}$ & 59 & 175 & 390 & 9.9 & 40 \\
\hline 46 & 1968 & 1970 & 17 & $\begin{array}{r}80-111 \\
159-180\end{array}$ & $\begin{array}{c}63-94 \\
142-163\end{array}$ & 52 & 175 & 540 & 24.2 & 22 \\
\hline 47 & 1957 & 1961 & 24 & $56-135$ & $32-111$ & 79 & 125 & 200 & 35.5 & 5.6 \\
\hline 48 & 1957 & 1961 & 26 & $82-134$ & $56-108$ & 52 & 125 & 150 & 11.1 & 13.6 \\
\hline 49 & 1957 & 1961 & 23 & $79-130$ & $55-107$ & 52 & 125 & 190 & 18.8 & 10 \\
\hline & 1957 & 1961 & 23 & $78-130$ & $55-107$ & 52 & 125 & 186 & 56.6 & $3.3 * * *$ \\
\hline 50 & 1960 & 1961 & 25 & $63-120$ & $38-05$ & 57 & 125 & 407 & 22.4 & 18 \\
\hline 51 & 1960 & 1961 & 21 & $\begin{array}{c}61-81 \\
104-135\end{array}$ & $\begin{array}{l}40-60 \\
83-114\end{array}$ & 51 & 155 & 307 & 21.0 & 15 \\
\hline 52 & 1960 & 1961 & 22 & $\begin{array}{c}60-85 \\
140-170\end{array}$ & $\begin{array}{c}38-63 \\
118-148\end{array}$ & 55 & 200 & 355 & 15.5 & 23 \\
\hline 53 & 1968 & 1968 & 23 & $56-97$ & $33-74$ & 41 & 110 & 280 & 14.9 & 19 \\
\hline 54 & 1968 & 1969 & 23 & $55-102$ & $32-79$ & 47 & 98 & 280 & 13.9 & 20 \\
\hline 55 & 1968 & 1968 & 24 & $53-97$ & $29-73$ & 44 & 98 & 280 & 11.8 & 24 \\
\hline 56 & 1968 & 1968 & 26 & $58-100$ & $32-74$ & 42 & 94 & 280 & 14.8 & 19 \\
\hline 57 & 1968 & 1968 & 23 & $58-100$ & $35-77$ & 42 & 102 & 310 & 15.0 & 21 \\
\hline 58 & 1968 & 1968 & 24 & $63-111$ & $39-97$ & 48 & 110 & 350 & 19.0 & 18 \\
\hline 59 & 1980 & 1981 & 24 & $83-130$ & $59-106$ & 47 & 135 & 450 & 17.5 & 26 \\
\hline 60 & 1980 & 1981 & 64 & $123-155$ & $59-91$ & 32 & 160 & 450 & 27.0 & 17 \\
\hline
\end{tabular}

* Upper well screen was sleeved off, October 1981; specific capacity measured prior to sleeving.

** Lower screen only; specific capacity measured in October 1981.

*** Specific capacity measured May 13, 1981. 
Table 11.--Construction data for profile and test wells in the coastal dunes aquifer.

\begin{tabular}{|c|c|c|c|c|c|}
\hline Well & $\begin{array}{l}\text { Summarized } \\
\text { description }\end{array}$ & $\begin{array}{l}\text { Range in el } \\
\text { of tube so } \\
\text { or well scre }\end{array}$ & $\begin{array}{l}\text { levation } \\
\text { creens } \\
\text { een (feet) }\end{array}$ & $\begin{array}{l}\text { Drilling } \\
\text { method }\end{array}$ & $\begin{array}{l}\text { Approximate } \\
\text { date } \\
\text { completed }\end{array}$ \\
\hline \multicolumn{6}{|c|}{ Horstall Beach Section } \\
\hline B1 & $\begin{array}{l}\text { 32 tube screens, .25-inch 1.D. } \\
\text { central } 1.25 \text {-inch PVC pipe with screen }\end{array}$ & $\begin{array}{r}-3 \text { to } \\
-142 \text { to }\end{array}$ & $\begin{array}{l}-223 \\
-147\end{array}$ & cable tool & $8 / 79$ \\
\hline B2 & $\begin{array}{l}30 \text { tube screens, } .25-\text { inch } 1.0 \text {. } \\
\text { central } 1.25 \text {-inch PVC pipe with screen }\end{array}$ & $\begin{array}{r}0 \text { to } \\
-146 \text { to }\end{array}$ & $\begin{array}{l}-179 \\
-151\end{array}$ & do. & $9 / 79$ \\
\hline B3 & $\begin{array}{l}33 \text { tube screens, } 25-\text { inch } 1.0 \text {. } \\
\text { central } 1.25-\text { inch PVC pipe with screen }\end{array}$ & $\begin{array}{r}+4 \text { to } \\
-147 \text { to }\end{array}$ & $\begin{array}{l}-210 \\
-153\end{array}$ & do. & $10 / 79$ \\
\hline B4 & $\begin{array}{l}10 \text { inch casing with well screen } \\
\text { well modified in December } 1979\end{array}$ & $\begin{array}{l}-152 \text { to } \\
-147 \text { to }\end{array}$ & $\begin{array}{l}-162 \\
-162\end{array}$ & $\begin{array}{l}\text { do. } \\
\text { do. }\end{array}$ & $\begin{array}{r}9 / 79 \\
12 / 79\end{array}$ \\
\hline B5 & $\begin{array}{l}27 \text { tube screens, } 25-\text { inch } 1.0 \text {. } \\
\text { central } 1.25 \text {-inch PVC pipe with screen }\end{array}$ & $\begin{array}{r}+10 \text { to } \\
-157 \text { to }\end{array}$ & $\begin{array}{l}-170 \\
-162\end{array}$ & do. & $8 / 81$ \\
\hline P1 & 9 tube screens, .25-inch I.D. & +6 to & -26 & jetting rig & $6 / 78$ \\
\hline P2 & 10 tube screens, .25-inch I.D. & +4 to & -41 & do. & $8 / 78$ \\
\hline P3 & 11 tube screens, .25-inch 1.0. & +13 to & -27 & do. & do. \\
\hline P4 & 11 tube screens, .25-inch 1.0. & +11 to & -29 & do. & do. \\
\hline P5 & 10 tube screens, .25-inch I.D. & +6 to & -35 & do. & $9 / 78$ \\
\hline P6 & 9 tube screens, .25-inch 1.0. & +5 to & -31 & do. & do. \\
\hline P7 & 7 tube screens, .25-inch 1.D. & +8 to & -18 & do. & do. \\
\hline P41A & $\begin{array}{l}2 \text { inch galvanized pipe } \\
\text { with } 3-f t \text { well point }\end{array}$ & -129 to & -132 & cable tool & 1968 \\
\hline P44 & $\begin{array}{l}2 \text { inch galvanized pipe } \\
\text { with 3-ft well point }\end{array}$ & -138 to & -141 & do. & do. \\
\hline P45 & $\begin{array}{l}2 \text { inch galvanized pipe } \\
\text { with 3-ft well point }\end{array}$ & -138 to & -141 & do. & do. \\
\hline 205 & $\begin{array}{l}2 \text { inch galvanized pipe } \\
\text { with } 3-f t \text { well point }\end{array}$ & -138 to & -141 & cable tool & 1957 \\
\hline HB & 10 inch casing with well screen & -20 to & -35 & do. & 1975 \\
\hline $100 \mathrm{~W}$ & $\begin{array}{l}1.25 \text { to } 2 \text { inch galvanized pipe } \\
\text { with } 3-f t \text { well point }\end{array}$ & -9 to & -12 & jetting rig & do. \\
\hline $200 \mathrm{~N}$ & $\begin{array}{l}1.25 \text { to } 2 \text { inch galvanized pipe } \\
\text { with } 3-f+\text { well point }\end{array}$ & -12 to & -15 & do. & do. \\
\hline $200 W$ & $\begin{array}{l}1.25 \text { to } 2 \text { inch galvanized pipe } \\
\text { with } 3-f+\text { well point }\end{array}$ & -9 to & -12 & do. & do. \\
\hline \multirow[t]{2}{*}{$400 W$} & $\begin{array}{l}1.25 \text { to } 2 \text { inch galvanized pipe } \\
\text { with } 3-f t \text { well point }\end{array}$ & -5 to & -8 & do. & do. \\
\hline & North Coastal Section & west of Saun & nders Lake & & \\
\hline NTW & $\begin{array}{l}10 \text { inch casing with stainless } \\
\text { well screen }\end{array}$ & -16 to & -26 & cable tool & 1978 \\
\hline $100 E$ & $\begin{array}{l}1.25 \text { to } 2 \text { inch galvanized pipe } \\
\text { with } 3-f t \text { well point }\end{array}$ & -10 to & -13 & jetting rig & do. \\
\hline $100 \mathrm{~S}$ & $\begin{array}{l}1.25 \text { to } 2 \text { inch galvanized pipe } \\
\text { with } 3-f t \text { well point }\end{array}$ & -21 to & -24 & do. & do. \\
\hline $100 \mathrm{~W}$ & do. & -22 to & -25 & do. & do. \\
\hline $250 W$ & $\begin{array}{l}1.25 \text { to } 2 \text { inch galvanized pipe } \\
\text { with } 3-f t \text { well point }\end{array}$ & -6 to & -9 & do. & do. \\
\hline $400 \mathrm{~N}$ & $\begin{array}{l}1.25 \text { to } 2 \text { inch galvanized pipe } \\
\text { with } 3-f+\text { well point }\end{array}$ & 0 to & -3 & do. & do. \\
\hline TIDE & $\begin{array}{l}1.25 \text { to } 2 \text { inch galvanized pipe } \\
\text { with } 3-f t \text { well point }\end{array}$ & -3 to & -6 & do. & do. \\
\hline P8 & 10 tube screens, .25-inch 1.0. & +6 to & -39 & do. & $7 / 79$ \\
\hline P11 & 7 tube screens, .25-inch I.D. & 8 to & -22 & do. & $8 / 79$ \\
\hline
\end{tabular}

